\title{
The Index Bundle for Gap-Continuous Families, Morse-Type Index Theorems and Bifurcation
}

\author{
Dissertation \\ zur Erlangung des mathematisch-naturwissenschaftlichen Doktorgrades \\ "Doctor rerum naturalium" \\ der Georg-August-Universität Göttingen
}

vorgelegt von

Nils Waterstraat

aus Wolfsburg

Göttingen 2011 
Referent: Prof. Dr. Thomas Schick

Korreferent: Prof. Dr. Ralf Meyer

Tag der mündlichen Prüfung: 31.10.2011 
To the memory of Margarete Waterstraat 


\section{Introduction}

Unbounded linear operators arise naturally in the study of differential equations and hence are often motivated from physical and geometrical problems. In many important cases the obtained abstract operators turn out to be Fredholm and formulas for their corresponding indices are part of deep theorems in mathematics like the Atiyah-Singer index theorem for elliptic differential operators on closed manifolds with all its caused insight of modern geometry.

A particular extension of this index theory can be obtained by considering families of differential equations and, accordingly, families of Fredholm operators acting between linear spaces. By a construction that has been developed independently by Atiyah and Jänich, the integer valued Fredholm index can now be replaced by a $K$-theory class of the parameter space which is called the index bundle. Moreover, Atiyah and Singer proved that also their index formula for elliptic differential operators on closed manifolds generalises to this situation (cf. [LM89]). However, as the ordinary Fredholm index, the index bundle vanishes for families of selfadjoint Fredholm operators. But Atiyah and Singer constructed a variant of the index bundle and later proved in collaboration with Patodi that also the Atiyah-Singer index theorem, appropriately restated, continuous to be true for selfadjoint operators (cf. [APS76]). Moreover, they observed that if the families of selfadjoint Fredholm operators are parametrised by a circle, then the selfadjoint index bundle can be identified with an integer that has an interpretation in terms of the spectra of the operators. This so called spectral flow is nowadays a popular concept in mathematics having many applications like for instance in index theory and Floer homology but also in more applied mathematics (cf. [FPR99] and the references given there). Further, besides its original definition by means of the selfadjoint index bundle, it has been redefined in analytical ways (cf. [Ph96], FPR99]).

When talking about families we need first of all a topology on the underlying spaces of operators in order to make sense of continuity. The index bundle constructions we have mentioned so far are all restricted to norm continuous families of bounded operators. Accordingly, in the index theorems it is assumed that the considered differential operators can be transformed into norm continuous families of bounded operators in a certain way. This particular assumption can often be verified easily by assuming that the differential operators act on bundles over closed manifolds and so have typically a constant domain space. However, these transformations become usually much more involved when considering, for example, families of boundary value problems hav- 
ing varying boundary conditions (cf. $\mathrm{Ni07}$ ). On the other hand, the set of all not necessarily bounded but closed operators acting between two given Banach spaces can be turned canonically into a metric space by the so called gap metric which has been studied intensively during the last decades. Since by definition any Fredholm operator is closed, we obtain that the space of generally unbounded Fredholm operators actig between two Banach spaces is a metric space. Moreover, all types of families we have mentioned above are continuous with respect to this metric.

The aim of this thesis is to extend the construction of the index bundle and its selfadjoint variant to families of generally unbounded Fredholm operators that are just assumed to be continuous with respect to the gap metric. On our way we define an index bundle for Fredholm morphisms between Banach bundles and prove two index theorems for families of boundary value problems of ordinary differential operators. Furthermore we extend results from bifurcation theory of nonlinear operator equations that were obtained by the classical index bundle to equations involving unbounded operators.

We now give a detailed exposition of the content of the thesis and its structure.

In the first part we provide some preliminaries which are mainly known but apparently not well-established. In the first chapter we study Banach bundles and their morphisms, where we mainly follow the presentation in the survey article [ZKKP75] but include rigorous proofs and adapt the presentation to our purposes. In particular we introduce some results that can not be found in [ZKKP75] but will be needed in later sections. The main result of this chapter asserts that any Banach bundle over a paracompact base space having an infinite dimensional model space has a nowhere vanishing section. Moreover, we draw several conclusions which are as surprising as this result itself and that will be needed later in the definition of the index bundle. The second chapter is devoted to the metric space of closed operators with respect to the gap distance where our main reference is the famous monograph Ka76. After its definition and some basic observations in the first section, we prove an estimate for bounded perturbations of closed operators and discuss its consequences in the second section. In particular we show the continuity of certain inclusions of the space of bounded operators into the closed operators which played a role in the development of spectral flow some years ago. In the third section we turn towards spectral theory and the stability of spectra with respect to the gap topology. Besides the upper semicontinuity of the whole spectrum we also show the continuity of eigenvalues of finite multiplicity and the upper semicontinuity of the essential spectrum. Our results in this second and third section can either be found in Ka76] or we expect them to be well known even though we could not find suitable references in the literature. However, at least our methods in the proofs in the third section may be of some originality since they carry over techniques from the corresponding study of selfadjoint operators in BLP05 to general closed operators acting on Banach spaces. In a final fourth section of this second chapter we prove a well known formula that computes the gap distance of two closed operators acting on a Hilbert space in terms of the 
norms of the orthogonal projections onto their graphs.

In the third chapter we deal with the Lebesgue covering dimension and concepts of algebraic topology in order to construct maps that can be used to find lower bounds of the dimension of subsets of topological spaces. Our constructions partially follow FP91] and Pe12b, where lower bounds on the dimension of all bifurcation points for families of nonlinear operator equations were obtained. Nevertheless, here we abstract from this concrete case to general subsets of topological spaces and develop some new methods. Finally we point out in a simple example our intention for applying these ideas in later chapters.

We begin the second part of the thesis with a chapter that introduces several fundamentals from the classical index bundle of Atiyah and Singer up to recent results on the space of Fredholm operators with respect to the gap topology. In the first two sections we consider the classical index bundle and its selfadjoint version. We state the deep theorem that the space of bounded Fredholm operators acting on a separable Hilbert space is a classifying space of the $K$-theory functor, where the corresponding map is given by the index bundle and which is usually known as the Atiyah-Jänich theorem. Moreover, we state the corresponding result for the selfadjoint index bundle which asserts that the space of bounded selfadjoint Fredholm operators consists of three connected components and two of them are contractible whereas the third one is a classifying space of the odd $K$-theory functor. The third section is devoted to generally unbounded selfadjoint Fredholm operators. After having compared the so called Riesz topology on the space of selfadjoint operators with the gap topology we give a rigorous definition of spectral flow for gap continuous paths which was obtained for the first time quite recently by Bernhelm Booss-Bavnbek, Matthias Lesch and John Phillips in BLP05. The construction is a straight transcription of the analytic definition of spectral flow for bounded operators from [Ph96] by using results on the behaviour of spectra of selfadjoint operators with respect to the gap topology. Here we make use of our results obtained in the first part of the thesis instead of quoting [BLP05] and so obtain a self contained exposition. We conclude this introductory chapter by stating some recent results which assert that certain subspaces of the space of generally unbounded Fredholm operators are still classifying spaces for even and odd $K$-theory, respectively. However, the methods of proof to obtain these theorems have nothing in common with the classical ones and in particular make no use of an index bundle for unbounded operators.

With the second chapter of the second part we are approaching the core of the thesis. We consider Fredholm morphisms acting between Banach bundles and define an index bundle as $K$-theory class of the underlying base space. Whereas it turns out to be a moderate argument to allow non constant domains of the operators, a non trivial bundle of target spaces causes serious difficulties. Here we will need the full strength of the theory of Banach bundles as developed in the first part in order to construct the index bundle and to prove its well definedness. After having overcome these obstacles in the first section, we elaborate the basic properties of the index bundle in the second section and in particular show that in the case of families of operators 
acting on a separable Hilbert space we get back the classical index bundle of Atiyah and Jänich. Further, we prove a reduction property which strongly uses that we can work on bundles instead of families defined on fixed spaces. In the final third section we apply the theory developed in the last chapter of the first part. We consider Fredholm morphisms of index 0 and obtain from the index bundle a lower bound on the dimension of the set of points in the base over which the morphism is not bijective.

The third chapter deals with gap continuous families of generally unbounded Fredholm operators acting on a Hilbert space. In a first section we make the main observation for defining the index bundle in this situation by showing that the domains of any gap continuous family of Fredholm operators fit together to a Hilbert bundle over the parametrising space. Although it is by definition a quite abstract object, this domain bundle turns out to be understandable in situations that appear frequently in applications. The domain bundle has several pleasant properties and among them we want to emphasize that the family of operators itself defines a bundle morphism into the product bundle induced by the underlying Hilbert space without any transformation. This observation in combination with the corresponding definition for bundle morphisms from the first section now leads to the definition of the index bundle for gap continuous families of Fredholm operators which we state in the second section. Moreover, we transfer the properties of the index bundle for Fredholm morphisms to this case and in the final third section we obtain from our index bundle lower bounds on the set of those points of the parameter space at which the corresponding operator does not have a bounded inverse.

In the fourth and last chapter of the second part of the thesis we generalise the classical index bundle for bounded selfadjoint Fredholm operators to generally unbounded gap continuous families. Besides the definition and some usual properties, we state in the first section a significant result that follows from the reduction property of the index bundle invented before and that will be of high importance in the proof of one of our index theorems in the subsequent third part of the thesis. In the second section we again obtain lower bounds as for the index bundle for general Fredholm operators. Finally, in the third section of the fourth chapter we show that our definition coincides with the classical one when restricted to bounded operators. Moreover, we prove that in case of families which are parametrised by an interval, our selfadjoint index bundle can be identified with the spectral flow as defined in [BLP05] and which we presented in the first chapter of this second part. Hence the spectral flow for gap continuous paths of selfadjoint Fredholm operators [BLP05] which is defined analytically can be obtained from our selfadjoint index bundle in the same way as the spectral flow for bounded operators from the classical selfadjoint index bundle as constructed by Atiyah and Singer.

The third part of the thesis discusses two index theorems for families of ordinary differential operators. In the first chapter we consider first order systems of ordinary differential operators having varying domains which are defined by families of invertible matrices acting on the boundary values. Now our first index theorem states that the corresponding unbounded operators on 
$L^{2}\left(I, \mathbb{C}^{n}\right)$ build a gap continuous family of Fredholm operators of (numerical) index 0. Moreover, the associated domain bundle is a subbundle of the product $X \times H^{1}\left(I, \mathbb{C}^{n}\right)$ and we obtain an explicit formula of the index bundle in the $K$-theory of the parameter space in terms of the matrix family defining the boundary conditions.

In the second chapter we consider families of selfadjoint second order systems of ordinary differential operators having constant Dirichlet boundary conditions. Besides the selfadjoint index bundle we define two further $K$-theoretic indices of our family. The first one is the selfadjoint index bundle of a corresponding family of bounded selfadjoint operators that appear as Riesz representations of a quadratic form associated canonically to the differential operators. The second index is constructed directly from the family of fundamental solutions of the differential equations. Now our second index theorem states the equality of these three indices and its proof is based on the properties of the index bundle we discussed in the second part of the thesis and moreover uses the index theorem for first order families from the foregoing chapter.

We conclude the third part of the thesis with a chapter that deals with a geometric interpretation of our second index theorem. We consider families of geodesics starting at a fixed point in a semi-Riemannian manifold and which are parametrised by elements of the corresponding tangent space at the initial point. By using parallel frames along the geodesics we can transform their associated Hessians and Jacobi equations to a family of quadratic forms and a family of differential equations, respectively. We then obtain an index theorem for families of geodesics from our abstract index theorem from the foregoing chapter. Subsequently we consider examples and note that our index theorem reduces to the Morse index theorem for geodesics in semi-Riemannian manifolds [MPP05] due to Monica Musso, Jacobo Pejsachowicz and Alessandro Portaluri if we consider the special case of a single geodesic. Whereas the proof of this theorem in [MPP05] uses methods from functional analysis, we have already given a topological proof of one of its equalities by means of the selfadjoint index bundle in Wa12. Now our family index theorem not only generalises from a single geodesics to suitable parametrised families, but also gives a proof of the remaining equality by using elementary properties of the index bundle. The original proof of this equality in [MPP05, which we just recalled in Wa12], uses quite hard perturbation results for spectra of selfadjoint Fredholm operators which are taken from [RS95] and [FPR99] and exclusively hold for paths of selfadjoint operators.

The families of geodesics we consider in the geometric index theorem are, however, quite restricted due to their particular parametrisation. In a separate section we explain how we want to use our index theorem for families of differential operators in order to find index theorems for more general families of geodesics in future research.

In a final section we come back to the ideas of the last chapter of the first part and estimate the dimension of the conjugate locus in the tangent spaces of semi-Riemannian manifolds.

The fourth part of the thesis is devoted to bifurcation theory for families of nonlinear equations. We follow ideas of Jacobo Pejsachowicz who discovered in Pe88] that the index bundle for 
families of bounded Fredholm operators can be used in order to prove the existence of bifurcation from a given branch of solutions for equations that are nonlinear perturbations of the considered linear family. These ideas were developed further in [FP91 and Pe01] and it is still a topic of active research $\mathrm{Pe} 12 \mathrm{a}$, Pe12b].

Here we try to combine his fundamental ideas from the early work [Pe88] with our constructions of index bundles and accordingly consider families of operator equations that are defined by nonlinear perturbations of families of generally unbounded Fredholm operators. In the first section we give precise definitions and develop a few fundamentals of bifurcation theory for perturbations of unbounded operators. A significant difference between [Pe88] and our investigations is that in the bounded case the linear operators have a constant domain which is in addition assumed to be the domain of the nonlinearity. In contrast, if we consider families of unbounded operators we have a whole family of domains and to make sense of an addition of the linear and the nonlinear part, it is natural to assume that the nonlinearity is defined on a linear space which contains all domains of the linear operators. Since we allow this space to have its own norm and want to consider the linear operators as bounded operators on their domain bundle, we need a certain compatibility condition between the different topologies involved which is our main object of study in the first section. In the second section we introduce compact nonlinear operators and recall the definition of the $J$-homomorphism from algebraic topology. Afterwards we state two bifurcation theorems which assert, roughly speaking, the existence of bifurcation points if the image of the index bundle under $J$ is non trivial. Finally we draw some conclusions in terms of characteristic classes which are more accessible in concrete applications and prove a lower bound of the dimension of the set of all bifurcation points. The third section is entirely devoted to the proof of our theorems. The proof of the first theorem, which deals with Fredholm operators of index 0 , is quite moderate because we just use our domain bundle in order to reduce to the situation of one of Pejsachowicz' results in $\mathrm{Pe} 88$. In contrast the proof of the second result, which deals with perturbations of selfadjoint operators, is much more technical and involves several subsections. To our best knowledge our results are original in the sense that the existence of bifurcation points for nonlinear perturbations of unbounded operators having varying domains has not been investigated before.

In the second chapter of the fourth part we consider two examples of our theorems. At first we study nonlinear perturbations of a family of first order systems having varying boundary conditions parametrised by maps into a Grassmannian. We use our first bifurcation theorem in order to show the existence of bifurcation points under the assumption that, roughly speaking, the total Chern class of a certain pullback bundle of the tautological bundle over the Grassmannian is odd. Afterwards we consider a nonlinear perturbation of a family of selfadjoint first order operators having varying boundary conditions parametrised by maps into a symplectic group. We obtain the existence of bifurcation points if the spectral flow of the composition of any path in the parameter space and the family of linear operators is odd. 
The last part is an appendix which we recommend to browse through before reading the thesis in order to become familiar with our notation. In its first chapter we recall basics of functional analysis and in particular deal with generally unbounded Fredholm operators and spectral theory. Here we omit almost all proofs but give references to the literature.

In the second chapter we consider $K$-theory for pairs of locally compact spaces, where we use Segal's definition from Se68. Since this construction is less known than the usual definition of $K$-theory with compact supports, we give a fairly detailed exposition including the basic properties that we need throughout the thesis and, moreover, we show that it is equivalent to the ordinary one. Finally we construct an explicit isomorphism between the odd $K$-theory of the unit interval relative to its boundary and the integers which corresponds to the first Chern number and is defined by means of the well known winding number for self-maps of the unit circle. 


\section{Notation and Symbols}

We briefly introduce some basic notations that we will use throughout the thesis without further reference. More advanced notations can be found in the appendix.

First of all, $\mathbb{N}=\{1,2, \ldots\}$ denotes the natural numbers and the symbols $\mathbb{Z}, \mathbb{Q}, \mathbb{R}$ and $\mathbb{C}$ are used as common. $I$ denotes either the unit interval $[0,1]$ or the identity operator on a normed linear space. In the latter case we sometimes add the space $E$ on which the identity acts by writing $I_{E}$. Unless otherwise stated we assume all linear spaces we consider to be defined over the complex numbers. For two normed linear spaces $E$ and $F$ we denote by $\mathcal{L}(E, F)$ the space of bounded linear operators with the usual operator norm. For the sake of simplicity we assume all topological spaces to be Hausdorff. Hence a space is paracompact if and only if it admits partitions of unity subordinated to any open cover. For a locally compact space $X$ we denote by $X^{+}$its one point compactification and set $X^{+}=X \cup\{*\}$ if $X$ is compact, where $*$ is a disjoint point. Finally, if we consider vector bundles over a fixed base space $X$, we frequently shorten notation by writing $\Theta(W)$ for the product bundle over $X$ with fibre $W$. 


\section{Acknowledgements}

I would like to express my gratitude to my supervisor, Prof. Dr. Thomas Schick, who has made available his support in a number of ways and in particular gave me the freedom to follow my own mathematical ways. Moreover, I am grateful to Prof. Dr. Ralf Meyer (University of Göttingen) and Prof. Dr. Alberto Abbondandolo (University of Pisa) who have agreed to act as further referees for this thesis. I gratefully acknowledge the financial support of the research training group 1493 "Mathematical Structures in Modern Quantum Physics" of the German Research Foundation. Further thanks go to Prof. Dr. Andreas Thom (University of Leipzig), Dr. Charlotte Wahl (Leibniz archive Hannover) and Prof. Dr. Antonio di Scala (Polytechnic University of Turin) for fruitful discussions, hints and help regarding the thesis. Further, I thank Lynn Wells for helping me with the English of the thesis. I want to point out that all remaining mistakes are my fault, due to changes after she finished the reviewing process.

I owe my deepest gratitude to Prof. Dr. Jacobo Pejsachowicz (Polytechnic University of Turin) for countless nice discussions about mathematical and non-mathematical subjects, his active interest in my work, many invitations to excellent restaurants and hundreds of coffees. Moreover, I would like to thank his family for their kind hospitality. During my PhD time I spent two and a half months at Torino which was mainly enabled due to financial support of the VIGONI program of the German Academic Exchange Service to which I am greatly indebted.

I would also like to thank my family for the support they provided me through my entire life and in particular, I must acknowledge my girl friend and best friend, Nancy, without whose love, encouragement and editing assistance, I would not have finished this thesis. Thank you for the last nine years! 


\section{Contents}

I Opening: Meet some good Friends 1

1 Banach Bundles 3

1.1 Definitions . . . . . . . . . . . . . . . . . . . . . . . . 4

1.2 Banach Bundle Morphisms $\ldots \ldots \ldots \ldots \ldots \ldots$

1.3 Sections and Finite Dimensional Subbundles . . . . . . . . . . . . . . . . . . . . 17

\begin{tabular}{|lll}
2 & The Space of Closed Operators & 23
\end{tabular}

2.1 Definition of the Gap Metric . . . . . . . . . . . . . . . . . . . . . . . 24

2.2 An Estimate for Bounded Perturbations . . . . . . . . . . . . . . . . . . . . 34

2.3 On the Spectral Theory of Closed Operators . . . . . . . . . . . . . . . . . 40

2.4 The Special Case of Hilbert Spaces $\ldots \ldots \ldots \ldots$

\begin{tabular}{|ll|}
\hline & On the Dimension of Exceptional Sets
\end{tabular}

3.1 Two Definitions of Topological Dimension . . . . . . . . . . . . . . 57

3.2 Dimension Measured by Homology $\ldots \ldots \ldots \ldots$

3.3 Dimension Measured by Homotopy . . . . . . . . . . . . . . . . . . . . . . . . . 61

3.4 First Examples . . . . . . . . . . . . . . . . . . . . . . . . . . 66

\begin{tabular}{lll}
\hline II & The Index Bundles ind and s-ind & 69
\end{tabular}

4 Preliminaries: Fredholm Operators, Index Bundles and Spectral Flow $\quad 71$

4.1 The Atiyah-Jänich Bundle . . . . . . . . . . . . . . . . . . . . . 71

4.2 The Selfadjoint Index Bundle and Spectral Flow . . . . . . . . . . . . . . . 74

4.3 Unbounded Selfadjoint Operators and Spectral Flow . . . . . . . . . . . . . 76

$4.3 .1 \quad$ Gap versus Riesz-topology $\ldots \ldots \ldots \ldots$. . . . . . . . . . . . 76

$4.3 .2 \quad$ Spectral Flow in the Gap Topology . . . . . . . . . . . . . . . . . . . . 79

$4.4 \quad$ Further Results . . . . . . . . . . . . . . . . . . . . . . . . . . . . . . 86 
\begin{tabular}{|lll}
5 & Fredholm Morphisms and the Index Bundle & 89
\end{tabular}

5.1 Construction of the Index Bundle . . . . . . . . . . . . . . . . . . 90

5.2 Main Properties . . . . . . . . . . . . . . . . . . . . . . . . . . . . . 98

5.3 On the Dimension of the Support . . . . . . . . . . . . . . . . . . . . . . 104

6 The Index Bundle for Families of Fredholm Operators in the Gap Topology 109

6.1 The Domain Bundle . . . . . . . . . . . . . . . . . . . . . . . . . . . . 110

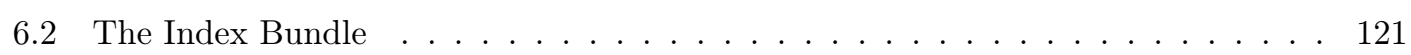

$6.3 \quad$ On the Dimension of the Singular Set $\ldots \ldots \ldots$. . . . . . . . . . . 126

$\begin{array}{lll}7 \text { s-ind and Spectral Flow } & 129\end{array}$

7.1 The Selfadjoint Index Bundle . . . . . . . . . . . . . . . . . . . . . . . . . . . . . 129

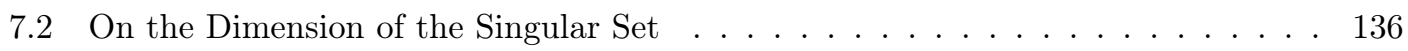

7.3 s-ind, ind ind and the Spectral Flow $\ldots \ldots \ldots \ldots . \ldots \ldots$

\begin{tabular}{lll}
\hline III & Family Index Theorems and Applications & 143
\end{tabular}

8 An Index Theorem for a Floer Family of Elliptic Boundary Value Problems 145

8.1 The Index Theorem . . . . . . . . . . . . . . . . . . . . . 145

8.2 Proof of the Index Theorem . . . . . . . . . . . . . . . . . . . . . . . . . . 147

9 The Morse Index Theorem for Families $\quad 157$

9.1 Around the Index Theorem . . . . . . . . . . . . . . . . . . . . . . . . 157

9.2 The $\operatorname{Index} \operatorname{sind}(\mathcal{A}) \ldots \ldots \ldots \ldots \ldots$

$9.3 \quad$ The Index $\operatorname{s-ind}(q)] \ldots \ldots \ldots \ldots$. . . . . . . . . . . . . . . . . 164

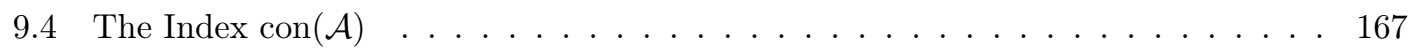

9.5 The Proof $\ldots \ldots \ldots \ldots$. . . . . . . . . . . . . . . . . . . . . . . 169

$9.5 .1 \quad \operatorname{s-ind}(q)=\operatorname{sind}(\mathcal{A}) \ldots \ldots \ldots \ldots \ldots$

$9.5 .2 \quad \operatorname{sind}(\mathcal{A})=\operatorname{con}(\mathcal{A})] \ldots \ldots \ldots \ldots \ldots \ldots$

$\begin{array}{ll}10 \text { Applications to semi-Riemannian Geodesics } & 181\end{array}$

10.1 The Theorem . . . . . . . . . . . . . . . . . . . . . . . . . . . . . . . 181

10.2 Examples . . . . . . . . . . . . . . . . . . . . . . . . . 189

10.3 Outlook: A Generalisation and the Porta Recht Theorem . . . . . . . . . . . . . 191

10.4 On the Size and Shape of the Conjugate Locus $\ldots \ldots \ldots$

\begin{tabular}{lll}
\hline IV & Bifurcation & 199
\end{tabular}

\begin{tabular}{ll}
\hline 11 The Bifurcation Theorems & 201
\end{tabular}

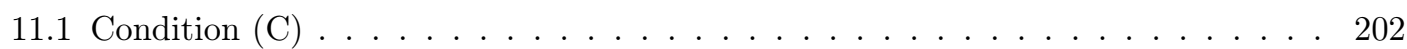


11.2 The Bifurcation Theorems . . . . . . . . . . . . . . . . . . . . . . . . . 205

11.3 The Proof of the Theorems . . . . . . . . . . . . . . . . . . . . . . . . 210

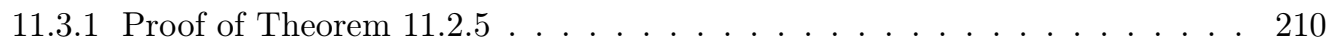

11.3 .2 A Finite Dimensional Approximation to C . . . . . . . . . . . . . . . . . . 212

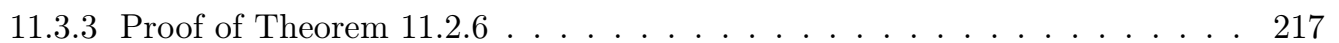

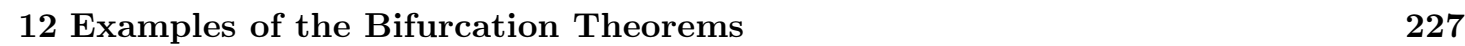

12.1 Example I: A First Order Family Parametrised by a Grassmannian . . . . . . . . 227

12.2 Example II: A Nonlinear Floer Family of Elliptic Boundary Value Problems . . . 231

\begin{tabular}{lll}
\hline V Appendix & 241
\end{tabular}

\begin{tabular}{|ll}
\hline A A Few Basic Definitions and Theorems of Functional Analysis & 243
\end{tabular}

A.1 Projections and Subspaces . . . . . . . . . . . . . . . . . . . . . 243

A.2 Closed Operators . . . . . . . . . . . . . . . . . . . . . . . . . 245

A.3 A Little Spectral Theory . . . . . . . . . . . . . . . . . . . . . . . . . . . . 250

\begin{tabular}{ll}
\hline B $K$-Theory & 255
\end{tabular}

B.1 Basic Definitions and Properties . . . . . . . . . . . . . . . . . . 255

B.2 Relation to other Definitions of $K$-Theory . . . . . . . . . . . . . . . . . . . 259

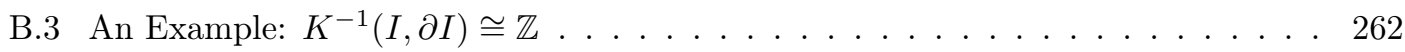




\section{Part I}

Opening: Meet some good Friends 



\section{Chapter 1}

\section{Banach Bundles}

In this first chapter we consider the category of Banach bundles over a topological space, which are a natural generalisation to infinite fibre dimensions of classical vector bundles. The results of this chapter play a crucial role in the following and will be used thoroughly in the second part of the thesis. The arguments we present here are occasionally quite long and technical, but some of the obtained results are really surprising which may compensate the inconvenience.

Our main reference in this chapter is the article [ZKKP75] which contains a survey on Banach bundles in its first part. Since, being a survey, proofs are throughout just sketched or even omitted, the aim of this chapter is to give a rigorous introduction to the principles of Banach bundles and their morphisms which is based on this article. However, in some parts we have changed the presentation completely and enriched it by some results which are mainly taken from [St51] and La95] and which we will need in later chapters of the thesis.

The chapter is divided into three sections. In the first one we define Banach bundles as fibre bundles (in the sense of [St51]) having a Banach space as typical fibre and clarify how a fibrewise norm can be obtained on them which is, accordingly, not part of the definition. Moreover, we define subbundles of Banach- and Hilbert bundles and discover that the latter ones are usually trivial; that is, equivalent to a product bundle. In the second section we build the category of Banach bundles by defining morphisms between them. We prove that idempotent endomorphisms of a Banach bundle $\mathcal{E}$ are in one to one correspondence with decompositions $\mathcal{E}=\mathcal{F} \oplus \mathcal{G}$ into subbundles and demonstrate how the fibrewise kernels and images of bundle morphisms give rise to subbundles. In the third section we concentrate on sections and finite dimensional subbundles of Banach bundles and obtain some surprising results which are in clear contrast to ordinary vector bundles. They are all concerned with the fundamental result that on every Banach bundle exists a nowhere vanishing section. 


\subsection{Definitions}

In this section we present some basic definitions and properties along $\S 2$ of [ZKKP75]. Apart from some minor modifications in the order of the presentation we mainly follow this reference.

1.1.1 Definition. Let $\mathcal{E}$ and $X$ be topological spaces and $p: \mathcal{E} \rightarrow X$ a continuous surjection, such that any fibre $\mathcal{E}_{\lambda}:=p^{-1}(\lambda)$ is a linear space.

- Let $I$ be an index set. An open cover $\left\{U_{\alpha}\right\}_{\alpha \in I}$ is called trivialising if for each $\alpha$ there is a Banach space $E_{\alpha}$ and a homeomorphism $\varphi_{\alpha}: p^{-1}\left(U_{\alpha}\right) \rightarrow U_{\alpha} \times E_{\alpha}$ having the following properties:

i) The diagram

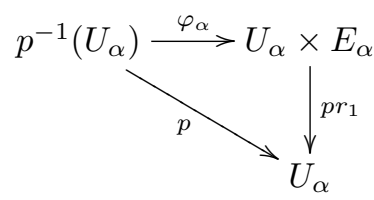

commutes and for each $\lambda \in U_{\alpha}$ the restricted map $\varphi_{\alpha, \lambda}:=\left.\varphi_{\alpha}\right|_{\mathcal{E}_{\lambda}}: \mathcal{E}_{\lambda} \rightarrow E_{\alpha}$ is linear.

ii) For any two elements $U_{\alpha}$ and $U_{\beta}$ of the cover, the map

$$
\varphi_{\alpha \beta}: U_{\alpha} \cap U_{\beta} \rightarrow \mathcal{L}\left(E_{\alpha}, E_{\beta}\right), \quad \lambda \mapsto \varphi_{\beta, \lambda} \circ \varphi_{\alpha, \lambda}^{-1}
$$

is continuous with respect to the operator norm.

We call the maps $\varphi_{\alpha}$ trivialisations and $\varphi_{\alpha \beta}$ transition maps.

- Two trivialising covers are equivalent if their union is still a trivialising cover. An equivalence class with respect to this relation gives the structure of a Banach bundle $p: \mathcal{E} \rightarrow X$, where $\mathcal{E}$ is the bundle space and $X$ the base space.

- We call a Banach bundle $p: \mathcal{E} \rightarrow X$ of finite type, if there exists a finite trivialising cover of $X$.

We continue by giving a thorough list of remarks concerning this definition.

1.1.2 Remark. $\quad$ i) In the following, we will mostly shorten notation and just mention the bundle space $\mathcal{E}$ instead of writing $p: \mathcal{E} \rightarrow X$.

ii) Note that we allow each trivialising neighbourhood $U_{\alpha}$ to have its own model space $E_{\alpha}$. If the base space $X$ is connected, it is clear that we can identify all these spaces $E_{\alpha}$ to a single Banach space E. Hence, in order to simplify notation, we will mostly assume in the following that we just have a single model space E. Moreover, we will denote the total spaces of Banach bundles by calligraphic letters as $\mathcal{E}, \mathcal{F}$ and the model spaces by the corresponding latin letters $E, F$ without further reference. 
iii) It is easy to show that condition ii) in the definition of a Banach bundle holds in any case if the model spaces are finite dimensional (compare for example [La95, III.1.1]). Hence all (finite dimensional) vector bundles are in particular Banach bundles.

iv) If $\mathcal{E}$ and $X$ are (in general infinite dimensional) $C^{k}$-manifolds, the trivialisations $\varphi_{\alpha}$ are $C^{k}$-diffeomorphisms and the transition maps $\varphi_{\alpha \beta}: U_{\alpha} \cap U_{\beta} \rightarrow \mathcal{L}\left(E_{\alpha}, E_{\beta}\right)$ are $C^{k}$, then we obtain in the same way the notion of a $C^{k}$ Banach bundle (compare [La95]). Nevertheless, since we only need the definition as stated above we will also follow [ZKKP75] here and will not include the differentiable category in our discussion.

Note that we do not require the fibres $\mathcal{E}_{\lambda}$ to be Banach spaces explicitly. However, as the following obvious result shows, they are Banach spaces with respect to the norm in the model space transported by a trivialisation to the fibres.

1.1.3 Lemma. Let $\mathcal{E}$ be a Banach bundle. Then any fibre $\mathcal{E}_{\lambda}$ can be given a norm $\|\cdot\|_{\lambda}$ which induces the given topology on it and such that $\mathcal{E}_{\lambda}$ is a Banach space with respect to $\|\cdot\|_{\lambda}$.

Moreover, if the base space $X$ is paracompact, we can find a continuous function $\|\cdot\|: \mathcal{E} \rightarrow \mathbb{R}$ such that $\|\cdot\|_{\lambda}:=\left.\|\cdot\|\right|_{\mathcal{E}_{\lambda}}: \mathcal{E}_{\lambda} \rightarrow \mathbb{R}, \lambda \in X$, is a norm as above.

Proof. In order to prove the first assertion take $\alpha \in I$ such that $\lambda \in U_{\alpha}$ and define $\|\cdot\|_{\lambda}$ as the unique norm such that $\varphi_{\alpha, \lambda}: \mathcal{E}_{\lambda} \rightarrow E$ is an isometry. If we denote the normed space by $\left(\mathcal{E}_{\lambda},\|\cdot\|_{\lambda}\right)$ and the fibre with the subspace topology of $\mathcal{E}$ by $\mathcal{E}_{\lambda}$, then both maps $\varphi_{\alpha, \lambda}: \mathcal{E}_{\lambda} \rightarrow E$ and $\varphi_{\alpha, \lambda}:\left(\mathcal{E}_{\lambda},\|\cdot\|_{\lambda}\right) \rightarrow E$ are homeomorphisms. We infer that the identity map $\left(\mathcal{E}_{\lambda},\|\cdot\|\right) \rightarrow \mathcal{E}_{\lambda}$ is a homeomorphism as well and hence both topologies coincide.

In order to prove the second assertion we note at first that our fibrewise norms constructed above give rise to functions $\|\cdot\|_{\alpha, \lambda}: p^{-1}\left(U_{\alpha}\right) \rightarrow \mathbb{R}, \alpha \in I$, having the required properties. Since $X$ is paracompact we obtain by [MS74, Lemma 5.9] that we can assume without loss of generality that $\left\{U_{\alpha}\right\}_{\alpha \in I}$ is a locally finite covering of $X$ and that there is a subordinated partition of unity $\left\{\eta_{\alpha}\right\}_{\alpha \in I}$. Now we define

$$
\|\cdot\|: \mathcal{E} \rightarrow \mathbb{R}, \quad\|\cdot\|_{\lambda}=\sum_{\alpha \in I} \eta_{\alpha}(\lambda)\|\cdot\|_{\alpha, \lambda}
$$

which is first of all continuous because it is continuous on any trivialising neighbourhood $U_{\alpha}$. We fix some $\lambda \in X$ and note at first that $\|\cdot\|_{\lambda}$ is obviously a norm. Moreover, if we set

$$
J:=\left\{\alpha \in I: \eta_{\alpha}(\lambda) \neq 0\right\},
$$

then all norms $\|\cdot\|_{\alpha, \lambda}, \alpha \in J$, induce the same topology on $\mathcal{E}_{\lambda}$ and hence are pairwise equivalent 1 . We infer that $\|\cdot\|_{\lambda}$ is equivalent to $\|\cdot\|_{\alpha, \lambda}$ for some $\alpha \in J$ and hence $\left(\mathcal{E}_{\lambda},\|\cdot\|_{\lambda}\right)$ is complete and $\|\cdot\|_{\lambda}$ induces the given topology on $\mathcal{E}_{\lambda}$.

\footnotetext{
${ }^{1} \mathrm{~A}$ linear map on a normed linear space is continuous if and only it is bounded. As a simple consequence, two norms on a linear space are equivalent if and only if they induce the same topology
} 
The following well known result will be important in later parts for the construction of bundles. We include it for the sake of completeness but omit its proof which can be found for example in [St51, Theorem 3.2].

1.1.4 Lemma. Let $p: \mathcal{E} \rightarrow X$ be a surjective mapping from some set $\mathcal{E}$ onto a topological space $X$ and $I$ an index set. Let $\left\{U_{\alpha}\right\}_{\alpha \in I}$ be an open covering of $X$, and suppose that we are given for each $\alpha$ a Banach space $E_{\alpha}$ and a bijection

$$
\varphi_{\alpha}: p^{-1}\left(U_{\alpha}\right) \rightarrow U_{\alpha} \times E_{\alpha}
$$

commuting with the projection on $U_{\alpha}$. Moreover, we assume that for each pair $\alpha, \beta \in I$ the map

$$
U_{\alpha} \cap U_{\beta} \rightarrow G L\left(E_{\alpha}, E_{\beta}\right), \quad \lambda \mapsto\left(\varphi_{\beta} \circ \varphi_{\alpha}^{-1}\right)_{\lambda}
$$

is continuous with respect to the operator norm.

Then there exists a unique topology on $\mathcal{E}$, making this set into the total space of a Banach bundle with projection $p$ and trivialising covering $\left\{\left(U_{\alpha}, \varphi_{\alpha}\right)\right\}_{\alpha \in I}$.

Recall that a subbundle $\mathcal{F}$ of a finite dimensional vector bundle $\mathcal{E}$ over $X$ is by definition a vector bundle such that each fibre $\mathcal{F}_{\lambda}$ is a subspace of the corresponding fibre $\mathcal{E}_{\lambda}$ of $\mathcal{E}$. In the case of Banach bundles we want to require more, but later we will see in corollary 1.2.11 that our new assumption becomes evident in the finite dimensional case.

1.1.5 Definition. Let $p: \mathcal{E} \rightarrow X$ be a Banach bundle and $\mathcal{F} \subset \mathcal{E}$ be a subspace, such that for each $\lambda \in X$ the intersection $\mathcal{E}_{\lambda} \cap \mathcal{F}$ is a closed linear subspace of $\mathcal{E}_{\lambda}$. Assume that a Banach bundle structure $\left.p\right|_{\mathcal{F}}: \mathcal{F} \rightarrow X$ is given. $\mathcal{F}$ is called a subbundle of $\mathcal{E}$ if any $\lambda \in X$ is contained in a trivialising neighbourhood of $\mathcal{F}$, such that the corresponding trivialisation can be extended to a trivialisation of $\mathcal{E}$, that is, for each $\lambda \in X$ there are trivialisations

$$
\varphi: p^{-1}(U) \rightarrow U \times E, \quad \psi:(p \mid \mathcal{F})^{-1}(U) \rightarrow U \times F
$$

such that the map $\lambda \mapsto \varphi_{\lambda} \circ \psi_{\lambda}^{-1}$ is constant.

Moreover, $\mathcal{F}$ is called direct subbundle if each $\mathcal{F}_{\lambda}$ is a complemented subspace of $\mathcal{E}_{\lambda}$.

Finally, we define a special case of Banach bundles, which appears quite often in applications and is usually much easier to handle.

1.1.6 Definition. We call $\mathcal{E}$ a Hilbert bundle, if the model space $E$ is a Hilbert space.

Note that we obtain from the construction in the proof of lemma 1.1.3 that each fibre $\mathcal{E}_{\lambda}$ of a Hilbert bundle can be made into a Hilbert space as well. A first interesting property of Hilbert bundles is given by the following result. 
1.1.7 Lemma. Every subbundle $\mathcal{F}$ of a Hilbert bundle $\mathcal{E}$ is direct.

Proof. The assertion follows immediately from A.1.5 since we can choose a scalar product on $\mathcal{E}_{\lambda}$ making this space into a Hilbert space and since $\mathcal{F}_{\lambda} \subset \mathcal{E}_{\lambda}$ is closed by definition.

1.1.8 Theorem. If $X$ is either compact or a $C W$-complex and $\mathcal{E}$ is an infinite dimensional Hilbert bundle over $X$, then $\mathcal{E}$ is trivial.

Proof. Since the structure group of $\mathcal{E}$ is $G L(E)$ and this group is contractible by Kuiper's theorem Il65 the assertion follows from [St51, p. $54 \mathrm{f}$.].

1.1.9 Remark. It is an interesting question to ask for the homotopy type of $G L(E)$ if $E$ is a Banach space. We want to mention [Mi70], where one can find many results and examples concerning this question. For example, the linear group of the space $C(K)$ of continuous functions on a compact space $K$ with the usual sup-norm is not contractible in general. However, $G L(C(K))$ is contractible if $K$ is a manifold. Moreover, the linear groups of the Lebesgue spaces $L^{p}[0,1], 1 \leq p \leq \infty$, are contractible.

\subsection{Banach Bundle Morphisms}

In order to build a category of Banach bundles we now define morphisms. Compared with the finite dimensional case, we again have an additional assumption.

1.2.1 Definition. A morphism $L: \mathcal{E} \rightarrow \mathcal{F}$ between two Banach bundles $p_{1}: \mathcal{E} \rightarrow X$ and $p_{2}: \mathcal{F} \rightarrow X$ is a continuous map such that:

- The diagram

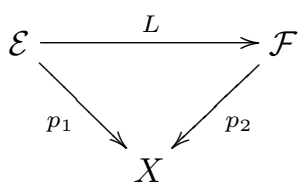

commutes.

- The restricted maps $L_{\lambda}:=\left.L\right|_{\mathcal{E}_{\lambda}}: \mathcal{E}_{\lambda} \rightarrow \mathcal{F}_{\lambda}, \lambda \in X$, are linear and bounded.

- For any $\lambda_{0} \in X$ we have an open neighbourhood $U$ of $\lambda_{0}$ and trivialisations

$$
\varphi: p_{1}^{-1}(U) \rightarrow U \times E, \quad \psi: p_{2}^{-1}(U) \rightarrow U \times F
$$

\footnotetext{
${ }^{2}$ In the usually quoted original work of Kuiper Kui65, the Hilbert space $H$ is assumed to be separable. But, according to the cited reference, the contractability of $G L(E)$, and hence our theorem 1.1.8 holds for any Hilbert space.
} 
such that the map

$$
U \rightarrow \mathcal{L}(E, F), \quad \lambda \mapsto \psi_{\lambda} \circ L_{\lambda} \circ \varphi_{\lambda}^{-1}
$$

is continuous with respect to the operator norm.

We denote the space of all bundle morphisms between $\mathcal{E}$ and $\mathcal{F}$ by $\mathcal{L}(\mathcal{E}, \mathcal{F})$. If $\mathcal{E}=\mathcal{F}$ we write $\mathcal{L}(\mathcal{E})=\mathcal{L}(\mathcal{E}, \mathcal{F})$.

It is easy to show that the last assumption becomes redundant if $\operatorname{dim} \mathcal{E}, \operatorname{dim} \mathcal{F}<\infty$. Moreover, we obtain immediately that the composition $M \circ L: \mathcal{E} \rightarrow \mathcal{G}$ of two Banach bundle morphisms $M: \mathcal{F} \rightarrow \mathcal{G}$ and $L: \mathcal{E} \rightarrow \mathcal{F}$ is again a Banach bundle morphism. Hence the Banach bundles and their bundle morphisms indeed build a category.

The following result can be seen as counterpart of lemma 1.1.4 for morphisms.

1.2.2 Lemma. Let $\mathcal{E}, \mathcal{F}$ be Banach bundles over $X$ and suppose that we are given for each $\lambda \in X$ a continuous linear map

$$
L_{\lambda}: \mathcal{E}_{\lambda} \rightarrow \mathcal{F}_{\lambda}
$$

such that assumption (1.1) is satisfied for any $\lambda_{0} \in X$. Then $L: \mathcal{E} \rightarrow \mathcal{F}$, defined by $L_{\lambda}$ in any fibre, is a Banach bundle morphism.

Moreover, if each $L_{\lambda}$ is bijective, then $L$ is actually a Banach bundle isomorphism.

Proof. We just have to show that $L: \mathcal{E} \rightarrow \mathcal{F}$ is continuous. But since this is a local question, the continuity can be checked with respect to trivialisations. Accordingly, if $\lambda_{0} \in X$ and $\varphi, \psi$ are charts around $\lambda_{0}$ as in 1.1 we obtain

$$
\psi \circ L \circ \varphi^{-1}: U \times E \rightarrow U \times F, \quad(\lambda, u) \mapsto\left(\lambda,\left(\psi_{\lambda} \circ L_{\lambda} \circ \varphi_{\lambda}^{-1}\right) u\right)
$$

Now we denote $\hat{L}_{\lambda}=\psi_{\lambda} \circ L_{\lambda} \circ \varphi_{\lambda}^{-1} \in \mathcal{L}(E, F)$ and consider some $\left(\lambda_{1}, u\right) \in U \times E$. We define

$$
\hat{U}:=\left\{\lambda \in U:\left\|\hat{L}_{\lambda}-\hat{L}_{\lambda_{1}}\right\|<1\right\} \subset U
$$

which is open by condition (1.1). By the reverse triangle inequality we infer $\left\|\hat{L}_{\lambda}\right\| \leq 1+\left\|\hat{L}_{\lambda_{1}}\right\|$ for all $\lambda \in \hat{U}$ and we obtain for all $\lambda \in \hat{U}$ and all $v \in E$

$$
\begin{aligned}
\left\|\hat{L}_{\lambda} u-\hat{L}_{\lambda_{1}} v\right\| & \leq\left\|\hat{L}_{\lambda} u-\hat{L}_{\lambda} v\right\|+\left\|\hat{L}_{\lambda} v-\hat{L}_{\lambda_{1}} v\right\| \\
& \leq\left\|\hat{L}_{\lambda}\right\|\|u-v\|+\left\|\hat{L}_{\lambda}-\hat{L}_{\lambda_{1}}\right\|\|v\| \\
& \leq\left(1+\left\|\hat{L}_{\lambda_{1}}\right\|\right)\|u-v\|+\left\|\hat{L}_{\lambda}-\hat{L}_{\lambda_{1}}\right\|\|v\|,
\end{aligned}
$$


showing the continuity of $\psi \circ L \circ \varphi^{-1}$ in $\left(\lambda_{1}, u\right)$.

It remains to prove the second assertion. First of all, if $L_{\lambda}$ is bijective, then it is a Banach space isomorphism by corollary A.2.5. Hence we have a bounded linear operator $L_{\lambda}^{-1}: \mathcal{F}_{\lambda} \rightarrow \mathcal{E}_{\lambda}$ in any fibre. Let now $U_{\lambda_{0}}$ be a neighbourhood of some $\lambda_{0} \in X$ and $\varphi, \psi$ charts such that

$$
\psi \circ L \circ \varphi^{-1}: U_{\lambda_{0}} \rightarrow \mathcal{L}(E, F)
$$

is continuous. Since each $\psi_{\lambda} \circ L_{\lambda} \circ \varphi_{\lambda}^{-1}$ is invertible, we obtain from the well known continuity of the inversion on $G L(E, F)$ (cf. [Ka76, I.(4.24)]) that

$$
\varphi \circ L^{-1} \circ \psi^{-1}=\left(\psi \circ L \circ \varphi^{-1}\right)^{-1}: U_{\lambda_{0}} \rightarrow \mathcal{L}(F, E)
$$

is continuous. Now the claim follows from the first assertion of the lemma.

1.2.3 Remark. Note that we have shown in the proof of the foregoing result that any bijective Banach bundle morphism is actually an isomorphism. Hence corollary A.2.5 holds for Banach bundles as well.

Before discussing the first important result concerning morphisms on Banach bundles, we want to prepare its proof by stating two well known facts. At first we want to remind the following elementary result.

1.2.4 Lemma. Let $V$ be vector space and $U \subset V$ a subspace. Then the set of all projections onto $U$ is convex.

Proof. If $P$ and $Q$ are two projections onto $U$, we obtain

$$
\begin{aligned}
((1-t) P+t Q)^{2} & =(1-t)^{2} P^{2}+t(1-t) P Q+t(1-t) Q P+t^{2} Q^{2} \\
& =(1-t)^{2} P+t(1-t) Q+t(1-t) P+t^{2} Q=(1-t) P+t Q
\end{aligned}
$$

and hence $(1-t) P+t Q$ is a projection in $V$. Moreover, it is clear that $((1-t) P+t Q)(V) \subset U$ and since we have $((1-t) P+t Q) u=u$ for all $u \in U$, this inclusion is actually an equality.

A little bit more advanced, is the following result, which is problem 4.12 in the first part of Kato's book [Ka76]. 
1.2.5 Lemma. Let $P$ and $Q$ be projections in a Banach space $E$ such that $\|P-Q\|<1$. Then we have a decomposition

$$
E=P E \oplus(I-P) E=Q E \oplus(I-P) E
$$

and the map

$$
W=I-P+Q P: E \rightarrow E
$$

is a Banach space isomorphism, sending PE to QE bijectively and which acts as the identity on $(I-P) E$.

Proof. We begin with the main part of the proof showing that $W \in G L(E)$.

We abbreviate $(P-Q)^{2}$ to $R$ and obtain at first that $R$ commutes with $P$ and $Q$ by

$$
\begin{aligned}
& P R=P(P+Q-P Q-Q P)=P-P Q P=(P+Q-P Q-Q P) P=R P \\
& Q R=Q(P+Q-P Q-Q P)=Q-Q P Q=(P+Q-P Q-Q P) Q=R Q .
\end{aligned}
$$

Next we compute

$$
\begin{aligned}
(I-P+Q P)(I-Q+P Q) & =I-Q+P Q-P+P Q-P^{2} Q+Q P-Q P Q+Q P^{2} Q \\
& =I-Q+P Q-P+Q P=I-(P-Q)^{2}=I-R .
\end{aligned}
$$

and since $R$ is symmetric in $P$ and $Q$ this also gives

$$
(I-Q+P Q)(I-P+Q P)=I-R
$$

By the well known theorem on the Neumann series (cf. [Ka76, III.3.2]), we know that $I-R$ is invertible, because $\|R\| \leq\|P-Q\|^{2}<1$. Now we obtain from 1.3

$$
(I-R)^{-1}(I-Q+P Q)(I-P+Q P)=I .
$$

Moreover, by using the commutativity of $R$ with $P$ and $Q$ and again the Neumann series

$$
(I-R)^{-1}=\sum_{k=0}^{\infty} R^{k}
$$

we obtain that also $(I-R)^{-1}$ commutes with $P$ and $Q$. Hence 1.2 yields 


$$
(I-P+Q P)(I-R)^{-1}(I-Q+P Q)=(I-R)^{-1}(I-P+Q P)(I-Q+P Q)=I
$$

and, setting $W:=I-P+Q P$ as in the assertion, we infer from (1.4) and 1.5 that $W \in G L(E)$ and

$$
W^{-1}=(I-R)^{-1}(I-Q+P Q)
$$

As next step we prove that $W$ maps $P E$ to $Q E$ bijectively. Since $(I-P) P=0$, it is clear that $W$ maps $P E$ into $Q E$. Moreover, as already noted above, $(I-R)^{-1}$ commutes with $P$ and so maps $P E$ to $P E$. Since $I-Q+P Q$ maps $Q E$ into $P E$, we infer that $W^{-1}$ maps $Q E$ into $P E$ as well. Finally, since $W$ and $W^{-1}$ are inverse to each other, we obtain that $W$ maps indeed $P E$ onto $Q E$ bijectively.

Moreover, from $P(I-P)=0$ it is clear that $W$ acts as identity on $(I-P) E$.

Finally, from $E=P E \oplus(I-P) E$ and the fact that $W$ maps $P E$ bijectively onto $Q E$ and $(I-P) E$ bijectively onto itself, we obtain that $E=Q E \oplus(I-P) E$. Indeed, because of the surjectivity of $W$ the corresponding sum spans the whole space $X$ and because of the injectivity, the sum is direct.

We are now able to prove the following important result which clarifies the relation between families of projections and Banach bundles.

1.2.6 Proposition. Let $\mathcal{E}$ be a Banach bundle and $P \in \mathcal{L}(\mathcal{E})$ an idempotent element. Then

$$
\operatorname{im}(P)=\{u \in \mathcal{E}: P u=u\} \subset \mathcal{E}
$$

is a direct subbundle of $\mathcal{E}$.

Conversely, if the base space is paracompact and $\mathcal{F} \subset \mathcal{E}$ is a direct subbundle of $\mathcal{E}$, then there exists an idempotent morphism $P \in \mathcal{L}(\mathcal{E})$ such that $\mathcal{F}=\operatorname{im}(P)$.

Proof. We begin with the first assertion and hence have to show that the image of $P$ is a direct subbundle of $\mathcal{E}$. The strategy of the proof is to modify a given set of trivialisations of $\mathcal{E}$ such that the obtained ones restrict to trivialisations of $\operatorname{im}(P)$.

Let $\lambda_{0} \in X$ and consider a trivialisation around $\lambda_{0}$

$$
\varphi^{\lambda_{0}}: \pi^{-1}\left(U_{\lambda_{0}}\right) \rightarrow U_{\lambda_{0}} \times E_{\lambda_{0}}
$$

By the definition of bundle morphisms, we obtain a continuous family of projections on $E_{\lambda_{0}}$ by $\hat{P}_{\lambda}^{\lambda_{0}}=\varphi_{\lambda}^{\lambda_{0}} P_{\lambda}\left(\varphi_{\lambda}^{\lambda_{0}}\right)^{-1}$. We now define 


$$
\hat{U}_{\lambda_{0}}=\left\{\lambda \in U_{\lambda_{0}}:\left\|\hat{P}_{\lambda}^{\lambda_{0}}-\hat{P}_{\lambda_{0}}^{\lambda_{0}}\right\|<1\right\}
$$

and

$$
\psi^{\lambda_{0}}: p^{-1}\left(\hat{U}_{\lambda_{0}}\right) \rightarrow \hat{U}_{\lambda_{0}} \times E_{\lambda_{0}}, \quad \psi_{\lambda}^{\lambda_{0}}(u)=\left(\lambda,\left(I_{E_{\lambda_{0}}}-\hat{P}_{\lambda}^{\lambda_{0}}+\hat{P}_{\lambda_{0}}^{\lambda_{0}} \hat{P}_{\lambda}^{\lambda_{0}}\right) \varphi_{\lambda}^{\lambda_{0}} u\right) .
$$

Since $I_{E_{\lambda_{0}}}-\hat{P}_{\lambda}^{\lambda_{0}}+\hat{P}_{\lambda_{0}}^{\lambda_{0}} \hat{P}_{\lambda}^{\lambda_{0}}: E_{\lambda_{0}} \rightarrow E_{\lambda_{0}}$ is a continuous family of isomorphisms by lemma 1.2.5, we infer that $\psi^{\lambda_{0}}$ is a homeomorphism which is an isomorphism in each fibre.

If

$$
\psi^{\lambda_{1}}: p^{-1}\left(\hat{U}_{\lambda_{1}}\right) \rightarrow \hat{U}_{\lambda_{1}} \times E_{\lambda_{1}}
$$

is a further homeomorphism constructed in this way such that $\hat{U}_{\lambda_{0}} \cap \hat{U}_{\lambda_{1}} \neq \emptyset$, we obtain

$$
\psi_{\lambda}^{\lambda_{1}} \circ\left(\psi_{\lambda}^{\lambda_{0}}\right)^{-1}=\left(I_{E_{\lambda_{1}}}-\hat{P}_{\lambda}^{\lambda_{1}}+\hat{P}_{\lambda_{1}}^{\lambda_{1}} \hat{P}_{\lambda}^{\lambda_{1}}\right) \varphi_{\lambda}^{\lambda_{1}}\left(\varphi_{\lambda}^{\lambda_{0}}\right)^{-1}\left(I_{E_{\lambda_{0}}}-\hat{P}_{\lambda}^{\lambda_{0}}+\hat{P}_{\lambda_{0}}^{\lambda_{0}} \hat{P}_{\lambda}^{\lambda_{0}}\right)^{-1} \in \mathcal{L}\left(E_{\lambda_{0}}, E_{\lambda_{1}}\right)
$$

which is continuous because the family $\varphi_{\lambda}^{\lambda_{1}}\left(\varphi_{\lambda}^{\lambda_{0}}\right)^{-1} \in \mathcal{L}\left(E_{\lambda_{0}}, E_{\lambda_{1}}\right)$ is continuous by the definition of a Banach bundle and $\left(I_{E_{\lambda_{0}}}-\hat{P}_{\lambda}^{\lambda_{0}}+\hat{P}_{\lambda_{0}}^{\lambda_{0}} \hat{P}_{\lambda}^{\lambda_{0}}\right)^{-1} \in \mathcal{L}\left(E_{0}\right)$ is continuous by the continuity of the inversion in $G L\left(E_{0}\right)$ (cf. [Ka76, I.(4.24)]). Moreover, using the same argument, we obtain that also the families of bounded linear operators $\varphi_{\lambda}^{\lambda_{1}} \circ\left(\psi_{\lambda}^{\lambda_{0}}\right)^{-1} \in \mathcal{L}\left(E_{\lambda_{0}}, E_{\lambda_{1}}\right)$ and $\psi_{\lambda}^{\lambda_{0}} \circ\left(\varphi_{\lambda}^{\lambda_{1}}\right)^{-1} \in \mathcal{L}\left(E_{\lambda_{1}}, E_{\lambda_{0}}\right)$ are continuous if $\hat{U}_{\lambda_{0}} \cap U_{\lambda_{1}} \neq \emptyset$.

Hence the maps $\psi$ define a trivialising cover for the bundle $\mathcal{E}$ which is, moreover, equivalent to the original cover defined by the maps $\varphi$.

Now we just have to observe that by lemma 1.2 .5

$$
\begin{aligned}
\psi_{\lambda}^{\lambda_{0}} P_{\lambda} \mathcal{E}_{\lambda} & =\left(I_{E_{\lambda_{0}}}-\hat{P}_{\lambda}^{\lambda_{0}}+\hat{P}_{\lambda_{0}}^{\lambda_{0}} \hat{P}_{\lambda}^{\lambda_{0}}\right) \varphi_{\lambda}^{\lambda_{0}} P_{\lambda} \mathcal{E}_{\lambda} \\
& =\left(I_{E_{\lambda_{0}}}-\hat{P}_{\lambda}^{\lambda_{0}}+\hat{P}_{\lambda_{0}}^{\lambda_{0}} \hat{P}_{\lambda}^{\lambda_{0}}\right) \hat{P}_{\lambda}^{\lambda_{0}} E_{\lambda_{0}} \\
& =\hat{P}_{\lambda_{0}}^{\lambda_{0}} E_{\lambda_{0}}, \quad \lambda \in \hat{U}_{\lambda_{0}}
\end{aligned}
$$

and hence we have found a trivialising cover of $\mathcal{E}$ that restricts to a trivialising cover of im $P$. Finally, that each fibre $\operatorname{im}(P)_{\lambda}$ is complemented in $\mathcal{E}_{\lambda}$ is an immediate consequence of lemma A.1.2

Now we turn to the proof of the second assertion and assume that $\mathcal{F}$ is a direct subbundle of $\mathcal{E}$. Given $\lambda_{0} \in X$ we can by definition choose a neighbourhood $U_{\lambda_{0}}$ of $\lambda_{0}$ and a trivialisation $\varphi: p^{-1}\left(U_{\lambda_{0}}\right) \rightarrow U_{\lambda_{0}} \times E_{\lambda_{0}}$ of $\mathcal{E}$ that restricts to a trivialisation of $\mathcal{F}$ on $U_{\lambda_{0}}$. Since $\mathcal{F}$ is a direct subbundle, its model space $F_{\lambda_{0}} \subset E_{\lambda_{0}}$ is complemented and hence there exists a bounded projection $\hat{P} \in \mathcal{L}\left(E_{\lambda_{0}}\right)$ such that im $\hat{P}=F_{\lambda_{0}}$ by lemma A.1.2. If we define 


$$
P_{\lambda}^{\lambda_{0}}=\varphi_{\lambda}^{-1} \hat{P} \varphi_{\lambda}, \quad \lambda \in U_{\lambda_{0}}
$$

we obtain an idempotent bundle morphism of $\mathcal{E}$ restricted to $U_{\lambda_{0}}$, such that $P_{\lambda}^{\lambda_{0}} \mathcal{E}_{\lambda}=\mathcal{F}_{\lambda}$ for all $\lambda \in U_{\lambda_{0}}$.

Now, since $X$ is paracompact, we can find by [MS74, Lemma 5.9] a locally finite open cover $\left\{U_{\lambda_{i}}\right\}_{i \in I}$ of $X$ consisting of trivialising neighbourhoods as considered above. We choose a partition of unity $\left\{\eta_{i}\right\}_{i \in I}$ subordinated to $\left\{U_{\lambda_{i}}\right\}$ and define

$$
P=\sum_{i \in I} \eta_{i} P^{\lambda_{i}}
$$

Now $P$ is bundle morphism of $\mathcal{E}$ and, moreover, each $P_{\lambda}$ is a projection onto $\mathcal{F}_{\lambda}$ by lemma 1.2 .4 .

As next step we use the obtained proposition in order to show the following perfect analogy with subspaces of Banach spaces as discussed in section A.1

1.2.7 Proposition. Let $\mathcal{E}$ be a Banach bundle over the paracompact base space $X$ and $\mathcal{F}, \mathcal{G}$ be subbundles of $\mathcal{E}$. Then $\mathcal{E} \cong \mathcal{F} \oplus \mathcal{G}$ if and only if there exists an idempotent morphism $P \in \mathcal{L}(\mathcal{E})$ such that $\operatorname{im}(P)=\mathcal{F}$ and $\operatorname{ker}(P)=\mathcal{G}$.

Proof. We begin by proving the existence of $P \in \mathcal{L}(\mathcal{E})$ if $\mathcal{E}=\mathcal{F} \oplus \mathcal{G}$ which is just a refinement of the argument in the proof of the second part of proposition 1.2.6. Since $\mathcal{F}$ and $\mathcal{G}$ are subbundles of $\mathcal{E}$ we can find for any $\lambda_{0} \in X$ an open neighbourhood $U$ and trivialisations

$$
\varphi:\left(\left.p\right|_{\mathcal{F}}\right)^{-1}(U) \rightarrow U \times F, \quad \psi:\left(\left.p\right|_{\mathcal{G}}\right)^{-1}(U) \rightarrow U \times G
$$

which extends to trivialisations of $\mathcal{E}$. Although $F$ and $G$ have a non trivial intersection in general, we can consider the Banach space $U \times V$ according to section A.1. which is isomorphic to $E$, and obtain a trivialisation of $\mathcal{E}$ over $U$ by

$$
\varphi \times \psi: \pi^{-1}(U)=\left(\left.p\right|_{\mathcal{F}}\right)^{-1}(U) \oplus\left(\left.p\right|_{\mathcal{G}}\right)^{-1}(U) \rightarrow U \times(F \times G) .
$$

Since $F$ and $G$ are closed subspaces of $F \times G$, the projection onto $F$ with respect to this decomposition is bounded. By invoking lemma 1.2.4 we now can construct as in the proof of proposition 1.2.6 an idempotent morphism having $\mathcal{F}$ as image and, moreover, whose kernel is $\mathcal{G}$. If, on the other hand, $P \in \mathcal{L}(\mathcal{E})$ is given, then $\mathcal{F}:=\operatorname{im} P$ and $\mathcal{G}:=\operatorname{ker} P=\operatorname{im}\left(I_{\mathcal{E}}-P\right)$ are direct subbundles of $\mathcal{E}$ according to proposition 1.2.6 Now a bundle isomorphism is given by

$$
\mathcal{E} \rightarrow \mathcal{F} \oplus \mathcal{G}, \quad u \mapsto\left(P u,\left(I_{\mathcal{E}}-P\right) u\right)
$$


1.2.8 Corollary. Let $\mathcal{E}$ be a Banach bundle over the paracompact base $X$ and $\mathcal{F}$ a direct subbundle. Then $\mathcal{F}$ is complemented, that is, there exists a direct subbundle $\mathcal{G} \subset \mathcal{E}$, such that

$$
\mathcal{E} \cong \mathcal{F} \oplus \mathcal{G}
$$

Proof. By proposition 1.2.6 we find an idempotent $P \in \mathcal{L}(\mathcal{E})$, such that $\operatorname{im}(P)=\mathcal{F}$ and, moreover, $\mathcal{G}=\operatorname{ker} P=\operatorname{im}\left(I_{\mathcal{E}}-P\right)$ is a subbundle of $\mathcal{E}$. Now the result follows from proposition 1.2 .7 .

As final results of this section we want to study the question when the kernel and image of a Banach bundle morphism yield a subbundle of its domain and target bundle, respectively. At first, we need the following lemma whose following corollaries will answer this question.

1.2.9 Lemma. Let $\mathcal{E}, \mathcal{F}$ be Banach bundles over the paracompact base $X$ and $L \in \mathcal{L}(\mathcal{E}, \mathcal{F})$ a morphism.

(i) If each $L_{\lambda}$ has a bounded right inverse, then there exists $M \in \mathcal{L}(\mathcal{F}, \mathcal{E})$ such that

$$
L \circ M=I \in \mathcal{L}(\mathcal{F})
$$

(ii) If each $L_{\lambda}$ has a bounded left inverse, then there exists $N \in \mathcal{L}(\mathcal{F}, \mathcal{E})$ such that

$$
N \circ L=I \in \mathcal{L}(\mathcal{E})
$$

Proof. We only prove the first assertion because the proof of the second one is very similar.

Let $\lambda_{0} \in X, U$ an open neighbourhood of $\lambda_{0}$ and $\varphi, \psi$ trivialisations on $U$ as in 1.1. By assumption there exists $S_{0} \in \mathcal{L}\left(\mathcal{F}_{\lambda_{0}}, \mathcal{E}_{\lambda_{0}}\right)$ such that $L_{\lambda_{0}} S_{0}=I_{\mathcal{F}_{\lambda_{0}}}$. Hence $\psi_{\lambda_{0}} L_{\lambda_{0}} \varphi_{\lambda_{0}}^{-1} \varphi_{\lambda_{0}} S_{0} \psi_{\lambda_{0}}^{-1}=$ $I_{F}$ and, since $G L(F) \subset \mathcal{L}(F)$ is open, we can find an open neighbourhood $U_{0} \subset U$ such that

$$
\psi_{\lambda} L_{\lambda} \varphi_{\lambda}^{-1} \varphi_{\lambda_{0}} S_{0} \psi_{\lambda_{0}}^{-1} \in G L(F), \quad \lambda \in U_{0}
$$

Then a continuous right inverse on $U_{0}$ is given by

$$
M_{0, \lambda}=\varphi_{\lambda}^{-1} \varphi_{\lambda_{0}} S_{0} \psi_{\lambda_{0}}^{-1}\left(\psi_{\lambda} L_{\lambda} \varphi_{\lambda}^{-1} \varphi_{\lambda_{0}} S_{0} \psi_{\lambda_{0}}^{-1}\right)^{-1} \psi_{\lambda}, \quad \lambda \in U_{0}
$$

because

$$
L_{\lambda} M_{0, \lambda}=\psi_{\lambda}^{-1} \underbrace{\psi_{\lambda} L_{\lambda} \varphi_{\lambda}^{-1} \varphi_{\lambda_{0}} S_{0} \psi_{\lambda_{0}}^{-1}\left(\psi_{\lambda} L_{\lambda} \varphi_{\lambda}^{-1} \varphi_{\lambda_{0}} S_{0} \psi_{\lambda_{0}}^{-1}\right)^{-1}}_{=I_{F}} \psi_{\lambda}=I_{\mathcal{F}_{\lambda}}
$$


For the global case we choose a cover $\left\{U_{\alpha}\right\}$ of $X$ such that we have a continuous right inverse $M_{\alpha}$ over each $U_{\alpha}$. With a subordinated partition of unity $\left\{\eta_{\alpha}\right\}$ a right inverse to $L$ on $X$ is given by

$$
M_{\lambda}=\sum_{\alpha} \eta_{\alpha}(\lambda) M_{\alpha, \lambda}
$$

As a corollary we now obtain the following important result.

1.2.10 Corollary. Let $\mathcal{E}, \mathcal{F}$ be Banach bundles over the paracompact base $X$ and $L \in \mathcal{L}(\mathcal{E}, \mathcal{F})$ a morphism.

(i) If the kernels $\operatorname{ker} L_{\lambda}, \lambda \in X$, form a direct subbundle of $\mathcal{E}$ and each $L_{\lambda}$ has a complemented image, then the spaces $\operatorname{im} L_{\lambda}$ form a direct subbundle of $\mathcal{F}$.

(ii) If the images $\operatorname{im} L_{\lambda}$ form a direct subbundle of $\mathcal{F}$ and each $L_{\lambda}$ has a complemented kernel, then the spaces $\operatorname{ker} L_{\lambda}$ form a direct subbundle of $\mathcal{E}$.

Proof. (i) By corollary 1.2 .8 we can assume without loss of generality that each $L_{\lambda}$ is injective. Moreover, since each $L_{\lambda}$ has a complemented image, by lemma A.1.8, there exists a bounded left inverse $M_{\lambda}, \lambda \in X$. By the previous lemma we can assume that these left inverses build a morphism $M \in \mathcal{L}(\mathcal{F}, \mathcal{E})$. Now the claim follows from proposition 1.2 .6 by observing that $P:=L \circ M \in \mathcal{L}(\mathcal{F})$ is an idempotent morphism and

$$
\operatorname{im}\left(P_{\lambda}\right)=\operatorname{im}\left(L_{\lambda} \circ M_{\lambda}\right)=\operatorname{im}\left(L_{\lambda}\right), \quad \lambda \in X
$$

because of the surjectivity of $M_{\lambda}, \lambda \in X$.

(ii) We can assume that $L$ is surjective and, moreover, we will argue very similar to the case (i). Since $L_{\lambda}$ has a complemented kernel by assumption, for each $\lambda \in X$ there exists a bounded right inverse $M_{\lambda}$ to $L_{\lambda}$ by lemma A.1.8. Now we use again the previous lemma and assume that we have a global right inverse $M \in \mathcal{L}(\mathcal{F}, \mathcal{E})$. Then $P:=M \circ L \in \mathcal{L}(\mathcal{E})$ is an idempotent morphism whose kernel is given by

$$
\operatorname{ker}\left(P_{\lambda}\right)=\operatorname{ker}\left(M_{\lambda} \circ L_{\lambda}\right)=\operatorname{ker}\left(L_{\lambda}\right), \quad \lambda \in X,
$$

where we use the injectivity of $M_{\lambda}$. Finally, $I_{\mathcal{E}}-P$ gives the required idempotent in $\mathcal{L}(\mathcal{E})$. 
We conclude this section by two important corollaries of the foregoing one. Note that, in contrast to ordinary vector bundles (cf. [At89, 1.3.1]), the image of a bundle monomorphism $\iota: \mathcal{E} \rightarrow \mathcal{F}$ is not a subbundle of the target bundle $\mathcal{F}$ in general. For example, take $\mathcal{E}=X \times C[0,1]$ and $\mathcal{F}=X \times L^{2}[0,1]$ where the linear spaces carry their usual norms. Then $\mathcal{E}$ and $\mathcal{F}$ are Banach bundles and the canonical inclusion $\iota: \mathcal{E} \hookrightarrow \mathcal{F}$ is a Banach bundle monomorphism. However, the fibres of $\iota(\mathcal{E})$ are not closed in $\mathcal{F}$.

1.2.11 Corollary. Let $p: \mathcal{E} \rightarrow X$ be a Banach bundle over the paracompact base $X$ and $\mathcal{F} \subset \mathcal{E}$ be a subspace, such that $\left.p\right|_{\mathcal{F}}: \mathcal{F} \rightarrow X$ is a finite dimensional vector bundle. Then $\mathcal{F}$ is a direct subbundle of $\mathcal{E}$.

Proof. Since finite dimensional subspaces are complemented we just have to show that the inclusion map $\iota: \mathcal{F} \hookrightarrow \mathcal{E}$ defines a bundle morphism in order to conclude that $\mathcal{F}=\iota(\mathcal{F}) \subset \mathcal{E}$ is a direct subbundle.

We want to use lemma 1.2 .2 and hence have to show that for any trivialisations $\varphi$ of $\mathcal{F}$ and $\psi$ of $\mathcal{E}$ over $U \subset X$, the map

$$
U \ni \lambda \mapsto L_{\lambda}:=\psi_{\lambda} \circ \iota \circ \varphi_{\lambda}^{-1} \in \mathcal{L}(F, E)
$$

is continuous.

First of all, we infer from the continuity of $\iota: \mathcal{F} \rightarrow \mathcal{E}$ that

$$
U \times F \rightarrow U \times E, \quad(\lambda, u) \mapsto\left(\lambda,\left(\psi_{\lambda} \circ \iota \circ \varphi_{\lambda}^{-1}\right) u\right)
$$

is continuous. Moreover, since $F$ is finite dimensional, we can find a finite base $\left\{e_{1}, \ldots, e_{n}\right\}$ and can define a norm on $F$ by

$$
\|u\|_{F}:=\left(\sum_{i=1}^{n}\left|u_{i}\right|^{2}\right)^{\frac{1}{2}}, \quad u=\sum_{i=1}^{n} u_{i} e_{i},
$$

which induces the given topology on $F$. We obtain for any $\lambda, \lambda_{0} \in U$ and $u \in F$

$$
\begin{aligned}
\left\|L_{\lambda} u-L_{\lambda_{0}} u\right\| & =\left\|u_{1}\left(L_{\lambda}-L_{\lambda_{0}}\right) e_{1}+\cdots+u_{n}\left(L_{\lambda}-L_{\lambda_{0}}\right) e_{n}\right\| \\
& \leq\left|u_{1}\right|\left\|\left(L_{\lambda}-L_{\lambda_{0}}\right) e_{1}\right\|+\cdots+\left|u_{n}\right|\left\|\left(L_{\lambda}-L_{\lambda_{0}}\right) e_{n}\right\| \\
& \leq\left(\sum_{i=1}^{n}\left|u_{i}\right|^{2}\right)^{\frac{1}{2}}\left(\sum_{i=1}^{n}\left\|\left(L_{\lambda}-L_{\lambda_{0}}\right) e_{i}\right\|^{2}\right)^{\frac{1}{2}}=\left(\sum_{i=1}^{n}\left\|\left(L_{\lambda}-L_{\lambda_{0}}\right) e_{i}\right\|^{2}\right)^{\frac{1}{2}}\|u\|_{F} .
\end{aligned}
$$

Hence 


$$
\left\|L_{\lambda}-L_{\lambda_{0}}\right\| \leq\left(\sum_{i=1}^{n}\left\|\left(L_{\lambda}-L_{\lambda_{0}}\right) e_{i}\right\|^{2}\right)^{\frac{1}{2}}
$$

and the continuity of $L$ follows from the continuity of 1.6 .

Finally, we want to consider a result that we will need later in order to prove that a given Banach bundle $\mathcal{E}_{0}$ is a subbundle of another bundle $\mathcal{E}$. Note that the following assertion is not a tautology. The crucial point is that $\mathcal{E}_{0}$ needs to have the subspace topology of $\mathcal{E}$ in order to be a subbundle.

1.2.12 Lemma. Let $\mathcal{E}_{0}$ and $\mathcal{E}$ be two Banach bundles over the same paracompact base $X$. We assume that for each $\lambda \in X$ the fibre $\mathcal{E}_{0, \lambda}$ is a complemented subspace of $\mathcal{E}_{\lambda}$ and that the canonical injection $\iota: \mathcal{E}_{0} \rightarrow \mathcal{E}$ is a bundle morphism. Then $\mathcal{E}_{0}$ is a direct subbundle of $\mathcal{E}$.

Proof. Since $\iota$ is an injective bundle morphism having a complemented image in each fibre, we obtain by corollary 1.2 .10 that $\iota\left(\mathcal{E}_{0}\right)$ is a complemented subbundle of $\mathcal{E}$. It remains to show that $\mathcal{E}_{0}$ carries the subspace topology induced by $\mathcal{E}$. By assumption each $\mathcal{E}_{0, \lambda}$ is a complemented subspace of $\mathcal{E}_{\lambda}$ and hence we have a left inverse to $\iota$ in each fibre by lemma A.1.8. According to lemma 1.2.9, we can find a bundle morphism $M: \mathcal{E} \rightarrow \mathcal{E}_{0}$ such that $M \circ \iota$ is the identity on $\mathcal{E}_{0}$. Now the restriction of $M$ to $\iota\left(\mathcal{E}_{0}\right)$ shows that $\iota: \mathcal{E}_{0} \rightarrow \iota\left(\mathcal{E}_{0}\right)$ is a bundle isomorphism and so in particular a homeomorphism. Hence $\iota: \mathcal{E}_{0} \rightarrow \mathcal{E}$ is an embedding, showing that $\mathcal{E}_{0} \subset \mathcal{E}$ carries the subspace topology.

\subsection{Sections and Finite Dimensional Subbundles}

In this section we mainly consider finite dimensional subbundles of Banach bundles, where results can be obtained that are in marked contrast to the case of finite dimensional bundles. The reason for the appearance of unexpected phenomena is given by the following deep theorem which can be found in [ZKKP75] and the references given there.

1.3.1 Theorem. Every infinite dimensional Banach space $E$ is homeomorphic to $E \backslash\{0\}$.

We will apply this result in the proof of our main theorem of this section in combination with the following simple observation.

1.3.2 Lemma. Let $V$ be a topological vector space, $X$ a topological space and $\varphi: V \rightarrow X a$ homeomorphism. Then $X$ can be given a topological vector space structure such that $\varphi$ is an isomorphism.

Proof. For $x, y \in X$ and $\lambda \in \mathbb{C}$ we define

$$
x \hat{+} y:=\varphi\left(\varphi^{-1}(x)+\varphi^{-1}(y)\right), \quad \lambda \cdot x:=\varphi\left(\lambda \varphi^{-1}(x)\right), \quad 0:=\varphi\left(0_{V}\right) .
$$


We obtain, for example,

$$
\begin{aligned}
0 \hat{+} x & =\varphi\left(\varphi^{-1}\left(\varphi\left(0_{V}\right)\right)+\varphi^{-1}(x)\right)=\varphi\left(\varphi^{-1}(x)\right)=x \\
x \hat{+}(-x) & =\varphi\left(\varphi^{-1}(x)+\varphi^{-1}\left(\varphi\left(-\varphi^{-1}(x)\right)\right)\right)=\varphi\left(0_{V}\right)=0 \\
1 \cdot x & =\varphi\left(\varphi^{-1}(x)\right)=x
\end{aligned}
$$

and the remaining vector space axioms are simple computations as well. Moreover, since $\varphi$ is supposed to be a homeomorphism, it is clear that the addition and scalar multiplication introduced on $X$ are continuous.

Finally, we compute for $u, v \in V$ and $\lambda \in \mathbb{C}$

$$
\begin{aligned}
\varphi(u) \hat{+} \varphi(v) & =\varphi\left(\varphi^{-1}(\varphi(u))+\varphi^{-1}(\varphi(v))\right)=\varphi(u+v) \\
\lambda \cdot \varphi(u) & =\varphi\left(\lambda \varphi^{-1}(\varphi(u))\right)=\varphi(\lambda u)
\end{aligned}
$$

and hence $\varphi$ is a bijective homomorphism.

The following theorem is neither stated explicitly nor proved in [ZKKP75]. Instead, the related result that we state below as corollary $1.3 .5 i$ ) is considered and the special case $n=1$ of our following theorem is mentioned as a remark. The main ideas of the proofs are very similar. However, we give a slightly different approach that in particular allows us to avoid a transfinite induction argument.

1.3.3 Theorem. Let $p: \mathcal{E} \rightarrow X$ be an infinite dimensional Banach bundle over the paracompact base $X$ and $\left\{\delta_{1}, \ldots, \delta_{n}\right\}$ pointwise linear independent sections of $\mathcal{E}$ over an open set $U \subset X$. Then for every closed subspace $D \subset U$ there exist pointwise linear independent sections $\left\{\tilde{\delta}_{1}, \ldots, \tilde{\delta}_{n}\right\}$ of the whole bundle $\mathcal{E}$ such that $\left.\tilde{\delta}_{i}\right|_{D}=\delta_{i}, i=1, \ldots, n$.

Proof. At first we consider the special case $n=1$. Let $\left\{U_{k}\right\}_{k \in \mathbb{N}}$ be a locally finite countable cover of $X$ by trivialising neighbourhoods which exists for any fibre bundle over a paracompact base according to [MS74, 5.9]. We define $V_{0}:=U$ and $V_{k}=U_{k} \cap(X \backslash D), k \in \mathbb{N}$, and obtain a locally finite countable open cover $\left\{V_{k}\right\}_{k \in \mathbb{N} \cup\{0\}}$ of $X$ such that $V_{k} \cap D=\emptyset, k \in \mathbb{N}$. Moreover, we choose for each $k \in \mathbb{N}$ a trivialisation $\varphi_{k}: p^{-1}\left(V_{k}\right) \rightarrow V_{k} \times E$ of $\mathcal{E}$.

We now construct the required section by induction and show that whenever we have a nowhere vanishing section $\delta_{n}$ on

$$
W_{n}:=\bigcup_{0 \leq k \leq n} V_{k}, \quad n \in \mathbb{N} \cup\{0\},
$$

we can find a nowhere vanishing section $\delta_{n+1}$ on $W_{n+1}$ such that $\left.\delta_{n+1}\right|_{D}=\left.\delta_{n}\right|_{D}$. Since on $W_{0}=U$ we have by assumption a nowhere vanishing section that coincides with itself over $D$, 
this shows the existence of the desired section on each $W_{n}, n \in \mathbb{N}$. We will explain below why our construction actually yields a continuous section on all of $X$.

By theorem 1.3.1 and lemma 1.3.2 there exists a linear structure on $E \backslash\{0\}$ that is compatible with the given topology on this space. If now $\delta_{n}$ is constructed over $W_{n}$, we note that $W_{n+1}$ is a normal space as an open subset of the paracompact space $X$ and hence there exists a partition of unity $\left\{\eta_{1, n}, \eta_{2, n}\right\}$ subordinated to the open covering $\left\{W_{n}, V_{n+1}\right\}$ of $W_{n+1}$. We define

$$
\tilde{\delta}_{n+1}(\lambda)=\varphi_{n+1}^{-1}\left(\eta_{1, n}(\lambda) \cdot \varphi_{n+1}\left(\delta_{n}(\lambda)\right) \hat{+} \eta_{2, n}(\lambda) \cdot u\right), \quad \lambda \in V_{n+1}
$$

for some $u \in E \backslash\{0\}$. Then

$$
\delta_{n+1}(\lambda)= \begin{cases}\delta_{n}(\lambda), & \lambda \notin V_{n+1} \\ \tilde{\delta}_{n+1}(\lambda), & \lambda \in V_{n+1}\end{cases}
$$

is a nowhere vanishing continuous section over $W_{n+1}$. Moreover, $\delta_{n+1}$ coincides with $\delta_{1}$ on $D$ since $V_{k} \cap D=\emptyset$ for all $k \in \mathbb{N}$.

By the local finiteness of $\left\{V_{k}\right\}_{k \in \mathbb{N}}$, the construction is such that for any $\lambda_{0} \in X$ there exists an $n_{0} \in \mathbb{N}$ and a neighbourhood $U_{\lambda_{0}}$ of $\lambda_{0}$ such that $U_{\lambda_{0}} \cap V_{n}=\emptyset$ for all $n \geq n_{0}$. Accordingly, $\left.\delta_{n}\right|_{U_{\lambda_{0}}}=\left.\delta_{n_{0}}\right|_{U_{\lambda_{0}}}$ for all $n \geq n_{0}$. Hence any point $\lambda_{0}$ has a neighbourhood on which the construction of $\delta$ is finished after a finite number of steps and so we indeed obtain a nowhere vanishing continuous section.

Now we turn to the case $n>1$. By the special case already proven we can extend $\delta_{1}$ to a nowhere vanishing section $\tilde{\delta}_{1}$ of $\mathcal{E}$. Now $\tilde{\delta}_{1}$ defines a one dimensional vector bundle $\mathcal{F}_{1}$ over $X$ which is a subbundle of $\mathcal{E}$ by corollary 1.2.11. The same argument applies to the sections $\left\{\delta_{2}, \ldots, \delta_{n}\right\}$ over $U$ which accordingly build a subbundle of $\mathcal{E}$ restricted to $U$. Moreover, since $X$ is paracompact, we can choose an open subset $U_{1} \subset X$ such that $D \subset U_{1} \subset \bar{U}_{1} \subset U$. Arguing as in the proof of proposition 1.2 .7 , it is readily seen that we can construct a projection $P \in \mathcal{L}(\mathcal{E})$ having the one dimensional subbundle $\mathcal{F}_{1}$ of $\mathcal{E}$ as image and such that the remaining sections $\delta_{2}, \ldots, \delta_{n}$ are in its kernel over $U_{1}$. Hence we obtain a splitting $\mathcal{E}=\mathcal{F}_{1} \oplus \mathcal{G}_{1}$ such that $\delta_{1}$ is a section of the one dimensional bundle $\mathcal{F}_{1}$ and $\delta_{2}, \ldots, \delta_{n}$ are sections over $U_{1}$ of the infinite dimensional Banach bundle $\mathcal{G}_{1}$. Now we can apply the first part of our proof to $\delta_{2}$ as a section of $\mathcal{G}_{1}$ over $U_{1}$. Iterating this construction we obtain the desired sections $\tilde{\delta}_{1}, \ldots, \tilde{\delta}_{n}: X \rightarrow \mathcal{E}$.

1.3.4 Remark. One may also obtain the case $n=1$ of theorem 1.3 .3 in a different way by using well known facts of the theory of fibre bundles. Indeed, we can consider the bundle $\mathcal{E}$ with the zero section removed. This is a fibre bundle with fibre $E \backslash\{0\}$. Now by 1.3 .1 the fibre is contractibl ${ }^{3}$ and hence under the additional assumption that $(X, D)$ is a relative $C W$-complex,

\footnotetext{
${ }^{3}$ The contractibility of $E \backslash\{0\} \simeq S_{E}=\{u \in E:\|u\|=1\}, \operatorname{dim} E=\infty$, can also be proved along different lines, even if $E$ is not assumed to be complete BS83.
} 
we obtain that any section of $\mathcal{E} \backslash\{0\}$ on $D$ prolongs to a section of this bundle on all of $X$ (cf. [Hu94, Theorem 7.1]).

We note a couple of immediate, but important consequences.

1.3.5 Corollary. Let $p: \mathcal{E} \rightarrow X$ be a Banach bundle over a paracompact base such that $\operatorname{dim} E=$ $\infty$. Then

(i) There exists a nowhere vanishing section of $\mathcal{E}$.

(ii) For any $n \in \mathbb{N}, \mathcal{E}$ contains an $n$-dimensional trivial subbundle.

(iii) If $\mathcal{F}$ is a finite dimensional vector bundle of finite type over $X$, then $\mathcal{E}$ contains a subbundle isomorphic to $\mathcal{F}$.

(iv) If $\mathcal{F}$ is a finite dimensional subbundle of $\mathcal{E}$ of finite type, then there exists a further finite dimensional subbundle $\mathcal{F}^{\prime}$ of $\mathcal{E}$ such that $\mathcal{F} \oplus \mathcal{F}^{\prime}$ is a trivial subbundle of $\mathcal{E}$.

(v) Let $U \subset X$ be open and $\mathcal{F}$ a finite dimensional, trivial subbundle of $\mathcal{E}$ over $U$. Then for any closed subset $D \subset U$ there exists a finite dimensional trivial subbundle of $\mathcal{E}$ that coincides with $\mathcal{F}$ over $D$.

Proof. In the following, we use corollary 1.2 .11 without further reference.

Let $n \in \mathbb{N}$. We take some trivialising neighbourhood $U$ of $\mathcal{E}$ and transport $n$ linear independent elements of $E$ to an $n$-frame of $\mathcal{E}$ over $U$. Extending this frame to all of $X$ according to theorem 1.3 .3 gives an $n$ dimensional trivial subbundle of $\mathcal{E}$, which is the assertion of (ii), and proves (i) in the special case $n=1$.

In order to prove (iii), we just have to remember the well known fact that, since $\mathcal{F}$ is of finite type, we can embed it into a trivial vector bundle $\mathcal{G}$ over $X$ (cf. eg. [Ha09, Prop. 1.4] ). Now, choosing $N:=\operatorname{dim} \mathcal{G}<\infty$ pointwise linear independent sections of $\mathcal{G}$ and according to (ii) $N$ pointwise linear independent sections of $\mathcal{E}$, we can embed $\mathcal{G}$ into $\mathcal{E}$. Hence we can embed $\mathcal{F}$ into $\mathcal{E}$.

To prove (iv) we again embed $\mathcal{F}$ into a finite dimensional trivial vector bundle $\mathcal{G}$ over $X$ and now choose a complement $\mathcal{F}^{\perp}$ of $\mathcal{F}$ in $\mathcal{G}$, that is, $\mathcal{F} \oplus \mathcal{F}^{\perp}=\mathcal{G}$. Moreover, according to corollary 1.2.8, we can choose a subbundle $\mathcal{M}$ of $\mathcal{E}$ that complements $\mathcal{F}$ in $\mathcal{E}$. Now we obtain a bundle $\mathcal{F}^{\prime}$ as in the assertion by embedding $\mathcal{F}^{\perp}$ into $\mathcal{M}$ according to assertion (iii) of this corollary.

The assertion (v) can be proved by choosing $N:=\operatorname{dim} \mathcal{F}$ pointwise linear independent sections $\left\{\delta_{1}, \ldots, \delta_{N}\right\}$ over $U$ such that $\operatorname{span}\left\{\delta_{1}(\lambda), \ldots, \delta_{N}(\lambda)\right\}=\mathcal{F}_{\lambda}, \lambda \in U$. Then any pointwise linear independent extension of these sections to $X$ as in theorem 1.3 .3 defines a subbundle that coincides with $\mathcal{F}$ over $D$.

1.3.6 Corollary. Let $\mathcal{E}$ be an infinite dimensional Banach bundle over the paracompact base $X$, $U \subset X$ an open subset and $\mathcal{F}_{1}, \mathcal{F}_{2}$ subbundles of $\left.\mathcal{E}\right|_{U}$ such that $\left.\mathcal{E}\right|_{U}=\mathcal{F}_{1} \oplus \mathcal{F}_{2}$, where $\mathcal{F}_{1}$ is finite dimensional and trivial. Then for each closed set $D \subset U$ there is a decomposition $\mathcal{E}=\mathcal{E}_{1} \oplus \mathcal{E}_{2}$, where $\mathcal{E}_{1}$ is finite dimensional and trivial, such that $\left.\mathcal{E}_{i}\right|_{D}=\left.\mathcal{F}_{i}\right|_{D}, i=1,2$. 
Proof. Since $X$ is paracompact, we can find an open subset $U_{1} \subset X$ such that $D \subset U_{1} \subset \overline{U_{1}} \subset$ $U$. By the last part of corollary 1.3 .5 there is a finite dimensional trivial subbundle $\mathcal{E}_{1}$ which coincides with $\mathcal{F}_{1}$ on $\overline{U_{1}}$. We choose a partition of unity $\left\{\eta_{1}, \eta_{2}\right\}$ corresponding to the open cover $\left\{U_{1}, X \backslash D\right\}$ of $X$. Furthermore, we use the propositions 1.2.6 and 1.2 .7 in order to construct a projection $P \in \mathcal{L}(\mathcal{E})$ on the subbundle $\mathcal{E}_{1}$ and a projection $Q \in \mathcal{L}\left(\left.\mathcal{E}\right|_{\overline{U_{1}}}\right)$ on $\left.\mathcal{E}_{1}\right|_{\overline{U_{1}}}=\left.\mathcal{F}_{1}\right|_{\overline{U_{1}}}$ parallel to $\left.\mathcal{F}_{2}\right|_{\overline{U_{1}}}$, where we use that $\overline{U_{1}}$ is closed in $X$ and hence paracompact. Then, by lemma 1.2.4 the morphism $\eta_{1} Q+\eta_{2} P$ is a projection onto $\mathcal{E}_{1}$ and by corollary 1.2 .8 its kernel gives a subbundle $\mathcal{E}_{2}$ such that $\mathcal{E}=\mathcal{E}_{1} \oplus \mathcal{E}_{2}$. Moreover, $\mathcal{E}_{2}$ coincides with $\mathcal{F}_{2}$ over $D$ by construction.

Before stating a further corollary of theorem 1.3 .3 , we make the following definiton.

1.3.7 Definition. Let $\mathcal{E}$ be a Banach Bundle and denote by $\pi: X \times[0,1] \rightarrow X$ the projection onto the first component. Two subbundles $\mathcal{F}_{0}$ and $\mathcal{F}_{1}$ of $\mathcal{E}$ are said to be homotopic if there exists a subbundle $\mathcal{F}$ of $\pi^{*} \mathcal{E}$ such that

$$
\left.\mathcal{F}\right|_{X \times\{i\}}=\mathcal{F}_{i}, \quad i=0,1 .
$$

1.3.8 Remark. As in the case of finite fibre dimensions (cf. [Ha09, Prop 1.7]) one can show that homotopic bundles over a paracompact base space are isomorphic.

1.3.9 Corollary. Two trivial finite dimensional subbundles of the same dimension of a Banach bundle over a paracompact base are homotopic.

Proof. We denote by $\pi_{1}: X \times\left[0, \frac{1}{3}\right) \rightarrow X$ and $\pi_{2}: X \times\left(\frac{2}{3}, 1\right] \rightarrow X$ the projections on the first component. If $\mathcal{F}_{1}$ and $\mathcal{F}_{2}$ are two trivial finite dimensional bundles over $X$, we obtain a finite dimensional trivial bundle over $X \times\left(\left[0, \frac{1}{3}\right) \cup\left(\frac{2}{3}, 1\right]\right)$ by $\pi_{1}^{*} \mathcal{F}_{1} \cup \pi_{2}^{*} \mathcal{F}_{2}$. Setting $D=X \times(\{0\} \cup\{1\})$, the claim follows from the last part of corollary 1.3 .5 .

1.3.10 Remark. Using the same idea as in the proof of the foregoing corollary 1.3.9 and theorem 1.3 .3 one can show that any two nowhere vanishing sections $\delta_{0}, \delta_{1}$ of $\mathcal{E}$ are homotopic as maps $X \rightarrow \mathcal{E} \backslash\{0\}$.

The next goal is to improve this result and obtain some interesting consequences.

1.3.11 Proposition. Let $\mathcal{E}$ be an infinite dimensional Banach bundle over the paracompact base $X$ and $\mathcal{F}_{0}, \mathcal{F}_{1}$ two isomorphic finite dimensional subbundles of finite type. Then $\mathcal{F}_{0}$ and $\mathcal{F}_{1}$ are homotopic.

Proof. Since $\mathcal{F}_{0}$ and $\mathcal{F}_{1}$ are isomorphic and of finite type, we can find a finite dimensional bundle $\mathcal{M}$ of finite type over $X$ such that $\mathcal{F}_{0} \oplus \mathcal{M}$ and $\mathcal{F}_{1} \oplus \mathcal{M}$ are trivial vector bundles of some dimension $n \in \mathbb{N}$ (cf. [Ha09, Prop. 1.4]). Now, as in the proof of part (iii) of corollary 1.3.5. we can embed these bundles into $\mathcal{E}$ and obtain that $\mathcal{F}_{0}$ and $\mathcal{F}_{1}$ are contained in trivial subbundles $\mathcal{G}_{0}$ and $\mathcal{G}_{1}$ of $\mathcal{E}$, respectively, which are of the same finite dimension $n$. Then $\mathcal{G}_{0}$ and $\mathcal{G}_{1}$ are homotopic by 
corollary 1.3.9 and, moreover, since $\mathcal{G}_{0}$ and $\mathcal{G}_{1}$ are trivial, the corresponding subbundle $\mathcal{G}$ of $\pi^{*} \mathcal{E}$ is trivial as well. Now we use a complement of $\mathcal{G}$ in $\pi^{*} \mathcal{E}$ and part (ii) of corollary 1.3.5 in order to extend $\mathcal{G}$ to a $2 n$-dimensional trivial subbundle $\tilde{\mathcal{G}}$ of $\pi^{*} \mathcal{E}$. Since we can embed $\mathcal{F}_{0}$ and $\mathcal{F}_{1}$ into $\Theta\left(\mathbb{C}^{n}\right)$, there exist continuous maps $f_{0}, f_{1}: X \rightarrow G_{k}\left(\mathbb{C}^{n}\right), k=\operatorname{dim} \mathcal{F}_{i}$, such that $f_{i}^{*} \gamma_{k}^{n} \cong \mathcal{F}_{i}$, $i=0,1$ (cf. [Hu94, 3.5.3]). Then we obtain from [Hu94, 3.6.2] and the assumption that $\mathcal{F}_{0}$ and $\mathcal{F}_{1}$ are isomorphic that $j \circ f_{0}$ and $j \circ f_{1}$ are homotopic, where $j: G_{k}\left(\mathbb{C}^{n}\right) \hookrightarrow G_{k}\left(\mathbb{C}^{2 n}\right)$ denotes the canonical inclusion. Hence $\mathcal{F}_{0}$ and $\mathcal{F}_{1}$ are homotopic by a subbundle of $\tilde{\mathcal{G}}$.

1.3.12 Corollary. Let $\mathcal{E}$ be an infinite dimensional Banach bundle over a paracompact base and $\mathcal{F}_{0}, \mathcal{F}_{1}$ two isomorphic finite dimensional subbundles of finite type. Then any complements of $\mathcal{F}_{0}$ and $\mathcal{F}_{1}$ are homotopic as well and in particular isomorphic.

Proof. Let $\mathcal{F}_{0}^{\prime}$ and $\mathcal{F}_{1}^{\prime}$ denote complements to $\mathcal{F}_{0}$ and $\mathcal{F}_{1}$, respectively. By proposition $1.3 .11 \mathcal{F}_{0}$ and $\mathcal{F}_{1}$ are homotopic. Arguing as in the proof of proposition 1.2 .7 it is readily seen that we now can find an idempotent $P \in \mathcal{L}\left(\pi^{*} \mathcal{E}\right)$ having the homotopy between $\mathcal{F}_{0}$ and $\mathcal{F}_{1}$ as image and such that $\mathcal{F}_{0}^{\prime}$ and $\mathcal{F}_{1}^{\prime}$ are in the kernel of $P$ over $X \times\{0\} \cup X \times\{1\}$. Hence the image of $I-P$ gives a homotopy of the complements.

1.3.13 Corollary. Let $\mathcal{E}$ be a trivial infinite dimensional Banach bundle over a paracompact base and $\mathcal{F}$ a trivial finite dimensional subbundle. Then any complement to $\mathcal{F}$ is trivial as well.

Proof. Let $\varphi: \mathcal{E} \rightarrow X \times E$ be a global trivialisation of $\mathcal{E}$ and $F \subset E$ be a subspace having the same finite dimension than $\mathcal{F}$. Then the counterimage of $F$ under $\varphi$ defines a trivial subbundle $\mathcal{F}^{\prime}$ of $\mathcal{E}$. Moreover, by taking any complementary subspace of $F$ in $E$ we obtain in the same way a trivial subbundle of $\mathcal{E}$ which is complementary to $\mathcal{F}^{\prime}$. Now $\mathcal{F}$ and $\mathcal{F}^{\prime}$ are homotopic by corollary 1.3 .9 and so any subbundle complementary to $\mathcal{F}$ is trivial by corollary 1.3 .12 . 


\section{Chapter 2}

\section{The Space of Closed Operators}

In this second chapter we consider the space of closed operators $\mathcal{C}(E, F)$ acting between two Banach spaces. Most of the following parts of the thesis will deal with closed operators and the main aim of the present chapter is to extend the fundamentals from the appendix to a broader background that is needed in order to follow the subsequent investigations. The chapter is organised as follows:

In the first section we take into account that $\mathcal{C}(E, F)$ is not a linear space and hence, in contrast to the space of bounded operators, we can not topologise it by a norm. However, following Kato's book [Ka76], we introduce the so called gap metric on $\mathcal{C}(E, F)$ which we will use in the following throughout in order to regard $\mathcal{C}(E, F)$ as a topological space. Most of the presentation is based on parts of [Ka76, IV.2]. Nevertheless, we adapt some of the results to our needs and add that the multiplication by a fixed non-vanishing scalar is a continuous self map of $\mathcal{C}(E, F)$. The latter result may seem trivial at a first view and also its proof is not sophisticated, but it will serve us well in the following sections.

The main result of the second section estimates the gap distance of $T+A$ and $S+B$, where $T$ and $S$ are two closed operators which are perturbed by bounded operators $A$ and $B$, respectively, by the gap distance of $S$ and $T$ and the norm distance of $A$ and $B$. In the special case $A=B$ this result is theorem IV.2.17 of [Ka76]. Afterwards we discuss a particular application of the case $A \neq B$ which is inspired by Matthias Lesch's investigation of topologies on spaces of unbounded operators which are relevant for defining the notion of spectral flow [Le05].

The third section is concerned with the spectral theory of closed operators. At the beginning we generalise some arguments for selfadjoint operators, which appeared in the construction of spectral flow in BLP05, to $\mathcal{C}(E)$ where $E$ is a general Banach space. We obtain the continuous dependence of the resolvent map $R(\lambda, T)=(\lambda-T)^{-1}$ on $\lambda$ and $T$ with respect to the gap topology and as a consequence the continuity of the spectral projections $T \mapsto P_{\triangle}(T)$ as introduced in theorem A.3.3 in the appendix. Afterwards we define the Cayley transform, which is usually just considered for selfadjoint or at least symmetric operators (cf. [Yo95, Sec. VII.4]), on a certain 
open subset of $\mathcal{C}(E)$ and study how spectra are transformed under this map. Finally, we use the Cayley transform in order to transfer the established stability results for subsets of spectra of bounded operators to $\mathcal{C}(E)$.

In the final section of this chapter we discuss the gap metric on $\mathcal{C}(H)$, where $H$ is a Hilbert space. The aim is to obtain a well known result which allows to compute the gap distance between two given operators by the corresponding orthogonal projections onto their graphs. We will use this possibility of computing gap distances in our main constructions in the second chapter thoroughly.

\subsection{Definition of the Gap Metric}

Before we study closed operators, we define the so called gap metric in a more general setting on the set of all closed subspaces of a given Banach space $E$. In the following we denote for any closed subspace $M$ of $E$ by $S_{M}$ the unit sphere in $M$. Moreover, given $u \in E$ and a closed subspace $N$ of $E$ let $d(u, N)=\inf _{v \in N}\|u-v\|$ denote the distance.

If $M$ and $N$ are closed subspace of $E$ we define

$$
\delta(M, N)=\left\{\begin{array}{l}
0, \quad \text { if } \quad M=0 \\
\sup _{u \in S_{M}} d(u, N), \text { otherwise }
\end{array}\right.
$$

as well as

$$
\hat{\delta}(M, N)=\max \{\delta(M, N), \delta(N, M)\}
$$

Note that

$$
\delta(M, N)=\inf \{\delta: d(u, N) \leq \delta\|u\| \forall u \in M\}
$$

and since $d(u, N)=\inf _{v \in N}\|u-v\| \leq\|u\|$ for all $u \in M$ we infer

$$
0 \leq \hat{\delta}(M, N) \leq 1
$$

$\hat{\delta}$ is symmetric by definition and one can easily check that it is positive definite as well, but the triangle inequality does not hold in genera 1 . Since our aim is to have a metric on the set of all closed subspaces of $E$, we modify $\delta$ and $\hat{\delta}$ slightly and define

\footnotetext{
${ }^{1}$ Nevertheless, by corollary 2.4 .3 which we will prove below, the triangle inequality holds if $E$ is a Hilbert space. Hence $\hat{\delta}$ defines a metric on the set of all closed subspaces of $E$ in this case.
} 


$$
d(M, N)=\left\{\begin{array}{l}
0, \text { if } \quad M=0 \\
2, \text { if } \quad M \neq 0, N=0 \\
\sup _{u \in S_{M}} d\left(u, S_{N}\right) \text { otherwise }
\end{array}\right.
$$

and

$$
\hat{d}(M, N)=\max \{d(M, N), d(N, M)\} .
$$

Note that since $\|u-v\| \leq 2$ for any $u, v \in S_{E}$ we have

$$
0 \leq \hat{d}(M, N) \leq 2
$$

for any closed subspaces $M$ and $N$ of $E$.

2.1.1 Lemma. $\hat{d}$ defines a metric on the set of all closed subspaces of $E$.

Proof. At first, it is clear that $\hat{d}(M, N) \geq 0$ and so, in order to check that $\hat{d}$ is positive definite, it remains to show that $\hat{d}(M, N)=0$ if and only if $M=N$. Moreover, it suffices to prove that $d(M, N)=0$ if and only if $M \subset N$. Note at first that $M \subset N$ obviously implies $d(M, N)=0$. Conversely, if $d(M, N)=0$ and $M \neq 0$, we infer $d\left(u, S_{N}\right)=0$ for all $u \in S_{M}$ and so $S_{M} \subset S_{N}$ since $S_{N}$ is closed. But $S_{M} \subset S_{N}$ implies $M \subset N$ and hence we have shown that $\hat{d}$ is positive definite. Moreover, the symmetry of $\hat{d}$ is a direct consequence of its definition.

Finally we have to check the triangle inequality, which is a little bit cumbersome due to the case-by-case definition of $d$. Note at first that it is enough to show that

$$
d(L, N) \leq d(L, M)+d(M, N)
$$

for all closed subspaces $L, M$ and $N$ of $E$. Now the following table, which is self-explanatory, covers all cases in which at least one of the three spaces in 2.4 is trivial.

\begin{tabular}{|c|c|c|c|c|}
$L$ & $N$ & $M$ & $d(L, N)$ & $d(L, M)+d(M, N)$ \\
\hline 0 & 0 & 0 & 0 & 0 \\
$\neq 0$ & 0 & 0 & 2 & 2 \\
0 & $\neq 0$ & 0 & 0 & 0 \\
0 & 0 & $\neq 0$ & 0 & 2 \\
$\neq 0$ & $\neq 0$ & 0 & $\leq 2$ & 2 \\
0 & $\neq 0$ & $\neq 0$ & 0 & $\geq 0$ \\
$\neq 0$ & 0 & $\neq 0$ & 2 & $\geq 2$
\end{tabular}


Now we turn to the case that all three spaces $L, N$ and $M$ are non trivial. From the triangle inequality

$$
\|u-v\| \leq\|u-w\|+\|w-v\|, \quad u \in S_{L}, v \in S_{N}, w \in S_{M}
$$

we infer

$$
d\left(u, S_{N}\right) \leq\|u-w\|+d\left(w, S_{N}\right) \leq\|u-w\|+\sup _{w \in S_{M}} d\left(w, S_{N}\right)
$$

for all $u \in S_{L}, w \in S_{M}$ and hence

$$
d\left(u, S_{N}\right) \leq d\left(u, S_{M}\right)+d(M, N), \quad u \in S_{L} .
$$

Finally, this implies

$$
d(L, N) \leq d(L, M)+d(M, N)
$$

2.1.2 Remark. In [Ka76, IV.2.1] it is mentioned without proof that the set of all closed subspaces of $E$ is complete with respect to $\hat{d}$.

Next we show that $\hat{d}$ and $\hat{\delta}$ are strictly related. This will be important in the sequel because, even if $\hat{\delta}$ is not a metric, it is often much more convenient to work with it instead of using $\hat{d}$.

2.1.3 Lemma. Let $M, N \subset E$ be closed subspaces. Then

$$
\hat{\delta}(M, N) \leq \hat{d}(M, N) \leq 2 \hat{\delta}(M, N) .
$$

Proof. First of all, it is enough to show that

$$
\delta(M, N) \leq d(M, N) \leq 2 \delta(M, N)
$$

for all closed subspaces $M$ and $N$ of $E$ and this inequality holds by definition if $M=\{0\}$. Hence we assume that $M \neq\{0\}$ in the following and also note that the first inequality follows immediately from the definitions since

$$
d(u, N) \leq d\left(u, S_{N}\right), \quad u \in M,
$$


if $N \neq 0$.

In order to show the second inequality of $(2.5)$ we consider the special case that $N$ is trivial at first. Since we assume that $M$ is not trivial, we have $d(M, 0)=2=2 \delta(M, 0)$ and hence 2.5 holds.

We finally treat the case $N \neq\{0\}$ and note that the assertion follows once we can show that

$$
d\left(u, S_{N}\right) \leq 2 d(u, N)
$$

for all $u \in M$ such that $\|u\|=1$. In order to prove 2.6), we fix $u \in S_{M}$ and let $\varepsilon>0$ be arbitrary. Then there exists $v \in N$ such that

$$
\|u-v\|<d(u, N)+\varepsilon
$$

and since a small perturbation of $v$ does not affect this inequality we can even assume $v$ to be non zero. Hence

$$
v_{0}=\frac{v}{\|v\|} \in S_{N}
$$

is defined and we obtain

$$
\begin{aligned}
\left\|v-v_{0}\right\| & =\left\|v-\frac{v}{\|v\|}\right\|=\left|1-\frac{1}{\|v\|}\right|\|v\|=|\|v\|-1| \\
& =|\|v\|-\|u\|| \leq\|v-u\| .
\end{aligned}
$$

Hence

$$
d\left(u, S_{N}\right) \leq\left\|u-v_{0}\right\| \leq\|u-v\|+\left\|v-v_{0}\right\| \leq 2\|u-v\| \leq 2 d(u, N)+2 \varepsilon
$$

which implies (2.6) since $\varepsilon>0$ is arbitrary.

Now we turn to closed operators and state the main definition of this section.

2.1.4 Definition. Let $E, F$ be Banach spaces. We define the gap metric on $\mathcal{C}(E, F)$ by

$$
d_{G}\left(\mathcal{A}_{1}, \mathcal{A}_{2}\right)=\hat{d}\left(\operatorname{graph}\left(\mathcal{A}_{1}\right), \operatorname{graph}\left(\mathcal{A}_{2}\right)\right) .
$$

$d_{G}$ defines indeed a metric on $\mathcal{C}(E, F)$ by lemma 2.1.1. Note that the set of all graphs of closed operators in $E \times F$ is just a proper subspace of the set of all closed subspaces of $E \times F$. More precisely, a subspace $U \subset E \times F$ is the graph of a linear operator if and only if $U \cap(\{0\} \times F)=\{0\}$. 
As a first step of our investigation of $\mathcal{C}(E, F)$ we want to use this observation to show that at least in the case $E=F, \mathcal{C}(E)$ is not complete with respect to the gap metrif ${ }^{2}$

Consider the sequence of operators $T_{n}=n \cdot I_{E} \in \mathcal{L}(E) \subset \mathcal{C}(E)$. From

$$
\operatorname{graph}\left(T_{n}\right)=\{(u, n u): u \in E\}=\left\{\left(\frac{1}{n} u, u\right): u \in E\right\}
$$

we find for any $(0, v) \in\{0\} \times E \subset E \times E$

$$
\begin{aligned}
d\left((0, v), \operatorname{graph} T_{n}\right) & =\inf _{u \in E}\left\{\left\|(0, v)-\left(\frac{1}{n} u, u\right)\right\|\right\}=\inf _{u \in E} \sqrt{\frac{1}{n^{2}}\|u\|^{2}+\|u-v\|^{2}} \\
& \leq \frac{1}{n}\|v\|
\end{aligned}
$$

and hence

$$
\delta\left(\{0\} \times E, \operatorname{graph} T_{n}\right)=\sup _{(0, v) \in S_{\{0\} \times E}} d\left((0, v), \operatorname{graph} T_{n}\right) \leq \frac{1}{n} .
$$

Similarly, we obtain for any $\left(\frac{1}{n} u, u\right), u \in E$,

$$
\begin{aligned}
\left.d\left(\left(\frac{1}{n} u, u\right)\right),\{0\} \times E\right) & =\inf _{v \in E}\left\{\left\|\left(\frac{1}{n} u, u\right)-(0, v)\right\|\right\}=\inf _{v \in E} \sqrt{\frac{1}{n^{2}}\|u\|^{2}+\|u-v\|^{2}} \\
& \leq \frac{1}{n}\|u\| \leq \frac{1}{n} \sqrt{\frac{1}{n^{2}}\|u\|^{2}+\|u\|^{2}}=\frac{1}{n}\left\|\left(\frac{1}{n} u, u\right)\right\|
\end{aligned}
$$

implying

$$
\delta\left(\operatorname{graph} T_{n},\{0\} \times E\right)=\sup _{\left(\frac{1}{n} u, u\right) \in S_{\operatorname{graph} T_{n}}} d\left(\left(\frac{1}{n} u, u\right),\{0\} \times E\right) \leq \frac{1}{n} .
$$

Accordingly, $\operatorname{graph}\left(T_{n}\right) \rightarrow\{0\} \times E$ with respect to $\hat{\delta}$ and hence $T_{n} \in \mathcal{C}(E)$ is a Cauchy sequence with respect to $d_{G}$ by lemma 2.1.3 But, since $\{0\} \times E$ is not the graph of an operator, $\left\{T_{n}\right\}$ does not converge in $\mathcal{C}(E)$. Hence $\mathcal{C}(E)$ is not complete with respect to the graph norm. We can even learn a little bit more from this example: Since all $T_{n}, n \in \mathbb{N}$, are actually elements of $\mathcal{L}(E)$, we infer that the metric space of the bounded operators $\mathcal{L}(E)$ with the gap metric is not complete and hence the metric $d_{G}$ and the metric induced by the operator norm are not uniformly equivalent in the sense that there exist constants $c_{1}, c_{2}>0$ such that

$$
c_{1} d_{G}(A, B) \leq\|A-B\| \leq c_{2} d_{G}(A, B), \quad A, B \in \mathcal{L}(E) .
$$

Nevertheless, at least the first of these inequalities holds by the following simple result.

\footnotetext{
${ }^{2}$ As announced in [Ka76, Remark 2.10], one can even show this in the general case of different spaces $E$ and $F$. However, we are content with the special case $E=F$ in which we are mainly interested anyway.
} 
2.1.5 Lemma. Let $A, B \in \mathcal{L}(E, F)$. Then

$$
d_{G}(A, B) \leq 2\|A-B\| .
$$

Proof. For any fixed $u \in E$ we find

$$
\begin{aligned}
d((u, A u), \operatorname{graph}(B)) & =\inf _{v \in E}\|(u, A u)-(v, B v)\|=\inf _{v \in E}\|(u-v, A u-B v)\| \\
& \leq\|A u-B u\| \leq\|A-B\|\|u\| .
\end{aligned}
$$

If $u \in S_{\operatorname{graph}(A)}$, we have by definition $\|u\|^{2}+\|A u\|^{2}=1$ and so in particular $\|u\| \leq 1$. Hence we infer from 2.8

$$
\delta(\operatorname{graph}(A), \operatorname{graph}(B))=\sup _{(u, A u) \in S_{\operatorname{graph}(A)}} d((u, A u), \operatorname{graph}(B)) \leq\|A-B\|
$$

and by symmetry of the right hand side we also have

$$
\delta(\operatorname{graph}(B), \operatorname{graph}(A)) \leq\|A-B\| .
$$

Using lemma 2.1.3, we finally obtain

$$
d_{G}(A, B) \leq 2 \hat{\delta}(\operatorname{graph}(A), \operatorname{graph}(B)) \leq 2\|A-B\| .
$$

Our next aim is to prove that nevertheless the remaining inequality of 2.7) holds locally. As a consequence, we will show in a subsequent corollary that the topologies induced by the norm and the gap topology coincide on $\mathcal{L}(E, F)$.

The proof of the following result is a combination and simple modification of Lemma IV.2.11 and Theorem IV.2.13 of [Ka76].

2.1.6 Lemma. Let $A \in \mathcal{L}(E, F)$ be given. If $B \in \mathcal{L}(E, F)$ is a further bounded operator such that $d_{G}(A, B)<\left(1+\|A\|^{2}\right)^{-\frac{1}{2}}$, then

$$
\|A-B\| \leq \frac{1+\|A\|^{2}}{1-d_{G}(A, B) \sqrt{1+\|A\|^{2}}} d_{G}(A, B)
$$

Proof. In order to simplify the quite technical proof, we want to note two simple observations before.

At first, for any real number $0 \leq a \leq 1$ and any $b \in \mathbb{R}$ we obviously have 


$$
\underbrace{1-\sqrt{1-a^{2}}}_{\geq 0}+\underbrace{a\left(\sqrt{1+b^{2}}-b\right)}_{\geq 0} \geq 0
$$

and hence

$$
b a+\sqrt{1-a^{2}} \leq 1+a \sqrt{1+b^{2}} .
$$

If moreover $a \sqrt{1+b^{2}}<1$, we infer

$0<\left(1-a \sqrt{1+b^{2}}\right)\left(b a+\sqrt{1-a^{2}}\right) \leq\left(1-a \sqrt{1+b^{2}}\right)\left(1+a \sqrt{1+b^{2}}\right)=1-a^{2}\left(1+b^{2}\right)$.

Secondly, if $a, c>0, b \geq 0$, we note that all positive real numbers $x$ satisfying the inequality

$$
a x^{2}-b x-c \leq 0
$$

are given by

$$
x \in\left[0, \frac{1}{2 a}\left(b+\sqrt{b^{2}+4 a c}\right)\right) .
$$

Now we start to prove lemma 2.1.6

Let $\varphi \in S_{\operatorname{graph}(B)}$ and $u \in E$ such that $\varphi=(u, B u),\|u\|^{2}+\|B u\|^{2}=\|\varphi\|^{2}=1$. We fix some $\delta^{\prime}>0$ such that

$$
\hat{\delta}(\operatorname{graph}(A), \operatorname{graph}(B)) \leq d_{G}(A, B)<\delta^{\prime}<\left(1+\|A\|^{2}\right)^{-\frac{1}{2}} \leq 1 .
$$

Then

$$
d(\varphi, \operatorname{graph}(A)) \leq \hat{\delta}(\operatorname{graph}(A), \operatorname{graph}(B))<\delta^{\prime}
$$

and thus we can choose $\psi=(v, A v) \in \operatorname{graph}(A)$ such that $\|\varphi-\psi\|<\delta^{\prime}$.

We obtain

$$
\|u-v\|^{2}+\|B u-A v\|^{2}=\|\varphi-\psi\|^{2}<\left(\delta^{\prime}\right)^{2}
$$

and hence, by using the Cauchy-Schwartz inequality, 


$$
\begin{aligned}
\|(A-B) u\|^{2} & =\|B u-A v-A(u-v)\|^{2} \leq(\|B u-A v\|+\|A\|\|u-v\|)^{2} \\
& \leq\left(1+\|A\|^{2}\right)\left(\|B u-A v\|^{2}+\|u-v\|^{2}\right) \leq\left(1+\|A\|^{2}\right)\left(\delta^{\prime}\right)^{2} .
\end{aligned}
$$

Moreover,

$$
\begin{aligned}
1 & =\|u\|^{2}+\|B u\|^{2}=\|u\|^{2}+\|A u+(B-A) u\|^{2} \\
& \leq\|u\|^{2}+(\|A u\|+\|(B-A) u\|)^{2} \\
& \leq\|u\|^{2}+\|A u\|^{2}+2\|A\|\|u\|\|(B-A) u\|+\|(B-A) u\|^{2} \\
& \leq\left(1+\|A\|^{2}\right)\|u\|^{2}+2\|A\|\|u\|\|(B-A) u\|+\|(B-A) u\|^{2}
\end{aligned}
$$

Combining 2.11) and 2.12) we find

$$
\begin{aligned}
\|(A-B) u\|^{2} & \leq\left(\delta^{\prime}\right)^{2}\left(1+\|A\|^{2}\right) \\
& \leq\left(\delta^{\prime}\right)^{2}\left(1+\|A\|^{2}\right)\left(\left(1+\|A\|^{2}\right)\|u\|^{2}+2\|A\|\|u\|\|(B-A) u\|+\|(B-A) u\|^{2}\right)
\end{aligned}
$$

or equivalently

$$
\begin{aligned}
0 & \geq\left(1-\left(\delta^{\prime}\right)^{2}\left(1+\|A\|^{2}\right)\right)\|(B-A) u\|^{2}-2\|A\|\|u\|\left(\delta^{\prime}\right)^{2}\left(1+\|A\|^{2}\right)\|(B-A) u\| \\
& -\left(\delta^{\prime}\right)^{2}\left(1+\|A\|^{2}\right)^{2}\|u\|^{2} .
\end{aligned}
$$

Now we make use of formula 2.10 and set

$$
\begin{aligned}
& a=1-\left(\delta^{\prime}\right)^{2}\left(1+\|A\|^{2}\right) \\
& b=2\|A\|\|u\|\left(\delta^{\prime}\right)^{2}\left(1+\|A\|^{2}\right) \\
& c=\left(\delta^{\prime}\right)^{2}\left(1+\|A\|^{2}\right)^{2}\|u\|^{2} .
\end{aligned}
$$

Note that obviously $b \geq 0$ and $c>0$ while $a>0$ follows from the choice of $\delta^{\prime}$. We compute

$$
\begin{aligned}
b^{2}+4 a c & =4\|A\|^{2}\|u\|^{2}\left(\delta^{\prime}\right)^{4}\left(1+\|A\|^{2}\right)^{2}+4\left(1-\left(\delta^{\prime}\right)^{2}\left(1+\|A\|^{2}\right)\right)\left(\delta^{\prime}\right)^{2}\left(1+\|A\|^{2}\right)^{2}\|u\|^{2} \\
& =4\left(\delta^{\prime}\right)^{2}\|u\|^{2}\left(1+\|A\|^{2}\right)^{2}\left(\|A\|^{2}\left(\delta^{\prime}\right)^{2}+1-\left(\delta^{\prime}\right)^{2}\left(1+\|A\|^{2}\right)\right) \\
& =4\left(\delta^{\prime}\right)^{2}\|u\|^{2}\left(1+\|A\|^{2}\right)^{2}\left(1-\left(\delta^{\prime}\right)^{2}\right)
\end{aligned}
$$

and obtain

$$
\begin{aligned}
\|(B-A) u\| & \leq \frac{2\|A\|\|u\|\left(\delta^{\prime}\right)^{2}\left(1+\|A\|^{2}\right)+2 \delta^{\prime}\|u\|\left(1+\|A\|^{2}\right) \sqrt{1-\left(\delta^{\prime}\right)^{2}}}{\left.2\left(1-\delta^{\prime}\right)^{2}\left(1+\|A\|^{2}\right)\right)} \\
& =\frac{\delta^{\prime}\left(1+\|A\|^{2}\right)\|u\|\left(\delta^{\prime}\|A\|+\sqrt{1-\left(\delta^{\prime}\right)^{2}}\right)}{1-\left(\delta^{\prime}\right)^{2}\left(1+\|A\|^{2}\right)} .
\end{aligned}
$$


Finally, by using the inequality 2.9$]$, we end up at

$$
\|(B-A) u\| \leq \frac{\delta^{\prime}\left(1+\|A\|^{2}\right)\|u\|\left(\delta^{\prime}\|A\|+\sqrt{1-\left(\delta^{\prime}\right)^{2}}\right)}{\left(\delta^{\prime}\|A\|+\sqrt{1-\left(\delta^{\prime}\right)^{2}}\right)\left(1-\delta^{\prime} \sqrt{1+\|A\|^{2}}\right)} \leq \frac{\delta^{\prime}\left(1+\|A\|^{2}\right)}{1-\delta^{\prime} \sqrt{1+\|A\|^{2}}}\|u\|,
$$

where we use again that $\delta^{\prime}\left(1+\|A\|^{2}\right)^{\frac{1}{2}}<1$ by assumption.

Since $d_{G}(A, B)<\delta^{\prime}<\left(1+\|A\|^{2}\right)^{-\frac{1}{2}}$ is arbitrary, we obtain

$$
\|(B-A) u\| \leq \frac{d_{G}(A, B)\left(1+\|A\|^{2}\right)}{1-d_{G}(A, B) \sqrt{1+\|A\|^{2}}}\|u\| .
$$

Now we just have to remember that $\varphi=(u, B u)$ is an arbitrary element of $S_{\operatorname{graph}(B)}$. Accordingly, since the inequality (2.14) is obviously homogenous in $u$, we infer that it holds for all $u \in E$, implying finally

$$
\|B-A\| \leq \frac{1+\|A\|^{2}}{1-d_{G}(A, B) \sqrt{1+\|A\|^{2}}} d_{G}(A, B)
$$

2.1.7 Corollary. For all $A \in \mathcal{L}(E, F)$ there exists a neighbourhood $U \subset \mathcal{L}(E, F)$ with respect to the gap topology and a constant $c>0$ such that

$$
\|B-A\| \leq c d_{G}(A, B), \quad B \in U
$$

Proof. We set

$$
U=\left\{B \in \mathcal{L}(E, F): d_{G}(A, B)<\frac{1}{2}\left(1+\|A\|^{2}\right)^{-\frac{1}{2}}\right\}
$$

and obtain from lemma 2.1 .6

$$
\|B-A\| \leq 2\left(1+\|A\|^{2}\right) d_{G}(A, B)
$$

for all $B \in U$.

Note that this corollary and lemma 2.1.5 imply that the topologies induced by the norm and the gap metric on $\mathcal{L}(E, F)$ coincide.

So far we have only considered bounded operators in the gap topology. Now we turn for the first time to the unbounded case and show that the simplest non-constant map $\mathcal{C}(E, F) \rightarrow \mathcal{C}(E, F)$ is continuous with respect to the gap topology. Note that, by lemma A.2.2, $\alpha T$ is a closed operator if $T$ is closed and $\alpha \neq 0$. 
2.1.8 Lemma. Let $\alpha \in \mathbb{C} \backslash\{0\}$ and $S, T \in \mathcal{C}(E, F)$. Then we have

$$
d_{G}(\alpha S, \alpha T) \leq 2 \max \left\{|\alpha|, \frac{1}{|\alpha|}\right\} d_{G}(S, T)
$$

Proof. At first, note that the assertion holds if $\mathcal{D}(S)=\{0\}$ or $\mathcal{D}(T)=\{0\}$ and hence we assume in the following that both domains are non trivial.

Now for every $u \in \mathcal{D}(S), u \neq 0$, we obtain

$$
\begin{aligned}
d((u, \alpha S u), \operatorname{graph}(\alpha T)) & =\inf _{v \in \mathcal{D}(T)} \sqrt{\|u-v\|^{2}+\|\alpha(S u-T v)\|^{2}} \\
& \leq \max \{1,|\alpha|\} d((u, S u), \operatorname{graph}(T)) \\
& \leq \max \{1,|\alpha|\} d\left(\frac{(u, S u)}{\|(u, S u)\|}, \operatorname{graph}(T)\right)\|(u, S u)\| \\
& \leq \max \{1,|\alpha|\} \underset{(w, S w) \in S_{\operatorname{graph}(S)}}{\sup } d((w, S w), \operatorname{graph}(T))\|(u, S u)\| \\
& =\max \{1,|\alpha|\} \delta(\operatorname{graph}(S), \operatorname{graph}(T))\|(u, S u)\| .
\end{aligned}
$$

From the obvious inequality

$$
\|u\|^{2}+\|S u\|^{2} \leq \max \left\{1, \frac{1}{|\alpha|^{2}}\right\}\left(\|u\|^{2}+|\alpha|^{2}\|S u\|^{2}\right), \quad u \in \mathcal{D}(S),
$$

we infer

$$
\|(u, S u)\| \leq \max \left\{1, \frac{1}{|\alpha|}\right\}\|(u, \alpha S u)\|
$$

and hence 2.15 yields

$$
d((u, \alpha S u), \operatorname{graph}(\alpha T)) \leq \max \left\{|\alpha|, \frac{1}{|\alpha|}\right\} \delta(\operatorname{graph}(S), \operatorname{graph}(T))\|(u, \alpha S u)\| .
$$

We conclude

$$
\begin{aligned}
\delta(\operatorname{graph}(\alpha S), \operatorname{graph}(\alpha T)) & =\sup _{(u, \alpha S u) \in S_{\operatorname{graph}(\alpha S)}} d((u, \alpha S u), \operatorname{graph}(\alpha T)) \\
& \leq \max \left\{|\alpha|, \frac{1}{|\alpha|}\right\} \delta(\operatorname{graph}(S), \operatorname{graph}(T))
\end{aligned}
$$

and by interchanging $S$ and $T$ we infer

$$
\hat{\delta}(\operatorname{graph}(\alpha S), \operatorname{graph}(\alpha T)) \leq \max \left\{|\alpha|, \frac{1}{|\alpha|}\right\} \hat{\delta}(\operatorname{graph}(S), \operatorname{graph}(T))
$$


From lemma 2.1 .3 we finally obtain

$$
d_{G}(\alpha S, \alpha T) \leq 2 \max \left\{|\alpha|, \frac{1}{|\alpha|}\right\} d_{G}(S, T)
$$

\subsection{An Estimate for Bounded Perturbations}

Given a closed operator $\mathcal{A} \in \mathcal{C}(E, F)$ and a bounded operator $B \in \mathcal{L}(E, F)$, we know by lemma A.2.2 that $\mathcal{A}+B \in \mathcal{C}(E, F)$ as well, where $\mathcal{D}(\mathcal{A}+B)=\mathcal{D}(\mathcal{A})$. Theorem IV.2.17 of [Ka76] asserts that if $T, S \in \mathcal{C}(E, F)$ are closed operators and $A \in \mathcal{L}(E, F)$ is bounded, then

$$
d_{G}(S+A, T+A) \leq 4\left(1+\|A\|^{2}\right) d_{G}(S, T) .
$$

Our aim in this section is to generalise this theorem to the case that $S$ and $T$ are perturbed by different bounded operators $A$ and $B$. Moreover, this will lead to a result concerning the continuity of a certain type of canonical embeddings of $\mathcal{L}(E, F)$ with the norm topology into $\mathcal{C}(E, F)$. These embeddings appeared in the special case of selfadjoint Fredholm operators in the development of the spectral flow for unbounded operators some years ago. We will give details and references below.

We start by proving the main result of this section as announced above, which reads as follows:

2.2.1 Theorem. Let $S, T \in \mathcal{C}(E, F)$ be closed operators and $A, B \in \mathcal{L}(E, F)$ bounded. Then

$$
d_{G}(T+A, S+B) \leq 4 \sqrt{2} \sqrt{1+\|A\|^{2}} \sqrt{1+\|B\|^{2}}\left(d_{G}(S, T)+\|A-B\|\right) .
$$

Proof. Note at first that we can assume that $\mathcal{D}(T), \mathcal{D}(S) \neq\{0\}$ without loss of generality, because otherwise $d_{G}(T+A, S+B)=d_{G}(T, S)$ and the assertion holds trivially.

Let now $\varphi \in \operatorname{graph}(S+B),\|\varphi\|=1$. Then there exists $u \in \mathcal{D}(S)$, such that $\varphi=(u,(S+B) u)$ and $\|u\|^{2}+\|(S+B) u\|^{2}=\|\varphi\|^{2}=1$. Setting $r^{2}:=\|u\|^{2}+\|S u\|^{2}>0$ we obtain, incidentally, the following inequality

$$
\begin{aligned}
r^{2} & =\|u\|^{2}+\|(S+B) u-B u\|^{2} \leq\|u\|^{2}+2\|(S+B) u\|^{2}+2\|B u\|^{2} \\
& \leq 2(\underbrace{\|u\|^{2}+\|(S+B) u\|^{2}}_{=1})+2\|B\|^{2} \underbrace{\|u\|^{2}}_{\leq 1} \leq 2\left(1+\|B\|^{2}\right),
\end{aligned}
$$

which we will need below. Moreover, by the choice of $r, r^{-1}(u, S u)$ is an element of the unit $\operatorname{sphere}$ of $\operatorname{graph}(S)$. Hence we have for all $\delta^{\prime}>\hat{\delta}(\operatorname{graph}(S), \operatorname{graph}(T))$ 


$$
d\left(r^{-1}(u, S u), \operatorname{graph}(T)\right) \leq \sup _{w \in S_{\operatorname{graph}(S)}} d(w, \operatorname{graph}(T)) \leq \hat{\delta}(\operatorname{graph}(S), \operatorname{graph}(T))<\delta^{\prime},
$$

which implies $d((u, S u), \operatorname{graph}(T))<r \delta^{\prime}$ and so the existence of $v \in \mathcal{D}(T)$ such that

$$
\|u-v\|^{2}+\|S u-T v\|^{2}<r^{2} \delta^{2} .
$$

Setting $\psi=(v,(T+A) v) \in \operatorname{graph}(T+A)$, we obtain

$$
\begin{aligned}
\|\varphi-\psi\|^{2} & =\|(u,(S+B) u)-(v,(T+A) v)\|^{2}=\|u-v\|^{2}+\|S u-T v+B u-A v\|^{2} \\
& \leq\|u-v\|^{2}+2\|S u-T v\|^{2}+2\|B u-A v\|^{2} \\
& \leq 2\left(\|u-v\|^{2}+\|S u-T v\|^{2}\right)+2\|B u-A v\|^{2} .
\end{aligned}
$$

Now we use 2.18 twice and that $\|u\| \leq r$ by definition of $r$, and get

$$
\begin{aligned}
\|\varphi-\psi\|^{2} & \leq 2 r^{2} \delta^{\prime 2}+2\|B u-A v\|^{2} \leq 2 r^{2} \delta^{2}+2(\|A v-A u\|+\|A u-B u\|)^{2} \\
& \leq 2 r^{2} \delta^{\prime 2}+4\|A\|^{2}\|v-u\|^{2}+4\|A-B\|^{2}\|u\|^{2} \\
& \leq 2\left(1+2\|A\|^{2}\right) r^{2} \delta^{\prime 2}+4\|A-B\|^{2}\|u\|^{2} \\
& \leq 2\left(1+2\|A\|^{2}\right) r^{2} \delta^{\prime 2}+4 r^{2}\|A-B\|^{2} .
\end{aligned}
$$

Finally, by using (2.17), we conclude that

$$
\begin{aligned}
\|\varphi-\psi\|^{2} & \leq 4\left(1+2\|A\|^{2}\right)\left(1+\|B\|^{2}\right) \delta^{2}+8\left(1+\|B\|^{2}\right)\|A-B\|^{2} \\
& \leq 8\left(1+\|A\|^{2}\right)\left(1+\|B\|^{2}\right) \delta^{2}+8\left(1+\|A\|^{2}\right)\left(1+\|B\|^{2}\right)\|A-B\|^{2} \\
& \leq 8\left(1+\|A\|^{2}\right)\left(1+\|B\|^{2}\right)\left(\delta^{\prime 2}+\|A-B\|^{2}\right) \\
& \leq 8\left(1+\|A\|^{2}\right)\left(1+\|B\|^{2}\right)\left(\delta^{\prime}+\|A-B\|^{2}\right.
\end{aligned}
$$

and infer

$$
\|\varphi-\psi\| \leq 2 \sqrt{2} \sqrt{1+\|A\|^{2}} \sqrt{1+\|B\|^{2}}\left(\delta^{\prime}+\|A-B\|\right) .
$$

Now we are almost done.

Let us point out that in 2.19$) \varphi \in S_{\operatorname{graph}(S+B)}$ is arbitrary whereas $\psi \in \operatorname{graph}(T+A)$ depends on $\varphi$. Since $\psi \in \operatorname{graph}(T+A)$ we obtain from 2.19 .

$$
\begin{aligned}
d(\varphi, \operatorname{graph}(T+A)) & =\inf _{\tilde{\psi} \in \operatorname{graph}(T+A)}\|\varphi-\tilde{\psi}\| \leq\|\varphi-\psi\| \\
& \leq 2 \sqrt{2} \sqrt{1+\|A\|^{2}} \sqrt{1+\|B\|^{2}}\left(\delta^{\prime}+\|A-B\|\right)
\end{aligned}
$$


for any $\varphi \in \operatorname{graph}(S+B),\|\varphi\|=1$, and hence

$$
\begin{aligned}
\delta(\operatorname{graph}(S+B), \operatorname{graph}(T+A)) & =\sup _{\tilde{\varphi} \in S_{\operatorname{graph}(S+B)}} d(\tilde{\varphi}, \operatorname{graph}(T+A)) \\
& \leq 2 \sqrt{2} \sqrt{1+\|A\|^{2}} \sqrt{1+\|B\|^{2}}\left(\delta^{\prime}+\|A-B\|\right) .
\end{aligned}
$$

Remembering that $\delta^{\prime}$ is any fixed number greater than $\hat{\delta}(\operatorname{graph}(S)$, graph $(T))$, we infer

$\delta(\operatorname{graph}(S+B), \operatorname{graph}(T+A)) \leq 2 \sqrt{2} \sqrt{1+\|A\|^{2}} \sqrt{1+\|B\|^{2}}(\hat{\delta}(\operatorname{graph}(S), \operatorname{graph}(T))+\|A-B\|)$

and since the right hand side of this inequality is symmetric in $T+A$ and $S+B$, we obtain by interchanging $S+B$ and $T+A$

$\hat{\delta}(\operatorname{graph}(T+A), \operatorname{graph}(S+B)) \leq 2 \sqrt{2} \sqrt{1+\|A\|^{2}} \sqrt{1+\|B\|^{2}}(\hat{\delta}(\operatorname{graph}(S), \operatorname{graph}(T))+\|A-B\|)$.

Finally we use lemma 2.1 .3 and conclude

$$
d_{G}(T+A, S+B) \leq 4 \sqrt{2} \sqrt{1+\|A\|^{2}} \sqrt{1+\|B\|^{2}}\left(d_{G}(S, T)+\|A-B\|\right) .
$$

Before going on, we want to state some remarks regarding theorem 2.2.1.

2.2.2 Remark. i) Note that if $A=B$, theorem 2.2.1 and (2.16) differ by a constant $\sqrt{2}$ which is of course not very important for applications.

ii) The proof of theorem 2.2.1 follows the argument of the proof of 2.16 as presented in [Ka76, IV.2.17]. Hence we doubt strongly that 2.2.1 is unknown, however, we could not find any reference for it in the literature.

iii) An alternative way to prove theorem 2.2.1 should be as follows: By using the triangle inequality, we obtain

$$
d_{G}(T+A, S+B) \leq d_{G}(T+A, T+B)+d_{G}(T+B, S+B)
$$

and the second term can be estimated according to 2.16. However, the proof of 2.16 is not much more elementary than the proof of theorem 2.2.1. Moreover, we do not know if there is a way to estimate $d_{G}(T+A, T+B)$ by $\|A-B\|$ other than just using the argument of 2.2.1 in the special case $S=T$. 
We now want to consider a couple of applications of the foregoing theorem 2.2.1. The first one is just an obvious extension of lemma 2.1.8

2.2.3 Corollary. Let $\alpha, \beta \in \mathbb{C}, \alpha \neq 0$. Then the map

$$
\mathcal{C}(E, F) \rightarrow \mathcal{C}(E, F), \quad T \mapsto \alpha T+\beta
$$

is continuous.

Proof. If $T, S \in \mathcal{C}(E, F)$, we obtain by theorem 2.2.1 and lemma 2.1.8 that

$$
d_{G}(\alpha T+\beta, \alpha S+\beta) \leq 4 \sqrt{2}\left(1+|\beta|^{2}\right) d_{G}(\alpha T, \alpha S) \leq 8 \sqrt{2}\left(1+|\beta|^{2}\right) \max \left\{|\alpha|, \frac{1}{|\alpha|}\right\} d_{G}(T, S) .
$$

Let $T \in \mathcal{C}(E, F)$ be a fixed operator and consider the map

$$
\mathcal{T}_{T}: \mathcal{L}(E, F) \rightarrow \mathcal{C}(E, F), \quad C \mapsto T+C
$$

In the special case that $E=F=H$ is a Hilbert space and $T$ a selfadjoint operator, the continuity of the restriction of $\mathcal{T}_{T}$ to the selfadjoint bounded operators in $\mathcal{L}(H)$ is a direct consequence of [Le05, 2.2]. Moreover, an alternative proof, using quite advanced functional calculus, is sketched in [BoFu98, 4.10].

We now obtain the continuity of $\mathcal{T}_{T}$ on the whole domain $\mathcal{L}(E, F)$ in the general case where $E$ and $F$ are Banach spaces and $T \in \mathcal{C}(E, F)$ as a direct consequence of theorem 2.2.1.

2.2.4 Corollary. $\mathcal{T}_{T}: \mathcal{L}(E, F) \rightarrow \mathcal{C}(E, F)$ is continuous.

Proof. Let $A \in \mathcal{L}(E, F)$ be fixed. For any $B \in \mathcal{L}(E, F)$ such that $\|A-B\|<1$ we have

$$
\|B\| \leq\|A-B\|+\|A\| \leq 1+\|A\|
$$

and hence by theorem 2.2 .1

$$
\begin{aligned}
d_{G}\left(\mathcal{T}_{T}(B), \mathcal{T}_{T}(A)\right) & \leq 4 \sqrt{2} \sqrt{1+\|A\|^{2}} \sqrt{1+\|B\|^{2}}\|A-B\| \\
& \leq 4 \sqrt{2} \sqrt{1+\|A\|^{2}} \sqrt{1+(1+\|A\|)^{2}}\|A-B\| .
\end{aligned}
$$


Note that in general it is a difficult task to show the continuity of a given family of closed operators because of the very definition of the gap metric. Accordingly, corollary 2.2.4 is an important result for constructing gap continuous families.

Finally, we want to take a further look at the map $\mathcal{T}_{T}$ and ask about the relation between the topology that the injection $\mathcal{T}_{T}$ induces on its image in $\mathcal{C}(E, F)$ and the subspace topology induced by the gap metric on $\operatorname{im} \mathcal{T}_{T}$. Note that $\mathcal{T}_{T}$ is an embedding if $T \in \mathcal{L}(E, F)$ because the norm topology and the gap topology coincide on $\mathcal{L}(E, F)$ as shown in corollary 2.1.7. Our next aim is to show that the topology induced on its image can also be strictly finer than the gap topology. At first we need a technical result which is problem IV.1.2 in [Ka76].

2.2.5 Lemma. Let $T$ and $A$ be operators acting between the normed linear spaces $E$ and $F$ such that $\mathcal{D}(T) \subset \mathcal{D}(A)$ and

$$
\|A u\| \leq a\|u\|+b\|T u\|, \quad u \in \mathcal{D}(T),
$$

for some $a \geq 0$ and $0 \leq b<13$ Then the operator $S=T+A, \mathcal{D}(S)=\mathcal{D}(T)$, satisfies

$$
\|A u\| \leq a\|u\|+b\|T u\| \leq \frac{1}{1-b}(a\|u\|+b\|S u\|), \quad u \in \mathcal{D}(T) .
$$

Proof. By assumption we have

$$
-a\|u\|-b\|T u\| \leq-\|A u\|
$$

and hence for any $u \in \mathcal{D}(S)=\mathcal{D}(T)$

$$
-a\|u\|+(1-b)\|T u\| \leq\|T u\|-\|A u\| \leq|\|T u\|-\|A u\|| \leq\|T u+A u\|=\|S u\| .
$$

Using this inequality, we find

$$
\begin{aligned}
a\|u\|+b\|T u\| & =\frac{1}{1-b}(a(1-b)\|u\|+b(1-b)\|T u\|)=\frac{1}{1-b}(a\|u\|+b(-a\|u\|+(1-b)\|T u\|)) \\
& \leq \frac{1}{1-b}(a\|u\|+b\|S u\|) .
\end{aligned}
$$

The following result can also be obtained as a consequence of [Le05, 2.4]. There it is proved that the restriction of $\mathcal{T}_{T}$ to the selfadjoint elements in $\mathcal{L}(H)$ is not an embedding when viewed as a map into a certain space of unbounded selfadjoint operators which itself is continuously included in the subspace of selfadjoint elements in $\mathcal{C}(H)$. We do not want to introduce this intermediate space here and give a different proof in our setting instead.

\footnotetext{
${ }^{3}$ By definition, this just means that $A$ is $T$-bounded with $T$-bound smaller than 1 (cf. Ka76 IV.1.1]).
} 
2.2.6 Lemma. Let $H$ be a separable Hilbert space and $T$ a selfadjoint operator having a compact resolvent. Then

$$
\mathcal{T}_{T}: \mathcal{L}(H) \rightarrow \mathcal{C}(H)
$$

is not an embedding.

Proof. Since $T$ is selfadjoint and has a compact resolvent, we obtain from [GGK90, XVI.5.1] the existence of a complete orthonormal system $\left\{e_{n}\right\}_{n \in \mathbb{N}}$ of $H$ and a sequence $\left\{\lambda_{n}\right\}_{n \in \mathbb{N}}$ such that $\lambda_{n} \rightarrow \infty, n \rightarrow \infty$, and

$$
\begin{aligned}
\mathcal{D}(T) & =\left\{u \in H: \sum_{n \in \mathbb{N}}\left|\lambda_{n}\right|^{2}\left|\left\langle u, e_{n}\right\rangle\right|^{2}<\infty\right\} \\
T u & =\sum_{n \in \mathbb{N}} \lambda_{n}\left\langle u, e_{n}\right\rangle e_{n} .
\end{aligned}
$$

Moreover, consider the sequence $\left\{A_{n}\right\}_{n \in \mathbb{N}}$ of bounded operators on $H$ defined by

$$
A_{n} u=\sqrt{\left|\lambda_{n}\right|}\left\langle u, e_{n}\right\rangle e_{n}
$$

At first, note that

$$
\left\|A_{n}\right\|=\sqrt{\left|\lambda_{n}\right|}, \quad n \in \mathbb{N}
$$

and hence $\left\{A_{n}\right\}_{n \in \mathbb{N}}$ does not converge in $\mathcal{L}(H)$.

Now we consider the sequence of operators

$$
T_{n}=T+A_{n} \in \mathcal{C}(H), \quad \mathcal{D}\left(T_{n}\right)=\mathcal{D}(T), \quad n \in \mathbb{N},
$$

and our aim is to show that $T_{n}$ converges to $T$ in $\mathcal{C}(H)$ with respect to the gap topology. Keep in mind that once we have shown this assertion we are done, that is, $\mathcal{T}_{T}$ is not an embedding of $\mathcal{L}(H)$ into $\mathcal{C}(H)$.

We choose an $n_{0} \in \mathbb{N}$ such that $\left|\lambda_{n}\right|>1$ for all $n \geq n_{0}$ and obtain

$$
\begin{aligned}
\left\|A_{n} u\right\| & =\sqrt{\left|\lambda_{n}\right|}\left|\left\langle u, e_{n}\right\rangle\right|=\frac{\left|\lambda_{n}\right|}{\sqrt{\left|\lambda_{n}\right|}}\left|\left\langle u, e_{n}\right\rangle\right| \leq \frac{1}{\sqrt{\left|\lambda_{n}\right|}}\left(\sum_{k \in \mathbb{N}}\left|\lambda_{k}\right|^{2}\left|\left\langle u, e_{k}\right\rangle\right|^{2}\right)^{\frac{1}{2}} \\
& =\frac{1}{\sqrt{\left|\lambda_{n}\right|}}\left\|\sum_{k \in \mathbb{N}} \lambda_{k}\left\langle u, e_{k}\right\rangle e_{k}\right\|=\frac{1}{\sqrt{\left|\lambda_{n}\right|}}\|T u\|
\end{aligned}
$$


for all $n \geq n_{0}$ as well as

$$
\left\|A_{n} u\right\| \leq \frac{1}{1-\frac{1}{\sqrt{\left|\lambda_{n}\right|}}} \frac{1}{\sqrt{\left|\lambda_{n}\right|}}\left\|T_{n} u\right\|=\frac{1}{\sqrt{\left|\lambda_{n}\right|}-1}\left\|T_{n} u\right\|, \quad n \geq n_{0},
$$

where we use lemma 2.2.5.

Now we obtain from 2.20 for any $u \in \mathcal{D}(T)$

$$
\begin{aligned}
d\left((u, T u), \operatorname{graph}\left(T_{n}\right)\right) & =\inf _{v \in \mathcal{D}\left(T_{n}\right)}\left\|(u, T u)-\left(v,\left(T+A_{n}\right) v\right)\right\|=\inf _{v \in \mathcal{D}\left(T_{n}\right)}\left\|\left(u-v, T(u-v)-A_{n} v\right)\right\| \\
& \leq\left\|A_{n} u\right\| \leq \frac{1}{\sqrt{\left|\lambda_{n}\right|}-1}\|T u\|, \quad n \geq n_{0} .
\end{aligned}
$$

Since $\|T u\| \leq 1$ for any $(u, T u) \in S_{\operatorname{graph}(T)}$, we have

$$
\delta\left(\operatorname{graph}(T), \operatorname{graph}\left(T_{n}\right)\right)=\sup _{(u, T u) \in S_{\operatorname{graph}(T)}} d\left((u, T u), \operatorname{graph}\left(T_{n}\right)\right) \leq \frac{1}{\sqrt{\left|\lambda_{n}\right|}-1}, \quad n \geq n_{0} .
$$

Moreover, by 2.21 we have for any $u \in \mathcal{D}\left(T_{n}\right)$ and $n \geq n_{0}$

$$
\begin{aligned}
d\left(\left(u, T_{n} u\right), \operatorname{graph} T\right) & =\inf _{v \in \mathcal{D}(T)}\left\|\left(u,\left(T+A_{n}\right) u\right)-(v, T v)\right\|=\inf _{v \in \mathcal{D}(T)}\left\|\left(u-v, T(u-v)+A_{n} u\right)\right\| \\
& \leq\left\|A_{n} u\right\| \leq \frac{1}{\sqrt{\left|\lambda_{n}\right|}-1}\left\|T_{n} u\right\|
\end{aligned}
$$

and obtain as above

$$
\delta\left(\operatorname{graph}\left(T_{n}\right), \operatorname{graph}(T)\right)=\sup _{\left(u, T_{n} u\right) \in S_{\operatorname{graph}\left(T_{n}\right)}} d\left(\left(u, T_{n} u\right), \operatorname{graph}(T)\right) \leq \frac{1}{\sqrt{\left|\lambda_{n}\right|}-1}, \quad n \geq n_{0} .
$$

Hence we have found

$$
d_{G}\left(T, T_{n}\right) \leq \frac{2}{\sqrt{\left|\lambda_{n}\right|}-1}, \quad n \geq n_{0},
$$

showing that $T_{n} \rightarrow T, n \rightarrow \infty$, with respect to the gap topology.

\subsection{On the Spectral Theory of Closed Operators}

We begin by presenting the result [Ka76, IV.2.20] which will be important for us in the sequel.

2.3.1 Lemma. Let $T, S \in \mathcal{C}(E, F)$ be invertible. Then

$$
d_{G}(S, T)=d_{G}\left(S^{-1}, T^{-1}\right)
$$


Proof. We define the inverse graph of an operator $T: \mathcal{D}(T) \subset E \rightarrow F$ by

$$
\operatorname{graph}^{\prime}(T)=\{(T u, u) \in F \times E: u \in \mathcal{D}(T)\} \subset F \times E
$$

and note that

$\operatorname{graph}^{\prime}\left(T^{-1}\right)=\left\{\left(T^{-1} u, u\right) \in E \times F: u \in F\right\}=\{(u, T u) \in E \times F: u \in \mathcal{D}(T)\}=\operatorname{graph}(T)$.

If now $T, S \in \mathcal{C}(E, F)$ are invertible, we obtain

$$
\begin{aligned}
d\left(\operatorname{graph}\left(T^{-1}\right), \operatorname{graph}\left(S^{-1}\right)\right) & =\sup _{\left(u, T^{-1} u\right) \in S_{\operatorname{graph}\left(T^{-1}\right)}} \inf _{\left(v, S^{-1} v\right) \in S_{\operatorname{graph}\left(S^{-1}\right)}}\left\|\left(u, T^{-1} u\right)-\left(v, S^{-1} v\right)\right\| \\
& \left.=\inf _{\left(u, T^{-1} u\right) \in S_{\operatorname{graph}\left(T^{-1}\right)}}\left\|\sup _{\left(S^{-1} v\right) \in S_{\operatorname{graph}\left(S^{-1}\right)}}\right\| T^{-1} u, u\right)-\left(S^{-1} v, v\right) \| \\
& =\inf _{\left(T^{-1} u, u\right) \in S_{\operatorname{graph}^{\prime}\left(T^{-1}\right)}}\left(S^{-1} v, v\right) \in S_{\operatorname{graph}^{\prime}\left(S^{-1}\right)}\left\|\left(T^{-1} u, u\right)-\left(S^{-1} v, v\right)\right\| \\
& =d\left(\operatorname{graph}^{\prime}\left(T^{-1}\right), \operatorname{graph}^{\prime}\left(S^{-1}\right)\right)=d(\operatorname{graph}(T), \operatorname{graph}(S))
\end{aligned}
$$

and infer by interchanging $S$ and $T$

$$
d_{G}\left(T^{-1}, S^{-1}\right)=\hat{d}\left(\operatorname{graph}\left(T^{-1}\right), \operatorname{graph}\left(S^{-1}\right)\right)=\hat{d}(\operatorname{graph}(T), \operatorname{graph}(S))=d_{G}(T, S) .
$$

We now turn to spectral theory and assume from now on that $E=F$. Moreover, we define for any $z_{0} \in \mathbb{C}$

$$
\Omega_{z_{0}}=\left\{T \in \mathcal{C}(E): z_{0} \notin \sigma(T)\right\} .
$$

Even if the following simple result is not explicitly stated in [Ka76], it is at least used in the proof of theorem IV.2.23.

2.3.2 Lemma. Let $z_{0} \in \mathbb{C}$ and $S, T \in \Omega_{z_{0}}$. Then

$$
\begin{aligned}
& d_{G}\left(\left(S-z_{0}\right)^{-1},\left(T-z_{0}\right)^{-1}\right) \leq 4 \sqrt{2}\left(1+\left|z_{0}\right|^{2}\right) d_{G}(S, T), \\
& d_{G}(S, T) \leq 8 \sqrt{2}\left(1+\left|z_{0}\right|^{2}\right)\left\|\left(S-z_{0}\right)^{-1}-\left(T-z_{0}\right)^{-1}\right\| .
\end{aligned}
$$


Proof. By using theorem 2.2.1 and lemma 2.3.1 we obtain

$$
d_{G}\left(\left(S-z_{0}\right)^{-1},\left(T-z_{0}\right)^{-1}\right)=d_{G}\left(S-z_{0}, T-z_{0}\right) \leq 4 \sqrt{2}\left(1+\left|z_{0}\right|^{2}\right) d_{G}(S, T)
$$

and

$$
\begin{aligned}
d_{G}(S, T) & =d_{G}\left(S-z_{0}+z_{0}, T-z_{0}+z_{0}\right) \leq 4 \sqrt{2}\left(1+\left|z_{0}\right|^{2}\right) d_{G}\left(S-z_{0}, T-z_{0}\right) \\
& =4 \sqrt{2}\left(1+\left|z_{0}\right|^{2}\right) d_{G}\left(\left(S-z_{0}\right)^{-1},\left(T-z_{0}\right)^{-1}\right) \\
& \leq 8 \sqrt{2}\left(1+\left|z_{0}\right|^{2}\right)\left\|\left(S-z_{0}\right)^{-1}-\left(T-z_{0}\right)^{-1}\right\|,
\end{aligned}
$$

where we additionally use lemma 2.1.5

Note that the second inequality is of interest for proving the gap continuity of families of operators having non empty resolvent sets. Accordingly, one can check the continuity by considering bounded operators in the operator norm.

An easy variation of the first inequality even allows to estimate the norm distance of the resolvents locally.

2.3.3 Corollary. Let $z_{0} \in \mathbb{C}$ and $T \in \Omega_{z_{0}}$. Then for any $S \in \Omega_{z_{0}}$ such that

$$
d_{G}(S, T)<\frac{1}{8 \sqrt{2}\left(1+\left|z_{0}\right|^{2}\right)}\left(1+\left\|\left(T-z_{0}\right)^{-1}\right\|^{2}\right)^{-\frac{1}{2}}
$$

we have

$$
\left\|\left(S-z_{0}\right)^{-1}-\left(T-z_{0}\right)^{-1}\right\| \leq 8 \sqrt{2}\left(1+\left|z_{0}\right|^{2}\right)\left(1+\left\|\left(T-z_{0}\right)^{-1}\right\|^{2}\right) d_{G}(S, T) .
$$

Proof. Under the given assumptions we obtain by lemma 2.3 .2 from above

$$
d_{G}\left(\left(S-z_{0}\right)^{-1},\left(T-z_{0}\right)^{-1}\right) \leq 4 \sqrt{2}\left(1+\left|z_{0}\right|^{2}\right) d_{G}(S, T)<\frac{1}{2}\left(1+\left\|\left(T-z_{0}\right)^{-1}\right\|^{2}\right)^{-\frac{1}{2}}
$$

Hence we can apply lemma 2.1.6 which yields

$$
\begin{aligned}
\left\|\left(S-z_{0}\right)^{-1}-\left(T-z_{0}\right)^{-1}\right\| & \leq \frac{\left(1+\left\|\left(T-z_{0}\right)^{-1}\right\|^{2}\right) d_{G}\left(\left(S-z_{0}\right)^{-1},\left(T-z_{0}\right)^{-1}\right)}{1-d_{G}\left(\left(S-z_{0}\right)^{-1},\left(T-z_{0}\right)^{-1}\right) \sqrt{1+\left\|\left(T-z_{0}\right)^{-1}\right\|^{2}}} \\
& \leq 2\left(1+\left\|\left(T-z_{0}\right)^{-1}\right\|^{2}\right) d_{G}\left(\left(S-z_{0}\right)^{-1},\left(T-z_{0}\right)^{-1}\right) \\
& \leq 8 \sqrt{2}\left(1+\left|z_{0}\right|^{2}\right)\left(1+\left\|\left(T-z_{0}\right)^{-1}\right\|^{2}\right) d_{G}(S, T),
\end{aligned}
$$

where we use once again lemma 2.3 .2 in the last inequality. 
Note that the map

$$
d_{\rho}: \Omega_{z_{0}} \rightarrow \mathbb{R}, \quad d_{\rho}(T, S)=\left\|\left(T-z_{0}\right)^{-1}-\left(S-z_{0}\right)^{-1}\right\|
$$

defines a metric on $\Omega_{z_{0}}$ and by lemma 2.3 .2 and corollary $2.3 .3 d_{\rho}$ and $d_{G}$ induce the same topology on $\Omega_{z_{0}}$. This is also stated in an equivalent way in [Ka76, IV.2.25].

In the special case that $E$ is a Hilbert space the subset $\mathcal{C}^{s a}(E) \subset \Omega_{-i}$ consisting of all selfadjoint operators was studied in [BLP05]. Besides $d_{G}^{4}$ and $d_{\rho}$ they defined a further metric by

$$
d_{C}(T, S)=\|\kappa(T)-\kappa(S)\|,
$$

where $\kappa(T)$ denotes the Cayley transform of $T$. In [BLP05, 1.1] it is proved that all three metrics are mutually uniformly equivalent. Moreover, these constructions are used in BLP05] and [Le05] in order to study the space of selfadjoint Fredholm operators and the spectral flow with respect to the gap metric.

Below we will adapt some of the results of [BLP05] to the more general case of closed operators on Banach spaces at first. Afterwards we will use them in order to study the stability of spectra for closed operators.

In order to prove our first result we need the following stability theorem for Fredholm operators [Ka76, IV.5.17].

2.3.4 Theorem. Let $T \in \mathcal{C}(E, F)$ be Fredholm. There exists $\delta^{\prime}=\delta^{\prime}(T)>0$ such that if $S \in \mathcal{C}(E, F)$ and $d_{G}(S, T)<\delta^{\prime}$, then $S$ is Fredholm and

$$
\operatorname{dim} \operatorname{ker} S \leq \operatorname{dim} \operatorname{ker} T
$$

$\operatorname{dim}$ coker $S \leq \operatorname{dim} \operatorname{coker} T$

as well as

$$
\operatorname{ind}(S)=\operatorname{ind}(T)
$$

We obtain the following principle of stability of bounded invertibility which can also be proved independently in a more elementary way (see [Ka76, Theorem 2.21]).

2.3.5 Corollary. Let $T \in \mathcal{C}(E, F)$ be invertible. There exists $\delta^{\prime}>0$ such that if $S \in \mathcal{C}(E, F)$ and $d_{G}(S, T)<\delta^{\prime}$ then $S$ is invertible.

\footnotetext{
${ }^{4}$ Note that in BLP05] a different but equivalent definition of $d_{G}$ is used that we will consider below in a subsequent section.
} 
Proof. If $S^{-1}$ exists, then it is closed by lemma A.2.2 and hence bounded by the closed graph theorem A.2.4 Accordingly, we just have to show that under the given assumptions $S: \mathcal{D}(S) \rightarrow$ $F$ is bijective. But, since $T$ is invertible, both $\operatorname{ker} T$ and coker $T$ are trivial. By theorem 2.3.4 we conclude that there exists $\delta^{\prime}>0$ such that the same holds for any $S \in \mathcal{C}(E, F)$ which is closer to $T$ than $\delta^{\prime}$.

2.3.6 Lemma. For $z_{0} \in \mathbb{C}$ the sets

$$
\begin{aligned}
\Omega_{z_{0}} & =\left\{T \in \mathcal{C}(E): z_{0} \notin \sigma(T)\right\} \\
\Omega_{z_{0}}^{\text {ess }} & =\left\{T \in \mathcal{C}(E): z_{0} \notin \sigma_{\text {ess }}(T)\right\}
\end{aligned}
$$

are open in $\mathcal{C}(E)$.

Proof. At first, $\Omega_{z_{0}}$ is open by corollary 2.3.5.

If $T \in \Omega_{z_{0}}^{\text {ess }}, T-z_{0}$ is by definition a Fredholm operator. Since by theorem 2.2 .1

$$
d_{G}\left(S-z_{0}, T-z_{0}\right) \leq 4 \sqrt{2} \sqrt{1+\left|z_{0}\right|^{2}} d_{G}(S, T)
$$

for any $S \in \mathcal{C}(E)$, we obtain from theorem 2.3 .4 the existence of $\delta^{\prime}=\delta^{\prime}\left(T-z_{0}\right)>0$ such that $S-z_{0}$ is Fredholm if

$$
d_{G}(S, T)<\frac{\delta^{\prime}}{4 \sqrt{2} \sqrt{1+\left|z_{0}\right|^{2}}}
$$

2.3.7 Remark. The openness of the sets

$$
\begin{aligned}
& \left\{T \in \mathcal{C}^{s a}(H): \lambda \notin \sigma(T)\right\} \\
& \left\{T \in \mathcal{C}^{s a}(H): \lambda \notin \sigma_{e s s}(T)\right\}
\end{aligned}
$$

of selfadjoint operators for $\lambda \in \mathbb{R}$ is proved in [BLP05, Prop.1.7] by using the Cayley transform instead of theorem 2.3.4

2.3.8 Lemma. The map

$$
\Omega_{z_{0}} \ni T \mapsto\left(T-\overline{z_{0}}\right)\left(T-z_{0}\right)^{-1} \in \mathcal{L}(E)
$$

is continuous for any $z_{0} \in \mathbb{C}$. 
Proof. At first we note that $\operatorname{im}\left(T-z_{0}\right)=E$ and hence $\left(T-\overline{z_{0}}\right)\left(T-z_{0}\right)^{-1}$ is indeed bounded by corollary A.2.6. Moreover, we can assume that $z_{0} \notin \mathbb{R}$ because otherwise $\left(T-\overline{z_{0}}\right)\left(T-z_{0}\right)^{-1}=I$ for all $T \in \Omega_{z_{0}}$ and the assertion is trivial in this case.

So let us require that $z_{0} \neq \overline{z_{0}}$. Then we have

$$
\left(T-\overline{z_{0}}\right)\left(T-z_{0}\right)^{-1}=\left(T-z_{0}+\left(z_{0}-\overline{z_{0}}\right)\right)\left(T-z_{0}\right)^{-1}=I+\left(z_{0}-\overline{z_{0}}\right)\left(T-z_{0}\right)^{-1}
$$

and by theorem 2.2.1, lemma 2.1.8 and lemma 2.3 .2 we obtain

$$
\begin{aligned}
d_{G}\left(\left(T-\overline{z_{0}}\right)\left(T-z_{0}\right)^{-1},\right. & \left.\left(S-\overline{z_{0}}\right)\left(S-z_{0}\right)^{-1}\right) \\
& =d_{G}\left(I+\left(z_{0}-\overline{z_{0}}\right)\left(T-z_{0}\right)^{-1}, I+\left(z_{0}-\overline{z_{0}}\right)\left(S-z_{0}\right)^{-1}\right) \\
& \leq 8 \sqrt{2} d_{G}\left(\left(z_{0}-\overline{z_{0}}\right)\left(T-z_{0}\right)^{-1},\left(z_{0}-\overline{z_{0}}\right)\left(S-z_{0}\right)^{-1}\right) \\
& \leq 16 \sqrt{2} \max \left\{\left|z_{0}-\overline{z_{0}}\right|, \frac{1}{\left|z_{0}-\overline{z_{0}}\right|}\right\} d_{G}\left(\left(T-z_{0}\right)^{-1},\left(S-z_{0}\right)^{-1}\right) \\
& \leq 256\left(1+\left|z_{0}\right|^{2}\right) \max \left\{\left|z_{0}-\overline{z_{0}}\right|, \frac{1}{\left|z_{0}-\overline{z_{0}}\right|}\right\} d_{G}(S, T)
\end{aligned}
$$

Now the assertion follows since the norm- and the gap topology coincide on $\mathcal{L}(E)$ according to corollary 2.1 .7 .

The proof of the next result is precisely along the lines of its analogue for selfadjoint operators in [BLP05, Lemma 2.8]. Remind before that the composition ST of two operators

$$
T: \mathcal{D}(T) \subset E \rightarrow E, \quad S: \mathcal{D}(S) \subset E \rightarrow E
$$

is defined usually on the domain $\mathcal{D}(S T)=T^{-1} \mathcal{D}(S)$. Note that if $S$ and $T$ are invertible, then $S T$ is invertible as well and its inverse is given by $T^{-1} S^{-1}: E \rightarrow E$.

2.3.9 Theorem. Let $\emptyset \neq K \subset \mathbb{C}$ and $\Omega_{K}=\{T \in \mathcal{C}(E): K \subset \rho(T)\}$. Then the map

$$
R: K \times \Omega_{K} \rightarrow \mathcal{L}(E), \quad(\lambda, T) \mapsto(T-\lambda)^{-1}
$$

is continuous.

Proof. We fix $z_{0} \in K$ and note that for $(\lambda, T) \in K \times \Omega_{K}$ we have

$$
\begin{aligned}
R(\lambda, T) & =(T-\lambda)^{-1}=\left(\left(T-z_{0}\right)-\left(\lambda-z_{0}\right)\right)^{-1} \\
& =\left(\left(T-z_{0}\right)\left(I-\left(\lambda-z_{0}\right)\left(T-z_{0}\right)^{-1}\right)\right)^{-1} \\
& =\left(I-\left(\lambda-z_{0}\right)\left(T-z_{0}\right)^{-1}\right)^{-1}\left(T-z_{0}\right)^{-1}=F(G(\lambda, T))
\end{aligned}
$$


where the maps $F$ and $G$ are defined by ${ }^{5}$

$$
\begin{aligned}
G: K \times \Omega_{K} & \rightarrow K \times\left\{S \in \mathcal{L}(E):\left(K-z_{0}\right)^{-1} \subset \rho(S)\right\} \\
(\lambda, T) & \mapsto\left(\lambda,\left(T-z_{0}\right)^{-1}\right)
\end{aligned}
$$

and

$$
\begin{aligned}
F: & K \times\left\{S \in \mathcal{L}(E):\left(K-z_{0}\right)^{-1} \subset \rho(S)\right\} \rightarrow \mathcal{L}(E), \\
& (\lambda, S) \mapsto\left(I-\left(\lambda-z_{0}\right) S\right)^{-1} S
\end{aligned}
$$

respectively. Now $G$ is continuous by corollary 2.3.3 Furthermore, the continuity of $F$ is a simple computation using the continuity of the inversion on $G L(E)$ (cf.[Ka76, I.(4.24),III.3.1]).

As an important corollary we obtain the continuity of the spectral projections as introduced in A.3.3.

2.3.10 Corollary. Let $\triangle \subset \mathbb{C}$ be a bounded Cauchy domain with boundary $\Gamma$ and denote

$$
\Omega_{\triangle}=\{T \in \mathcal{C}(E): \Gamma \subset \rho(T)\}
$$

Then the map

$$
\Omega_{\triangle} \rightarrow \mathcal{L}(E), \quad T \mapsto P_{\triangle}(T)=\frac{1}{2 \pi i} \int_{\Gamma}(\lambda-T)^{-1} d \lambda
$$

is continuous.

Proof. For any $T, S \in \Omega_{\triangle}$ we have by [He92, (97.4)]

$$
\left\|P_{\triangle}(T)-P_{\triangle}(S)\right\| \leq \frac{1}{2 \pi}|\Gamma| \max _{\lambda \in \Gamma}\left\|(\lambda-T)^{-1}-(\lambda-S)^{-1}\right\|,
$$

where $|\Gamma|$ denotes the length of $\Gamma$. The rest of the proof is a standard argument in calculus. Let $T \in \Omega_{\triangle}$ and $\varepsilon>0$. By theorem 2.3.9 there exists a $\delta\left(\lambda^{\prime}\right)>0$ for any $\lambda^{\prime} \in \Gamma$ such that

$$
\left\|(\lambda-S)^{-1}-\left(\lambda^{\prime}-T\right)^{-1}\right\|<\frac{\pi \varepsilon}{|\Gamma|}
$$

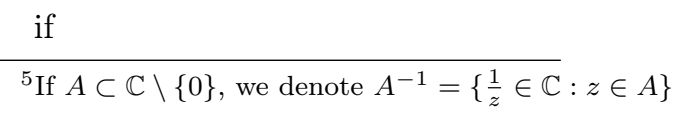




$$
\lambda \in U\left(\lambda^{\prime}, \delta\left(\lambda^{\prime}\right)\right):=\left\{\lambda \in \Gamma:\left|\lambda-\lambda^{\prime}\right|<\delta\left(\lambda^{\prime}\right)\right\} \text { and } d_{G}(S, T)<\delta\left(\lambda^{\prime}\right)
$$

Since $\Gamma$ is compact we can find $\lambda_{1}, \ldots, \lambda_{n} \in \Gamma$ such that $\bigcup_{i=1}^{n} U\left(\lambda_{i}, \delta\left(\lambda_{i}\right)\right)=\Gamma$ and define $\delta:=\min _{1 \leq i \leq n} \delta\left(\lambda_{i}\right)$.

Now, for any $\lambda \in \Gamma$ there exists an $1 \leq i \leq n$ such that $\lambda \in U\left(\lambda_{i}, \delta\left(\lambda_{i}\right)\right)$ and hence we obtain for $S \in \Omega_{\triangle}, d_{G}(S, T)<\delta$,

$$
\left\|(\lambda-T)^{-1}-(\lambda-S)^{-1}\right\| \leq\left\|(\lambda-T)^{-1}-\left(\lambda_{i}-T\right)^{-1}\right\|+\left\|\left(\lambda_{i}-T\right)^{-1}-(\lambda-S)^{-1}\right\|<\frac{2 \pi \varepsilon}{|\Gamma|} .
$$

We conclude by 2.23

$$
\left\|P_{\triangle}(T)-P_{\triangle}(S)\right\| \leq \frac{1}{2 \pi}|\Gamma| \max _{\lambda \in \Gamma}\left\|(\lambda-T)^{-1}-(\lambda-S)^{-1}\right\|<\varepsilon
$$

for all $S \in \Omega_{\triangle}$ such that $d_{G}(S, T)<\delta$.

We now turn to the study of spectra of closed operators by means of the Cayley transform

$$
\kappa: \Omega_{-i} \rightarrow \mathcal{L}(X), \quad \kappa(T)=(T-i)(T+i)^{-1}
$$

Note that $\kappa$ is continuous by lemma 2.3.8. In addition, since we consider $\Omega_{-i}$ we implicitly assume throughout that all operators have a non empty resolvent set.

Our first result shows how to reconstruct an operator in $\Omega_{-i}$ from its Cayley transform. The proof does not differ from the corresponding statement for selfadjoint operators (cf. A.3.8).

2.3.11 Lemma. If $T \in \Omega_{-i}$, then

$$
T=i(I+\kappa(T))(I-\kappa(T))^{-1}
$$

Proof. Using the equality

$$
\kappa(T)=(T-i)(T+i)^{-1}=(T+i-2 i)(T+i)^{-1}=I-2 i(T+i)^{-1}
$$

we obtain at first that

$$
I-\kappa(T)=2 i(T+i)^{-1}
$$

and hence 


$$
(I-\kappa(T))^{-1}=\frac{1}{2 i}(T+i)
$$

is defined on $\mathcal{D}(T)$.

We compute

$$
\begin{aligned}
i(I+\kappa(T))(I-\kappa(T))^{-1} & =i(I+\kappa(T)) \frac{1}{2 i}(T+i) \\
& =\frac{1}{2}\left(I+(T-i)(T+i)^{-1}\right)(T+i) \\
& =\frac{1}{2}(T+i+T-i)=T .
\end{aligned}
$$

Below we will study the relation between the spectrum of a closed operator in $\Omega_{-i}$ and the spectrum of its Cayley transform. Since $1 \in \mathbb{C}$ is the only point having no counterimage in $\mathbb{C}$ under the classical Cayley transform

$$
\kappa(\lambda)=\frac{\lambda-i}{\lambda+i},
$$

we have to treat this special case separately.

2.3.12 Lemma. Let $T \in \Omega_{-i}$ be densely defined. Then

i) $1 \in \rho(\kappa(T)) \Longleftrightarrow \mathcal{D}(T)=E$ and this is true if and only if $T$ is bounded.

ii) $1 \in \sigma_{\text {ess }}(\kappa(T)) \Longleftrightarrow \mathcal{D}(T) \neq E$ and this is true if and only if $T$ is unbounded.

Proof. The assertions regarding the boundedness and unboundedness of $T$ follow by A.2.1 and the assumption that $T$ is densely defined.

By 2.25 we have

$$
I-\kappa(T)=2 i(T+i)^{-1} \in \mathcal{L}(E)
$$

mapping $E$ bijectively onto $\mathcal{D}(T)$. Accordingly, if $1 \in \rho(\kappa(T))$, we infer $E=\operatorname{im}(I-\kappa(T))=$ $\mathcal{D}(T)$. Conversely, if $\mathcal{D}(T)=E$, then $I-\kappa(T)$ maps $E$ bijectively onto $E$ showing $1 \in \rho(\kappa(T))$ by the closed graph theorem A.2.4. Hence assertion $i$ ) is proved.

In order to show $i i)$ we note at first that by $i), 1 \in \sigma(\kappa(T))$ if and only if $\mathcal{D}(T) \neq E$. Now it remains to show that if $1 \in \sigma(T)$, then we actually have $1 \in \sigma_{\text {ess }}(T)$. But, if $\mathcal{D}(T) \neq E$, we obtain that $\operatorname{im}(I-\kappa(T))=\mathcal{D}(T)$ is a proper dense subspace of $E$ and hence in particular not closed. Accordingly, $I-\kappa(T)$ is not a Fredholm operator. 
2.3.13 Lemma. If $T \in \Omega_{-i}$ and $\lambda \neq-i$, then

$$
\lambda-T=(\lambda+i)(\kappa(\lambda)-\kappa(T))(I-\kappa(T))^{-1}
$$

Proof. We obtain by lemma 2.3.11

$$
\begin{aligned}
\lambda-T & =\lambda-i(I+\kappa(T))(I-\kappa(T))^{-1}=(\lambda(I-\kappa(T))-i(I+\kappa(T)))(I-\kappa(T))^{-1} \\
& =(\lambda-\lambda \kappa(T)-i-i \kappa(T))(I-\kappa(T))^{-1}=((\lambda-i)-(\lambda+i) \kappa(T))(I-\kappa(T))^{-1} \\
& =(\lambda+i)\left((\lambda-i)(\lambda+i)^{-1}-\kappa(T)\right)(I-\kappa(T))^{-1}=(\lambda+i)(\kappa(\lambda)-\kappa(T))(I-\kappa(T))^{-1} .
\end{aligned}
$$

As a consequence we obtain the following important corollary, illustrating the relation between the spectrum of an operator in $\Omega_{-i}$ and the spectrum of its Cayley transform.

2.3.14 Corollary. Let $T \in \Omega_{-i}$. If $\lambda \neq-i$, then

1. $\operatorname{ker}(\lambda-T) \neq\{0\}$ if and only if $\operatorname{ker}(\kappa(\lambda)-\kappa(T)) \neq\{0\}$. Moreover the dimensions of both spaces coincide.

2. $\operatorname{im}(\lambda-T)=\operatorname{im}(\kappa(\lambda)-\kappa(T))$

In particular,

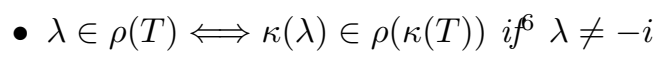

- $\lambda \in \sigma(T) \Longleftrightarrow \kappa(\lambda) \in \sigma(\kappa(T))$

- $\lambda \in \sigma_{p}(T) \Longleftrightarrow \kappa(\lambda) \in \sigma_{p}(\kappa(T))$

- $\lambda \in \sigma_{\text {ess }}(T) \Longleftrightarrow \kappa(\lambda) \in \sigma_{\text {ess }}(\kappa(T))$.

Proof. By the foregoing lemma 2.3.13 we know that if $\lambda \neq-i$ and $T \in \Omega_{-i}$, then

$$
\lambda-T=(\lambda+i)(\kappa(\lambda)-\kappa(T))(I-\kappa(T))^{-1}
$$

and by 2.26$)(I-\kappa(T))^{-1}$ maps $\mathcal{D}(T)$ bijectively onto $H$. This implies the assertions on $\operatorname{ker}(\lambda-T)$ and $\operatorname{im}(\lambda-T)$ and the remaining part of the corollary is an immediate consequence of them. Note that we do not need to exclude the case $\lambda=-i$ at the results concerning the spectra, because by assumption $T \in \Omega_{-i}$ and hence $-i \notin \sigma(T)$.

2.3.15 Corollary. Let $T \in \Omega_{-i}$ be densely defined.

\footnotetext{
${ }^{6}$ Note that $-i \in \rho(T)$ anyway, since $T \in \Omega_{-i}$
} 
i) If $T$ is bounded, then $\kappa(\sigma(T))=\sigma(\kappa(T))$

ii) If $T$ is unbounded, then $\kappa(\sigma(T)) \cup\{1\}=\sigma(\kappa(T))$

Both conclusions hold true if we replace $\sigma$ by $\sigma_{\text {ess }}$.

Proof. Since $\kappa$ maps $\mathbb{C} \backslash\{-i\}$ bijectively onto $\mathbb{C} \backslash\{1\}$, from the equivalences stated in corollary 2.3 .14 it is clear that either $\kappa\left(\sigma_{(e s s)}(T)\right)=\sigma_{(e s s)}(\kappa(T))$ or $\kappa\left(\sigma_{(e s s)}(T)\right) \cup\{1\}=\sigma_{(e s s)}(\kappa(T))$. Now the assertions follow from lemma 2.3.12.

We finally can study the stability of spectra. But before that, we want to recall the corresponding results for bounded operators that we will subsequently extend to $\mathcal{C}(E)$ by the Cayley transform.

2.3.16 Theorem. Let $A \in \mathcal{L}(E)$ and $\Omega \subset \mathbb{C}$ be an open neighbourhood of $\sigma(A)$. Then there exists $\varepsilon>0$ such that $\sigma(B) \subset \Omega$ for any $B \in \mathcal{L}(E)$ with $\|A-B\|<\varepsilon$. Moreover, the same conclusion holds true if we replace $\sigma$ by $\sigma_{\text {ess }}$.

Proof. The assertion on the spectrum is proved in [He92, 96.5] for any complex unital Banach algebra $R$. Hence the first assertion follows by setting $R=\mathcal{L}(E)$. The second assertion follows by setting $R$ to be the Calkin algebra $\operatorname{Cal}(E)$ of $E$ and the continuity of the quotient map $q: \mathcal{L}(E) \rightarrow \operatorname{Cal}(E)$.

The second theorem concerns the continuity of eigenvalues of finite type.

2.3.17 Theorem. Let $\sigma$ be a finite set of eigenvalues of finite type of $A \in \mathcal{L}(E)$ and let $\triangle$ be a Cauchy domain such that $\sigma \subset \triangle$ and $\bar{\triangle} \cap(\sigma(A) \backslash \sigma)=\emptyset$. Then there exists $\varepsilon>0$ such that for any $B \in \mathcal{L}(E)$ with $\|A-B\|<\varepsilon$ :

i) $\sigma(B) \cap \partial \triangle=\emptyset$,

ii) $\sigma(B) \cap \triangle$ is a finite set of eigenvalues of finite type,

iii)

$$
\sum_{\lambda \in \triangle} m(\lambda ; B)=\sum_{\lambda \in \triangle} m(\lambda ; A),
$$

where $m(\lambda ; B)=\operatorname{dim} \operatorname{ker}(\lambda-B)$ for any $B \in \mathcal{L}(E)$.

Proof. [GGK90, II.4.2]

Next we transfer these results to closed operators.

2.3.18 Theorem. Let $T \in \mathcal{C}(E)$ be densely defined and unbounded. Moreover, let $\Omega \subset \mathbb{C}$ be an open neighbourhood of $\infty$ such that $\sigma(T) \subset \Omega$. Then there exists $\delta>0$ such that $\sigma(S) \subset \Omega$ for all $S \in \mathcal{C}(E)$ with $d_{G}(T, S)<\delta$.

The same conclusion holds if we replace $\sigma$ by $\sigma_{\text {ess }}$ under the additional assumption that $\rho(T) \neq \emptyset$. 
Proof. First of all, we can assume without loss of generality that $\rho(T) \neq \emptyset$ in both cases, because if $\sigma(T)=\mathbb{C}$, then we have $\Omega=\mathbb{C}$ necessarily and the first assertion is trivial.

We choose $z_{0} \in \rho(T)$ and $\delta_{1}>0$ such that $S \in \Omega_{z_{0}}$ for all $S \in \mathcal{C}(E)$ such that $d_{G}(S, T)<\delta_{1}$, where we use that $\Omega_{z_{0}} \subset \mathcal{C}(E)$ is open by lemma 2.3.6 We define

$$
F: \Omega_{z_{0}} \rightarrow \Omega_{-i}, \quad F(T)=T-z_{0}-i
$$

which is continuous by corollary 2.2.3 Then $\kappa \circ F: \Omega_{z_{0}} \rightarrow \mathcal{L}(E)$ is defined and continuous as well because $z_{0} \in \rho(T)$.

Now, since $\sigma_{(e s s)}\left(T-z_{0}-i\right)=\sigma_{(e s s)}(T)-z_{0}-i \subset \Omega-z_{0}-i:=\Omega^{\prime}$, we obtain from corollary 2.3 .15 and our assumptions that

$$
\sigma_{(e s s)}\left(\kappa(F(T))=\kappa\left(\sigma_{(e s s)}(F(T))\right) \cup\{1\} \subset \kappa\left(\Omega^{\prime}\right) \cup\{1\}\right.
$$

Note that $\kappa\left(\Omega^{\prime}\right) \cup\{1\}$ is open in $\mathbb{C}$ because $\Omega^{\prime}$ is an open neighbourhood of $\infty$ and, accordingly, 1 is an interior point of $\kappa\left(\Omega^{\prime}\right) \cup\{1\}$.

Now we use theorem 2.3 .16 to obtain an $\varepsilon>0$ such that $\sigma_{(e s s)}(A)$ is contained in $\kappa\left(\Omega^{\prime}\right) \cup\{1\}$ whenever $A \in \mathcal{L}(E)$ satisfies $\|A-\kappa(F(T))\|<\varepsilon$. Moreover, since $\kappa \circ F$ is continuous, there exists $\delta_{2}>0$ such that

$$
\|\kappa(F(S))-\kappa(F(T))\|<\varepsilon
$$

for all $S \in \Omega_{z_{0}}$ such that $d_{G}(S, T)<\delta_{2}$.

Setting $\delta=\min \left\{\delta_{1}, \delta_{2}\right\}$, we finally obtain the assertion.

The following corollary is just an equivalent formulation of the theorem.

2.3.19 Corollary. Let $T \in \mathcal{C}(E)$ be densely defined and unbounded. Moreover, let $K \subset \mathbb{C}$ be compact and $z_{0} \in \mathbb{C}$. Then the sets

$$
\begin{aligned}
& \{T \in \mathcal{C}(E): K \subset \rho(T)\} \\
& \left\{T \in \Omega_{z_{0}}: K \subset \rho_{\text {ess }}(T)\right\}
\end{aligned}
$$

are open subsets of $\mathcal{C}(E)$ with respect to the gap topology.

2.3.20 Remark. We expect our result 2.3 .18 to be well known. At least, the stability of the whole spectrum is proved by different methods in [Ka76, IV.3.1]. On the other hand we could not find any reference concerning the stability of the essential spectrum with respect to the gap topology in this generality. Both results are proved in the special case of selfadjoint operators on a Hilbert space in [BLP05, Lemma 2.7]. 
Finally, we consider eigenvalues of finite type.

2.3.21 Theorem. Let $T \in \mathcal{C}(E)$ and $\sigma \subset \mathbb{C}$ be a finite set of eigenvalues of finite type of $T$. We assume that there exists a bounded Cauchy domain $\triangle$ such that $\sigma \subset \triangle$ and $\bar{\triangle} \cap(\sigma(T) \backslash \sigma)=\emptyset$. Then there exists $\delta>0$ such that for any $S \in C(E)$ with $d_{G}(T, S)<\delta$, we have $\sigma(S) \cap \partial \triangle=\emptyset$, $\sigma(S) \cap \triangle$ is a finite set of eigenvalues of finite type and

$$
\sum_{\lambda \in \triangle} m(\lambda ; T)=\sum_{\lambda \in \triangle} m(\lambda ; S)
$$

Proof. We use the same idea as in the proof of theorem 2.3.18 in order to reduce to the corresponding result for bounded operators.

By assumption we have $\partial \triangle \cap \sigma(T)=\emptyset$ and since $\sigma(T) \backslash \sigma$ is closed and $\bar{\triangle}$ compact, we can choose a $z_{0} \in \rho(T)$ such that $z_{0} \notin \bar{\triangle}$. By using lemma 2.3.6 we can find a $\delta_{1}>0$ such that $S \in \Omega_{z_{0}}$ for all $S \in \mathcal{C}(E)$ satisfying $d_{G}(S, T)<\delta_{1}$. Moreover, we set again

$$
F: \Omega_{z_{0}} \rightarrow \Omega_{-i}, \quad F(T)=T-z_{0}-i
$$

and note that $\kappa \circ F: \Omega_{z_{0}} \rightarrow \mathcal{L}(E)$ is defined and continuous. Since $z_{0} \notin \bar{\triangle}$, we infer that $-i \notin \bar{\triangle}-z_{0}-i=F(\bar{\triangle})$ and hence $\kappa(F(\triangle)) \subset \mathbb{C} \backslash\{1\}$ is a bounded Cauchy domain having boundary $\partial \kappa(F(\triangle))=\kappa(F(\partial \triangle))$ and containing $\kappa(F(\sigma))$.

Now, by theorem 2.3.17 there exists an $\varepsilon>0$ such that for any $A \in \mathcal{L}(E)$ with $\|\kappa(F(T))-A\|<\varepsilon$ we have $\sigma(A) \cap \partial \kappa(F(\triangle))=\emptyset, \sigma(A) \cap \kappa(F(\triangle))$ is a finite set of eigenvalues of finite multiplicity and

$$
\sum_{\lambda \in \kappa(F(\triangle))} m(\lambda ; \kappa(F(T)))=\sum_{\lambda \in \kappa(F(\triangle))} m(\lambda ; A) .
$$

Due to the continuity of $\kappa \circ F$ we can find $\delta_{2}>0$ such that

$$
\|\kappa(F(S))-\kappa(F(T))\|<\varepsilon
$$

for all $S \in \Omega_{z_{0}}$ such that $d_{G}(T, S)<\delta_{2}$. Setting $\delta=\min \left\{\delta_{1}, \delta_{2}\right\}$ we finally obtain the assertion from corollary 2.3 .14 .

2.3.22 Remark. i) A different proof of theorem 2.3.21 can be found in [Ka76, §IV.3.5].

ii) Note that under the assumptions of the foregoing theorem the map

$$
S \mapsto P_{\triangle}(S)
$$

is continuous on the set of all $S \in \mathcal{C}(E)$ such that $d_{G}(S, T)<\delta$ by corollary 2.3.10. 


\subsection{The Special Case of Hilbert Spaces}

In this section we consider $\hat{\delta}(M, N)$ in the special case of closed subspaces $M, N$ of a Hilbert space $H$. The crucial difference to the case of Banach spaces is that any closed subspace $M$ of $H$ is complemented by its orthogonal complement and, accordingly, there exists a distinguished bounded projection onto $M$ (cf. theorem A.1.5). The key result of this section shows that we now can express $\hat{\delta}(M, N)$ by means of the orthogonal projections $P_{M}$ and $P_{N}$, respectively. Before we can discuss this result, we need the following lemma, whose first assertion is problem I.6.33 of Kato's book [Ka76].

2.4.1 Lemma. Let $M, N \subset H$ be closed subspaces and denote by $P_{M}$ and $P_{N}$ the orthogonal projections onto $M$ and $N$, respectively. Then

i) $\left\|P_{M}-P_{N}\right\| \leq 1$

ii) $\delta(M, N)=\left\|\left(I-P_{N}\right) P_{M}\right\|$

iii) $\hat{\delta}(M, N) \leq\left\|P_{M}-P_{N}\right\|$

Proof. i) We compute

$$
\begin{aligned}
\left(P_{M}-P_{N}\right)^{2} & +\left(I-P_{M}-P_{N}\right)^{2} \\
& =\left(P_{M}-P_{N}\right)\left(P_{M}-P_{N}\right)+\left(I-P_{M}-P_{N}\right)\left(I-P_{M}-P_{N}\right) \\
& =P_{M}-P_{M} P_{N}-P_{N} P_{M}+P_{N}+I-P_{M}-P_{N}-P_{M} \\
& +P_{M}+P_{M} P_{N}-P_{N}+P_{N} P_{M}+P_{N}=I
\end{aligned}
$$

and obtain for every $u \in H$

$$
\begin{aligned}
\|u\|^{2} & =\left\langle\left(P_{M}-P_{N}\right)^{2} u, u\right\rangle+\left\langle\left(I-P_{M}-P_{N}\right)^{2} u, u\right\rangle \\
& =\left\|\left(P_{M}-P_{N}\right) u\right\|^{2}+\left\|\left(I-P_{M}-P_{N}\right) u\right\|^{2},
\end{aligned}
$$

where we use that $P_{M}-P_{N}$ and $I-P_{M}-P_{N}$ are selfadjoint. Hence

$$
\left\|\left(P_{M}-P_{N}\right) u\right\| \leq \sqrt{\left\|\left(P_{M}-P_{N}\right) u\right\|^{2}+\left\|\left(I-P_{M}-P_{N}\right) u\right\|^{2}}=\|u\|, \quad u \in H
$$

ii) Any $v \in H$ can be decomposed as $v=P_{M} v+P_{M}^{\perp} v$, where $P_{M}^{\perp}=I-P_{M}$ denotes the complementary projection of $P_{M}$. Since $\left\|P_{M} u+P_{M}^{\perp} u\right\|=\sqrt{\left\|P_{M} u\right\|^{2}+\left\|P_{M}^{\perp} u\right\|^{2}}$ and $P_{M} w=$ 0 for all $w \in M^{\perp}$, we obtain

$$
\begin{aligned}
\left\{P_{M} v: v \in H,\|v\|=1\right\} & =\left\{P_{M} v: v \in H,\left\|P_{M} v\right\|^{2}+\left\|P_{M}^{\perp} v\right\|^{2}=1\right\} \\
& =\left\{P_{M} v: v \in H,\left\|P_{M} v\right\| \leq 1\right\}=\{u \in M:\|u\| \leq 1\} .
\end{aligned}
$$


Hence

$$
\left\|\left(I-P_{N}\right) P_{M}\right\|=\sup _{\|v\|=1}\left\|\left(I-P_{N}\right) P_{M} v\right\|=\sup _{\substack{u \in M \\\|u\| \leq 1}}\left\|\left(I-P_{N}\right) u\right\|=\sup _{\substack{u \in M \\\|u\|=1}}\left\|\left(I-P_{N}\right) u\right\|,
$$

where the last equality follows from the linearity of $I-P_{N}$ in the usual way.

Now we just have to use the well known fact that $\left\|u-P_{N} u\right\|=d(u, N)$ (cf. [He92, 22.3]) and finally obtain

$$
\left\|\left(I-P_{N}\right) P_{M}\right\|=\sup _{\substack{u \in M \\\|u\|=1}}\left\|u-P_{N} u\right\|=\sup _{\substack{u \in M \\\|u\|=1}} d(u, N)=\delta(M, N)
$$

iii) Since $P_{M} u=u$ for any $u \in M$, we obtain from 2.27

$$
\begin{gathered}
\delta(M, N)=\sup _{\substack{u \in M \\
\|u\|=1}}\left\|u-P_{N} u\right\|=\sup _{\substack{u \in M \\
\|u\|=1}}\left\|P_{M} u-P_{N} u\right\| \\
\leq \sup _{\substack{u \in M \\
\|u\|=1}}\left\|P_{M}-P_{N}\right\|\|u\|=\left\|P_{M}-P_{N}\right\|
\end{gathered}
$$

and by interchanging $M$ and $N$

$$
\delta(N, M) \leq\left\|P_{N}-P_{M}\right\|=\left\|P_{M}-P_{N}\right\|
$$

Hence

$$
\hat{\delta}(M, N)=\max \{\delta(M, N), \delta(N, M)\} \leq\left\|P_{M}-P_{N}\right\|
$$

In order to proof our main result of this section we need the following theorem which can be found in $[\mathrm{Ka} 76$, I.6.34].

2.4.2 Theorem. Let $P, Q$ be two orthogonal projections with $M=\operatorname{im}(P), N=\operatorname{im}(Q)$ such that

$$
\|(I-Q) P\|=\delta<1 .
$$

Then there are the following alternatives. Either 
i) $Q$ maps $M$ onto $N$ one to one and bicontinuously, and

$$
\|P-Q\|=\|(I-P) Q\|=\|(I-Q) P\|=\delta ; \quad \text { or }
$$

ii) $Q$ maps $M$ onto a proper subspace $N_{0}$ of $N$ one to one and bicontinuously and, if $Q_{0}$ is the orthogonal projection onto $N_{0}$,

$$
\begin{aligned}
\left\|P-Q_{0}\right\| & =\left\|(I-P) Q_{0}\right\|=\left\|\left(I-Q_{0}\right) P\right\|=\|(I-Q) P\|=\delta \\
\|P-Q\| & =\|(I-P) Q\|=1 .
\end{aligned}
$$

Our main result of this section is the following corollary of theorem 2.4.2 which is mentioned in [Ka76] as a footnote on page 198 with reference to the theorem.

2.4.3 Corollary. If $M, N \subset H$ are closed subspaces and $P_{M}, P_{N}$ denote the orthogonal projections onto these spaces, then

$$
\hat{\delta}(M, N)=\left\|P_{M}-P_{N}\right\|
$$

Proof. By lemma 2.4.1 we know that $\hat{\delta}(M, N) \leq\left\|P_{M}-P_{N}\right\| \leq 1$ and hence the assertion is true if $\hat{\delta}(M, N)=1$. If, however, $\hat{\delta}(M, N)<1$, we obtain from lemma 2.4.1 that

$$
\left\|\left(I-P_{N}\right) P_{M}\right\|=\delta(M, N) \leq \hat{\delta}(M, N)<1
$$

and

$$
\left\|\left(I-P_{M}\right) P_{N}\right\|=\delta(N, M) \leq \hat{\delta}(M, N)<1 .
$$

Hence in 2.4 .2 only the first possibility can hold true and we infer

$$
\left\|P_{M}-P_{N}\right\|=\left\|\left(I-P_{M}\right) P_{N}\right\|=\left\|\left(I-P_{N}\right) P_{M}\right\|=\max \{\delta(N, M), \delta(M, N)\}=\hat{\delta}(M, N) .
$$

For later reference we want to mention that in the case of a Hilbert space, the space of all closed subspaces $\operatorname{Gr}(H)$ of $H$ with respect to the gap topology is not only a metric space but can even be given the structure of an analytic Banach manifold (cf. [AM09]) which is called the Hilbert Grassmannian.

For any closed subspace $M \subset H$ a chart can be defined by 


$$
\Psi_{M}: \mathcal{L}\left(M, M^{\perp}\right) \rightarrow B_{1}(M), \quad A \mapsto \operatorname{graph}(A),
$$

where $B_{1}(M)$ denotes the open unit ball around $M$ in $G r(H)$ with respect to the gap topology. Moreover, it can be shown that the inverse of $\Psi_{M}$ is

$$
\Psi_{M}^{-1}: B_{1}(M) \rightarrow \mathcal{L}\left(M, M^{\perp}\right), \quad N \mapsto P_{M^{\perp}}\left(\left.P_{M}\right|_{N}\right)^{-1}
$$

and the transition maps are given by

$$
\left(\Psi_{M}^{-1} \circ \Psi_{N}\right)(A)=P_{M^{\perp}}(I+A)\left(P_{M}(I+A)\right)^{-1} .
$$




\section{Chapter 3}

\section{On the Dimension of Exceptional Sets}

Throughout this chapter let $X \neq \emptyset$ be a fixed topological space and $\Sigma \subset X$ be a closed subspace which we call the exceptional set. In the following we assume that certain maps are given which are, roughly speaking, defined on a subset of the power set of $X$. We suppose that these maps have a couple of properties regarding $X$ and $\Sigma$ and our aim is to infer results about the Lebesgue covering dimension of $\Sigma$ from them. Constructions in later sections will naturally lead to such maps and interesting exceptional sets $\Sigma$ will appear whose dimension can be studied by the results of this chapter.

We start by reminding two different definitions of a topological dimension and compare them in the first section. Afterwards, in the second section, we obtain a result on the dimension of $\Sigma$ by assuming the existence of maps which assign singular homology classes to compact subspaces of $X$. In the third section we consider maps that are closer to homotopy theory and which assign elements of $\mathbb{Z}_{2}$ to homotopy classes of mappings from cubes into $X$. In our main result we estimate the dimension of $\Sigma$ from below by studying homotopy classes of paths in $X$. Finally, in the fourth section we consider first simple examples of parts of our theory.

\subsection{Two Definitions of Topological Dimension}

There are several inequivalent definitions of dimension in mathematics being defined on different kinds of spaces like, among others, the vector space dimension, the dimensions of manifolds, $\mathrm{CW}$-complexes and polyhedra, the Hausdorff dimension of metric spaces and the small and large inductive dimension of general topological spaces. Here we will consider two further definitions of dimension for arbitrary topological spaces that will be of interest in the following. We consider at first the Lebesgue covering dimension which is defined as follows: 
3.1.1 Definition. The (covering) dimension $\operatorname{dim} X$ of a topological space $X$ is the minimal value of $n \in \mathbb{N}$ such that every finite open cover of $X$ has a finite open refinement in which no point is included in more than $n+1$ elements. $X$ is called infinite dimensional if no such $n$ exists.

3.1.2 Remark. It follows immediately from the definition that the dimension is hereditary which means that for any closed subspace $A \subset X$ we have $\operatorname{dim} A \leq \operatorname{dim} X$.

We do not want to discuss examples, but mention that this definition coincides with the dimension of polyhedra (cf. [Fed90, 2.2]). The definition is, however, in general not very comfortable for explicit computations. In the following, our aim is to show that the exceptional sets $\Sigma$ we want to consider are large in the sense that they have a large dimension. Accordingly, it suffices for us to estimate the dimension from below.

3.1.3 Definition. The cohomological dimension $\operatorname{dim}_{G} X$ of the topological space $X$ with respect to an abelian coefficient group $G$ is the largest integer $n$ such that there exists a closed subset $A \subset X$ with $\check{H}^{n}(X, A ; G) \neq 0$. If there is no such number we set $\operatorname{dim}_{G} X=\infty$.

The following result gives the connection between the Lebesgue covering dimension and the cohomological dimension for compact spaces $X$. Usually it is left as an exercise for the reader in the literature but we want to include its proof here for the sake of completeness.

3.1.4 Lemma. Let $X$ be compact and Hausdorff. Then

$$
\operatorname{dim}_{G} X \leq \operatorname{dim}_{\mathbb{Z}} X \leq \operatorname{dim} X
$$

for any abelian group $G$.

Proof. We begin by proving the first inequality. By the universal coefficient theorem for Čhech cohomology (cf. Sp81, Theorem 6.11]), we have for any closed subspace $A$ of $X, n \in \mathbb{Z}$ and abelian group $G$ a short exact sequence

$$
0 \rightarrow \check{H}^{n}(X, A) \otimes G \rightarrow \check{H}^{n}(X, A ; G) \rightarrow \operatorname{Tor}\left(\check{H}^{n+1}(X, A), G\right) \rightarrow 0
$$

Now set $n:=\operatorname{dim}_{\mathbb{Z}} X$ which we can assume to be a finite number without loss of generality. Then $\check{H}^{m}(X, A)=0$ for any closed subspace $A \subset X$ and any $m \geq n+1$ and by 3.1 we infer that all $\check{H}^{m}(X, A ; G)$ are trivial as well. Hence $\operatorname{dim}_{G} X \leq n=\operatorname{dim}_{\mathbb{Z}} X$.

In order to prove the second inequality, we want to recall the definition of Čhech cohomology for compact spaces at first: The set of all finite open coverings of $X$ is directed by defining $\sigma<\tau$ if $\tau$ is a refinement of $\sigma$. With each finite open covering $\sigma$ of $X$ we can consider its nerve $N(\sigma)$, which is a simplicial complex made of the elements of $\sigma$ in an abstract way and having the order of $\sigma$ as dimension. Accordingly, the dimension of $N(\sigma)$ is the largest integer $n$ such that there exist 
$n+1$ elements of $\sigma$ having a non-empty intersection. Moreover, if $A \subset X$ is a closed subspace, any covering $\sigma$ of $X$ induces a covering of $A$ and so a nerve $N\left(\left.\sigma\right|_{A}\right)$ which is a subcomplex of $N(\sigma)$. We now consider the relative simplicial cohomology groups $H^{n}\left(N(\sigma), N\left(\left.\sigma\right|_{A}\right)\right)$ for some $n \in \mathbb{N}$. If $\sigma<\tau$, we can construct a simplicial map $N(\tau) \rightarrow N(\sigma)$ which yields a well defined homomorphism $h_{\sigma, \tau}: H^{n}\left(N(\sigma), N\left(\left.\sigma\right|_{A}\right)\right) \rightarrow H^{n}\left(N(\tau), N\left(\left.\tau\right|_{A}\right)\right)$. Finally, one can show that these data build a direct system of groups and the corresponding direct limit is defined to be the Čhech cohomology group $\check{H}^{n}(X, A)$.

Now we begin to prove the assertion, set $n:=\operatorname{dim} X$ and suppose that $A \subset X$ is a closed subspace. We define $\mathcal{T}_{n}$ as the subset of the set of all finite open coverings of $X$ which have an order less than or equal to $n$. By the very definition of $\operatorname{dim} X$, for any finite open covering $\sigma$ of $X$ there exists $\tau \in \mathcal{T}_{n}$ such that $\sigma<\tau$. Moreover, for any $m \in \mathbb{Z}$, the subsystem of $\left\{H^{m}\left(N(\sigma), N\left(\left.\sigma\right|_{A}\right)\right)\right\}$ obtained by restricting the set of all finite open coverings of $X$ to $\mathcal{T}_{n}$ is again a direct system and by [HW48, VIII.4.A)] its direct limit is isomorphic to $\check{H}^{m}(X, A)$.

Now the rest of the prove is quite immediate: Since the dimension of $N(\tau)$ is bounded above by $n$ for any $\tau \in \mathcal{T}_{n}$ we infer that the simplicial cohomology group $H^{m}\left(N(\tau), N\left(\left.\tau\right|_{A}\right)\right)$ is trivial for all $m>n$. Accordingly, its direct limit over $\mathcal{T}_{n}$, and so $\check{H}^{m}(X, A)$, is trivial for any $m>n$. Since the closed subspace $A \subset X$ was assumed to be arbitrary, we finally infer $\operatorname{dim}_{\mathbb{Z}} X \leq n=\operatorname{dim} X$.

3.1.5 Remark. One can show that even the equality $\operatorname{dim}_{\mathbb{Z}} X=\operatorname{dim} X$ holds in lemma 3.1.4 if $\operatorname{dim} X<\infty$ (cf. [Fed90, Theorem 5.2], [Dr99, Example 1.3]).

\subsection{Dimension Measured by Homology}

Let $\nu: \mathbb{N} \rightarrow \mathbb{N}$ be a map, $G$ an abelian group and we assume throughout in this section that $X$ is compact. We denote by $\mathcal{K}(X) \subset 2^{X}$ the set of all compact subspaces of $X$ and assume that we have for any $k \in \mathbb{N}$ a map

$$
X^{\prime} \mapsto \sigma_{k}\left(X^{\prime}\right) \in H^{\nu(k)}\left(X^{\prime} ; G\right), \quad X^{\prime} \in \mathcal{K}(X),
$$

such that

i) $\sigma_{k}\left(X^{\prime}\right)=0 \in H^{\nu(k)}\left(X^{\prime} ; G\right)$ whenever $X^{\prime} \cap \Sigma=\emptyset$.

ii) If $X^{\prime}, X^{\prime \prime} \in \mathcal{K}(X), X^{\prime} \subset X^{\prime \prime} \subset X$ and $\iota: X^{\prime} \hookrightarrow X^{\prime \prime}$ denotes the inclusion, then

$$
\sigma_{k}\left(X^{\prime}\right)=\iota^{*} \sigma_{k}\left(X^{\prime \prime}\right) \in H^{\nu(k)}\left(X^{\prime} ; G\right)
$$

Here $H^{*}(X ; G)$ denotes the singular cohomology of $X$ with coefficients in $G$. 
3.2.1 Theorem. Let $G$ be a principal ideal domain and $X$ a connected, compact and $G$-orientable manifold of dimension $n \in \mathbb{N}$. If there exists $k \in \mathbb{N}$ such that $1 \leq \nu(k) \leq n-1, \sigma_{k}(X) \neq 0 \in$ $H^{\nu(k)}(X ; G)$ and $H_{\nu(k)-1}(X ; G)$ is free, then

i) $\operatorname{dim} \Sigma \geq n-\nu(k)$.

ii) $\Sigma$ cannot be deformed in $X$ into a point, which means that the inclusion $\iota: \Sigma \hookrightarrow X$ is not homotopic to a constant map.

3.2.2 Remark. The assumption that $H_{\nu(k)-1}(X ; G)$ is free holds in particular if $G$ is a field or if $\nu(k)=1$ and $G$ is free.

Proof. According to [Gr73, Corollary 23.14] the Kronecker homomorphism

$$
H^{\nu(k)}(X ; G) \rightarrow H_{\nu(k)}(X ; G)^{*}
$$

is bijective because $G$ is a principal ideal domain and $H_{\nu(k)-1}(X ; G)$ is free by assumption. Hence we can find $0 \neq \alpha \in H_{\nu(k)}(X ; G)$ such that $\left\langle\sigma_{k}(X), \alpha\right\rangle \neq 0 \in G$. Denote by $\eta \in$ $H^{n-\nu(k)}(X ; G)$ its Poincaré dual, where we use that $X$ is compact and $G$-oriented. According to [Br93, Cor. VI.8.4] and the fact that Čech cohomology and singular cohomology coincide for manifolds (cf. [Br93, E.6]), we have a commutative diagram

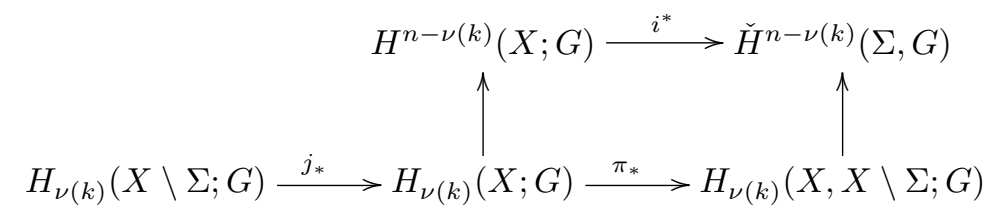

where the vertical arrows are isomorphisms given by Poincaré-Lefschetz duality and the lower horizontal sequence is part of the long exact homology sequence of the pair $(X, X \backslash \Sigma)$. Because of the commutativity, the class $i^{*} \eta$ is dual to $\pi_{*} \alpha$ and we now assume by contradiction the triviality of the latter one.

Then, by exactness, there exists $\beta \in H_{\nu(k)}(X \backslash \Sigma ; G)$ such that $\alpha=j_{*} \beta$. Moreover, since homology is compactly supported (cf. [Ma99, sect. 20.4]) we can find a compact subset $L \subset X \backslash \Sigma$ and a class $\gamma \in H_{\nu(k)}(L ; G)$ such that $\iota_{*} \gamma=\beta$, where $\iota$ denotes the inclusion. But since $\Sigma \cap L=\emptyset$, we obtain from the properties of $\sigma_{k}$ and the Kronecker pairing

$$
0=\left\langle\sigma_{k}(L), \gamma\right\rangle=\left\langle(j \circ \iota)^{*} \sigma_{k}(X), \gamma\right\rangle=\left\langle\sigma_{k}(X), j_{*} \iota_{*} \gamma\right\rangle=\left\langle\sigma_{k}(X), \alpha\right\rangle \neq 0
$$

a contradiction. Hence $\pi_{*} \alpha$ and so $i^{*} \eta \in \check{H}^{n-\nu(k)}(\Sigma ; \tilde{G})$ is non trivial. Now the assertion on the dimension of $\Sigma$ follows from lemma 3.1.4. Furthermore, the non triviality of $\iota^{*} \eta \in$ $\check{H}^{n-\nu(k)}(\Sigma ; G)$, where $\eta \in H^{n-\nu(k)}(X ; G)$, is clearly an obstruction to a deformation of $\Sigma$ to a point inside $X$. 
3.2.3 Remark. $\quad$ i) The main ideas of the proof of theorem 3.2.1 are taken from the proof of theorem 3.1 in [FP91] which claims an estimate of the covering dimension of the set of bifurcation points from a branch of zeroes of nonlinear operator families parametrized by compact manifolds.

ii) It is not difficult to generalise theorem 3.2.1 to the case that we have a closed subspace $Y \subset X$ and for any $k \in \mathbb{N}$ a map

$$
\sigma_{k}:\left\{X^{\prime} \in \mathcal{K}(X): Y \subset X^{\prime}\right\} \rightarrow A B E L, \quad X^{\prime} \mapsto \sigma_{k}\left(X^{\prime}\right) \in H^{\nu(k)}\left(X^{\prime}, Y ; G\right)
$$

However, the proof is a little bit more technical due to the fact that one has to work on the quotient $X / Y$ which is now assumed to be a compact G-orientable manifold. Since we will not need this more general result in the sequel, we have restricted our considerations here to the case that $Y=\emptyset$.

\subsection{Dimension Measured by Homotopy}

We want to begin this section by demonstrating its heuristic idea. We consider as before an exceptional set $\Sigma$ which we assume to be subset of a manifold $X$. Suppose that we have a map $\Gamma$ which assigns to each path $\gamma: I \rightarrow X$ having ends in $X \backslash \Sigma$ an element of $\mathbb{Z}_{2}$. We require that $\Gamma(\gamma)=0$ if $\gamma$ does not meet $\Sigma$ and that $\Gamma$ is homotopy invariant under homotopies having fixed ends. Now assume that we can find a path $\gamma$ in $X$ such that $\Gamma(\gamma)=1$. By our assumptions we know that $\gamma$ meets $\Sigma$ and that every path homotopic to $\gamma$ with fixed ends has to meet $\Sigma$ as well. Since $X$ is a manifold it cannot be singular at some point and hence we have space in order to deform $\gamma$ through $X$ and so to infer that $\Sigma$ needs to be large.

Before introducing the rigorous setup for our main theorems of this section, we prove a result about the dimension of subsets $\Sigma$ of manifolds whose first part is well known, although we could not find any proof in the literature. Here we give a proof which uses Poincaré-Lefschetz duality and, moreover, by this method we get some insight in the topology of $\Sigma$ for free.

3.3.1 Lemma. Let $X$ be a connected manifold of dimension $n \geq 2$ and $\Sigma \subset X$ compact.

i) If $X \backslash \Sigma$ is not path connected, then $\operatorname{dim} \Sigma \geq n-1$.

ii) Assume that $X$ is moreover orientable and $2 \leq k \leq n-1$ is a fixed natural number such that $H_{j}(X)=0$ for all $1 \leq j \leq k-1$. If now

- $\pi_{1}(X \backslash \Sigma)$ is abelian and

- $\pi_{k-1}(X \backslash \Sigma) \neq 0$,

then $\operatorname{dim} \Sigma \geq n-k$. 
In both cases $\Sigma$ is not contractible.

Proof. We prove the assertions successively and hence consider at first i):

Since $X \backslash \Sigma$ is not path connected, we obtain that the reduced homology group $\tilde{H}_{0}\left(X \backslash \Sigma ; \mathbb{Z}_{2}\right)$ is non trivial. Since $X$ is path connected by assumption, the long exact sequence of homology gives

$$
\cdots \rightarrow H_{1}\left(X, X \backslash \Sigma ; \mathbb{Z}_{2}\right) \rightarrow \tilde{H}_{0}\left(X \backslash \Sigma ; \mathbb{Z}_{2}\right) \rightarrow \tilde{H}_{0}\left(X ; \mathbb{Z}_{2}\right)=0
$$

and hence yields a surjective map $H_{1}\left(X, X \backslash \Sigma ; \mathbb{Z}_{2}\right) \rightarrow \tilde{H}_{0}\left(X \backslash \Sigma ; \mathbb{Z}_{2}\right)$ implying the non triviality of $H_{1}\left(X, X \backslash \Sigma ; \mathbb{Z}_{2}\right)$. Since $\Sigma$ is compact, we obtain by Poincaré-Lefschetz duality (cf. Do80, Prop. 7.2]) an isomorphism

$$
H_{1}\left(X, X \backslash \Sigma ; \mathbb{Z}_{2}\right) \stackrel{\cong}{\rightrightarrows} \check{H}^{n-1}\left(\Sigma ; \mathbb{Z}_{2}\right)
$$

showing that $\check{H}^{n-1}\left(\Sigma ; \mathbb{Z}_{2}\right) \neq 0$. Hence $\Sigma$ is not contractible and, moreover, the estimate $\operatorname{dim} \Sigma \geq n-1$ follows from lemma 3.1.4.

We now turn to the prove of ii). If $\pi_{0}(X \backslash \Sigma) \neq 0$, we obtain from the already proved first assertion that $\operatorname{dim} \Sigma \geq n-1>n-k$.

If $\pi_{0}(X \backslash \Sigma)=0$ we use the assumption that $\pi_{k-1}(X \backslash \Sigma)$ is non trivial and accordingly we can find a minimal natural number $2 \leq l \leq k$ such that $\pi_{l-1}(X \backslash \Sigma) \neq 0$ but $\pi_{j}(X \backslash \Sigma)=0$ for all $0 \leq j \leq l-2$. Hence in this case $X \backslash \Sigma$ is $l-2$ connected and we obtain from the Hurewicz theorem, that $H_{l-1}(X \backslash \Sigma) \cong \pi_{l-1}(X \backslash \Sigma) \neq 0$, where we use that $\pi_{1}(X \backslash \Sigma)$ is assumed to be abelian if $l=2$. Since $H_{l-1}(X)=0$ by assumption, the long exact sequence of homology yields a surjection

$$
H_{l}(X, X \backslash \Sigma) \rightarrow H_{l-1}(X \backslash \Sigma) \rightarrow H_{l-1}(X)=0
$$

showing that $H_{l}(X, X \backslash \Sigma) \neq 0$. Because $X$ is orientable and $\Sigma$ is compact, we can use Poincaré-Lefschetz duality once again and obtain that

$$
\check{H}^{n-l}(\Sigma) \cong H_{l}(X, X \backslash \Sigma) \neq 0
$$

is non trivial. Hence $\Sigma$ is not contractible and, by using lemma 3.1.4, we obtain that $\operatorname{dim} \Sigma \geq$ $n-l \geq n-k$.

In applications we can of course not assume to have any information about $\pi_{1}(X \backslash \Sigma)$. Hence we want to reformulate 3.3 .1 ii) as the following alternative. 
3.3.2 Corollary. Let $X$ be a connected orientable manifold of dimension $n$ and $\Sigma \subset X$ compact. Moreover, let $2 \leq k \leq n-1$ be a fixed natural number such that $H_{j}(X)=0$ for all $1 \leq j \leq k-1$ and $\pi_{k-1}(X \backslash \Sigma) \neq 0$. Then $\pi_{1}(X \backslash \Sigma)$ is not abelian or $\operatorname{dim} \Sigma \geq n-k$.

The point in this alternative is that a non abelian group is in particular not trivial which can also be interpreted as a result about the size of $\Sigma$ if, for example, $X$ is simply connected or even contractible. In the latter case it seems that by geometric intuition one needs $\Sigma$ "of dimension $n-2$ " in order to make $\pi_{1}(X \backslash \Sigma)$ non trivial which is in accordance with lemma 3.3 .1

3.3.3 Remark. The second part of lemma 3.3.1 also holds under the weaker assumption that $\pi_{1}(X \backslash \Sigma)$ is either trivial or not a perfect group. However, in view of the alternative which we formulated in the corollary above, this does not improve our result much.

Let now $Y$ be a subspace of $X$ such that $Y \cap \Sigma=\emptyset$ and define for $k \in \mathbb{N}$

$$
\Omega^{k}(X, Y)=\left\{f:\left(I^{k}, \partial I^{k}\right) \rightarrow(X, Y): f \text { continuous }\right\}
$$

Throughout, we assume that $Y \neq \emptyset$ such that in particular the case $X=\Sigma$ is excluded.

If $f_{1}, f_{2} \in \Omega^{k}(X, Y)$ such that $f_{1}\left(t_{1}, \ldots, t_{k-1}, 1\right)=f_{2}\left(t_{1}, \ldots, t_{k-1}, 0\right)$, we define their product $f_{1} * f_{2} \in \Omega^{k}(X, Y)$ by

$$
\left(f_{1} * f_{2}\right)\left(t_{1}, \ldots, t_{k}\right)=\left\{\begin{array}{l}
f_{1}\left(t_{1}, \ldots, t_{k-1}, 2 t_{k}\right), 0 \leq t_{k} \leq \frac{1}{2} \\
f_{2}\left(t_{1}, \ldots, t_{k-1}, 2 t_{k}-1\right), \frac{1}{2} \leq t_{k} \leq 1
\end{array}\right.
$$

and $\check{f}_{1} \in \Omega^{k}(X, Y)$ by

$$
\check{f}_{1}\left(t_{1}, \ldots, t_{k}\right)=f_{1}\left(t_{1}, \ldots, t_{k-1}, 1-t_{k}\right)
$$

In the following we assume that for any $k \in \mathbb{N}$ we have a map

$$
\Gamma_{k}: \Omega^{k}(X, Y) \rightarrow \mathbb{Z}_{2}
$$

such that

i) $\Gamma_{k}(f)=0 \in \mathbb{Z}_{2}$ if $f\left(I^{k}\right) \cap \Sigma=\emptyset$,

ii) $\Gamma_{k}\left(f_{1} * f_{2}\right)=\Gamma_{k}\left(f_{1}\right)+\Gamma_{k}\left(f_{2}\right)$ whenever $f_{1} * f_{2}$ exists,

iii) $\Gamma_{k}(f)=\Gamma_{k}(g)$ if $f$ and $g$ are homotopic in $\Omega^{k}(X, Y)$. 
3.3.4 Lemma. Let $\pi_{k}(X)$ be trivial and $f_{1}, f_{2} \in \Omega^{k}(X, Y)$ be such that $\left.f_{1}\right|_{\partial I^{k}}=\left.f_{2}\right|_{\partial I^{k}}$. Then

$$
\Gamma_{k}\left(f_{1} * \check{f}_{2}\right)=0
$$

Hence we obtain in particular

$$
\Gamma_{k}\left(f_{1} * \check{f}_{1}\right)=0 .
$$

Proof. At first we want to show that $\left.f_{1} * \check{f}_{2}\right|_{\partial I^{k}}: \partial I^{k} \rightarrow Y$ is homotopic to a constant map by a homotopy in $Y$. Note that $f_{1} * \check{f}_{2}$ is given by

$$
\left(f_{1} * \check{f}_{2}\right)\left(t_{1}, \ldots, t_{k}\right)=\left\{\begin{array}{l}
f_{1}\left(t_{1}, \ldots, t_{k-1}, 2 t_{k}\right), \quad 0 \leq t_{k} \leq \frac{1}{2} \\
f_{2}\left(t_{1}, \ldots, t_{k-1}, 2-2 t_{k}\right), \quad \frac{1}{2} \leq t_{k} \leq 1 .
\end{array}\right.
$$

Then, by using the homotopy $H: I \times \partial I^{k} \rightarrow Y$

$$
H\left(\lambda, t_{1}, \ldots, t_{k}\right)=\left\{\begin{array}{l}
f_{1}\left(t_{1}, \ldots, t_{k-1}, 2 t_{k}(1-\lambda)\right), \quad 0 \leq t_{k} \leq \frac{1}{2} \\
f_{2}\left(t_{1}, \ldots, t_{k-1},\left(2-2 t_{k}\right)(1-\lambda)\right), \quad \frac{1}{2} \leq t_{k} \leq 1,
\end{array}\right.
$$

we can deform $\left.f_{1} * \check{f}_{2}\right|_{\partial I^{k}}$ into

$$
\left(t_{1}, \ldots, t_{k}\right) \mapsto f_{1}\left(t_{1}, \ldots, t_{k-1}, 0\right)=f_{2}\left(t_{1}, \ldots, t_{k-1}, 0\right), \quad\left(t_{1}, \ldots, t_{k}\right) \in \partial I^{k} .
$$

Furthermore we can deform the latter map to a constant one by

$$
\tilde{H}: I \times \partial I^{k} \rightarrow Y, \quad\left(\lambda, t_{1}, \ldots, t_{k}\right) \mapsto f_{1}\left((1-\lambda) \cdot t_{1}, \ldots,(1-\lambda) \cdot t_{k-1}, 0\right) .
$$

Since $\left(I^{k}, \partial I^{k}\right)$ has the homotopy extension property, we infer that $f_{1} * \breve{f}_{2}$ is homotopic inside $\Omega^{k}(X, Y)$ to a map $g \in \Omega^{k}(X, Y)$ such that $g\left(\partial I^{k}\right)=y_{0}$ for some $y_{0} \in Y$. Because of the assumed triviality of $\pi_{k}(X)$ we finally obtain that $g$ is homotopic (inside $\Omega^{k}(X, Y)$ ) to the constant map $g^{\prime} \in \Omega^{k}(X, Y), g^{\prime}\left(I^{k}\right)=y_{0}$. Hence

$$
\Gamma_{k}\left(f_{1} * \check{f}_{2}\right)=\Gamma_{k}(g)=\Gamma_{k}\left(g^{\prime}\right)=0
$$

because $y_{0} \in Y$ and $Y \cap \Sigma=\emptyset$.

The following result is the main observation in order to use lemma 3.3 .1 to obtain estimates on the dimension of $\Sigma$ by homotopy. 
3.3.5 Lemma. Let $f \in \Omega^{k}(X, Y)$ be such that $\Gamma_{k}(f)=1 \in \mathbb{Z}_{2}$ and $\pi_{k}(X)=0$. Then $\pi_{k-1}(X \backslash$ $\Sigma) \neq 0$.

Proof. Assume that there exists $g \in \Omega^{k}(X, Y)$ such that $\left.g\right|_{\partial I^{k}}=\left.f\right|_{\partial I^{k}}$ and $g\left(I^{k}\right) \cap \Sigma=\emptyset$. Then by the properties (i),(ii) of $\Gamma_{k}$ and lemma 3.3 .4 we infer

$$
0 \neq \Gamma_{k}(f)=\Gamma_{k}(f)+\Gamma_{k}(\check{g})=\Gamma_{k}(f * \check{g})=0 ;
$$

a contradiction. Hence a map $g$ with the required properties cannot exist. Since $f\left(\partial I^{k}\right) \subset Y$ and $Y \cap \Sigma=\emptyset$, we conclude that there exists no continuous extension of $\left.f\right|_{\partial I^{k}}: \partial I^{k} \rightarrow X \backslash \Sigma$ to $I^{k}$. Consequently, $\left.f\right|_{\partial I^{k}}$ is not homotopic to a constant map (cf. [StZi94, 2.3.3]) and so in particular defines a non trivial element in $\pi_{k-1}(X \backslash \Sigma)$.

3.3.6 Proposition. Let $X$ be a simply connected manifold of dimension $n \geq 2$. If $\Sigma$ is compact and there exists $f \in \Omega^{1}(X, Y)$ such that $\Gamma_{1}(f)=1 \in \mathbb{Z}_{2}$, then

i) $\operatorname{dim} \Sigma \geq n-1$

ii) $\Sigma$ is not contractible.

Proof. By lemma 3.3.5. $X \backslash \Sigma$ is not path connected and hence the assertion follows from the first part of lemma 3.3.1

We now turn to the case $k \geq 2$ where, according to lemma 3.3.1, we need more assumptions in order to conclude results on the dimension of $\Sigma$.

3.3.7 Proposition. Let $X$ be a connected orientable manifold of dimension $n$ and $2 \leq k \leq n-1$ a fixed natural number such that $H_{j}(X)=0$ for all $1 \leq j \leq k-1$ and $\pi_{k}(X)=0$. Assume that the exceptional set $\Sigma \subset X$ is compact and that $\pi_{1}(X \backslash \Sigma)$ is abelian. Moreover, assume that there exists $f \in \Omega^{k}(X, Y)$ such that $\Gamma_{k}(f)=1$. Then

i) $\operatorname{dim} \Sigma \geq n-k$.

ii) $\Sigma$ is not contractible.

Proof. By lemma 3.3.5, we have $\pi_{k-1}(X \backslash \Sigma) \neq 0$ and hence the assertion follows from the second part of 3.3 .1

3.3.8 Remark. Note that theorem 3.2.1 states that the canonical inclusion $\iota: \Sigma \hookrightarrow X$ is not homotopic to a constant map, whereas the propositions 3.3.6 and 3.3 .7 assert that the identity map $\Sigma \rightarrow \Sigma$ is not homotopic to a constant map. Hence the conclusion of theorem 3.2.1 is stronger. On the other hand, exceptional sets $\Sigma$ which are contractible in $X$ cannot be detected by theorem 3.2.1. 
3.3.9 Remark. We want to point out that $X$ is not assumed to be compact in the propositions 3.3.6 and 3.3.7. On the other hand we need several other restrictive assumptions on the topology of $X$. This suggests to use these propositions as a kind of local version of 3.2.1. Since $X$ is by assumption a manifold, we can use our results in open coordinate balls $B$ of $X$. Then $B$ is contractible and hence all assumptions on the triviality of the homology and homotopy groups of $B$ hold. Note that estimates on the dimension of $B \cap \Sigma$ yield estimates on the dimension of all of $\Sigma$ by remark 3.1.2.

\subsection{First Examples}

In this section we want to consider first examples of the ideas developed above. We want to restrict to section 3.3 and consider only maps

$$
\Gamma: \Omega^{1}(X, Y) \rightarrow \mathbb{Z}_{2}
$$

Moreover, our examples are such that we can actually use the following adapted version of lemma 3.3 .5 in which we can avoid to assume $X$ to be simply connected.

3.4.1 Lemma. Let $\Gamma: \Omega^{1}(X, Y) \rightarrow \mathbb{Z}_{2}$ be such that condition i) from section 3.3 holds as well as

ii') $\Gamma\left(\gamma_{1}\right)=\Gamma\left(\gamma_{2}\right)$ if $\left.\gamma_{1}\right|_{\partial I}=\left.\gamma_{2}\right|_{\partial I}$

If there exists $\gamma \in \Omega^{1}(X, Y)$ such that $\Gamma(\gamma)=1 \in \mathbb{Z}_{2}$, then $X \backslash \Sigma$ is not path connected.

Proof. Assume that $X \backslash \Sigma$ is path connected. Since $\gamma(0), \gamma(1) \in Y \subset X \backslash \Sigma$, we can find a path $\gamma^{\prime}: I \rightarrow X \backslash \Sigma$ such that $\gamma(0)=\gamma^{\prime}(0), \gamma^{\prime}(1)=\gamma(1)$. We obtain $\Gamma\left(\gamma^{\prime}\right)=\Gamma(\gamma)=1$ by condition ii') and, moreover, $\Gamma\left(\gamma^{\prime}\right)=0$ by condition i) since $\gamma^{\prime}(I)$ does not meet $\Sigma$.

3.4.2 Remark. We want to point out that in our later applications of section 3.3 condition ii') does not hold in general and hence we really need the theory developed there.

Note that if we assume in addition that $X$ is a connected manifold of dimension $n \geq 2$ and $\Sigma$ is compact, we obtain that $\Sigma$ is not contractible and $\operatorname{dim} \Sigma \geq n-1$ by lemma 3.3.1.

We will now give two applications of these simple observations. At first we consider continuous functions $f: X \rightarrow \mathbb{R}$, where $X$ is a general topological space, and study for some $c \in \mathbb{R}$ the closed level set

$$
\Sigma:=f^{-1}(c)=\{x \in X: f(x)=c\}
$$

which we assume not to be empty or the whole space $X$. If we set $Y=X \backslash \Sigma$ and define 


$$
\Gamma: \Omega^{1}(X, Y) \rightarrow \mathbb{Z}_{2}, \quad \Gamma(\gamma)=\frac{|\operatorname{sgn}(f(\gamma(1))-c)-\operatorname{sgn}(f(\gamma(0))-c)|}{2},
$$

then clearly ii') holds for $\Gamma$. Moreover, if $\gamma: I \rightarrow X$ is such that $\gamma(I) \cap \Sigma=\emptyset$, we obtain by the intermediate value theorem that $\Gamma(\gamma)=0$. Hence the condition i) is satisfied as well and we obtain the following generalised intermediate value theorem whose second assertion makes think of the regular value theorem of differential topology.

3.4.3 Proposition. If $f: X \rightarrow \mathbb{R}$ is a continuous function on a topological space $X$ and there exists $x_{0}, x_{1} \in X$ such that $f\left(x_{0}\right)<c$ and $f\left(x_{1}\right)>c$ for some $c \in \mathbb{R}$, then $X \backslash f^{-1}(c)$ is not path connected. If, moreover, $X$ is a connected manifold of dimension $n \geq 2$ and $f^{-1}(c)$ compact, then $\operatorname{dim}\left(f^{-1}(c)\right) \geq n-1$ and $f^{-1}(c)$ is not contractible.

It is easy to verify proposition 3.4 .3 for concrete manifolds and functions. For example, consider the compact manifold $S^{2}$ and define by using spherical coordinates

$$
f: S^{2} \rightarrow \mathbb{R}, f(\varphi, \theta)=\theta-\frac{\pi}{2},
$$

where $\theta \in[0, \pi]$ denotes the inclination angle. Then the conditions of proposition 3.4.3 are fulfilled for $c=0$ if we take $x_{0}$ and $x_{1}$ to be the North- and South Pole of $S^{2}$, respectively. On the other hand, we see immediately that $f^{-1}(0)$ is given by $S^{1} \subset S^{2}$ which is not contractible and of dimension 1 as predicted by proposition 3.4 .3

Similarly, an example of a function on a non compact manifold can be obtained by setting $X=\mathbb{R}^{n}$ and

$$
f: X \rightarrow \mathbb{R}, \quad f(x)=1-\|x\| .
$$

Then $f^{-1}(0)=S^{n-1}$ is $n$-1-dimensional and non contractible.

Note that in both examples the exceptional sets are contractible inside the manifold $X$ and hence they cannot be detected by theorem 3.2.1. On the other hand it is easy to construct level sets of continuous functions on manifolds $X$ which are not contractible in $X$. An example is given by the height function on the 2-torus (cf. [Mi69, §1]).

We now consider our second example which actually is a step in the direction of our more sophisticated applications in later chapters. Let $X$ be a topological space and $L: X \rightarrow \mathcal{S}(n)$ a continuous family of symmetric $n \times n$ matrices. Then the spectrum of each $L_{x}$ is non-empty, real and for each $x \in X$ we can define

$$
\mu_{-}\left(L_{x}\right)=\#\left\{\lambda \in \sigma\left(L_{x}\right): \lambda<0\right\}
$$


We are interested in the dimension of the singular set

$$
\Sigma=\left\{x \in X: \operatorname{ker} L_{x} \neq\{0\}\right\}
$$

which is closed by the continuity of the determinant.

We set again $Y=X \backslash \Sigma$, assume $Y \neq \emptyset$ and define

$$
\Gamma_{1}: \Omega^{1}(X, Y) \rightarrow \mathbb{Z}_{2}, \Gamma_{1}(\gamma)=\left|\operatorname{sgn}\left(\mu_{-}\left(L_{\gamma(1)}\right)-\mu_{-}\left(L_{\gamma(0)}\right)\right)\right| .
$$

Note that again ii') holds obviously for $\Gamma_{1}$. Moreover, the remaining property i) follows from the continuity of eigenvalues for paths of symmetric matrices (cf. [Ka76, II.5.1]) and the intermediate value theorem.

3.4.4 Proposition. Let $L: X \rightarrow \mathcal{S}(n)$ be a continuous family of symmetric matrices parametrised by a topological space $X$. If there exists a path $\gamma: I \rightarrow X$ such that $\gamma(0), \gamma(1) \notin \Sigma$ and

$$
\mu_{-}\left(L_{\gamma(1)}\right) \neq \mu_{-}\left(L_{\gamma(0)}\right),
$$

then $X \backslash \Sigma$ is not path connected. If $X$ is moreover a connected manifold of dimension $n \geq 2$ and $\Sigma$ is compact, then $\operatorname{dim} \Sigma \geq n-1$ and $\Sigma$ is not contractible. 


\section{Part II}

\section{The Index Bundles ind and s-ind}





\section{Chapter 4}

\section{Preliminaries: Fredholm Operators, Index Bundles and Spectral Flow}

The aim of this second part of the thesis, consisting of the chapters 4-7, is to generalise the celebrated Atiyah-Jänich index bundle (cf. [At89, Appendix] ) and its variant for selfadjoint operators (cf. [AS69]) to gap continuous families of unbounded operators. Before we begin with our investigations in the subsequent chapters, we want to prepare ourselves by recalling the classical definitions and presenting some modern results that trace back to them in a certain manner.

The chapter is structured as follows: In the first two sections we give a survey of the classical constructions and deep results which are related to them. Afterwards, in the third chapter, we introduce the quite recent construction of spectral flow for gap continuous paths of unbounded selfadjoint Fredholm operators [BLP05] and state an associated uniqueness result due to Lesch [Le05]. The latter one will later ensure that the spectral flow of a path of unbounded selfadjoint operators can be computed from our selfadjoint index bundle in the same way as in the classical setting of bounded operators. In the fourth section we briefly mention recent results that have appeared in connection with the investigation of spectral flow for unbounded operators.

In this chapter we assume throughout that all Hilbert spaces we consider are separable.

\subsection{The Atiyah-Jänich Bundle}

In this section we recall the construction of the celebrated Atiyah-Jänich index bundle for families of bounded Fredholm operators acting on a Hilbert space as developed independently by Atiyah and Jänich around 1964. Nowadays there are many references concerning the index bundle. Besides the probably most often cited one At89] we want to mention that a quite accessible discussion can be found in BoBl85, where, however, the assumption that the operators act on Hilbert spaces is strongly used. Our main reference here is [ZKKP75], where the original 
construction is considered for families of Fredholm operators acting on Banach spaces. It turns out that this weaker assumption on the underlying linear space does not affect the construction of the index bundle as we now want to present.

Accordingly let $E$ be a Banach space, $X$ a compact topological space and $L: X \rightarrow \mathcal{B F}(E)$ a family of bounded Fredholm operators which we assume to be continuous with respect to the norm topology on $\mathcal{B F}(E)$. We repeat the following well known fact which is the main observation for defining the index bundle.

4.1.1 Lemma. There exists a closed subspace $E_{1} \subset E$ of finite codimension such that $\operatorname{ker} L_{\lambda} \cap$ $E_{1}=\{0\}$ for all $\lambda \in X$.

Proof. Let $\lambda_{0} \in X$ and let $F_{0}$ be a closed subspace that complements the finite dimensional subspace $\operatorname{ker} L_{\lambda_{0}} \subset E$. Then $L_{\lambda_{0}}: F_{0} \rightarrow \operatorname{im} L_{\lambda_{0}}$ is a topological isomorphism onto the closed subspace im $L_{\lambda_{0}} \subset E$. Denote by $L_{\lambda_{0}}^{\prime}: \operatorname{im} L_{\lambda_{0}} \rightarrow F_{0}$ the continuous inverse and choose a bounded projection $P: E \rightarrow E$ onto im $L_{\lambda_{0}}$ which exists since im $L_{\lambda_{0}}$ is closed and has finite codimension (cf. A.1.2). Now consider the continuous family

$$
L_{\lambda_{0}}^{\prime} P L_{\lambda}: F_{0} \rightarrow F_{0}, \quad \lambda \in X
$$

and observe that for $\lambda=\lambda_{0}$ we have $L_{\lambda_{0}}^{\prime} P L_{\lambda_{0}}=I_{F_{0}}$. Since $G L\left(F_{0}\right) \subset \mathcal{L}\left(F_{0}\right)$ is open, we can find a neighbourhood $U_{\lambda_{0}}$ of $\lambda_{0}$ such that $L_{\lambda_{0}}^{\prime} P L_{\lambda} \in G L\left(F_{0}\right)$ for all $\lambda \in U_{\lambda_{0}}$. In particular we have ker $L_{\lambda} \cap F_{0}=\{0\}$ for all $\lambda \in U_{\lambda_{0}}$.

Now, since $X$ is compact by assumption, we can find a finite cover $X=\bigcup_{i=1}^{n} U_{i}$ and closed subspaces $F_{i}, i=1, \ldots, n$, of finite codimension such that $F_{i} \cap \operatorname{ker} L_{\lambda}=\{0\}$ for all $\lambda \in U_{i}$ and $i=1, \ldots, n$. Then $E_{1}=\bigcap_{i=1}^{n} F_{i}$ is closed, of finite codimension and $E_{1} \cap \operatorname{ker} L_{\lambda}=\{0\}$ for all $\lambda \in X$.

The index bundle of $L: X \rightarrow \mathcal{B F}(E)$ can now be defined as follows: Choose a subspace $E_{1}$ as in the foregoing lemma. Then the restriction $\left.L\right|_{E_{1}}$ of each $L_{\lambda}$ to $E_{1}$ defines a bundle monomorphism of $X \times E_{1}$ into $X \times E$. Since each im $\left.L_{\lambda}\right|_{E_{1}}$ is complemented by lemma A.2.11. $\left.\operatorname{im} L\right|_{E_{1}}$ defines a subbundle of $X \times E$ according to corollary 1.2.10. By proposition 1.2.6 we can find a family of projections $P: X \rightarrow \mathcal{L}(E)$ such that $\operatorname{im} P_{\lambda}=\left.\operatorname{im} L_{\lambda}\right|_{E_{1}}, \lambda \in X$.

We now define the Atiyah-Jänich bundle by

$$
\text { ind } L=\left[\Theta\left(E / E_{1}\right)\right]-[\operatorname{im}(I-P)] \in K(X) \text {. }
$$

Although it is a consequence of our constructions below, we want to show that the index bundle is well defined. First of all, if we have two families of projections $P_{0}, P_{1}$ such that $\operatorname{im}\left(P_{0}\right)=\operatorname{im}\left(P_{1}\right)=\operatorname{im}\left(\left.L\right|_{E_{1}}\right)$, then $\operatorname{im}\left(I-P_{0}\right)$ and $\operatorname{im}\left(I-P_{1}\right)$ are complements of one and the same subbundle in $\Theta(E)$ and hence isomorphic. Accordingly it is enough to show that ind $L$ does not depend on the choice of $E_{1}$. If $E_{0}, E_{1}$ are two closed subspaces of finite codimension having 
trivial intersection with the kernels of $L$, then $E_{0} \cap E_{1}$ has these properties as well. Hence we can assume that $E_{0} \subset E_{1}$ and take a finite dimensional subspace $E_{0}^{\prime} \subset E_{1}$ such that $E_{0} \oplus E_{0}^{\prime}=E_{1}$. Let $P^{0}$ and $P^{1}$ denote the families of projections onto im $\left.L\right|_{E_{0}}$ and $\left.\operatorname{im} L\right|_{E_{1}}$, respectively. Taking into account that $\operatorname{im}\left(I-P^{1}\right) \subset \operatorname{im}\left(I-P^{0}\right)$ it is easily seen that we obtain an exact sequence

$$
0 \rightarrow \Theta\left(E_{0}^{\prime}\right) \stackrel{\left(I-P^{0}\right) L}{\longrightarrow} \operatorname{im}\left(I-P^{0}\right) \stackrel{I-P^{1}}{\longrightarrow} \operatorname{im}\left(I-P^{1}\right) \rightarrow 0
$$

and hence a bundle isomorphism $\operatorname{im}\left(I-P^{0}\right) \cong \operatorname{im}\left(I-P^{1}\right) \oplus \Theta\left(E_{0}^{\prime}\right)$ showing

$$
\left[\operatorname{im}\left(I-P^{0}\right)\right]-\left[\operatorname{im}\left(I-P^{1}\right)\right]=\left[\Theta\left(E_{0}^{\prime}\right)\right] \in K(X) .
$$

On the other hand, by dimension counting we clearly have an isomorphism $E_{0}^{\prime} \oplus E / E_{0} \cong E / E_{1}$ and infer

$$
\left[\Theta\left(E_{0}^{\prime}\right)\right]=\left[\Theta\left(E / E_{0}\right)\right]-\left[\Theta\left(E / E_{1}\right)\right] .
$$

We obtain

$$
\left[\Theta\left(E / E_{0}\right)\right]-\left[\operatorname{im}\left(I-P^{0}\right)\right]=\left[\Theta\left(E / E_{1}\right)\right]-\left[\operatorname{im}\left(I-P^{1}\right)\right] \in K(X)
$$

and hence the index bundle is indeed well defined.

For the sake of completeness we want to mention the main properties of the index bundle which are as follows:

- If $L_{1} \simeq L_{2}: X \rightarrow \mathcal{B F}(E)$ are homotopy equivalent, then

$$
\operatorname{ind}\left(L_{1}\right)=\operatorname{ind}\left(L_{2}\right),
$$

- $\operatorname{ind}(L)=0$ if and only if $L \simeq L^{\prime}: X \rightarrow \mathcal{B F}(E)$ where $L_{\lambda}^{\prime} \in G L(E)$ for all $\lambda \in X$,

- $\operatorname{ind}\left(L_{1} L_{2}\right)=\operatorname{ind}\left(L_{1}\right)+\operatorname{ind}\left(L_{2}\right) \in K(X)$ for all $L_{1}, L_{2}: X \rightarrow \mathcal{B F}(E)$.

One can summarise all these properties of the index bundle to the statement that for any compact topological space $X$ and Banach space $E$ we have a short exact sequence of monoids

$$
0 \rightarrow[X, G L(E)] \rightarrow[X, \mathcal{B F}(E)] \stackrel{\text { ind }}{\longrightarrow} K(X) .
$$

Moreover, one can show that ind is surjective whenever $E$ is a separable Hilbert space $H$ (cf. [ZKKP75, Theorem 2.3], where this assertion is even proved under weaker conditions for a 
certain class of Banach spaces which contains all separable Hilbert spaces). By using Kuiper's theorem we obtain that ind actually defines an isomorphism

$$
\text { ind : }[X, \mathcal{B F}(H)] \rightarrow K(X)
$$

and infer that for any separable Hilbert space $H, \mathcal{B F}(H)$ is a classifying space for the $K$ functor. This result is commonly known as the Atiyah-Jänich theorem.

\subsection{The Selfadjoint Index Bundle and Spectral Flow}

Let $H$ be a separable Hilbert space and $L: X \rightarrow \mathcal{B F}^{s a}(H)$ a family of selfadjoint bounded Fredholm operators which is continuous with respect to the norm topology. By a simple argument (which we will repeat below in section 7.1) one can prove that the selfadjointness of the operators $L$ forces the index bundle ind $L$ to be trivial. In AS69] Atiyah and Singer constructed a variant of the index bundle that allows to study the selfadjoint bounded Fredholm operators as well and which we want to recall in the following.

First of all let us remind the well known fact that $\mathcal{B F}^{s a}(H)$ has three connected components

$$
\mathcal{B F}^{s a}(H)=\mathcal{B F}_{+}^{s a}(H) \cup \mathcal{B F}_{-}^{s a}(H) \cup \mathcal{B F}_{*}^{s a}(H),
$$

where

$$
\begin{aligned}
& \mathcal{B F}_{+}^{s a}(H)=\left\{L \in \mathcal{B F}^{s a}(H): \sigma_{\text {ess }}(L) \subset(0, \infty)\right\}, \\
& \mathcal{B F}_{-}^{s a}(H)=\left\{L \in \mathcal{B F}^{s a}(H): \sigma_{\text {ess }}(L) \subset(-\infty, 0)\right\}, \\
& \mathcal{B F}_{*}^{s a}(H)=\mathcal{B F}^{s a}(H) \backslash\left(\mathcal{B F}_{+}^{s a}(H) \cup \mathcal{B F}_{-}^{s a}(H)\right)
\end{aligned}
$$

It is easy to see that $\mathcal{B F}_{+}^{s a}(H)$ and $\mathcal{B F}_{-}^{s a}(H)$ are contractible spaces. All this is well known and a detailed exposition can be found for example in [Wa07, Section 4.2.1].

The remaining component $\mathcal{B F}_{*}^{s a}(H)$ turned out to be topological non trivial and the crucial tool in order to study its topology is given by the selfadjoint index bundle whose construction can be sketched as follows:

We consider the map

$$
\alpha: \mathcal{B F}_{*}^{s a}(H) \rightarrow \Omega \mathcal{B F}_{0}(H), \quad \alpha[L](t)=\left\{\begin{array}{l}
\cos (2 \pi t)+i L \sin (2 \pi t), \quad t \in\left[0, \frac{1}{2}\right] \\
\cos (2 \pi t)+i \sin (2 \pi t), \quad t \in\left[\frac{1}{2}, 1\right]
\end{array},\right.
$$

where $\Omega \mathcal{B F}_{0}(H)$ denotes the loop space of $\mathcal{B F}_{0}(H)$. The main theorem of [AS69] states that $\alpha$ is a homotopy equivalence but its proof is far from being elementary. 
Now, since the loop functor $\Omega$ and the suspension functor $S$ are adjoint to each other, where $S X=X \times I /(X \times\{0\}) \cup(X \times\{1\})$, we obtain a monoid isomorphism $F:\left[X, \Omega \mathcal{B F}_{0}(H)\right] \rightarrow$ $\left[S X, \mathcal{B F}_{0}(H)\right]$. Using the results of the foregoing section this yields for any compact topological space a sequence of isomorphisms

$$
\left[X, \mathcal{B F}_{*}^{s a}(H)\right] \stackrel{\alpha}{\rightarrow}\left[X, \Omega \mathcal{B} \mathcal{F}_{0}(H)\right] \stackrel{F}{\longrightarrow}\left[S X, \mathcal{B F}_{0}(H)\right] \stackrel{\text { ind }}{\longrightarrow} \tilde{K}(S X)=K^{-1}(X) .
$$

Hence the following selfadjoint index bundle

$$
\operatorname{ind}_{s}:\left[X, \mathcal{B F}_{*}^{s a}(H)\right] \rightarrow K^{-1}(X), \quad L \mapsto \operatorname{ind}(F(\alpha(L)))
$$

defines a bijection and shows that $\mathcal{B F}_{*}^{s a}(H)$ is a classifying space for odd $K$-theory. A further outcome of these investigations are the identities

$$
\pi_{k}\left(\mathcal{B F}_{*}^{s a}(H)\right)=\left\{\begin{array}{ll}
0, & \text { if } k \text { is even } \\
\mathbb{Z}, & \text { if } k \text { is odd }
\end{array} \quad, \quad k \in \mathbb{N} \cup\{0\}\right.
$$

(cf. [Wa07, 4.2.26]) and later Atiyah, Singer and Patodi [APS76] constructed an explicit description of

$$
\operatorname{ind}_{s}:\left[S^{1}, \mathcal{B F}_{*}^{s a}\right] \rightarrow K^{-1}\left(S^{1}\right) \cong \mathbb{Z}
$$

which is called spectral flow and whose main idea can be sketched briefly as follows:

For any path of selfadjoint Fredholm operators which is continuous with respect to the norm topology one can show that there exists a neighbourhood $(-\varepsilon, \varepsilon)$ of $0 \in \mathbb{R}$ such that the eigenvalues around 0 can be represented as the graphs of continuous functions in $I \times(-\varepsilon, \varepsilon)$. Given a closed path $L: S^{1} \rightarrow \mathcal{B F}^{s a}(H)$ the spectral flow counts, roughly speaking, the number of negative eigenvalues of $L_{0}$ that become positive as the parameter $\lambda$ travels along the circle $S^{1}$ positively oriented minus the number of positive eigenvalues of $L_{0}$ that become negative. Of course, the described process of counting is just meant heuristically here because the functions that represent the eigenvalues are only continuous in general and hence may be difficult to handle. But it turned out that the integer obtained from the first Chern number of $\operatorname{ind}_{s}(L) \in K^{-1}\left(S^{1}\right)$ gives a precise definition of this heuristic idea.

A more detailed exposition can be found in [BW85]. Moreover, since the space of invertible selfadjoint operators is path connected it is easy to generalise the definition along these lines from closed paths to general paths having invertible ends (cf. Wa07]).

Finally, the spectral flow can also be constructed without using topological concepts as shown in Ph96]. The aim of the following section is to present this latter analytic definition of spectral flow for unbounded operators as obtained in [BLP05]. 


\subsection{Unbounded Selfadjoint Operators and Spectral Flow}

\subsubsection{Gap versus Riesz-topology}

In the discussion of spectral flow in the foregoing section we considered throughout bounded selfadjoint Fredholm operators. However, in many applications one is faced with unbounded operators. For example, in [APS76], where spectral flow was introduced for the first time, Atiyah, Patodi and Singer studied selfadjoint elliptic operators on manifolds.

A possible solution of this issue is to define a metric on $\mathcal{C} \mathcal{F}^{s a}(H)$ by requiring the map

$$
\mathcal{C F}^{s a}(H) \rightarrow \mathcal{B F}^{s a}(H), \mathcal{A} \mapsto \mathcal{A}\left(I+\mathcal{A}^{2}\right)^{-\frac{1}{2}}
$$

to be an isometry. The resulting topology is usually called the Riesz topology on $\mathcal{C F}^{s a}(H)$. Remembering the spectral mapping theorem, one defines the spectral flow of a path $\mathcal{A}:(I, \partial I) \rightarrow$ $\left(\mathcal{C F}^{s a}(H), G^{s a}(H)\right)$ which is continuous with respect to the Riesz topology by

$$
\operatorname{sf}(\mathcal{A})=\operatorname{sf}\left(\mathcal{A}\left(I+\mathcal{A}^{2}\right)^{-\frac{1}{2}}\right)
$$

However, continuity proofs with respect to the Riesz topology turned out to be delicate even for simple maps. An illustrative example is given by the main result of section 4.2 of [BoFu98], where a long computation using hard functional calculus is needed in order to prove that the map

$$
\mathcal{B}^{s a}(H) \rightarrow \mathcal{C F}^{s a}(H), \quad C \mapsto \mathcal{A}+C
$$

is continuous with respect to the Riesz topology for any fixed selfadjoint Fredholm operator $\mathcal{A}$ having a compact resolvent ${ }^{1}$. A further, but nevertheless quite long and technical, proof of this fact can be found in [Le05, Prop. 2.2].

In the second chapter we defined the gap topology on the set of all closed operators $\mathcal{C}(H)$ and hence the corresponding subspace topology yields another topology on $\mathcal{C F}^{s a}(H)$. Note that in corollary 2.2.4 we obtained the continuity of the map 4.1 with respect to the gap topology as a simple consequence of the basic theorem 2.2 .1 .

This observation is in accordance with the following result which is due to Nicolaescu [Ni07].

4.3.1 Lemma. The Riesz topology on $\mathcal{C F}^{s a}(H)$ is strictly finer than the gap topology.

Proof. At first we note that

$$
\left(I+T^{2}\right)^{-\frac{1}{2}} T \subset T\left(I+T^{2}\right)^{-\frac{1}{2}}
$$

\footnotetext{
${ }^{1}$ The assumption of a compact resolvent is actually not needed in the proof of the continuity but it ensures that the sum $\mathcal{A}+C$ is a Fredholm operator.
} 
for any selfadjoint operator $T$. Indeed, the obvious relation $\left(I+T^{2}\right)^{-1} T^{2} \subset T^{2}\left(I+T^{2}\right)^{-1}$ implies by the construction of the continuous functional calculus for bounded selfadjoint operators that $\left(I+T^{2}\right)^{-\frac{1}{2}} T^{2} \subset T^{2}\left(I+T^{2}\right)^{-\frac{1}{2}}$. Finally, it is proved in [Ka76, Theorem V.3.35] that $T$ commutes with any bounded operator that commutes with $T^{2}$.

Now we show that the Riesz topology is finer than the gap topology and in order to do so we let $\left\{T_{n}\right\}_{n \in \mathbb{N}} \subset \mathcal{C F}^{s a}(H)$ be a sequence such that $T_{n} \rightarrow T \in \mathcal{C F}^{s a}(H), n \rightarrow \infty$, with respect to the Riesz topology. We have to show that this sequence converges to $T$ with respect to the gap topology as well.

From the very definition of the Riesz topology we infer that

$$
T_{n}\left(I+T_{n}^{2}\right)^{-\frac{1}{2}} \rightarrow T\left(I+T^{2}\right)^{-\frac{1}{2}}, \quad n \rightarrow \infty,
$$

with respect to the operator norm. Hence by using 4.2 we obtain

$$
\begin{aligned}
\left(I+T_{n}^{2}\right)^{-1} & =\left(I+T_{n}^{2}-T_{n}^{2}\right)\left(I+T_{n}^{2}\right)^{-1}=I-T_{n}^{2}\left(I+T_{n}^{2}\right)^{-1} \\
& =I-T_{n}\left(I+T_{n}^{2}\right)^{-\frac{1}{2}} T_{n}\left(I+T_{n}^{2}\right)^{-\frac{1}{2}} \\
& \rightarrow I-T\left(I+T^{2}\right)^{-\frac{1}{2}} T\left(I+T^{2}\right)^{-\frac{1}{2}}=\left(I+T^{2}\right)^{-1}
\end{aligned}
$$

and moreover we get from elementary properties of the functional calculus for bounded selfadjoint operators

$$
\left(I+T_{n}^{2}\right)^{-\frac{1}{2}} \rightarrow\left(I+T^{2}\right)^{-\frac{1}{2}}, \quad n \rightarrow \infty .
$$

Finally, by using (4.2), 4.3) and (4.4), we infer

$$
\begin{aligned}
\left(T_{n}+i\right)^{-1} & =\left(T_{n}-i\right)\left(\left(T_{n}+i\right)\left(T_{n}-i\right)\right)^{-1}=\left(T_{n}-i\right)\left(I+T_{n}^{2}\right)^{-1} \\
& =T_{n}\left(I+T_{n}^{2}\right)^{-1}-i\left(I+T_{n}^{2}\right)^{-1} \\
& =\left(I+T_{n}^{2}\right)^{-\frac{1}{2}} T_{n}\left(I+T_{n}^{2}\right)^{-\frac{1}{2}}-i\left(I+T_{n}^{2}\right)^{-1} \\
& \rightarrow\left(I+T^{2}\right)^{-\frac{1}{2}} T\left(I+T^{2}\right)^{-\frac{1}{2}}-i\left(I+T^{2}\right)^{-1} \\
& =(T+i)^{-1}
\end{aligned}
$$

We obtain from lemma 2.3 .2

$$
d_{G}\left(T_{n}, T\right) \leq 16 \sqrt{2}\left\|\left(T_{n}+i\right)^{-1}-(T+i)^{-1}\right\| \rightarrow 0
$$

and hence $T_{n} \rightarrow T, n \rightarrow \infty$ with respect to the gap topology.

In order to show that the topologies do not coincide we consider an example that is due to Fuglede 
(cf. [Ni07, Rem.1.5]). Let $\left\{e_{n}\right\}_{n \in \mathbb{N}}$ be a complete orthonormal system of $H$ and $\left\{\lambda_{n}\right\}_{n \in \mathbb{N}} \subset \mathbb{R} \backslash\{0\}$ a sequence such that $\lambda_{n} \rightarrow \infty, n \rightarrow \infty$. We consider the unbounded operator

$$
D u=\sum_{k=1}^{\infty} \lambda_{k}\left\langle u, e_{k}\right\rangle e_{k}, \quad u \in \mathcal{D}(D)=\left\{u \in H: \sum_{k=1}^{\infty} \lambda_{k}^{2}\left|\left\langle u, e_{k}\right\rangle\right|^{2}<\infty\right\}
$$

which is easily seen to be selfadjoint and invertible by noting that its inverse is given by the compact selfadjoint operator

$$
K u=\sum_{k=1}^{\infty} \frac{1}{\lambda_{k}}\left\langle u, e_{k}\right\rangle e_{k}, \quad u \in H
$$

Moreover, we define a sequence of bounded selfadjoint operators by

$$
C_{n} u=-2 \lambda_{n}\left\langle u, e_{n}\right\rangle e_{n}, \quad u \in H, n \in \mathbb{N} .
$$

A simple computation shows

$$
\begin{aligned}
\left(D+C_{n}+i\right)^{-1} u & =\sum_{\substack{k \in \mathbb{N} \\
k \neq n}} \frac{1}{\lambda_{k}+i}\left\langle u, e_{k}\right\rangle e_{k}+\frac{1}{i-\lambda_{n}}\left\langle u, e_{n}\right\rangle e_{n} \\
(D+i)^{-1} u & =\sum_{k \in \mathbb{N}} \frac{1}{\lambda_{k}+i}\left\langle u, e_{k}\right\rangle e_{k}
\end{aligned}
$$

for any $u \in H$ and hence

$$
\begin{aligned}
\left\|\left(D+C_{n}+i\right)^{-1} u-(D+i)^{-1} u\right\| & =\left|\left(i-\lambda_{n}\right)^{-1}-\left(i+\lambda_{n}\right)^{-1}\right|\left|\left\langle u, e_{n}\right\rangle\right| \\
& \leq\left|\left(i-\lambda_{n}\right)^{-1}-\left(i+\lambda_{n}\right)^{-1}\right|\|u\|, \quad n \in \mathbb{N} .
\end{aligned}
$$

which shows

$$
\left\|\left(D+C_{n}+i\right)^{-1}-(D+i)^{-1}\right\| \leq\left|\left(i-\lambda_{n}\right)^{-1}-\left(i+\lambda_{n}\right)^{-1}\right| .
$$

Now, by lemma 2.3.2, we obtain

$$
d_{G}\left(D+C_{n}, D\right) \leq 16 \sqrt{2}\left\|\left(D+C_{n}+i\right)^{-1}-(D+i)^{-1}\right\| \rightarrow 0, \quad n \rightarrow \infty .
$$

On the other hand, by a further simple computation we infer that 


$$
\begin{aligned}
\left(D+C_{n}\right)\left(I+\left(D+C_{n}\right)^{2}\right)^{-\frac{1}{2}} u & =\sum_{\substack{k \in \mathbb{N} \\
k \neq n}} \frac{\lambda_{k}}{\left(1+\lambda_{k}^{2}\right)^{\frac{1}{2}}}\left\langle u, e_{k}\right\rangle e_{k}-\frac{\lambda_{n}}{\left(I+\lambda_{n}^{2}\right)^{\frac{1}{2}}}\left\langle u, e_{n}\right\rangle e_{n} \\
D\left(I+D^{2}\right)^{-\frac{1}{2}} u & =\sum_{k \in \mathbb{N}} \frac{\lambda_{k}}{\left(1+\lambda_{k}^{2}\right)^{\frac{1}{2}}}\left\langle u, e_{k}\right\rangle e_{k}
\end{aligned}
$$

for any $u \in H$ and so

$$
\left\|\left(D+C_{n}\right)\left(I+\left(D+C_{n}\right)^{2}\right)^{-\frac{1}{2}}-D\left(I+D^{2}\right)^{-\frac{1}{2}}\right\|=\left|2 \frac{\lambda_{n}}{\left(1+\lambda_{n}^{2}\right)^{\frac{1}{2}}}\right| \rightarrow 2, \quad n \rightarrow \infty .
$$

\subsubsection{Spectral Flow in the Gap Topology}

In BLP05, Booss-Bavnbek, Lesch and Phillips gave two equivalent constructions of the spectral flow for paths of unbounded selfadjoint operators which are assumed to be continuous with respect to the gap topology. In the first construction they used the Cayley transform in order to convert the given path into a path of unitary operators (cf. A.3.8). Then crossings through 0 of eigenvalues of the original path correspond to crossings through $-1 \in S^{1}$ of the path of unitary operators. Now a generalisation of the winding number for a certain class of unitary operators introduced before in [KL04] serves in order to count these crossings and leads to a first definition of spectral flow. The second approach is along the lines of the analytical construction of spectral flow for bounded operators as described in [Ph96]. We essentially present their construction in this section. But, instead of using their investigations on spectra of selfadjoint operators, we demonstrate that our general results on spectra of closed operators in Banach spaces from section 2.3 are enough to proceed along their lines. Moreover, in BLP05] some details are skipped with reference to the bounded case in [Ph96] which we will work out here in detail as well.

In this section we set for notational convenience

$$
\chi_{[a, b]}(T):=P_{D\left(\frac{a+b}{2}, \frac{b-a}{2}\right)}(T) \in \mathcal{L}(H)
$$

for any $T \in \mathcal{C}^{s a}(H)$ such that $a, b \in \rho(T)$, where $D(z, r)$ denotes the disc around $z \in \mathbb{C}$ of radius $r>0$.

Before we begin with the construction of spectral flow, we prove the following well known lemma.

4.3.2 Lemma. For $T \in \mathcal{C F}^{s a}(H), 0$ is either in the resolvent set $\rho(T)$ or it is an isolated eigenvalue of finite multiplicity.

Proof. First of all, since $T$ is Fredholm by assumption, we obtain from lemma 2.3 .6 that there exists $\varepsilon>0$ such that $\lambda-T$ is Fredholm as well for all $\lambda \in(-\varepsilon, \varepsilon)$. Hence $(-\varepsilon, \varepsilon) \cap \sigma_{\text {ess }}(T)=\emptyset$ 
and it just remains to show that 0 is isolated in $\sigma(T)$ if it is not in the resolvent set.

Define $X:=(\operatorname{ker} T)^{\perp}=\operatorname{im} T$ which is a Hilbert space being a closed subspace of $H$ by assumption. It is easy to see that the operator

$$
T^{\prime}:=\left.T\right|_{(\operatorname{ker} T)^{\perp}}: \mathcal{D}\left(T^{\prime}\right)=X \cap \mathcal{D}(T) \subset X \rightarrow X
$$

is closed. Since $T^{\prime}$ is moreover bijective, we obtain from the closed graph theorem A.2.4 that $\left(T^{\prime}\right)^{-1}: X \rightarrow X$ is bounded and hence $0 \in \rho\left(T^{\prime}\right)$. Accordingly, there exists a neighbourhood of 0 in $\mathbb{C}$ belonging entirely to the resolvent set of $T^{\prime}$.

Now assume that there exists $\lambda \in(-\varepsilon, \varepsilon) \backslash\{0\}$ such that $\lambda \in \rho\left(T^{\prime}\right)$ and there exists $u=$ $u_{1}+u_{2} \in \mathcal{D}(T)=(\operatorname{ker} T \oplus \operatorname{im} T) \cap \mathcal{D}(T)$ such that $\lambda u-T u=\lambda u_{1}+\lambda u_{2}-T u_{2}=0$. Then $\lambda u_{1}=(T-\lambda) u_{2}=\left(T^{\prime}-\lambda\right) u_{2}$ and since the right hand side is in $X=\operatorname{im} T$ and the left hand side in $\operatorname{ker} T$, we infer that both sides vanish. But because of $0 \neq \lambda \in \rho\left(T^{\prime}\right)$ we obtain that $u_{1}=u_{2}=0$ and hence $u=0$ which shows that $\lambda \in \rho(T)$.

Let $\mathcal{A}:(I, \partial I) \rightarrow\left(\mathcal{C F}^{s a}(H), G \mathcal{C}^{s a}(H)\right)$ be a continuous path with respect to the gap topology. By the foregoing lemma and theorem 2.3.21 we infer that for every $t \in I$ there exists $a>0$ and an open neighbourhood $N_{t, a} \subset \mathcal{C}(H)$ such that $\partial D(0, a) \subset \rho(T)$ for all $T \in N_{t, a}$ and the map

$$
N_{t, a} \rightarrow \mathcal{L}(H), \quad T \mapsto \chi_{[-a, a]}(T)
$$

is continuous. Moreover, all $\chi_{[-a, a]}(T), T \in N_{t, a}$, have the same finite rank. Now the counterimages of the $N_{t, a}$ under $\mathcal{A}$ define an open covering of the unit interval and by using the Lebesgue number of this covering we can find $0=t_{0} \leq t_{1} \leq \ldots \leq t_{n}=1$ and $a_{i}>0, i=1, \ldots n$, such that the maps

$$
\left[t_{i-1}, t_{i}\right] \ni t \mapsto \chi_{\left[-a_{i}, a_{i}\right]}\left(\mathcal{A}_{t}\right) \in \mathcal{L}(H)
$$

are continuous and have constant rank. We want to define the spectral flow of $\mathcal{A}:(I, \partial I) \rightarrow$ $\left(\mathcal{C F}^{s a}(H), G^{s a}(H)\right)$ by

$$
\operatorname{sf}(\mathcal{A})=\sum_{i=1}^{n} \operatorname{dimim} \chi_{\left[0, a_{i}\right]}\left(\mathcal{A}_{t_{i}}\right)-\operatorname{dimim} \chi_{\left[0, a_{i}\right]}\left(\mathcal{A}_{t_{i-1}}\right)
$$

but we have to prove its well definedness at first.

4.3.3 Lemma. $\operatorname{sf}(\mathcal{A})$ depends only on the continuous map $\mathcal{A}$.

Proof. We decompose the proof into three steps. In the first two steps we consider special variations of our choices and in the final step we explain why they already ensure the well 
definedness of the definition above.

In the first step we consider $t_{1}, \ldots, t_{n-1}$ and $a_{1}, \ldots, a_{n}$ as in 4.5 and assume that a further instant $t^{*} \in(0,1)$ is given such that $t_{i-1}<t<t_{i}$ for some $i$. If we now take the two maps

$$
\begin{aligned}
{\left[t_{i-1}, t^{*}\right] \ni t } & \mapsto \chi_{\left[-a_{i}, a_{i}\right]}\left(\mathcal{A}_{t}\right) \\
{\left[t^{*}, t_{i}\right] \ni t } & \mapsto \chi_{\left[-a_{i}, a_{i}\right]}\left(\mathcal{A}_{t}\right)
\end{aligned}
$$

instead of the map

$$
\left[t_{i-1}, t_{i}\right] \ni t \mapsto \chi_{\left[-a_{i}, a_{i}\right]}\left(\mathcal{A}_{t}\right)
$$

for the computation of the sum 4.5, then 4.5 does not change because the two new appearing terms cancel each other.

In the second step we now consider the case that we do not change the partition of the interval but instead the numbers $a_{i}$. Let $[c, d] \subset[0,1]$ be any subinterval and $t \mapsto \chi_{\left[-a_{1}, a_{1}\right]}\left(\mathcal{A}_{t}\right)$, $t \mapsto \chi_{\left[-a_{2}, a_{2}\right]}\left(\mathcal{A}_{t}\right)$ be two continuous maps as in 4.5 which are defined on $[c, d]$. We assume without loss of generality that $a_{1} \geq a_{2}$. Since $a_{1}, a_{2} \notin \sigma\left(\mathcal{A}_{t}\right)$ for all $t \in[c, d]$ we obtain by theorem A.3.3 that

$$
\operatorname{dimim} \chi_{\left[0, a_{1}\right]}\left(\mathcal{A}_{t}\right)-\operatorname{dimim} \chi_{\left[0, a_{2}\right]}\left(\mathcal{A}_{t}\right)=\operatorname{dimim} \chi_{\left[a_{2}, a_{1}\right]}\left(\mathcal{A}_{t}\right)
$$

which is constant on $[c, d]$ by theorem 2.3 .21 . We conclude

$$
\begin{aligned}
\operatorname{dimim} \chi_{\left[0, a_{1}\right]}\left(\mathcal{A}_{d}\right)-\operatorname{dimim} \chi_{\left[0, a_{1}\right]}\left(\mathcal{A}_{c}\right) & =\left(\operatorname{dimim} \chi_{\left[0, a_{2}\right]}\left(\mathcal{A}_{d}\right)+\operatorname{dimim} \chi_{\left[a_{2}, a_{1}\right]}\left(\mathcal{A}_{d}\right)\right) \\
& -\left(\operatorname{dimim} \chi_{\left[0, a_{2}\right]}\left(\mathcal{A}_{c}\right)+\operatorname{dimim} \chi_{\left[a_{2}, a_{1}\right]}\left(\mathcal{A}_{c}\right)\right) \\
& =\operatorname{dimim} \chi_{\left[0, a_{2}\right]}\left(\mathcal{A}_{d}\right)-\operatorname{dimim} \chi_{\left[0, a_{2}\right]}\left(\mathcal{A}_{c}\right) .
\end{aligned}
$$

As a third step let us consider the general case that we have two partitions $t_{1}, \ldots, t_{n}$ and $t_{1}^{\prime}, \ldots, t_{m}^{\prime}$ having associated numbers $a_{1}, \ldots, a_{n}$ and $a_{1}^{\prime}, \ldots, a_{m}^{\prime}$, respectively, as in (4.5). We build the union of both partitions in order to obtain a third one $\left\{t_{1}^{\prime \prime}, \ldots, t_{m+n}^{\prime \prime}\right\}$ which is finer than $t_{1}, \ldots, t_{n}$ and $t_{1}^{\prime}, \ldots, t_{m}^{\prime}$. By our first step of this proof we obtain

$$
\begin{aligned}
& \sum_{i=1}^{n} \operatorname{dimim} \chi_{\left[0, a_{i}\right]}\left(\mathcal{A}_{t_{i}}\right)-\operatorname{dimim} \chi_{\left[0, a_{i}\right]}\left(\mathcal{A}_{t_{i-1}}\right)=\sum_{i=1}^{m+n} \operatorname{dimim} \chi_{\left[0, b_{i}\right]}\left(\mathcal{A}_{t_{i}^{\prime \prime}}\right)-\operatorname{dimim} \chi_{\left[0, b_{i}\right]}\left(\mathcal{A}_{t_{i-1}^{\prime \prime}}\right) \\
& \sum_{i=1}^{m} \operatorname{dimim} \chi_{\left[0, a_{i}^{\prime}\right]}\left(\mathcal{A}_{t_{i}^{\prime}}\right)-\operatorname{dimim} \chi_{\left[0, a_{i}^{\prime}\right]}\left(\mathcal{A}_{t_{i-1}^{\prime}}\right)=\sum_{i=1}^{m+n} \operatorname{dimim} \chi_{\left[0, b_{i}^{\prime}\right]}\left(\mathcal{A}_{t_{i}^{\prime \prime}}\right)-\operatorname{dimim} \chi_{\left[0, b_{i}^{\prime}\right]}\left(\mathcal{A}_{t_{i-1}^{\prime \prime}}\right),
\end{aligned}
$$

for suitable $b_{1}, \ldots, b_{m+n}, b_{1}^{\prime}, \ldots, b_{m+n}^{\prime} \in\left\{a_{1}, \ldots, a_{n}, a_{1}^{\prime}, \ldots a_{m}^{\prime}\right\}$. Now the same partition is used in the sums on the right hand sides and we obtain from the second step of our proof that they actually agree. 
During the rest of this section we concern ourselves with the basic properties of the spectral flow. We begin with some simple observations that all follow quite immediately from its construction.

4.3.4 Lemma. Let $N \subset \mathcal{C F}^{\text {sa }}(H)$ be a neighbourhood as in the construction of the spectral flow, that is, there exists $a>0$ such that $-a, a \in \rho(T)$ for all $T \in N$, the map

$$
N \ni T \mapsto \chi_{[-a, a]}(T) \in \mathcal{L}(H)
$$

is continuous and all $\chi_{[-a, a]}(T)$ have the same finite rank.

If $\mathcal{A}_{1}, \mathcal{A}_{2}:(I, \partial I) \rightarrow\left(\mathcal{C F}^{s a}(H), G \mathcal{C}^{s a}(H)\right)$ are gap continuous such that

$$
\mathcal{A}_{1}(I), \mathcal{A}_{2}(I) \subset N, \quad \mathcal{A}_{1}(0)=\mathcal{A}_{2}(0), \quad \mathcal{A}_{1}(1)=\mathcal{A}_{2}(1),
$$

then $\operatorname{sf}\left(\mathcal{A}_{1}\right)=\operatorname{sf}\left(\mathcal{A}_{2}\right)$.

Proof. We compute

$$
\begin{aligned}
\operatorname{sf}\left(\mathcal{A}_{1}\right) & =\operatorname{dimim} \chi_{[0, a]}\left(\mathcal{A}_{1}(1)\right)-\operatorname{dimim} \chi_{[0, a]}\left(\mathcal{A}_{1}(0)\right) \\
& =\operatorname{dimim} \chi_{[0, a]}\left(\mathcal{A}_{2}(1)\right)-\operatorname{dimim} \chi_{[0, a]}\left(\mathcal{A}_{2}(0)\right)=\operatorname{sf}\left(\mathcal{A}_{2}\right) .
\end{aligned}
$$

4.3.5 Lemma. i) If $\mathcal{A}_{1}, \mathcal{A}_{2}:(I, \partial I) \rightarrow\left(\mathcal{C F}^{s a}(H), G \mathcal{C}^{s a}(H)\right)$ are two gap continuous paths such that $\mathcal{A}_{2}(0)=\mathcal{A}_{1}(1)$, then

$$
\operatorname{sf}\left(\mathcal{A}_{1} * \mathcal{A}_{2}\right)=\operatorname{sf}\left(\mathcal{A}_{1}\right)+\operatorname{sf}\left(\mathcal{A}_{2}\right) .
$$

ii) If $\mathcal{A}:(I, \partial I) \rightarrow\left(\mathcal{C F}^{s a}(H), G \mathcal{C}^{s a}(H)\right)$ is gap continuous and $\mathcal{A}^{\prime}$ is defined by $\mathcal{A}_{t}^{\prime}=\mathcal{A}_{1-t}$, then

$$
\operatorname{sf}\left(\mathcal{A}^{\prime}\right)=-\operatorname{sf}(\mathcal{A})
$$

iii) If $\mathcal{A}: I \rightarrow G \mathcal{C}^{s a}(H)$ is gap continuous, then $\operatorname{sf}(\mathcal{A})=0$.

Proof. The first two assertions follow immediately from the definition 4.5. For the third assertion we just have to observe that by theorem 2.3.21 we can find $\delta>0$ such that $\sigma\left(\mathcal{A}_{t}\right) \cap[-\delta, \delta]=\emptyset$ for all $t \in[0,1]$. Then

$$
\operatorname{sf}(\mathcal{A})=\operatorname{dimim} \chi_{[0, \delta]}\left(\mathcal{A}_{1}\right)-\operatorname{dimim} \chi_{[0, \delta]}\left(\mathcal{A}_{0}\right)=0 .
$$


The probably most important property of spectral flow is its homotopy invariance which we prove in the following lemma.

4.3.6 Lemma. Let $H: I \times I \rightarrow \mathcal{C F}^{s a}(H)$ be a continuous map such that

$$
H(I \times \partial I) \subset G \mathcal{C}^{s a}(H)
$$

Then

$$
\operatorname{sf}(H(0, \cdot))=\operatorname{sf}(H(1, \cdot))
$$

Proof. Since $H([0,1] \times[0,1]) \subset \mathcal{C F}^{s a}(H)$ is compact, we can find a finite open covering

$$
H([0,1] \times[0,1]) \subset \bigcup_{i=1}^{N} N_{i},
$$

where the $N_{i} \subset \mathcal{C}(H)$ are open sets as in the construction of the spectral flow. Accordingly, for each $N_{i}$ there exists $a_{i}>0$ such that $-a_{i}, a_{i} \in \rho(T)$ for all $T \in N_{i}$, the map

$$
N_{i} \ni T \mapsto \chi_{\left[-a_{i}, a_{i}\right]}(T) \in \mathcal{L}(H)
$$

is continuous and all $\chi_{\left[-a_{i}, a_{i}\right]}(T)$ are projections of the same finite rank.

Let $\varepsilon_{0}>0$ be a Lebesgue number of the open covering

$$
[0,1] \times[0,1]=\bigcup_{i=1}^{N} H^{-1}\left(N_{i}\right),
$$

and note that now the image of each subset of $[0,1] \times[0,1]$ of diameter less than $\varepsilon_{0}$ is entirely contained in one of the $N_{i}$.

We choose instants $0=\lambda_{0}<\lambda_{1}<\ldots<\lambda_{m}=1$ such that $\left|\lambda_{i}-\lambda_{i-1}\right|<\frac{\varepsilon_{0}}{\sqrt{2}}, 1 \leq i \leq m$. Then for each pair $1 \leq i, j \leq m, H\left(\left[\lambda_{i-1}, \lambda_{i}\right] \times\left[\lambda_{j-1}, \lambda_{j}\right]\right)$ is contained entirely in one of the $N_{k}$. Now we obtain for any $\left.H\right|_{\left[\lambda_{i-1}, \lambda_{i}\right] \times\left[\lambda_{j-1}, \lambda_{j}\right]}$ by arguing as in lemma 4.3 .4 and 4.3 .5

$$
\begin{aligned}
\operatorname{sf}\left(\left.H\left(\lambda_{i-1}, \cdot\right)\right|_{\left[\lambda_{j-1}, \lambda_{j}\right]}\right) & =\operatorname{sf}\left(\left.H\left(\cdot, \lambda_{j-1}\right)\right|_{\left[\lambda_{i-1}, \lambda_{i}\right]}\right)+\operatorname{sf}\left(\left.H\left(\lambda_{i}, \cdot\right)\right|_{\left[\lambda_{j-1}, \lambda_{j}\right]}\right) \\
& -\operatorname{sf}\left(\left.H\left(\cdot, \lambda_{j}\right)\right|_{\left[\lambda_{i-1}, \lambda_{i}\right]}\right) .
\end{aligned}
$$

But

$$
\operatorname{sf}\left(\left.H(\cdot, 0)\right|_{\left[\lambda_{i-1}, \lambda_{i}\right]}\right)=\operatorname{sf}\left(\left.H(\cdot, 1)\right|_{\left[\lambda_{i-1}, \lambda_{i}\right]}\right)=0, \quad i=1, \ldots, m,
$$


by the third part of lemma 4.3.5. By using the first part of 4.3 .5 once again, we obtain

$$
\begin{aligned}
\operatorname{sf}\left(H\left(\lambda_{i-1}, \cdot\right)\right) & =\sum_{j=1}^{m} \operatorname{sf}\left(\left.H\left(\lambda_{i-1}, \cdot\right)\right|_{\left[\lambda_{j-1}, \lambda_{j}\right]}\right) \\
& =\sum_{j=1}^{m} \operatorname{sf}\left(\left.H\left(\cdot, \lambda_{j-1}\right)\right|_{\left[\lambda_{i-1}, \lambda_{i}\right]}\right)+\operatorname{sf}\left(\left.H\left(\lambda_{i}, \cdot\right)\right|_{\left[\lambda_{j-1}, \lambda_{j}\right]}\right)-\operatorname{sf}\left(\left.H\left(\cdot, \lambda_{j}\right)\right|_{\left[\lambda_{i-1}, \lambda_{i}\right]}\right) \\
& =\sum_{j=1}^{m} \operatorname{sf}\left(\left.H\left(\lambda_{i}, \cdot\right)\right|_{\left[\lambda_{j-1}, \lambda_{j}\right]}\right)=\operatorname{sf}\left(H\left(\lambda_{i}, \cdot\right)\right) .
\end{aligned}
$$

Hence

$$
\operatorname{sf}(H(0, \cdot))=\operatorname{sf}\left(H\left(\lambda_{0}, \cdot\right)\right)=\operatorname{sf}\left(H\left(\lambda_{1}, \cdot\right)\right)=\operatorname{sf}(H(1, \cdot))
$$

We now want to discuss another property of the spectral flow which is a kind of normalisation and needed in order to discuss the uniqueness of spectral flow. Let $\left\{e_{k}\right\}_{k \in \mathbb{Z}}$ be a complete orthonormal system of the Hilbert space $H$, which is still assumed to be separable. Denote by $P_{+}$the orthogonal projection onto the closure of the space spanned by $\left\{e_{k}\right\}_{k \in \mathbb{N}}$, by $P_{-}$ the orthogonal projection onto the closure of the space spanned by $\left\{e_{-k}\right\}_{k \in \mathbb{N}}$ and by $P_{0}$ the orthogonal projection onto the span of $e_{0}$. Then

$$
P_{+}+P_{-}+P_{0}=I
$$

and moreover the operator

$$
L_{t}=\left(t-\frac{1}{2}\right) P_{0}+P_{+}-P_{-}
$$

is for each $t \in I$ a bounded selfadjoint Fredholm operator. To be more precise, $L_{t} \in G L(H)$ as long as $t \neq \frac{1}{2}$ and in the remaining case $L_{\frac{1}{2}}$ has a one dimensional kernel and cokernel which are both given by the span of $e_{0}$. Moreover, $L_{t}$ is obviously a continuous path with respect to the norm topology and thus by lemma 2.1.6 continuous with respect to the gap topology as well. Hence the spectral flow of $L$ is well defined. Since

$$
\sigma\left(L_{t}\right)=\left\{-1,1, t-\frac{1}{2}\right\}, \quad t \in I,
$$

it is immediate from the definition that

$$
\operatorname{sf}(L)=1
$$


Moreover, we note that if we set $T_{0}=P_{+}+P_{0}-P_{-}$and $P=P_{0}$, then

$$
\begin{aligned}
(I-P) T_{0}(I-P) & =\left(I-P_{0}\right)\left(P_{+}+P_{0}-P_{-}\right)\left(I-P_{0}\right)=\left(I-P_{0}\right)\left(P_{+}-P_{-}\right) \\
& =P_{+}-P_{-}
\end{aligned}
$$

In particular, $(I-P) T_{0}(I-P)$ defines a bounded, invertible and selfadjoint operator on ker $P$ such that the path

$$
\left(t-\frac{1}{2}\right) P+(I-P) T_{0}(I-P)=L_{t}, \quad t \in I
$$

has spectral flow 1 .

Now we finally discuss briefly the so called uniqueness of spectral flow which is the main result of [Le05]. We use the notation $\Omega^{1}(X, Y)$ as defined in section 3.3

\subsubsection{Theorem. Let}

$$
\mu: \Omega^{1}\left(\mathcal{C F}^{s a}(H), G \mathcal{C}^{s a}(H)\right) \rightarrow \mathbb{Z}
$$

be a map which is additive with respect to concatenation of paths, invariant under gap continuous homotopies inside $\Omega^{1}\left(\mathcal{C F}^{s a}(H), G \mathcal{C}^{s a}(H)\right)$ and which satisfies the following normalisation condition:

There is a rank one orthogonal projection $P \in \mathcal{L}(H)$ and a selfadjoint operator $T_{0} \in G \mathcal{B F}_{*}^{\text {sa }}(H)$ having $\sigma\left(T_{0}\right)=\{-1,1\}$ such that $(I-P) T_{0}(I-P)$ defines a bounded, invertible and selfadjoint operator on $\operatorname{ker} P$ and

$$
\mu\left(\left(t-\frac{1}{2}\right) P+(I-P) T_{0}(I-P)\right)=1
$$

Then $\mu$ equals the spectral flow.

Note that we have verified in this section that the spectral flow indeed has all the properties mentioned in its uniqueness theorem. In the proof of theorem 4.3.7, it is shown that any gap continuous path can be deformed into a path in a certain normal form in which the spectral flow can be computed by considering finite dimensional matrices. Then the uniqueness of spectral flow follows from a corresponding result in finite dimensions which can also be found in [Le05]. 


\subsection{Further Results}

The aim of this final section is to report briefly about some recent results about the topology of $\mathcal{C F}^{s a}(H)$ which are also partially connected with the uniqueness question of spectral flow.

At first, we go back for a moment to the space of bounded selfadjoint Fredholm operators $\mathcal{B F}^{s a}(H)$ with the norm topology. By the results of section 4.2 we know that $\mathcal{B F}^{s a}(H)$ consists of three connected components and that two of them are contractible whereas the third one, namely the subspace $\mathcal{B F}_{*}^{s a}(H)$ consisting of essentially indefinite operators, represents the odd $K$-theory.

If we restrict the construction of spectral flow to the bounded members of $\mathcal{C F}^{s a}(H)$ and recall lemma 2.1.6, we get a definition of spectral flow for norm continuous paths of bounded selfadjoint operators. Moreover, this spectral flow is homotopy invariant with respect to homotopies that are continuous in the norm topology, it is additive under concatenation and it satisfies the normalisation condition of theorem 4.3.7. Actually it is just the construction of spectral flow in the bounded case as presented in Ph96.

We now want to demonstrate how to prove a uniqueness statement like 4.3.7 by using the fact that the fundamental group of $\mathcal{B F}_{*}^{s a}(H)$ is infinite cyclic (cf. [Le05, Theorem 5.4] or [Wa07]). However, we want to point out that in Le05, Section 5.3] also an elementary proof is given which does not use any information about $\pi_{1}\left(\mathcal{B F}_{*}^{s a}(H)\right)$.

\subsubsection{Theorem. Let}

$$
\mu: \Omega^{1}\left(\mathcal{B F}_{*}^{s a}(H), G_{*}^{s a}(H)\right) \rightarrow \mathbb{Z}
$$

be a map which is additive with respect to concatenation of paths, invariant under norm continuous homotopies inside $\Omega^{1}\left(\mathcal{B F}_{*}^{s a}(H), G \mathcal{B}_{*}^{s a}(H)\right)$ and which satisfies the following normalisation condition:

There is a rank one orthogonal projection $P \in \mathcal{L}(H)$ and a selfadjoint operator $T_{0} \in G \mathcal{B F}_{*}^{\text {sa }}(H)$ having $\sigma\left(T_{0}\right)=\{-1,1\}$ such that $(I-P) T_{0}(I-P)$ defines a bounded, invertible and selfadjoint operator on $\operatorname{ker} P$ and

$$
\mu\left(\left(t-\frac{1}{2}\right) P+(I-P) T_{0}(I-P)\right)=1
$$

Then $\mu$ equals the spectral flow.

Proof. The proof needs the following two ingredients:

- The fundamental group of $\mathcal{B F}_{*}^{s a}(H)$ is infinite cyclic (cf. section 4.2).

- $\mathcal{B F}_{*}^{s a}$ is path connected. 
The well known latter assertion can be found for example in Wa07. The rest of the proof is quite simple.

We denote by $\mu: \Omega^{1}\left(\mathcal{B F}_{*}^{s a}(H), G \mathcal{B} \mathcal{F}_{*}^{s a}(H)\right) \rightarrow \mathbb{Z}$ a further candidate satisfying the homotopy, concatenation and normalisation condition. Moreover, we set $L_{t}^{\prime}=\left(t-\frac{1}{2}\right) P+(I-P) T_{0}(I-P)$, $t \in I$. By the homotopy and concatenation condition we obtain that sf and $\mu$ both define a group homomorphism

$$
\mu, \mathrm{sf}: \pi_{1}\left(\mathcal{B F}_{*}^{s a}(H), L_{0}^{\prime}\right) \rightarrow \mathbb{Z}
$$

Since $\operatorname{GBF}^{s a}(H)_{*}$ is path connected, we can choose a path $M: I \rightarrow G \mathcal{B F}_{*}^{s a}(H)$ connecting $L_{1}^{\prime}$ and $L_{0}^{\prime}$. Moreover, since $M$ is homotopic to a constant path in $\Omega^{1}\left(\mathcal{B F}_{*}^{s a}(H), G \mathcal{B} \mathcal{F}_{*}^{s a}(H)\right)$ we obtain $\mu(M)=\mu\left(M_{0}\right)$ and $\operatorname{sf}(M)=\operatorname{sf}\left(M_{0}\right)$ by using the homotopy properties. Note that the right hand sides of these equalities vanish by the concatenation property. Hence we obtain

$$
\mu\left(M * L^{\prime}\right)=\mu(M)+\mu\left(L^{\prime}\right)=\mu\left(L^{\prime}\right)=1=\operatorname{sf}\left(L^{\prime}\right)=\operatorname{sf}(M)+\operatorname{sf}\left(L^{\prime}\right)=\operatorname{sf}\left(M * L^{\prime}\right)
$$

and thus

$$
\mu=\operatorname{sf}: \pi_{1}\left(\mathcal{B F}_{*}^{s a}(H), L_{0}\right) \rightarrow \mathbb{Z},
$$

where we use that $\pi_{1}\left(\mathcal{B F}_{*}^{s a}(H), L_{0}^{\prime}\right)$ is infinite cyclic.

If now $L \in \Omega^{1}\left(\mathcal{B F}_{*}^{s a}(H), G \mathcal{B F}_{*}^{s a}(H)\right)$ is a general path, we can use again that $G \mathcal{B} \mathcal{F}_{*}^{s a}(H)$ is path connected and choose two paths $M^{1}, M^{2}: I \rightarrow \operatorname{GBF}_{*}^{s a}(H)$ such that

$$
M^{1}(0)=L_{0}^{\prime}, M^{1}(1)=L_{0}, M^{2}(0)=L_{1}, M^{2}(1)=L_{0}^{\prime} .
$$

Then $M^{1} * L * M^{2}$ is a closed path crossing $L_{0}^{\prime}$ and finally we obtain

$$
\begin{aligned}
\mu(L) & =\mu\left(M^{1}\right)+\mu(L)+\mu\left(M^{2}\right)=\mu\left(M^{1} * L * M^{2}\right)=\operatorname{sf}\left(M^{1} * L * M^{2}\right) \\
& =\operatorname{sf}\left(M^{1}\right)+\operatorname{sf}(L)+\operatorname{sf}\left(M^{2}\right)=\operatorname{sf}(L) .
\end{aligned}
$$

We now turn towards unbounded selfadjoint Fredholm operators and will consider the Riesz topology on $\mathcal{C} \mathcal{F}^{s a}(H)$ at first. Here the foregoing result concerning the uniqueness of spectral flow in the bounded case carries over to the unbounded case verbatim due to the following theorem which can be found in Le05]. 
4.4.2 Theorem. The natural inclusion of the pair

$$
j:\left(\mathcal{B F}^{s a}(H), G^{s a}(H)\right) \hookrightarrow\left(\mathcal{C F}^{s a}(H), G \mathcal{C}^{s a}(H)\right)
$$

is a homotopy equivalence if $\mathcal{C F}^{s a}(H)$ carries the Riesz topology.

In particular, $\mathcal{C} \mathcal{F}^{s a}$ with the Riesz topology consists of three path components

$$
\mathcal{C F}^{s a}(H)=\mathcal{C F}_{+}^{s a}(H) \cup \mathcal{C F}_{-}^{s a}(H) \cup \mathcal{C F}_{*}^{s a}(H),
$$

where $\mathcal{C F}_{+}^{\text {sa }}(H)$ and $\mathcal{C} \mathcal{F}_{-}^{s a}(H)$ are contractible and $\mathcal{C F}_{*}^{s a}(H)$ represents the odd $K$-theory. Now we turn to the general case of $\mathcal{C F}^{s a}(H)$ with the gap topology. We already stated the uniqueness theorem 4.3.7 proved by Lesch without using any information about $\pi_{1}\left(\mathcal{C F}^{s a}(H)\right)$. Moreover, the topology of $\mathcal{C F}^{s a}(H)$ is well understood by the following result due to Michael Joachim Jo03.

4.4.3 Theorem. Consider $\mathcal{C} \mathcal{F}(H)$ and $\mathcal{C F}^{s a}(H)$ with the gap topology. Then

- $\mathcal{C F}(H)$ represents $K$.

- $\mathcal{C F}^{s a}(H)$ represents $K^{1}$.

Note in particular that the whole space $\mathcal{C F}^{s a}(H)$ is connected in contrast to the spaces we have considered before. This was already observed before in a more elementary way in [BLP05, Theorem 1.10].

Finally we want to mention a recent construction due to Charlotte Wahl [W08], who defined a topology on $\mathcal{C F}^{s a}(H)$ that is weaker than the gap topology and proved that also the corresponding result 4.4 .3 remains true. However, all our following investigations base strictly on the gap topology and hence we will not introduce her results here. 


\section{Chapter 5}

\section{Fredholm Morphisms and the Index Bundle}

In section 4.1 we recalled the definition of the Atiyah-Jänich index bundle for families $L: X \rightarrow$ $\mathcal{B F}(E)$ which are continuous with respect to the norm topology and where $X$ is assumed to be compact. In this chapter we want to make a first important generalisation of this construction that allows to assign an index bundle to any Fredholm morphism $L: \mathcal{E} \rightarrow \mathcal{F}$ between Banach bundles $\mathcal{E}$ and $\mathcal{F}$ over the same compact base $X$. The result is an element of the $K$-theory of the base space $X$ and, as we will discuss below in section 5.2, it has the same basic properties as the classical Atiyah-Jänich bundle. Moreover, in the special case of families $L: X \rightarrow \mathcal{B F}(E)$ both constructions lead to the same K-theory class.

In the first section of this chapter we establish the index bundle for Fredholm morphisms where we assume in general that the base space of the underlying Banach bundles is compact. It turns out that the constructions are quite technical which is mainly due to the fact that $\mathcal{F}$ is just locally trivial. However, we consider also the special case that $\mathcal{F}$ is globally trivial and show that we can drop the compactness assumption on the base $X$ which will allow us in later chapters to consider Fredholm morphisms on Banach bundles over products $X \times \mathbb{R}$ where $X$ is again assumed to be compact. In the second section we elaborate the main properties of the index bundle. As already announced above the basic properties of the classical Atiyah-Jänich bundle carry over verbatim to the generalised case. But, due to the fact that we work on bundles instead of single spaces, we also obtain properties that are not available in the classical setting. In the final third section we use the results from the third chapter on the dimension of exceptional sets in order to estimate the dimension of the support of Banach bundle morphisms from below. 


\subsection{Construction of the Index Bundle}

If $\mathcal{E}$ and $\mathcal{F}$ are Banach bundles over the space $X$, we denote by $\mathfrak{F}_{k}(\mathcal{E}, \mathcal{F}), k \in \mathbb{Z}$, the space of morphisms between $\mathcal{E}$ and $\mathcal{F}$ that are bounded Fredholm operators of index $k$ in each fibre and by $\mathfrak{F}(\mathcal{E}, \mathcal{F})$ the union of all these spaces. As in the first chapter we will use the convention to denote the model spaces of $\mathcal{E}$ and $\mathcal{F}$ by $E$ and $F$, respectively.

5.1.1 Definition. The support of the morphism $L \in \mathcal{L}(\mathcal{E}, \mathcal{F})$ is defined by

$$
\operatorname{supp} L=\left\{\lambda \in X: L_{\lambda} \notin G L\left(\mathcal{E}_{\lambda}, \mathcal{F}_{\lambda}\right)\right\}
$$

As next step we prove that $\operatorname{supp} L$ actually is a closed subset of $X$.

5.1.2 Lemma. Let $L \in \mathcal{L}(\mathcal{E}, \mathcal{F})$ be a morphism between the Banach bundles $p: \mathcal{E} \rightarrow X$ and $q: \mathcal{F} \rightarrow X$. Then the set

$$
\left\{\lambda \in X: L_{\lambda} \in G L\left(\mathcal{E}_{\lambda}, \mathcal{F}_{\lambda}\right)\right\} \subset X
$$

is open.

Proof. At first we note that if $G L(E, F)$ is not empty, we have a Banach space isomorphism $\mathcal{L}(F) \rightarrow \mathcal{L}(E, F)$ which maps $G L(F)$ bijectively onto $G L(E, F)$. Hence we obtain that $G L(E, F)$ is open from the well known fact that $G L(F)$ is open for any Banach space $F$. Moreover, in order to prove the lemma we can assume without loss of generality that $\operatorname{supp} L \neq X$ which in particular implies that $G L(E, F) \neq \emptyset$.

Now, given $\lambda_{0} \notin \operatorname{supp} L$, we can choose an open neighbourhood $U$ of $\lambda_{0}$ and trivialisations $\varphi, \psi$ as in (1.1) such that

$$
U \mapsto \mathcal{L}(E, F), \quad \lambda \mapsto \psi_{\lambda} \circ L_{\lambda} \circ \varphi_{\lambda}^{-1}
$$

is continuous. Since $\psi_{\lambda_{0}} \circ L_{\lambda_{0}} \circ \varphi_{\lambda_{0}}^{-1} \in G L(E, F)$ and $G L(E, F)$ is open, we can find a neighbourhood $V \subset U$ of $\lambda_{0}$ such that $\psi_{\lambda} \circ L_{\lambda} \circ \varphi_{\lambda}^{-1} \in G L(E, F)$ and hence $L_{\lambda} \in G L\left(\mathcal{E}_{\lambda}, \mathcal{F}_{\lambda}\right)$ for all $\lambda \in V$.

Now we begin the construction of the index bundle and assume in the following that $X$ is a paracompact space unless otherwise stated. Let $L \in \mathfrak{F}(\mathcal{E}, \mathcal{F})$ be a Fredholm morphism and $\mathcal{V} \subset \mathcal{F}$ be a direct subbundle that is transversal to $\operatorname{im}(L)$ in the sense that

$$
\operatorname{im}\left(L_{\lambda}\right)+\mathcal{V}_{\lambda}=\mathcal{F}_{\lambda} \quad \text { for all } \lambda \in X
$$


Using proposition 1.2 .6 there exists a fibrewise projection $P \in \mathcal{L}(\mathcal{F})$ such that $\operatorname{im}(P)=\mathcal{V}$ and $\operatorname{im}(I-P)$ is a direct subbundle of $\mathcal{F}$ as well. By the property (5.1) of $\mathcal{V}$ we obtain a surjective bundle morphism

$$
\mathcal{E} \stackrel{L}{\rightarrow} \mathcal{F} \stackrel{I-P}{\longrightarrow} \operatorname{im}(I-P) .
$$

If moreover the kernels of this morphism are complemented subspaces of the corresponding fibres, then they build a direct subbundle $E(L, \mathcal{V})$ of $\mathcal{E}$ by corollary 1.2 .10 whose total space is given by

$$
\coprod_{\lambda \in X}\left\{u \in \mathcal{E}_{\lambda}: L_{\lambda} u \in \mathcal{V}_{\lambda}\right\}
$$

Note that this is in particular the case if we can choose a finite dimensional transversal bundle $\mathcal{V}$. The next theorem ensures that such bundles really exist already under quite moderate assumptions. It is the first of two technical results which we need in order to construct the index bundle.

5.1.3 Theorem. Let the base space $X$ be compact and $L \in \mathfrak{F}(\mathcal{E}, \mathcal{F})$ a Fredholm morphism. Then there exists a finite dimensional trivial subbundle $\mathcal{V} \subset \mathcal{F}$ such that 5.1) holds over $X$.

Proof. As a first step we show that the assertion holds if $\mathcal{F}$ is trivial.

Let $\psi$ be a global trivialisation of $\mathcal{F}$. Let $\lambda_{0} \in X$ and $U_{\lambda_{0}} \subset X$ be an open neighbourhood of $\lambda_{0}$ such that $\mathcal{E}$ is trivial on $U_{\lambda_{0}}$ by means of a trivialisation $\varphi$. Let

$$
\tilde{L}=p r_{2} \circ \psi \circ L \circ \varphi^{-1}: U_{\lambda_{0}} \times E \rightarrow F
$$

denote the corresponding family of bounded Fredholm operators with respect to these trivialisations. Since $\tilde{L}_{\lambda_{0}}$ is Fredholm, there exists $V_{\lambda_{0}} \subset F, \operatorname{dim} V_{\lambda_{0}}<\infty$, and $W_{\lambda_{0}} \subset E$ closed such that

$$
\operatorname{im}\left(\tilde{L}_{\lambda_{0}}\right) \oplus V_{\lambda_{0}}=F, \quad \operatorname{ker}\left(\tilde{L}_{\lambda_{0}}\right) \oplus W_{\lambda_{0}}=E
$$

Now consider

$$
A_{\lambda}: W_{\lambda_{0}} \times V_{\lambda_{0}} \rightarrow F, \quad A_{\lambda}(w, v)=\tilde{L}_{\lambda} w+v .
$$

Because of $A_{\lambda_{0}} \in G L\left(W_{\lambda_{0}} \times V_{\lambda_{0}}, F\right)$ and the continuity of $A: U_{\lambda_{0}} \rightarrow \mathcal{L}\left(W_{\lambda_{0}} \times V_{\lambda_{0}}, F\right)$, there exists a neighbourhood $\tilde{U}_{\lambda_{0}} \subset U_{\lambda_{0}}$ of $\lambda_{0}$ such that $A_{\lambda} \in G L\left(W_{\lambda_{0}} \times V_{\lambda_{0}}, F\right)$ and hence 


$$
\operatorname{im}\left(\tilde{L}_{\lambda}\right)+V_{\lambda_{0}}=F \quad \text { for all } \lambda \in \tilde{U}_{\lambda_{0}} .
$$

By compactness we can now cover $X$ by a finite number of neighbourhoods $\tilde{U}_{\lambda_{i}}, i=1, \ldots, n$, such that for each $i$ there exists a finite dimensional subspace $V_{\lambda_{i}}$ such that

$$
\operatorname{im}\left(p r_{2} \circ \psi_{\lambda} \circ L_{\lambda}\right)+V_{\lambda_{i}}=F \text { for all } \lambda \in \tilde{U}_{\lambda_{i}}, \quad i=1, \ldots, n .
$$

Finally, $V:=V_{1}+\ldots+V_{n}$ defines a finite dimensional subspace of $F$ such that

$$
\operatorname{im}\left(p r_{2} \circ \psi_{\lambda} \circ L_{\lambda}\right)+V=F \text { for all } \lambda \in X \text {. }
$$

Then $\psi^{-1}(X \times V)$ is a finite dimensional trivial subbundle of $\mathcal{F}$ such that (5.1) holds on all of $X$ and the assertion is proved in the special case that $\mathcal{F}$ is trivial.

We now turn to the general case. Let $U_{k}^{0}, k=1, \ldots, N$, be a finite open covering of $X$ such that $\mathcal{F}$ is trivial over each $U_{k}^{0}$. Moreover, we choose open sets $U_{k}^{i}, i=1,2, k=1, \ldots, N$, such that $\overline{U_{k}^{i+1}} \subset U_{k}^{i}, i=0,1$, and $\left\{U_{k}^{2}\right\}$ is still an open covering of $X$ (cf. [Br93, I.12.9]).

Consider $U_{1}^{0}$. Since $\mathcal{F}$ is trivial on the compact subspace $\overline{U_{1}^{1}}$, we obtain from the special case in which we proved the assertion already that there is a finite dimensional trivial subbundle $\mathcal{V}^{\prime}$ of $\mathcal{F}$ over $\overline{U_{1}^{1}}$ such that 5 .5.1 holds. We choose a complement $\mathcal{W}^{\prime}$ to $\mathcal{V}^{\prime}$ in $\left.\mathcal{F}\right|_{\overline{U_{1}^{1}}}$. By corollary 1.3 .6 there exist bundles $\mathcal{V}_{1}$ and $\mathcal{W}_{1}$ over $X$ such that $\mathcal{F}=\mathcal{V}_{1} \oplus \mathcal{W}_{1}, \mathcal{V}_{1}$ is finite dimensional, trivial and

$$
\left.\mathcal{V}_{1}\right|_{\overline{U_{1}^{2}}}=\left.\mathcal{V}^{\prime}\right|_{\overline{U_{1}^{2}}},\left.\quad \mathcal{W}_{1}\right|_{\overline{U_{1}^{2}}}=\left.\mathcal{W}^{\prime}\right|_{\overline{U_{1}^{2}}}
$$

Note that $\mathcal{V}_{1}$ is a trivial bundle over all of $X$ satisfying (5.1) on $U_{1}^{2}$. Moreover, since $\mathcal{F}$ is trivial on $\overline{U_{k}^{1}}, k=1, \ldots N$, we obtain by corollary 1.3 .13 that the bundle $\mathcal{W}_{1}$ is trivial on all $\overline{U_{k}^{1}}$ as well.

Next we consider $U_{2}^{0}$ and let $P \in \mathcal{L}(\mathcal{F})$ denote a projection onto $\mathcal{W}_{1}$. Consider $P \circ L: \mathcal{E} \rightarrow \mathcal{W}_{1}$ which is again a Fredholm morphism. Since $\mathcal{W}_{1}$ is trivial on $\overline{U_{2}^{1}}$ we can argue as on $U_{1}^{0}$ above and obtain a decomposition $\mathcal{W}_{1}=\mathcal{V}_{2} \oplus \mathcal{W}_{2}$ such that 5.1 holds for $P \circ L$ and $\mathcal{V}_{2}$ on $U_{2}^{2}$. Moreover, $\mathcal{V}_{2}$ is trivial on $X$ and $\mathcal{W}_{2}$ is trivial on each $\overline{U_{k}^{1}}, k=1, \ldots, N$. Finally, note that $\mathcal{V}_{1} \oplus \mathcal{V}_{2}$ is a subbundle of $\mathcal{F}$ which is transversal to $\operatorname{im} L$ over $U_{1}^{2} \cup U_{2}^{2}$.

Continuing this process we eventually arrive at a finite dimensional trivial subbundle $\mathcal{V}=\bigoplus_{i=1}^{N} \mathcal{V}_{i}$ of $\mathcal{F}$ over $X$ such that 5.1 holds over all of $X=\bigcup_{k=1}^{N} U_{k}^{2}$.

5.1.4 Corollary. Let $X$ be a general topological space and $L \in \mathfrak{F}(\mathcal{E}, \mathcal{F})$ a Fredholm morphism having a compact support where we assume the Banach bundle $\mathcal{F}$ to be trivial. Then there exists a finite dimensional trivial subbundle $\mathcal{V} \subset \mathcal{F}$ which is transversal to $\operatorname{im} L$ in the sense of (5.1). 
Proof. Arguing as in the first part of the proof of theorem 5.1.3, we can find a finite dimensional trivial subbundle $\mathcal{V}$ of $\mathcal{F}$ which is transversal to im $L$ over the compact subspace $\operatorname{supp} L$. But, since $L$ is an isomorphism outside $\operatorname{supp} L$, it is clear that $\mathcal{V}$ is actually transversal to $\operatorname{im} L$ on all of $X$.

By using the same argument as in the foregoing proof of corollary 5.1.4 in the special case that $\mathcal{F}$ is a product bundle, we obtain the following important observation.

5.1.5 Corollary. If under the assumptions of corollary 5.1.4 the bundle $\mathcal{F}$ is a product $X \times F$ for some Banach space $F$, then there exists a finite dimensional subspace $V \subset F$ such that $X \times V$ is transversal to $\operatorname{im} L$.

Before going on in the definition of the index bundle, we note the following result on the dimension of the bundle $E(L, \mathcal{V})$.

5.1.6 Lemma. Let $L \in \mathfrak{F}(\mathcal{E}, \mathcal{F})$ be a Fredholm morphism between the Banach bundles $\mathcal{E}$ and $\mathcal{F}$ and $\mathcal{V}$ be a finite dimensional subbundle of $\mathcal{F}$ which is transversal to the image of $L$. Then

$$
\operatorname{dim} E(L, \mathcal{V})=\operatorname{ind}(L)+\operatorname{dim} \mathcal{V} .
$$

Proof. Consider $L_{\lambda}$ for some $\lambda \in X$ as a map between the finite dimensional vector spaces $E(L, \mathcal{V})_{\lambda}=L_{\lambda}^{-1}\left(\mathcal{V}_{\lambda}\right)$ and $\mathcal{V}_{\lambda}$. We obtain

$$
\begin{aligned}
\operatorname{dim} E(L, \mathcal{V})_{\lambda} & =\operatorname{dim} \operatorname{ker}\left(\left.L_{\lambda}\right|_{E(L, \mathcal{V})_{\lambda}}\right)+\operatorname{dim} \operatorname{im}\left(\left.L_{\lambda}\right|_{E(L, \mathcal{V})_{\lambda}}\right) \\
& =\operatorname{dim} \operatorname{ker} L_{\lambda}+\operatorname{dim}\left(\operatorname{im} L_{\lambda} \cap V_{\lambda}\right) \\
& =\operatorname{ind} L_{\lambda}+\operatorname{dim} \operatorname{coker} L_{\lambda}+\operatorname{dim}\left(\operatorname{im} L_{\lambda} \cap \mathcal{V}_{\lambda}\right) \\
& =\operatorname{ind} L_{\lambda}+\operatorname{dim} \operatorname{coker} L_{\lambda}+\operatorname{dim} \mathcal{V}_{\lambda}-\operatorname{dim} \operatorname{coker} L_{\lambda} \\
& =\operatorname{ind} L_{\lambda}+\operatorname{dim} \mathcal{V}_{\lambda} .
\end{aligned}
$$

If $L \in \mathfrak{F}(\mathcal{E}, \mathcal{F})$ is a Fredholm morphism and $\mathcal{V}$ a finite dimensional subbundle of $\mathcal{F}$ which is transversal to im $L$ as in $(5.1)$, then $E(L, \mathcal{V})$ is by construction a subbundle of $\mathcal{E}$ and hence the restriction of $L$ defines a bundle morphism

$$
\left.L\right|_{E(L, \mathcal{V})}: E(L, \mathcal{V}) \rightarrow \mathcal{V}
$$

which is actually an isomorphism outside the subspace $\operatorname{supp} L \subset X$. Hence, if we assume that $X$ is in addition locally compact, $L$ has a compact support and $A \subset X$ is a closed subspace such that $A \cap \operatorname{supp} L=\emptyset$, we obtain a $K$-theory class 


$$
\left[E(L, \mathcal{V}), \mathcal{V},\left.L\right|_{E(L, \mathcal{V})}\right] \in K(X, A)
$$

Our next aim is to show that this element is independent of the choice of the bundle $\mathcal{V}$. Before we need the following lemma.

5.1.7 Lemma. Let $\mathcal{E}, \mathcal{F}$ be Banach bundles over $X, L \in \mathfrak{F}(\mathcal{E}, \mathcal{F})$ a Fredholm morphism and $\mathcal{V} \subset \mathcal{F}$ a finite dimensional subbundle such that 5.1 holds. Moreover, let $Y$ be a further paracompact space and $f: Y \rightarrow X$ a continuous map. Then $f^{*} \mathcal{V}$ is transversal to the image of the pullback morphism $f^{*} L: f^{*} \mathcal{E} \rightarrow f^{*} \mathcal{F}$ and we have

$$
E\left(f^{*} L, f^{*} \mathcal{V}\right)=f^{*} E(L, \mathcal{V})
$$

Proof. By the definition of the pullback it is clear that

$$
\operatorname{im}\left(\left(f^{*} L\right)_{\lambda}\right)+\left(f^{*} \mathcal{V}\right)_{\lambda}=\left(f^{*} \mathcal{F}\right)_{\lambda} \quad \text { for all } \lambda \in X
$$

Moreover,

$$
\left\{u \in\left(f^{*} \mathcal{E}\right)_{\lambda}:\left(f^{*} L\right)_{\lambda} u \in\left(f^{*} \mathcal{V}\right)_{\lambda}\right\}=\left\{u \in \mathcal{E}_{f(\lambda)}: L_{f(\lambda)} u \in \mathcal{V}_{f(\lambda)}\right\}, \quad \lambda \in Y,
$$

and hence the total spaces of $E\left(f^{*} L, f^{*} \mathcal{V}\right)$ and $f^{*} E(L, \mathcal{V})$ coincide as sets. Since both bundles are subbundles of $f^{*} \mathcal{E}$ this implies that they actually coincide as bundles.

From the foregoing lemma, we obtain immediately the following corollary.

5.1.8 Corollary. Let $A \subset X$ be a closed subspace of the paracompact and locally compact space $X$ and $L \in \mathfrak{F}(\mathcal{E}, \mathcal{F})$ a Fredholm morphism with compact support such that $A \cap \operatorname{supp} L=\emptyset$. If $\mathcal{V}$ is a finite dimensional subbundle of $\mathcal{F}$ which is transversal to $\operatorname{im} L$ and $f:(Y, B) \rightarrow(X, A)$ is proper, then

$$
\left[E\left(f^{*} L, f^{*} \mathcal{V}\right), f^{*} \mathcal{V},\left.f^{*} L\right|_{E\left(f^{*} L, f^{*} \mathcal{V}\right)}\right]=f^{*}\left[E(L, \mathcal{V}), \mathcal{V},\left.L\right|_{E(L, \mathcal{V})}\right] \in K(X, A)
$$

Now we can prove that the element $\left[E(L, \mathcal{V}), \mathcal{V},\left.L\right|_{E(L, \mathcal{V})}\right] \in K(X, A)$ does not depend on the particular choice of a finite dimensional subbundle of $\mathcal{F}$ which is transversal to im $L$. 
5.1.9 Theorem. Let $L \in \mathfrak{F}(\mathcal{E}, \mathcal{F})$ be a Fredholm morphism with compact support acting between the Banach bundles $\mathcal{E}$ and $\mathcal{F}$ and $A \subset X$ a closed subspace such that $A \cap \operatorname{supp} L=\emptyset$. We assume that

- either $X$ is compact, or

- $X$ is locally compact and paracompact and $\mathcal{F}$ a trivial bundle.

If $\mathcal{V}, \mathcal{W} \subset \mathcal{F}$ are two finite dimensional subbundles of finite type that are transversal to the image of $L$ in the sense of (5.1), then

$$
\left[E(L, \mathcal{V}), \mathcal{V},\left.L\right|_{E(L, \mathcal{V})}\right]=\left[E(L, \mathcal{W}), \mathcal{W},\left.L\right|_{E(L, \mathcal{W})}\right] \in K(X, A)
$$

\section{Proof. Step 1}

In a first step we consider the case that $\mathcal{V} \subset \mathcal{W}$ is a subbundle.

Then $E(L, \mathcal{V}) \subset E(L, \mathcal{W})$ is a subbundle as well and hence, using the paracompactness of the base space, we can find a complement bundle $E(L, \mathcal{V})^{\perp}$, such that

$$
E(L, \mathcal{W})=E(L, \mathcal{V}) \oplus E(L, \mathcal{V})^{\perp}
$$

Since $\left.L\right|_{E(L, \mathcal{V})^{\perp}}$ is injective by the definition of $E(L, \mathcal{V})$, we conclude that $L\left(E(L, \mathcal{V})^{\perp}\right) \subset \mathcal{W}$ is a subbundle and the restriction of $L$ defines a bundle isomorphism

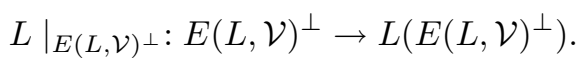

Moreover, we have $\mathcal{V}_{\lambda} \oplus L\left(E(L, \mathcal{V})^{\perp}\right)_{\lambda}=\mathcal{W}_{\lambda}$ for all $\lambda \in X$. Indeed, if $v \in \mathcal{V}_{\lambda} \cap L\left(E(L, \mathcal{V})_{\lambda}^{\perp}\right)$, then there exists $u \in E(L, \mathcal{V})_{\lambda}^{\perp} \subset E(L, \mathcal{W})_{\lambda}$ such that $L_{\lambda} u=v$. Since $v \in \mathcal{V}_{\lambda}$ we infer $u \in$ $E(L, \mathcal{V})_{\lambda} \cap E(L, \mathcal{V})_{\lambda}^{\perp}=\{0\}$ and hence $v=0$. Moreover, we obtain from lemma 5.1.6 that

$$
\begin{aligned}
\operatorname{dim} \mathcal{W}-\operatorname{dim} \mathcal{V} & =\operatorname{dim} E(L, \mathcal{W})-\operatorname{dim} E(L, \mathcal{V})=\operatorname{dim} E(L, \mathcal{V})^{\perp} \\
& =\operatorname{dim} L\left(E(L, \mathcal{V})^{\perp}\right)
\end{aligned}
$$

Now we have a commutative diagram

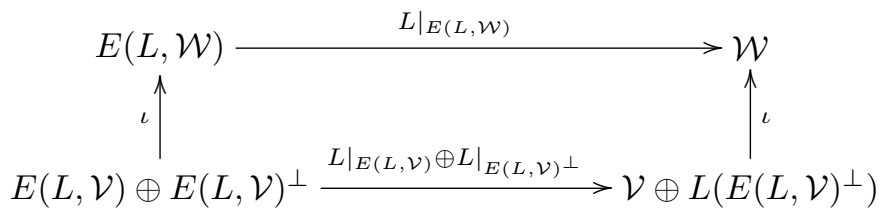


where $\iota$ denotes the canonical isomorphism. Hence we obtain

$$
\begin{aligned}
{\left[E(L, \mathcal{W}), \mathcal{W},\left.L\right|_{E(L, \mathcal{W})}\right] } & =\left[E(L, \mathcal{V}) \oplus E(L, \mathcal{V})^{\perp}, \mathcal{V} \oplus L\left(E(L, \mathcal{V})^{\perp}\right),\left.L\right|_{E(L, \mathcal{V}) \oplus E(L, \mathcal{V})^{\perp}}\right] \\
& =\left[E(L, \mathcal{V}), \mathcal{V},\left.L\right|_{E(L, \mathcal{V})}\right]
\end{aligned}
$$

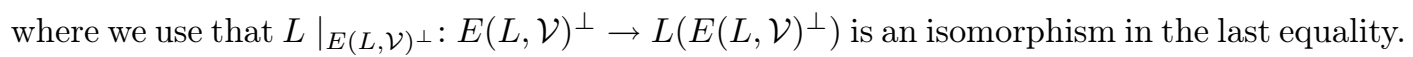

\section{Step 2}

In the second step of the proof we now turn to the general case and consider two finite dimensional bundles $\mathcal{V}, \mathcal{W}$ of finite type as in the assertion. Our aim is to use the special case we already proved above in order to show that we can assume without loss of generality that $\mathcal{V}$ and $\mathcal{W}$ are trivial and of the same dimension. In order to do so, note at first that $\mathcal{V}$ and $\mathcal{W}$ are contained in trivial finite dimensional subbundles of $\mathcal{E}$ by corollary 1.3.5. Hence by the special case proved above, we can assume without loss of generality that $\mathcal{V}$ and $\mathcal{W}$ are trivial. If now $\operatorname{dim} \mathcal{V}=\operatorname{dim} \mathcal{W}$, we are done. If, however, they are not of the same dimension, say $\operatorname{dim} \mathcal{V}<\operatorname{dim} \mathcal{W}$, we can choose a subbundle $\mathcal{M}$ of $\mathcal{E}$ which is complementary to $\mathcal{V}$ in $\mathcal{E}$ by corollary 1.2.8. According to corollary 1.3.5 we now can find a $(\operatorname{dim} \mathcal{W}-\operatorname{dim} \mathcal{V})$-dimensional trivial subbundle of $\mathcal{M}$ and the direct sum of this bundle and $\mathcal{V}$ yields a trivial subbundle of $\mathcal{E}$ of the same dimension than $\mathcal{W}$. By using the first step of our proof once again, we finally obtain that it suffices to prove the assertion of the theorem under the additional assumption that $\mathcal{V}$ and $\mathcal{W}$ are trivial and of the same dimension.

\section{Step 3}

By corollary 1.3.9 $\mathcal{V}$ and $\mathcal{W}$ are homotopic and hence there exists a finite dimensional subbundle $\mathcal{M}$ of $\pi^{*} \mathcal{F}$ such that $\left.\mathcal{M}\right|_{X \times\{0\}}=\mathcal{V}$ and $\left.\mathcal{M}\right|_{X \times\{1\}}=\mathcal{W}$. Moreover, $\mathcal{M}$ is trivial by [Hu94, 3.4.4] since its restriction to $X \times\{0\}$ is trivial. Consider the bundle morphism $\pi^{*} L: \pi^{*} \mathcal{E} \rightarrow \pi^{*} \mathcal{F}$. Our next aim is to extend $\mathcal{M}$ to a larger bundle over $X \times I$ which is transversal to $\operatorname{im}\left(\pi^{*} L\right)$ over $X \times I$. Since we have to argue differently for both kinds of base spaces we consider, we split the proof at this point.

\section{Step 3 a): $X$ compact}

Consider $P \pi^{*} L: \pi^{*} \mathcal{E} \rightarrow \mathcal{M}^{\prime}$, where $P: \pi^{*} \mathcal{F} \rightarrow \mathcal{M}^{\prime}$ denotes a projection onto a complement $\mathcal{M}^{\prime}$ of $\mathcal{M}$ in $\pi^{*} \mathcal{F}$ which exists according to corollary 1.2.8. $P \pi^{*} L$ is a Fredholm morphism and by theorem 5.1.3, we can find a finite dimensional subbundle $\mathcal{M}^{\prime \prime}$ of $\mathcal{M}^{\prime}$ which is transversal to $\operatorname{im}\left(P \pi^{*} L: \pi^{*} \mathcal{E} \rightarrow \mathcal{M}^{\prime}\right)$ over $X \times I$. Taking the direct sum of $\mathcal{M}^{\prime \prime}$ and $\mathcal{M}$ we finally obtain a finite dimensional subbundle of $\pi^{*} \mathcal{F}$ that is transversal to $\operatorname{im}\left(\pi^{*} L: \pi^{*} \mathcal{E} \rightarrow \pi^{*} \mathcal{F}\right)$ and contains $\mathcal{M}$ as a subbundle. 


\section{Step 3 b): $X$ non compact but $\mathcal{F}$ trivial}

We consider again $P \pi^{*} L: \pi^{*} \mathcal{E} \rightarrow \mathcal{M}^{\prime}$, where $P: \pi^{*} \mathcal{F} \rightarrow \mathcal{M}^{\prime}$ is as in step 3 a). Now $P \pi^{*} L$ is a Fredholm morphism but its support is non compact unless $X$ is compact. But since $\pi^{*} \mathcal{F}$ and $\mathcal{M}$ are trivial, $\mathcal{M}^{\prime}$ is trivial as well according to corollary 1.3.13. Now we can argue as in the first part of the proof of theorem 5.1.3 and obtain a finite dimensional trivial subbundle $\mathcal{M}^{\prime \prime}$ of $\mathcal{M}^{\prime}$ that is transversal to im $P \pi^{*} L$ over the compact set $(\operatorname{supp} L) \times I \subset X \times I$. But since $P \pi^{*} L$ is surjective outside $(\operatorname{supp} L) \times I$, we infer that $\mathcal{M} \oplus \mathcal{M}^{\prime \prime}$ is transversal to im $\pi^{*} L$ over all of $X \times I$.

\section{Step 4}

We denote by $\iota_{0}: X \hookrightarrow X \times\{0\} \subset X \times I$ and $\iota_{1}: X \hookrightarrow X \times\{1\} \subset X \times I$ the inclusions and note that

$$
\iota_{0}^{*}=\iota_{1}^{*}: K(X \times I, A \times I) \rightarrow K(X, A) .
$$

Using corollary 5.1 .8 and once again the first step of our proof, we finally obtain

$$
\begin{aligned}
{\left[E(L, \mathcal{V}), \mathcal{V},\left.L\right|_{E(L, \mathcal{V})}\right] } & =\left[E\left(L, \iota_{0}^{*}\left(\mathcal{M} \oplus \mathcal{M}^{\prime \prime}\right)\right), \iota_{0}^{*}\left(\mathcal{M} \oplus \mathcal{M}^{\prime \prime}\right),\left.L\right|_{E\left(L, \iota_{0}^{*}\left(\mathcal{M} \oplus \mathcal{M}^{\prime \prime}\right)\right)}\right] \\
& =\left[E\left(\iota_{0}^{*} \pi^{*} L, \iota_{0}^{*}\left(\mathcal{M} \oplus \mathcal{M}^{\prime \prime}\right)\right), \iota_{0}^{*}\left(\mathcal{M} \oplus \mathcal{M}^{\prime \prime}\right),\left.\iota_{0}^{*} \pi^{*} L\right|_{E\left(\iota_{0}^{*} \pi^{*} L, \iota_{0}^{*}\left(\mathcal{M} \oplus \mathcal{M}^{\prime \prime}\right)\right)}\right] \\
& =\iota_{0}^{*}\left[E\left(\pi^{*} L, \mathcal{M} \oplus \mathcal{M}^{\prime \prime}\right), \mathcal{M} \oplus \mathcal{M}^{\prime \prime},\left.\pi^{*} L\right|_{E\left(\pi^{*} L, \mathcal{M} \oplus \mathcal{M}^{\prime \prime}\right)}\right] \\
& =\iota_{1}^{*}\left[E\left(\pi^{*} L, \mathcal{M} \oplus \mathcal{M}^{\prime \prime}\right), \mathcal{M} \oplus \mathcal{M}^{\prime \prime},\left.\pi^{*} L\right|_{E\left(\pi^{*} L, \mathcal{M} \oplus \mathcal{M}^{\prime \prime}\right)}\right] \\
& =\left[E\left(\iota_{1}^{*} \pi^{*} L, \iota_{1}^{*}\left(\mathcal{M} \oplus \mathcal{M}^{\prime \prime}\right)\right), \iota_{1}^{*}\left(\mathcal{M} \oplus \mathcal{M}^{\prime \prime}\right),\left.\iota_{1}^{*} \pi^{*} L\right|_{E\left(\iota_{1}^{*} \pi^{*} L, \iota_{1}^{*}\left(\mathcal{M} \oplus \mathcal{M}^{\prime \prime}\right)\right)}\right] \\
& =\left[E\left(L, \iota_{1}^{*}\left(\mathcal{M} \oplus \mathcal{M}^{\prime \prime}\right)\right), \iota_{1}^{*}\left(\mathcal{M} \oplus \mathcal{M}^{\prime \prime}\right),\left.L\right|_{E\left(L, \iota_{1}^{*}\left(\mathcal{M} \oplus \mathcal{M}^{\prime \prime}\right)\right)}\right] \\
& =\left[E(L, \mathcal{W}), \mathcal{W},\left.L\right|_{E(L, \mathcal{W})}\right]
\end{aligned}
$$

Because of theorem 5.1 .3 we now can define the index bundle over compact base spaces as follows:

5.1.10 Definition. Let $L \in \mathfrak{F}(\mathcal{E}, \mathcal{F})$ be a Fredholm morphism between the Banach bundles $\mathcal{E}$ and $\mathcal{F}$ over the compact base $X$ and $A \subset X$ a closed subspace such that $A \cap \operatorname{supp} L=\emptyset$. We call the element

$$
\operatorname{ind}(L)=\left[E(L, \mathcal{V}), \mathcal{V},\left.L\right|_{E(L, \mathcal{V})}\right] \in K(X, A)
$$

the index bundle of $L$ with respect to $A$, where $\mathcal{V} \subset \mathcal{F}$ is any finite dimensional subbundle such that (5.1) holds. 
5.1.11 Remark. Note that $\operatorname{supp} L=X$ if $L \in \mathfrak{F}_{k}(\mathcal{E}, \mathcal{F}), k \neq 0$. Hence the case $A \neq \emptyset$ is only of interest if $L \in \mathfrak{F}_{0}(\mathcal{E}, \mathcal{F})$.

Finally, in the special case that $\mathcal{F}$ is a trivial bundle, we can extend the definition of the index bundle to more general base spaces as follows.

5.1.12 Definition. Let $X$ be locally compact and paracompact, $A \subset X$ closed, $\mathcal{F}$ a trivial bundle and $L \in \mathfrak{F}_{0}(\mathcal{E}, \mathcal{F})$ a Fredholm morphism of index 0 having a compact support such that $A \cap \operatorname{supp} L=\emptyset$. Then the index bundle of $L$ with respect to $A$ is defined by

$$
\operatorname{ind}(L)=\left[E(L, \mathcal{V}), \mathcal{V},\left.L\right|_{E(L, \mathcal{V})}\right] \in K(X, A),
$$

where $\mathcal{V} \subset \mathcal{F}$ is any finite dimensional subbundle of finite type which is transversal to im $L$ in the sense of 5.1.

5.1.13 Remark. Since $\operatorname{supp} L=X$ for any $L \in \mathfrak{F}_{k}(\mathcal{E}, \mathcal{F}), k \neq 0$, we restrict to $\mathfrak{F}_{0}(\mathcal{E}, \mathcal{F})$ in definition 5.1.12.

\subsection{Main Properties}

In this section we want to discuss the basic properties of the index bundle as defined by 5.1 .10 and 5.1.12, respectively. Besides the basic properties of the classical Atiyah-Jänich bundle we mentioned in section 4.1 and which we carry over here to the case of Fredholm morphisms between Banach bundles, we also obtain results that are only possible because we work with bundles instead of fixed spaces.

In the following we consider both definitions 5.1 .10 and 5.1.12 in parallel. Accordingly, we assume unless otherwise stated that $X$ is a locally compact and paracompact topological space and $A \subset X$ a closed subspace. Moreover, we suppose that $\mathcal{E}$ and $\mathcal{F}$ are general Banach bundles over $X$ and that $L \in \mathfrak{F}_{k}(\mathcal{E}, \mathcal{F})$ is a Fredholm morphism of index $k$ having a compact support which has a trivial intersection with $A$. However, we implicitly assume throughout that $\mathcal{F}$ is trivial and $k=0$ if $X$ is not compact. On the other hand, remember that $A=\emptyset$ necessarily if $X$ is compact and $k \neq 0$.

5.2.1 Lemma (Normalisation). Let $L \in \mathfrak{F}_{0}(\mathcal{E}, \mathcal{F})$ be a bundle isomorphism, that is $\operatorname{supp} L=\emptyset$. Then $\operatorname{ind}(L)=0 \in K(X, A)$.

Proof. Just observe that the image of the zero section in $\mathcal{F}$ satisfies 5.1 .

5.2.2 Lemma (Naturality). Let $f:(Y, B) \rightarrow(X, A)$ be proper, where $Y$ is a locally compact and paracompact space and $B \subset Y$ a closed subspace. Then

$$
\operatorname{ind}\left(f^{*} L\right)=f^{*} \operatorname{ind}(L) \in K(Y, B) .
$$


Proof. This is an immediate consequence of corollary 5.1.8.

We obtain as immediate consequence of the foregoing lemma and B.1.2 that $\operatorname{ind}\left(f^{*} L\right)=$ $\operatorname{ind}\left(g^{*} L\right) \in K(Y, B)$ if $f \simeq g:(Y, B) \rightarrow(X, A)$.

5.2.3 Lemma (Homotopy Invariance Property). Let $\mathcal{E}$ and $\mathcal{F}$ be Banach bundles over $X \times I$ and $L \in \mathfrak{F}(\mathcal{E}, \mathcal{F})$ a Fredholm morphism having compact support such that $(A \times I) \cap \operatorname{supp} L=\emptyset$. Moreover, denote by

$$
\begin{aligned}
& \iota_{0}:(X, A) \hookrightarrow(X \times\{0\}, A \times\{0\}) \subset(X \times I, A \times I), \\
& \iota_{1}:(X, A) \hookrightarrow(X \times\{1\}, A \times\{1\}) \subset(X \times I, A \times I)
\end{aligned}
$$

the canonical inclusions. Then

$$
\operatorname{ind}\left(i_{0}^{*} L\right)=\operatorname{ind}\left(i_{1}^{*} L\right) \in K(X, A) .
$$

Proof. Since $\iota_{0}$ and $\iota_{1}$ are homotopic by a proper homotopy, we obtain by using lemma 5.2 .2 and the homotopy invariance of $K$

$$
\operatorname{ind}\left(\iota_{0}^{*} L\right)=\iota_{0}^{*} \operatorname{ind}(L)=\iota_{1}^{*} \operatorname{ind}(L)=\operatorname{ind}\left(\iota_{1}^{*} L\right) \in K(X, A)
$$

We mention the following special case as a corollary.

5.2.4 Corollary. Let $L: \pi^{*} \mathcal{E} \rightarrow \pi^{*} \mathcal{F}$ be a Fredholm morphism with compact support such that $(A \times I) \cap \operatorname{supp} L=\emptyset$, where $\pi: X \times I \rightarrow X$ denotes as before the projection onto the first component. Then

$$
\operatorname{ind}\left(\iota_{0}^{*} L\right)=\operatorname{ind}\left(\iota_{1}^{*} L\right) \in K(X, A),
$$

where $\iota_{0}, \iota_{1}$ denote the canonical inclusions as in lemma 5.2.3

5.2.5 Corollary (Invariance under Compact Perturbations). Let $K \in \mathcal{L}(\mathcal{E}, \mathcal{F})$ be a compact operator in any fibre such that $L+t K \in \mathfrak{F}_{k}(\mathcal{E}, \mathcal{F})$ has compact support having trivial intersection with $A$ for all $t \in[0,1]$. Then

$$
\operatorname{ind}(L+K)=\operatorname{ind}(L) \in K(X, A) .
$$

Proof. Consider the Fredholm morphism $H: \pi^{*} \mathcal{E} \rightarrow \pi^{*} \mathcal{F}$ over $X \times I$ defined by $H(t, \lambda)=$ $L_{\lambda}+t \cdot K_{\lambda}$ and use homotopy invariance. 
5.2.6 Lemma (Direct Sum Property). Let $M \in \mathfrak{F}_{l}(\tilde{\mathcal{E}}, \tilde{\mathcal{F}})$ be a further Fredholm morphism with compact support between Banach bundles $\tilde{\mathcal{E}}$ and $\tilde{\mathcal{F}}$ over $X$ such that $A \cap \operatorname{supp} M=\emptyset$. Then

$$
\operatorname{ind}(L \oplus M)=\operatorname{ind}(L)+\operatorname{ind}(M) \in K(X, A) .
$$

Proof. Choose subbundles $\tilde{\mathcal{V}} \subset \mathcal{F}, \tilde{\mathcal{W}} \subset \tilde{\mathcal{F}}$ that are transversal to the images of the corresponding morphisms. Then

$$
\operatorname{im}\left(L_{\lambda} \oplus M_{\lambda}\right)+\tilde{\mathcal{V}}_{\lambda} \oplus \tilde{\mathcal{W}}_{\lambda}=\mathcal{F}_{\lambda} \oplus \tilde{\mathcal{F}}_{\lambda} \quad \text { for all } \lambda \in X
$$

and therefore

$$
\begin{aligned}
\operatorname{ind}(L \oplus M) & =[E(L \oplus M, \tilde{\mathcal{V}} \oplus \tilde{\mathcal{W}}), \tilde{\mathcal{V}} \oplus \tilde{\mathcal{W}}, L \oplus M] \\
& =[E(L, \tilde{\mathcal{V}}) \oplus E(M, \tilde{\mathcal{W}}), \tilde{\mathcal{V}} \oplus \tilde{\mathcal{W}}, L \oplus M] \\
& =[E(L, \tilde{\mathcal{V}}), \tilde{\mathcal{V}}, L] \oplus[E(M, \tilde{\mathcal{W}}), \tilde{\mathcal{W}}, M] \\
& =\operatorname{ind}(L)+\operatorname{ind}(M)
\end{aligned}
$$

5.2.7 Lemma (Logarithmic Property). Let $\mathcal{G}$ be a Banach bundle over $X$ and $M \in \mathfrak{F}(\mathcal{F}, \mathcal{G})$ a further Fredholm morphism whose compact support has a trivial intersection with $A$. Then

$$
\operatorname{ind}(M \circ L)=\operatorname{ind}(M)+\operatorname{ind}(L) \in K(X, A) .
$$

Proof. Let $\mathcal{W} \subset \mathcal{G}$ be a subbundle of finite type which is transversal to $\operatorname{im}(M \circ L)$. Then $\mathcal{W}$ is transversal to $\operatorname{im}(M)$ as well and hence $E(M, \mathcal{W})$ is defined. Our first aim is to show that $E(M, \mathcal{W})$ is transversal to $\operatorname{im}(L)$.

In order to do so, let $u \in \mathcal{F}_{\lambda}$ for some $\lambda \in X$. Then $M_{\lambda} u \in \mathcal{G}_{\lambda}$ and hence we can find $w_{0} \in \operatorname{im}\left(M_{\lambda} L_{\lambda}\right)$ and $w_{1} \in \mathcal{W}_{\lambda}$ such that $M_{\lambda} u=w_{0}+w_{1}$. Now we choose $u_{0} \in \operatorname{im}\left(L_{\lambda}\right)$ such that $M_{\lambda} u_{0}=w_{0}$ and set $u_{1}=u-u_{0} \in \mathcal{F}_{\lambda}$. Then $M_{\lambda} u_{1}=w_{1} \in \mathcal{W}_{\lambda}$ and hence $u=u_{0}+u_{1}$ where $u_{0} \in \operatorname{im}\left(L_{\lambda}\right)$ and $u_{1} \in M_{\lambda}^{-1}\left(\mathcal{W}_{\lambda}\right)$. Thus $\operatorname{im}\left(L_{\lambda}\right)+M_{\lambda}^{-1}\left(\mathcal{W}_{\lambda}\right)=\mathcal{F}_{\lambda}, \lambda \in X$, which proves that $E(M, \mathcal{W})$ is transversal to $\operatorname{im}(L)$.

Next we observe that

$$
\begin{aligned}
E(M \circ L, \mathcal{W})_{\lambda} & =\left\{u \in \mathcal{E}_{\lambda}: M_{\lambda} L_{\lambda} u \in \mathcal{W}_{\lambda}\right\}=\left\{u \in \mathcal{E}_{\lambda}: L_{\lambda} u \in M_{\lambda}^{-1}\left(\mathcal{W}_{\lambda}\right)\right\} \\
& =\left\{u \in \mathcal{E}_{\lambda}: L_{\lambda} u \in E(M, \mathcal{W})_{\lambda}\right\}=E(L, E(M, \mathcal{W}))_{\lambda}, \quad \lambda \in X,
\end{aligned}
$$

and hence 


$$
E(M \circ L, \mathcal{W})=E(L, E(M, \mathcal{W})) .
$$

Now the bundle morphism $L: E(M \circ L, \mathcal{W}) \rightarrow \mathcal{W}$ factorises as

$$
E(L, E(M, \mathcal{W})) \stackrel{L}{\rightarrow} E(M, \mathcal{W}) \stackrel{M}{\longrightarrow} \mathcal{W}
$$

and we obtain by using the logarithmic property B.1.5 of $K$

$$
\begin{aligned}
\operatorname{ind}(M \circ L) & =[E(M \circ L, \mathcal{W}), \mathcal{W}, M \circ L] \\
& =[E(L, E(M, \mathcal{W})), E(M, \mathcal{W}), L]+[E(M, \mathcal{W}), \mathcal{W}, M] \\
& =\operatorname{ind}(L)+\operatorname{ind}(M),
\end{aligned}
$$

where we have used theorem 5.1 .9 in the last equality. Note that indeed $E(M, \mathcal{W})$ is of finite type, since $\mathcal{W}$ is of finite type and $M$ is an isomorphism between $E(M, \mathcal{W})$ and $\mathcal{W}$ outside the compact subset $\operatorname{supp} M \subset X$.

Note that if $X$ is not compact in the foregoing lemma, we require $\mathcal{F}$ and $\mathcal{G}$ to be trivial.

5.2.8 Corollary. Let $L \in \mathfrak{F}_{0}(\mathcal{E}, \mathcal{F})$ be a Fredholm morphism with compact support such that $A \cap \operatorname{supp} L=\emptyset$ where we assume $\mathcal{F}$ to be trivial. Moreover, let $\mathcal{G}$ be a further Banach bundle which we assume not to be trivial and let $M: \mathcal{G} \rightarrow \mathcal{E}$ be a Banach bundle isomorphism. Then

$$
\operatorname{ind}(L \circ M)=\operatorname{ind}(L) \in K(X, A) .
$$

Proof. If $\mathcal{W} \subset \mathcal{F}$ is of finite type and transversal to $\operatorname{im}(L \circ M)$, we obtain verbatim as in the proof of lemma 5.2 .7

$$
\begin{aligned}
\operatorname{ind}(L \circ M) & =[E(L \circ M, \mathcal{W}), \mathcal{W}, L \circ M] \\
& =[E(M, E(L, \mathcal{W})), E(L, \mathcal{W}), M]+[E(L, \mathcal{W}), \mathcal{W}, L] \\
& =[E(M, E(L, \mathcal{W})), E(L, \mathcal{W}), M]+\operatorname{ind} L=\operatorname{ind} L,
\end{aligned}
$$

where we use that $M$ is an isomorphism in the last equality.

The following reduction property is in particular not available in this general form in the classical definition of the index bundle for families of operators acting between fixed Banach spaces. 
5.2.9 Lemma. Let $\mathcal{V}$ be a complemented subbundle of $\mathcal{F}$ which is transversal to $\operatorname{im}(L)$ in the sense of (5.1) and such that $L_{\lambda}^{-1}\left(\mathcal{V}_{\lambda}\right)$ is a complemented subspace of $\mathcal{E}_{\lambda}$ for all $\lambda \in X$. Then $\tilde{L}:=\left.L\right|_{E(L, \mathcal{V})}$ defines an element of $\mathfrak{F}(E(L, \mathcal{V}), \mathcal{V})$ with compact support and

$$
\operatorname{ind}(L)=\operatorname{ind}(\tilde{L}) \in K(X, A)
$$

Proof. Note at first that under the given assumptions $E(L, \mathcal{V})$ is indeed defined by our construction in section 5.1 .

Since $E(L, \mathcal{V})$ is a subbundle of $\mathcal{E}$ and $\mathcal{V}$ is a subbundle of $\mathcal{F}$, the restriction of $L$ is a bundle morphism. Hence in order to show the first assertion we just have to prove that $\tilde{L}$ is a Fredholm operator in each fibre which follows immediately from $\operatorname{ker}\left(L_{\lambda}\right)=\operatorname{ker}\left(\tilde{L}_{\lambda}\right)$ and $\operatorname{im}\left(\tilde{L}_{\lambda}\right)=\operatorname{im}\left(L_{\lambda}\right) \cap \mathcal{V}_{\lambda}$ for all $\lambda \in X$.

To prove the second assertion, let $\mathcal{W} \subset \mathcal{V}$ be a finite dimensional subbundle which is transversal to the image of $\tilde{L}$. Then $\mathcal{W}$ is transversal to the image of $L$ as well. Moreover, since $\mathcal{W} \subset \mathcal{V}$, we deduce that $E(L, \mathcal{W})=E(\tilde{L}, \mathcal{W})$ and the restrictions of $L$ and $\tilde{L}$ to this bundle coincide. Hence

$$
\operatorname{ind}(\tilde{L})=[E(\tilde{L}, \mathcal{W}), \mathcal{W}, \tilde{L}]=[E(L, \mathcal{W}), \mathcal{W}, L]=\operatorname{ind}(L)
$$

The following corollary of the reduction property turns out to be useful in explicit computations. We will use it below in order to prove the result 7.1 .12 whose corollary 7.1 .13 is half the battle of the proof of one part of our main index theorem 9.1.1 in the third part of the thesis.

5.2.10 Corollary. Let $L_{0} \in \mathfrak{F}_{k}\left(\mathcal{E}_{0}, \mathcal{F}_{0}\right)$ and $L_{1} \in \mathfrak{F}_{k}\left(\mathcal{E}_{1}, \mathcal{F}_{1}\right)$ be Fredholm morphisms having a compact support whose intersection with $A$ is trivial. Assume that there are bundle monomorphisms $i_{0}: \mathcal{E}_{1} \rightarrow \mathcal{E}_{0}$ and $i_{1}: \mathcal{F}_{1} \rightarrow \mathcal{F}_{0}$ such that the diagram

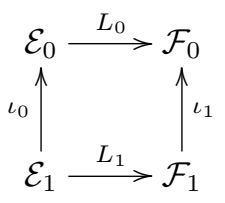

commutes. Moreover, assume that there exists a finite dimensional subbundle $\mathcal{V}_{1} \subset \mathcal{F}_{1}$ which is transversal to the image of $L_{1}$ and such that $\mathcal{V}_{0}=\iota_{1}\left(\mathcal{V}_{1}\right)$ is transversal to the image of $L_{0}$. Then

$$
\operatorname{ind}\left(L_{0}\right)=\operatorname{ind}\left(L_{1}\right) \in K(X, A)
$$


Proof. At first note that $E\left(L_{0}, \mathcal{V}_{0}\right)$ and $E\left(L_{1}, \mathcal{V}_{1}\right)$ are defined under the given assumptions. We obtain by the reduction property

$$
\operatorname{ind}\left(L_{0}\right)=\operatorname{ind}\left(\left.L_{0}\right|_{E\left(L_{0}, \mathcal{V}_{0}\right)}\right), \quad \operatorname{ind}\left(L_{1}\right)=\operatorname{ind}\left(\left.L_{1}\right|_{E\left(L_{1}, \mathcal{V}_{1}\right)}\right) .
$$

From the commutativity of 5.2 , we infer that $\iota_{0}$ maps $E\left(L_{1}, \mathcal{V}_{1}\right)$ into $E\left(L_{0}, \mathcal{V}_{0}\right)$. Indeed, if $u \in E\left(L_{1}, \mathcal{V}_{1}\right)$, then

$$
L_{0}\left(\iota_{0} u\right)=\iota_{1}\left(L_{1} u\right) \in \iota_{1}\left(\mathcal{V}_{1}\right)=\mathcal{V}_{0}
$$

and hence $\iota_{0} u \in E\left(L_{0}, \mathcal{V}_{0}\right)$. Moreover, since both bundles have the same finite dimension by lemma 5.1.6 and $\iota_{0}$ is injective, we obtain that $\iota_{0}$ is actually a bundle isomorphism. Now the diagram

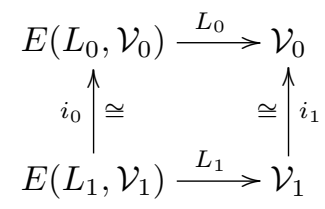

which commutes because of the commutativity of 5.2 , shows the assertion.

Finally, we want to show that in the case that $X$ is a compact space, $A=\emptyset$ and $\mathcal{E}=\mathcal{F}=$ $X \times E$, our definition of ind $(L)$ is just the classical Atiyah-Jänich bundle as described in section 4.1

Let $L: X \rightarrow \mathcal{B F}(E)$ be a norm continuous family of bounded Fredholm operators. Let $E_{1} \subset E$ be a finite codimensional closed subspace such that ker $L_{\lambda} \cap E_{1}=\{0\}$ for all $\lambda \in X$ and set $\mathcal{W}=\operatorname{im}(I-P) \subset \Theta(E)$, where $P$ denotes a projection onto the subbundle $\operatorname{im}\left(\left.L\right|_{X \times E_{1}}\right)$ of $\Theta(E)$. Then the Atiyah-Jänich bundle is by definition

$$
\left[\Theta\left(E / E_{1}\right)\right]-[\mathcal{W}] \in K(X) .
$$

Note that by construction

$$
\operatorname{im}\left(\left.L_{\lambda}\right|_{E_{1}}\right) \oplus \mathcal{W}_{\lambda}=E_{\lambda}
$$

which implies

$$
\operatorname{im} L_{\lambda}+\mathcal{W}_{\lambda}=E_{\lambda}, \quad \lambda \in X
$$


Hence we can use $\mathcal{W}$ in order to build the index bundle according to definition 5.1 .10 and obtain

$$
\operatorname{ind}(L)=[E(L, \mathcal{W})]-[\mathcal{W}] \in K(X)
$$

Now, since $E(L, \mathcal{W}) \subset \Theta(E)$ by definition, we have a well defined map

$$
F: E(L, \mathcal{W}) \rightarrow \Theta\left(E / E_{1}\right), \quad(\lambda, u) \mapsto(\lambda,[u])
$$

and in order to show that both definitions of the index bundles coincide we want to prove that $F$ is a bundle isomorphism.

Note at first that $F$ is obviously continuous and hence by well known facts about morphisms between vector bundles (cf. [MS74, Lemma 2.3]) it suffices to show that each $F_{\lambda}, \lambda \in X$, is bijective.

If $F_{\lambda}(u)=[u]=0$, then $u \in E_{1}$ because $E_{1}$ is closed. Hence $L_{\lambda} u \in \operatorname{im}\left(\left.L_{\lambda}\right|_{E_{1}}\right)$ and since $L_{\lambda} u \in \mathcal{W}_{\lambda}$ by definition of $E(L, \mathcal{W})$, we infer that $L_{\lambda} u=0$ by 5.3 . But using that $u \in E_{1}$ once again we conclude that $u=0$ and hence $F_{\lambda}$ is injective.

It remains to show that $\operatorname{dim} E(L, \mathcal{W})=\operatorname{dim} E / E_{1}$. By lemma 5.1.6 we have

$$
\operatorname{dim} E(L, \mathcal{W})=\operatorname{ind} L+\operatorname{dim} \mathcal{W}
$$

On the other hand we obtain from lemma A.2.11

$$
\operatorname{dim} \mathcal{W}_{\lambda}=\operatorname{dim} \operatorname{coker}\left(\left.L_{\lambda}\right|_{E_{1}}\right)=-\operatorname{ind}\left(\left.L_{\lambda}\right|_{E_{1}}\right)=-\left(\operatorname{ind} L_{\lambda}-\operatorname{dim} E / E_{1}\right)=\operatorname{dim} E / E_{1}-\operatorname{ind} L_{\lambda}
$$

and comparing both results yields the assertion.

Hence $F: E(L, \mathcal{W}) \rightarrow \Theta\left(E / E_{1}\right)$ is an isomorphism and the index bundle as defined by 5.1.10 and the classical Atiyah-Jänich bundle coincide.

\subsection{On the Dimension of the Support}

In this final section we want to use our results on the dimension of exceptional sets from the first part in order to study the dimension of the support $\operatorname{supp} L$ of a given bundle morphism $L \in \mathfrak{F}_{0}(\mathcal{E}, \mathcal{F})$. Note again that for $k \neq 0$ and $L \in \mathfrak{F}_{k}(\mathcal{E}, \mathcal{F})$ we have $\operatorname{supp}(L)=X$ and hence it is not interesting to study the dimension of $\operatorname{supp} L$.

We begin with applications of theorem 3.2.1. Let $L \in \mathfrak{F}_{0}(\mathcal{E}, \mathcal{F})$ be given where the base $X$ of $\mathcal{E}$ and $\mathcal{F}$ is assumed to be compact. Now we can use the Chern classes $c_{k}, k \in \mathbb{N}$, in order to define maps 


$$
\sigma_{k}\left(X^{\prime}\right)=c_{k}\left(\operatorname{ind}\left(\left.L\right|_{X^{\prime}}\right)\right) \in H^{2 k}\left(X^{\prime} ; \mathbb{Z}\right),
$$

where $X^{\prime}$ is any compact subset of $X$.

From the normalisation property 5.2.1 of the index bundle it is clear that

$$
\sigma_{k}\left(X^{\prime}\right)=0 \in H^{2 k}\left(X^{\prime} ; \mathbb{Z}\right)
$$

if $X^{\prime} \cap \operatorname{supp} L=\emptyset$. Moreover, if $X^{\prime \prime} \subset X^{\prime} \subset X$, where $X^{\prime \prime}$ and $X^{\prime}$ are compact, and $\iota: X^{\prime \prime} \hookrightarrow X^{\prime}$ denotes the inclusion, we obtain by lemma 5.2.2 and the naturality of the Chern classes

$$
\sigma_{k}\left(X^{\prime \prime}\right)=c_{k}\left(\operatorname{ind}\left(\left.L\right|_{X^{\prime \prime}}\right)\right)=c_{k}\left(\operatorname{ind}\left(\iota^{*}\left(\left.L\right|_{X^{\prime}}\right)\right)\right)=\iota^{*} c_{k}\left(\operatorname{ind}\left(\left.L\right|_{X^{\prime}}\right)\right)=\iota^{*} \sigma_{k}\left(X^{\prime}\right) .
$$

Hence the following result is an immediate consequence of theorem 3.2 .1 .

5.3.1 Proposition. Let $X$ be a compact orientable manifold of dimension $n$ such that $H_{2 k-1}(X ; \mathbb{Z})$ is free for some $1 \leq k \leq \frac{n-1}{2}$. If

$$
c_{k}(\operatorname{ind}(L)) \neq 0 \in H^{2 k}(X ; \mathbb{Z}),
$$

then the dimension of $\operatorname{supp} L$ is at least $n-2 k$ and $\operatorname{supp} L$ is not contractible to a point inside $X$.

5.3.2 Remark. In all of our constructions we have never used that the underlying Banach bundles are complex. Hence one could define in the same way the index bundle for Fredholm morphisms acting between real Banach bundles as an element in $K O(X, A)$. Then in the construction above the Chern classes can be substituted by the Stiefel-Whitney classes $w_{k}$ and proposition 5.3 .1 holds true even without the orientability assumption on $X$ as follows: If $X$ is a compact manifold of dimension $n$ and $w_{k}(X) \neq 0 \in H^{k}\left(X ; \mathbb{Z}_{2}\right)$ for some $1 \leq k \leq n-1$, then the dimension of $\operatorname{supp} L$ is at least $n-k$. In particular, the nonorientability of the index bundle forces $L$ to have a support of dimension at least $n-1$.

We now turn to apply proposition 3.3.7 to our situation. Accordingly, let $X$ be not necessarily compact, and $A \subset X$ be a closed subspace such that $A \cap \operatorname{supp} L=\emptyset$, where $L \in \mathfrak{F}_{0}(\mathcal{E}, \mathcal{F})$ is assumed to have compact support and $\mathcal{F}$ is trivial. We define for $k \in \mathbb{N}$

$$
\Gamma_{k}: \Omega^{k}(X, A) \rightarrow K\left(I^{k}, \partial I^{k}\right), \quad f \mapsto f^{*} \text { ind } L .
$$

Note in particular that $\Gamma_{k}(f)=0$ for all $f \in \Omega^{k}(X, A)$ if $k$ is odd. 
5.3.3 Lemma. Let $f_{1}, f_{2} \in \Omega^{k}(X, A)$ be such that $f_{1} * f_{2}$ exists and let $\mathcal{E}$ be a trivial bundle over $X$. Then

$$
\operatorname{ind}\left(\left(f_{1} * f_{2}\right)^{*} L\right)=\operatorname{ind}\left(f_{1}^{*} L\right)+\operatorname{ind}\left(f_{2}^{*} L\right) \in K\left(I^{k}, \partial I^{k}\right)
$$

Proof. The result is clear if $k$ is odd, because in this case $K\left(I^{k}, \partial I^{k}\right)=0$. Hence we assume in the following that $k$ is even.

We choose global trivialisations $\varphi$ of $\mathcal{E}$ and $\psi$ of $\mathcal{F}$ and denote $\hat{L}=\psi \circ L \circ \varphi^{-1}: X \times E \rightarrow X \times F$. From the properties 5.2.1 5.2.2 and 5.2.7 we obtain for any $f \in \Omega^{k}(X, A)$

$$
\begin{aligned}
\operatorname{ind}\left(f^{*} L\right)=f^{*}(\operatorname{ind}(L)) & =f^{*}\left(\operatorname{ind}(\psi)+\operatorname{ind}(L)+\operatorname{ind}\left(\varphi^{-1}\right)\right)=f^{*}\left(\operatorname{ind}\left(\psi \circ L \circ \varphi^{-1}\right)\right) \\
& =f^{*} \operatorname{ind}(\hat{L})=\operatorname{ind}\left(f^{*} \hat{L}\right) .
\end{aligned}
$$

Hence it is enough to prove the assertion for $\hat{L}$.

Let now $f_{1}, f_{2} \in \Omega^{k}(X, A)$ such that $f_{1} * f_{2}$ exists. We define $g, g_{1}, g_{2} \in \Omega^{k}(X, A)$ as follows:

$$
\begin{aligned}
g\left(t_{1}, \ldots, t_{k}\right) & =f_{1}\left(t_{1}, \ldots, t_{k-1}, 1\right)=f_{2}\left(t_{1}, \ldots, t_{k-1}, 0\right) \\
g_{1}\left(t_{1}, \ldots, t_{k}\right) & =\left\{\begin{array}{l}
f_{1}\left(t_{1}, \ldots, t_{k-1}, 2 t_{k}\right), 0 \leq t_{k} \leq \frac{1}{2} \\
f_{1}\left(t_{1}, \ldots, t_{k-1}, 1\right), \frac{1}{2} \leq t_{k} \leq 1
\end{array}\right. \\
g_{2}\left(t_{1}, \ldots, t_{k}\right) & =\left\{\begin{array}{l}
f_{2}\left(t_{1}, \ldots, t_{k-1}, 0\right) 0 \leq t_{k} \leq \frac{1}{2} \\
f_{2}\left(t_{1}, \ldots, t_{k-1}, 2 t_{k}-1\right) \frac{1}{2} \leq t_{k} \leq 1
\end{array}\right.
\end{aligned}
$$

Consider the homotopy

$$
H: I \times I^{k} \times E \oplus E \rightarrow F \oplus F
$$

defined by

$$
H_{\lambda}=\left\{\begin{array}{l}
\hat{L} \circ g_{1} \oplus \hat{L} \circ g_{2} \quad \text { if } 0 \leq t_{k} \leq \frac{1}{2} \\
\left(\begin{array}{cc}
\cos \left(\frac{\pi \lambda}{2}\right) & \sin \left(\frac{\pi \lambda}{2}\right) \\
-\sin \left(\frac{\pi \lambda}{2}\right) & \cos \left(\frac{\pi \lambda}{2}\right)
\end{array}\right)\left(\begin{array}{cc}
\hat{L} \circ g_{1} & 0 \\
0 & \hat{L} \circ g_{2}
\end{array}\right)\left(\begin{array}{cc}
\cos \left(\frac{\pi \lambda}{2}\right) & -\sin \left(\frac{\pi \lambda}{2}\right) \\
\sin \left(\frac{\pi \lambda}{2}\right) & \cos \left(\frac{\pi \lambda}{2}\right)
\end{array}\right), \text { if } \frac{1}{2} \leq t_{k} \leq 1
\end{array}\right.
$$

At first $H$ is continuous because $g_{1}\left(t_{1}, \ldots, t_{k-1}, \frac{1}{2}\right)=g_{2}\left(t_{1}, \ldots, t_{k-1}, \frac{1}{2}\right)$ and in this case the matrix product above reduces to 


$$
\left(\begin{array}{cc}
\hat{L} \circ g_{1} & 0 \\
0 & \hat{L} \circ g_{2}
\end{array}\right)
$$

Hence for $t_{k}=\frac{1}{2}$ both definitions of $H$ coincide for all $\lambda \in[0,1]$.

Next we note that

$$
H_{0}=\hat{L} \circ g_{1} \oplus \hat{L} \circ g_{2}, \quad H_{1}=\left\{\begin{array}{l}
\hat{L} \circ g_{1} \oplus \hat{L} \circ g_{2}, t_{k} \leq \frac{1}{2} \\
\hat{L} \circ g_{2} \oplus \hat{L} \circ g_{1}, t_{k} \geq \frac{1}{2}
\end{array}\right.
$$

and a closer look at the definitions shows that

$$
H_{1}=\hat{L} \circ\left(f_{1} * f_{2}\right) \oplus \hat{L} \circ g .
$$

Moreover, we note that, due to the invertibility of the rotation matrices in the definition of $H$, each $H\left(\lambda, t_{1}, \ldots, t_{k}\right)$ is a Fredholm operator of index 0 . Since the parameter space $I^{k}$ is itself compact, we finally have found a homotopy $H$ of Fredholm morphisms having compact support. Because $\hat{L}_{x} \in G L(E, F)$ for all $x \in g\left(I^{k}\right)$, we obtain

$$
\begin{aligned}
\operatorname{ind}\left(\left(f_{1} * f_{2}\right)^{*} \hat{L}\right) & =\operatorname{ind}\left(\left(f_{1} * f_{2}\right)^{*} \hat{L}\right)+\operatorname{ind}\left(g^{*} \hat{L}\right)=\operatorname{ind}\left(\left(f_{1} * f_{2}\right)^{*} \hat{L} \oplus g^{*} \hat{L}\right)=\operatorname{ind}\left(H_{1}\right) \\
& =\operatorname{ind}\left(H_{0}\right)=\operatorname{ind}\left(g_{1}^{*} \hat{L} \oplus g_{2}^{*} \hat{L}\right)=\operatorname{ind}\left(g_{1}^{*} \hat{L}\right)+\operatorname{ind}\left(g_{2}^{*} \hat{L}\right)=\operatorname{ind}\left(f_{1}^{*} \hat{L}\right)+\operatorname{ind}\left(f_{2}^{*} \hat{L}\right),
\end{aligned}
$$

where we use that $g_{i}$ is homotopic to $f_{i}, i=1,2$, in the last equation.

Since the other properties are clear by normalisation of ind according to lemma 5.2.1 and the homotopy invariance of $K$ according to lemma B.1.2 we obtain the following result.

5.3.4 Proposition. Let $X$ be a connected and orientable manifold and $k \in \mathbb{N}$ such that $k \leq \frac{n-1}{2}$, $H_{j}(X)=0,1 \leq j \leq 2 k-1$ and $\pi_{2 k}(X)=0$. Let $L \in \mathfrak{F}_{0}(\mathcal{E}, \mathcal{F})$ be a Fredholm morphism having compact support, where the bundles $\mathcal{E}$ and $\mathcal{F}$ are assumed to be trivial. If $\pi_{1}(X \backslash \operatorname{supp}(L))$ is abelian and there exists $f \in \Omega^{2 k}(X, A)$ such that

$$
f^{*} \text { ind } L \neq 0 \in K\left(I^{2 k}, \partial I^{2 k}\right) \cong \mathbb{Z},
$$

then $\operatorname{dim} \operatorname{supp} L \geq n-2 k$ and $\operatorname{supp} L$ is not contractible.

5.3.5 Remark. i) Our results on $\Gamma_{k}$ from section 3.3 are particular powerful if $k=1$. Unfortunately this case is excluded here because $K(I, \partial I)=0$.

ii) The assumption that $\mathcal{E}$ and $\mathcal{F}$ are trivial is not extremely restrictive in view of theorem 1.1.8. 


\section{Chapter 6}

\section{The Index Bundle for Families of Fredholm Operators in the Gap Topology}

In the sixth chapter of the thesis we connect the discussion of the gap topology on the space $\mathcal{C}(H)$ of all closed operators from the second chapter and the index bundle for Fredholm morphisms between Banach bundles as introduced in the foregoing fifth chapter. More precisely, the aim of this chapter is to assign an index bundle as a K-theory class in $K(X)$ to any gap continuous family $\mathcal{A}: X \rightarrow \mathcal{C F}(H)$ of generally unbounded Fredholm operators acting on $H$. In the special case that $\mathcal{A}: X \rightarrow \mathcal{B F}(H)$ is a family of bounded Fredholm operators, the resulting element is just the Atiyah-Jänich bundle which we recalled in section 4.1 . In the first section we consider general gap continuous families $\mathcal{A}: X \rightarrow \mathcal{C}(H)$ of closed operators and show that their domains $\mathcal{D}\left(\mathcal{A}_{x}\right), x \in X$, can be fit into a Hilbert bundle $\mathfrak{D}(\mathcal{A})$ which we call the domain bundle. The construction of the domain bundle is quite abstract but besides its main properties we also show that it can be understood well in special cases which appear frequently in applications. A particular observation is that the family $\mathcal{A}$ itself defines a bundle morphism from the domain bundle $\mathfrak{D}(\mathcal{A})$ into the product $X \times H$. It turns out that this morphism belongs to $\mathfrak{F}(\mathfrak{D}(\mathcal{A}), X \times H)$ if $\mathcal{A}: X \rightarrow \mathcal{C F}(H)$ is a family of Fredholm operators and hence we can use the theory developed in the foregoing fifth chapter in order to define the index bundle ind $\mathcal{A}$. Subsequently we discuss the properties of ind $\mathcal{A}$, where now most of them follow quite immediately from the corresponding results of section 5.2 . In the final third section we briefly consider estimates of the dimension of the so called singular set of a given gap continuous family $\mathcal{A}: X \rightarrow \mathcal{C F}(H)$, which is by definition the set of all $x \in X$ such that $\mathcal{A}_{x}$ does not have a bounded inverse. 


\subsection{The Domain Bundle}

In the following we assume throughout that $X$ is a topological space and $H$ a Hilbert space. Let us recall that by corollary 2.4 .3

$$
\hat{\delta}\left(\operatorname{graph} \mathcal{A}_{1}, \operatorname{graph} \mathcal{A}_{2}\right)=\left\|P_{\operatorname{graph}\left(\mathcal{A}_{1}\right)}-P_{\operatorname{graph}\left(\mathcal{A}_{2}\right)}\right\|
$$

for any closed operators $\mathcal{A}_{1}, \mathcal{A}_{2} \in \mathcal{C}(H)$, where $P_{\text {graph }\left(\mathcal{A}_{i}\right)}$ denotes the orthogonal projection onto $\operatorname{graph}\left(\mathcal{A}_{i}\right)$ in $H \times H, i=1,2$.

Let now $\mathcal{A}: X \rightarrow \mathcal{C}(H)$ be a gap continuous family. We define

$$
\mathcal{E}=\coprod_{x \in X} \mathcal{D}\left(\mathcal{A}_{x}\right)
$$

and denote by $\pi: \mathcal{E} \rightarrow X$ the obvious projection. Moreover, for any $x_{0} \in X$, let

$$
U_{x_{0}}=\left\{x \in X: d_{G}\left(\mathcal{A}_{x}, \mathcal{A}_{x_{0}}\right)<\frac{1}{3}\right\}
$$

which is an open subset of $X$ due to the assumed continuity of $\mathcal{A}$.

\subsubsection{Lemma.}

$$
P_{\operatorname{graph}\left(\mathcal{A}_{x_{0}}\right)} \mid \operatorname{graph}\left(\mathcal{A}_{x}\right): \operatorname{graph}\left(\mathcal{A}_{x}\right) \rightarrow \operatorname{graph}\left(\mathcal{A}_{x_{0}}\right)
$$

is an isomorphism for all $x \in U_{x_{0}}$. Moreover, if $\mathcal{A}: X \rightarrow \mathcal{C}(H)$ is gap continuous, then the map

$$
U_{x_{0}} \ni x \mapsto\left(P_{\operatorname{graph}\left(\mathcal{A}_{x_{0}}\right)} \mid \operatorname{graph}\left(\mathcal{A}_{x}\right)\right)^{-1} \in \mathcal{L}\left(\operatorname{graph}\left(\mathcal{A}_{x_{0}}\right), H \times H\right)
$$

is continuous with respect to the norm topology.

Proof. We compute

$$
\begin{aligned}
& I-\left(P_{\operatorname{graph}\left(\mathcal{A}_{x}\right)}-P_{\operatorname{graph}\left(\mathcal{A}_{x_{0}}\right)}\right)\left(P_{\operatorname{graph}\left(\mathcal{A}_{x}\right)}-P_{\left.\operatorname{graph}\left(\mathcal{A}_{x}\right)^{\perp}\right)}\right. \\
& =I-P_{\operatorname{graph}\left(\mathcal{A}_{x}\right)}+P_{\operatorname{graph}\left(\mathcal{A}_{x_{0}}\right)} P_{\operatorname{graph}\left(\mathcal{A}_{x}\right)}-P_{\operatorname{graph}\left(\mathcal{A}_{x_{0}}\right)} P_{\operatorname{graph}\left(\mathcal{A}_{x}\right)^{\perp}}
\end{aligned}
$$

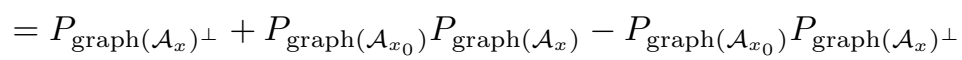

$$
\begin{aligned}
& =\left(I-P_{\operatorname{graph}\left(\mathcal{A}_{x_{0}}\right)}\right) P_{\operatorname{graph}\left(\mathcal{A}_{x}\right)^{\perp}}+P_{\operatorname{graph}\left(\mathcal{A}_{x_{0}}\right)} P_{\operatorname{graph}\left(\mathcal{A}_{x}\right)}
\end{aligned}
$$

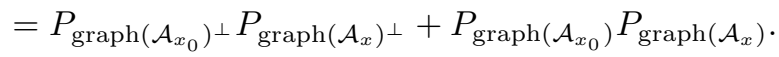

Since 


$$
\left\|P_{\operatorname{graph}\left(\mathcal{A}_{x}\right)}-P_{\operatorname{graph}\left(\mathcal{A}_{x}\right)^{\perp}}\right\|=\left\|P_{\operatorname{graph}\left(\mathcal{A}_{x}\right)}-\left(I-P_{\operatorname{graph}\left(\mathcal{A}_{x}\right)}\right)\right\| \leq 2\left\|P_{\operatorname{graph}\left(\mathcal{A}_{x}\right)}\right\|+1=3,
$$

we obtain from the well known Neumann series that

$$
P_{\operatorname{graph}\left(\mathcal{A}_{x_{0}}\right)^{\perp}} P_{\operatorname{graph}\left(\mathcal{A}_{x}\right)^{\perp}}+P_{\operatorname{graph}\left(\mathcal{A}_{x_{0}}\right)} P_{\operatorname{graph}\left(\mathcal{A}_{x}\right)}: H \rightarrow H
$$

is an isomorphism if $\left\|P_{\operatorname{graph}\left(\mathcal{A}_{x}\right)}-P_{\operatorname{graph}\left(\mathcal{A}_{x_{0}}\right)}\right\|<\frac{1}{3}$ which is in particular the case if $x \in U_{x_{0}}$ by lemma 2.1 .3 Now we just have to observe that

$$
H=\operatorname{graph}\left(\mathcal{A}_{x}\right) \oplus \operatorname{graph}\left(\mathcal{A}_{x}\right)^{\perp}=\operatorname{graph}\left(\mathcal{A}_{x_{0}}\right) \oplus \operatorname{graph}\left(\mathcal{A}_{x_{0}}\right)^{\perp}
$$

and the map 6.1 maps $\operatorname{graph}\left(\mathcal{A}_{x}\right) \operatorname{into} \operatorname{graph}\left(\mathcal{A}_{x_{0}}\right)$ and $\operatorname{graph}\left(\mathcal{A}_{x}\right)^{\perp} \operatorname{into} \operatorname{graph}\left(\mathcal{A}_{x_{0}}\right)^{\perp}$, respectively. Hence 6.1) maps graph $\left(\mathcal{A}_{x}\right)$ bijectively onto $\operatorname{graph}\left(\mathcal{A}_{x_{0}}\right)$ and $\operatorname{graph}\left(\mathcal{A}_{x}\right)^{\perp}$ bijectively $\operatorname{onto} \operatorname{graph}\left(\mathcal{A}_{x_{0}}\right)^{\perp}$. But the restriction of 6.1 to graph $\left(\mathcal{A}_{x}\right)$ is given by

$$
\left.\left.P_{\operatorname{graph}\left(\mathcal{A}_{x_{0}}\right)}\right|_{\operatorname{graph}\left(\mathcal{A}_{x}\right)}\right) \operatorname{graph}\left(\mathcal{A}_{x}\right) \rightarrow \operatorname{graph}\left(\mathcal{A}_{x_{0}}\right)
$$

which proves the first assertion.

In order to prove the second assertion note that if $X \ni x \mapsto P_{\operatorname{graph}\left(\mathcal{A}_{x}\right)} \in \mathcal{L}(H \times H)$ is continuous, then also

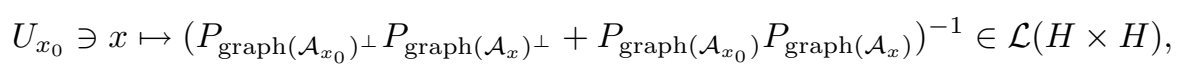

where we use that (6.1) is invertible for $x \in U_{x_{0}}$ and the well known continuity of the inversion in $G L(H \times H)$. But we observed above that the restriction of 6.1) to $\operatorname{graph}\left(\mathcal{A}_{x}\right)$ is given by $P_{\operatorname{graph}\left(\mathcal{A}_{x_{0}}\right)} \mid \operatorname{graph}\left(\mathcal{A}_{x}\right)$ which is moreover an isomorphism onto $\operatorname{graph}\left(\mathcal{A}_{x_{0}}\right)$. Hence

$$
\begin{aligned}
\left.\left(P_{\operatorname{graph}\left(\mathcal{A}_{x_{0}}\right)}{ }^{\perp} P_{\operatorname{graph}\left(\mathcal{A}_{x}\right)^{\perp}}+P_{\operatorname{graph}\left(\mathcal{A}_{x_{0}}\right)} P_{\operatorname{graph}\left(\mathcal{A}_{x}\right)}\right)^{-1}\right|_{\operatorname{graph}\left(\mathcal{A}_{x_{0}}\right)} & \\
=\left(P_{\operatorname{graph}\left(\mathcal{A}_{x_{0}}\right)}\right) & \left.{\operatorname{graph}\left(\mathcal{A}_{x}\right)}\right)^{-1} \in \mathcal{L}\left(\operatorname{graph}\left(\mathcal{A}_{x_{0}}\right), H \times H\right),
\end{aligned}
$$

depends continuously on $x \in U_{x_{0}}$.

Note that we have the following identities

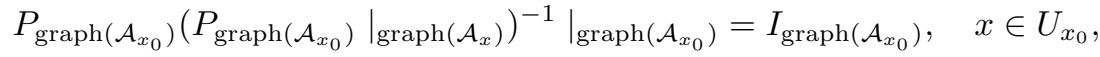

$$
\begin{aligned}
& \left(P_{\operatorname{graph}\left(\mathcal{A}_{x_{0}}\right)} \mid \operatorname{graph}\left(\mathcal{A}_{x}\right)\right)^{-1} P_{\operatorname{graph}\left(\mathcal{A}_{x_{0}}\right)} \mid \operatorname{graph}\left(\mathcal{A}_{x}\right)=I_{\operatorname{graph}\left(\mathcal{A}_{x}\right), \quad x \in U_{x_{0}} .}
\end{aligned}
$$


We define

$$
\tau_{x_{0}}: \pi^{-1}\left(U_{x_{0}}\right) \rightarrow U_{x_{0}} \times \operatorname{graph}\left(\mathcal{A}_{x_{0}}\right), \quad \tau_{x_{0}}(x, u)=\left(x, P_{\operatorname{graph}\left(\mathcal{A}_{x_{0}}\right)}\left(u, \mathcal{A}_{x} u\right)\right)
$$

and formally

$$
\tau_{x_{0}}^{-1}: U_{x_{0}} \times \operatorname{graph}\left(\mathcal{A}_{x_{0}}\right) \rightarrow \pi^{-1}\left(U_{x_{0}}\right), \quad \tau_{x_{0}}^{-1}(x, u)=P_{1}\left(\left.P_{\operatorname{graph}\left(\mathcal{A}_{x_{0}}\right)}\right|_{\operatorname{graph}\left(\mathcal{A}_{x}\right)}\right)^{-1} u,
$$

where $P_{1}=P_{H \times\{0\}}$.

We justify the latter notation by

$$
\begin{aligned}
& \left(\tau_{x_{0}} \circ \tau_{x_{0}}^{-1}\right)_{x}(u)=P_{\operatorname{graph}\left(\mathcal{A}_{x_{0}}\right)}\left(P_{1}\left(P_{\operatorname{graph}\left(\mathcal{A}_{x_{0}}\right)} \mid \operatorname{graph}\left(\mathcal{A}_{x}\right)\right)^{-1} u, \mathcal{A}_{x} P_{1}\left(\left.P_{\operatorname{graph}\left(\mathcal{A}_{x_{0}}\right)}\right|_{\operatorname{graph}\left(\mathcal{A}_{x}\right)}\right)^{-1} u\right) \\
& =\underbrace{P_{\operatorname{graph}\left(\mathcal{A}_{x_{0}}\right)}\left(P_{\operatorname{graph}\left(\mathcal{A}_{x_{0}}\right)} \operatorname{graph}\left(\mathcal{A}_{x}\right)\right)^{-1}}_{I_{\operatorname{graph}\left(\mathcal{A}_{0}\right)}} u=u
\end{aligned}
$$

for all $u \in \operatorname{graph}\left(\mathcal{A}_{x_{0}}\right)$, where we use that $\left(P_{1} u, \mathcal{A}_{x} P_{1} u\right)=u$ for all $u \in \operatorname{graph}\left(\mathcal{A}_{x}\right)$. Similarly we obtain

$$
\left(\tau_{x_{0}}^{-1} \circ \tau_{x_{0}}\right)_{x}(u)=P_{1} \underbrace{\left(\left.P_{\operatorname{graph}\left(\mathcal{A}_{x_{0}}\right)}\right|_{\operatorname{graph}\left(\mathcal{A}_{x}\right)}\right)^{-1} P_{\operatorname{graph}\left(\mathcal{A}_{x_{0}}\right)}}_{I_{\operatorname{graph}\left(\mathcal{A}_{x}\right)}}\left(u, \mathcal{A}_{x} u\right)=u
$$

for all $u \in \mathcal{D}\left(\mathcal{A}_{x}\right)$.

Moreover, if $x_{0}, x_{1} \in X$ are given such that $U_{x_{0}} \cap U_{x_{1}} \neq \emptyset$, we obtain by the same computation as above for any $x \in U_{x_{0}} \cap U_{x_{1}}$

$$
\left.\left(\tau_{x_{1}} \circ \tau_{x_{0}}^{-1}\right)_{x}=P_{\operatorname{graph}\left(\mathcal{A}_{x_{1}}\right)}\left(P_{\operatorname{graph}\left(\mathcal{A}_{x_{0}}\right)}\right) \operatorname{graph}\left(\mathcal{A}_{x}\right)\right)^{-1} \in \mathcal{L}\left(\operatorname{graph}\left(\mathcal{A}_{x_{0}}\right), \operatorname{graph}\left(\mathcal{A}_{x_{1}}\right)\right)
$$

and so

$$
U_{x_{0}} \cap U_{x_{1}} \ni x \mapsto\left(\tau_{x_{1}} \circ \tau_{x_{0}}^{-1}\right)_{x} \in \mathcal{L}\left(\operatorname{graph}\left(\mathcal{A}_{x_{0}}\right), \operatorname{graph}\left(\mathcal{A}_{x_{1}}\right)\right)
$$

is continuous by the second assertion of lemma 6.1.1. Finally, since $x \in U_{x_{0}} \cap U_{x_{1}}$, the maps

$$
\begin{aligned}
& \left(\left.P_{\operatorname{graph}\left(\mathcal{A}_{x_{0}}\right)}\right|_{\operatorname{graph}\left(\mathcal{A}_{x}\right)}\right)^{-1}: \operatorname{graph}\left(\mathcal{A}_{x_{0}}\right) \rightarrow \operatorname{graph}\left(\mathcal{A}_{x}\right), \\
& \left.P_{\operatorname{graph}\left(\mathcal{A}_{x_{1}}\right)}\right|_{\operatorname{graph}\left(\mathcal{A}_{x}\right)}: \operatorname{graph}\left(\mathcal{A}_{x}\right) \rightarrow \operatorname{graph}\left(\mathcal{A}_{x_{1}}\right)
\end{aligned}
$$

are topological isomorphisms by the first assertion of lemma 6.1.1 and hence 


$$
\left(\tau_{x_{1}} \circ \tau_{x_{0}}^{-1}\right)_{x} \in G L\left(\operatorname{graph}\left(\mathcal{A}_{x_{0}}\right), \operatorname{graph}\left(\mathcal{A}_{x_{1}}\right)\right), \quad x \in U_{0} \cap U_{1} .
$$

Thus we obtain by the bundle construction lemma 1.1 .4 a Hilbert bundle, which we denote by $\mathfrak{D}(\mathcal{A})$ and call the domain bundle of $\mathcal{A}$.

We now discuss the main properties of the domain bundle. In the following we denote, in accordance with the first chapter, the fibre of the domain bundle over $x \in X$ by $\mathfrak{D}(\mathcal{A})_{x}$. Note that we have to distinguish between $\mathcal{D}\left(\mathcal{A}_{x}\right)$ which is the domain of $\mathcal{A}_{x}$ as a subspace of $H$ and the fibre $\mathfrak{D}(\mathcal{A})_{x}$. The first result we want to mention explains the difference between these spaces.

6.1.2 Lemma. Let $\mathcal{A}: X \rightarrow \mathcal{C}(H)$ be gap continuous and $x \in X$. The fibre of $\mathfrak{D}(\mathcal{A})$ over $x$ is the domain of $\mathcal{A}_{x}$ with the topology induced by its graph norm.

Proof. By definition of the trivialisations and the first assertion of 6.1.1 the projection onto the first component

$$
P_{1}: \operatorname{graph}\left(\mathcal{A}_{x}\right) \rightarrow \mathfrak{D}(\mathcal{A})_{x}
$$

is a homeomorphism. This already shows the assertion; compare the discussion around A.2.7.

The following important property shows that we can use the domain bundle in order to transform a gap continuous family of closed operators to a family of bounded operators canonically.

6.1.3 Lemma. Let $\mathcal{A}: X \rightarrow \mathcal{C}(H)$ be gap continuous. Then $\mathcal{A}$ induces a bundle morphism

$$
\mathcal{A}: \mathfrak{D}(\mathcal{A}) \rightarrow X \times H
$$

Proof. We want to use lemma 1.2 .2 and note at first that each $\mathcal{A}_{x}: \mathfrak{D}(\mathcal{A})_{x} \rightarrow H$ is continuous according to the foregoing lemma 6.1.2. Hence it remains to show that for any $x_{0} \in X$ and trivialising neighbourhood $U_{x_{0}}$ around $x_{0}$, the map

$$
U_{x_{0}} \ni x \mapsto \mathcal{A}\left(\tau_{x_{0}}^{-1}(x, \cdot)\right) \in \mathcal{L}\left(\operatorname{graph}\left(\mathcal{A}_{x_{0}}\right), H\right)
$$

is continuous.

But since the map

$$
U_{x_{0}} \ni x \mapsto P_{2}\left(\left.P_{\operatorname{graph}\left(\mathcal{A}_{x_{0}}\right)}\right|_{\operatorname{graph}\left(\mathcal{A}_{x}\right)}\right)^{-1} \in \mathcal{L}\left(\operatorname{graph}\left(\mathcal{A}_{x_{0}}\right), H\right)
$$


is continuous by lemma 6.1.1 the assertion follows from

$$
\mathcal{A}\left(\tau_{x_{0}}^{-1}(x, \cdot)\right)=\mathcal{A}_{x} P_{1}\left(P_{\operatorname{graph}\left(\mathcal{A}_{x_{0}}\right)} \mid \operatorname{graph}\left(\mathcal{A}_{x}\right)\right)^{-1}=P_{2}\left(\left.P_{\operatorname{graph}\left(\mathcal{A}_{x_{0}}\right)}\right|_{\operatorname{graph}\left(\mathcal{A}_{x}\right)}\right)^{-1},
$$

where $P_{2}=P_{\{0\} \times H}$ and we use that $\mathcal{A}_{x} P_{1} u=P_{2} u$ for all $u \in \operatorname{graph}\left(\mathcal{A}_{x}\right)$.

6.1.4 Remark. The support of the induced bundle morphism $\mathcal{A}: \mathfrak{D}(\mathcal{A}) \rightarrow X \times H$ is given by

$$
\left\{x \in X: 0 \in \sigma\left(\mathcal{A}_{x}\right)\right\}
$$

Indeed, $0 \in \rho\left(\mathcal{A}_{x}\right)$ if and only if $\mathcal{A}_{x}: \mathcal{D}\left(\mathcal{A}_{x}\right) \rightarrow H$ is bijective by the closed graph theorem A.2.4 And, since $\mathcal{A}_{x}: \mathfrak{D}(\mathcal{A})_{x} \rightarrow H$ is continuous, this is the case if and only if the latter map is a Banach space isomorphism by lemma A.2.5.

According to lemma 6.1.2 we can include each fibre $\mathfrak{D}(\mathcal{A})_{x}$ of the domain bundle continuously into $H$. The next result shows that this is even globally true.

6.1.5 Lemma. Let $\mathcal{A}: X \rightarrow \mathcal{C}(H)$ be gap continuous. Then the canonical inclusion

$$
\iota: \mathfrak{D}(\mathcal{A}) \hookrightarrow X \times H
$$

is a Banach bundle morphism.

Proof. Given $x_{0} \in X, U_{x_{0}}$ the trivialising neighbourhood and $\tau_{x_{0}}$ the corresponding trivialisation according to 6.2, we know by lemma 6.1.1 that the map

$$
\iota \circ \tau_{x_{0}}^{-1}: U_{x_{0}} \rightarrow \mathcal{L}\left(\operatorname{graph}\left(\mathcal{A}_{x_{0}}\right), H\right)
$$

is continuous. Since $\iota$ is moreover in every fibre a continuous operator by lemma 6.1.2, we obtain the assertion from lemma 1.2.2.

6.1.6 Remark. Note that lemma 6.1.5 does not imply that $\iota(\mathfrak{D}(\mathcal{A})) \subset X \times H$ is a subbundle because $\iota\left(\mathfrak{D}(\mathcal{A})_{x}\right) \subset H$ is not even closed in general.

The following corollary is an immediate consequence of the last three lemmata.

6.1.7 Corollary. The map

$$
\|\cdot\|_{\mathcal{D}(\mathcal{A})}: \mathfrak{D}(\mathcal{A}) \rightarrow \mathbb{R}, \quad u \mapsto \sqrt{\|u\|_{H}^{2}+\left\|\mathcal{A}_{x} u\right\|_{H}^{2}}
$$

defines a bundle metric on $\mathfrak{D}(\mathcal{A})$ which induces the given topology in any fibre. 
The next lemma shows the naturality of the domain bundle.

6.1.8 Lemma. Let $X, Y$ be topological spaces, $f: Y \rightarrow X$ continuous and $\mathcal{A}: X \rightarrow \mathcal{C}(H)$ gap continuous. Then

$$
\mathfrak{D}\left(f^{*} \mathcal{A}\right)=f^{*} \mathfrak{D}(\mathcal{A}) .
$$

Proof. At first we note

$$
\mathfrak{D}\left(f^{*} \mathcal{A}\right)_{y}=\mathcal{D}\left(\left(f^{*} \mathcal{A}\right)_{y}\right)=\mathcal{D}\left(\mathcal{A}_{f(y)}\right)=\mathfrak{D}(\mathcal{A})_{f(y)}=\left(f^{*} \mathfrak{D}(\mathcal{A})\right)_{y}, \quad y \in Y,
$$

and hence the total spaces of both bundles coincide as sets.

By definition a trivialising neighbourhood around $y_{0} \in Y$ of $\mathfrak{D}\left(f^{*} \mathcal{A}\right)$ is given by

$$
U_{y_{0}}=\left\{y \in Y: d_{G}\left(\left(f^{*} \mathcal{A}\right)_{y},\left(f^{*} \mathcal{A}\right)_{y_{0}}\right)<\frac{1}{3}\right\}=\left\{y \in Y: d_{G}\left(\mathcal{A}_{f(y)}, \mathcal{A}_{f\left(y_{0}\right)}\right)<\frac{1}{3}\right\}
$$

Since a trivialising neighbourhood of $\mathfrak{D}(\mathcal{A})$ around $f\left(y_{0}\right)$ is given by

$$
U_{f\left(y_{0}\right)}=\left\{x \in X: d_{G}\left(\mathcal{A}_{x}, \mathcal{A}_{f\left(y_{0}\right)}\right)<\frac{1}{3}\right\}
$$

we have

$$
U_{y_{0}}=f^{-1}\left(U_{f\left(y_{0}\right)}\right)
$$

and so the trivialising neighbourhoods of $\mathfrak{D}\left(f^{*} \mathcal{A}\right)$ and $f^{*} \mathfrak{D}(\mathcal{A})$ coincide by definition of the pullback bundle. Finally, again by definition of the pullback bundle, the trivialisation of $f^{*} \mathfrak{D}(\mathcal{A})$ corresponding to $f^{-1}\left(U_{f\left(y_{0}\right)}\right)$ is given by

$$
\tilde{\tau}_{y_{0}}(y, u)=\tau_{f\left(y_{0}\right)}(f(y), u)=P_{\operatorname{graph}\left(\mathcal{A}_{f\left(y_{0}\right)}\right)}\left(u, \mathcal{A}_{f(y)} u\right)
$$

where $\tau$ denotes the trivialisation of the domain bundle $\mathfrak{D}(\mathcal{A})$ as defined by 6.2 . But $\tilde{\tau}$ is by definition just the trivialisation of $\mathfrak{D}\left(f^{*} \mathcal{A}\right)$ over $U_{y_{0}}$.

Hence in sum both bundles have the same total spaces and a common atlas which proves the assertion.

Next we want to study the effect of a perturbation of a gap continuous family by a family of bounded operators. But before that, we have to show that the perturbed family is gap continuous in general. 
6.1.9 Lemma. Let $\mathcal{A}: X \rightarrow \mathcal{C}(H)$ be gap continuous and $B: X \rightarrow \mathcal{L}(H)$ be a continuous family of bounded operators. Then

$$
\mathcal{A}+B: X \rightarrow \mathcal{C}(H)
$$

is gap continuous.

Proof. Given $x_{0} \in X$, we can choose an open neighbourhood $U$ of $x_{0}$ such that $\left\|B_{x}-B_{x_{0}}\right\|<1$ for all $x \in U$. From $\left\|B_{x}\right\| \leq\left\|B_{x}-B_{x_{0}}\right\|+\left\|B_{x_{0}}\right\| \leq 1+\left\|B_{x_{0}}\right\|, x \in U$, we obtain by theorem 2.2 .1

$$
d_{G}\left(\mathcal{A}_{x}+B_{x}, \mathcal{A}_{x_{0}}+B_{x_{0}}\right) \leq 4 \sqrt{2} \sqrt{1+\left(1+\left\|B_{x_{0}}\right\|\right)^{2}} \sqrt{1+\left\|B_{x_{0}}\right\|^{2}}\left(d_{G}\left(\mathcal{A}_{x}, \mathcal{A}_{x_{0}}\right)+\left\|B_{x}-B_{x_{0}}\right\|\right) .
$$

for all $x \in U$ which shows the continuity.

6.1.10 Lemma. Let $\mathcal{A}: X \rightarrow \mathcal{C}(H)$ be gap continuous and $B: X \rightarrow \mathcal{L}(H)$ a continuous family of bounded operators. Then

$$
\mathfrak{D}(\mathcal{A}+B)=\mathfrak{D}(\mathcal{A}) .
$$

Proof. Note at first that the total spaces of both bundles coincide as sets. Moreover, since $B$ is bounded, the identity maps $\mathfrak{D}(\mathcal{A})_{x} \rightarrow \mathfrak{D}(\mathcal{A}+B)_{x}, x \in X$, are continuous with respect to the corresponding graph norms. We now want to use lemma 1.2 .2 in order to show that the identity is even a Banach bundle isomorphism.

Accordingly, if $x_{0} \in X$ is fixed and $U_{x_{0}}$ a common trivialising neighbourhood of both bundles around $x_{0}$ with respect to the trivialisations

$$
\begin{aligned}
& \left(\tau^{1}\right)^{-1}: U_{x_{0}} \times \operatorname{graph}\left(\mathcal{A}_{x_{0}}\right) \rightarrow \pi^{-1}\left(U_{x_{0}}\right) \subset \mathfrak{D}(\mathcal{A}) \\
& \left(\tau^{2}\right)^{-1}: U_{x_{0}} \times \operatorname{graph}\left(\mathcal{A}_{x_{0}}+B_{x_{0}}\right) \rightarrow \pi^{-1}\left(U_{x_{0}}\right) \subset \mathfrak{D}(\mathcal{A}+B)
\end{aligned}
$$

we have to prove that the map

$$
U_{x_{0}} \ni x \mapsto \tau_{x}^{2} \circ\left(\tau^{1}\right)_{x}^{-1} \in \mathcal{L}\left(\operatorname{graph}\left(\mathcal{A}_{x_{0}}\right), \operatorname{graph}\left(\mathcal{A}_{x_{0}}+B_{x_{0}}\right)\right)
$$

is continuous with respect to the norm topology. In order to check this, consider the following family of bounded operators

$$
\iota_{+}: X \rightarrow \mathcal{L}(H \times H) ; \quad \iota_{+}[x](u, v)=\left(u, v+B_{x} u\right) .
$$


Then the map (6.4) can be decomposed as

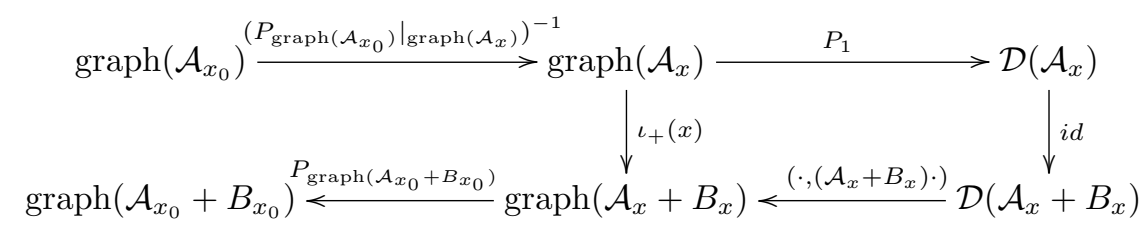

where the right square commutes. This shows that 6.4 is the composition of a family of maps from a fixed space into $H \times H$ which is continuous according to lemma 6.1.1, the continuous family $\iota_{+}: H \times H \rightarrow H \times H$ and a fixed bounded projection in $H \times H$.

6.1.11 Remark. It is natural to ask for a property like 6.1.10 in the more general case that $B: X \rightarrow \mathcal{C}(H)$ is gap continuous and each $B_{x}$ is $\mathcal{A}_{x}$-bounded (cf. [Ka76, \$IV.1.1]). However, note that we do not even have a result like lemma 6.1.9 at hand here and that we strongly used the boundedness of the operators $B_{x}$ in the proof of 6.1.10.

The definition of the domain bundle is quite abstract. Although its total space is precisely known as a set it seems to be hard to understand the bundle structure and so, for example, to verify that given maps on a domain bundle are continuous. As next step we prove some results that give a partial answer to this issue.

We begin by considering gap continuous families $\mathcal{A}: X \rightarrow \mathcal{C}(H)$ such that the domains are a constant subspace $D$ of $H$. In this case $\mathfrak{D}(\mathcal{A})$ and $X \times D$ coincide as sets. Moreover, since any two closed operators on $D$ have equivalent graph norms by lemma A.2.8, we obtain that all fibres of $\mathfrak{D}(\mathcal{A})$ carry the same topology. The following lemma now gives a condition under which $\mathfrak{D}(\mathcal{A})$ and $X \times D$ coincide also as Banach bundles. We denote in the following by $D_{x_{0}}$ the normed linear space $D$ with respect to the graph norm of $\mathcal{A}_{x_{0}}$ for some $x_{0} \in X$.

6.1.12 Lemma. Let $\mathcal{A}: X \rightarrow \mathcal{C}(H)$ be gap continuous and $\mathcal{D}\left(\mathcal{A}_{x}\right)=D \subset H, x \in X$. If the induced family of bounded operators

$$
\mathcal{A}: X \rightarrow \mathcal{L}\left(D_{x_{0}}, H\right)
$$

is continuous, then $\mathfrak{D}(\mathcal{A})=X \times D$ where $D$ has the topology induced by the graph norm of any of the operators $\mathcal{A}_{x}, x \in X$.

Proof. As we have already observed above, the identity map $X \times D \rightarrow \mathfrak{D}(\mathcal{A})$ is an isomorphism in any fibre. Moreover, the identity $X \times D_{x_{0}} \rightarrow X \times D$ is a global trivialisation of $X \times D$. If now $U_{x_{0}}$ is a trivialising neighbourhood of $\mathfrak{D}(\mathcal{A})$ and $\tau_{x_{0}}$ is the corresponding trivialisation according to 6.2 , then

$$
U_{x_{0}} \ni x \mapsto \tau_{x_{0}}(x)=P_{\operatorname{graph}\left(\mathcal{A}_{x_{0}}\right)}\left(\cdot, \mathcal{A}_{x} \cdot\right) \in \mathcal{L}\left(D_{x_{0}}, \operatorname{graph}\left(\mathcal{A}_{x_{0}}\right)\right)
$$


is continuous because of the continuity of 6.5. Hence the identity map $X \times D \rightarrow \mathfrak{D}(\mathcal{A})$ is a Banach bundle isomorphism according to lemma 1.2.2

6.1.13 Remark. $\quad$ i) We strongly believe that the continuity of 6.5 implies the gap continuity of $\mathcal{A}$ even if we have not found a proof yet. It is proved in [Le05, Prop. 2.2] that any family of selfadjoint operators such that 6.5 is continuous is actually continuous with respect to the Riesz metric on $\mathcal{C}^{s a}(H)$.

ii) It is shown in [Le05, Prop.2.4] that there exist gap continuous families $\mathcal{A}: X \rightarrow \mathcal{C}(H)$ having a constant domain such that 6.5 is not continuous. But since 6.5) is by definition continuous if $\mathcal{A}: X \times D \rightarrow X \times H$ is a bundle morphism, we infer by lemma 6.1.3 that there exist gap continuous families having a constant domain $D$ and $\mathfrak{D}(\mathcal{A})$ is not the product bundle $X \times D$.

Because a family of bounded operators is in particular gap continuous by lemma 2.1.5, we find an immediate consequence.

6.1.14 Corollary. If $\mathcal{A}: X \rightarrow \mathcal{L}(H)$ is a continuous family of bounded operators with respect to the norm topology, then

$$
\mathfrak{D}(\mathcal{A})=X \times H
$$

In view of the following results we want to point out that the domain bundle is a Hilbert bundle and hence every subbundle is direct.

The next observation is not very surprising when reminding that the fibres of the domain bundle carry the topologies induced by the graph norms of the corresponding operators.

6.1.15 Lemma. Let $X$ be paracompact and $\mathcal{A}: X \rightarrow \mathcal{C}(H)$ gap continuous. Then $\mathfrak{D}(\mathcal{A})$ is a subbundle of $X \times H$ if and only if each $\mathcal{A}_{x}$ is bounded.

Proof. If $\mathfrak{D}(\mathcal{A})$ is a subbundle of $X \times H$, then, by definition, each $\mathcal{D}\left(\mathcal{A}_{x}\right)=\mathfrak{D}(\mathcal{A})_{x} \subset H$ is closed with respect to the norm on $H$. Then $\mathcal{A}_{x}$ is a closed operator on $H$ having a closed domain and so it is bounded by lemma A.2.1

Conversely, if $\mathcal{A}_{x}$ is bounded, then the topology induced by the graph norm of $\mathcal{A}_{x}$ on $\mathcal{D}\left(\mathcal{A}_{x}\right)$ is just the topology induced by $H$. Moreover, each $\mathcal{D}\left(\mathcal{A}_{x}\right)$ is a closed subspace of $H$ by lemma A.2.1 which is complemented as every closed subspace in a Hilbert space. Since the inclusion $\iota: \mathfrak{D}(\mathcal{A}) \hookrightarrow X \times H$ is a bundle morphism by lemma 6.1.5, we obtain the assertion by lemma 1.2 .12

The following lemma considers a situation that appears frequently when dealing with families of unbounded operators and in combination with lemma 6.1 .12 it is our main result in order to describe the domain bundle in applications. 
6.1.16 Lemma. Let $X$ be paracompact and $\mathcal{A}_{1}, \mathcal{A}_{2}: X \rightarrow \mathcal{C}(H)$ be gap continuous families such that

$$
\mathcal{A}_{1, x} \subset \mathcal{A}_{2, x}, \quad x \in X
$$

Then $\mathfrak{D}\left(\mathcal{A}_{1}\right)$ is a direct subbundle of $\mathfrak{D}\left(\mathcal{A}_{2}\right)$.

Proof. Because of lemma 1.2 .12 it is enough to show that each fibre of $\mathfrak{D}\left(\mathcal{A}_{1}\right)$ is a closed subspace of the corresponding fibre of $\mathfrak{D}\left(\mathcal{A}_{2}\right)$ and that the canonical inclusion

$$
\iota: \mathfrak{D}\left(\mathcal{A}_{1}\right) \hookrightarrow \mathfrak{D}\left(\mathcal{A}_{2}\right)
$$

is a bundle morphism.

In order to prove the first assertion, note that $\operatorname{graph}\left(\mathcal{A}_{1, x}\right) \subset \operatorname{graph}\left(\mathcal{A}_{2, x}\right)$ is closed. Hence $\operatorname{graph}\left(\mathcal{A}_{1, x}\right)$ is mapped onto a closed set under the homeomorphism $P_{1}: \operatorname{graph}\left(\mathcal{A}_{2, x}\right) \rightarrow \mathfrak{D}\left(\mathcal{A}_{2}\right)_{x}$. But $P_{1}\left(\operatorname{graph}\left(\mathcal{A}_{1, x}\right)\right)$ is just $\mathfrak{D}\left(\mathcal{A}_{1}\right)_{x}$.

Let now $x_{0} \in X$ and $U_{x_{0}}$ be a common trivalising neighbourhood of $\mathfrak{D}\left(\mathcal{A}_{1}\right)$ and $\mathfrak{D}\left(\mathcal{A}_{2}\right)$ with respect to trivialisations

$$
\begin{gathered}
\tau_{1}: \pi^{-1}\left(U_{x_{0}}\right) \rightarrow U_{x_{0}} \times \operatorname{graph}\left(\mathcal{A}_{1, x_{0}}\right) \\
\tau_{2}: \pi^{-1}\left(U_{x_{0}}\right) \rightarrow U_{x_{0}} \times \operatorname{graph}\left(\mathcal{A}_{2, x_{0}}\right) .
\end{gathered}
$$

In order to prove the second assertion, we use lemma 1.2 .2 and hence have to show the continuity of

$$
\tau_{2} \circ \iota \circ \tau_{1}^{-1}=\tau_{2} \circ \tau_{1}^{-1}: U_{x_{0}} \rightarrow \mathcal{L}\left(\operatorname{graph}\left(\mathcal{A}_{1, x_{0}}\right), \operatorname{graph}\left(\mathcal{A}_{2, x_{0}}\right)\right) .
$$

By $6.3, \tau_{1}^{-1}$ is given by

$$
U_{x_{0}} \times \operatorname{graph}\left(\mathcal{A}_{1, x_{0}}\right) \ni(x, u) \mapsto P_{1}\left(P_{\operatorname{graph}\left(\mathcal{A}_{1, x_{0}}\right)} \mid \operatorname{graph}\left(\mathcal{A}_{1, x}\right)\right)^{-1} u \in \mathcal{D}\left(\mathcal{A}_{1, x}\right) .
$$

Since $\mathcal{A}_{1} \subset \mathcal{A}_{2}$, we have for $x \in U_{x_{0}}$

$$
\begin{aligned}
\mathcal{A}_{2, x} P_{1}\left(P_{\operatorname{graph}\left(\mathcal{A}_{1, x_{0}}\right)} \mid \operatorname{graph}\left(\mathcal{A}_{1, x}\right)\right)^{-1} & =\mathcal{A}_{1, x} P_{1}\left(P_{\operatorname{graph}\left(\mathcal{A}_{1, x_{0}}\right)} \mid \operatorname{graph}\left(\mathcal{A}_{1, x}\right)\right)^{-1} \\
& =\mathcal{A}_{1}\left(\tau_{1}^{-1}(x, \cdot)\right) \in \mathcal{L}\left(\operatorname{graph}\left(\mathcal{A}_{1, x_{0}}\right), H\right)
\end{aligned}
$$

and this is a continuous family of bounded operators since $\mathcal{A}_{1}: \mathfrak{D}\left(\mathcal{A}_{1}\right) \rightarrow X \times H$ is a bundle morphism by lemma 6.1.3. Hence 


$$
\begin{aligned}
& U_{x_{0}} \rightarrow \mathcal{L}\left(\operatorname{graph}\left(\mathcal{A}_{1, x_{0}}\right), \operatorname{graph}\left(\mathcal{A}_{2, x_{0}}\right)\right) \\
& x \mapsto\left(\tau_{2} \circ \tau_{1}^{-1}\right)_{x}=P_{\operatorname{graph}\left(\mathcal{A}_{2, x_{0}}\right)}\left(\cdot, \mathcal{A}_{2, x} P_{1}\left(\left.P_{\operatorname{graph}\left(\mathcal{A}_{1, x_{0}}\right)}\right|_{\operatorname{graph}\left(\mathcal{A}_{1, x}\right)}\right)^{-1} \cdot\right)
\end{aligned}
$$

is continuous as well and we are done.

The utility of the foregoing result can be indicated as follows: Let $H$ be the Hilbert space of square integrable functions on a certain domain, $D$ some suitable Sobolev space and $\mathcal{A}: D \rightarrow H$ a differential operator which is bounded with respect to the Sobolev norm and closed as an operator on $H$ with domain $D$. By imposing boundary conditions we can now create families $\mathcal{A}_{x}, x \in X$, of operators with domains $D_{x} \subset D$ where $X$ is any paracompact topological space. If the family $\left\{\mathcal{A}_{x}\right\}_{x \in X}$ of restrictions of $\mathcal{A}$ is now gap continuous, then its domain bundle is a subbundle of the product $X \times D$ by the foregoing lemma and 6.1.12.

As final property we note the following consequence of lemma 6.1.16.

6.1.17 Corollary. Let $X$ be paracompact and $\mathcal{A}: X \rightarrow \mathcal{C}(H)$ be a gap continuous family of densely defined symmetric operators. Then $\mathfrak{D}(\mathcal{A})$ is a direct subbundle of $\mathfrak{D}\left(\mathcal{A}^{*}\right)$.

Proof. By [Ka76, IV.2.18] we have

$$
\hat{\delta}(\operatorname{graph}(T), \operatorname{graph}(S))=\hat{\delta}\left(\operatorname{graph}\left(T^{*}\right), \operatorname{graph}\left(S^{*}\right)\right)
$$

for all densely defined operators $S, T \in \mathcal{C}(H)$. Hence $\mathcal{A}^{*}: X \rightarrow \mathcal{C}(H)$ is gap continuous. Now the assertion follows from lemma 6.1 .16 because $\mathcal{A}_{x} \subset \mathcal{A}_{x}^{*}, x \in X$.

6.1.18 Remark. $\quad$ i) The foregoing lemma imposes the question if there is a general way to compute the codimension of $\mathfrak{D}(\mathcal{A})$ in $\mathfrak{D}\left(\mathcal{A}^{*}\right)$.

ii) Assume that $\mathcal{A}: X \rightarrow \mathcal{C}(H)$ is a gap continuous family of densely defined operators. Then by lemma A.2.15 and 6.7), $\mathcal{A}^{*}: X \rightarrow \mathcal{C}(H)$ is a gap continuous family of densely defined operators as well. Now one may ask about relations between $\mathfrak{D}(\mathcal{A})$ and $\mathfrak{D}\left(\mathcal{A}^{*}\right)$.

Finally we want to sketch an alternative way to construct $\mathfrak{D}(\mathcal{A})$.

If $\mathcal{A}: X \rightarrow \mathcal{C}(H)$ is gap continuous, then the family of associated graphs in $H \times H$ is continuous in the Hilbert Grassmannian $\operatorname{Gr}(H \times H)$ which we introduced in section 2.4. We obtain a tautological Hilbert bundle $\gamma(H \times H)$ over $G r(H \times H)$ by defining its total space to be

$$
\{(W, u) \in G r(H \times H) \times(H \times H): u \in W\}
$$

as in the case of tautological bundles over finite dimensional Grassmannians (cf. [MS74, §5]). Then the pullback bundle $(\mathcal{A})^{*} \gamma(H \times H)$ is a Hilbert bundle over $X$ having the graphs of $\mathcal{A}$ as 
fibres and we can define $\mathfrak{D}(\mathcal{A})$ uniquely by requiring the projection $X \times(H \times H) \rightarrow X \times H$ of sets to induce a homeomorphism $(\mathcal{A})^{*} \gamma(H \times H) \rightarrow \mathfrak{D}(\mathcal{A})$. In view of the atlas of $\operatorname{Gr}(H \times H)$ we indicated in section 2.4 it should be easy to show that this construction leads to the same trivialisations 6.1 that we introduced directly at the beginning of this section.

\subsection{The Index Bundle}

In this section we give a second generalisation of the well known Atiyah-Jänich bundle. Assume that $\mathcal{A}: X \rightarrow \mathcal{C F}(H)$ is a gap continuous family of Fredholm operators parametrised by a paracompact and locally compact space $X$. We define the singular set of $\mathcal{A}$ to be

$$
\Sigma(\mathcal{A})=\left\{x \in X: 0 \in \sigma\left(\mathcal{A}_{x}\right)\right\},
$$

which is closed by lemma 2.3.6. Since the topology in the domain has no influence on the properties required in the definition of a Fredholm operator, the bundle morphism $\mathcal{A}: \mathfrak{D}(\mathcal{A}) \rightarrow$ $X \times H$ according to lemma 6.1 .3 is fibrewise a Fredholm operator. Therefore we can regard $\mathcal{A}$ as an element in $\mathfrak{F}(\mathfrak{D}(\mathcal{A}), X \times H)$ whose support is given by the singular set of $\mathcal{A}$ according to remark 6.1.4 Now, if $\Sigma(\mathcal{A})$ is compact, we obtain by the results of section 5.1 for any closed subspace $A \subset X$ such that $A \cap \Sigma(\mathcal{A})=\emptyset$ an element in $K$-theory

$$
\operatorname{ind}(\mathcal{A}) \in K(X, A),
$$

which we call the index bundle of $\mathcal{A}$.

Note at first that, if $\mathcal{A}: X \rightarrow \mathcal{B F}(H)$ is a continuous family of bounded Fredholm operators, we obtain the classical Atiyah-Jänich bundle because of corollary 6.1.14 and the discussion at the end of section 5.2

We now discuss the main properties of the index bundle in the case of families of generally unbounded Fredholm operators. Of course most of them follow immediately from the corresponding properties in section 5.2 and the properties of the domain bundle established in section 6.1

6.2.1 Lemma. Let $\mathcal{A}: X \rightarrow G \mathcal{C}(H) \subset \mathcal{C F}_{0}(H)$. Then

$$
\operatorname{ind}(\mathcal{A})=0 \in K(X, A) .
$$

Proof. Since the induced Banach bundle morphism has empty support, this is an immediate consequence of lemma 5.2 .1 .

6.2.2 Lemma. Let $Y$ be a paracompact and locally compact space, $B \subset Y$ closed and $f$ : $(Y, B) \rightarrow(X, A)$ proper. Then 


$$
\operatorname{ind}\left(f^{*} \mathcal{A}\right)=f^{*} \operatorname{ind}(\mathcal{A}) \in K(Y, B) .
$$

Proof. By lemma 6.1.8 we have $\mathfrak{D}\left(f^{*} \mathcal{A}\right)=f^{*} \mathfrak{D}(\mathcal{A})$ and hence $f^{*} \mathcal{A}$ is a bundle morphism between the bundles $f^{*} \mathfrak{D}(\mathcal{A})$ and $f^{*}(X \times H)=Y \times H$. Now the assertion follows from lemma 5.2.2.

6.2.3 Lemma. Let $H: I \times X \rightarrow \mathcal{C F}(H)$ be gap continuous with a compact singular set such that $A \cap \Sigma\left(H_{\lambda}\right)=\emptyset$ for all $\lambda \in I$. Then

$$
\operatorname{ind}\left(H_{0}\right)=\operatorname{ind}\left(H_{1}\right) \in K(X, A) .
$$

Proof. We consider the induced Banach bundle morphism

$$
H: \mathfrak{D}(H) \rightarrow(I \times X) \times H
$$

and obtain from lemma 5.2 .3

$$
\operatorname{ind}\left(\iota_{0}^{*} H\right)=\operatorname{ind}\left(\iota_{1}^{*} H\right) \in K(X, A),
$$

where

$$
\begin{aligned}
& \iota_{0}^{*}: \iota_{0}^{*} \mathfrak{D}(H) \rightarrow \iota_{0}^{*}((I \times X) \times H)=X \times H, \\
& \iota_{1}^{*}: \iota_{1}^{*} \mathfrak{D}(H) \rightarrow \iota_{1}^{*}((I \times X) \times H)=X \times H
\end{aligned}
$$

are to be interpreted as bundle morphisms. But since

$$
\iota_{0}^{*} \mathfrak{D}(H)=\mathfrak{D}\left(\iota_{0}^{*} H\right)=\mathfrak{D}\left(H_{0}\right), \quad \iota_{1}^{*} \mathfrak{D}(H)=\mathfrak{D}\left(\iota_{1}^{*} H\right)=\mathfrak{D}\left(H_{1}\right)
$$

by lemma 6.1 .8 , we infer

$$
\operatorname{ind}\left(\iota_{0}^{*} H\right)=\operatorname{ind}\left(H_{0}\right), \quad \operatorname{ind}\left(\iota_{1}^{*} H\right)=\operatorname{ind}\left(H_{1}\right),
$$

where the maps on the left hand sides are to be interpreted as bundle morphisms while the maps on the right hand sides are families of unbounded operators.

6.2.4 Corollary. If $\mathcal{A} \rightarrow \mathcal{C F}^{\text {sa }}(H)$ is a gap continuous family of selfadjoint operators having a compact singular set such that $A \cap \Sigma(\mathcal{A})=\emptyset$, then

$$
\operatorname{ind}(\mathcal{A})=0 \in K(X, A)
$$


Proof. Consider the homotopy

$$
H: I \times X \rightarrow \mathcal{C F}(H), \quad H(\lambda, x)=\mathcal{A}_{x}+\lambda i I
$$

which is gap continuous by theorem 2.2.1. Since each $\mathcal{A}_{x}$ is selfadjoint we infer $\Sigma\left(H_{\lambda}\right)=\emptyset$ for all $\lambda>0$ and so ind $\left(H_{\lambda}\right)=0 \in K(X, A)$ by lemma 6.2.1. Now the assertion follows from the foregoing lemma 6.2 .3 .

6.2.5 Lemma. Let $K: X \rightarrow \mathcal{K}(H)$ be a continuous family of compact linear operators such that $\mathcal{A}+\lambda K$ has a compact singular set in $X \times I$ and $A \cap \Sigma(\mathcal{A}+\lambda \cdot K)=\emptyset$ for all $\lambda \in I$. Then

$$
\operatorname{ind}(\mathcal{A}+K)=\operatorname{ind}(\mathcal{A}) \in K(X, A)
$$

Proof. Since $\mathfrak{D}(\mathcal{A}+K)=\mathfrak{D}(\mathcal{A})$, the perturbed family defines a Banach bundle morphism $\mathcal{A}+K$ : $\mathfrak{D}(\mathcal{A}) \rightarrow X \times H$ which is the sum of a Fredholm operator and a compact operator in each fibre. Now the assertion follows from corollary 5.2 .5

6.2.6 Lemma. Let $\mathcal{A}_{1}: X \rightarrow \mathcal{C F}(H)$ and $\mathcal{A}_{2}: X \rightarrow \mathcal{C F}(H)$ be gap continuous with compact singular sets such that $A \cap\left(\Sigma\left(\mathcal{A}_{1}\right) \cup \Sigma\left(\mathcal{A}_{2}\right)\right)=\emptyset$. Then

$$
\operatorname{ind}\left(\mathcal{A}_{1} \oplus \mathcal{A}_{2}\right)=\operatorname{ind}\left(\mathcal{A}_{1}\right)+\operatorname{ind}\left(\mathcal{A}_{2}\right) \in K(X, A)
$$

Proof. Since obviously

$$
\mathfrak{D}\left(\mathcal{A}_{1} \oplus \mathcal{A}_{2}\right)=\mathfrak{D}\left(\mathcal{A}_{1}\right) \oplus \mathfrak{D}\left(\mathcal{A}_{2}\right),
$$

the result follows immediately from lemma 5.2 .6

Remembering the results of section 5.2, one may ask for the logarithmic property. Note that by theorem A.2.12 the product of two densely defined Fredholm operators is again Fredholm. Moreover, in [Ne68, Cor.2.5] it is proved that the product of two gap continuous families of such operators is continuous as well. However, to establish a logarithmic property seems to be a quite delicate question. Because in order to use the corresponding result 5.2.7 for Banach bundle morphisms, we need to consider the product $\mathcal{A}_{2} \mathcal{A}_{1}$ of densely defined Fredholm operators as a composition of bundle morphisms

$$
\mathfrak{D}\left(\mathcal{A}_{2} \mathcal{A}_{1}\right) \stackrel{\mathcal{A}_{1}}{\longrightarrow} \mathfrak{D}\left(\mathcal{A}_{2}\right) \stackrel{\mathcal{A}_{1}}{\longrightarrow} X \times H
$$

But it is neither clear that 


$$
\mathcal{A}_{1}: \mathfrak{D}\left(\mathcal{A}_{2} \mathcal{A}_{1}\right) \rightarrow \mathfrak{D}\left(\mathcal{A}_{2}\right)
$$

is a bundle morphism nor if its index bundle coincides with the index bundle of the original family $\mathcal{A}_{1}$. Nevertheless we can at least prove the following result.

6.2.7 Lemma. Let $U_{1}, U_{2}: X \rightarrow \mathcal{U}(H)$ be continuous families of unitary operators. Then

$$
U_{1} \mathcal{A} U_{2}: X \rightarrow \mathcal{C F}(H)
$$

is gap continuous and

$$
\operatorname{ind}\left(U_{1} \mathcal{A} U_{2}\right)=\operatorname{ind}(\mathcal{A}) \in K(X, A) .
$$

Proof. The graph of $U_{1} \mathcal{A} U_{2}$ is given by

$$
\begin{aligned}
\operatorname{graph}\left(U_{1} \mathcal{A} U_{2}\right) & =\left\{\left(u, U_{1} \mathcal{A} U_{2} u\right): u \in U_{2}^{*} \mathcal{D}(\mathcal{A})\right\} \\
& =\left\{\left(U_{2}^{*} v, U_{1} \mathcal{A} v\right): v \in \mathcal{D}(\mathcal{A})\right\} \\
& =\left(\begin{array}{cc}
U_{2}^{*} & 0 \\
0 & U_{1}
\end{array}\right) \operatorname{graph}(\mathcal{A})=: U \operatorname{graph}(\mathcal{A}) .
\end{aligned}
$$

Since the orthogonal projection onto $U \operatorname{graph}(\mathcal{A})$ is given by $U P_{\operatorname{graph}(\mathcal{A})} U^{*}$, we obtain for any $x, x_{0} \in X$

$$
\begin{aligned}
& d_{G}\left(U_{1, x} \mathcal{A}_{x} U_{2, x}, U_{1, x_{0}} \mathcal{A}_{x_{0}} U_{2, x_{0}}\right)=\left\|P_{\operatorname{graph}\left(U_{1, x} \mathcal{A}_{x} U_{2, x}\right)}-P_{\operatorname{graph}\left(U_{1, x_{0}} \mathcal{A}_{x_{0}} U_{2, x_{0}}\right)}\right\| \\
& =\left\|U_{x} P_{\operatorname{graph}\left(\mathcal{A}_{x}\right)} U_{x}^{*}-U_{x_{0}} P_{\operatorname{graph}\left(\mathcal{A}_{x_{0}}\right)} U_{x_{0}}^{*}\right\| \leq\left\|U_{x} P_{\operatorname{graph}\left(\mathcal{A}_{x}\right)} U_{x}^{*}-U_{x} P_{\operatorname{graph}\left(\mathcal{A}_{x}\right)} U_{x_{0}}^{*}\right\| \\
& +\left\|U_{x} P_{\operatorname{graph}\left(\mathcal{A}_{x}\right)} U_{x_{0}}^{*}-U_{x_{0}} P_{\operatorname{graph}\left(\mathcal{A}_{x}\right)} U_{x_{0}}^{*}\right\|+\left\|U_{x_{0}} P_{\operatorname{graph}\left(\mathcal{A}_{x}\right)} U_{x_{0}}^{*}-U_{x_{0}} P_{\operatorname{graph}\left(\mathcal{A}_{x_{0}}\right)} U_{x_{0}}^{*}\right\| \\
& \leq\left\|P_{\operatorname{graph}\left(\mathcal{A}_{x}\right)}-P_{\operatorname{graph}\left(\mathcal{A}_{x_{0}}\right)}\right\|+2\left\|U_{x}-U_{x_{0}}\right\| \\
& \leq d_{G}\left(\mathcal{A}_{x}, \mathcal{A}_{x_{0}}\right)+2\left\|U_{x}-U_{x_{0}}\right\|,
\end{aligned}
$$

where we use corollary 2.4.3 and lemma 2.1.3 in the last inequality. Hence the first assertion is proved.

In order to prove the second assertion, we use Kuiper's theorem as already quoted in the proof of theorem 1.1.8 in order to choose two homotopies

$$
H_{1}, H_{2}: I \times X \rightarrow \mathcal{U}(H)
$$

such that 


$$
H_{1}(0, \cdot)=U_{1}, \quad H_{2}(0, \cdot)=U_{2}, \quad H_{1}(1, \cdot)=H_{2}(1, \cdot)=I .
$$

Since the family

$$
H: I \times X \rightarrow \mathcal{C F}(H), \quad H(t, x)=H_{1}(t, x) \mathcal{A}_{x} H_{2}(t, x)
$$

is continuous with respect to the gap topology by our first assertion, we obtain by the homotopy invariance property 6.2 .3

$$
\operatorname{ind}\left(U_{1} \mathcal{A} U_{2}\right)=\operatorname{ind}(H(0))=\operatorname{ind}(H(1))=\operatorname{ind}(\mathcal{A}) \in K(X, A) .
$$

6.2.8 Remark. As we already mentioned above, one can prove that the product of two gap continuous families of densely defined Fredholm operators is again continuous in the gap topology. Since $\mathcal{U}(H)$ is a deformation retract of $G L(H)$, we obtain that lemma 6.2.7 actually holds true if $U_{1}$ and $U_{2}$ are families of bounded invertible operators.

We finally want to mention three constructions that are related to our definition of $\operatorname{ind}(\mathcal{A})$ for gap continuous families of closed operators.

In [Ne68] Gerhard Neubauer studied certain metric spaces consisting of graphs of closed operators between Banach spaces with the metric $\hat{\delta}$ we introduced in the first section of the second chapter. He constructed a set valued index for families of such graphs parametrized by a compact space and showed that in the case that all graphs correspond to Fredholm operators, the obtained set is actually a group which turned out to be isomorphic to $K(X)$. Finally, he even proved that his construction yields an isomorphism

$$
[X, \mathcal{C F}(H)] \rightarrow K(X)
$$

showing that $\mathcal{C} \mathcal{F}(H)$ with the gap topology represents the $K$-functor (compare theorem 4.4.3). We conjecture that Neubauer's index coincides with our construction. However, compared to our definition his index is difficult to compute because every map into the space of graphs has to be deformed into a canonical form before the element in $K(X)$ can be built. We do not know how complicate it can be to prove that both constructions yield the same element in $K(X)$ and leave this question open for future research.

A further related construction is due to Patrick M. Fitzpatrick and Maria Testa [FT94] who generalised the so called parity to unbounded Fredholm operators that had been defined before in the bounded case in [FP88]. They considered gap continuous families $\mathcal{A}: X \rightarrow \mathcal{C F}_{0}(H)$ of 
Fredholm operators of index 0 parametrised by a compact metric space and constructed for any choice of a subspace $V \subset H$ such that

$$
\operatorname{im}\left(\mathcal{A}_{x}\right)+V=H, \quad x \in X,
$$

a continuous family of projections $P: X \rightarrow \mathcal{L}(H)$ such that im $P_{x}=\mathcal{A}_{x}^{-1}(V)$.

We now can use proposition 1.2 .6 in order to obtain a finite dimensional bundle over $X$ from this family which has the same total space than our $E(\mathcal{A}, V)$ as defined in section 5.1. Moreover, it seems that assuming the base space to have a metric and restricting to Fredholm operators of index 0 is unnecessary in the construction in [FT94]. However, we loose the feature to view $\mathcal{A}$ as a bundle morphism between Banach bundles and to obtain $E(\mathcal{A}, V)$ from it as the kernel of an explicit bundle epimorphism. Moreover, it seems to be hard to prove the properties of the index bundle from their definition. Conversely, since our construction transforms a gap continuous family $\mathcal{A}: X \rightarrow \mathcal{C F}(H)$ to a family of bounded Fredholm operators, one should try to transfer the definition of parity from [FP88 to unbounded Fredholm operators by using the domain bundle instead of the family of projections $P$ from above.

Finally we want to mention the work by Michael Joachim [Jo03] which we already quoted in theorem 4.4.3. He constructed a different bijection as in 6.8 which is however far from being elementary and relies on a result due to himself, Bunke and Stolz [BJS03. Also here we do not know yet if this map coincides with the corresponding one induced by our index bundle ind and leave this question open for future research.

\subsection{On the Dimension of the Singular Set}

By definition of ind $(\mathcal{A})$ for a gap continuous family $\mathcal{A}: X \rightarrow \mathcal{C F}_{0}(H)$, it is clear that the results from section 5.3 on the dimension of the support are verbatim true also in this case.

Hence we can state without further work the following theorem which is an immediate consequence of proposition 5.3 .1

6.3.1 Proposition. Let $X$ be a compact orientable manifold of dimension $n$ such that $H_{2 k-1}(X ; \mathbb{Z})$ is free for some $1 \leq k \leq \frac{n-1}{2}$. If $\mathcal{A}: X \rightarrow \mathcal{C F}_{0}(H)$ is gap continuous and

$$
c_{k}(\operatorname{ind}(\mathcal{A})) \neq 0 \in H^{2 k}(X ; \mathbb{Z}),
$$

then the dimension of the singular set $\Sigma(\mathcal{A})$ is at least $n-2 k$ and it is not contractible to a point inside $X$.

Moreover, we obtain from proposition 5.3.4 and theorem 1.1.8.

6.3.2 Proposition. Let $X$ be a connected and orientable manifold having a $C W$-structure. Let $k \in \mathbb{N}$ be a natural number such that $k \leq \frac{n-1}{2}, H_{j}(X)=0,1 \leq j \leq 2 k-1$, and $\pi_{2 k}(X)=0$. 
Finally, let $\mathcal{A}: X \rightarrow \mathcal{C F}_{0}(H)$ be gap continuous having a compact singular set and $A \subset X$ be a closed subspace such that $A \cap \Sigma(\mathcal{A})=\emptyset$. If $\pi_{1}(X \backslash \Sigma(\mathcal{A}))$ is abelian and there exists $f \in \Omega^{2 k}(X, A)$ such that

$$
f^{*} \text { ind } \mathcal{A} \neq 0 \in K\left(I^{2 k}, \partial I^{2 k}\right) \cong \mathbb{Z}
$$

then $\operatorname{dim} \Sigma \geq n-2 k$ and $\Sigma(\mathcal{A})$ is not contractible.

6.3.3 Remark. As in section 5.3 we want to point out that our estimates can be improved when working on real Hilbert spaces and defining the index bundle in KO-theory. 


\section{Chapter 7}

\section{s-ind and Spectral Flow}

In this chapter we introduce a variant of the index bundle defined for families of generally unbounded selfadjoint Fredholm operators which corresponds to ind in the same way as the classical Atiyah-Jänich bundle to ind ${ }_{s}$ which we introduced in section 4.2 .

In the first section we give the definitions and prove basic properties. The second section is concerned with estimates of the dimension of the singular set of a given family of selfadjoint Fredholm operators. Here we can apply proposition 3.3.6 for the first time in order to conclude results on the dimension by homotopy classes of paths. In the final third section we show at first that the restriction of our selfadjoint index bundle to bounded families can be identified with ind ${ }_{s}$ canonically. Afterwards we prove the important result that the first Chern number of our selfadjoint index bundle of paths of Fredholm operators is the spectral flow BLP05] as introduced in section 4.3 .

\subsection{The Selfadjoint Index Bundle}

Let $X$ be a compact topological space and $\mathcal{A}: X \rightarrow \mathcal{C F}^{s a}(H)$ a gap continuous family of selfadjoint Fredholm operators. Then $\operatorname{ind}(\mathcal{A})=0$ by lemma 6.2.4 and our aim is to modify the construction of the index bundle in order to make it accessible for selfadjoint operators as well. We denote by $\Sigma(\mathcal{A}) \subset X$ the singular set of $\mathcal{A}$ and define

$$
\overline{\mathcal{A}}_{(x, s)} u=\mathcal{A}_{x} u+i s u, \quad \mathcal{D}\left(\overline{\mathcal{A}}_{(x, s)}\right)=\mathcal{D}\left(\mathcal{A}_{x}\right), \quad(x, s) \in X \times \mathbb{R} .
$$

Then $\overline{\mathcal{A}}$ is a gap continuous family of closed operators on $H$ according to theorem 2.2.1

7.1.1 Lemma. $\overline{\mathcal{A}}$ is a family of Fredholm operators of index 0 whose singular set is

$$
\Sigma(\overline{\mathcal{A}})=\Sigma(\mathcal{A}) \times\{0\} \subset X \times \mathbb{R}
$$


Proof. Let $(x, s) \in X \times \mathbb{R}$. If $s=0$, then $\overline{\mathcal{A}}_{(x, 0)}=\mathcal{A}_{x}$ is a selfadjoint Fredholm operator and so in particular of index 0 . Moreover, $\overline{\mathcal{A}}_{(x, 0)}$ does not have a bounded inverse if and only if $x \in \Sigma(\mathcal{A})$. If $s \neq 0$, then $\overline{\mathcal{A}}_{(x, s)} u=\mathcal{A}_{x} u+i s u$ has a bounded inverse because otherwise $0 \neq-i s \in \sigma\left(\mathcal{A}_{x}\right)$ in contradiction to the selfadjointness of $\mathcal{A}_{x}$.

7.1.2 Definition. Let $\mathcal{A}: X \rightarrow \mathcal{C F}^{s a}(H)$ be a gap continuous family of selfadjoint operators parametrised by a compact space $X$ and $A \subset X$ a closed subspace such that $A \cap \Sigma(\mathcal{A})=\emptyset$. The selfadjoint index bundle of $\mathcal{A}$ is defined by

$$
\operatorname{s-ind}(\mathcal{A})=\operatorname{ind}(\overline{\mathcal{A}}) \in K(X \times \mathbb{R}, A \times \mathbb{R})=K^{-1}(X, A) .
$$

Note that we do not give the most general definition here but restrict to compact spaces $X$ for the sake of simplicity. Accordingly the singular set $\Sigma(\mathcal{A})$ is automatically compact.

We assume throughout that $\mathcal{A}: X \rightarrow \mathcal{C F}^{s a}(H)$ is a gap continuous family and $A \subset X$ a closed subspace of the compact space $X$ such that $A \cap \operatorname{supp}(\mathcal{A})=\emptyset$.

7.1.3 Lemma (Normalisation). If $\mathcal{A}(X) \subset G \mathcal{C}^{s a}(H)$, then $\operatorname{sind}(\mathcal{A})=0 \in K^{-1}(X, A)$.

Proof. Since $\Sigma(\overline{\mathcal{A}})=\emptyset$ this is an immediate consequence of lemma 6.2.1

7.1.4 Lemma. Let $Y$ be compact, $B \subset Y$ closed and $f:(Y, B) \rightarrow(X, A)$ continuous. Then

$$
\operatorname{s-ind}\left(f^{*} \mathcal{A}\right)=\bar{f}^{*} \operatorname{s-ind}(\mathcal{A}) \in K^{-1}(Y, B),
$$

where

$$
\bar{f}: Y \times \mathbb{R} \rightarrow X \times \mathbb{R}, \quad \bar{f}(y, s)=(f(y), s)
$$

Proof. At first observe that

$$
{\overline{\left(f^{*} \mathcal{A}\right)_{(y, s)}}}=\mathcal{A}_{f(y)}+i s \cdot I=\left(\bar{f}^{*} \overline{\mathcal{A}}\right)_{(y, s)}, \quad(y, s) \in Y \times \mathbb{R} .
$$

Now we obtain

$$
\operatorname{s-ind}\left(f^{*} \mathcal{A}\right)=\operatorname{ind}\left(\overline{f^{*} \mathcal{A}}\right)=\operatorname{ind}\left(\bar{f}^{*} \overline{\mathcal{A}}\right)=\bar{f}^{*} \operatorname{ind}(\overline{\mathcal{A}})=\bar{f}^{*} \operatorname{s-ind}(\mathcal{A}),
$$

where the third equality follows by the corresponding property 6.2 .2 of ind.

7.1.5 Lemma. If $H: I \times X \rightarrow \mathcal{C F}^{s a}(H)$ is gap continuous and $A \cap \Sigma\left(H_{\lambda}\right)=\emptyset$ for all $\lambda \in I$, then

$$
\operatorname{s-ind}\left(H_{0}\right)=\operatorname{s-ind}\left(H_{1}\right) \in K^{-1}(X, A) .
$$


Proof. Define

$$
\bar{H}: I \times X \times \mathbb{R} \rightarrow \mathcal{C}(H), \quad \bar{H}(t, x, s)=H(t, x)+i s \cdot I
$$

which is a gap continuous family of closed operators by theorem 2.2.1. Moreover, we infer as in the proof of lemma 7.1.1 that $\bar{H}(t, x, s) \in \mathcal{C F}_{0}(H)$ for all $(t, x, s) \in I \times X \times \mathbb{R}$ and $\Sigma(\bar{H})=\Sigma(H) \times\{0\}$. Now we finally obtain by lemma 6.2 .3

$$
\operatorname{s-ind}\left(H_{0}\right)=\operatorname{ind}\left(\bar{H}_{0}\right)=\operatorname{ind}\left(\bar{H}_{1}\right)=\operatorname{s-ind}\left(H_{1}\right)
$$

By the corresponding discussion in section 6.2 we obtain the following invariance under compact perturbations.

7.1.6 Corollary. If $K: X \rightarrow \mathcal{K}(H)$ is a family of compact selfadjoint operators such that $A \cap \Sigma(\mathcal{A}+\lambda \cdot K)=\emptyset$ for any $0 \leq \lambda \leq 1$, then

$$
\operatorname{s-ind}(\mathcal{A}+K)=\operatorname{s-ind}(\mathcal{A}) \in K^{-1}(X, A)
$$

7.1.7 Lemma. Let $\mathcal{A}_{1}, \mathcal{A}_{2}: X \rightarrow \mathcal{C F}^{s a}(H)$ be two gap continuous families such that their singular sets are disjoint from $A$. Then

$$
\operatorname{s-ind}\left(\mathcal{A}_{1} \oplus \mathcal{A}_{2}\right)=\operatorname{s-ind}\left(\mathcal{A}_{1}\right)+\operatorname{s-ind}\left(\mathcal{A}_{2}\right) \in K^{-1}(X, A) \text {. }
$$

Proof. Since $\overline{\mathcal{A}_{1} \oplus \mathcal{A}_{2}}=\overline{\mathcal{A}_{1}} \oplus \overline{\mathcal{A}_{2}}$, the assertion follows from 6.2 .6

Note that the logarithmic property makes no sense here, because the product of two selfadjoint operators is in general not even symmetric. Nevertheless we have the following result which is an immediate consequence of lemma 6.2.7.

7.1.8 Lemma. If $U: X \rightarrow \mathcal{U}(H)$ is a continuous family of unitary operators, then $U^{*} \mathcal{A} U$ is gap continuous and

$$
\operatorname{s-ind}\left(U^{*} \mathcal{A} U\right)=\operatorname{s-ind}(\mathcal{A}) \in K^{-1}(X, A)
$$

Now we discuss properties that hold exclusively in the selfadjoint case.

7.1.9 Lemma. If $\mathcal{A}: X \rightarrow \mathcal{B F}^{s a}(H)$ is a family of bounded selfadjoint Fredholm operators, then

$$
\operatorname{s-ind}(-\mathcal{A})=-\operatorname{s-ind}(\mathcal{A}) \in K^{-1}(X, A) \text {. }
$$


Proof. We compute for $z=(x, s) \in X \times \mathbb{R}$

$$
\overline{-\mathcal{A}}_{z} \overline{\mathcal{A}}_{z}=\left(-\mathcal{A}_{x}+i s I\right)\left(\mathcal{A}_{x}+i s I\right)=-\mathcal{A}_{x}^{2}-s^{2} I .
$$

By using the homotopy

$$
H: I \times X \times \mathbb{R} \rightarrow \mathcal{C F}(H), \quad H(\lambda, x, s)=-\mathcal{A}_{x}^{2}-\left(s^{2}+\lambda\right) I
$$

and the homotopy invariance 6.2 .3 we infer that $\operatorname{ind}(\overline{-\mathcal{A}} \overline{\mathcal{A}})=0$. Since

$$
\mathfrak{D}(\overline{-\mathcal{A}} \overline{\mathcal{A}})=\mathfrak{D}(\overline{-\mathcal{A}})=\mathfrak{D}(\overline{\mathcal{A}})=X \times H
$$

according to corollary 6.1 .14 , we now can use the logarithmic property 5.2 .7 and finally obtain

$$
\operatorname{s-ind}(-\mathcal{A})=\operatorname{ind}(\overline{-\mathcal{A}})=-\operatorname{ind}(\overline{\mathcal{A}})=-\operatorname{s-ind} \mathcal{A} \in K^{-1}(X, A) .
$$

7.1.10 Remark. $\quad$ i) Since (7.1) is essential in the proof of lemma 7.1.9. we have not found a proof of the corresponding property for general gap continuous families $\mathcal{A}: X \rightarrow \mathcal{C F}^{\text {sa }}(H)$ yet.

ii) Note that in contrast to lemma 7.1.9 we have $\operatorname{ind}(-\mathcal{A})=\operatorname{ind}(\mathcal{A})$ for all $\mathcal{A}: X \rightarrow \mathcal{C F}(H)$ which follows from the homotopy

$$
H: I \times X \rightarrow \mathcal{C F}(H), \quad H(\lambda, x)=e^{i \pi \lambda} \mathcal{A}_{x} .
$$

Before we can go on, we need an auxiliary result about selfadjoint operators.

7.1.11 Lemma. Let $\mathcal{A}$ be a selfadjoint operator acting on the Hilbert space $H$.

i) If $B \in G L(H)$ is a bounded, invertible and selfadjoint operator, then $B \mathcal{A} B$ is selfadjoint.

ii) If $B \in G L(H)$ is bounded, selfadjoint and either positive or negative, then $\mathcal{A}+i B$ has a bounded inverse.

Proof. i) Note at first that $B \mathcal{A} B$ is densely defined because $\mathcal{D}(B \mathcal{A} B)=B^{-1} \mathcal{D}(\mathcal{A})$ and hence its adjoint is defined.

If $u, v \in \mathcal{D}(B \mathcal{A} B)$, then 


$$
\langle B \mathcal{A} B u, v\rangle=\langle\mathcal{A} B u, B v\rangle=\langle B u, \mathcal{A} B v\rangle=\langle u, B \mathcal{A} B v\rangle,
$$

where we use in the second equality that $B v \in \mathcal{D}(\mathcal{A})$. Hence $B \mathcal{A} B$ is symmetric, which means

$$
B^{-1} \mathcal{D}(\mathcal{A})=\mathcal{D}(B \mathcal{A} B) \subset \mathcal{D}\left((B \mathcal{A} B)^{*}\right)
$$

Now, let $v \in \mathcal{D}\left((B \mathcal{A} B)^{*}\right)$ be given. By definition this means that

$$
\mathcal{D}(B \mathcal{A} B) \ni u \mapsto\langle B \mathcal{A} B u, v\rangle \in \mathbb{C}
$$

is bounded and hence there exists a constant $c>0$ such that

$$
|\langle B \mathcal{A} B u, v\rangle| \leq c\|u\|, \quad u \in \mathcal{D}(B \mathcal{A} B) .
$$

Since $B$ is invertible and $\mathcal{D}(B \mathcal{A} B)=B^{-1} \mathcal{D}(\mathcal{A})$ we obtain for any $u \in \mathcal{D}(\mathcal{A})$

$$
|\langle\mathcal{A} u, B v\rangle|=|\langle B \mathcal{A} u, v\rangle|=\left|\left\langle B \mathcal{A} B B^{-1} u, v\right\rangle\right| \leq c\left\|B^{-1} u\right\| \leq c\left\|B^{-1}\right\|\|u\| .
$$

Hence $B v \in \mathcal{D}\left(\mathcal{A}^{*}\right)=\mathcal{D}(\mathcal{A})$ implying $v \in B^{-1} \mathcal{D}(\mathcal{A})=\mathcal{D}(B \mathcal{A} B)$.

To sum up, $B \mathcal{A} B$ is symmetric and $\mathcal{D}\left((B \mathcal{A} B)^{*}\right)=\mathcal{D}(B \mathcal{A} B)$ which implies that $B \mathcal{A} B$ is selfadjoint.

ii) First of all note that we can assume without loss of generality that $B$ is positive because otherwise we consider $-(-\mathcal{A}+i(-B))$.

If we assume $B$ to be positive we can build its square root $B^{\frac{1}{2}} \in \mathcal{L}(H)$ which is selfadjoint and invertible (cf. [Ka76, V.3.11]). We obtain

$$
\mathcal{A}+i B=\mathcal{A}+i B^{\frac{1}{2}} B^{\frac{1}{2}}=B^{\frac{1}{2}}\left(B^{-\frac{1}{2}} \mathcal{A} B^{-\frac{1}{2}}+i \cdot I\right) B^{\frac{1}{2}}
$$

and since the operator $B^{-\frac{1}{2}} \mathcal{A} B^{-\frac{1}{2}}$ is selfadjoint on the domain $B^{\frac{1}{2}} \mathcal{D}(\mathcal{A})$ by the first part of this lemma, we infer that $B^{-\frac{1}{2}} \mathcal{A} B^{-\frac{1}{2}}+i \cdot I$ has a bounded inverse.

Now we consider the bounded operator

$$
B^{-\frac{1}{2}}\left(B^{-\frac{1}{2}} \mathcal{A} B^{-\frac{1}{2}}+i \cdot I\right)^{-1} B^{-\frac{1}{2}} \in \mathcal{L}(H)
$$


on $H$ which maps $H$ onto $\mathcal{D}(\mathcal{A})$. By using $(7.2)$, we obtain

$$
\begin{aligned}
& (\mathcal{A}+i B) B^{-\frac{1}{2}}\left(B^{-\frac{1}{2}} \mathcal{A} B^{-\frac{1}{2}}+i \cdot I\right)^{-1} B^{-\frac{1}{2}} \\
& =B^{\frac{1}{2}}\left(B^{-\frac{1}{2}} \mathcal{A} B^{-\frac{1}{2}}+i \cdot I\right)\left(B^{-\frac{1}{2}} \mathcal{A} B^{-\frac{1}{2}}+i \cdot I\right)^{-1} B^{-\frac{1}{2}}=I_{H}
\end{aligned}
$$

and

$$
\begin{aligned}
& B^{-\frac{1}{2}}\left(B^{-\frac{1}{2}} \mathcal{A} B^{-\frac{1}{2}}+i \cdot I\right)^{-1} B^{-\frac{1}{2}}(\mathcal{A}+i B) \\
& =B^{-\frac{1}{2}}\left(B^{-\frac{1}{2}} \mathcal{A} B^{-\frac{1}{2}}+i \cdot I\right)^{-1}\left(B^{-\frac{1}{2}} \mathcal{A} B^{-\frac{1}{2}}+i \cdot I\right) B^{\frac{1}{2}}=I_{\mathcal{D}(\mathcal{A})} .
\end{aligned}
$$

Hence $\mathcal{A}+i B$ has a bounded inverse.

The following result will be of high importance in the sequel. It shows that we actually have more freedom in the definition of s-ind which can be very useful in computations as we will see in the proof of the first part of theorem 9.1.1 below.

As before, we assume $\mathcal{A}: X \rightarrow \mathcal{C F}^{s a}(H)$ to be gap continuous and $A \subset X$ to be closed such that $A \cap \Sigma(\mathcal{A})=\emptyset$.

7.1.12 Proposition. Let $B: X \rightarrow \mathcal{L}(H)$ be a continuous family of selfadjoint non negative operators such that $\mathcal{A}_{x}+i s B_{x}$ has a bounded inverse for all $s \neq 0$. Then

$$
\operatorname{sind}(\mathcal{A})=\operatorname{ind}(\mathcal{A}+i s B) \in K^{-1}(X, A) .
$$

Proof. Consider the homotopy

$$
H(\lambda, x, s)=\mathcal{A}_{x}+i s\left(\lambda I+(1-\lambda) B_{x}\right), \quad \mathcal{D}(H(\lambda, x, s))=\mathcal{D}\left(\mathcal{A}_{x}\right), \quad(\lambda, x, s) \in I \times X \times \mathbb{R},
$$

which is gap continuous by theorem 2.2.1. Since $\lambda I+(1-\lambda) B_{x}$ is selfadjoint for each $\lambda \in I$, $x \in X$, and

$$
\sigma\left(\lambda I+(1-\lambda) B_{x}\right)=\lambda+(1-\lambda) \sigma\left(B_{x}\right), \quad \lambda \in I, x \in X,
$$

these operators are positive if $\lambda \neq 0$. We obtain from lemma 7.1.11 that $H(\lambda, x, s)$ has a bounded inverse whenever $\lambda \neq 0$ and $s \neq 0$. Since $H(0, x, s)=\mathcal{A}_{x}+i s B_{x}$ has by assumption a bounded inverse for all $s \neq 0$ we conclude that $\Sigma(H)=I \times \Sigma(\mathcal{A}) \times\{0\}$. Hence the homotopy $H$ has a constant compact singular set in $I \times X \times \mathbb{R}$ and we obtain from the homotopy invariance property 6.2 .3 


$$
\operatorname{ind}(\mathcal{A}+i s B)=\operatorname{ind}(H(0))=\operatorname{ind}(H(1))=\operatorname{ind}(\mathcal{A}+i s \cdot I)=\operatorname{s-ind}(\mathcal{A}) \in K^{-1}(X, A) .
$$

7.1.13 Corollary. If $B: X \rightarrow \mathcal{L}(H)$ is a continuous family of selfadjoint positive operators, then

$$
\operatorname{s-ind}(\mathcal{A})=\operatorname{ind}(\mathcal{A}+i s B) \in K^{-1}(X, A) .
$$

Proof. We only have to show that any $\mathcal{A}_{x}+i s B_{x}$ has a bounded inverse if $s \neq 0$ which follows from lemma 7.1.11.

If we assume for the moment that $\mathcal{A}: X \rightarrow \mathcal{C F}^{s a}(H)$ is continuous with respect to the Riesz topology, then we can build s-ind $(\mathcal{A})$ because every Riesz continuous family is gap continuous as well by lemma 4.3.1. On the other hand, we can also proceed as usual in the case of Riesz continuous families and consider s-ind of the bounded transform $\mathcal{A}\left(I+\mathcal{A}^{2}\right)^{-\frac{1}{2}}: X \rightarrow \mathcal{B F}^{s a}(H)$. The next lemma ensures that we obtain the same elements in $K$-theory in both cases.

7.1.14 Lemma. Let $\mathcal{A}: X \rightarrow \mathcal{C F}^{s a}(H)$ be Riesz continuous. Then

$$
\operatorname{s-ind}(\mathcal{A})=\operatorname{s-ind}\left(\mathcal{A}\left(I+\mathcal{A}^{2}\right)^{-\frac{1}{2}}\right)
$$

Proof. First of all, by definition of the Riesz topology and (4.4) we know that the maps

$$
\begin{aligned}
& X \ni x \mapsto \mathcal{A}_{x}\left(I+\mathcal{A}_{x}^{2}\right)^{-\frac{1}{2}} \in \mathcal{L}(H) \\
& X \ni x \mapsto\left(I+\mathcal{A}_{x}^{2}\right)^{-\frac{1}{2}} \in \mathcal{L}(H)
\end{aligned}
$$

are continuous. Next we want to show that the map

$$
M: X \times \mathbb{R} \times H \rightarrow \mathfrak{D}(\bar{A}), \quad(x, s, u) \mapsto\left(I+\mathcal{A}_{x}^{2}\right)^{-\frac{1}{2}} u
$$

is a Banach bundle isomorphism. We note at first that each $M_{z}, z=(x, s) \in X \times \mathbb{R}$, is bijective and by lemma A.2.6 continuous with respect to the topology in $\mathfrak{D}(\overline{\mathcal{A}})_{z}$ which is induced by the norm $\|u\|_{H}+\left\|\left(\mathcal{A}_{x}+i s I\right) u\right\|_{H}$. Moreover, if $\tau_{z_{0}}$ denotes the trivialisation of $\mathfrak{D}(\overline{\mathcal{A}})$ at some $z_{0} \in X \times \mathbb{R}$ as defined in 6.2 , we obtain for all $z \in U_{z_{0}}$

$$
\left(\tau_{z_{0}} \circ M\right)_{z}=P_{\operatorname{graph}\left(\overline{\mathcal{A}}_{z_{0}}\right)}\left(\left(I+\mathcal{A}_{x}^{2}\right)^{-\frac{1}{2}} \cdot, \mathcal{A}_{x}\left(I+\mathcal{A}_{x}^{2}\right)^{-\frac{1}{2}} \cdot+i s\left(I+\mathcal{A}_{x}^{2}\right)^{-\frac{1}{2}} \cdot\right) \in \mathcal{L}\left(H, \operatorname{graph}\left(\overline{\mathcal{A}}_{z_{0}}\right)\right)
$$


which is continuous in $z$ due to the continuity of the maps 7.3 . Now lemma 1.2 .2 shows that $M$ is indeed a Banach bundle isomorphism.

Now we conclude from lemma 5.2 .7 that

$$
\operatorname{s-ind}(\mathcal{A})=\operatorname{ind}(\overline{\mathcal{A}})=\operatorname{ind}(\overline{\mathcal{A}})+\operatorname{ind}(M)=\operatorname{ind}(\overline{\mathcal{A}} \circ M) \in K^{-1}(X, A),
$$

where

$$
(\overline{\mathcal{A}} \circ M)_{z}=\mathcal{A}_{x}\left(I+\mathcal{A}_{x}^{2}\right)^{-\frac{1}{2}}+i s\left(I+\mathcal{A}_{x}^{2}\right)^{-\frac{1}{2}}
$$

and each $\left(I+\mathcal{A}_{x}^{2}\right)^{-\frac{1}{2}}$ is bounded and non negative. Since $(\overline{\mathcal{A}} \circ M)_{z}$ has for each $s \neq 0$ the inverse $\left(I+\mathcal{A}_{x}^{2}\right)^{\frac{1}{2}}\left(\mathcal{A}_{x}+i s\right)^{-1}$ which is bounded by corollary A.2.6. we obtain from proposition 7.1 .12

$$
\operatorname{ind}(\overline{\mathcal{A}} \circ M)=\operatorname{s-ind}\left(\mathcal{A}\left(I+\mathcal{A}_{x}^{2}\right)^{-\frac{1}{2}}\right) \in K^{-1}(X, A) .
$$

\subsection{On the Dimension of the Singular Set}

We now study which information we can gain from the selfadjoint index about the dimension of the singular set of $\mathcal{A}$.

We have for any compact topological space $X$ an isomorphism $K^{-1}(X) \cong \tilde{K}\left(\Sigma\left(X^{+}\right)\right)$by lemma B.2.1 where, according to our notation, $X^{+}$denotes the union of $X$ and a disjoint point.

7.2.1 Definition. For a compact space $X$ we define the odd $k$-th Chern class $\hat{c}_{k}, k \in \mathbb{N}$, as the composition of the following maps

$$
\hat{c}_{k}: K^{-1}(X)=K(X \times \mathbb{R}) \stackrel{\cong}{\rightrightarrows} \tilde{K}\left(\Sigma\left(X^{+}\right)\right) \stackrel{c_{k}}{\longrightarrow} H^{2 k}\left(\Sigma\left(X^{+}\right)\right) \stackrel{\cong}{\rightrightarrows} H^{2 k-1}(X) .
$$

We obtain immediately from the definition that $\hat{c}_{k}$ is indeed a characteristic class in the ordinary sense:

7.2.2 Lemma. If $X, Y$ are compact spaces and $f: X \rightarrow Y$ continuous, then

$$
\hat{c}_{k}\left(f^{*} \eta\right)=f^{*} \hat{c}_{k}(\eta), \quad \eta \in K^{-1}(Y) .
$$

Proof. This follows from the corresponding property of $c_{k}$, the naturality of the suspension isomorphism $H^{n+1}(\Sigma X) \stackrel{\cong}{\longrightarrow} H^{n}(X), n \in \mathbb{N}$, and lemma B.2.1 
7.2.3 Proposition. Let $X$ be a compact orientable manifold of dimension $n$ and $1 \leq k \leq \frac{n}{2}$ a natural number such that $H_{2 k-2}(X)$ is free. Let $\mathcal{A}: X \rightarrow \mathcal{C F}^{s a}(H)$ be a gap continuous family such that

$$
\hat{c}_{k}(\operatorname{s-ind}(\mathcal{A})) \neq 0 \in H^{2 k-1}(X) .
$$

Then $\operatorname{dim} \Sigma \geq n-2 k+1$ and $\Sigma$ is not contractible to a point in $X$.

Proof. We define for any compact subspace $X^{\prime} \subset X$

$$
\sigma_{k}\left(X^{\prime}\right)=\hat{c}_{k}\left(\operatorname{s-ind}\left(\mathcal{A} \circ \iota_{X^{\prime}}\right)\right) \in H^{2 k-1}\left(X^{\prime}\right)
$$

where $\iota_{X^{\prime}}: X^{\prime} \hookrightarrow X$ denotes the inclusion. It is clear by lemma 7.1.3 7.1.4 and 7.2.2 that $\sigma_{k}$ satisfies the properties required in theorem 3.2 .1

Let $\mathcal{A}:(X, A) \rightarrow\left(\mathcal{C F}^{s a}(H), \mathcal{G C}^{s a}(H)\right)$ be a gap continuous family and let $f \in \Omega^{2 k-1}(X, A)$ be given, where $A$ is a closed subspace of the compact space $X$. Then $\Gamma_{2 k-1}(f):=\operatorname{sind}\left(f^{*} \mathcal{A}\right)$ is an element in $K^{-1}\left(I^{2 k-1}, \partial I^{2 k-1}\right) \cong \mathbb{Z}$. In order to study the dimension of its singular set by this construction, we need as in section 5.3 the following technical result.

7.2.4 Lemma. Let $f_{1}, f_{2} \in \Omega^{k}(X, Y)$ be such that $f_{1} * f_{2}$ exists. Moreover, assume that $\mathfrak{D}(\overline{\mathcal{A}})$ is a trivial bundle. Then

$$
\operatorname{s-ind}\left(\left(f_{1} * f_{2}\right)^{*} \mathcal{A}\right)=\operatorname{s-ind}\left(f_{1}^{*} \mathcal{A}\right)+\operatorname{s-ind}\left(f_{2}^{*} \mathcal{A}\right) .
$$

Proof. We observe at first that by lemma 7.1 .4 and the definition of s-ind

$$
\operatorname{s-ind}\left(\left(f_{1} * f_{2}\right)^{*} \mathcal{A}\right)={\overline{f_{1} * f_{2}}}^{*} \operatorname{s-ind}(\mathcal{A})={\overline{f_{1} * f_{2}}}^{*} \operatorname{ind}(\overline{\mathcal{A}}) .
$$

Hence as in the proof of lemma 5.3 .3 we can use the normalisation and logarithmic property 5.2.1 and 5.2 .8 in order to assume without loss of generality that $\mathfrak{D}(\overline{\mathcal{A}})$ is a product $X \times H^{\prime}$ for some Hilbert space $H^{\prime}$.

Now the rest of the proof is verbatim as the proof of lemma 5.3.3 if we add a further component $s \in \mathbb{R}$ to each of the maps $f_{1}, f_{2}, f_{1} * f_{2}, g, g_{1}$ and $g_{2}$ which is, moreover, not even affected by the homotopies.

Note that $\mathfrak{D}(\overline{\mathcal{A}})$ is in particular trivial if $X$ is a CW-complex by theorem 1.1.8. Because the remaining assumptions of proposition 3.3.6 and 3.3.7 are clear for $\Gamma_{2 k-1}$ by lemma 7.1 .3 and lemma B.1.2, we obtain immediately the following two results. 
7.2.5 Proposition. Let $X$ be a simply connected manifold of dimension $n \geq 2$ possessing a $C W$-complex structure and $\mathcal{A}:(X, A) \rightarrow\left(\mathcal{C F}^{s a}(H), G \mathcal{C}^{s a}(H)\right)$ a gap continuous family. If there exists $f \in \Omega^{1}(X, A)$ such that $\operatorname{s-ind}\left(f^{*} \mathcal{A}\right) \neq 0 \in \mathbb{Z}$ then $\operatorname{dim} \Sigma \geq n-1$ and $\Sigma$ is not contractible.

7.2.6 Proposition. Let $X$ be a connected orientable manifold of dimension $n$ possessing a $C W$ structure and $\mathcal{A}:(X, A) \rightarrow\left(\mathcal{C F}^{s a}(H), G \mathcal{C}^{s a}(H)\right)$ be gap continuous. Assume that for some $k \in \mathbb{N}, 2 \leq k \leq \frac{n}{2}, H_{j}(X)=0$ for all $1 \leq j \leq 2 k-2, \pi_{2 k-1}(X)=0$ and that there exists $f \in \Omega^{2 k-1}(X, A)$ such that $\operatorname{sind}\left(f^{*} \mathcal{A}\right) \neq 0 \in \mathbb{Z}$. If $\pi_{1}(X \backslash \Sigma)$ is abelian, then $\operatorname{dim} \Sigma \geq n-2 k+1$ and $\Sigma$ is not contractible.

\section{3 s-ind, ind ind $_{s}$ the Spectral Flow}

In this final section on the selfadjoint index bundle s-ind we have two different aims. At first we want to compare our definition with the classical one due to Atiyah and Singer which we reminded of in section 4.2. We obtain that both coincide under a suitable isomorphism between the $K$ theory groups $\tilde{K}(S X)$ and $K(X \times \mathbb{R})$ to which the indices belong to. Our second aim is to compute the spectral flow of gap continuous paths in $\mathcal{C F}^{s a}(H)$ as introduced in section 4.3 by means of s-ind. Recall from section 4.2 that Atiyah, Patodi and Singer originally defined the spectral flow for paths of bounded selfadjoint Fredholm operators as the first Chern number of the selfadjoint index bundle $\operatorname{ind}_{s}$. We obtain that the first Chern number of the selfadjoint index bundle s-ind $\mathcal{A} \in K^{-1}(I, \partial I)$ gives for any gap continuous path $\mathcal{A}:(I, \partial I) \rightarrow\left(\mathcal{C F}^{s a}(H), G \mathcal{C}^{s a}(H)\right)$ the spectral flow $\operatorname{sf}(\mathcal{A})$ as defined by Booss-Bavnbek, Lesch, Phillips and introduced in section 4.3 .

In order to explain our first aim more detailed we let $X$ be a compact space and note that the one point compactification of $X \times \mathbb{R}$ is given by

$$
(X \times \mathbb{R})^{+}=X \times S^{1} / X \times\{1\}=S X
$$

which is the ordinary unreduced suspension of $X$ with the cusps identified to a single point. Remember the isomorphism $\varphi: \tilde{K}\left((X \times \mathbb{R})^{+}\right) \rightarrow K(X \times \mathbb{R})$ which we defined in lemma B.2.1. Now our aim is to show that the diagram

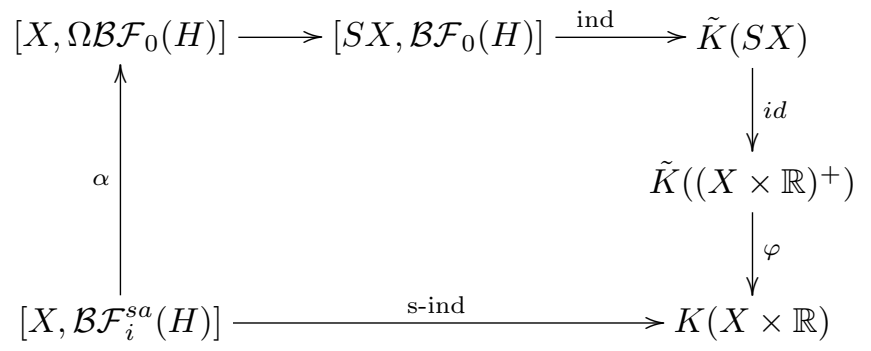


is commutative, where $\alpha$ is defined in section 4.2 and $\Omega \mathcal{B} \mathcal{F}_{0}(H)$ denotes the space of loops $s: S^{1} \rightarrow \mathcal{B F}_{0}(H)$. From the commutativity we infer

$$
\varphi\left(\operatorname{ind}_{s}(\mathcal{A})\right)=\text { s-ind } \mathcal{A}
$$

for any continuous family $\mathcal{A}: X \rightarrow \mathcal{B F}_{i}^{s a}(H)$ and hence we have shown that both definitions are equivalent in the bounded case.

Let $\mathcal{A}: X \rightarrow \mathcal{B F}_{i}^{s a}(H)$ be a continuous family. At first, the image of $\alpha(\mathcal{A})$ in $\left[S X, \mathcal{B F}_{0}\right]$ is given by

$$
\beta(\mathcal{A})(x, t)=\left\{\begin{array}{l}
\cos (t)+i \mathcal{A}_{x} \sin (t), \quad t \in[0, \pi] \\
\cos (t)+i \sin (t), \quad t \in[\pi, 2 \pi]
\end{array}\right.
$$

Now let $V \subset H$ be a finite dimensional subspace of $H$ which is transversal to $\operatorname{im} \beta(\mathcal{A})$ over $(X \times \mathbb{R})^{+}$in the sense of $(5.1)$. Since $\beta(\mathcal{A})$ defines a trivialisation of the bundle $E(\beta(\mathcal{A}), V)$ in a neighbourhood of $X \times\{1\} \subset X \times S^{1}$, we infer that $\varphi$ maps the class $[E(\beta(\mathcal{A}), V)]-[V]$ we obtained in $\tilde{K}\left((X \times \mathbb{R})^{+}\right)$to

$$
[E(\beta(\mathcal{A}), V), V, \beta(\mathcal{A})] \in K\left(X \times S^{1} \backslash X \times\{1\}\right)
$$

which is the index bundle of $\beta(\mathcal{A})$ regarded as a family on $X \times S^{1} \backslash X \times\{1\}=X \times(0,2 \pi)$. Finally we just have to identify this class with s-ind $(\mathcal{A}) \in K(X \times \mathbb{R})$. The homotopy $H$ : $I \times\left(X \times S^{1} \backslash X \times\{1\}\right) \rightarrow \mathcal{B F}_{0}(H)$ defined by

$$
H(\lambda, x, t)=\left\{\begin{array}{l}
\cos \frac{t}{\lambda+1}+i \mathcal{A}_{x} \sin \frac{t}{\lambda+1}, t \in(0, \pi(\lambda+1)) \\
\cos \frac{t}{\lambda+1}+i \sin \frac{t}{\lambda+1}, \quad t \in[\pi(\lambda+1), 2 \pi) .
\end{array}\right.
$$

shows that the index bundle we are studying is the index bundle of the family

$$
H(1, x, t)=\cos \frac{t}{2}+i \mathcal{A}_{x} \sin \frac{t}{2}, \quad(x, t) \in X \times S^{1} \backslash X \times\{1\}=X \times(0,2 \pi) .
$$

By using an obvious homotopy and $\sin (t)>0, t \in(0, \pi)$, we can deform $H(1, \cdot, \cdot)$ to

$$
\mathcal{A}_{x}-i \cot \frac{t}{2}, \quad(x, t) \in X \times S^{1} \backslash X \times\{1\}=X \times(0,2 \pi) .
$$

Finally, the isomorphism $f^{*}: K\left(X \times S^{1} \backslash X \times\{1\}\right) \rightarrow K(X \times \mathbb{R})$ induced by the homeomorphism 


$$
f: X \times \mathbb{R} \rightarrow X \times(0,2 \pi), \quad(x, s) \mapsto\left(x, \cot ^{-1}(-s)\right)
$$

$\operatorname{maps} \operatorname{ind}\left(\mathcal{A}-i \cot \frac{t}{2}\right) \in K\left(X \times S^{1} \backslash X \times\{0\}\right)$ to the index bundle of the family

$$
\mathcal{A}_{x}+i s \cdot I, \quad(x, s) \in X \times \mathbb{R},
$$

which is by definition s-ind $\mathcal{A} \in K(X \times \mathbb{R})=K^{-1}(X)$.

By using the results we mentioned in section 4.2 we in particular obtain from the commutativity of 7.4 that we can compute the spectral flow of closed paths of bounded selfadjoint Fredholm operators by using s-ind. However, the following result shows, by using Lesch's uniqueness theorem 4.3.7, that we even can compute the spectral flow of any gap continuous path of selfadjoint Fredholm operators with invertible ends by means of our selfadjoint index bundle s-ind.

7.3.1 Proposition. Let $H$ be separable and $\mathcal{A}:(I, \partial I) \rightarrow\left(\mathcal{C F}^{s a}(H), G \mathcal{C}^{s a}(H)\right)$ gap continuous. Then

$$
c_{1}(\operatorname{s-ind}(\mathcal{A}))=\operatorname{sf}(\mathcal{A}),
$$

where we use $c_{1}: K^{-1}(I, \partial I) \rightarrow \mathbb{Z}$ as defined in section B.3.

Proof. We have to check the conditions from theorem 4.3.7

The homotopy invariance follows from the homotopy invariance of s-ind proved in lemma 7.1.5. Moreover, the concatenation property is a consequence of lemma 7.2.4 Indeed, if $\mathcal{A}_{1}$ and $\mathcal{A}_{2}$ are two paths having invertible ends and such that $\mathcal{A}_{1} * \mathcal{A}_{2}$ exists, we define

$$
f_{1}, f_{2}: I \rightarrow I, \quad f_{1}(t)=\frac{t}{2}, f_{2}(t)=\frac{1}{2}(t+1) .
$$

Then $f_{1} * f_{2}=i d, f_{i}^{*}\left(\mathcal{A}_{1} * \mathcal{A}_{2}\right)=\mathcal{A}_{i}, i=1,2$, and the assertion follows from lemma 7.2.4 applied to $\mathcal{A}:=\mathcal{A}_{1} * \mathcal{A}_{2}:\left(I,\left\{0, \frac{1}{2}, 1\right\}\right) \rightarrow\left(\mathcal{C F}^{s a}(H), G \mathcal{C}^{s a}(H)\right)$.

Accordingly, it remains to show the normalisation property. As in the discussion around theorem 4.3 .7 we choose a complete orthonormal system $\left\{e_{k}\right\}_{k \in \mathbb{Z}}$ of $H$ and denote by $H_{+}$and $H_{-}$the closure of the infinite dimensional subspaces of $H$ which are spanned by all $e_{k}$ having positive or negative $k \in \mathbb{Z}$, respectively. Moreover, we denote by $H_{0}$ the one dimensional subspace spanned by $e_{0}$ and by $P_{+}, P_{-}$and $P_{0}$ the corresponding orthogonal projections.

Now we consider the path

$$
L:(I, \partial I) \rightarrow\left(\mathcal{C F}^{s a}(H), G \mathcal{C}^{s a}(H)\right), \quad L_{t}=\left(t-\frac{1}{2}\right) P_{0}+P_{+}-P_{-},
$$


whose spectral flow we determined in section 4.3 to be 1 . Hence the assertion is proved once we can show that also the first Chern number of s-ind $(L)$ is 1 .

Since $L_{t}$ is not invertible if and only if $t=\frac{1}{2}$, we obtain that $\operatorname{ker} L_{\frac{1}{2}}=\operatorname{im} P_{0}=H_{0}$ is a subspace of $H$ which is transversal to $L$ in the sense of (5.1). Moreover, from the $L$-invariant decomposition $H=H_{0} \oplus H_{+} \oplus H_{-}$it is clear that $\left(L_{t}+i s I\right)^{-1}\left(H_{0}\right)=H_{0}$ for all $t \in I, s \in \mathbb{R}$. We infer

$$
\operatorname{s-ind}(L)=\left[\Theta\left(H_{0}\right), \Theta\left(H_{0}\right),\left(t-\frac{1}{2}\right) P_{0}+i s \cdot I\right]=[\Theta(\mathbb{C}), \Theta(\mathbb{C}), \kappa] \in K^{-1}(I, \partial I),
$$

where the map $\kappa: I \times \mathbb{R} \rightarrow M(1, \mathbb{C})$ is defined by $\kappa(t, s)=t-\frac{1}{2}+i s$. Now it follows from section B.3 that

$$
c_{1}(\operatorname{s-ind}(L))=1 \in \mathbb{Z}
$$

7.3.2 Corollary. Let $\gamma:(I, \partial I) \rightarrow(X, A)$ be a continuous path and

$$
\mathcal{A}:(X, A) \rightarrow\left(\mathcal{C F}^{s a}(H), G \mathcal{C}^{s a}(H)\right)
$$

be gap continuous. Then

$$
c_{1}\left(\gamma^{*} \operatorname{s-ind}(\mathcal{A})\right)=\operatorname{sf}(\mathcal{A} \circ \gamma) .
$$

Finally, we obtain immediately from proposition 7.2.5 and the foregoing corollary

7.3.3 Corollary. Let $X$ be a simply connected manifold of dimension $n \geq 2$ possessing a $C W$ complex structure and $\mathcal{A}:(X, A) \rightarrow\left(\mathcal{C F}^{s a}(H), G \mathcal{C}^{s a}(H)\right)$ a gap continuous family. If there exists $\gamma \in \Omega^{1}(X, A)$ such that $\operatorname{sf}(\mathcal{A} \circ \gamma) \neq 0 \in \mathbb{Z}$ then $\operatorname{dim} \Sigma \geq n-1$ and $\Sigma$ is not contractible. 
Part III

\section{Family Index Theorems and Applications}





\section{Chapter 8}

\section{An Index Theorem for a Floer Family of Elliptic Boundary Value Problems}

In this first section of the third part we consider a family of ordinary differential operators of first order having varying domains parametrised by a compact topological space $X$. The particular type of family we consider is a special case of what is called Floer families of elliptic boundary value problems in the literature.

During this section we show that our family is gap continuous and consists of Fredholm operators of vanishing (numerical) index. We determine its domain bundle completely as a subbundle of the product bundle $X \times H^{1}\left(I, \mathbb{C}^{2 n}\right)$ and finally compute its index as a $K$-theory class over $X$. The result will play a significant role in the proof of the family Morse index theorem 9.1.1 below.

\subsection{The Index Theorem}

In the following we denote by $H^{1}\left(I, \mathbb{C}^{n}\right)$ the linear space of all absolutely continuous functions on the interval having a square integrable derivative. Recall that absolutely continuous functions are differentiable almost everywhere and hence the definition is indeed meaningful. It is a routine exercise to show that $H^{1}\left(I, \mathbb{C}^{n}\right)$ becomes a Hilbert space with respect to the scalar product

$$
\langle u, v\rangle_{H^{1}\left(I, \mathbb{C}^{n}\right)}=\langle u, v\rangle_{L^{2}\left(I, \mathbb{C}^{n}\right)}+\left\langle u^{\prime}, v^{\prime}\right\rangle_{L^{2}\left(I, \mathbb{C}^{n}\right)}, \quad u, v \in H^{1}\left(I, \mathbb{C}^{n}\right) .
$$

Moreover, it can be shown that the induced norm of this scalar product is equivalent to either of the following two 


$$
\begin{aligned}
\|u\| & =\|u\|_{\infty}+\left\|u^{\prime}\right\|_{L^{2}\left(I, \mathbb{C}^{n}\right)}, \quad u \in H^{1}\left(I, \mathbb{C}^{n}\right), \\
\|u\| & =\left\|u\left(t_{0}\right)\right\|+\left\|u^{\prime}\right\|_{L^{2}\left(I, \mathbb{C}^{n}\right)}, \quad u \in H^{1}\left(I, \mathbb{C}^{n}\right),
\end{aligned}
$$

where as usual $\|u\|_{\infty}=\max _{t \in I}\|u(t)\|$ and $t_{0} \in I$ is arbitrary. Details can be found for example in [Wa07, §6.2.1].

Let now $X$ be a locally compact and paracompact space,

$$
A: X \rightarrow G L(2 n, \mathbb{C}), \quad A_{x}=\left(\begin{array}{ll}
a_{x} & b_{x} \\
c_{x} & d_{x}
\end{array}\right)
$$

a continuous family of matrices and $Y \subset X$ a closed subspace such that the matrix $b_{x}$ is invertible if $x \in Y \cup(X \backslash K)$ for some compact subspace $K \subset X$. Let $\sigma \in G L(2 n, \mathbb{C})$ be an invertible matrix. We consider the family of first order differential operators

$$
\mathcal{A}_{x}: \mathcal{D}\left(\mathcal{A}_{x}\right) \subset L^{2}\left(I, \mathbb{C}^{2 n}\right) \rightarrow L^{2}\left(I, \mathbb{C}^{2 n}\right), \quad u \mapsto \sigma \frac{d u}{d t}
$$

defined on the domains

$$
\mathcal{D}\left(\mathcal{A}_{x}\right)=\left\{u \in H^{1}\left(I, \mathbb{C}^{2 n}\right): u(0) \in\{0\} \times \mathbb{C}^{n}, u(1) \in A_{x}\left(\{0\} \times \mathbb{C}^{n}\right)\right\}
$$

In accordance with [Ni97, §5.3] and [Ni07], we call $\mathcal{A}$ a Floer family of elliptic boundary value problems. Our aim of this chapter is to prove the following theorem.

8.1.1 Theorem. $\mathcal{A}$ is a gap continuous family of Fredholm operators of index 0 and its domain bundle is a $2 n$-codimensional subbundle of the product bundle $X \times H^{1}\left(I, \mathbb{C}^{2 n}\right)$. Moreover, $\mathcal{A}_{x}$ has a bounded inverse if and only if $b_{x}$ is invertible and its index bundle is given by

$$
\text { ind } \mathcal{A}=\left[\Theta\left(\mathbb{C}^{n}\right), \Theta\left(\mathbb{C}^{n}\right), b\right] \in K(X, Y)
$$

where $b: X \rightarrow M(n, \mathbb{C})$ is the matrix family defined by $b(x):=b_{x}$.

Note that theorem 8.1.1 can be used in order to construct families having non trivial index bundles. For example, set $X=D^{2} \subset \mathbb{C}, Y=S^{1}, n=1$, and define for $k \in \mathbb{Z}$

$$
A: D^{2} \rightarrow G L(2, \mathbb{C}), \quad z \mapsto\left(\begin{array}{cc}
1 & \rho(z) z^{k} \\
1 & \rho(z) z^{k}+1
\end{array}\right)
$$


where $\rho: D^{2} \rightarrow[0,1]$ is a cutoff function being identical to 1 outside a small ball around 0 and which vanishes at the origin. Then $\operatorname{det} A_{z}=1$ and hence $A_{z} \in G L(2, \mathbb{C})$ for all $z \in D^{2}$. Now we can compute as in section B.3

$$
\text { ind } \mathcal{A}=k \in \mathbb{Z} \cong K\left(D^{2}, S^{1}\right) \text {. }
$$

\subsection{Proof of the Index Theorem}

We prove the theorem step by step during this section.

\section{Step 1: Proof of the Closedness}

We start by showing that $\mathcal{A}_{x}, x \in X$, is a closed operator. Assume that $\left\{u_{n}\right\}_{n \in \mathbb{N}} \subset \mathcal{D}\left(\mathcal{A}_{x}\right)$ is a sequence and $u, v \in L^{2}\left(I, \mathbb{C}^{2 n}\right)$ are such that $u_{n} \rightarrow u$ and $\mathcal{A}_{x} u_{n} \rightarrow \sigma v$ in $L^{2}\left(I, \mathbb{C}^{2 n}\right)$. We have to show that $u \in \mathcal{D}\left(\mathcal{A}_{x}\right)$ and $\mathcal{A}_{x} u=\sigma v$.

Our first aim is to compute $u$. Since each $u_{n}$ is absolutely continuous, we have a representation

$$
u_{n}(t)=u_{n}(0)+\int_{0}^{t} u_{n}^{\prime}(s) d s, \quad t \in I, n \in \mathbb{N},
$$

and hence we obtain

$$
u_{n}(0)-u_{m}(0)=u_{n}(t)-u_{m}(t)+\int_{0}^{t}\left(u_{m}^{\prime}(s)-u_{n}^{\prime}(s)\right) d s, \quad t \in I, \quad m, n \in \mathbb{N} .
$$

By using the Minkowski inequality we now infer

$$
\begin{aligned}
\left\|u_{n}(0)-u_{m}(0)\right\| & =\left(\int_{0}^{1}\left\|u_{n}(0)-u_{m}(0)\right\|^{2} d s\right)^{\frac{1}{2}} \\
& \leq\left(\int_{0}^{1}\left\|u_{n}(s)-u_{m}(s)\right\|^{2} d s\right)^{\frac{1}{2}}+\left(\int_{0}^{1}\left\|\int_{0}^{s}\left(u_{m}^{\prime}(\tau)-u_{n}^{\prime}(\tau)\right) d \tau\right\|^{2} d s\right)^{\frac{1}{2}} \\
& \leq\left(\int_{0}^{1}\left\|u_{n}(s)-u_{m}(s)\right\|^{2} d s\right)^{\frac{1}{2}}+\left(\int_{0}^{1}\left(\int_{0}^{1}\left\|u_{m}^{\prime}(\tau)-u_{n}^{\prime}(\tau)\right\| d \tau\right)^{2} d s\right)^{\frac{1}{2}} \\
& =\left(\int_{0}^{1}\left\|u_{n}(s)-u_{m}(s)\right\|^{2} d s\right)^{\frac{1}{2}}+\int_{0}^{1}\left\|u_{m}^{\prime}(\tau)-u_{n}^{\prime}(\tau)\right\| d \tau \\
& \leq\left\|u_{n}-u_{m}\right\|_{L^{2}\left(I, \mathbb{C}^{2 n}\right)}+\left\|u_{n}^{\prime}-u_{m}^{\prime}\right\|_{L^{2}\left(I, \mathbb{C}^{2 n}\right)} .
\end{aligned}
$$

and hence $\left\{u_{n}(0)\right\}_{n \in \mathbb{N}}$ is a Cauchy sequence in $\mathbb{C}^{2 n}$ converging to some complex number $\alpha \in \mathbb{C}$.

We define 


$$
w(t)=\alpha+\int_{0}^{t} v(s) d s, \quad t \in I,
$$

and note that $w$ is absolutely continuous with derivative $v \in L^{2}\left(I, \mathbb{C}^{2 n}\right)$ and so an element of $H^{1}\left(I, \mathbb{C}^{2 n}\right)$. Moreover

$$
\begin{aligned}
\left\|u_{n}(t)-w(t)\right\| & \leq\left\|u_{n}(0)-\alpha\right\|+\left\|\int_{0}^{t} u_{n}^{\prime}(s) d s-\int_{0}^{t} v(s) d s\right\| \\
& \leq\left\|u_{n}(0)-\alpha\right\|+\int_{0}^{1}\left\|u_{n}^{\prime}(s)-v(s)\right\| d s \\
& \leq\left\|u_{n}(0)-\alpha\right\|+\left\|u_{n}^{\prime}-v\right\|_{L^{2}\left(I, \mathbb{C}^{2 n}\right)}
\end{aligned}
$$

and since $u_{n}(0) \rightarrow \alpha$ and $u_{n}^{\prime} \rightarrow v$, we conclude that $u_{n} \rightarrow w$ uniformly. This means in particular that $u_{n}$ converges to $w$ in $L^{2}\left(I, \mathbb{C}^{2 n}\right)$.

We infer that $w=u \in L^{2}\left(I, \mathbb{C}^{2 n}\right)$ because of $u_{n} \rightarrow u \in L^{2}\left(I, \mathbb{C}^{2 n}\right)$ by assumption. Hence we have found a representative $w$ of $u$ explicitly and now it remains to show that $w \in \mathcal{D}\left(\mathcal{A}_{x}\right)$ and $\mathcal{A}_{x} w=\sigma v$.

At first we have $\mathcal{A}_{x} w=\sigma w^{\prime}=\sigma v$ by definition of $w \in H^{1}\left(I, \mathbb{C}^{2 n}\right)$. In order to show that $w \in \mathcal{D}\left(\mathcal{A}_{x}\right)$, note that $w(0)=\alpha \in\{0\} \times \mathbb{C}^{n}$ since $\alpha=\lim _{n \rightarrow \infty} u_{n}(0)$ and $u_{n}(0) \in\{0\} \times \mathbb{C}^{n}$ for all $n \in \mathbb{N}$. Moreover, from 8.2 we obtain $u_{n}(1) \rightarrow w(1)$ and hence also $w(1) \in A_{x}\left(\{0\} \times \mathbb{C}^{n}\right)$ because of $u_{n}(1) \in A_{x}\left(\{0\} \times \mathbb{C}^{n}\right)$ for all $n \in \mathbb{N}$.

To sum up, we have shown $u=w \in \mathcal{D}\left(\mathcal{A}_{x}\right)$ and $\mathcal{A}_{x} u=\mathcal{A}_{x} w=\sigma v$ and hence we are done.

\section{Step 2: Proof of the Gap Continuity}

The aim of this second part of the proof is to show that $\mathcal{A}$ is a gap continuous family.

Let $x_{0} \in X$ be fixed and $\varepsilon>0$. At first we obtain

$$
\begin{aligned}
\left\|\left(u, \mathcal{A}_{x} u\right)-\left(v, \mathcal{A}_{x_{0}} v\right)\right\|_{L^{2}\left(I, \mathbb{C}^{2 n}\right) \oplus L^{2}\left(I, \mathbb{C}^{2 n}\right)} & =\left\|\left(u-v, \sigma\left(u^{\prime}-v^{\prime}\right)\right)\right\|_{L^{2}\left(I, \mathbb{C}^{2 n}\right) \oplus L^{2}\left(I, \mathbb{C}^{2 n}\right)} \\
& =\left(\|u-v\|_{L^{2}\left(I, \mathbb{C}^{2 n}\right)}^{2}+\left\|\sigma\left(u^{\prime}-v^{\prime}\right)\right\|_{L^{2}\left(I, \mathbb{C}^{2 n}\right)}^{2}\right)^{\frac{1}{2}} \\
& \leq(1+\|\sigma\|)\left(\|u-v\|_{L^{2}\left(I, \mathbb{C}^{2 n}\right)}^{2}+\left\|u^{\prime}-v^{\prime}\right\|_{L^{2}\left(I, \mathbb{C}^{2 n}\right)}^{2}\right)^{\frac{1}{2}} \\
& =(1+\|\sigma\|)\|u-v\|_{H^{1}\left(I, \mathbb{C}^{2 n}\right)},
\end{aligned}
$$

for all $u, v \in H^{1}\left(I, \mathbb{C}^{2 n}\right)$.

Let now $\tilde{P}: X \rightarrow M(2 n, \mathbb{C})$ denote the family of orthogonal projections onto the $n$-dimensional subspaces $A_{x}\left(\{0\} \times \mathbb{C}^{n}\right) \subset \mathbb{C}^{2 n}$. We define a family of operators by

$$
\left(P_{x} w\right)(t)=w(t)-(1-t) P_{\mathbb{C}^{n} \times\{0\}}(w(0))-t\left(I-\tilde{P}_{x}\right)(w(1)), \quad x \in X .
$$


It is readily seen that $P$ is a continuous family of operators in $H^{1}\left(I, \mathbb{C}^{2 n}\right)$ and $P_{x}$ is a projection onto $\mathcal{D}\left(\mathcal{A}_{x}\right), x \in X$. However, the only fact that we need here is that $\operatorname{im} P_{x} \subset \mathcal{D}\left(\mathcal{A}_{x}\right)$ which is clear from the definition of $P_{x}$. Now we infer

$$
\inf _{v \in \mathcal{D}\left(\mathcal{A}_{x_{0}}\right)}\|u-v\|_{H^{1}\left(I, \mathbb{C}^{2 n}\right)} \leq\left\|u-P_{x_{0}} u\right\|_{H^{1}\left(I, \mathbb{C}^{2 n}\right)}=\left\|\left(I-P_{x_{0}}\right) u\right\|_{H^{1}\left(I, \mathbb{C}^{2 n}\right)}, \quad u \in \mathcal{D}\left(\mathcal{A}_{x}\right) .
$$

Since

$\left(\left(I-P_{x_{0}}\right) u\right)(t)=(1-t) P_{\mathbb{C}^{n} \times\{0\}}(u(0))+t\left(I-\tilde{P}_{x_{0}}\right)(u(1))=t\left(I-\tilde{P}_{x_{0}}\right)(u(1)), \quad u \in \mathcal{D}\left(\mathcal{A}_{x}\right)$,

we obtain

$$
\begin{aligned}
\left\|\left(I-P_{x_{0}}\right) u\right\|_{H^{1}\left(I, \mathbb{C}^{2 n}\right)} & \leq 2\left\|\left(I-\tilde{P}_{x_{0}}\right)(u(1))\right\|_{\mathbb{C}^{2 n}}=2\left\|\left(I-\tilde{P}_{x_{0}}\right) \tilde{P}_{x}(u(1))\right\|_{\mathbb{C}^{2 n}} \\
& \leq 2\left\|\left(I-\tilde{P}_{x_{0}}\right) \tilde{P}_{x}\right\|\|u(1)\|_{\mathbb{C}^{2 n}}, \quad u \in \mathcal{D}\left(\mathcal{A}_{x}\right),
\end{aligned}
$$

where we use that $\tilde{P}_{x}(u(1))=u(1)$ because $u(1) \in \operatorname{im} \tilde{P}_{x}$.

Finally, since the point evaluation is continuous on $H^{1}\left(I, \mathbb{C}^{2 n}\right)$ according to 8.1 , there exists $c>0$ such that

$$
\begin{aligned}
\|u(1)\|_{\mathbb{C}^{2 n}} & \leq c\|u\|_{H^{1}\left(I, \mathbb{C}^{2 n}\right)}=c\left(\|u\|_{L^{2}\left(I, \mathbb{C}^{2 n}\right)}^{2}+\left\|u^{\prime}\right\|_{L^{2}\left(I, \mathbb{C}^{2 n}\right)}^{2}\right)^{\frac{1}{2}} \\
& =c\left(\|u\|_{L^{2}\left(I, \mathbb{C}^{2 n}\right)}^{2}+\left\|\sigma^{-1} \sigma u^{\prime}\right\|_{L^{2}\left(I, \mathbb{C}^{2 n}\right)}^{2}\right)^{\frac{1}{2}} \\
& \leq c\left(1+\left\|\sigma^{-1}\right\|\right)\left(\|u\|_{L^{2}\left(I, \mathbb{C}^{2 n}\right)}^{2}+\left\|\sigma u^{\prime}\right\|_{L^{2}\left(I, \mathbb{C}^{2 n}\right)}^{2}\right)^{\frac{1}{2}}
\end{aligned}
$$

for all $u \in H^{1}\left(I, \mathbb{C}^{2 n}\right)$.

To sum up, we have found

$$
\begin{aligned}
& \delta\left(\operatorname{graph}\left(\mathcal{A}_{x}\right), \operatorname{graph}\left(\mathcal{A}_{x_{0}}\right)\right)=\sup _{\substack{u \in \mathcal{D}\left(\mathcal{A}_{x}\right),\|u\|^{2}+\left\|\sigma u^{\prime}\right\|^{2}=1}} d\left(\left(u, \mathcal{A}_{x} u\right), \operatorname{graph}\left(\mathcal{A}_{x_{0}}\right)\right) \\
& \quad \leq 2 c(1+\|\sigma\|)\left(1+\left\|\sigma^{-1}\right\|\right)\left\|\left(I-\tilde{P}_{x_{0}}\right) \tilde{P}_{x}\right\| \quad \sup _{\substack{u \in \mathcal{D}\left(\mathcal{A}_{x}\right),\|u\|^{2}+\left\|\sigma u^{\prime}\right\|^{2}=1}}\left(\|u\|_{L^{2}\left(I, \mathbb{C}^{2 n}\right)}^{2}+\left\|\sigma u^{\prime}\right\|_{L^{2}\left(I, \mathbb{C}^{2 n}\right)}^{2}\right)^{\frac{1}{2}} \\
& \quad=2 c(1+\|\sigma\|)\left(1+\left\|\sigma^{-1}\right\|\right)\left\|\left(I-\tilde{P}_{x_{0}}\right) \tilde{P}_{x}\right\|, \quad x \in X,
\end{aligned}
$$

and if we interchange $x$ and $x_{0}$ we obtain by the very same argument

$$
\delta\left(\operatorname{graph}\left(\mathcal{A}_{x_{0}}\right), \operatorname{graph}\left(\mathcal{A}_{x}\right)\right) \leq 2 c(1+\|\sigma\|)\left(1+\left\|\sigma^{-1}\right\|\right)\left\|\left(I-\tilde{P}_{x}\right) \tilde{P}_{x_{0}}\right\|, \quad x \in X .
$$


Since $\left(I-\tilde{P}_{x_{0}}\right) \tilde{P}_{x_{0}}=0$ we can find an open neighbourhood $U$ of $x_{0}$ such that $\left\|\left(I-\tilde{P}_{x_{0}}\right) \tilde{P}_{x}\right\|<1$ for all $x \in U$. Moreover, we take an open neighbourhood $V$ of $x_{0}$ such that

$$
\left\|\tilde{P}_{x}-\tilde{P}_{x_{0}}\right\|<\min \left\{1, \frac{\varepsilon}{4 c(1+\|\sigma\|)\left(1+\left\|\sigma^{-1}\right\|\right)}\right\}, \quad x \in V .
$$

Now we obtain by theorem 2.4 .2 that

$$
\left\|\left(I-\tilde{P}_{x_{0}}\right) \tilde{P}_{x}\right\|=\left\|\left(I-\tilde{P}_{x}\right) \tilde{P}_{x_{0}}\right\|=\left\|\tilde{P}_{x}-\tilde{P}_{x_{0}}\right\|, \quad x \in U \cap V
$$

and hence

$$
d_{G}\left(\mathcal{A}_{x}, \mathcal{A}_{x_{0}}\right) \leq 2 \hat{\delta}\left(\operatorname{graph}\left(\mathcal{A}_{x}\right), \operatorname{graph}\left(\mathcal{A}_{x_{0}}\right)\right) \leq 4 c(1+\|\sigma\|)\left(1+\left\|\sigma^{-1}\right\|\right)\left\|\tilde{P}_{x}-\tilde{P}_{x_{0}}\right\|<\varepsilon
$$

for all $x \in U \cap V$.

\section{Step 3: The Fredholm Property}

Next we want to show that each $\mathcal{A}_{x}$ is a Fredholm operator of index 0. At first we consider its kernel. If $u \in H^{1}\left(I, \mathbb{C}^{n}\right)$ lies in the kernel of $\mathcal{A}_{x}$, it is a constant function. Hence we infer immediately from the boundary conditions

$$
\operatorname{dim} \operatorname{ker} \mathcal{A}_{x}=\operatorname{dim}\left(\left(\{0\} \times \mathbb{C}^{n}\right) \cap A_{x}\left(\{0\} \times \mathbb{C}^{n}\right)\right) .
$$

Now we study the cokernel of $\mathcal{A}_{x}$. For any $y \in L^{2}\left(I, \mathbb{C}^{2 n}\right)$, the functions

$$
u(t)=\sigma^{-1} \int_{0}^{t} y(s) d s+c, t \in I, \quad c \in \mathbb{C}^{2 n},
$$

satisfy $\sigma u^{\prime}=y$ and the boundary conditions require

$$
\begin{aligned}
& u(0)=c \in\{0\} \times \mathbb{C}^{n} \\
& u(1)=\sigma^{-1} \int_{0}^{1} y(s) d s+c \in A_{x}\left(\{0\} \times \mathbb{C}^{n}\right) .
\end{aligned}
$$

By writing $y(x)=\left(y(x)-\int_{0}^{1} y(s) d s\right)+\int_{0}^{1} y(s) d s$ it is clear that we have an orthogonal decomposition

$$
L^{2}\left(I, \mathbb{C}^{2 n}\right)=Y_{1} \oplus Y_{2}
$$


where $Y_{1}=\mathbb{C}^{2 n}$ denotes the $2 n$-dimensional space of constant functions and

$$
Y_{2}=\left\{y \in L^{2}\left(I, \mathbb{C}^{2 n}\right): \int_{0}^{1} y(s) d s=0\right\} .
$$

At first we observe that 8.3 holds for any $y \in Y_{2}$ just by setting $c=0$. If, however, $y \in Y_{1}$ we can find $v \in \mathbb{C}^{2 n}$ such that $y=\sigma v$ and now 8.3 reduces to the conditions

$$
c \in\{0\} \times \mathbb{C}^{n}, \quad v+c \in A_{x}\left(\{0\} \times \mathbb{C}^{n}\right) .
$$

It is readily seen that there exists $c \in\{0\} \times \mathbb{C}^{n}$ such that 8.5 holds for a given $v \in \mathbb{C}^{2 n}$ if and only if

$$
v \in \operatorname{span}\left\{\left(\{0\} \times \mathbb{C}^{n}\right) \cup A_{x}\left(\{0\} \times \mathbb{C}^{n}\right)\right\}=\left(\{0\} \times \mathbb{C}^{n}\right)+\left(A_{x}\left(\{0\} \times \mathbb{C}^{n}\right)\right) .
$$

By using the well known dimension formula for subspaces from elementary linear algebra we finally infer

$$
\operatorname{dim} \operatorname{coker} \mathcal{A}=\operatorname{dim}\left(\left(\{0\} \times \mathbb{C}^{n}\right) \cap A_{x}\left(\{0\} \times \mathbb{C}^{n}\right)\right) .
$$

Hence each $\mathcal{A}_{x}$ is a Fredholm operator of index 0.

We want to conclude this third step of the proof by showing that $\mathcal{A}_{x}$ has a bounded inverse if and only if $b_{x}$ is invertible. We just have shown that $\mathcal{A}_{x}$ is a Fredholm operator of index 0 which accordingly has a bounded inverse if and only if

$$
\operatorname{dim} \operatorname{ker} \mathcal{A}_{x}=\operatorname{dim}\left(\left(\{0\} \times \mathbb{C}^{n}\right) \cap A_{x}\left(\{0\} \times \mathbb{C}^{n}\right)\right)=0 .
$$

Now

$$
A_{x}\left(\begin{array}{l}
0 \\
u
\end{array}\right)=\left(\begin{array}{l}
b_{x} u \\
d_{x} u
\end{array}\right), \quad u \in \mathbb{C}^{n}
$$

and since ker $b_{x} \cap \operatorname{ker} d_{x}=\{0\}$ due to the invertibility of the matrix $A_{x}$, we infer that $\left(\{0\} \times \mathbb{C}^{n}\right) \cap A_{x}\left(\{0\} \times \mathbb{C}^{n}\right)=\{0\}$ if and only if $b_{x}$ is invertible. 


\section{Step 4: The Domain Bundle}

We consider the unbounded operator on $L^{2}\left(I, \mathbb{C}^{2 n}\right)$ given by

$$
\mathcal{A}^{\prime}: \mathcal{D}\left(\mathcal{A}^{\prime}\right)=H^{1}\left(I, \mathbb{C}^{2 n}\right) \subset L^{2}\left(I, \mathbb{C}^{2 n}\right) \rightarrow L^{2}\left(I, \mathbb{C}^{2 n}\right), \quad \mathcal{A}^{\prime} u=\sigma \frac{d u}{d t}
$$

$\mathcal{A}^{\prime}$ is closed which can be proved almost verbatim as for $\mathcal{A}_{x}$ in the first step above but can also easily be found in the literature (cf. eg. [We05, Bsp.IV.4.(c)]). Hence the constant family

$$
\mathcal{A}^{\prime}: X \rightarrow \mathcal{C}\left(L^{2}\left(I, \mathbb{C}^{2 n}\right)\right), \quad \mathcal{A}_{x}^{\prime}=\mathcal{A}^{\prime}
$$

is a gap continuous family of closed operators and according to lemma 6.1 .12 its domain bundle $\mathfrak{D}\left(\mathcal{A}^{\prime}\right)$ is the product $X \times H^{1}\left(I, \mathbb{C}^{2 n}\right)$ where $H^{1}\left(I, \mathbb{C}^{2 n}\right)$ carries the topology induced by the graph norm of $\mathcal{A}^{\prime}$. But, since the graph norm of $\mathcal{A}^{\prime}$ is equivalent to the standard Sobolev norm, this means that $H^{1}\left(I, \mathbb{C}^{2 n}\right)$ carries its usual topology.

Because of $\mathcal{A}_{x} \subset \mathcal{A}_{x}^{\prime}, x \in X$, and lemma 6.1.16 we now infer that $\mathfrak{D}(\mathcal{A})$ is a subbundle of the product $X \times H^{1}\left(I, \mathbb{C}^{2 n}\right)$.

In order to determine the codimension of $\mathfrak{D}(\mathcal{A})$ in $X \times H^{1}\left(I, \mathbb{C}^{2 n}\right)$ we consider a fixed $x \in X$ and define two $n$-dimensional subspaces of $H^{1}\left(I, \mathbb{C}^{2 n}\right)$ by

$$
\begin{aligned}
& H_{0}=\left\{u \in H^{1}\left(I, \mathbb{C}^{2 n}\right): u(t)=(1-t) v, v \in \mathbb{C}^{n} \times\{0\}\right\} \\
& H_{1}=\left\{u \in H^{1}\left(I, \mathbb{C}^{2 n}\right): u(t)=t v, v \in\left(A_{x}\left(\{0\} \times \mathbb{C}^{n}\right)\right)^{\perp}\right\},
\end{aligned}
$$

where $\left(A_{x}\left(\{0\} \times \mathbb{C}^{n}\right)\right)^{\perp}$ denotes the orthogonal complement of $A_{x}\left(\{0\} \times \mathbb{C}^{n}\right)$ in $\mathbb{C}^{2 n}$ with respect to the usual scalar product. Note that $\mathcal{D}\left(\mathcal{A}_{x}\right), H_{0}$ and $H_{1}$ have mutually trivial intersections.

Now let $P_{1}$ denote the orthogonal projection in $\mathbb{C}^{2 n}$ onto $\mathbb{C}^{n} \times\{0\}$ and $P_{2}$ the orthogonal projection onto $\left(A_{x}\left(\{0\} \times \mathbb{C}^{n}\right)\right)^{\perp}$. Then, given any $w \in H^{1}\left(I, \mathbb{C}^{2 n}\right)$, we have

$$
w(t)=\underbrace{\left(w(t)-(1-t) P_{1}(w(0))-t P_{2}(w(1))\right)}_{\in \mathcal{D}\left(\mathcal{A}_{x}\right)}+\underbrace{(1-t) P_{1}(w(0))}_{\in H_{0}}+\underbrace{t P_{2}(w(1))}_{\in H_{1}}
$$

and infer $H^{1}\left(I, \mathbb{C}^{2 n}\right)=\mathcal{D}\left(\mathcal{A}_{x}\right) \oplus H_{0} \oplus H_{1}$. Hence the codimension of $\mathfrak{D}(\mathcal{A})$ in $X \times H^{1}\left(I, \mathbb{C}^{2 n}\right)$ is indeed $2 n$. 


\section{Step 5: Computation of the Index Bundle}

Now we finally come to the computation of the index bundle ind $\mathcal{A}$. Recall that the domain of $\mathcal{A}_{x}$ is given by

$$
\mathcal{D}\left(\mathcal{A}_{x}\right)=\left\{u \in H^{1}\left(I, \mathbb{C}^{2 n}\right): u(0) \in\{0\} \times \mathbb{C}^{n}, u(1) \in A_{x}\left(\{0\} \times \mathbb{C}^{n}\right)\right\} .
$$

Let $Y_{1}, Y_{2} \subset L^{2}\left(I, \mathbb{C}^{2 n}\right)$ be defined as in (8.4) and $v \in Y_{2}$ be given. We define $u \in H^{1}\left(I, \mathbb{C}^{2 n}\right)$ by

$$
u(t)=\sigma^{-1} \int_{0}^{t} v(s) d s, \quad t \in I
$$

and from

$$
u(0)=u(1)=0 \in\left(\{0\} \times \mathbb{C}^{n}\right) \cap A_{x}\left(\{0\} \times \mathbb{C}^{n}\right)
$$

we infer that $u \in \mathcal{D}\left(\mathcal{A}_{x}\right)$. Since $\mathcal{A}_{x} u=v$, we have $\mathcal{A}_{x}\left(\mathcal{D}\left(\mathcal{A}_{x}\right)\right) \supset Y_{2}, x \in X$, and hence

$$
\operatorname{im} \mathcal{A}_{x}+Y_{1}=L^{2}\left(I, \mathbb{C}^{2 n}\right), \quad x \in X .
$$

Now the index bundle of $\mathcal{A}$ is given by

$$
\text { ind } \mathcal{A}=\left[E\left(\mathcal{A}, \Theta\left(Y_{1}\right)\right), \Theta\left(Y_{1}\right), \mathcal{A}\right] \in K(X, Y),
$$

where we use that we already have proved in the third step that $\mathcal{A}_{x}$ has a bounded inverse for all $x \in Y$. Note that the total space of $E\left(\mathcal{A}, \Theta\left(Y_{1}\right)\right)$ is just

$$
\begin{aligned}
& \left\{(x, w) \in X \times H^{1}\left(I, \mathbb{C}^{2 n}\right): w \in \mathcal{D}\left(\mathcal{A}_{x}\right), \sigma w^{\prime} \in Y_{1}\right\} \\
& =\left\{(x, w) \in X \times H^{1}\left(I, \mathbb{C}^{2 n}\right): w \in \mathcal{D}\left(\mathcal{A}_{x}\right), w^{\prime}=\text { const. }\right\} \\
& =\left\{(x, w) \in X \times H^{1}\left(I, \mathbb{C}^{2 n}\right): w(t)=(1-t) a+t b, a \in\{0\} \times \mathbb{C}^{n}, b \in A_{x}\left(\{0\} \times \mathbb{C}^{n}\right)\right\}
\end{aligned}
$$

We consider the bundle isomorphisms

$$
\begin{aligned}
& \Phi_{1}: \Theta\left(Y_{1}\right) \rightarrow \Theta\left(\mathbb{C}^{2 n}\right), \quad \Phi_{1}(x, u)=(x, u(0)), \\
& \Phi_{2}: E\left(\mathcal{A}, \Theta\left(Y_{1}\right)\right) \rightarrow \Theta\left(\mathbb{C}^{2 n}\right), \quad \Phi_{2}(x, w)=\left(x, p r_{2}(w(0)), p r_{2}\left(A_{x}^{-1} w(1)\right)\right),
\end{aligned}
$$

where $p r_{2}: \mathbb{C}^{2 n} \rightarrow \mathbb{C}^{n},(u, v) \mapsto v$, and the bundle morphism 


$$
\tilde{\mathcal{A}}: \Theta\left(\mathbb{C}^{2 n}\right) \rightarrow \Theta\left(\mathbb{C}^{2 n}\right), \quad \tilde{\mathcal{A}}(x, u, v)=\sigma\left(A_{x}(0, v)-(0, u)\right) .
$$

Let $w \in E\left(\mathcal{A}, \Theta\left(Y_{1}\right)\right)_{x}$, where $w(t)=(1-t) a+t b$ for some $a \in\{0\} \times \mathbb{C}^{n}, b \in A_{x}\left(\{0\} \times \mathbb{C}^{n}\right)$. We compute

$$
\Phi_{1}\left(\mathcal{A}_{x} w\right)=\Phi_{1}(\sigma(b-a))=\sigma(b-a)
$$

and

$$
\begin{aligned}
\tilde{\mathcal{A}}_{x} \Phi_{2, x} w & =\tilde{\mathcal{A}}\left(x, p r_{2}(a), p r_{2}\left(A_{x}^{-1} b\right)\right)=\sigma\left(A_{x}\left(0, p r_{2}(\underbrace{A_{x}^{-1} b}_{\in\{0\} \times \mathbb{C}^{n}})\right)-\left(0, p r_{2}(a)\right)\right) \\
& =\sigma(b-a) .
\end{aligned}
$$

Hence we have a commutative diagram

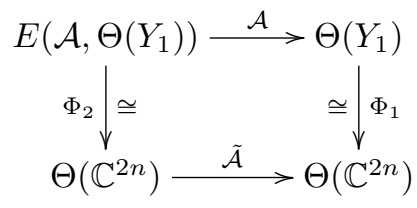

and infer

$$
\text { ind } \mathcal{A}=\left[\Theta\left(\mathbb{C}^{2 n}\right), \Theta\left(\mathbb{C}^{2 n}\right), \tilde{\mathcal{A}}\right] \in K(X, Y) \text {. }
$$

$\tilde{\mathcal{A}}$ is given explicitly by

$$
\tilde{A}(x, u, v)=\sigma\left(\left(\begin{array}{ll}
a_{x} & b_{x} \\
c_{x} & d_{x}
\end{array}\right)\left(\begin{array}{l}
0 \\
v
\end{array}\right)-\left(\begin{array}{l}
0 \\
u
\end{array}\right)\right)=\sigma\left(\left(\begin{array}{l}
b_{x} v \\
d_{x} v
\end{array}\right)-\left(\begin{array}{l}
0 \\
u
\end{array}\right)\right)=\sigma\left(\begin{array}{cc}
0 & b_{x} \\
-I & d_{x}
\end{array}\right)\left(\begin{array}{l}
u \\
v
\end{array}\right)
$$

and now we want to deform this family to a simpler one in two steps.

At first, since $G L(2 n, \mathbb{C})$ is path connected and $\sigma \in G L(2 n, \mathbb{C})$ is a constant matrix, we can find a path $\gamma: I \rightarrow G L(2 n, \mathbb{C})$ such that $\gamma(0)=\sigma$ and

$$
\gamma(1)=\left(\begin{array}{cc}
0 & -I \\
I & 0
\end{array}\right)
$$

By the product rule of the determinant we obtain that 


$$
H_{1}: I \times X \rightarrow M(2 n, \mathbb{C}), \quad H_{1}(\lambda, x)=\gamma(\lambda)\left(\begin{array}{cc}
0 & b_{x} \\
-I & d_{x}
\end{array}\right)
$$

is invertible if and only if $b_{x}$ is invertible, $x \in X$. Hence we deduce by the homotopy invariance of $K$-theory B.1.3

$$
\operatorname{ind}(\mathcal{A})=\left[\Theta\left(\mathbb{C}^{2 n}\right), \Theta\left(\mathbb{C}^{2 n}\right), \tilde{\mathcal{A}}\right]=\left[\Theta\left(\mathbb{C}^{2 n}\right), \Theta\left(\mathbb{C}^{2 n}\right), H_{1}(1, \cdot)\right] \in K(X, Y)
$$

where

$$
H_{1}(1, x)=\gamma(1)\left(\begin{array}{cc}
0 & b_{x} \\
-I & d_{x}
\end{array}\right)=\left(\begin{array}{cc}
0 & -I \\
I & 0
\end{array}\right)\left(\begin{array}{cc}
0 & b_{x} \\
-I & d_{x}
\end{array}\right)=\left(\begin{array}{cc}
I & -d_{x} \\
0 & b_{x}
\end{array}\right), \quad x \in X .
$$

Next we consider

$$
H_{2}: I \times X \rightarrow M(2 n, \mathbb{C}), \quad H_{2}(\lambda, x)=\left(\begin{array}{cc}
I & (\lambda-1) d_{x} \\
0 & b_{x}
\end{array}\right),
$$

shifting $-d_{x}$ linearly to 0 . Note that

$$
\operatorname{det}\left(H_{1}(1, x)\right)=\operatorname{det}\left(H_{2}(0, x)\right)=\operatorname{det}\left(H_{2}(\lambda, x)\right), \quad \lambda \in I, x \in X,
$$

and we obtain by using the homotopy invariance B.1.3 once again

$$
\text { ind } \mathcal{A}=\left[\Theta\left(\mathbb{C}^{2 n}\right), \Theta\left(\mathbb{C}^{2 n}\right), H_{2}(1, \cdot)\right] \in K(X, Y)
$$

where

$$
H_{2}(1, x)=\left(\begin{array}{cc}
I & 0 \\
0 & b_{x}
\end{array}\right)
$$

Finally we split off a trivial bundle according to the equivalence relation in $K(X, Y)$ and obtain the assertion

$$
\operatorname{ind}(\mathcal{A})=\left[\Theta\left(\mathbb{C}^{n}\right), \Theta\left(\mathbb{C}^{n}\right), b\right] \in K(X, Y),
$$

where $b: X \rightarrow M(n, \mathbb{C})$ is the matrix family defined by $b(x)=b_{x}$. 


\section{Chapter 9}

\section{The Morse Index Theorem for Families}

\subsection{Around the Index Theorem}

Let $X$ be a compact topological space, $Y \subset X$ a closed subspace and $S: I \times X \rightarrow M(n, \mathbb{R})$ a continuous family of real symmetric matrices such that $t \mapsto S_{x}(t)$ is smooth for any $x \in X$. We fix any norm $\|\cdot\|$ on $M(n, \mathbb{C})$ and denote

$$
\|M\|_{\infty}=\sup _{t \in I}\|M(t)\|
$$

for functions $M: I \rightarrow M(n, \mathbb{C})$. Furthermore, let $J=\operatorname{diag}(1, \ldots, 1,-1, \ldots,-1)$ be a diagonal matrix with 1 and -1 on the diagonal. We consider the family of unbounded differential operators

$$
\begin{gathered}
\mathcal{A}_{x}: \mathcal{D}\left(\mathcal{A}_{x}\right)=H^{2}\left(I, \mathbb{C}^{n}\right) \cap H_{0}^{1}\left(I, \mathbb{C}^{n}\right) \subset L^{2}\left(I, \mathbb{C}^{n}\right) \rightarrow L^{2}\left(I, \mathbb{C}^{n}\right), \\
\left(\mathcal{A}_{x} u\right)(t)=J u^{\prime \prime}(t)+S_{x}(t) u(t),
\end{gathered}
$$

where we assume that the singular set of $\mathcal{A}$ has an empty intersection with $Y$. In the following first section of this chapter we show that under the given assumptions $\mathcal{A}$ is a gap continuous family of selfadjoint Fredholm operators and so we can build its selfadjoint index bundle

$$
\operatorname{s-ind}(\mathcal{A})=\operatorname{ind}(\overline{\mathcal{A}}) \in K^{-1}(X, Y)
$$

which is defined according to section 7.1

The aim of this chapter is to find different ways to compute this $K$-theory class which we now describe briefly.

A first way is to consider the scalar products 


$$
\left\langle\mathcal{A}_{x} u, u\right\rangle_{L^{2}\left(I, \mathbb{C}^{n}\right)}=\int_{0}^{1}\left\langle J u^{\prime \prime}(t)+S_{x}(t) u(t), u(t)\right\rangle d t .
$$

Using integration by parts and a change of sign, we obtain a family of bounded quadratic forms

$$
q_{x}: H_{0}^{1}\left(I, \mathbb{C}^{n}\right) \rightarrow \mathbb{R}, \quad q_{x}(u)=\int_{0}^{1}\left\langle J u^{\prime}(t), u^{\prime}(t)\right\rangle d t-\int_{0}^{1}\left\langle S_{x}(t) u(t), u(t)\right\rangle d t
$$

which will turn out to be continuous in $x \in X$. Then by the Riesz representation theorem we can find a continuous family of bounded selfadjoint Fredholm operators $L: X \rightarrow \mathcal{L}\left(H_{0}^{1}\left(I, \mathbb{C}^{n}\right)\right)$ such that

$$
q_{x}(u)=\left\langle L_{x} u, u\right\rangle_{H_{0}^{1}\left(I, \mathbb{C}^{n}\right)}, \quad u \in H_{0}^{1}\left(I, \mathbb{C}^{n}\right), x \in X .
$$

It turns out that $L$ and $\mathcal{A}$ have the same singular set and hence we can define an index by

$$
\operatorname{s-ind}(q):=-\operatorname{s-ind}(L) \in K^{-1}(X, Y)
$$

where the right hand side is again defined according to section 7.1

A third way to assign a $K$-theory element to the family 9.1 is by using certain matrix valued solutions $b_{z}: I \rightarrow M(n, \mathbb{C}), z=(x, s) \in X \times \mathbb{R}$, of the family of differential equations

$$
J u^{\prime \prime}(t)+S_{x}(t) u(t)+i s u(t)=0
$$

such that $b_{z}(1)$ is not invertible if and only if $z=(x, s)=(x, 0) \in X \times \mathbb{R}$ and ker $\mathcal{A}_{x} \neq\{0\}$. Then we can define a $K$-theory class by

$$
\operatorname{con}(\mathcal{A})=\left[\Theta\left(\mathbb{C}^{n}\right), \Theta\left(\mathbb{C}^{n}\right), b^{T}\right] \in K^{-1}(X, Y),
$$

where

$$
b^{T}: X \times \mathbb{R} \rightarrow M(n, \mathbb{C}), \quad z \mapsto b_{z}(1)^{T}
$$

and ${ }^{T}$ denotes the transpose of a matrix instead of the conjugate transpose although $b_{z}$ has complex entries in general. We call $\operatorname{con}(\mathcal{A})$ the conjugate index of the family $\mathcal{A}$. With all this said, our main theorem reads as follows. 
9.1.1 Theorem. Let $X$ be a compact topological space, $Y \subset X$ a closed subspace, $J$ a diagonal matrix as above and $S: I \times X \rightarrow M(n, \mathbb{R})$ a continuous family of real symmetric matrices such that $t \mapsto S_{x}(t)$ is smooth for all $x \in X$. If the singular set of $\mathcal{A}$ intersects $Y$ trivially, then

$$
\operatorname{s-ind}(q)=\operatorname{s-ind}(\mathcal{A})=\operatorname{con}(\mathcal{A}) \in K^{-1}(X, Y) .
$$

We will give the proof of theorem 9.1.1 in section 9.5 below. Before that, we make the definitions of the indices precise during the following sections 9.2 .9 .4 and, first of all, we conclude this section by a few remarks on theorem 9.1.1.

The Morse index theorem is widely known as the equality of the Morse index of a geodesic in a Riemannian manifold and the number of conjugate points along the geodesic counted with multiplicities (cf. Mi69]). However, up to a canonical identification, the Morse index theorem is an assertion about differential equations which generalises parts of the well known Sturm theorems to systems of ordinary differential equations. In this form it can be found in the original reference [Mo34] due to Marston Morse and it follows from our theorem 9.1.1 in the special case that $X$ is the unit interval $I, Y$ its boundary $\partial I$ and $J$ the identity matrix. In the nineties the physicist Adam Helfer brought up the question if the classical Morse index theorem can be generalised to geodesics in general semi-Riemannian manifolds (cf. [Hel94]). The arising differential equations are now families like 9.1) where $X$ and $Y$ are again the unit interval and its boundary but $J$ is no longer necessarily the identity matrix. Helfer showed that the properties of the system of differential equations 9.1 which correspond to the geometric quantities in the classical Morse index theorem now change dramatically and that the classical result can no longer hold in the general semi-Riemannian case. However, he gave a first generalisation and later his ideas were developed further by Paolo Piccione, Daniel Victor Tausk and some coauthors in a series of papers (cf. PT02 and the references therein). In 2005 Monica Musso, Jacobo Pejsachowicz and Alessandro Portaluri obtained a further generalisation of the classical Morse index theorem to semi-Riemannian manifolds in [MPP05] by different methods and showed that their result comprises the former ones. If $X=I$ and $Y=\partial I$ our theorem 9.1.1 is precisely the index theorem [MPP05] under the identification $K^{-1}(X, Y) \cong \mathbb{Z}$ by the first Chern number. We will consider this case in the geometric framework of geodesics more precisely below in section 10.2. However, we want to point out that our proof of 9.1 .1 is completely different from the proof in [MPP05] which uses functional analysis instead of topological methods. First applications of theorem 9.1.1 to geodesics where $X$ is not assumed to be the unit interval will be given in the following eleventh chapter.

Our "topological" proof of the equality s-ind $(\mathcal{A})=\operatorname{con}(\mathcal{A})$ for $X=I$ and $Y=\partial I$ is the main result of Wha12]. But at that time we had not even found a way to connect the $K$-theory classes of s-ind $(q)$ and s-ind $(\mathcal{A})$ in this special case. Instead we just cited the proof in [MPP05] where perturbation results due to Robbin and Salamon (cf. [RS95]) and Fitzpatrick, Pejsachowicz, Recht (cf. [FPR99]) were used that hold exclusively for paths of operators. The proof of the 
family index theorem 9.1.1 now shows the corresponding equality for general families without any perturbation just by using results on the selfadjoint index bundle which we obtained in section 7.1 .

Finally we want to present briefly that our family index theorem 9.1.1 makes the Morse index theorem reminiscent of the Atiyah-Singer index theorem for families of selfadjoint elliptic operators. More details on the constructions which are described here only quite roughly can be found for example in BW85.

Let $X$ be a compact topological space and $\mathcal{A}: X \rightarrow \operatorname{Ell}(E ; M)$ be a family of selfadjoint elliptic operators acting on the smooth sections of a Hermitian vector bundle $E$ over the closed Riemannian manifold $M$. Denoting by

$$
\sigma_{x}(p, v): E_{p} \rightarrow E_{p} \quad p \in M, v \in T_{p}^{*} M,\|v\|=1
$$

the symbols of the operators $\mathcal{A}_{x}$, we define analogous to the map $\alpha$ in section 4.2 the two parameter family

$$
\sigma_{(x, t)}^{\prime}(p, v)=\left\{\begin{array}{l}
\cos (t)+i \sigma_{x}(p, v) \sin (t), \quad t \in[0, \pi] \\
\cos (t)+i \sin (t), \quad t \in[\pi, 2 \pi]
\end{array}\right.
$$

of elliptic symbols over $M$. Now this defines an automorphism of $\pi^{*} E$, where $\pi: S^{1} \times X \times$ $S(M) \rightarrow M$ is the projection and $S(M)$ the unit sphere bundle of the cotangent bundle of $M$. We obtain an element in $K\left(S^{1} \times X \times D(M), S^{1} \times X \times S(M)\right) \cong K\left(S^{1} \times X \times T^{*} M\right)$ which is trivial on $\{0\} \times X \times T^{*} M$ and hence can be regarded as element $\left[\sigma^{\prime}(\mathcal{A})\right] \in K^{-1}\left(X \times T^{*} M\right)$. $\left[\sigma^{\prime}(\mathcal{A})\right]$ is called the stable symbol class of the family $\mathcal{A}$. Moreover, by applying the topological index from the Atiyah-Singer index theorem to $\left[\sigma^{\prime}(\mathcal{A})\right] \in K^{-1}\left(X \times T^{*} M\right)$ regarded as an element of $K\left(S^{1} \times X \times T^{*} M\right)$, we obtain a class in $K\left(S^{1} \times X\right)$ which lies in fact in $K^{-1}(X)$. This yields a topological index

$$
t-\text { ind }: K^{-1}\left(X \times T^{*} M\right) \rightarrow K^{-1}(X) .
$$

Now the Atiyah-Singer index theorem for families of selfadjoint elliptic operators APS76, Theorem 3.4] reads as follows.

9.1.2 Theorem. Let $X$ be a compact topological space and $\mathcal{A}: X \rightarrow \operatorname{Ell}(E ; M)$ be a family of selfadjoint elliptic operators acting on a smooth Hermitian vector bundle over the closed Riemannian manifold $M$ such that the induced family $\mathcal{A}: X \rightarrow L^{2}(M, E)$ is Riesz continuous. Then

$$
\operatorname{ind}_{s} \mathcal{A}\left(I+\mathcal{A}^{2}\right)^{-\frac{1}{2}}=t-i n d\left(\left[\sigma^{\prime}(\mathcal{A})\right]\right) \in K^{-1}(X) \text {. }
$$


In view of lemma 7.1.14 we see that the family Morse index theorem 9.1.1 is indeed of a similar form as the Atiyah-Singer theorem for selfadjoint operators 9.1.2 On the other hand one can also see clear differences between them: in 9.1.1 we consider operators with constant top order coefficients which guarantees triviality in the Atiyah-Singer theorem. On the other hand we consider boundary value problems in 9.1.1 whereas the Atiyah-Singer theorem deals with families of differential operators on closed manifolds.

\subsection{The Index s-ind $(\mathcal{A})$}

We consider the family of operators 9.1], which is

$$
\left(\mathcal{A}_{x} u\right)(t)=J u^{\prime \prime}(t)+S_{x}(t) u(t), \quad u \in H^{2}\left(I, \mathbb{C}^{n}\right) \cap H_{0}^{1}\left(I, \mathbb{C}^{n}\right) .
$$

Recall that $H^{2}\left(I, \mathbb{C}^{n}\right)$ consists of all functions $u: I \rightarrow \mathbb{C}^{n}$ which are differentiable with an absolutely continuous derivative and such that the almost everywhere existing function $u^{\prime \prime}$ belongs to $L^{2}\left(I, \mathbb{C}^{n}\right)$ (cf. Wei80, §6.4]).

We want to show at first that for any $x \in X, \mathcal{A}_{x}$ is a selfadjoint Fredholm operator. Afterwards we prove that the family $\mathcal{A}$ is continuous with respect to the gap topology.

Before we start the discussion, we state a common result that we will need several times in the sequel.

9.2.1 Lemma. Let $S: I \times X \rightarrow M(n, \mathbb{C})$ be a continuous family of matrices, where $X$ is any topological space. Then the map

$$
X \ni x \mapsto\left\|S_{x}\right\|_{\infty}=\sup _{t \in I}\left\|S_{x}(t)\right\| \in \mathbb{R}
$$

is continuous.

Proof. Let $x_{0} \in X$ and $\varepsilon>0$ be fixed.

If $t^{\prime} \in I$ is arbitrary, we can find an open neighbourhood $V_{t^{\prime}} \times U_{t^{\prime}} \subset I \times X$ of $\left(t^{\prime}, x_{0}\right)$ such that

$$
\left\|S_{x}(t)-S_{x_{0}}\left(t^{\prime}\right)\right\|<\frac{\varepsilon}{2}, \quad(t, x) \in V_{t^{\prime}} \times U_{t^{\prime}} .
$$

Now choose $t_{1}, \ldots, t_{N}$ and neighbourhoods $V_{t_{i}} \times U_{t_{i}}$ as above such that $I=\bigcup_{i=1}^{N} V_{t_{i}}$. We define $U_{x_{0}}=\bigcap_{i=1}^{N} U_{t_{i}} \subset X$ which is an open neighbourhood of $x_{0}$.

If now $t \in I$, there exists $t_{i}$ such that $t \in V_{t_{i}}$ and we obtain

$$
\left\|S_{x}(t)-S_{x_{0}}(t)\right\| \leq\left\|S_{x}(t)-S_{x_{0}}\left(t_{i}\right)\right\|+\left\|S_{x_{0}}\left(t_{i}\right)-S_{x_{0}}(t)\right\|<\varepsilon, \quad x \in U_{x_{0}} .
$$


Hence

$$
\left|\left\|S_{x}\right\|_{\infty}-\left\|S_{x_{0}}\right\|_{\infty}\right| \leq\left\|S_{x}-S_{x_{0}}\right\|_{\infty}<\varepsilon, \quad x \in U_{x_{0}}
$$

Now we show that each $\mathcal{A}_{x}$ is a selfadjoint Fredholm operator. We note at first the well known result that the operator

$$
T: \mathcal{D}(T)=H^{2}\left(I, \mathbb{C}^{n}\right) \cap H_{0}^{1}\left(I, \mathbb{C}^{n}\right) \subset L^{2}\left(I, \mathbb{C}^{n}\right) \rightarrow L^{2}\left(I, \mathbb{C}^{n}\right), \quad u \mapsto J u^{\prime \prime}
$$

is selfadjoint which can be found for example in [Wei80, Th. 8.26]. Hence $\mathcal{A}_{x}, x \in X$, is the sum of the selfadjoint operator $T$ and the bounded selfadjoint operator

$$
B_{x}: L^{2}\left(I, \mathbb{C}^{n}\right) \rightarrow L^{2}\left(I, \mathbb{C}^{n}\right), \quad u \mapsto S_{x} u
$$

and so is itself selfadjoint by the well known Rellich perturbation theorem (cf. [Ka76, V.4.3]). In order to show that $\mathcal{A}_{x}$ is Fredholm, we want to use lemma A.3.5 and accordingly have to show that $T$ has a compact resolvent. In order to do so we formally define

$$
T^{-1}: L^{2}\left(I, \mathbb{C}^{n}\right) \rightarrow L^{2}\left(I, \mathbb{C}^{n}\right), \quad\left(T^{-1} u\right)(t)=\int_{0}^{t} \int_{0}^{s} J u(\tau) d \tau d s-t \int_{0}^{1} \int_{0}^{s} J u(\tau) d \tau d s
$$

and note at first that $T^{-1} u \in H^{2}\left(I, \mathbb{C}^{n}\right) \cap H_{0}^{1}\left(I, \mathbb{C}^{n}\right)$ for all $u \in L^{2}\left(I, \mathbb{C}^{n}\right)$. Moreover, we obtain $T\left(T^{-1} u\right)=u$ for all $u \in L^{2}\left(I, \mathbb{C}^{n}\right)$ and

$$
\begin{aligned}
\left(T^{-1}(T u)\right)(t) & =\int_{0}^{t} \int_{0}^{s} u^{\prime \prime}(\tau) d \tau d s-t \int_{0}^{1} \int_{0}^{s} u^{\prime \prime}(\tau) d \tau d s \\
& =\int_{0}^{t} u^{\prime}(s)-u^{\prime}(0) d s-t \int_{0}^{1} u^{\prime}(s)-u^{\prime}(0) d s \\
& =u(t)-t u^{\prime}(0)+t u^{\prime}(0)=u(t), \quad t \in I,
\end{aligned}
$$

for all $u \in \mathcal{D}(T)$. Hence $T^{-1}$ is indeed the inverse of $T$ and the following lemma now finally shows that $\mathcal{A}_{x}, x \in X$, is a Fredholm operator.

9.2.2 Lemma. There exists a constant $c>0$ such that

$$
\left\|T^{-1} u\right\|_{H^{1}\left(I, \mathbb{C}^{n}\right)} \leq c\|u\|_{L^{2}\left(I, \mathbb{C}^{n}\right)}, \quad u \in L^{2}\left(I, \mathbb{C}^{n}\right),
$$

and $T^{-1}: L^{2}\left(I, \mathbb{C}^{n}\right) \rightarrow L^{2}\left(I, \mathbb{C}^{n}\right)$ is a compact operator. 
Proof. In order to prove the first assertion we take the second norm of (8.1) with $t_{0}=0$ and obtain

$$
\begin{aligned}
\left\|T^{-1} u\right\|_{H^{1}\left(I, \mathbb{C}^{n}\right)}^{2} & =\int_{0}^{1}\left\|\int_{0}^{t} J u(s) d s-\int_{0}^{1} \int_{0}^{s} J u(\tau) d \tau d s\right\|^{2} d t \\
& \leq \int_{0}^{1}\left(\left\|\int_{0}^{t} J u(s) d s\right\|+\left\|\int_{0}^{1} \int_{0}^{s} J u(\tau) d \tau d s\right\|\right)^{2} d t \\
& \leq \int_{0}^{1}\left(\int_{0}^{1}\|u(s)\| d s+\int_{0}^{1} \int_{0}^{1}\|u(\tau)\| d \tau d s\right)^{2} d t=4\left(\int_{0}^{1}\|u(s)\| d s\right)^{2} \\
& \leq 4\|u\|_{L^{2}\left(I, \mathbb{C}^{n}\right)}^{2}
\end{aligned}
$$

where we use that $J$ is a unitary matrix.

The second assertion now follows from the well known compactness of the embedding $H^{1}\left(I, \mathbb{C}^{n}\right) \hookrightarrow$ $L^{2}\left(I, \mathbb{C}^{n}\right)$ which is proved, for example, in [Wa07, 6.2.15].

Finally we want to show the gap continuity of the family $\mathcal{A}$. Note that according to theorem 2.2 .1 it is enough to prove the continuity of the family of bounded operators

$$
B: X \rightarrow \mathcal{L}\left(L^{2}\left(I, \mathbb{C}^{n}\right)\right),\left(B_{x} u\right)(t)=S_{x}(t) u(t)
$$

with respect to the norm topology. If $x_{0} \in X$ and $u \in L^{2}\left(I, \mathbb{C}^{n}\right)$ we obtain

$$
\left\|B_{x} u-B_{x_{0}} u\right\|_{L^{2}\left(I, \mathbb{C}^{n}\right)}^{2} \leq \int_{0}^{1} \|\left(S_{x}(t)-S_{x_{0}}(t)\left\|^{2}\right\| u(t)\left\|^{2} d t \leq\right\| S_{x}-S_{x_{0}}\left\|_{\infty}^{2}\right\| u \|_{L^{2}\left(I, \mathbb{C}^{n}\right)}^{2}\right.
$$

and hence

$$
\left\|B_{x}-B_{x_{0}}\right\|_{\mathcal{L}\left(L^{2}\left(I, \mathbb{C}^{n}\right)\right)} \leq\left\|S_{x}-S_{x_{0}}\right\|_{\infty}, \quad x \in X
$$

which shows the assertion because of lemma 9.2.1.

To sum up, we can define the selfadjoint index bundle

$$
\operatorname{s-ind}(\mathcal{A})=\operatorname{ind}(\overline{\mathcal{A}}) \in K^{-1}(X, Y)
$$

for any closed subspace $Y \subset X$ which intersects the singular set of $\mathcal{A}$ trivially. 


\subsection{The Index s-ind $(q)$}

In this section we consider the family

$$
q_{x}: H_{0}^{1}(I, \mathbb{C}) \rightarrow \mathbb{R}, \quad q_{x}(u)=\int_{0}^{1}\left\langle J u^{\prime}(t), u^{\prime}(t)\right\rangle d t-\int_{0}^{1}\left\langle S_{x}(t) u(t), u(t)\right\rangle d t
$$

of quadratic forms. In the following, we use the scalar product

$$
\langle u, v\rangle_{H_{0}^{1}\left(I, \mathbb{C}^{n}\right)}=\left\langle u^{\prime}, v^{\prime}\right\rangle_{L^{2}\left(I, \mathbb{C}^{n}\right)}, \quad u, v \in H_{0}^{1}\left(I, \mathbb{C}^{n}\right)
$$

whose induced norm is equivalent to the restriction of the usual Sobolev norm on $H^{1}\left(I, \mathbb{C}^{n}\right)$ by (8.1). In particular $H_{0}^{1}\left(I, \mathbb{C}^{n}\right)$ is a Hilbert space with respect to $\langle\cdot, \cdot\rangle_{H_{0}^{1}\left(I, \mathbb{C}^{n}\right)}$.

By the Riesz representation theorem there exists a family of bounded selfadjoint operators $L_{x}$ such that

$$
q_{x}(u)=\left\langle L_{x} u, u\right\rangle_{H_{0}^{1}\left(I, \mathbb{C}^{n}\right)}, \quad u \in H_{0}^{1}\left(I, \mathbb{C}^{n}\right), x \in X .
$$

One can show that $L_{x}$ is a continuous family of Fredholm operators just by using the theory of quadratic forms as in MPP05, Prop. 3.1]. Nevertheless here we want to compute $L_{x}$ explicitly instead. At first, we obtain for any $u \in H_{0}^{1}\left(I, \mathbb{C}^{n}\right)$ and $c_{1} \in \mathbb{C}^{n}$

$$
\begin{aligned}
0=\int_{0}^{1} \frac{d}{d t}\left\langle\int_{0}^{t} S_{x}(\tau) u(\tau) d \tau+c_{1}, u(t)\right\rangle d t & =\int_{0}^{1}\left\langle S_{x}(t) u(t), u(t)\right\rangle d t \\
& +\int_{0}^{1}\left\langle\int_{0}^{t} S_{x}(\tau) u(\tau) d \tau+c_{1}, u^{\prime}(t)\right\rangle d t
\end{aligned}
$$

and hence

$$
\begin{aligned}
q_{x}(u) & =\int_{0}^{1}\left\langle J u^{\prime}(t), u^{\prime}(t)\right\rangle d t-\int_{0}^{1}\left\langle S_{x}(t) u(t), u(t)\right\rangle d t \\
& =\int_{0}^{1}\left\langle J u^{\prime}(t), u^{\prime}(t)\right\rangle d t+\int_{0}^{1}\left\langle\int_{0}^{t} S_{x}(\tau) u(\tau) d \tau-\int_{0}^{1} \int_{0}^{s} u(\tau) d \tau d s, u^{\prime}(t)\right\rangle d t \\
& =\int_{0}^{1}\left\langle J u^{\prime}(t)+\int_{0}^{t} S_{x}(\tau) u(\tau) d \tau-\int_{0}^{1} \int_{0}^{s} u(\tau) d \tau d s, u^{\prime}(t)\right\rangle d t \\
& =\int_{0}^{1}\left\langle\frac{d}{d t}\left(J u(t)+\int_{0}^{t} \int_{0}^{s} S_{x}(\tau) u(\tau) d \tau d s-t \int_{0}^{1} \int_{0}^{s} S_{x}(\tau) u(\tau) d \tau d s\right), u^{\prime}(t)\right\rangle d t .
\end{aligned}
$$

Since the functions $J u(t)+\int_{0}^{t} \int_{0}^{s} S_{x}(\tau) u(\tau) d \tau d s-t \int_{0}^{1} \int_{0}^{s} S_{x}(\tau) u(\tau) d \tau d s$ obviously belong to $H_{0}^{1}\left(I, \mathbb{C}^{n}\right)$, we obtain that the Riesz representations of $q_{x}, x \in X$, are given by 


$$
\begin{gathered}
L_{x}: H_{0}^{1}\left(I, \mathbb{C}^{n}\right) \rightarrow H_{0}^{1}\left(I, \mathbb{C}^{n}\right), \\
\left(L_{x} u\right)(t)=J u(t)+\int_{0}^{t} \int_{0}^{s} S_{x}(\tau) u(\tau) d \tau d s-t \int_{0}^{1} \int_{0}^{s} S_{x}(\tau) u(\tau) d \tau d s .
\end{gathered}
$$

Since the operators $L_{x}$ are selfadjoint by definition, it remains to check that each $L_{x}$ is a Fredholm operator and that $L$ is a continuous family with respect to the norm topology.

9.3.1 Lemma. Each operator $L_{x}$ is a compact perturbation of a bounded invertible operator and hence in particular Fredholm.

Proof. Consider for a fixed $x \in X$ the two operators

$$
\begin{aligned}
& K_{x}^{1}: C\left(I, \mathbb{C}^{n}\right) \rightarrow H^{1}\left(I, \mathbb{C}^{n}\right), \quad\left(K_{x}^{1} u\right)(t)=\int_{0}^{t} \int_{0}^{s} S_{x}(\tau) u(\tau) d \tau d s \\
& K_{x}^{2}: C\left(I, \mathbb{C}^{n}\right) \rightarrow H^{1}\left(I, \mathbb{C}^{n}\right), \quad\left(K_{x}^{2} u\right)(t)=t \int_{0}^{1} \int_{0}^{s} S_{x}(\tau) u(\tau) d \tau d s .
\end{aligned}
$$

Note that $K_{x}^{1} u \in C^{2}\left(I, \mathbb{C}^{n}\right)$ and $K_{x}^{2} u \in C^{\infty}\left(I, \mathbb{C}^{n}\right)$ for any $u \in C\left(I, \mathbb{C}^{n}\right)$ such that indeed both operators map into $H^{1}\left(I, \mathbb{C}^{n}\right)$. Since the inclusion $\iota: H_{0}^{1}\left(I, \mathbb{C}^{n}\right) \hookrightarrow C\left(I, \mathbb{C}^{n}\right)$ is compact (cf. eg. Wa07, 6.2.14]), we obtain that the compositions $K_{x}^{1} \circ \iota, K_{x}^{2} \circ \iota$ are compact once we can show the boundedness of $K_{x}^{1}$ and $K_{x}^{2}$. In this case also the difference of their restrictions

$$
\left(K_{x}^{1}-K_{x}^{2}\right) \circ \iota: H_{0}^{1}\left(I, \mathbb{C}^{n}\right) \rightarrow H^{1}\left(I, \mathbb{C}^{n}\right)
$$

is compact and since the range of this map is actually contained in the subspace $H_{0}^{1}\left(I, \mathbb{C}^{2 n}\right)$, we obtain the assertion.

Hence we now concentrate on the proof of the continuity of $K_{x}^{1}, K_{x}^{2}$ on the domain $C\left(I, \mathbb{C}^{n}\right)$. Note at first that $K_{x}^{2}$ has finite rank and so is obviously bounded. Finally, for any $u \in C\left(I, \mathbb{C}^{n}\right)$ we find

$$
\begin{aligned}
\left\|K_{x}^{1} u\right\|_{H^{1}\left(I, \mathbb{C}^{n}\right)}^{2} & =\int_{0}^{1}\left\|\int_{0}^{t} S_{x}(\tau) u(\tau) d \tau\right\|^{2} d t \\
& \leq \int_{0}^{1}\left(\int_{0}^{t}\left\|S_{x}(\tau)\right\|\|u(\tau)\| d \tau\right)^{2} d t \\
& \leq\left\|S_{x}\right\|_{\infty}^{2}\|u\|_{\infty}^{2},
\end{aligned}
$$

where we use the second norm in 8.1 with $t_{0}=0$. Hence $K_{x}^{1}$ is bounded as well.

9.3.2 Lemma. The family $L: X \rightarrow \mathcal{L}\left(H_{0}^{1}\left(I, \mathbb{C}^{n}\right)\right)$ is continuous with respect to the norm topology. 
Proof. If $x_{0} \in X$ is fixed, we find for any $u \in H_{0}^{1}\left(I, \mathbb{C}^{n}\right)$

$$
\begin{aligned}
\| L_{x} u- & L_{x_{0}} u \|_{H_{0}^{1}\left(I, \mathbb{C}^{n}\right)}^{2} \\
& =\int_{0}^{1}\left\|\int_{0}^{t}\left(S_{x}(\tau)-S_{x_{0}}(\tau)\right) u(\tau) d \tau-\int_{0}^{1} \int_{0}^{s}\left(S_{x}(\tau)-S_{x_{0}}(\tau)\right) u(\tau) d \tau d s\right\|^{2} d t \\
& \leq \int_{0}^{1}\left(\int_{0}^{t}\left\|S_{x}(\tau)-S_{x_{0}}(\tau)\right\|\|u(\tau)\| d \tau+\int_{0}^{1} \int_{0}^{s}\left\|S_{x}(\tau)-S_{x_{0}}(\tau)\right\|\|u(\tau)\| d \tau d s\right)^{2} d t \\
& \leq\left\|S_{x}-S_{x_{0}}\right\|_{\infty}^{2} \int_{0}^{1}\left(\int_{0}^{t}\|u(\tau)\| d \tau+\int_{0}^{1} \int_{0}^{s}\|u(\tau)\| d \tau d s\right)^{2} d t \\
& \leq\left\|S_{x}-S_{x_{0}}\right\|_{\infty}^{2} \int_{0}^{1}\left(\int_{0}^{1}\|u(\tau)\| d \tau+\int_{0}^{1} \int_{0}^{1}\|u(\tau)\| d \tau d s\right)^{2} d t \\
& =4\left\|S_{x}-S_{x_{0}}\right\|_{\infty}^{2}\left(\int_{0}^{1}\|u(\tau)\| d \tau\right)^{2} \leq 4\left\|S_{x}-S_{x_{0}}\right\|_{\infty}^{2} \int_{0}^{1}\|u(\tau)\|^{2} d \tau \\
& =4\left\|S_{x}-S_{x_{0}}\right\|_{\infty}^{2}\|u\|_{L^{2}\left(I, \mathbb{C}^{n}\right)}^{2} \leq 4 c^{2}\left\|S_{x}-S_{x_{0}}\right\|_{\infty}^{2}\|u\|_{H_{0}^{1}\left(I, \mathbb{C}^{n}\right)}^{2},
\end{aligned}
$$

where we have used in the last step that

$$
\begin{aligned}
\|u\|_{L^{2}\left(I, \mathbb{C}^{n}\right)} & \leq\left(\|u\|_{L^{2}\left(I, \mathbb{C}^{n}\right)}^{2}+\left\|u^{\prime}\right\|_{L^{2}\left(I, \mathbb{C}^{n}\right)}^{2}\right)^{\frac{1}{2}}=\|u\|_{H^{1}\left(I, \mathbb{C}^{n}\right)} \\
& \leq c\|u\|_{H_{0}^{1}\left(I, \mathbb{C}^{n}\right)}, \quad u \in H_{0}^{1}\left(I, \mathbb{C}^{n}\right),
\end{aligned}
$$

for some $c>0$, which follows from (8.1) for $t_{0}=0$. We obtain

$$
\left\|L_{x}-L_{x_{0}}\right\| \leq 2 c\left\|S_{x}-S_{x_{0}}\right\|_{\infty}
$$

and hence the assertion by lemma 9.2.1

As the final result of this section we show that the singular sets of $\mathcal{A}$ and $L$ coincide.

9.3.3 Lemma. $\operatorname{ker} \mathcal{A}_{x}=\operatorname{ker} L_{x}, x \in X$.

Proof. By definition of $L_{x}$ we have

$$
\left\langle\mathcal{A}_{x} u, v\right\rangle_{L^{2}\left(I, \mathbb{C}^{n}\right)}=\left\langle L_{x} u, v\right\rangle_{H_{0}^{1}\left(I, \mathbb{C}^{n}\right)}, \quad u \in H^{2}\left(I, \mathbb{C}^{n}\right) \cap H_{0}^{1}\left(I, \mathbb{C}^{n}\right), v \in H_{0}^{1}\left(I, \mathbb{C}^{n}\right) .
$$

If now $u \in \operatorname{ker} \mathcal{A}_{x}$, then 9.5 gives $\left\langle L_{x} u, v\right\rangle_{H_{0}^{1}\left(I, \mathbb{C}^{n}\right)}=0$ for all $v \in H_{0}^{1}\left(I, \mathbb{C}^{n}\right)$ and hence $L_{x} u=$ 0 . Conversely, if $u \in \operatorname{ker} L_{x}$, then $u$ is obviously in $H^{2}\left(I, \mathbb{C}^{n}\right)$ by 9.4 . Hence $\left\langle\mathcal{A}_{x} u, v\right\rangle_{L^{2}\left(I, \mathbb{C}^{n}\right)}=0$ for all $v \in H_{0}^{1}\left(I, \mathbb{C}^{n}\right)$ and since $H_{0}^{1}\left(I, \mathbb{C}^{n}\right)$ is dense in $L^{2}\left(I, \mathbb{C}^{n}\right)$ we actually infer $\mathcal{A}_{x} u=0$.

We now can conclude this section by defining the index of $q$ as announced in section 9.1 
9.3.4 Definition. If $X$ is compact and $Y \subset X$ closed having a trivial intersection with the singular set of $\mathcal{A}$, then we define

$$
\text { s-ind } q=- \text { s-ind } L \in K^{-1}(X, Y) \text {. }
$$

\subsection{The $\operatorname{Index} \operatorname{con}(\mathcal{A})$}

We remember at first that the extended family

$$
\overline{\mathcal{A}}_{z} u=J u^{\prime \prime}+S_{x}(t) u+i s u, \quad \mathcal{D}\left(\overline{\mathcal{A}}_{z}\right)=H^{2}\left(I, \mathbb{C}^{n}\right) \cap H_{0}^{1}\left(I, \mathbb{C}^{n}\right), \quad z=(x, s) \in X \times \mathbb{R}
$$

is needed in the construction of $\operatorname{s-ind}(\mathcal{A})$.

Let now $b_{z}^{1}, \ldots, b_{z}^{n}: I \rightarrow \mathbb{C}^{n}, z=(x, s) \in X \times \mathbb{R}$, be the solutions of the differential equations

$$
J u^{\prime \prime}+S_{x}(t) u+i s u=0
$$

such that $b_{z}^{i}(0)=0$ and $\left(b_{z}^{i}\right)^{\prime}(0)=e_{i}, i=1, \ldots, n$. We denote by $b_{z}(t), t \in I$, the matrix $\left(b_{z}^{1}(t), \ldots, b_{z}^{n}(t)\right)$ and $b_{z}:=b_{z}(1)$. The crucial observation for the definition of the conjugate index is given by the following lemma.

9.4.1 Lemma. $b_{z}, z=(x, s) \in X \times \mathbb{R}$, is not invertible if and only if $s=0$ and ker $\mathcal{A}_{x} \neq\{0\}$.

Proof. If $b_{z}$ is not invertible, take $0 \neq v \in \operatorname{ker} b_{z}$ and define $w(t)=b_{z}(t) v$. Then

$$
J w^{\prime \prime}(t)+S_{x}(t) w(t)=\left(J b_{z}^{\prime \prime}(t)+S_{x}(t) b_{z}(t)+i s b_{z}(t)\right) v=0, \quad t \in I
$$

and $w(0)=b_{z}(0) v=0$ as well as $w(1)=b_{z} v=0$. We infer $w \in \operatorname{ker} \overline{\mathcal{A}}_{z}$ and since $w \neq 0 \in$ $\mathcal{D}\left(\overline{\mathcal{A}}_{z}\right)$ this is just possible if $s=0$ by the selfadjointness of $\mathcal{A}_{x}$.

Conversely, if $u \in \operatorname{ker} \overline{\mathcal{A}}_{z}$ we obtain the equality $u(t)=b_{z}(t) u^{\prime}(0), t \in I$, by the uniqueness of solutions of initial value problems, where we use that $b_{z}(0)=0$ and $b_{z}^{\prime}(0)=I$. Therefore $0=u(1)=b_{z} u^{\prime}(0)$, and if $\operatorname{det} b_{z} \neq 0$ this implies $u^{\prime}(0)=0$ and so $u=0$.

As a consequence of the foregoing result, the points $z=(x, s) \in X \times \mathbb{R}$ at which $b_{z}$ is not invertible lie in $X$ and coincide with the compact singular set of $\mathcal{A}$. Hence we can make the following definition.

9.4.2 Definition. The conjugate index of $\mathcal{A}$ is defined by

$$
\operatorname{con}(\mathcal{A})=\left[\Theta\left(\mathbb{C}^{n}\right), \Theta\left(\mathbb{C}^{n}\right), b^{T}\right] \in K^{-1}(X, Y)
$$

where $b$ denotes the map $\mathbb{C} \ni z \mapsto b_{z} \in M(n, \mathbb{C})$ and $\cdot^{T}$ the transpose of a matrix. 
As we already mentioned in section 9.1 we gave a proof of the second equality of theorem 9.1.1 for $X=I$ and $Y=\partial I$ in Wa12. In this special case the conjugate index is given by

$$
\left[\Theta\left(\mathbb{C}^{n}\right), \Theta\left(\mathbb{C}^{n}\right), b\right] \in K^{-1}(I, \partial I)
$$

which comes from the fact that the isomorphism $c_{1}: K^{-1}(I, \partial I) \rightarrow \mathbb{Z}$ we defined in section B.3 uses the winding number of the determinant of $b$ and hence we have

$$
\left[\Theta\left(\mathbb{C}^{n}\right), \Theta\left(\mathbb{C}^{n}\right), b^{T}\right]=\left[\Theta\left(\mathbb{C}^{n}\right), \Theta\left(\mathbb{C}^{n}\right), b\right] \in K^{-1}(I, \partial I) .
$$

We want to conclude this section by explaining why we do not expect this equality to hold in general and that accordingly 9.4 .2 is the "real" conjugate index.

In the following we denote by $\overline{b_{z}}$ the complex conjugate matrix of $b_{z}$. Then it is clear that $\overline{b_{z}} b_{z}^{T}$ is a selfadjoint matrix and hence the homotopy

$$
H: I \times X \times \mathbb{R} \rightarrow M(n, \mathbb{C}), \quad(\lambda, x, s) \mapsto \overline{b_{z}} b_{z}^{T}+\lambda i I
$$

consists of invertible matrices if $\lambda \neq 0$. We infer from the homotopy invariance B.1.2 of $K$ that

$$
\left[\Theta\left(\mathbb{C}^{n}\right), \Theta\left(\mathbb{C}^{n}\right), \bar{b} b^{T}\right]=0 \in K^{-1}(X, Y)
$$

and now the logarithmic property B.1.5 implies

$$
\left[\Theta\left(\mathbb{C}^{n}\right), \Theta\left(\mathbb{C}^{n}\right), b^{T}\right]=-\left[\Theta\left(\mathbb{C}^{n}\right), \Theta\left(\mathbb{C}^{n}\right), \bar{b}\right] \in K^{-1}(X, Y) .
$$

According to [MS74, Lemma 14.9] we strongly believe that

$$
c_{k}\left(\left[\Theta\left(\mathbb{C}^{n}\right), \Theta\left(\mathbb{C}^{n}\right), \bar{b}\right]\right)=(-1)^{k} c_{k}\left(\left[\Theta\left(\mathbb{C}^{n}\right), \Theta\left(\mathbb{C}^{n}\right), b\right]\right),
$$

where $c_{k}$ denotes the $k$-th Chern number which can be defined for non compact spaces as in [Fe91, §3.2]. But then

$$
c_{k}\left(\left[\Theta\left(\mathbb{C}^{n}\right), \Theta\left(\mathbb{C}^{n}\right), b^{T}\right]\right)=-c_{k}\left(\left[\Theta\left(\mathbb{C}^{n}\right), \Theta\left(\mathbb{C}^{n}\right), \bar{b}\right]\right)=(-1)^{k+1} c_{k}\left(\left[\Theta\left(\mathbb{C}^{n}\right), \Theta\left(\mathbb{C}^{n}\right), b\right]\right)
$$

and by using theorem 9.1.1 any family having an index bundle with a non trivial even Chern class shows that $b^{T}$ can not be replaced by $b$ in the definition of the conjugate index 9.4 .2 . 


\subsection{The Proof}

In this section we give the proof of theorem 9.1.1. We prove the two equalities s-ind $(q)=\operatorname{sind}(\mathcal{A})$ and s-ind $(\mathcal{A})=\operatorname{con}(\mathcal{A})$ independently in two subsections and each of these subsections is itself decomposed into several subsections.

Before we begin, we want to point out that we will use corollary 5.1.5 in the following without further reference.

\subsection{1 $\quad \operatorname{s-ind}(q)=\operatorname{s-ind}(\mathcal{A})$}

We consider the operator

$$
K: L^{2}\left(I, \mathbb{C}^{n}\right) \rightarrow H_{0}^{1}\left(I, \mathbb{C}^{n}\right), \quad\left(K_{0} u\right)(t)=\int_{0}^{t} \int_{0}^{s} u(\tau) d \tau d s-t \int_{0}^{1} \int_{0}^{s} u(\tau) d \tau d s
$$

and its restriction to $H_{0}^{1}\left(I, \mathbb{C}^{n}\right)$, which we denote by $K_{0}$ in order to avoid confusion. Arguing verbatim as in section 9.2 , we find that $K$ is the inverse of the operator

$$
T: \mathcal{D}(T)=H^{2}\left(I, \mathbb{C}^{n}\right) \cap H_{0}^{1}\left(I, \mathbb{C}^{n}\right) \subset L^{2}\left(I, \mathbb{C}^{n}\right) \rightarrow L^{2}\left(I, \mathbb{C}^{n}\right), \quad u \mapsto u^{\prime \prime}
$$

if we regard it as operator into $L^{2}\left(I, \mathbb{C}^{n}\right)$. Accordingly, we note that im $K=H^{2}\left(I, \mathbb{C}^{n}\right) \cap$ $H_{0}^{1}\left(I, \mathbb{C}^{n}\right)$ and $K$ as well as $K_{0}$ is injective.

We now denote by $\iota: H^{2}\left(I, \mathbb{C}^{n}\right) \cap H_{0}^{1}\left(I, \mathbb{C}^{n}\right) \hookrightarrow H_{0}^{1}\left(I, \mathbb{C}^{n}\right)$ the canonical inclusion and obtain a diagram

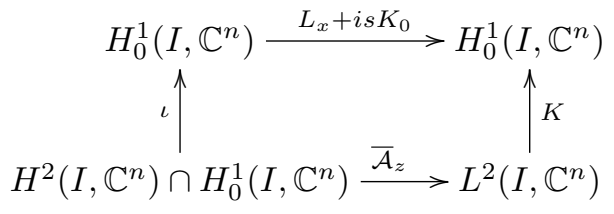

which is commutative because 


$$
\begin{aligned}
\left(K\left(\overline{\mathcal{A}}_{z} u\right)\right)(t) & =\left(K\left(J u^{\prime \prime}+S_{x} u+i s u\right)\right)(t) \\
& =\int_{0}^{t} \int_{0}^{s} J u^{\prime \prime}(\tau)+S_{x}(\tau) u(\tau) d \tau d s-t \int_{0}^{1} \int_{0}^{s} J u^{\prime \prime}(\tau)+S_{x}(\tau) u(\tau) d \tau d s+(K(i s u))(t) \\
& =\int_{0}^{t} J u^{\prime}(s)-J u^{\prime}(0) d s-t \int_{0}^{1} J u^{\prime}(s)-J u^{\prime}(0) d s \\
& +\int_{0}^{t} \int_{0}^{s} S_{x}(\tau) u(\tau) d \tau d s-t \int_{0}^{1} \int_{0}^{s} S_{x}(\tau) u(\tau) d \tau d s+(K(i s u))(t) \\
& =J u(t)-J u(0)-t J u^{\prime}(0)-t J u(1)+t J u(0)+t J u^{\prime}(0) \\
& +\int_{0}^{t} \int_{0}^{s} S_{x}(\tau) u(\tau) d \tau d s-t \int_{0}^{1} \int_{0}^{s} S_{x}(\tau) u(\tau) d \tau d s+i s(K(u))(t) \\
& =\left(L_{x}(\iota u)\right)(t)+i s K_{0}(\iota(u))(t), \quad t \in I
\end{aligned}
$$

for all $u \in H^{2}\left(I, \mathbb{C}^{n}\right) \cap H_{0}^{1}\left(I, \mathbb{C}^{n}\right)$.

The proof of the equality s-ind $(q)=\operatorname{s-ind}(\mathcal{A})$ now proceeds as follows: In a first step we use the property 7.1 .12 in order to infer that s-ind $(q)=\operatorname{ind}\left(L_{x}+i s K_{0}\right)$. Afterwards we apply the reduction property 5.2 .10 and obtain $\operatorname{ind}\left(L_{x}+i s K_{0}\right)=\operatorname{ind}(\overline{\mathcal{A}})=\operatorname{s-ind}(\mathcal{A})$ from the commutativity of 9.6 .

Step 1.: $\operatorname{s-ind}(q)=\operatorname{ind}\left(L_{x}+i s K_{0}\right)$

We begin by studying the operator $K_{0}: H_{0}^{1}\left(I, \mathbb{C}^{n}\right) \rightarrow H_{0}^{1}\left(I, \mathbb{C}^{n}\right)$ more precisely.

9.5.1 Lemma. The operator

$$
K_{0}: H_{0}^{1}\left(I, \mathbb{C}^{n}\right) \rightarrow H_{0}^{1}\left(I, \mathbb{C}^{n}\right)
$$

is selfadjoint, compact and non positive.

Proof. In 9.4 we introduced a bounded selfadjoint operator $L_{x}$ on $H_{0}^{1}\left(I, \mathbb{C}^{n}\right)$ for any real symmetric matrix $S_{x} . L_{x}$ is of the form $L_{x} u=J u+K_{x} u$ where, according to lemma 9.3.1, $K_{x}$ is a compact operator. Moreover, since $L_{x}$ and the multiplication by the symmetric matrix $J$ are selfadjoint operators, we infer that $K_{x}$ is selfadjoint as well. But $K_{x}=K_{0}$ in the special case that $S_{x}=I$ and so we obtain that $K_{0}$ is compact and selfadjoint as well.

It remains to study the spectrum of $K_{0}$ which consists of 0 and a possibly empty discrete set of eigenvalues due to the compactness. Suppose $K_{0} u=\lambda u$ for some $\lambda \neq 0$ and $u \in H_{0}^{1}\left(I, \mathbb{C}^{n}\right)$. Then $u$ is smooth and by differentiating twice we obtain

$$
u^{\prime \prime}(t)-\frac{1}{\lambda} u(t)=0 .
$$


If $\lambda>0$, then all solutions of this differential equation are of the form

$$
u(t)=c_{1} e^{\frac{1}{\sqrt{\lambda}} t}+c_{2} e^{-\frac{1}{\sqrt{\lambda}} t}, \quad t \in I, \quad c_{1}, c_{2} \in \mathbb{C},
$$

and such a function belongs to $H_{0}^{1}\left(I, \mathbb{C}^{n}\right)$ if and only if $c_{1}=c_{2}=0$. Hence $K_{0}$ has no positive eigenvalues and, accordingly, is non positive ${ }^{1}$

We now verify the remaining assumption of proposition 7.1.12.

9.5.2 Lemma. The operator $L_{x}-i s K_{0}$ has for any $s \neq 0$ a bounded inverse.

Proof. In the foregoing lemma we have shown that $K_{0}$ is compact and hence $L_{x}-i s K_{0}$ is a Fredholm operator of index 0 . Accordingly, $L_{x}-i s K_{0}$ has a bounded inverse if and only if its kernel is trivial.

Now suppose $L_{x} u-i s K_{0} u=0$ for some $u \in H_{0}^{1}\left(I, \mathbb{C}^{n}\right)$. We infer

$$
u(t)=J \int_{0}^{t} \int_{0}^{s}\left(i s I-S_{x}(\tau)\right) u(\tau) d \tau d s-t J \int_{0}^{1} \int_{0}^{s}\left(i s I-S_{x}(\tau)\right) u(\tau) d \tau d s,
$$

and see that the right hand side is an element of $H^{2}\left(I, \mathbb{C}^{n}\right) \cap H_{0}^{1}\left(I, \mathbb{C}^{n}\right)$. Then 9.6 implies that $\mathcal{A}_{x} u-i s u=0$ because of the injectivity of $K$. Since $\mathcal{A}_{x}$ is selfadjoint and $s \neq 0$ by assumption, we infer $u=0$.

Now we finally can apply proposition 7.1 .12 and lemma 7.1 .9 and obtain

$$
\operatorname{s-ind}(q)=-\operatorname{s-ind}(L)=\operatorname{s-ind}(-L)=\operatorname{ind}\left(-L_{x}+i s I\right)=\operatorname{ind}\left(-L_{x}-i s K_{0}\right)=\operatorname{ind}\left(L_{x}+i s K_{0}\right) .
$$

Step 2: $\operatorname{ind}\left(L_{x}+i s K_{0}\right)=\operatorname{s-ind}(\mathcal{A})$

We now have to verify the assumptions of the reduction property 5.2 .10 and note at first that the domain bundle of the family $L_{x}+i s K_{0}$ is given by the product $\Theta\left(H_{0}^{1}\left(I, \mathbb{C}^{n}\right)\right)$ according to corollary 6.1.14 Moreover, since

$$
\begin{aligned}
\left\|\overline{\mathcal{A}}_{z} u-\overline{\mathcal{A}}_{z_{0}} u\right\|_{L^{2}\left(I, \mathbb{C}^{n}\right)} & \leq\left\|S_{x} u-S_{x_{0}} u\right\|_{L^{2}\left(I, \mathbb{C}^{n}\right)}+\left|s-s_{0}\right|\|u\|_{L^{2}\left(I, \mathbb{C}^{n}\right)} \\
& \leq\left(\left\|S_{x}-S_{x_{0}}\right\|_{\infty}+\left|s-s_{0}\right|\right)\|u\|_{L^{2}\left(I, \mathbb{C}^{n}\right)} \\
& \leq\left(\left\|S_{x}-S_{x_{0}}\right\|_{\infty}+\left|s-s_{0}\right|\right)\left(\|u\|_{L^{2}\left(I, \mathbb{C}^{n}\right)}^{2}+\left\|\overline{\mathcal{A}}_{z_{1}} u\right\|_{L^{2}\left(I, \mathbb{C}^{n}\right)}^{2}\right)^{\frac{1}{2}}
\end{aligned}
$$

we obtain from lemma 9.2.1 that $\overline{\mathcal{A}}$ defines a continuous family of operators in $\mathcal{L}\left(\mathfrak{D}(\overline{\mathcal{A}})_{z_{1}}, L^{2}\left(I, \mathbb{C}^{n}\right)\right)$ for any $z_{1} \in X \times \mathbb{R}$. Hence the domain bundle of $\overline{\mathcal{A}}$ is the product $\Theta\left(H^{2}\left(I, \mathbb{C}^{n}\right) \cap H_{0}^{1}\left(I, \mathbb{C}^{n}\right)\right)$ according to lemma 6.1 .12 and we conclude that the canonical inclusion $\iota$ induces an injective bundle morphism from $\mathfrak{D}(\overline{\mathcal{A}})$ into $\Theta\left(H_{0}^{1}\left(I, \mathbb{C}^{n}\right)\right)$. Now the following lemma shows the remaining assumption of corollary 5.2.10.

\footnotetext{
${ }^{1}$ A simple computation shows that the eigenvalues of $K_{0}$ are given by $\lambda=-\frac{1}{k^{2} \pi^{2}}, k \in \mathbb{N}$.
} 
9.5.3 Lemma. There exists a finite dimensional subspace $V \subset L^{2}\left(I, \mathbb{C}^{n}\right)$ such that

$$
\operatorname{im}\left(\mathcal{A}_{x}+i s \cdot I\right)+V=L^{2}\left(I, \mathbb{C}^{n}\right),(x, s) \in X \times \mathbb{R},
$$

and

$$
\operatorname{im}\left(L_{x}+i s K_{0}\right)+K(V)=H_{0}^{1}\left(I, \mathbb{C}^{n}\right),(x, s) \in X \times \mathbb{R} .
$$

Proof. At first we consider the case $s=0$. Since $L_{x}$ is selfadjoint and Fredholm, we have $\operatorname{im} L_{x} \oplus \operatorname{ker} L_{x}=H$ for any $x \in X$ and, moreover, it follows from (9.4) that $\operatorname{ker} L_{x} \subset H^{2}\left(I, \mathbb{C}^{n}\right) \cap$ $H_{0}^{1}\left(I, \mathbb{C}^{n}\right)$. From the first part of the proof of the existence of transversal subspaces 5.1 .3 it is now readily seen that we can find a finite dimensional subspace $W \subset H^{2}\left(I, \mathbb{C}^{n}\right) \cap H_{0}^{1}\left(I, \mathbb{C}^{n}\right)$ such that

$$
\operatorname{im}\left(L_{x}\right)+W=H_{0}^{1}\left(I, \mathbb{C}^{n}\right), \quad x \in X .
$$

In the case that $s \neq 0$ we know from lemma 9.5 .2 that $L_{x}+i s K_{0}$ is surjective and hence we deduce

$$
\operatorname{im}\left(L_{x}+i s K_{0}\right)+W=H_{0}^{1}\left(I, \mathbb{C}^{n}\right), \quad(x, s) \in X \times \mathbb{R} .
$$

We now choose any finite dimensional subspace $V_{1} \subset L^{2}\left(I, \mathbb{C}^{n}\right)$ such that $K\left(V_{1}\right) \supset W$, which indeed exists because of $\operatorname{im} K=H^{2}\left(I, \mathbb{C}^{n}\right) \cap H_{0}^{1}\left(I, \mathbb{C}^{n}\right)$ and the injectivity of $K$. Finally, the assertion follows by taking any finite dimensional subspace $V_{2} \subset L^{2}\left(I, \mathbb{C}^{n}\right)$ such that

$$
\operatorname{im}\left(\mathcal{A}_{x}+i s I\right)+V_{2}=L^{2}\left(I, \mathbb{C}^{n}\right), \quad(x, s) \in X \times \mathbb{R},
$$

and setting $V=V_{1}+V_{2} \subset L^{2}\left(I, \mathbb{C}^{n}\right)$.

Now we finally obtain from the commutativity of 9.6 and the reduction property of the index bundle 5.2 .10

$$
\operatorname{ind}\left(L_{x}+i s K_{0}\right)=\operatorname{ind}(\overline{\mathcal{A}})=\operatorname{s-ind}(\mathcal{A}) \in K^{-1}(X, Y)
$$

and so the first equality from our theorem 9.1 .1 is proved. 


\subsection{2 $\quad \operatorname{sind}(\mathcal{A})=\operatorname{con}(\mathcal{A})$}

We split the proof into three steps.

Step 1: $\operatorname{s-ind}(\mathcal{A})=\operatorname{ind}(M)$

At first we want to show that the selfadjoint index of $\mathcal{A}$ is equal to the index bundle of the family

$$
\begin{aligned}
& M_{z}: \mathcal{H} \rightarrow L^{2}\left(I, \mathbb{C}^{2 n}\right), \quad z \in X \times \mathbb{R}, \\
& \left(M_{z} w\right)(t)=\sigma w^{\prime}(t)+H_{z}(t) w(t),
\end{aligned}
$$

where $\mathcal{H}=\left\{w \in H^{1}\left(I, \mathbb{C}^{2 n}\right): w(0), w(1) \in\{0\} \times \mathbb{C}^{n}\right\}$,

$$
H_{z}(t)=\left(\begin{array}{cc}
-S_{z}(t) & 0 \\
0 & -J
\end{array}\right), \quad S_{z}(t)=S_{x}(t)+i s I, z=(x, s) \in X \times \mathbb{R},
$$

and $S_{x}, x \in X$, is the matrix family already introduced in 9.1 . Note that we have two ways to make a family out of the operators $M_{z}$ in our framework. The first way is to consider the $M_{z}$ as unbounded operators on $L^{2}\left(I, \mathbb{C}^{2 n}\right)$ having the constant domain $\mathcal{H}$. Then it is not difficult to show that $M$ defines a gap continuous family of Fredholm operators having the product $\Theta(\mathcal{H})$ as domain bundle. However, here we want to use a second way by regarding $M$ directly as a bundle morphism between the products $\Theta(\mathcal{H})$ and $\Theta\left(L^{2}\left(I, \mathbb{C}^{2 n}\right)\right)$, where the continuity of $M$ as family of bounded operators follows easily from lemma 9.2.1. Hence all we have to do is to show that each $M_{z}: \mathcal{H} \rightarrow L^{2}\left(I, \mathbb{C}^{2 n}\right)$ is a Fredholm operator. At first, we need some preparations. If $w=\left(w_{1}, w_{2}\right) \in \mathcal{H}$, we have explicitly

$$
M_{z} w=\left(\begin{array}{cc}
0 & -I \\
I & 0
\end{array}\right)\left(\begin{array}{c}
w_{1}^{\prime} \\
w_{2}^{\prime}
\end{array}\right)+\left(\begin{array}{cc}
-S_{z} & 0 \\
0 & -J
\end{array}\right)\left(\begin{array}{l}
w_{1} \\
w_{2}
\end{array}\right)=\left(\begin{array}{c}
-w_{2}^{\prime}-S_{z} w_{1} \\
w_{1}^{\prime}-J w_{2}
\end{array}\right) .
$$

We define operators $j: H^{2}\left(I, \mathbb{C}^{n}\right) \cap H_{0}^{1}\left(I, \mathbb{C}^{n}\right) \hookrightarrow \mathcal{H}$ and $\iota: L^{2}\left(I, \mathbb{C}^{n}\right) \hookrightarrow L^{2}\left(I, \mathbb{C}^{2 n}\right)$ by

$$
j(u)=\left(u, J u^{\prime}\right), \quad \iota(u)=(-u, 0)
$$

and obtain a diagram

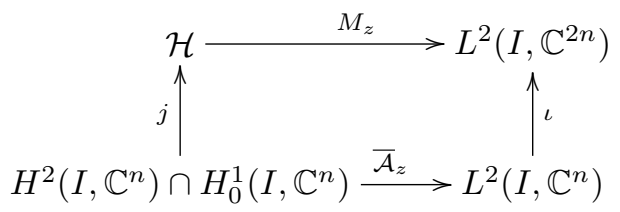

The diagram is commutative because 


$$
\iota\left(\overline{\mathcal{A}}_{z} u\right)=\left(-J u^{\prime \prime}-S_{z} u, 0\right)=M_{z}(j(u))
$$

for $u \in H^{2}\left(I, \mathbb{C}^{n}\right) \cap H_{0}^{1}\left(I, \mathbb{C}^{n}\right)$.

9.5.4 Lemma. Let $V \subset L^{2}\left(I, \mathbb{C}^{n}\right)$ be any subspace such that

$$
\operatorname{im}\left(\overline{\mathcal{A}}_{z}\right)+V=L^{2}\left(I, \mathbb{C}^{n}\right), \quad z \in X \times \mathbb{R}
$$

Then

$$
\operatorname{im}\left(M_{z}\right)+\iota(V)=L^{2}\left(I, \mathbb{C}^{2 n}\right), \quad z \in X \times \mathbb{R} .
$$

Proof. At first, setting $w_{2}=J w_{1}^{\prime}$, we obtain from 9.7

$$
M_{z}\left(w_{1}, J w_{1}^{\prime}\right)=\left(-J w_{1}^{\prime \prime}-S_{z} w_{1}, 0\right), \quad w_{1} \in H^{2}\left(I, \mathbb{C}^{n}\right) \cap H_{0}^{1}\left(I, \mathbb{C}^{n}\right)
$$

and

$$
\left\{\left(-J w_{1}^{\prime \prime}-S_{z} w_{1}, 0\right): w_{1} \in H^{2}\left(I, \mathbb{C}^{n}\right) \cap H_{0}^{1}\left(I, \mathbb{C}^{n}\right)\right\}+\iota(V)=L^{2}\left(I, \mathbb{C}^{n}\right) \oplus\{0\}
$$

by the assumed transversality of $V$ with respect to the image of $\overline{\mathcal{A}}$.

Moreover, if $v \in L^{2}\left(I, \mathbb{C}^{n}\right)$, we have

$$
v=\frac{d}{d t}\left(\int_{0}^{t} v(s) d s-t \int_{0}^{1} v(s) d s\right)-J\left(-J \int_{0}^{1} v(s) d s\right)=: w_{1}^{\prime}-J w_{2},
$$

where $w_{1}(0)=w_{1}(1)=0$ and hence

$$
\left\{w_{1}^{\prime}-J w_{2}:\left(w_{1}, w_{2}\right) \in \mathcal{H}\right\}=L^{2}\left(I, \mathbb{C}^{n}\right),
$$

where the left hand side is the image of the second component of $M_{z}$ according to 9.7 .

Let now $(u, v) \in L^{2}\left(I, \mathbb{C}^{2 n}\right)$ be arbitrary. We want to use 9.9$)$ and 9.10 in order to construct $w \in \mathcal{H}$ and $w_{0} \in \iota(V)$ such that $M_{z} w+w_{0}=(u, v)$ as follows:

- We use 9.10$)$ in order to choose $\left(w_{1}, w_{2}\right) \in \mathcal{H}$ such that $M_{z}\left(w_{1}, w_{2}\right)=(\tilde{u}, v)$ for some $\tilde{u} \in L^{2}\left(I, \mathbb{C}^{n}\right)$. 
- We use 9.9$)$ in order to choose $\tilde{v} \in V$ and $\left(\tilde{w}_{1}, \tilde{w}_{2}\right) \in \mathcal{H}$ such that $M_{z}\left(\tilde{w}_{1}, \tilde{w}_{2}\right)+\iota(\tilde{v})=$ $(u-\tilde{u}, 0)$.

Setting $w=\left(w_{1}+\tilde{w}_{1}, w_{2}+\tilde{w}_{2}\right) \in \mathcal{H}$ and $w_{0}=\iota(\tilde{v}) \in \iota(V)$, we obtain

$$
M_{z} w+w_{0}=M_{z}\left(w_{1}, w_{2}\right)+M_{z}\left(\tilde{w}_{1}, \tilde{w}_{2}\right)+\iota(\tilde{v})=(\tilde{u}, v)+(u-\tilde{u}, 0)=(u, v) .
$$

9.5.5 Lemma. $\operatorname{ker}\left(M_{z}\right)=j\left(\operatorname{ker} \overline{\mathcal{A}}_{z}\right), z=(x, s) \in X \times \mathbb{R}$.

Proof. At first we have $j\left(\operatorname{ker} \overline{\mathcal{A}}_{z}\right) \subset \operatorname{ker}\left(M_{z}\right)$ by the commutativity of 9.8). Conversely, if $\left(w_{1}, w_{2}\right) \in \operatorname{ker}\left(M_{z}\right)$, then $w_{1}^{\prime}=J w_{2}$ by (9.7) and hence $\left(w_{1}, w_{2}\right)=\left(w_{1}, J w_{1}^{\prime}\right) \in \operatorname{im} j$. Now the assertion follows by using the commutativity of 9.8 once again.

Now we discuss at first the Fredholm property of the operators $M_{z}$.

In order to do so we consider the operator $M_{z}^{\prime}: \mathcal{H} \rightarrow L^{2}\left(I, \mathbb{C}^{2 n}\right)$ which is defined to be the operator $M_{z}$ under the assumption that $S_{x}=0$. The operator $\overline{\mathcal{A}}_{z}^{\prime}$ corresponding to $M_{z}^{\prime}$ has a bounded inverse as we have shown in section 9.2 Hence we obtain from lemma 9.5.5 and lemma 9.5 .4 with $V=\{0\}$ that $M_{z}^{\prime}$ is bijective and hence a Banach space isomorphism by the open mapping principle A.2.5

A general operator $M_{z}$ is of the form $M_{z}=M_{z}^{\prime}+K_{z}$ where

$$
K_{z}: \mathcal{H} \rightarrow L^{2}\left(I, \mathbb{C}^{2 n}\right), \quad u \mapsto\left(\begin{array}{cc}
-S_{x}(\cdot) & 0 \\
0 & 0
\end{array}\right) u
$$

But since $S_{x}$ is smooth, $K_{z}$ maps $\mathcal{H}$ into $H^{1}\left(I, \mathbb{C}^{2 n}\right)$ continuously and hence it is a compact operator by the compactness of the embedding $H^{1}\left(I, \mathbb{C}^{2 n}\right) \hookrightarrow L^{2}\left(I, \mathbb{C}^{2 n}\right)$. Thus $M_{z}$ is the sum of a Banach space isomorphism and a compact operator and hence a Fredholm operator of index 0 .

With the results we have shown so far we are now very close to our aim of this first step of the proof. Since all $M_{z}$ are Fredholm operators of index 0 we obtain from lemma 9.5.5 that $\operatorname{supp} M=\operatorname{supp} \overline{\mathcal{A}}$ and hence $M$ has in particular a compact support. Thus we can build the index bundle ind $M$ as defined for Fredholm morphisms in section 5.1 We now take a finite dimensional subspace $V \subset L^{2}\left(I, \mathbb{C}^{n}\right)$ such that

$$
\operatorname{im} \overline{\mathcal{A}}_{z}+V=L^{2}\left(I, \mathbb{C}^{2 n}\right), \quad z \in X \times \mathbb{R},
$$

and note that $\iota(V)$ is transversal to im $M$ by lemma 9.5.4. Finally, we have already shown in section 9.5 .1 that $\mathfrak{D}(\overline{\mathcal{A}})$ is the product $\Theta\left(H^{2}\left(I, \mathbb{C}^{n}\right) \cap H_{0}^{1}\left(I, \mathbb{C}^{n}\right)\right)$ and so $j$ induces a bundle morphism between $\mathfrak{D}(\overline{\mathcal{A}})$ and $\Theta(\mathcal{H})$. Now the equality s-ind $(\mathcal{A})=\operatorname{ind}(M)$ follows from the commutativity of 9.8 by the reduction property 5.2 .10 of the index bundle. 
Step 2: $\operatorname{ind}(M)=\operatorname{ind}(N)$

Let $\Psi_{z}: I \rightarrow G L(n, \mathbb{C}), z \in X \times \mathbb{R}$, be the solution of the initial value problem

$$
\left\{\begin{array}{l}
\Psi_{z}^{\prime}(t)=\sigma H_{z}(t) \Psi_{z}(t) \\
\Psi_{z}(0)=I
\end{array} .\right.
$$

We note at first that each $\Psi_{z}(t)$ is a symplectic matrix in the following sense.

9.5.6 Lemma. Let $z \in X \times \mathbb{R}$ be fixed. Then

$$
\Psi_{z}^{T}(t) \sigma \Psi_{z}(t)=\sigma
$$

for all $t \in I$, where ${ }^{T}$ denotes the transpose of a matrix.

Proof. Note at first that $H_{z}^{T}(t)=H_{z}(t)$ because $S_{x}(t)$ is by assumption real and symmetric for any $x \in X, t \in I$. Now we obtain by differentiation

$$
\begin{aligned}
\left(\Psi_{z}^{T}(t) \sigma \Psi_{z}(t)\right)^{\prime} & =\left(\Psi_{z}^{\prime}(t)\right)^{T} \sigma \Psi_{z}(t)+\Psi_{z}^{T}(t) \sigma \Psi_{z}^{\prime}(t) \\
& =\left(\sigma H_{z}(t) \Psi_{z}(t)\right)^{T} \sigma \Psi_{z}(t)+\Psi_{z}^{T}(t) \sigma^{2} H_{z}(t) \Psi_{z}(t) \\
& =\Psi_{z}^{T}(t) H_{z}^{T}(t) \sigma^{T} \sigma \Psi_{z}(t)+\Psi_{z}^{T}(t) \sigma^{2} H_{z}(t) \Psi_{z}(t) \\
& =\Psi_{z}^{T}(t)\left(H_{z}^{T}(t)-H_{z}(t)\right) \Psi_{z}(t)=0
\end{aligned}
$$

and since

$$
\Psi_{z}^{T}(0) \sigma \Psi_{z}(0)=\sigma
$$

we obtain the assertion.

We define families of topological isomorphisms by

$$
\begin{array}{lc}
U: X \times \mathbb{R} \rightarrow G L\left(L^{2}\left(I, \mathbb{C}^{2 n}\right)\right) & \left(U_{z} w\right)(t)=\Psi_{z}(t) w(t) \\
U^{T}: X \times \mathbb{R} \rightarrow G L\left(L^{2}\left(I, \mathbb{C}^{2 n}\right)\right) & \left(U_{z}^{T} w\right)(t)=\Psi_{z}^{T}(t) w(t) .
\end{array}
$$

Note that $\Psi_{z}(t)$ depends continuously on $(z, t) \in X \times \mathbb{R} \times I$ by well known results on the continuous dependence of solutions of ordinary differential equations (cf. [He95, 13.1] ) and so it is clear by lemma 9.2.1 that $U$ and $U^{T}$ are continuous.

Moreover, we define a family

$$
N: \tilde{H}_{z} \rightarrow L^{2}\left(I, \mathbb{C}^{2 n}\right), \quad N_{z} w=U_{z}^{T} M_{z} U_{z}, \quad z \in X \times \mathbb{R},
$$


where

$$
\tilde{H}_{z}=\left\{w \in H^{1}\left(I, \mathbb{C}^{2 n}\right): w(0) \in\{0\} \times \mathbb{C}^{n}, w(1) \in \Psi_{z}^{-1}\left(\{0\} \times \mathbb{C}^{n}\right)\right\}
$$

and $\Psi_{z}:=\Psi_{z}(1)$. Note that $U_{z}\left(\tilde{H}_{z}\right)=\mathcal{H}$ for all $z \in X \times \mathbb{R}$ such that the operators $N_{z}$ indeed are well defined. From the definition of $N_{z}$, we obtain

$$
\begin{aligned}
N_{z} w & =U_{z}^{T} M_{z} U_{z}=\Psi_{z}^{T}(t)\left(\sigma\left(\Psi_{z}(t) w(t)\right)^{\prime}+H_{z}(t) \Psi_{z}(t) w(t)\right) \\
& =\Psi_{z}^{T}(t)\left(\sigma \Psi_{z}^{\prime}(t) w(t)+\sigma \Psi_{z}(t) w^{\prime}(t)+H_{z}(t) \Psi_{z}(t) w(t)\right) \\
& =\Psi_{z}^{T}(t)\left(-H_{z}(t) \Psi_{z}(t) w(t)+\sigma \Psi_{z}(t) w^{\prime}(t)+H_{z}(t) \Psi_{z}(t) w(t)\right) \\
& =\Psi_{z}^{T}(t) \sigma \Psi_{z}(t) w^{\prime}(t)=\sigma w^{\prime}(t), \quad w \in \tilde{H}_{z} .
\end{aligned}
$$

By theorem 8.1.1 $N$ defines a gap continuous family of closed operators and its domain bundle $\mathfrak{D}(N)$ is a subbundle of $\Theta\left(H^{1}\left(I, \mathbb{C}^{2 n}\right)\right)$. Hence we can in particular regard $U$ as a bundle isomorphism between $\mathfrak{D}(N)$ and $\Theta(\mathcal{H})$. Moreover, since we can regard $U^{T}$ as a bundle automorphism of $\Theta\left(L^{2}\left(I, \mathbb{C}^{2 n}\right)\right)$, we obtain by lemma 5.2.1 and lemma 5.2.7

$$
\operatorname{ind}(N)=\operatorname{ind}\left(U^{T} M U\right)=\operatorname{ind}\left(U^{T}\right)+\operatorname{ind}(M)+\operatorname{ind}(U)=\operatorname{ind}(M) \in K^{-1}(X, Y) .
$$

Step 3: $\operatorname{ind}(N)=\operatorname{con}(\mathcal{A})$

The index bundle of a family like $N$ is known to us by theorem 8.1.1 and the aim of this third and final step is to show that the result is indeed the conjugate index.

9.5.7 Lemma. The matrix $\Psi_{z}$ is of the form

$$
\Psi_{z}=\left(\begin{array}{cc}
a_{z} & b_{z} J \\
c_{z} & d_{z}
\end{array}\right)
$$

where $b_{z}$ is the matrix family defined in the construction of the conjugate index.

Proof. We denote

$$
\Psi_{z}(t)=\left(\begin{array}{cc}
a_{z}(t) & b_{z}(t) J \\
c_{z}(t) & d_{z}(t)
\end{array}\right), \quad t \in I,
$$

and obtain by inserting into the differential equation 9.11

$$
\left(\begin{array}{cc}
a_{z}^{\prime}(t) & b_{z}^{\prime}(t) J \\
c_{z}^{\prime}(t) & d_{z}^{\prime}(t)
\end{array}\right)=\left(\begin{array}{cc}
0 & J \\
-S_{z}(t) & 0
\end{array}\right)\left(\begin{array}{cc}
a_{z}(t) & b_{z}(t) J \\
c_{z}(t) & d_{z}(t)
\end{array}\right)=\left(\begin{array}{cc}
J c_{z}(t) & J d_{z}(t) \\
-S_{z}(t) a_{z}(t) & -S_{z}(t) b_{z}(t) J
\end{array}\right) .
$$


We infer

$$
\begin{aligned}
b_{z}^{\prime}(t) & =J d_{z}(t) J \\
d_{z}^{\prime}(t) & =-S_{z}(t) b_{z}(t) J .
\end{aligned}
$$

Inserting the first equality of 9.12 in the second one, we obtain

$$
J b_{z}^{\prime \prime}(t)+S_{z}(t) b_{z}(t)=0, t \in I,
$$

and using $\Psi_{z}(0)=I$ and the first equation of 9.12 once again, we also find

$$
\begin{aligned}
& b_{z}(0)=0 \\
& b_{z}^{\prime}(0)=J d_{z}(0) J=J^{2}=I .
\end{aligned}
$$

Hence, by the uniqueness of solutions of initial value problems, $b_{z}$ is the matrix family we already introduced in the definition of the conjugate index.

Note that by lemma 9.5 .6 we have $\Psi_{z}^{-1}=-\sigma \Psi_{z}^{T} \sigma, z \in X \times \mathbb{R}$, because

$$
\begin{aligned}
& -\sigma \Psi_{z}^{T} \sigma \Psi_{z}=-\sigma^{2}=I \\
& -\Psi_{z} \sigma \Psi_{z}^{T} \sigma=-\sigma^{2}=I .
\end{aligned}
$$

Hence

$$
\begin{aligned}
\Psi_{z}^{-1} & =-\sigma\left(\begin{array}{cc}
a_{z} & b_{z} J \\
c_{z} & d_{z}
\end{array}\right)^{T} \sigma=\left(\begin{array}{cc}
0 & I \\
-I & 0
\end{array}\right)\left(\begin{array}{cc}
a_{z}^{T} & c_{z}^{T} \\
J b_{z}^{T} & d_{z}^{T}
\end{array}\right)\left(\begin{array}{cc}
0 & -I \\
I & 0
\end{array}\right) \\
& =\left(\begin{array}{cc}
0 & I \\
-I & 0
\end{array}\right)\left(\begin{array}{cc}
c_{z}^{T} & -a_{z}^{T} \\
d_{z}^{T} & -J b_{z}^{T}
\end{array}\right)=\left(\begin{array}{cc}
d_{z}^{T} & -J b_{z}^{T} \\
-c_{z}^{T} & a_{z}^{T}
\end{array}\right)
\end{aligned}
$$

and we obtain from theorem 8.1 .1 that the index bundle of $N$ is given by

$$
\operatorname{ind}(N)=\left[\Theta\left(\mathbb{C}^{n}\right), \Theta\left(\mathbb{C}^{n}\right),-J b^{T}\right]
$$

By using the homotopy

$$
H: I \times X \times \mathbb{R} \rightarrow M(n, \mathbb{C}), \quad(\lambda, x, s) \mapsto \operatorname{diag}\left(-e^{i \pi \lambda}, \ldots,-e^{i \pi \lambda}, 1, \ldots, 1\right) b^{T}
$$

we finally infer by the homotopy invariance of $K$ stated in lemma B.1.3 that the right hand side of 9.13 , and hence s-ind $(\mathcal{A})$, is given by 
$\left[\Theta\left(\mathbb{C}^{n}\right), \Theta\left(\mathbb{C}^{n}\right), b^{T}\right]$

which is by definition $\operatorname{con}(\mathcal{A})$. 


\section{Chapter 10}

\section{Applications to semi-Riemannian Geodesics}

In this final chapter of the third part of the thesis we study certain families of geodesics in semiRiemannian manifolds. In the first section we construct the families and state a Morse index theorem which is obtained from 9.1.1. In the second section we consider examples and show in particular that the semi-Riemannian Morse index theorem [MPP05] for a single geodesics is a special case of our Morse index theorem. The third section deals with the question how to extend our index theorem to more general families of geodesics in view of the Morse index theorem for families of Riemannian geodesics due to Horacio Porta and Lazaro Recht [PR82]. In the final fourth section we come back to the first part of the thesis and obtain estimates from below for the dimension of the conjugate locus in tangent spaces of semi-Riemannian manifolds.

\subsection{The Theorem}

At first we want to fix notations and briefly describe the setting. We refer to Wa07 as basic reference and accordingly we argue less detailed as before.

Let $(M, g)$ be a semi-Riemannian manifold of dimension $n$ and index $0 \leq \nu \leq n$. We call a path $\gamma: I \rightarrow M$ a geodesic, if it fulfils the equation

$$
\frac{\nabla}{d t} \gamma^{\prime}(t)=0, \quad t \in I,
$$

where $\nabla$ denotes the Levi-Civita connection. A Jacobi field along a geodesic $\gamma$ is a vector field $\xi$ along $\gamma$ satisfying the so called Jacobi equation

$$
\frac{\nabla^{2}}{d t^{2}} \xi(t)-R\left(\gamma^{\prime}(t), \xi(t)\right) \gamma^{\prime}(t)=0
$$


and, moreover, $t \in I$ is called a conjugate instant if there exists a non trivial Jacobi field $\xi$ along $\gamma$ satisfying $\xi(0)=0$ and $\xi(t)=0$.

Let $H^{1}(I, M)$ be the Hilbert manifold consisting of paths in $M$ of Sobolev regularity $H^{1,2}$ and, for fixed points $p, q \in M, H_{p q}^{1}(I, M)$ the submanifold consisting of those paths that connect $p$ and $q$. The smooth function, that is of interest in view of Morse theory for geodesics in semi-Riemannian manifolds, is the action functional defined by

$$
\mathcal{A}(\gamma)=\frac{1}{2} \int_{0}^{1} g\left(\gamma^{\prime}(t), \gamma^{\prime}(t)\right) d t, \quad \gamma \in H_{p q}^{1}(I, M)
$$

The critical points of this functional are precisely the geodesics joining $p$ and $q$ and the Hessian

$$
\operatorname{Hess}_{\gamma}\left(\mathcal{A}_{p q}\right): T_{\gamma} H_{p q}^{1}(I, M) \times T_{\gamma} H_{p q}^{1}(I, M) \rightarrow \mathbb{R}
$$

at such a critical point $\gamma$ is given by

$$
\begin{aligned}
\operatorname{Hess}_{\gamma}\left(\mathcal{A}_{p q}\right)(\xi, \eta) & =\int_{0}^{1} g_{\gamma(t)}\left(\frac{\nabla}{d t} \xi(t), \frac{\nabla}{d t} \eta(t)\right) d t \\
& +\int_{0}^{1} g_{\gamma(t)}\left(R\left(\gamma^{\prime}(t), \xi(t)\right) \gamma^{\prime}(t), \eta(t)\right) d t
\end{aligned}
$$

where

$$
\xi, \eta \in T_{\gamma} H_{p, q}^{1}(I, M)=\left\{\xi \in H^{1}(I, T M): \xi(t) \in T_{\gamma(t)} M \forall t \in I, \xi(0)=0, \xi(1)=0\right\} .
$$

These definitions suffice to state the Riemannian version of the Morse index theorem as the well known equality of the finite Morse index of the Hessian 10.2 and the number of conjugate points along the geodesic counted with multiplicity.

We now want to consider families of geodesics in $M$ in the following sense. We fix a point $p \in M$ and let $\Omega_{p} \subset T_{p} M$ denote the open and star shaped subset of the tangent space consisting of all $v \in T_{p} M$ such that $\exp (v)$ is defined (cf. [Le97, Prop. 5.7]). Let $X \subset \Omega_{p}$ be any subset. We obtain a family of geodesics by

$$
X \ni v \mapsto \gamma_{v}(\cdot) \in H_{p, \gamma_{v}(1)}^{1}(I, M),
$$

where $\gamma_{v}(\cdot)$ is defined by

$$
I \ni t \mapsto \gamma_{v}(t)=\exp _{p}(t \cdot v) \in M .
$$


So $X$ induces a family

$$
\Gamma: X \rightarrow H^{1}(I, M), \quad \Gamma(v)=\gamma_{v}
$$

where $\gamma_{v}$ is a critical point of the smooth function $\mathcal{A}_{v}: H_{p, \gamma_{v}(1)}^{1}(I, M) \rightarrow \mathbb{R}$ given by

$$
\mathcal{A}_{v}(\gamma)=\frac{1}{2} \int_{0}^{1} g\left(\gamma^{\prime}(t), \gamma^{\prime}(t)\right) d t
$$

We obtain a family of associated quadratic forms of the Hessians

$$
h e s s_{\gamma_{v}}\left(\mathcal{A}_{p \gamma_{v}(1)}\right): T_{\gamma_{v}} H_{p \gamma_{v}(1)}^{1}(I, M) \rightarrow \mathbb{R}
$$

which we now want to transform to a family of quadratic forms on the fixed Hilbert space $H_{0}^{1}\left(I, \mathbb{R}^{n}\right)$ by using local coordinates.

We fix a $g$-orthonormal basis $\left\{e^{1}, \ldots, e^{n}\right\}$ of $T_{p} M$ and define mappings

$$
e^{i}: I \times X \rightarrow T M, \quad i=1, \ldots, n
$$

such that $e^{i}(t, v)$ is obtained from $e^{i}$ by parallel transport along $\gamma_{v}$ to $T_{\gamma_{v}(t)} M$. From the well known formula

$$
\frac{d}{d t} g(\xi, \eta)=g\left(\frac{\nabla}{d t} \xi, \eta\right)+g\left(\xi, \frac{\nabla}{d t} \eta\right)
$$

for any vector fields $\xi, \eta \in \Gamma(\gamma)$ along $\gamma$, we obtain

$$
\frac{d}{d t} g\left(e^{i}(t, v), e^{j}(t, v)\right)=0, \quad i, j=1, \ldots n, \quad v \in X
$$

since $e^{i}(\cdot, v), i=1, \ldots, n$, is parallel along $\gamma_{v}$. Hence

$$
g\left(e^{i}(t, v), e^{i}(t, v)\right)=\left\{\begin{array}{l}
1, i \leq n-\nu \\
-1, i>n-\nu
\end{array} \quad, \quad g\left(e^{i}(t, v), e^{j}(t, v)\right)=0, i \neq j, \quad t \in I, v \in X\right.
$$

and any vector field $\xi: I \rightarrow T M$ along $\gamma_{v}$ can be written uniquely as

$$
\xi(t)=\sum_{i=1}^{n} u_{i}(t) e^{i}(t, v)
$$


where $u_{i}: I \rightarrow \mathbb{R}$ are functions which are as smooth as $\xi$. Using these trivialisations, we can transform the family of quadratic forms $\operatorname{hess}_{\gamma_{v}}\left(\mathcal{A}_{p \gamma_{v}(1)}\right)$ into a family of quadratic forms on $H_{0}^{1}\left(I, \mathbb{R}^{n}\right)$ as follows. Plugging 10.3 into 10.2 , we obtain by using again that each $e^{i}(\cdot, v)$ is parallel along $\gamma_{v}$

$$
\begin{aligned}
& \int_{0}^{1} g_{\gamma_{v}(t)}\left(\frac{\nabla}{d t} \xi, \frac{\nabla}{d t} \xi\right) d t+\int_{0}^{1} g_{\gamma_{v}(t)}\left(R\left(\gamma_{v}^{\prime}(t), \xi(t)\right) \gamma_{v}^{\prime}(t), \xi(t)\right) d t \\
& =\int_{0}^{1} g_{\gamma_{v}(t)}\left(\sum_{i=1}^{n} u_{i}^{\prime}(t) e^{i}(t, v), \sum_{j=1}^{n} u_{j}^{\prime}(t) e^{j}(t, v)\right) d t+\int_{0}^{1} g_{\gamma_{v}(t)}\left(R\left(\gamma_{v}^{\prime}(t), \xi(t)\right) \gamma_{v}^{\prime}(t), \xi(t)\right) d t \\
& =\int_{0}^{1} \sum_{i, j=1}^{n} u_{i}^{\prime}(t) u_{j}^{\prime}(t) g_{\gamma_{v}(t)}\left(e^{i}(t, v), e^{j}(t, v)\right) d t \\
& +\int_{0}^{1} \sum_{i, j=1}^{n} u_{i}(t) u_{j}(t) g_{\gamma_{v}(t)}\left(R\left(\gamma_{v}^{\prime}(t), e^{i}(t, v)\right) \gamma_{v}^{\prime}(t), e^{j}(t, v)\right) d t \\
& =\int_{0}^{1} \sum_{i=1}^{n} u_{i}^{\prime}(t) u_{i}^{\prime}(t) g_{\gamma_{v}(t)}\left(e^{i}(t, v), e^{i}(t, v)\right) d t \\
& +\int_{0}^{1} \sum_{i, j=1}^{n} u_{i}(t) u_{j}(t) g_{\gamma_{v}(t)}\left(R\left(\gamma_{v}^{\prime}(t), e^{i}(t, v)\right) \gamma_{v}^{\prime}(t), e^{j}(t, v)\right) d t, \quad \xi \in T_{\gamma_{v}} H_{p \gamma_{v}(1)}^{1}(I, M) .
\end{aligned}
$$

Hence the resulting family is given by

$$
\begin{gathered}
q_{v}: H_{0}^{1}\left(I, \mathbb{R}^{n}\right) \rightarrow \mathbb{R}, \\
q_{v}(u)=\int_{0}^{1}\left\langle J u^{\prime}(t), u^{\prime}(t)\right\rangle d t-\int_{0}^{1}\left\langle S_{v}(t) u(t), u(t)\right\rangle d t,
\end{gathered}
$$

where

$$
J=\left(\begin{array}{cc}
I_{n-\nu} & 0 \\
0 & -I_{\nu}
\end{array}\right)
$$

and

$$
S_{v}(t)=\left\{S_{i j}(v, t)\right\}_{i, j}, \quad S_{i j}(v, t)=-g\left(R\left(\gamma_{v}^{\prime}(t), e^{i}(t, v)\right) \gamma_{v}^{\prime}(t), e^{j}(t, v)\right) .
$$

10.1.1 Lemma. Each $S_{v}(t),(t, v) \in I \times \Omega_{p}$, is a symmetric matrix and the map $S: I \times \Omega_{p} \rightarrow$ $M(n, \mathbb{R})$ is smooth.

Proof. At first we obtain for any $1 \leq i, j \leq n$

$$
S_{i j}(v, t)=-g\left(R\left(\gamma_{v}^{\prime}(t), e^{j}(t, v)\right) \gamma_{v}^{\prime}(t), e^{i}(t, v)\right)=-g\left(R\left(\gamma_{v}^{\prime}(t), e^{i}(t, v)\right) \gamma_{v}^{\prime}(t), e^{j}(t, v)\right)=S_{j i}(v, t)
$$


by elementary properties of the curvature tensor $R$ and hence $S_{v}(t)$ is symmetric. In order to show the second assertion we note at first that the map

$$
I \times \Omega_{p} \ni(t, v) \mapsto \gamma_{v}^{\prime}(t) \in T M
$$

is smooth because the exponential map is smooth on $\Omega_{p}$. Moreover the map

$$
I \times \Omega_{p} \ni(t, v) \mapsto e^{i}(t, v) \in T M,
$$

obtained by parallel transport of the vector $e^{i}$ along the geodesics $\gamma_{v}$, is smooth by [Mi88, 1.5]. Now the assertion follows from the smoothness of the curvature tensor

$$
g(R(\cdot, \cdot) \cdot, \cdot): T M \otimes T M \otimes T M \otimes T M \rightarrow \mathbb{R} .
$$

We now consider the Jacobi equation along $\gamma_{v}$ for a fixed $v \in X$ which is

$$
\frac{\nabla^{2}}{d x^{2}} \xi(x)-R\left(\gamma_{v}^{\prime}(t), \xi(t)\right) \gamma_{v}^{\prime}(t)=0, \quad \xi \in \Gamma\left(\gamma_{v}\right) .
$$

Inserting 10.3 into 10.5, we obtain

$$
\begin{aligned}
& \frac{\nabla^{2}}{d t^{2}}\left(\sum_{i=1}^{n} u_{i}(t) e^{i}(t, v)\right)-R\left(\gamma_{v}^{\prime}(t), \sum_{i=1}^{n} u_{i}(t) e^{i}(t, v)\right) \gamma_{v}^{\prime}(t) \\
= & \sum_{i=1}^{n} u_{i}^{\prime \prime}(t) e^{i}(t, v)-\sum_{i=1}^{n} u^{i}(t) R\left(\gamma_{v}^{\prime}(t), e^{i}(t, v)\right) \gamma_{v}^{\prime}(t)=0, \quad i=1, \ldots n .
\end{aligned}
$$

If we finally insert the result into the scalar products $g\left(\cdot, e^{j}(t, v)\right), j=1, \ldots, n$, we obtain $g\left(e^{i}(t, v), e^{j}(t, v)\right) u_{i}^{\prime \prime}(t)-\sum_{j=1}^{n} g\left(R\left(\gamma_{v}^{\prime}(t), e^{i}(t, v)\right) \gamma_{v}^{\prime}(t), e^{j}(t, v)\right) u_{j}(t)=0, \quad t \in I, \quad i=1, \ldots n$, and so the Jacobi equation of $\gamma_{v}$ is equivalent to

$$
J u^{\prime \prime}(t)+S_{v}(t) u(t)=0, \quad t \in I,
$$

where $u=\left(u_{1}, \ldots, u_{n}\right): I \rightarrow \mathbb{R}$ is a vector valued function and $J=\operatorname{diag}(1, \ldots, 1,-1, \ldots,-1)$ as well as $S_{v}: I \rightarrow M(n, \mathbb{R})$ are as above. Hence the Jacobi equations associated to our family of geodesics induce a family of differential operators 


$$
\begin{aligned}
\mathcal{A}_{v}: \mathcal{D}\left(\mathcal{A}_{v}\right) & =H^{2}\left(I, \mathbb{R}^{n}\right) \cap H_{0}^{1}\left(I, \mathbb{R}^{n}\right) \subset L^{2}\left(I, \mathbb{R}^{n}\right) \rightarrow L^{2}\left(I, \mathbb{R}^{n}\right) \\
\left(\mathcal{A}_{v} u\right)(t) & =J u^{\prime \prime}(t)+S_{v}(t) u(t), \quad v \in X,
\end{aligned}
$$

which corresponds to the quadratic forms 10.4 in the same way as 9.1 to 9.2 in our family Morse index theorem 9.1.1. We want to point out that we denote the family 10.6 by the same symbol than the action functional above. However, since we will not meet the action functional again in the following, there will be very little opportunity to confuse the two.

The following result now shows the expected relation between the operators 10.6 and conjugate instants.

10.1.2 Lemma. $\exp _{p}(v)$ is conjugate to $p$ along $\gamma_{v}$ if and only if $\operatorname{ker} \mathcal{A}_{v} \neq 0$.

Proof. By definition $\exp _{p}(v)$ is conjugate to $p$ if and only if there exists a non trivial Jacobi field $\xi \in \Gamma\left(\gamma_{v}\right)$ along $\gamma_{v}$ vanishing at 0 and 1. By construction each such Jacobi field yields a nontrivial element of $\operatorname{ker} \mathcal{A}_{v}$. Conversely, if $u \in \operatorname{ker} \mathcal{A}_{v}$, it is clear that $u$ is actually a smooth function vanishing at 0 and 1 . Hence by construction it yields a non trivial Jacobi field along $\gamma_{v}$ vanishing at its endpoints.

10.1.3 Definition. We call $v \in \Omega_{p} \subset T_{p} M$ a conjugate point if $\gamma_{v}(1)=\exp _{p}(v) \in M$ is conjugate to $p$ along $\gamma_{v}$.

In order to apply our results from the foregoing section, we now consider the complexified families

$$
\begin{aligned}
& q^{\mathbb{C}}: H_{0}^{1}\left(I, \mathbb{C}^{n}\right) \rightarrow \mathbb{R} \\
& \mathcal{A}^{\mathbb{C}}: \mathcal{D}\left(\mathcal{A}^{\mathbb{C}}\right)=H^{2}\left(I, \mathbb{C}^{n}\right) \cap H_{0}^{1}\left(I, \mathbb{C}^{n}\right) \subset L^{2}\left(I, \mathbb{C}^{n}\right) \rightarrow L^{2}\left(I, \mathbb{C}^{n}\right) .
\end{aligned}
$$

Note that lemma 10.1 .2 holds verbatim if we replace $\mathcal{A}_{v}$ by $\mathcal{A}_{v}^{\mathbb{C}}$ because $\operatorname{ker} \mathcal{A}^{\mathbb{C}}=\operatorname{ker} \mathcal{A} \oplus$ $i \operatorname{ker} \mathcal{A}$.

10.1.4 Definition. Let $X \subset \Omega_{p}$ be compact and $Y \subset X$ be a closed subset containing no conjugate points. Then we define

$$
\begin{aligned}
\mu_{\text {quad }}(X, Y) & =\operatorname{s-ind}\left(q^{\mathbb{C}}\right) \in K^{-1}(X, Y) \\
\mu_{\text {spec }}(X, Y) & =\operatorname{s-ind}\left(\mathcal{A}^{\mathbb{C}}\right) \in K^{-1}(X, Y) \\
\mu_{\text {con }}(X, Y) & =\operatorname{con}\left(\mathcal{A}^{\mathbb{C}}\right) \in K^{-1}(X, Y) .
\end{aligned}
$$

Note that by lemma 10.1 .1 and lemma 10.1 .2 these elements are indeed defined if $Y$ does not contain conjugate points. Now we obtain our Morse index theorem for families of geodesics which are parametrized by compact subspaces of $T_{p} M$ immediately from 9.1.1 
10.1.5 Theorem. Let $X \subset \Omega_{p}$ be compact and $Y \subset X$ a closed subset containing no conjugate points. Then

$$
\mu(X, Y):=\mu_{\text {quad }}(X, Y)=\mu_{\text {spec }}(X, Y)=\mu_{\text {con }}(X, Y) \in K^{-1}(X, Y)
$$

Moreover, if $\mu(X, Y)$ is non trivial, we can infer the existence of a conjugate point in $X \backslash Y$.

Note that the second assertion follows immediately from the normalisation property 6.2.1 of s-ind.

So far we have not proved that the indices in theorem 10.1.5 are well defined in the sense that they do not depend on the choices involved. However, by checking our constructions we notice that the only choice we made is a $g$-orthonormal frame $\left\{e^{1}, \ldots, e^{n}\right\}$ in $T_{p} M$. Moreover, by theorem 10.1 .5 it suffices to prove well definedness for one of the indices.

10.1.6 Lemma. Let $(X, Y)$ be as above. Then $\mu_{\text {quad }}(X, Y)$ does not depend on the choice of the $g$-orthonormal basis $\left\{e_{1}, \ldots, e_{n}\right\}$ in $T_{p} M$.

Proof. Let $\left\{e_{1}, \ldots, e_{n}\right\}$ and $\left\{\tilde{e}_{1}, \ldots, \tilde{e}_{n}\right\}$ be two $g$-orthonormal basis of $T_{p} M$ and $M$ a $g$-orthogonal matrix such that $M e_{i}=\tilde{e}_{i}, i=1, \ldots, n$. Then the parallel transported basis vectors are given by

$$
e^{i}(t, v)=P(t, v) e_{i} \quad \text { and } \quad \tilde{e}^{i}(t, v)=P(t, v) M e_{i},
$$

respectively, where $P(t, v): T_{p} M \rightarrow T_{\gamma_{v}(t)} M$ denotes the operator of parallel transport along $\gamma_{v}$.

We now consider the matrices $S_{v}(t)$ and $\tilde{S}_{v}(t)$ for some $(v, t) \in X \times I$, where

$$
\begin{aligned}
& S_{i j}(v, t)=-g\left(R\left(\gamma_{v}^{\prime}(t), e^{j}(t, v)\right) \gamma_{v}^{\prime}(t), e^{i}(t, v)\right)=-g\left(R\left(\gamma_{v}^{\prime}(t), P(t, v) e^{j}\right) \gamma_{v}^{\prime}(t), P(t, v) e^{i}\right) \\
& \tilde{S}_{i j}(v, t)=-g\left(R\left(\gamma_{v}^{\prime}(t), \tilde{e}^{j}(t, v)\right) \gamma_{v}^{\prime}(t), \tilde{e}^{i}(t, v)\right)=-g\left(R\left(\gamma_{v}^{\prime}(t), P(t, v) \tilde{e}^{j}\right) \gamma_{v}^{\prime}(t), P(t, v) \tilde{e}^{i}\right) .
\end{aligned}
$$

Since the representing matrix for the change of basis from $\left\{\tilde{e}_{i}\right\}$ to $\left\{e_{i}\right\}$ is $M$ and $g\left(R\left(\gamma_{v}^{\prime}(t), P(t, v) \cdot\right) \gamma_{v}^{\prime}(t), P(t, v) \cdot\right)$ is bilinear, we infer that $\tilde{S}_{v}(t)=M^{T} S_{v}(t) M$. Now the Riesz representation of the quadratic form (10.4) with respect to the matrix family $\tilde{S}_{v}$ is given according to 9.4 by

$$
\begin{aligned}
\left(\tilde{L}_{v} u\right)(t) & =J u(t)+\int_{0}^{t} \int_{0}^{s} \tilde{S}_{v}(\tau) u(\tau) d \tau d s-t \int_{0}^{1} \int_{0}^{s} \tilde{S}_{v}(\tau) u(\tau) d \tau d s \\
& =J u(t)+\int_{0}^{t} \int_{0}^{s} M^{T} S_{v}(\tau) M u(\tau) d \tau d s-t \int_{0}^{1} \int_{0}^{s} M^{T} S_{v}(\tau) M u(\tau) d \tau d s .
\end{aligned}
$$


Since $M$ is $g$-orthogonal, we have $M^{T} J M=J$ and now we obtain from 10.7

$$
\left(\tilde{L}_{v} u\right)(t)=\left(M^{T} L_{v} M u\right)(t), \quad u \in H_{0}^{1}\left(I, \mathbb{C}^{n}\right) .
$$

We choose a path $\Psi: I \rightarrow G L(n, \mathbb{C})$ connecting $M$ and the identity matrix $I$ and define a homotopy

$$
H: I \times X \rightarrow \mathcal{B F}^{s a}\left(H_{0}^{1}\left(I, \mathbb{C}^{n}\right)\right), \quad H(\lambda, v)=\Psi(\lambda)^{*} L_{v} \Psi(\lambda),
$$

where now $\cdot^{*}$ denotes the conjugate transpose of a matrix. Then $H$ is a homotopy of bounded selfadjoint Fredholm operators which is continuous with respect to the norm topology on $\mathcal{B F}^{s a}\left(H_{0}^{1}\left(I, \mathbb{C}^{n}\right)\right)$ and we finally infer

$$
\operatorname{s-ind}(\tilde{L})=\operatorname{s-ind}(L) \in K^{-1}(X, Y)
$$

by the homotopy invariance property 7.1 .5 .

We conclude this section by first properties of $\mu$.

10.1.7 Lemma. For any $\left(X^{\prime}, Y^{\prime}\right) \subset(X, Y)$ as in the definition of $\mu$ we have

$$
\mu\left(X^{\prime}, Y^{\prime}\right)=\iota^{*} \mu(X, Y) \in K^{-1}\left(X^{\prime}, Y^{\prime}\right) .
$$

Proof. The assertion follows from the functoriality of the selfadjoint index bundle as proved in lemma 7.1.4.

Hence no information gets lost as far as $\iota^{*}: K^{-1}(X, Y) \rightarrow K^{-1}\left(X^{\prime}, Y^{\prime}\right)$ is injective. Moreover, we infer the following important corollary.

10.1.8 Corollary. If $\mu\left(X^{\prime}, Y^{\prime}\right) \neq 0$, then $\mu(X, Y) \neq 0$.

This is certainly a pleasant property, because once we infer the existence of a conjugate point in $X^{\prime} \backslash Y^{\prime}$ by the non triviality of $\mu\left(X^{\prime}, Y^{\prime}\right)$, this information will not disappear when considering larger pairs of spaces. Nevertheless, we can loose information and it would be much better to have a more powerful result that ensures not only the existence of a single conjugate point but the existence of lots of them. This will be the topic of section 10.4 below.

10.1.9 Lemma. Let $X \subset T_{p} M$ be compact. Then

i) $\mu(X, \emptyset)=0$,

ii) 0 is not a conjugate point and $\mu(X,\{0\})=0$ if $0 \in X$. 
Proof. We recall at first that the operator family $L$ in 9.4 from the definition of $\mu_{\text {quad }}$ is of the form $L_{v}=J I+K_{v}$ where $J$ is a constant invertible matrix, $I$ the identity operator on $H_{0}^{1}\left(I, \mathbb{C}^{n}\right)$ and $K$ a family of compact operators. Now the first assertion follows immediately from corollary 7.1.6.

In order to show the second assertion we note that $\gamma_{0}(t)=p, t \in I$, and so $R\left(\gamma_{0}^{\prime}(t), e^{i}(t, 0)\right) \gamma_{0}^{\prime}(t)=$ $0, i=1, \ldots, n$, for all $t \in I$. Accordingly, $S_{0}=0$ and $\mathcal{A}_{0}$ is the operator mapping a function $u \in H^{2}\left(I, \mathbb{R}^{n}\right) \cap H_{0}^{1}\left(I, \mathbb{R}^{n}\right)$ to $J u^{\prime \prime}$. Hence $\operatorname{ker} \mathcal{A}_{0}=\{0\}$ and so $0 \in T_{p} M$ is not a conjugate point. Moreover, from $S_{0}=0$ we infer by 9.4 that $K_{0}=0$ and hence $J I+\lambda K_{v}$ is invertible for $v \in Y=\{0\}$ for all $\lambda \in[0,1]$. Now corollary 7.1.6 shows again that $\mu(X,\{0\})=0$.

10.1.10 Remark. $\mathcal{A}$ is a gap continuous family of selfadjoint Fredholm operators by the results of section 9.2 and, moreover, the invertible elements are open in $\mathcal{C}\left(L^{2}\left(I, \mathbb{C}^{n}\right)\right)$ according to lemma 2.3.6. Since 0 is not a conjugate point according to the second part of lemma 10.1.9 we now obtain from lemma 10.1.2 the existence of an open neighbourhood of $0 \in T_{p} M$ which contains no conjugate points.

\subsection{Examples}

In the following we consider some examples which show in particular that our indices depend heavily on the choice of $Y$ and are far from being a reliable detector for conjugate points.

\section{Example I: Single Geodesics}

Let $\gamma: I \rightarrow M$ be a geodesic in $M$ such that 1 is not a conjugate instant and define

$$
\begin{aligned}
& X=\left\{x \cdot \gamma^{\prime}(0): 0 \leq x \leq 1\right\} \subset T_{\gamma(0)} M \\
& Y=\left\{0, \gamma^{\prime}(0)\right\} \subset X .
\end{aligned}
$$

Then for $(v, t)=\left(x \cdot \gamma^{\prime}(0), t\right) \in X \times I$ the components of the matrix $S_{v}(t)$ are given by

$$
\begin{aligned}
S_{i j}(v, t) & =-g\left(R\left(\gamma_{x \gamma^{\prime}(0)}^{\prime}(t), e^{i}\left(t, x \cdot \gamma^{\prime}(0)\right)\right) \gamma_{x \gamma^{\prime}(0)}^{\prime}(t), e^{j}\left(t, x \cdot \gamma^{\prime}(0)\right)\right) \\
& =-g\left(R\left(x \cdot \gamma_{\gamma^{\prime}(0)}^{\prime}(x \cdot t), e^{i}\left(x \cdot t, \gamma^{\prime}(0)\right)\right) x \cdot \gamma_{\gamma^{\prime}(0)}^{\prime}(x \cdot t), e^{j}\left(x \cdot t, \gamma^{\prime}(0)\right)\right) \\
& =-g\left(R\left(x \cdot \gamma^{\prime}(x \cdot t), e^{i}\left(x \cdot t, \gamma^{\prime}(0)\right)\right) x \cdot \gamma^{\prime}(x \cdot t), e^{j}\left(x \cdot t, \gamma^{\prime}(0)\right)\right) \\
& =-x^{2} g\left(R\left(\gamma^{\prime}(x \cdot t), e^{i}\left(x \cdot t, \gamma^{\prime}(0)\right)\right) \gamma^{\prime}(x \cdot t), e^{j}\left(x \cdot t, \gamma^{\prime}(0)\right)\right)
\end{aligned}
$$

and we now regard $S$ as parametrised by $x \in[0,1]$. Then our operator families $L$, which represents the quadratic forms 10.4, and $\mathcal{A}$ defined in 10.6 are just paths of selfadjoint Fredholm operators. Moreover, the matrix family $b$ constructed in the definition of the conjugate index can be regarded as parametrized by $I \times \mathbb{R}$. So all our indices are elements of $K^{-1}(I, \partial I)$ and 
this group is isomorphic to the integers by the isomorphism $c_{1}: K^{-1}(I, \partial I) \rightarrow \mathbb{Z}$ constructed in section B.3. We obtain

$$
c_{1}\left(\mu_{\text {con }}(X, Y)\right)=w(\operatorname{det} b \circ \varphi, 0) \in \mathbb{Z},
$$

where $\varphi: S^{1} \rightarrow I \times \mathbb{R}$ is any simple positively oriented curve surrounding $(0,1) \times\{0\}$. Moreover, from proposition 7.3 .1 we deduce that

$$
\begin{aligned}
& c_{1}\left(\mu_{\text {spec }}(X, Y)\right)=\operatorname{sf}(\mathcal{A}) \in \mathbb{Z} \\
& c_{1}\left(\mu_{\text {quad }}(X, Y)\right)=\operatorname{sf}(L) \in \mathbb{Z}
\end{aligned}
$$

Now the right hand side of 10.8 is by definition the conjugate index $\mu_{\text {con }}(\gamma)$ and the right hand side of 10.10 the generalised Morse index $\mu_{\text {spec }}(\gamma)$ of the geodesic $\gamma$ as defined in MPP05. The main result of [MPP05] states the equality of $\mu_{\text {spec }}(\gamma)$ and $\mu_{\text {con }}(\gamma)$ which we now have obtained as a consequence of theorem 10.1.5.

The integer 10.9 does not appear in the semi-Riemannian Morse index theorem in [MPP05] explicitly but in its proof, where it is shown in a first step that $\mu_{\text {spec }}(\gamma)=\operatorname{sf}(\mathcal{A})$ and afterwards the equality $\operatorname{sf}(\mathcal{A})=\mu_{\text {con }}(\gamma)$ is proven. Note that the first of these equalities precisely corresponds to the first part of theorem 9.1.1 which asserts $\operatorname{sind}(q)=\operatorname{sind}(\mathcal{A})$ and which is proved by the property 7.1 .13 of the selfadjoint index bundle and the reduction property 5.2.10. In contrast, in the proofs in [MPP05] and Wa12], $\mu_{\text {spec }}(\gamma)=\operatorname{sf}(\mathcal{A})$ is proved by using quite involved principles on the spectral flow obtained by Robbin and Salamon in RS95] and by Fitzpatrick, Pejsachowicz and Recht in [FPR99]. These results deal with spectra of perturbations of smooth paths of selfadjoint operators and are in particular not available for families as in theorem 9.1.1. The equality $\operatorname{sf}(\mathcal{A})=\mu_{\text {con }}(\gamma)$ corresponds to the second part of our theorem 9.1.1 which shows that s-ind $(\mathcal{A})$ and $\operatorname{con}(\mathcal{A})$ coincide. Our $K$-theoretic proof of this equality is just a straightforward modification of the corresponding result in Wa12 in the case that $X$ is an interval. In contrast, the proof of $\operatorname{sf}(\mathcal{A})=\mu_{\text {con }}(\gamma)$ in MPP05 is completely functional analytic and restricted to this special case.

\section{Example II}

Let $X$ and $Y$ be as in definition 10.1.4 and assume that $0 \in Y$ and that there exists $v \in Y$ such that $\gamma_{v}$ has a non vanishing generalised Morse index $\mu_{\text {spec }}(\gamma) \in \mathbb{Z}$ as defined in example I above. Then $c_{1}\left(\mu\left(X^{\prime}, Y^{\prime}\right)\right)=\mu_{\text {spec }}(\gamma) \neq 0$, where $X^{\prime}$ is the line given by the vector $\gamma_{v}^{\prime}(0)$ in $T_{p} M$ and $Y^{\prime}$ its boundary. Now corollary 10.1 .8 implies

$$
\mu(X, Y) \neq 0 .
$$


This shows that, as far as $\{0\} \subset Y$ is a proper subset, it can be easy to see the non triviality of $\mu(X, Y)$. However, note that it is important to assume $\{0\}$ to be a proper subset by lemma 10.1.9

For example, if $(M, g)$ is Riemannian, then $\mu_{\text {spec }}(\gamma)$ is the Morse index of $\gamma$ by MPP05, Prop. 3.4] and now the existence of a single conjugate point $v \in X$ is sufficient in order to conclude that $\mu(X, Y) \neq 0$ if $0 \in Y$ and $t v \in Y$ for some instant $t>1$.

\section{Example III: Stars of Geodesics}

Let $N \in \mathbb{N}$ and $\gamma_{i}, i=1, \ldots, N$, be geodesics such that $\gamma_{i}(0)=p$ and $\gamma_{i}(1)$ is not conjugate to $p$ along $\gamma_{i}(t), i=1, \ldots, N$. Moreover, assume that all $\gamma_{i}^{\prime}(0)$ are pairwise linearly independent. Define

$$
\begin{aligned}
& X=\bigcup_{i=1}^{N}\left\{t \gamma_{i}^{\prime}(0): 0 \leq t \leq 1\right\} \\
& Y=\{0\} \cup \bigcup_{i=1}^{N}\left\{\gamma_{i}^{\prime}(0)\right\} .
\end{aligned}
$$

Then it is an easy exercise in elementary $K$-theory to show that

$$
\mu(X, Y)=\left(\mu_{\text {spec }}\left(\gamma_{1}\right), \ldots, \mu_{\text {spec }}\left(\gamma_{N}\right)\right) \in \bigoplus_{i=1}^{N} \mathbb{Z}
$$

where it is used that $X / Y$ is a wedge product of 1 -spheres and

$$
K^{-1}\left(S^{1} \vee S^{1}\right) \cong K^{-1}\left(S^{1}\right) \oplus K^{-1}\left(S^{1}\right) \cong \mathbb{Z} \oplus \mathbb{Z}
$$

If the star is degenerated in the sense that some of the $\gamma_{i}^{\prime}(0)$ are pairwise linearly dependent, one has to modify the result slightly in the obvious way.

\subsection{Outlook: A Generalisation and the Porta Recht The- orem}

Our index theorem 10.1 .5 treats families of geodesics only in a very restrictive sense and the aim of this section is to indicate briefly a possible way to apply the family index theorem 9.1.1 for differential equations to more general families of geodesics.

Our main reference here is the article [PR82] by H. Porta and L. Recht who generalised the classical Morse index theorem to families of Riemannian geodesics. Instead of a single geodesic in a Riemannian manifold they considered a compact topological space $X$ and maps $\gamma: I \times X \rightarrow M$ 
such that each $\gamma_{x}=\gamma(\cdot, x): I \rightarrow M$ is a geodesic in $M$. For a sufficiently fine decomposition $0=t_{0}<t_{1}<\ldots<t_{n}=1$ of the unit interval one can show that the continuous vector fields along $\gamma_{x}$, which are piecewise Jacobi fields with respect to the chosen decomposition and which vanish at 0 and $\tau \in(0,1]$, are in one-to-one correspondence with the space $\bigoplus_{i=1}^{k-1} T_{\gamma\left(t_{i}, x\right)} M$, where $k$ is chosen such that $t_{k-1}<\tau \leq t_{k}$. Now we consider the vector bundles

$$
\xi\left(t_{0}, \ldots, t_{k-1}, \tau\right)=\bigoplus_{i=1}^{k-1} \gamma\left(t_{i}, \cdot\right)^{*}(T M)
$$

over $X$ and by the correspondence just mentioned above the bilinear forms 10.2 induce a map on $\xi\left(t_{0}, \ldots, t_{k-1}, \tau\right)$. By using the metric induced by $g$ on $\xi\left(t_{0}, \ldots, t_{k-1}, \tau\right)$, each fibre splits as a direct sum

$$
\xi_{x}\left(t_{0}, \ldots, t_{k-1}, \tau\right)=\xi_{x}^{-}\left(t_{0}, \ldots, t_{k-1}, \tau\right) \oplus \xi_{x}^{0}\left(t_{0}, \ldots, t_{k-1}, \tau\right) \oplus \xi_{x}^{+}\left(t_{0}, \ldots, t_{k-1}, \tau\right), \quad x \in X
$$

in the corresponding negative subspace, kernel and positive subspace of the corresponding bilinear map. Porta and Recht now defined a family $\gamma: I \times X \rightarrow M$ of geodesics to be special if for each $\tau \in(0,1]$ the kernels $\xi_{x}^{0}\left(t_{0}, \ldots, t_{k-1}, \tau\right)$ fit together to a vector bundle over $X$. It is a common exercise to show that if a geodesic family is special, then the spaces $\xi_{x}^{-}\left(t_{0}, \ldots, t_{k-1}, \tau\right)$ and $\xi_{x}^{+}\left(t_{0}, \ldots, t_{k-1}, \tau\right)$ build vector bundles for any $\tau \in(0,1]$ as well such that we actually obtain bundle decompositions of each $\xi\left(t_{0}, \ldots, t_{k-1}, \tau\right)$ into three subbundles. The main theorem of PR82 asserts that for a special family $\gamma: I \times X \rightarrow M$ of geodesics the isomorphism classes of the bundles according to the splitting 10.12 do not depend on the choice of the decomposition $0=t_{0}<t_{1}<\ldots<t_{n}=1$ once it is sufficiently fine and, moreover, in this case we have a bundle isomorphism

$$
\xi^{-}\left(t_{0}, \ldots, t_{n}\right) \cong \bigoplus_{0<\tau<1} \xi^{0}\left(t_{0}, \ldots, t_{k}, \tau\right)
$$

In the case that $X$ is a single point, 10.13 is just the classical Riemannian Morse Index theorem.

We strongly believe that one can find from our index theorem 9.1.1 for differential equations an index theorem for general families of geodesics $\gamma: I \times X \rightarrow M$ which not only removes the restrictive assumption in 10.13 that $\gamma$ has to be special but also holds in general semi-Riemannian manifolds $(M, g)$ as our theorem 10.1.5. Hence the result we are looking for consolidates the Morse index theorems for geodesics mentioned so far in a single one, which even remedies unpleasant assumptions.

Let us recall that the definition of special means that the family of kernels considered in 10.12 fit together to a bundle over $X$. The index bundle, which we use in the construction of our 
indices in the abstract index theorem 9.1.1. can also be defined in a straightforward way as long as the kernels of the family of operators fit together to a bundle (cf. [BoBl85, §3]). However, in the general case this restrictive condition can be avoided by considering stable equivalence of bundles, that is, $K$-theory. Accordingly, to work in $K$-theory as we do in the abstract family index theorem is supposed to be the right setting in order to consider families of geodesics which are not special in the sense of Porta and Recht.

Finally we want to describe difficulties to overcome. In the family index theorem for geodesics 10.1.5 we have to choose parallel frames along the geodesics which allows to translate all geometric data to the abstract families (10.4) and (10.6). Since the frame is obtained by parallel translation from a base of $T_{\gamma(0)} M$, in our situation it actually suffices to find a way how to assign a base of the tangent space $T_{\gamma(x, 0)} M$ to each $x \in X$ such that the arising operator families in our constructions become continuous and the associated $K$-theory elements are independent of the choice made. So far we have no definite answer to this problem but it seems to be possible at least under the additional assumption that the map $\gamma(\cdot, 0): X \rightarrow M$ of initial points can be lifted to the frame bundle associated to $T M$. Then we obtain from a family $\gamma: I \times X \rightarrow M$ of semi-Riemannian geodesics abstract families as in 10.4 and $(10.6)$ in the same way as they were obtained in theorem 10.1.5. However, the corresponding $K$-theory classes according to our abstract family index theorem are elements of $K^{-1}(X \times \mathbb{R})$ which is by definition $K\left(X \times \mathbb{R}^{2}\right)$. Hence we obtain virtual bundles over $X \times \mathbb{R}^{2}$ whereas the elements constructed by Porta and Recht are bundles over $X$. Nevertheless, we strongly expect that in the case of a special family of Riemannian geodesics both definitions coincide under the celebrated Bott periodicity isomorphism

$$
\mu: K(X) \stackrel{\cong}{\rightrightarrows} K\left(X \times \mathbb{R}^{2}\right) .
$$

Note again that we work with $K$-theory and Porta and Recht used isomorphism classes of vector bundles instead. However, we expect that for special families of geodesics we can reconstruct the elements of Porta and Recht from our definitions of the $K$-theoretic indices in a unique way.

\subsection{On the Size and Shape of the Conjugate Locus}

Throughout this section let $(M, g)$ be a semi-Riemannian manifold of dimension $n, p \in M$ a fixed point and $\Omega_{p}$ defined as in section 10.1 . The aim of this section is to obtain results on the dimension and shape of the set of conjugate points

$$
\Sigma_{p}=\left\{v \in \Omega_{p}: \exp _{p}(v) \text { is conjugate to } p \text { along } \gamma_{v}\right\} \subset T_{p} M .
$$

Frank Warner studied in Wa65 analytic properties of $\Sigma_{p}$ if $M$ is a Riemannian manifold. He proved that if $\Sigma_{p}$ is not empty, then it contains a submanifold of $T_{p} M$ of codimension 1. 
As a consequence we obtain that $\operatorname{dim} \Sigma_{p} \geq n-1$. In the recent work [Sz08] David Szeghy showed that Warner's result holds locally in semi-Riemannian manifolds in neighbourhoods of conjugate points in $T_{p} M$ that are not degenerated in a certain sense. However, as he states in the introduction of [Sz08], it is not clear how exceptional it is for a conjugate point to be non degenerate. Note that again the existence of a non degenerate conjugate point implies in particular $\operatorname{dim} \Sigma_{p} \geq n-1$.

We now want to study the dimension of $\Sigma_{p}$ by using the indices from the semi-Riemannian Morse index theorem and the ideas we developed in the third chapter of the first part of the thesis. Hence we use topological methods instead of analytical ones. We use in the following the notations from section 10.1 freely.

10.4.1 Lemma. $\Sigma_{p} \subset \Omega_{p}$ is closed.

Proof. By lemma 10.1.2 $\Sigma_{p}$ is the singular set of the operator family $\mathcal{A}: \Omega_{p} \rightarrow \mathcal{C F}^{s a}\left(L^{2}\left(I, \mathbb{C}^{n}\right)\right)$ introduced in (10.6) which is continuous in the gap topology by the results of section 9.2 and lemma 10.1.1. Since the invertible elements are open in the gap topology by lemma 2.3.6, we infer that $\Omega_{p} \backslash \Sigma_{p}=\mathcal{A}^{-1}\left(G \mathcal{C}^{s a}\left(L^{2}\left(I, \mathbb{R}^{n}\right)\right)\right.$ is open.

Note that we also could obtain the following result as a consequence of lemma 3.3.5. Here we prefer a more direct proof instead which allows to shorten the argumentation. We want to point out that we do not have to distinguish between path- and connected components because of lemma 10.4.1.

10.4.2 Lemma. Let $v_{1}, v_{2} \in \Omega_{p}$ be not conjugate and assume that $\mu_{\text {spec }}\left(\gamma_{v_{1}}\right) \neq \mu_{\text {spec }}\left(\gamma_{v_{2}}\right)$. Then $v_{1}$ and $v_{2}$ lie in different components of $\Omega_{p} \backslash \Sigma_{p}$.

Proof. We assume on the contrary that $v_{1}$ and $v_{2}$ lie in the same path component.

Consider the paths $\gamma_{i}: I \rightarrow \Omega_{p}, i=1,2,3$, where

$$
\begin{array}{r}
\gamma_{1}(t)=t v_{1} \\
\gamma_{3}(t)=(1-t) v_{2}
\end{array}
$$

and $\gamma_{2}$ is a path in $\Omega_{p}$ connecting $v_{1}$ and $v_{2}$ without intersecting $\Sigma_{p}$. Note that we use that $\Omega_{p}$ is star shaped with respect to 0 in the definition of $\gamma_{1}$ and $\gamma_{3}$.

We denote by $L$ the family of Riesz representations of 10.4 and note that the path

$$
L^{\mathbb{C}} \circ \gamma_{2}: I \rightarrow \mathcal{B F}^{s a}\left(H_{0}^{1}\left(I, \mathbb{C}^{n}\right)\right)
$$

has vanishing spectral flow because it consists solely of invertible operators. Hence 


$$
\begin{aligned}
\operatorname{sf}\left(L^{\mathbb{C}} \circ\left(\gamma_{1} * \gamma_{2} * \gamma_{3}\right)\right) & =\operatorname{sf}\left(L^{\mathbb{C}} \circ \gamma_{1}\right)+\operatorname{sf}\left(L^{\mathbb{C}} \circ \gamma_{2}\right)+\operatorname{sf}\left(L^{\mathbb{C}} \circ \gamma_{3}\right)=\operatorname{sf}\left(L^{\mathbb{C}} \circ \gamma_{1}\right)+\operatorname{sf}\left(L^{\mathbb{C}} \circ \gamma_{3}\right) \\
& =\operatorname{sf}\left(L^{\mathbb{C}} \circ \gamma_{1}\right)-\operatorname{sf}\left(L^{\mathbb{C}} \circ \gamma_{3}^{-1}\right)=\mu_{\text {spec }}\left(\gamma_{v_{1}}\right)-\mu_{\text {spec }}\left(\gamma_{v_{2}}\right) \neq 0,
\end{aligned}
$$

where we use that $\mu_{\text {spec }}\left(\gamma_{v_{1}}\right) \neq \mu_{\text {spec }}\left(\gamma_{v_{2}}\right)$ by assumption.

On the other hand, $\gamma=\gamma_{1} * \gamma_{2} * \gamma_{3}$ is a closed path with initial point $0 . L_{0}^{\mathbb{C}}$ is invertible since 0 is not conjugate by lemma 10.1 .9 and, moreover, $L^{\mathbb{C}} \circ \gamma(t)-L_{0}^{\mathbb{C}}$ is a compact selfadjoint operator as shown in section 9.3 Hence we can now define a homotopy

$$
H: I \times I \rightarrow \mathcal{B F}^{s a}\left(H_{0}^{1}\left(I, \mathbb{C}^{n}\right)\right), \quad H(\lambda, t)=L_{0}^{\mathbb{C}}+(1-\lambda)\left(L^{\mathbb{C}} \circ \gamma(t)-L_{0}^{\mathbb{C}}\right)
$$

such that $H(0)$ is $L^{\mathbb{C}} \circ \gamma$ and $H(1)$ is the constant path given by $L_{0}^{\mathbb{C}}$. Note that moreover $H(\lambda, 0)=H(\lambda, 1)=L_{0}^{\mathbb{C}} \in G L\left(H_{0}^{1}\left(I, \mathbb{C}^{n}\right)\right)$ for all $\lambda \in I$. Hence

$$
\operatorname{sf}\left(L^{\mathbb{C}} \circ\left(\gamma_{1} * \gamma_{2} * \gamma_{3}\right)\right)=\operatorname{sf}(H(0, \cdot))=\operatorname{sf}(H(1, \cdot))=0
$$

contradicting 10.14.

So, roughly speaking, the foregoing lemma asserts that if an observer in $T_{p} M$ can see a single conjugate point on the horizon (which means "in the direction of $\infty$ "), then he is already surrounded by them.

10.4.3 Corollary. If there exists $v \in \Omega_{p} \backslash \Sigma_{p}$ such that $\mu_{\text {spec }}\left(\gamma_{v}\right) \neq 0$, then $\Omega_{p} \backslash \Sigma$ is not connected.

10.4.4 Corollary. If $M$ is compact and there exists $v \in T_{p} M \backslash \Sigma_{p}$ such that $\mu_{\text {spec }}\left(\gamma_{v}\right) \neq 0$, then

$$
\operatorname{dim} \Sigma_{p} \geq n-1 .
$$

Proof. Note at first that we can not apply lemma 3.3 .1 because we can not assume $\Sigma$ to be compact in general.

We choose any norm on $T_{p} M$ and take a ball $B$ around $0 \in T_{p} M$ which contains $v$. Then $B \subset \Omega_{p}=T_{p} M$ because $M$ is compact and according to the foregoing lemma 0 and $v$ lie in different components of $B \backslash(B \cap \Sigma)$. Hence $\Sigma$ disconnects a ball of dimension $n$ which shows that $\operatorname{dim} \Sigma \geq n-1$ by [Fed90, Prop. 5, Prop. 6].

Now we want to study the shape of the set of conjugate points. To be more precise, we will study the shape of a subset of $\Sigma_{p}$ which turns out to be all of $\Sigma_{p}$ in important cases. At first, we recall some definitions.

In the following, we denote by $\mathcal{I}$ the set of all Jacobi fields along $\gamma$ that vanish at $0 \in I$ and by 


$$
m(t)=\operatorname{dim}\{\xi \in \mathcal{I}: \xi(t)=0\}
$$

the multiplicity of $t \in I$ as a conjugate instant. Moreover, we denote $\mathcal{I}[t]=\{\xi(t): \xi \in \mathcal{I}\}$ and note that $m(t)=\operatorname{dim} \mathcal{I}[t]^{\perp}$, where $\perp$ denotes the orthogonal complement with respect to $g$.

10.4.5 Definition. We call a conjugate instant $t \in I$ regular, if the restriction of the quadratic form $g_{\gamma(t)}$ to the space $\mathcal{I}[t]^{\perp}$ is non degenerated. Moreover, we call $t \in I$ strictly regular if $\operatorname{sig}\left(\left.g\right|_{\mathcal{I}[t]}\right) \neq 0$.

Note that all conjugate instants of a Riemannian geodesic are strictly regular because in this case $\operatorname{sig}\left(\left.g\right|_{\mathcal{I}[t]^{\perp}}\right)=m(t)$. Moreover, it is well known (cf. eg. MPP05]) that regular conjugate instants are isolated and if all conjugate instants along a geodesic are regular, we can compute the conjugate index by

$$
\mu_{\text {con }}(\gamma)=\sum_{t \in I} \operatorname{sig}\left(\left.g\right|_{\mathcal{I}[t]^{\perp}}\right) .
$$

Finally we want to mention that all conjugate points in a semi-Riemannian manifold are regular if the metric is analytic and that strictly conjugate points are in particular of interest in the bifurcation theory of geodesics MPP07, 1

10.4.6 Definition. We call a subset $U \subset T_{p} M$ radially convex if for any $v \in U$ the set

$$
\{t \in \mathbb{R}: t v \in U\}
$$

is connected, that is, an interval.

Note that if $U$ is radially convex and $0 \in T_{p} M$ is an interior point of $U$, then $U$ is star shaped with respect to 0 . Conversely, it is clear that any subset $U \subset T_{p} M$ which is star shaped with respect to 0 is radially convex.

Now let $v \in \Omega_{p} \backslash \Sigma_{p}$ be such that $\gamma_{v}$ is a regular geodesic and let

$$
t_{1} v, \ldots, t_{n} v, \quad \text { for some } 0<t_{1}<\ldots<t_{n}<1
$$

be the conjugate points of $\gamma_{v}$. We define a function $F_{v}: I \rightarrow \mathbb{R}$ by

$$
F_{v}(t)=\mu_{\text {spec }}\left(\gamma_{t v}\right), \quad t \neq t_{i}, \quad i=1, \ldots, n
$$

and the assumption that $F_{v}$ is upper semi-continuous.

\footnotetext{
${ }^{1}$ Note that our definition of a strictly conjugate point differs slightly from the one in MPP07.
} 
10.4.7 Lemma. Let $\Omega_{p}^{\prime} \subset \Omega_{p}$ be star shaped with respect to $0 \in T_{p} M$ and such that all conjugate points in $\Omega_{p}^{\prime}$ are strictly regular. Then the connected components of the set

$$
\left\{v \in \Omega_{p}^{\prime} \backslash\left(\Omega_{p}^{\prime} \cap \Sigma_{p}\right): F_{v} \text { monotone }\right\} \subset T_{p} M
$$

are radially convex.

Proof. Let $A$ be a connected component and assume on the contrary that $A$ is not radially convex. Then we can find an element $v \in A$ and $0 \leq t<t^{\prime}<1$ such that $t v \in A$ but $t^{\prime} v \notin A$. We claim that we can even choose $t^{\prime}$ such that $t^{\prime} v \in \Sigma_{p}$. Indeed, if this is not the case, then $\lambda v \notin \Sigma_{p}$ for all $\lambda \in[t, 1]$. Accordingly, $F_{v}$ is constant on $[t, 1]$ and hence each $F_{\lambda v}, \lambda \in[t, 1]$, monotone because $F_{t v}$ is monotone. Then $\lambda v, \lambda \in[t, 1]$, defines a path in $\left\{v \in \Omega_{p}^{\prime} \backslash\left(\Omega_{p}^{\prime} \cap \Sigma_{p}\right): F_{v}\right.$ monotone $\}$ connecting $t v$ and $v$. We conclude $\lambda v \in A$ for all $\lambda \in[t, 1]$ which is a contradiction.

Hence we can assume that $t^{\prime} v \in \Sigma_{p}$ and since $\Omega_{p}^{\prime}$ is star shaped with respect to 0 we infer that $t^{\prime} v \in \Omega_{p}^{\prime}$ and so $t^{\prime} v$ is a strictly regular conjugate point. Since $F_{v}$ is monotone, we obtain by 10.15

$$
\mu_{\text {spec }}\left(\gamma_{t v}\right)=F_{v}(t) \neq F_{v}(1)=\mu_{\text {spec }}\left(\gamma_{v}\right)
$$

Then $t v$ and $v$ lie in different components of $\Omega_{p}^{\prime} \backslash\left(\Sigma_{p} \cap \Omega_{p}^{\prime}\right)$ by lemma 10.4 .2 which contradicts that they both belong to $A$.

10.4.8 Corollary. Let $(M, g)$ be a Riemannian manifold. Then the connected components of $\Omega_{p} \backslash \Sigma_{p}$ are radially convex. In particular, the connected component of 0 is star shaped.

Proof. Since $\Omega_{p}$ is star shaped with respect to 0 and any conjugate point in a Riemannian manifold is strictly regular, we obtain from the foregoing lemma that

$$
\left\{v \in \Omega_{p} \backslash \Sigma_{p}: F_{v} \text { monotone }\right\}
$$

is radially convex. But since

$$
F_{v}(t)=\sum_{0 \leq t^{\prime} \leq t} m\left(t^{\prime}\right)
$$

any $F_{v}, v \in \Omega_{p} \backslash \Sigma_{p}$ is monotone.

The following corollary can be proved in the same way as the foregoing one just by noting that timelike geodesics in Lorentzian manifolds behave essentially as geodesics in Riemannian manifolds (cf. [BEE96]). 
10.4.9 Corollary. Let $(M, g)$ be a Lorentzian manifold and set

$$
\Omega_{p}^{\prime}=\left\{v \in \Omega_{p}: v \text { timelike }\right\}
$$

Then the connected components of $\Omega_{p}^{\prime} \backslash\left(\Omega_{p}^{\prime} \cap \Sigma_{p}\right)$ are radially convex. 
Part IV

\section{Bifurcation}





\section{Chapter 11}

\section{The Bifurcation Theorems}

In the following we assume throughout that $X$ is a compact topological space and $H$ a Hilbert space. We let $\mathcal{A}: X \rightarrow \mathcal{C}(H)$ be a gap continuous family of closed operators and $C: X \times \tilde{H} \rightarrow H$ be a continuous map such that $C_{x}(0)=0$ for all $x \in X$, where $\tilde{H} \subset H$ is a linear subspace such that $\mathcal{D}\left(\mathcal{A}_{x}\right) \subset \tilde{H}$ for all $x \in X$. We assume that $\tilde{H}$ carries a norm in its own right which need not be induced by the norm on $H$ in general. However, we assume throughout that $\tilde{H}$ is complete and the inclusion $\iota: \tilde{H} \hookrightarrow H$ is continuous.

We consider the family of maps

$$
f_{x}: \mathcal{D}\left(\mathcal{A}_{x}\right) \rightarrow H, \quad f_{x}(u)=\mathcal{A}_{x} u+C_{x}(u), \quad x \in X
$$

and call $x^{*} \in X$ a bifurcation point of (11.1), if there exists a sequence

$$
\left\{\left(x_{n}, u_{n}\right)\right\}_{n \in \mathbb{N}} \subset\left\{(x, u) \in X \times H: u \in \mathcal{D}\left(\mathcal{A}_{x}\right)\right\}
$$

such that $u_{n} \neq 0, f_{x_{n}}\left(u_{n}\right)=0$ for all $n \in \mathbb{N}$ and $\left(x_{n}, u_{n}\right) \rightarrow\left(x^{*}, 0\right)$ in $X \times \tilde{H}$.

Note that we require $\left(x_{n}, u_{n}\right)$ to converge with respect to the norm on $\tilde{H}$ which is in general a stronger assumption than requiring convergence with respect to the norm on $H$ because of the assumed continuity of the inclusion $\tilde{H} \hookrightarrow H$.

The chapter is structured as follows: In the first section we define and study a certain compatibility condition which ensures that the operators $C$ descend in a suitable way to continuous maps on the domain bundle $\mathfrak{D}(\mathcal{A})$ of $\mathcal{A}$. In the second section we define compact nonlinear operators and state our main theorems of this chapter. The subsequent sections 3-5 are devoted to the proofs of these theorems.

Finally, we want to point out that the precise definition of the trivialisations of the domain bundle $\mathfrak{D}(\mathcal{A})$ which we constructed in section 6.1 are of minor interest in the following. Hence we will usually shorten notation by denoting the model space of the domain bundle $\mathfrak{D}(\mathcal{A})$ by $H^{\prime}$. 
Moreover, since $X$ is throughout assumed to be compact, $\mathfrak{D}(\mathcal{A})$ is trivial according to theorem 1.1.8. However, we will not use this fact more often than necessary.

\subsection{Condition (C)}

The main observation that will allow us to prove existence results for bifurcation points of 11.1 is that $\mathcal{A}$ can be interpreted as a bundle morphism between Hilbert bundles according to the results of section 6.1. The strategy is now to consider the whole family of maps $f$ in (11.1) on the domain bundle $\mathfrak{D}(\mathcal{A})$ of $\mathcal{A}$ and to study the bifurcation problem in this setting. To achieve this we have to require of course certain compatibility conditions between the topologies on $\mathfrak{D}(\mathcal{A})$ and $X \times \tilde{H}$ in order to ensure that $C$ descends at least to a continuous map on $\mathfrak{D}(\mathcal{A})$.

The strongest assumption that one may have in mind here is to require that $\mathfrak{D}(\mathcal{A})$ is a subbundle of the product bundle $X \times \tilde{H}$. But in this case each fibre $\mathfrak{D}(\mathcal{A})_{x}$, i.e. $\mathcal{D}\left(\mathcal{A}_{x}\right)$ with the topology induced by the graph norm of $\mathcal{A}_{x}$, needs to carry the topology induced by $\tilde{H}$ which may be a quite restrictive condition in applications. Fortunately, it will turn out that the following slightly weaker condition is sufficient for our purposes:

(C) The canonical inclusion $\iota: \mathfrak{D}(\mathcal{A}) \hookrightarrow X \times \tilde{H}$ is a bundle morphism.

Note that by definition of a bundle morphism (C) not only means that $\iota$ is continuous, but also requires that for any trivialisation $\psi: \pi^{-1}(U) \rightarrow U \times H^{\prime}$ of $\mathfrak{D}(\mathcal{A})$, the map

$$
U \ni x \mapsto \iota_{x} \circ \psi_{x}^{-1}=\psi_{x}^{-1} \in \mathcal{L}\left(H^{\prime}, \tilde{H}\right)
$$

is continuous.

For example, if $\tilde{H}=H$ carries the norm of $H$, then we obtain from lemma 6.1.15 that $\mathfrak{D}(\mathcal{A})$ is a subbundle of $X \times \tilde{H}$ if and only if each $\mathcal{A}_{x}$ is bounded. In contrast, the weaker assumption (C) turns out to hold without any further restrictions in this case by lemma 6.1.5.

Remember that we proved in lemma6.1.12 that the domain bundle of a gap continuous family $\mathcal{A}: X \rightarrow \mathcal{C}(H)$ having a constant domain $D$ is the product $X \times D$ if the induced family

$$
\mathcal{A}: X \rightarrow \mathcal{L}\left(D_{x_{0}}, H\right)
$$

is continuous, where $D_{x_{0}}$ denotes the space $D$ with the graph norm of $\mathcal{A}_{x_{0}}$ for some $x_{0} \in X$.

11.1.1 Lemma. Let $\mathcal{A}_{1}: X \rightarrow \mathcal{C}(H)$ and $\mathcal{A}_{2}: X \rightarrow \mathcal{C}(H)$ be gap continuous and such that $\mathcal{A}_{1, x} \subset \mathcal{A}_{2, x}$ for all $x \in X$. Moreover, assume that $\mathcal{D}\left(\mathcal{A}_{2, x}\right)=D$ for all $x \in X$, the map 11.2 induced by $\mathcal{A}_{2}$ is continuous with respect to some $x_{0} \in X$ and that we have a continuous inclusion $\iota: D_{x_{0}} \hookrightarrow \tilde{H}$. Then condition $(C)$ holds for $\mathcal{A}_{1}$. 
Proof. Since we have $\mathcal{A}_{1, x} \subset \mathcal{A}_{2, x}$ for all $x \in X$ by assumption, we know by lemma 6.1.16 that $\mathfrak{D}\left(\mathcal{A}_{1}\right) \subset \mathfrak{D}\left(\mathcal{A}_{2}\right)$ is a subbundle. By lemma 6.1.12 we have $\mathfrak{D}\left(\mathcal{A}_{2}\right)=X \times D$ where $D$ carries the topology induced by $D_{x_{0}}$. Hence we infer from the remaining assumption that (C) holds for $\mathcal{A}_{1}$.

11.1.2 Remark. If $D_{x_{0}}=\tilde{H}$, we even get the stronger result that $\mathfrak{D}\left(\mathcal{A}_{1}\right)$ is a subbundle of $X \times \tilde{H}$.

Setting $\mathcal{A}_{1}=\mathcal{A}_{2}$, we obtain the following immediate consequence of lemma 11.1.1.

11.1.3 Corollary. Let $\mathcal{A}: X \rightarrow \mathcal{C}(H)$ be a gap continuous family having the constant domain $D$ such that the induced map 11.2 is continuous for some $x_{0}$ and such that $D_{x_{0}}$ can be included continuously into $\tilde{H}$. Then $(C)$ holds.

After having studied when $(\mathrm{C})$ holds, we will now take a look at some simple consequences of $(\mathrm{C})$ that we will need below.

11.1.4 Lemma. Let $\mathcal{A}: X \rightarrow \mathcal{C}(H)$ be gap continuous and $\tilde{H}$ be a Banach space such that condition $(C)$ holds. Then for any closed subset $K \subset X$ and trivialisation

$$
\psi: \pi^{-1}(K) \rightarrow K \times H^{\prime}
$$

of the domain bundle $\mathfrak{D}(\mathcal{A})$ over $K$ there exists a constant $c=c(K, \psi)>0$ such that

$$
\left\|\left(\iota \circ \psi^{-1}\right)(x, u)\right\|_{\tilde{H}} \leq c\|u\|_{H^{\prime}}, \quad u \in H^{\prime}, x \in K .
$$

Proof. By assumption (C) the map

$$
K \ni x \mapsto\left\|\iota_{x} \circ \psi_{x}^{-1}\right\|_{\mathcal{L}\left(H^{\prime}, \tilde{H}\right)} \in \mathbb{R}
$$

is continuous. Using the compactness of $K$, we infer that

$$
c:=\sup _{x \in K}\left\|\iota_{x} \circ \psi_{x}^{-1}\right\|_{\mathcal{L}\left(H^{\prime}, \tilde{H}\right)}<\infty .
$$

Hence we obtain

$$
\left\|\left(\iota \circ \psi^{-1}\right)(x, u)\right\|_{\tilde{H}} \leq\left\|\iota_{x} \circ \psi_{x}^{-1}\right\|_{\mathcal{L}\left(H^{\prime}, \tilde{H}\right)}\|u\|_{H^{\prime}} \leq c\|u\|_{H^{\prime}}, \quad u \in H^{\prime}, x \in K .
$$


In the following we will sometimes need to work with derivatives. We note at first that we have two natural ways to define derivatives of maps $f: \Omega \rightarrow F$, where $\Omega \subset E$ is open and $E, F$ are complex Banach spaces. The first way is to use the ordinary Fréchet derivative which, however, leads to the notion of holomorphy and so its existence is an extremely stringent assumption on $f$. Instead we will usually assume our maps to be real differentiable, which means that $f$ is Fréchet differentiable as a map between the realifications $\Omega^{\mathbb{R}} \subset E^{\mathbb{R}} \rightarrow F^{\mathbb{R}}$. Accordingly, unless otherwise stated we denote by $D_{u} f \in \mathcal{L}\left(E^{\mathbb{R}}, F^{\mathbb{R}}\right)$ the real derivative at $u \in \Omega$. Since $E^{\mathbb{R}}$ and $E$ are essentially the same objects we will not distinguish between them very carefully in the following but note that $D_{u} f: E \rightarrow F, u \in \Omega$, is only $\mathbb{R}$-linear in contrast to the ordinary Fréchet derivative.

Let $x \in X$ be fixed and assume that $\iota_{x}: \mathcal{D}\left(\mathcal{A}_{x}\right) \rightarrow \tilde{H}$ is continuous, which is in particular the case if (C) holds. If $U \subset \tilde{H}$ is an open neighbourhood of some $u \in \iota_{x}\left(\mathcal{D}\left(\mathcal{A}_{x}\right)\right)=\iota_{x}\left(\psi_{x}^{-1}\left(H^{\prime}\right)\right) \subset \tilde{H}$ such that $C_{x}$ is real continuously differentiable on $U$, then there exists an open neighbourhood $V \subset H^{\prime}$ of $\left(\iota_{x} \circ \psi_{x}^{-1}\right)^{-1}(u)$ such that

$$
\mathcal{A}_{x} \circ \psi_{x}^{-1}+C_{x} \circ \iota_{x} \circ \psi_{x}^{-1}: H^{\prime} \rightarrow H
$$

is real continuously differentiable on $V$. Indeed, at first we have that $\mathcal{A}_{x} \circ \psi_{x}^{-1}$ is the composition of the bounded linear operators $\psi_{x}^{-1}: H^{\prime} \rightarrow \mathfrak{D}(\mathcal{A})_{x}$ and $\mathcal{A}_{x}: \mathfrak{D}(\mathcal{A})_{x} \rightarrow H$. Hence $\mathcal{A}_{x} \circ \psi_{x}^{-1}$ is continuously differentiable and $D_{v}\left(\mathcal{A}_{x} \circ \psi_{x}^{-1}\right)$ is just $\mathcal{A}_{x} \circ \psi_{x}^{-1}$ itself for all $v \in H^{\prime}$. Moreover, $C_{x} \circ \iota_{x} \circ \psi_{x}^{-1}: H^{\prime} \rightarrow H$ is the composition of the bounded linear maps $\psi_{x}^{-1}: H^{\prime} \rightarrow \mathfrak{D}(\mathcal{A})_{x}$, $\iota_{x}: \mathfrak{D}(\mathcal{A})_{x} \rightarrow \tilde{H}$ and the map $C_{x}: \tilde{H} \rightarrow H$. Setting $V=\left(\iota_{x} \circ \psi_{x}^{-1}\right)^{-1}(U)$ we now obtain

$$
D_{v}\left(\mathcal{A}_{x} \circ \psi_{x}^{-1}+C_{x} \circ \iota_{x} \circ \psi_{x}^{-1}\right)=\mathcal{A}_{x} \circ \psi_{x}^{-1}+\left(D_{\left(\iota_{x} \circ \psi_{x}^{-1}\right)(v)} C_{x}\right) \circ \iota_{x} \circ \psi_{x}^{-1}
$$

which depends continuously on $v \in V$.

Under the stronger assumption that $\mathfrak{D}(\mathcal{A})$ is a subbundle of $X \times \tilde{H}$, the following result about the position of the set of all bifurcation points of $f$ holds true which corresponds to well known results in the bounded case (cf. eg. [Dei85, Prop. 28.1]).

11.1.5 Lemma. Assume that $\mathfrak{D}(\mathcal{A})$ is a subbundle of $X \times \tilde{H}$ and $C: X \times \tilde{H} \rightarrow H$ is real continuously differentiable with respect to $u \in \tilde{H}$. If $x^{*} \in X$ is a bifurcation point of (11.1), then

$$
\mathcal{A}_{x^{*}}+\left(D_{0} C_{x^{*}}\right) \circ \iota_{x^{*}} \notin G L\left(\mathfrak{D}(\mathcal{A})_{x^{*}}^{\mathbb{R}}, H^{\mathbb{R}}\right)
$$

Proof. By definition there exists a sequence $\left\{\left(x_{n}, u_{n}\right)\right\}_{n \in \mathbb{N}}$ such that $\left(x_{n}, u_{n}\right) \rightarrow\left(x^{*}, 0\right)$ in $X \times \tilde{H}$, $u_{n} \in \mathcal{D}\left(\mathcal{A}_{x_{n}}\right)$ and $f\left(x_{n}, u_{n}\right)=0, n \in \mathbb{N}$. Let $U_{x^{*}}$ be a trivialising neighbourhood of $\mathfrak{D}(\mathcal{A})$ around $x^{*}$ and $\psi: \pi^{-1}\left(U_{x^{*}}\right) \rightarrow U_{x^{*}} \times H^{\prime}$ a trivialisation. We choose $n_{0} \in \mathbb{N}$ such that $x_{n} \in U_{x^{*}}$ for all $n \geq n_{0}$ and define $\left(x_{n}, v_{n}\right)=\psi\left(x_{n}, u_{n}\right) \in U_{x^{*}} \times H^{\prime}, n \geq n_{0}$. Since by assumption $\mathfrak{D}(\mathcal{A})$ is a 
subbundle of $X \times \tilde{H}$ we infer that $v_{n} \rightarrow 0$ in $H^{\prime}$.

Now we define $\tilde{\mathcal{A}}_{x}=\mathcal{A}_{x} \circ \psi_{x}^{-1}, \tilde{C}_{x}=C_{x} \circ \iota_{x} \circ \psi_{x}^{-1}$ and consider the family

$$
\tilde{\mathcal{A}}_{x}+\tilde{C}_{x}: H^{\prime} \rightarrow H, \quad x \in U_{x^{*}} .
$$

Note that $\tilde{\mathcal{A}}_{x_{n}} v_{n}+\tilde{C}_{x_{n}}\left(v_{n}\right)=0$ for all $n \geq n_{0}$ and, since $v_{n} \rightarrow 0$ in $H^{\prime}, x^{*}$ is a bifurcation point for this family. Moreover, by using 11.3 it is clear that $\tilde{\mathcal{A}}+\tilde{C}$ is real continuously differentiable with respect to $u \in H^{\prime}$ under the given assumptions.

If now $\mathcal{A}_{x^{*}}+\left(D_{0} C_{x^{*}}\right) \circ \iota_{x^{*}} \in G L\left(\mathfrak{D}(\mathcal{A})_{x^{*}}^{\mathbb{R}}, H^{\mathbb{R}}\right)$, then using $(11.3)$, we have $D_{0}\left(\tilde{\mathcal{A}}_{x^{*}}+\tilde{C}_{x^{*}}\right) \in$ $G L\left(\left(H^{\prime}\right)^{\mathbb{R}}, H^{\mathbb{R}}\right)$ and by the implicit function theorem [Dei85, Th. 15.1] there exists a neighbourhood of $\left(x^{*}, 0\right) \in U_{x^{*}} \times H^{\prime}$ such that the only solutions of $\tilde{\mathcal{A}}_{x} u+\tilde{C}_{x}(u)=0$ in this neighbourhood are of the form $(x, 0)$. Hence we obtain a contradiction to the existence of the sequence $\left\{\left(x_{n}, u_{n}\right)\right\}$.

\subsection{The Bifurcation Theorems}

11.2.1 Definition. Let $X$ be a compact topological space, $E, F$ normed linear spaces and $M \subset E$. We call a map

$$
C: X \times M \rightarrow F
$$

compact if it is continuous and for any bounded subset $B \subset M, C(X \times B) \subset F$ is relatively compact.

11.2.2 Remark. $C: X \times M \rightarrow F$ is compact if and only if it is continuous and for any sequence $\left\{\left(x_{n}, u_{n}\right)\right\}_{n \in \mathbb{N}}$ in $X \times M$ such that $\left\|u_{n}\right\|<c, n \in \mathbb{N}$, the sequence $C\left(x_{n}, u_{n}\right)$ has a convergent subsequence in $F$.

The next result gives a first example of a compact map in this sense.

11.2.3 Lemma. Let $X$ be a compact metric space and $E, F$ normed linear spaces. Then any norm continuous family $K: X \rightarrow \mathcal{K}(E, F)$ is compact.

Proof. Let $\left\{\left(x_{n}, u_{n}\right)\right\}_{n \in \mathbb{N}}$ be a sequence in $X \times E$ such that $\left\|u_{n}\right\| \leq c, n \in \mathbb{N}$, for some $c>0$. Since $X$ is sequentially compact we can find a subsequence $\left\{x_{n_{k}}\right\}_{k \in \mathbb{N}} \subset\left\{x_{n}\right\}_{n \in \mathbb{N}}$ converging to some $x^{*} \in X$. Moreover, by the compactness of $K_{x^{*}}$ we can thin out further and obtain a subsequence $\left\{\left(x_{n_{l}}, u_{n_{l}}\right)\right\}_{l \in \mathbb{N}} \subset\left\{\left(x_{n}, u_{n}\right)\right\}_{n \in \mathbb{N}}$ such that $x_{n_{l}} \rightarrow x^{*}$ and $K\left(x^{*}, u_{n_{l}}\right)$ converges to some $v \in F$. Then

$$
\begin{aligned}
\left\|K\left(x_{n_{l}}, u_{n_{l}}\right)-v\right\| & \leq\left\|K\left(x_{n_{l}}, u_{n_{l}}\right)-K\left(x^{*}, u_{n_{l}}\right)\right\|+\left\|K\left(x^{*}, u_{n_{l}}\right)-v\right\| \\
& \leq\left\|K_{x_{n_{l}}}-K_{x^{*}}\right\|_{\mathcal{L}(E, F)}\left\|u_{n_{l}}\right\|+\left\|K\left(x^{*}, u_{n_{l}}\right)-v\right\| \\
& \leq c\left\|K_{x_{n_{l}}}-K_{x^{*}}\right\|_{\mathcal{L}(E, F)}+\left\|K\left(x^{*}, u_{n_{l}}\right)-v\right\| \rightarrow 0,
\end{aligned}
$$


where the first term converges to zero because of the continuity of the family $K$ with respect to the norm topology.

As next step we show how compact maps fit in our setting described in section 11.1 .

11.2.4 Lemma. Let $\mathcal{A}: X \rightarrow \mathcal{C}(H)$ be gap continuous, $K \subset X$ a closed subspace and $\psi:$ $\pi^{-1}(K) \rightarrow K \times H^{\prime}$ any trivialisation of $\mathfrak{D}(\mathcal{A})$ over $K$. Let $C: X \times \tilde{H} \rightarrow H$ be a compact map such that $(C)$ holds. Then the composition

$$
C \circ \iota \circ \psi^{-1}: K \times H^{\prime} \rightarrow H
$$

is compact.

Proof. Let $\left\{\left(x_{n}, u_{n}\right)\right\}_{n \in \mathbb{N}}$ be any sequence in $K \times H^{\prime}$ such that $\left\|u_{n}\right\|_{H^{\prime}}<c^{\prime}, n \in \mathbb{N}$, for some $c^{\prime}>0$. According to lemma 11.1 .4 we have a constant $c>0$ such that

$$
\left\|\left(\iota_{x_{n}} \circ \psi_{x_{n}}^{-1}\right)\left(u_{n}\right)\right\|_{\tilde{H}} \leq c\left\|u_{n}\right\| \leq c c^{\prime}, \quad n \in \mathbb{N},
$$

and hence $\left\|\left(\iota_{x_{n}} \circ \psi_{x_{n}}^{-1}\right)\left(u_{n}\right)\right\|_{\tilde{H}}$ is a bounded sequence in $\tilde{H}$. Since $C: X \times \tilde{H} \rightarrow H$ is compact we obtain the assertion.

We want to point out that the choice of a norm on $\tilde{H}$ usually strongly affects the number of nonlinear compact maps from $\tilde{H}$ to $H$. Typical examples of nonlinear maps are given by the so called Nemytskij operators which act on function spaces by mapping a function $u$ which is defined on a bounded domain $\Omega \subset \mathbb{R}^{n}$ to the composition $F(\cdot, u(\cdot))$ for a given function $F: \Omega \times \mathbb{R} \rightarrow \mathbb{R}$. Under certain assumptions on the regularity of $F$, such operators map common function spaces to themselves. But one can show that for some Banach spaces, like $C[0,1]$ and $L^{2}(0,1)$, a Nemytskij operator is compact if and only if the function $F$ is constant (cf. ApVä05, Satz 9.5]). Hence if we assume $\tilde{H}$ to carry the induced norm by $H$, then condition $(\mathrm{C})$ is satisfied by lemma 6.1 .5 but it can be difficult to find interesting nonlinear compact operators.

On the other hand, if we choose a norm on $\tilde{H}$ such that $\iota: \tilde{H} \hookrightarrow H$ is compact, then any bounded and continuous nonlinear operator $\tilde{H} \rightarrow \tilde{H}$ leads to a compact map $C: \tilde{H} \rightarrow H$. Of course, here we have the Rellich compactness theorem for Sobelev spaces in mind. For example, if we consider families of boundary value problems for semi-linear partial differential equations defined on a bounded domain $\Omega \subset \mathbb{R}^{n}$, then nonlinear lower order terms may define compact operators if $\tilde{H}$ is a suitable Sobolev space and $H=L^{2}(\Omega)$.

Before we state our main theorems regarding bifurcation, we want to recall the definition of the groups $J(X)$ (cf. At89, III,§3]).

Let $E, F$ be two vector bundles over the compact and connected base space $X$ which have Hermitian metrics. We call $E$ and $F$ fibre homotopy equivalent if there exist bundle maps 
$f: S(E) \rightarrow S(F)$ and $g: S(F) \rightarrow S(E)$ between their associated sphere bundles such that $f \circ g$ and $g \circ f$ are homotopic to the identity through fibre preserving maps. Note that the equivalence class of a given bundle is independent of the metric which we have used in order to build its sphere bundle. $E$ and $F$ are said to be stably fibre homotopy equivalent if there exist trivial bundles $V$ and $W$ such that $E \oplus V$ is fibre homotopy equivalent to $F \oplus W$. Now the set of all stable fibre homotopy equivalence classes over $X$ form a group which is denoted by $J(X)$. Moreover, the map $\operatorname{Vect}(X) \rightarrow J(X)$ extends to an epimorphism $\widetilde{K}(X) \rightarrow J(X)$ which is denoted by $J$ as well.

$J$ can be constructed for real vector bundles and $\widetilde{K O}(X)$ in precisely the same way. In the following we will not distinguish between these cases very carefully and will usually denote both groups by $J(X)$ since there is little opportunity to confuse them. We only make the following exception in order to note that there is a canonical map

$$
J^{\mathbb{C}}(X) \rightarrow J^{\mathbb{R}}(X), \quad[E] \mapsto\left[E^{\mathbb{R}}\right]
$$

where $E^{\mathbb{R}}$ denotes the realification of the complex bundle $E$.

We now state the main theorems of the fourth part of the thesis.

11.2.5 Theorem. Let $X$ be a compact connected $C W$-complex, $\mathcal{A}: X \rightarrow \mathcal{C F}_{0}(H)$ be gap continuous and $C: X \times \tilde{H} \rightarrow H$ a compact map such that $C_{x}(0)=0, x \in X$, and condition $(C)$ holds. Assume that some $C_{x_{0}}$ is real continuously differentiable at 0 and $\mathcal{A}_{x_{0}}+\left(D_{0} C_{x_{0}}\right) \circ \iota_{x_{0}} \in$ $G L\left(\mathfrak{D}(\mathcal{A})_{x_{0}}^{\mathbb{R}}, H^{\mathbb{R}}\right)$.

If $J\left(\operatorname{ind}(\mathcal{A})^{\mathbb{R}}\right) \neq 0 \in J(X)$, then there exists a bifurcation point $x^{*} \in X$ for $f$ as defined by 11.1 .

Next we assume that $Y \subset X$ is a closed subspace and $\mathcal{A}:(X, Y) \rightarrow\left(\mathcal{C F}^{s a}(H), G \mathcal{C}^{s a}(H)\right)$ a gap continuous family of selfadjoint Fredholm operators. According to [Se68, Prop. A.4] the restriction defines an isomorphism $K^{-1}(X, Y) \cong K^{-1}(X \backslash Y)$ and by using the isomorphism

$$
\varphi: K^{-1}(X \backslash Y) \cong \tilde{K}\left((X \backslash Y \times \mathbb{R})^{+}\right)
$$

from lemma B.2.1 we can regard s-ind $(\mathcal{A})$ as an element in $\tilde{K}\left((X \backslash Y \times \mathbb{R})^{+}\right)$. Note that if $Y \neq \emptyset$, then

$$
(X \backslash Y \times \mathbb{R})^{+} \approx(X \backslash Y)^{+} \wedge S^{1} \approx X / Y \wedge S^{1} \approx \Sigma(X / Y)
$$

where $\Sigma(X / Y)$ denotes the reduced suspension of $X / Y$. 
11.2.6 Theorem. Let $X$ be a compact connected $C W$-complex, $\mathcal{A}:(X, Y) \rightarrow\left(\mathcal{C F}^{s a}(H), G \mathcal{C}^{s a}(H)\right)$ gap continuous and $C: X \times \tilde{H} \rightarrow H$ a compact map such that $C_{x}(0)=0, x \in X$, and condition (C) holds. We denote by $\tilde{B}_{r}(0)$ the ball of radius $r>0$ in $\tilde{H}$. Assume that

i) there exists $M>0$ and $r_{0}>0$ such that

$$
\left\|C_{x}(u)\right\|_{H} \leq M\|u\|_{\tilde{H}}^{2}
$$

for all $u \in \tilde{B}_{r_{0}}(0)$ and $x \in X$.

ii) there exists $\varepsilon>0$ and $r_{1}>0$ such that $\mathcal{A}_{x} u+C_{x}(u) \neq i$ su for all $u \in \tilde{B}_{r_{1}}(0) \cap\left(\mathcal{D}\left(\mathcal{A}_{x}\right) \backslash\{0\}\right)$, $s \in(-\varepsilon, \varepsilon) \backslash\{0\}, x \in X$.

iii) $(X \backslash Y \times \mathbb{R})^{+}$is a connected $C W$-complex and there exists a relatively compact open neighbourhood $V$ of the singular set $\Sigma(\mathcal{A}) \subset X \backslash Y \times\{0\}$ in $X \backslash Y \times \mathbb{R}$ such that $(X \backslash Y \times \mathbb{R})^{+} \backslash V$ is homeomorphic to a closed unit ball $B^{l}$ in $\mathbb{R}^{l}$ for some $l \in \mathbb{N}$.

If $J(\operatorname{s-ind}(\mathcal{A})) \neq 0 \in J\left((X \backslash Y \times \mathbb{R})^{+}\right)$, then there exists a bifurcation point $x^{*}$ for $f$ as defined by 11.1. Moreover, $x^{*}$ belongs to the singular set of $\mathcal{A}$.

11.2.7 Remark. $\quad$ i) The neighbourhood $V$ required in iii) exists in any case if $(X \backslash Y \times \mathbb{R})^{+}$ is a manifold.

ii) Assumption ii) in the theorem holds in particular if there exists $r_{0}>0$ such that any $C_{x}: \tilde{B}_{r_{0}}(0) \rightarrow H$ is two times real continuously differentiable, $C_{x}(0)=0, D_{0} C_{x}=0$ and $\left\|D_{u}^{2} C_{x}\right\|<M$ for all $(x, u) \in X \times \tilde{B}_{r_{0}}(0)$ and some $M>0$. This is an immediate consequence of Taylor's theorem (cf. [AmM07, §1]).

iii) We believe that the assumption iii) becomes superfluous if $Y=\emptyset$.

We now conclude this section by discussing conditions for the non-vanishing of the $J$-homomorphism which will lead to some corollaries of the theorems above.

The main observation for us is that according to [Pe12b, §3] the Stiefel-Whitney classes $w_{i}$ of a real vector bundle $E$ depend solely on the fibre homotopy class of $E$. Hence the $w_{i}, i \in \mathbb{N}$, can be defined on $J(X)$ and the nontriviality of some $w_{i}(E)$ ensures that $J(E) \neq 0 \in J(X)$. Moreover, if we consider complex bundles and denote by

$$
\kappa_{k}: H^{k}(X ; \mathbb{Z}) \rightarrow H^{k}\left(X ; \mathbb{Z}_{2}\right), \quad k \in \mathbb{Z}
$$

the coefficient homomorphism then we have

$$
\kappa_{2 k}\left(c_{k}(E)\right)=w_{2 k}\left(E^{\mathbb{R}}\right) \in H^{2 k}\left(X ; \mathbb{Z}_{2}\right), \quad k \in \mathbb{N},
$$


for every complex vector bundle $E$ over $X$ (cf. [Ha09, Prop. 3.8]), where $E^{\mathbb{R}}$ denotes the realification of $E$ as above.

11.2.8 Corollary. If the assumptions of theorem 11.2.5 hold and

$$
\kappa_{2 k}\left(c_{k}(\operatorname{ind} \mathcal{A})\right) \neq 0 \in H^{2 k}\left(X ; \mathbb{Z}_{2}\right)
$$

for some $k \in \mathbb{N}$, then there exists a bifurcation point $x^{*} \in X$ for $f$ as defined by (11.1).

Since the homomorphism (11.4) shows that the nontriviality of $J\left(\operatorname{sind}(\mathcal{A})^{\mathbb{R}}\right)$ implies that $J(\operatorname{sind}(\mathcal{A})) \neq 0$, we obtain the following corollary.

11.2.9 Corollary. If the assumptions of theorem 11.2.6 hold and

$$
\kappa_{2 k}\left(c_{k}(\text { s-ind } \mathcal{A})\right) \neq 0 \in H^{2 k}\left((X \backslash Y \times \mathbb{R})^{+} ; \mathbb{Z}_{2}\right)
$$

for some $k \in \mathbb{N}$, then there exists a bifurcation point for $f$ as defined by (11.1).

Note that $\kappa_{2 k}\left(c_{k}(\operatorname{sind}(\mathcal{A}))\right)$ can be regarded as element in

$$
H^{2 k}\left((X \backslash Y \times \mathbb{R})^{+} ; \mathbb{Z}_{2}\right) \cong H^{2 k}\left(\Sigma(X / Y) ; \mathbb{Z}_{2}\right) \cong H^{2 k-1}\left(X / Y ; \mathbb{Z}_{2}\right) \cong H^{2 k-1}\left(X, Y ; \mathbb{Z}_{2}\right)
$$

11.2.10 Corollary. Let $\mathcal{A}:(I, \partial I) \rightarrow\left(\mathcal{C F}^{s a}(H), G \mathcal{C}^{s a}(H)\right)$ be a gap continuous path and $C$ : $I \times \tilde{H} \rightarrow H$ such that $C_{t}(0)=0, t \in I$, and condition (C) as well as the assumptions $\left.i\right)$ and ii) of theorem 11.2.6 are satisfied. If $\operatorname{sf}(\mathcal{A}) \bmod 2 \neq 0$, then there exists a bifurcation point $t^{*} \in I \backslash \partial I$.

Proof. We set $X=I, Y=\partial I$ and note that assumption iii) of theorem 11.2.6 is satisfied since $((I \backslash \partial I) \times \mathbb{R})^{+}=S^{2}$. Now the assertion is an immediate consequence of proposition 7.3 .1 and the fact that the coefficient homomorphism $\mathbb{Z} \cong H^{1}(I, \partial I ; \mathbb{Z}) \rightarrow H^{1}\left(I, \partial I ; \mathbb{Z}_{2}\right) \cong \mathbb{Z}_{2}$ is given by mod 2 reduction according to the corresponding Bockstein sequence.

11.2.11 Remark. $\quad$ i) In the case of bounded selfadjoint Fredholm operators $\mathcal{A}$, the foregoing corollary 11.2.10 actually holds if $\operatorname{sf} \mathcal{A} \neq 0$ under the assumption that $\tilde{H}=H, D_{0} C=0$ and the corresponding map $f$ in 11.1) is the gradient of a $C^{2}$ functional on $I \times H$ (cf. [FPR99]). However, the method of proof is completely different from ours and strongly uses that the operators are parametrised by an interval.

ii) Corollary 11.2 .10 is also a variant of the classical Krasnoselski bifurcation theorem (cf. [AmM07, 3.31]) which deals with compact $C^{1}$ operators $C: E \rightarrow E$ acting on a real Banach space $E$ such that $C(0)=0$ and $D_{0} C=0$. If now $K \in \mathcal{L}(E)$ is compact, then every characteristic value (inverse of an eigenvalue) $\lambda^{*}$ of $K$ having odd algebraic multiplicity is a bifurcation point for the equation 


$$
f(\lambda, u)=(I-\lambda K) u+C(u), \quad(\lambda, u) \in \mathbb{R} \times E .
$$

Finally we come back to the dimensions of exceptional sets from the third chapter. Although we could obtain more results here, we just want to state the following one.

11.2.12 Proposition. Let $X$ be a simply connected and compact topological space, $Y \subset X$ a closed subspace and $\mathcal{A}:(X, Y) \rightarrow\left(\mathcal{C F}^{s a}(H), G \mathcal{C}^{s a}(H)\right)$ a gap continuous family. Moreover, let $C: X \times \tilde{H} \rightarrow H$ be compact such that $C_{x}(0)=0, x \in X$, and condition $(C)$ as well as the assumptions i) and ii) of theorem 11.2.6 are satisfied. If there exists $f \in \Omega^{1}(X, Y)$ such that

$$
\operatorname{sf}\left(f^{*} \mathcal{A}\right) \quad \bmod 2 \neq 0,
$$

then $X \backslash B$ is not path connected, where $B \subset X$ denotes the set of all bifurcation points. If moreover $X$ is manifold of dimension $n \geq 2$, then $B$ is not contractible and its dimension is at least $n-1$.

Proof. Setting

$$
\Gamma_{1}: \Omega^{1}(X, Y) \rightarrow \mathbb{Z}_{2}, \quad \Gamma_{1}(f)=\operatorname{sf}\left(f^{*} \mathcal{A}\right) \quad \bmod 2
$$

it is clear that $\Gamma_{1}(f)=\Gamma_{1}(g)$ if $f, g \in \Omega^{1}(X, Y)$ are homotopic and that $\Gamma_{1}(f * g)=\Gamma_{1}(f)+$ $\Gamma_{1}(g)$ whenever $f * g$ is defined. Since $\Gamma_{1}(f)=0$ if $f(I)$ does not contain a bifurcation point by corollary 11.2.10, we obtain the assertion from lemma 3.3.5 and proposition 3.3.6.

11.2.13 Remark. Note that the foregoing corollary is also an existence result because we obtain that $B \neq \emptyset$ from its assertion.

\subsection{The Proof of the Theorems}

\subsubsection{Proof of Theorem 11.2 .5}

We take a global trivialisation

$$
\psi: \mathfrak{D}(\mathcal{A}) \rightarrow X \times H^{\prime},
$$

of the domain bundle of $\mathcal{A}$ and consider the family of maps

$$
\tilde{\mathcal{A}}+\tilde{C}: X \times H^{\prime} \rightarrow H, \quad(\tilde{\mathcal{A}}+\tilde{C})(x, u):=\mathcal{A}_{x} \psi_{x}^{-1} u+C_{x}\left(\left(\iota_{x} \circ \psi_{x}^{-1}\right) u\right) .
$$


Then $\tilde{A}: X \rightarrow \mathcal{B F}_{0}\left(H^{\prime}, H\right)$ is a continuous family of Fredholm operators of index 0 because of lemma 6.1.3 Further, $\tilde{C}: X \times H^{\prime} \rightarrow H$ defines a compact map by condition (C) and lemma 11.2.4 Finally, by our assumptions on the differentiability of $C_{x_{0}}$ and 11.3 , we obtain that $\tilde{\mathcal{A}}_{x_{0}}+\tilde{C}_{x_{0}}$ is real continuously differentiable at $0 \in H^{\prime}$ and $D_{0}\left(\tilde{A}_{x_{0}}+\tilde{C}_{x_{0}}\right) \in G L\left(\left(H^{\prime}\right)^{\mathbb{R}}, H^{\mathbb{R}}\right)$. Now we can use the following result which can be found in [Pe88, Cor. 4].

11.3.1 Theorem. Let $E, F$ be real Banach spaces and $X$ a compact connected $C W$-complex. Let $f: X \times E \rightarrow F$ be a map of the form $f_{x}(u)=L_{x} u+C_{x}(u)$, where $L: X \rightarrow \mathcal{B F}_{0}(E, F)$ is continuous with respect to the norm topology and $C: X \times E \rightarrow F$ is compact such that $C_{x}(0)=0, x \in X$. Assume moreover that some $f_{x_{0}}$ is continuously differentiable at 0 and $D_{0} f_{x_{0}} \in G L(E, F)$.

If $J\left(\operatorname{ind}_{\mathbb{R}} L\right) \neq 0 \in J(X)$, then there exists a bifurcation point $x^{*} \in X$ of the family $f$.

Here $\operatorname{ind}_{\mathbb{R}}(L) \in K O(X)$ denotes the index bundle for families of bounded Fredholm operators $L: X \rightarrow \mathcal{B F}(E, F)$ acting between real Banach spaces $E$ and $F$. The index bundle for Fredholm morphisms between Banach bundles from section 5.1 can be constructed verbatim for Fredholm morphisms of real Banach bundles. In the special case of families acting between two fixed real Banach spaces the result coincides with the construction of $\operatorname{ind}_{\mathbb{R}}(L)$ in [Pe88]. Accordingly, it is readily seen that $\operatorname{ind}_{\mathbb{R}}\left(L^{\mathbb{R}}\right)=(\text { ind } L)^{\mathbb{R}} \in K O(X)$, where now $L^{\mathbb{R}}: E^{\mathbb{R}} \rightarrow F^{\mathbb{R}}$ is the realification of a family of bounded Fredholm operators acting between the complex Banach spaces $E$ and $F$. We now come back to the proof of our theorem and consider the realifications $\left(H^{\prime}\right)^{\mathbb{R}}$ and $H^{\mathbb{R}}$ and the realifications of the maps $\tilde{\mathcal{A}}^{\mathbb{R}}+\tilde{C}^{\mathbb{R}}$. Since $\tilde{\mathcal{A}}$ is a composition of bundle morphisms

$$
X \times H^{\prime} \stackrel{\psi^{-1}}{\longrightarrow} \mathfrak{D}(\mathcal{A}) \stackrel{\mathcal{A}}{\longrightarrow} X \times H
$$

we conclude by using lemma 5.2 .7 that

$$
\operatorname{ind}(\tilde{\mathcal{A}})=\operatorname{ind}\left(\mathcal{A} \circ \psi^{-1}\right)=\operatorname{ind}(\mathcal{A})+\operatorname{ind}\left(\psi^{-1}\right)=\operatorname{ind}(\mathcal{A})
$$

Hence

$$
J\left(\operatorname{ind}_{\mathbb{R}}\left(\tilde{\mathcal{A}}^{\mathbb{R}}\right)\right)=J\left(\operatorname{ind}(\tilde{\mathcal{A}})^{\mathbb{R}}\right)=J\left(\operatorname{ind}(\mathcal{A})^{\mathbb{R}}\right) \neq 0 \in J(X)
$$

and since all other assumptions of theorem 11.3.1 hold for $\tilde{A}^{\mathbb{R}}+\tilde{C}^{\mathbb{R}}$ we infer the existence of a sequence $\left\{\left(x_{n}, v_{n}\right)\right\}_{n \in \mathbb{N}} \subset X \times H^{\prime}, v_{n} \neq 0, n \in \mathbb{N}$, converging to $\left(x^{*}, 0\right)$ for some $x^{*} \in X$ such that

$$
\tilde{\mathcal{A}}_{x_{n}} v_{n}+\tilde{C}_{x_{n}}\left(v_{n}\right)=\mathcal{A}_{x_{n}} \psi_{x_{n}}^{-1} v_{n}+C_{x_{n}}\left(\left(\iota_{x_{n}} \circ \psi_{x_{n}}^{-1}\right) v_{n}\right)=0, \quad n \in \mathbb{N} .
$$

Setting $\left(x_{n}, u_{n}\right)=\psi^{-1}\left(x_{n}, v_{n}\right) \in \mathcal{D}\left(\mathcal{A}_{x_{n}}\right), n \in \mathbb{N}$, we finally obtain a sequence which converges to $\left(x^{*}, 0\right)$ in $X \times \tilde{H}$ because of the continuity of $\iota \circ \psi^{-1}: X \times H^{\prime} \rightarrow \tilde{H}$ which holds by condition $(\mathrm{C})$. 


\subsubsection{A Finite Dimensional Approximation to $C$}

In this section we prepare the proof of the bifurcation theorem 11.2.6.

Let $X$ be a compact topological space and $E, F$ Banach spaces. Let, moreover, $L: X \rightarrow$ $\mathcal{B F}_{0}(E, F)$ be a continuous family of Fredholm operators of index 0 and $C: X \times E \rightarrow F$ a compact map. We consider the family of maps

$$
f=L+C: X \times E \rightarrow F .
$$

The aim of this section is the proof of the following two theorems.

11.3.2 Theorem. If $X$ is a compact connected $C W$-complex and $B \subset E$ bounded and closed, then $f(X \times B) \subset F$ is closed.

11.3.3 Theorem. Let $X$ be a compact connected $C W$-complex and $B \subset E$ bounded. Let $B^{\prime} \subset B$ be closed such that $0 \notin f\left(X \times B^{\prime}\right)$. Then for any sufficiently small $\varepsilon>0$ there exists a continuous map $\tilde{C}: X \times B \rightarrow F$ and a finite dimensional subspace $W \subset F$ such that

$$
\begin{gathered}
\|C(x, u)-\tilde{C}(x, u)\|<\varepsilon, \quad(x, u) \in X \times B, \\
\tilde{C}(X \times B) \subset W
\end{gathered}
$$

and we still have $0 \notin \tilde{f}\left(X \times B^{\prime}\right)$, where

$$
\tilde{f}=L+\tilde{C}: X \times B \rightarrow F .
$$

The remaining section is entirely devoted to the proof of these theorems.

Before we can begin with the proof of theorem 11.3.2, we prepare it by discussing a helpful result which we already mentioned in section 4.1 without proof.

It is easy to see that an operator $L \in \mathcal{L}(E, F)$ is Fredholm of index 0 if and only if there exists an invertible operator $M \in G L(F, E)$ and a compact operator $K \in \mathcal{K}(E)$ such that $M L=I+K$ (cf. GGK90, Th. XI.5.3]). We now show that the same holds true for families of bounded Fredholm operators. The result can be found in [FP88, 1.6.3].

11.3.4 Proposition. Let $X$ be a compact topological space, $E, F$ Banach spaces and $L: X \rightarrow$ $\mathcal{B F}_{0}(E, F)$ be a continuous family of bounded Fredholm operators of index 0 . If $\operatorname{ind}(L)=0 \in$ $K(X)$, then there exist continuous families $M: X \rightarrow G L(F, E)$ and $K: X \rightarrow \mathcal{K}(E)$ such that

$$
M_{x} L_{x}=I_{E}+K_{x}, \quad x \in X .
$$


Proof. As a first step we want to show that under the assumption $\operatorname{ind}(L)=0$ we can find a subspace $V \subset F$ which is transversal to the image of $L$ and such that the bundle space $E(L, V)$ from the definition of the index bundle is trivial.

Let $V \subset F$ be any subspace transversal to the image of $L$. Since ind $(L)=[E(L, V)]-[\Theta(V)]=$ $0 \in \tilde{K}(X)$ we can find a trivial vector bundle $\mathcal{W}$ over $X$ such that

$$
E(L, V) \oplus \mathcal{W} \cong \Theta(V) \oplus \mathcal{W}
$$

and hence $E(L, V) \oplus \mathcal{W}$ is a trivial bundle. Now we choose a subspace $V^{\prime} \subset F$ such that $\operatorname{dim} V=\operatorname{dim} \mathcal{W}, V \cap V^{\prime}=\{0\}$ and consider the bundle $E\left(L, V \oplus V^{\prime}\right)$. Let $E(L, V)^{\perp}$ denote a complement of $E(L, V)$ in $E\left(L, V \oplus V^{\prime}\right)$ and $P_{V^{\prime}}: V \oplus V^{\prime} \rightarrow V \oplus V^{\prime}$ be the projection onto $V^{\prime}$ along $V$. Then the bundle map

$$
\left.P_{V^{\prime}} L\right|_{E(L, V)^{\perp}}: E(L, V)^{\perp} \rightarrow \Theta\left(V^{\prime}\right)
$$

is an isomorphism. Indeed, if $P_{V^{\prime}} L_{x} u=0$ for some $u \in E(L, V)_{x}^{\perp}$, we infer $L_{x} u \in V$ which implies $u \in E(L, V)_{x}$ and hence $u=0$. Moreover, since $\operatorname{dim} E\left(L, V \oplus V^{\prime}\right)=\operatorname{dim} V+\operatorname{dim} V^{\prime}$ by lemma 5.1 .6 and $\operatorname{dim} E(L, V)=\operatorname{dim} V$ we deduce that $\operatorname{dim} E(L, V)^{\perp}=\operatorname{dim} V^{\prime}$ and hence $\left.P_{V^{\prime}} L\right|_{E(L, V)^{\perp}}$ is an isomorphism.

Finally we summarise

$$
E\left(L, V \oplus V^{\prime}\right) \cong E(L, V) \oplus E(L, V)^{\perp} \cong E(L, V) \oplus \Theta\left(V^{\prime}\right) \cong E(L, V) \oplus \mathcal{W}
$$

where the last isomorphism exists because $\mathcal{W}$ is trivial and of the same dimension than $V^{\prime}$. Hence $E\left(L, V \oplus V^{\prime}\right)$ is a trivial bundle by 11.5.

Now we begin with the proof of the assertion. Let $V \subset F$ be a subspace which is transversal to the image of $L$ and such that $E(L, V)$ is a trivial bundle. Since $\operatorname{dim} E(L, V)=\operatorname{dim} V$ by lemma 5.1.6. we can find an isomorphism $a: E(L, V) \rightarrow \Theta(V)$. Now we use proposition 1.2.6 in order to choose a family of projections $P: X \rightarrow \mathcal{L}(E)$ such that im $P_{x}=E(L, V)_{x}, x \in X$, and, moreover, we take a fixed projection $Q \in \mathcal{L}(F)$ onto the finite dimensional space $V$. Then we can define a continuous family

$$
K: X \rightarrow \mathcal{K}(E, F), \quad K_{x}=a_{x} \circ P_{x}-Q \circ L_{x}
$$

which consists of finite rank operators. Now we consider

$$
L_{x}+K_{x}=(I-Q) L_{x}+a_{x} P_{x}
$$


which is a continuous family of bounded Fredholm operators of index 0 .

If $L_{x} u+K_{x} u=0$ for some $u \in E$, we infer $(I-Q) L_{x} u=a_{x} P_{x} u=0$ because $a_{x} P_{x} u \in V$ and $Q$ is a projection onto $V$. Now from $(I-Q) L_{x} u=0$ we obtain $L_{x} u \in V$ while $a_{x} P_{x} u=0$ gives $P_{x} u=0$ because $a_{x}$ is an isomorphism. But, since $P_{x}$ is a projection onto $E(L, V)_{x}=L_{x}^{-1}(V)$, we conclude from $L_{x} u \in V$ that $P_{x} u=u$ and so finally $u=0$.

Hence each $L_{x}+K_{x}$ is an injective Fredholm operator of index 0 and so an element of $G L(E, F)$. Accordingly, by using the continuity of the inversion in $G L(E)$, we obtain a continuous family

$$
M: X \rightarrow G L(F, E), \quad M_{x}=\left(L_{x}+K_{x}\right)^{-1}
$$

and now the assertion follows from

$$
M_{x} L_{x}=\left(L_{x}+K_{x}\right)^{-1} L_{x}=\left(L_{x}+K_{x}\right)^{-1}\left(L_{x}+K_{x}\right)-\left(L_{x}+K_{x}\right)^{-1} K_{x}=I_{E}+M_{x} K_{x}, x \in X,
$$

since $M_{x} K_{x}$ is a compact operator.

Now we can start with the proof of theorem 11.3.2 and our first aim is to prove it under the additional assumption that the base space $X$ is a sequentially compact and contractible.

Then ind $(L)=0 \in K(X)$ and hence by proposition 11.3.4 there exist continuous families $M: X \rightarrow G L(F, E)$ and $K: X \rightarrow \mathcal{K}(E)$ such that $M_{x} L_{x}=I_{E}+K_{x}, x \in X$. Now let $\left\{v_{n}\right\}_{n \in \mathbb{N}} \subset f(X \times B)$ be a sequence converging to some $v \in F$. We take a sequence $\left\{\left(x_{n}, u_{n}\right)\right\}_{n \in \mathbb{N}} \subset X \times B$ such that $f\left(x_{n}, u_{n}\right)=v_{n}$. Since $X$ is sequentially compact, $\left\{x_{n}\right\}_{n \in \mathbb{N}}$ contains a subsequence $\left\{x_{n_{k}}\right\}_{k \in \mathbb{N}}$ converging to some $x^{*} \in X$. Moreover, by using lemma 11.2.3 and the compactness of $C$ we can further thin out to a subsequence $\left\{x_{n_{l}}\right\}_{l \in \mathbb{N}}$ such that $\left\{K\left(x_{n_{l}}, u_{n_{l}}\right)\right\}_{l \in \mathbb{N}}$ and $\left\{C\left(x_{n_{l}}, u_{n_{l}}\right)\right\}_{l \in \mathbb{N}}$ converge in $F$.

From $f\left(x_{n_{l}}, u_{n_{l}}\right) \rightarrow v$ and the continuity of $M$ we infer that

$$
M_{x_{n_{l}}} f\left(x_{n_{l}}, u_{n_{l}}\right)=u_{n_{l}}+K_{x_{n_{l}}} u_{n_{l}}+M_{x_{n_{l}}} C\left(x_{n_{l}}, u_{n_{l}}\right)
$$

converges in $F$. But since $K_{x_{n_{l}}} u_{n_{l}}$ and $C\left(x_{n_{l}}, u_{n_{l}}\right)$ are convergent we obtain that also $\left\{u_{n_{l}}\right\}_{l \in \mathbb{N}}$ converges to some $u^{*} \in B$, where we use that $B$ is closed by assumption. Hence we obtain by the continuity of $f$

$$
v=\lim _{l \rightarrow \infty} f\left(x_{n_{l}}, u_{n_{l}}\right)=f\left(x^{*}, u^{*}\right) \in f(X \times B)
$$

and infer that $f(X \times B)$ is closed.

Now we consider the general case and assume that $X$ is a compact connected CW-complex. Note at first that $X$ is metrisable and hence each closed subspace of $X$ is sequentially compact. Moreover, arguing as in [Ha02, Prop. A.4], $X$ can be covered by a finite number of closed 
contractible subsets $U_{i}, i=1, \ldots, N$.

According to the special case we already proved above $f\left(U_{i} \times B\right) \subset E$ is closed, $i=1, \ldots N$, and hence we infer that

$$
f(X \times B)=f\left(\bigcup_{i=1}^{N} U_{i} \times B\right)=\bigcup_{i=1}^{N} f\left(U_{i} \times B\right)
$$

is closed as well.

In order to prove the second theorem 11.3 .3 we need again a helpful theorem before. It is a standard result in nonlinear functional analysis in the special case that $X$ is a single point. Here we need a generalisation to families but whose proof is almost the same than in the special case.

11.3.5 Theorem. Let $X$ be a compact topological space, $E, F$ Banach spaces, $B \subset E$ a bounded subset and $C: X \times B \rightarrow F$ compact. Then for any $\varepsilon>0$ there exists a compact map $\tilde{C}: X \times B \rightarrow$ $F$ such that $\tilde{C}(X \times B)$ is contained in a finite dimensional subspace of $F$ and

$$
\|C(x, u)-\tilde{C}(x, u)\|<\varepsilon \quad \text { for all } \quad(x, u) \in X \times B .
$$

Proof. Since $C$ is a compact operator, $C(X \times B)$ is relatively compact by definition. Accordingly we can find $\left(x_{i}, u_{i}\right) \in X \times B, i=1, \ldots N$, such that

$$
C(X \times B) \subset \bigcup_{i=1}^{N} B\left(C\left(x_{i}, u_{i}\right), \varepsilon\right),
$$

where $B(u, r)$ denotes the open Ball of radius $r>0$ around $u \in F$.

We define functions

$$
\alpha_{i}: X \times B \rightarrow \mathbb{R}, \quad \alpha_{i}(x, u)=\max \left\{\varepsilon-\left\|C(x, u)-C\left(x_{i}, u_{i}\right)\right\|, 0\right\}, \quad i=1, \ldots N .
$$

Note that by 11.6

$$
\sum_{i=1}^{N} \alpha_{i}(x, u)>0, \quad \text { for all } \quad(x, u) \in X \times B
$$

and hence we can define continuous maps by

$$
\lambda_{i}: X \times B \rightarrow \mathbb{R}, \quad \lambda_{i}(x, u)=\alpha_{i}(x, u)\left(\sum_{i=1}^{N} \alpha_{i}(x, u)\right)^{-1}, \quad i=1, \ldots N .
$$


Finally we define

$$
\tilde{C}: X \times B \rightarrow F, \quad \tilde{C}(x, u)=\sum_{i=1}^{N} \lambda_{i}(x, u) C\left(x_{i}, u_{i}\right)
$$

Using that $\lambda_{i}(x, u)>0$ implies $\left\|C(x, u)-C\left(x_{i}, u_{i}\right)\right\|<\varepsilon$ and, moreover, $\sum_{i=1}^{N} \lambda_{i}(x, u)=1$, $(x, u) \in X \times B$, we obtain

$$
\begin{aligned}
\|C(x, u)-\tilde{C}(x, u)\| & =\left\|\tilde{C}(x, u)-\sum_{i=1}^{N} \lambda_{i}(x, u) C(x, u)\right\| \leq \sum_{i=1}^{N} \lambda_{i}(x, u)\left\|C\left(x_{i}, u_{i}\right)-C(x, u)\right\| \\
& <\varepsilon \sum_{i=1}^{N} \lambda_{i}(x, u)=\varepsilon, \quad(x, u) \in X \times B .
\end{aligned}
$$

Hence $\tilde{C}: X \times B \rightarrow F$ indeed approximates $C$ as stated in the assertion.

We have by definition

$$
\operatorname{im} \tilde{C} \subset \operatorname{span}\left\{C\left(x_{1}, u_{1}\right), \ldots, C\left(x_{N}, u_{N}\right)\right\}
$$

and moreover $\tilde{C}: X \times B \rightarrow F$ is obviously continuous. Hence it remains to prove that $\tilde{C}(X \times B)$ is relatively compact. But from

$$
\|\tilde{C}(x, u)\| \leq\|\tilde{C}(x, u)-C(x, u)\|+\|C(x, u)\| \leq \varepsilon+\|C(x, u)\|, \quad(x, u) \in X \times B,
$$

and the fact that $C(X \times B)$ is relatively compact and so in particular bounded, we infer that $\tilde{C}(X \times B)$ is bounded. Accordingly, $\tilde{C}(X \times B)$ is a bounded subset of a finite dimensional subspace of $F$ and consequently relatively compact.

Now the proof of theorem 11.3 .3 is straightforward. By theorem 11.3.2, $f\left(X \times B^{\prime}\right)$ is closed in $F$ and hence

$$
\inf _{(x, u) \in X \times B^{\prime}}\|f(x, u)\|>2 \varepsilon>0 .
$$

for any sufficiently small $\varepsilon>0$. According to theorem 11.3 .5 we can find a compact map $\tilde{C}: X \times B \rightarrow F$ such that $\tilde{C}(X \times B)$ is contained in a finite dimensional subspace of $F$ and

$$
\|C(x, u)-\tilde{C}(x, u)\|<\varepsilon, \quad \text { for all }(x, u) \in X \times B .
$$

If now $(x, u) \in X \times B^{\prime}$ is an arbitrary element, we obtain 


$$
\begin{aligned}
\left\|\tilde{f}_{x}(u)\right\| & =\left\|L_{x} u+\tilde{C}(x, u)\right\|=\left\|L_{x} u+C(x, u)-C(x, u)+\tilde{C}(x, u)\right\| \\
& \geq\left\|f_{x}(u)\right\|-\|C(x, u)-\tilde{C}(x, u)\| \geq \varepsilon>0
\end{aligned}
$$

and hence theorem 11.3 .3 is proved.

\subsubsection{Proof of Theorem 11.2 .6}

\section{Step 1: Some More Preliminaries}

We begin with a well known result about Hilbert bundles whose proof we adapt here from a similar construction in [La95, VII.3.1].

11.3.6 Lemma. Let $\mathcal{H}$ be a Hilbert bundle with model space $H$ and $g: \mathcal{H} \rightarrow \mathbb{R}$ a bundle metric. Then, for any trivialisation

$$
\tau: \pi^{-1}(U) \rightarrow U \times H
$$

there exists a continuous map $B: U \rightarrow G L(H)$, such that

$$
B \tau: \pi^{-1}(U) \rightarrow U \times H, \quad(B \tau)(u)=B_{\pi(u)} \tau(u)
$$

is a trivialisation belonging to the same atlas as $\tau$ and each restriction to a fibre

$$
B_{x} \tau_{x}=\left.B \tau\right|_{\mathcal{H}_{x}}: \mathcal{H}_{x} \rightarrow H
$$

is a unitary operator with respect to $g_{x}$ and the scalar product in the model space.

Proof. We define a continuous family of metrics on $H$ by

$$
\tilde{g}_{x}(u, v)=g_{x}\left(\tau_{x}^{-1} u, \tau_{x}^{-1} v\right), \quad x \in U .
$$

According to the Riesz representation theorem, there exists a continuous family $A: U \rightarrow$ $G L(H)$ of selfadjoint positive operators, such that

$$
\tilde{g}_{x}(u, v)=\left\langle A_{x} u, v\right\rangle_{H}, \quad \forall u, v \in H, x \in U .
$$

Now we define a continuous family of invertible selfadjoint operators by $B_{x}:=A_{x}^{\frac{1}{2}}$ and obtain for any $x \in U$ and $u, v \in \mathcal{H}_{x}$ 


$$
\begin{aligned}
\left\langle B_{x} \tau_{x} u, B_{x} \tau_{x} v\right\rangle_{H} & =\left\langle A_{x}^{\frac{1}{2}} \tau_{x} u, A_{x}^{\frac{1}{2}} \tau_{x} v\right\rangle_{H}=\left\langle A_{x} \tau_{x} u, \tau_{x} u\right\rangle_{H} \\
& =\tilde{g}\left(\tau_{x} u, \tau_{x} v\right)=g_{x}(u, v) .
\end{aligned}
$$

11.3.7 Lemma. Let $X$ be a topological space, $\mathcal{A}: X \rightarrow \mathcal{C}(H)$ gap continuous and $\tilde{H}$ a linear space such that condition $(C)$ holds. If the singular set of $\mathcal{A}$ does not intersect the compact set $K$, then there exists a constant $\tilde{c}=\tilde{c}(K)>0$ such that

$$
\left\|\iota_{x} \circ \mathcal{A}_{x}^{-1}\right\|_{\mathcal{L}(H, \tilde{H})}<\tilde{c}, \quad x \in K .
$$

Proof. We consider the restriction $\mathcal{A}_{K}: K \rightarrow \mathcal{C}(H)$ to $K$. Then $\mathcal{A}_{K}$ induces a bundle morphism between $\mathfrak{D}\left(\mathcal{A}_{K}\right)$ and $K \times H$ which has empty support and hence is a bundle isomorphism according to lemma 1.2.2. The inverse of $\mathcal{A}_{K}$ is given by the bundle morphism induced by the operators $\mathcal{A}_{x}^{-1}: H \rightarrow \mathcal{D}\left(\mathcal{A}_{x}\right), x \in K$, and by condition (C) we infer that

$$
\iota \circ \mathcal{A}_{K}^{-1}: K \times H \rightarrow \mathfrak{D}\left(\mathcal{A}_{K}\right) \rightarrow K \times \tilde{H}
$$

is a bundle morphism. So in particular the map

$$
K \ni x \mapsto \iota_{x} \circ \mathcal{A}_{x}^{-1} \in \mathcal{L}(H, \tilde{H})
$$

is continuous and now the assertion follows by the compactness of $K$.

\section{Step 2: Extension and Approximation of $f$}

We consider a gap continuous family $\mathcal{A}: X \rightarrow \mathcal{C F}^{s a}(H)$ and a compact operator $C: X \times \tilde{H} \rightarrow H$ such that the condition $(\mathrm{C})$ holds as in our theorem 11.2.6.

In the definition of the selfadjoint index s-ind $(\mathcal{A})$ we considered the canonical extension of $\mathcal{A}$ by

$$
\overline{\mathcal{A}}: X \times \mathbb{R} \rightarrow \mathcal{C F}_{0}(H), \quad \overline{\mathcal{A}}_{(x, s)} u=\mathcal{A}_{x} u+i s u
$$

which is continuous with respect to the gap topology and hence has an associated domain bundle $\mathfrak{D}(\overline{\mathcal{A}})$. We now can extend the compact map $C$ continuously by

$$
\bar{C}: X \times \mathbb{R} \times \tilde{H} \rightarrow H, \quad \bar{C}(x, s, u)=C(x, u)
$$

and note that $\bar{C}$ is compact as well in the sense that $\bar{C}(X \times \mathbb{R} \times B)$ is relatively compact for any bounded subset $B \subset \tilde{H}$. Moreover, we have the following extension of the assumed condition (C). 
11.3.8 Lemma. The canonical inclusion

$$
\iota: \mathfrak{D}(\overline{\mathcal{A}}) \hookrightarrow X \times \mathbb{R} \times \tilde{H}
$$

is a bundle morphism.

Proof. Let $p: X \times \mathbb{R} \rightarrow X$ be the projection onto the first component. By using lemma 6.1.10 and lemma 6.1 .8 we infer

$$
\mathfrak{D}(\overline{\mathcal{A}})=\mathfrak{D}\left(p^{*} \mathcal{A}\right)=p^{*} \mathfrak{D}(\mathcal{A})
$$

and, since $\iota: \mathfrak{D}(\mathcal{A}) \hookrightarrow X \times \tilde{H}$ is a bundle morphism by assumption, we obtain the assertion from the representation

$$
\iota: \mathfrak{D}(\overline{\mathcal{A}})=p^{*} \mathfrak{D}(\mathcal{A}) \hookrightarrow p^{*}(X \times \tilde{H})=X \times \mathbb{R} \times \tilde{H}
$$

As an immediate consequence we see that $\bar{C}$ restricts to a continuous map $\bar{C} \circ \iota$ on $\mathfrak{D}(\overline{\mathcal{A}})$ and hence we obtain a continuous map

$$
\bar{f}=\overline{\mathcal{A}}+\bar{C} \circ \iota: \mathfrak{D}(\overline{\mathcal{A}}) \rightarrow X \times \mathbb{R} \times H
$$

We now choose once and for all a relatively compact open neighbourhood $V \subset X \backslash Y \times \mathbb{R}$ of the singular set of $\mathcal{A}$ as in assumption iii) in the theorem. Note, incidentally, that the closures of $V$ in $X \backslash Y \times \mathbb{R}$ and $X \times \mathbb{R}$ coincide which is readily seen from the relative compactness of $V$ in $X \backslash Y \times \mathbb{R}$.

As next aim we want to define a certain bundle metric on $\mathfrak{D}(\overline{\mathcal{A}})$. At first, we need the following result.

11.3.9 Lemma. There exists a continuous function $\rho: X \times \mathbb{R} \rightarrow[0,1]$ such that $\left.\rho\right|_{(X \times \mathbb{R}) \backslash V}=0$ and $\rho>0$ on $V$.

Proof. We can choose an $n \in \mathbb{N}$ such that $\bar{V} \subset X \times[-n, n]$ and since the latter space is a compact $\mathrm{CW}$-complex it is in particular metrisable. We obtain a continuous function

$$
\tilde{\rho}: X \times[-n, n] \rightarrow \mathbb{R}, \quad \tilde{\rho}(z)=d(z, \partial V)
$$

where $d$ denotes the distance function with respect to any metric on $X \times[-n, n]$ which is compatible with the given topology. Since $\partial V \subset X \times[-n, n]$ is closed, we obtain that $\tilde{\rho}(z)=0$ 
if and only if $z \in \partial V$. Moreover, because $\bar{V} \subset X \times[-n, n]$ is compact, $\tilde{\rho}$ has a maximum $m>0$ on this space. Now we define

$$
\rho: X \times \mathbb{R} \rightarrow[0,1], \quad \rho(z)=\left\{\begin{array}{l}
\frac{\tilde{\rho}(z)}{m}, z \in \bar{V} \\
0, \quad z \in X \times \mathbb{R} \backslash V
\end{array}\right.
$$

and note that $\rho>0$ on $V$. Moreover, $\rho$ is continuous because both definitions coincide on $(X \times \mathbb{R} \backslash V) \cap \bar{V}=\partial V$.

We now choose once and for all a function $\rho: X \times \mathbb{R} \rightarrow[0,1]$ as in the foregoing lemma.

11.3.10 Lemma. The map

$$
g: \mathfrak{D}(\overline{\mathcal{A}}) \rightarrow \mathbb{R}, \quad g_{z}(u, v)=\left\langle\overline{\mathcal{A}}_{z} u, \overline{\mathcal{A}}_{z} v\right\rangle_{H}+\rho(z)\langle u, v\rangle_{H}
$$

is a bundle metric on $\mathfrak{D}(\overline{\mathcal{A}})$ which induces the given topology in each fibre.

Proof. First of all $g$ is continuous because of the continuity of $\overline{\mathcal{A}}: \mathfrak{D}(\overline{\mathcal{A}}) \rightarrow X \times \mathbb{R} \times H$ which we proved in lemma 6.1.3 the continuity of the inclusion $\iota: \mathfrak{D}(\overline{\mathcal{A}}) \hookrightarrow X \times \mathbb{R} \times \tilde{H}$ proved in lemma 11.3 .8 and the continuity of $\iota: \tilde{H} \hookrightarrow H$.

Now we consider a fixed $z=(x, s) \in X \times \mathbb{R}$ and observe that $g_{z}$ has by definition all properties of a scalar product except that the definiteness may fail.

We distinguish two cases. At first assume that $z$ is in the interior of $V$ and hence $\rho(z) \neq 0$. Then we obtain for any $u \in \mathcal{D}(\overline{\mathcal{A}})_{z}$

$$
g_{z}(u, u)=\left\|\overline{\mathcal{A}}_{z} u\right\|^{2}+\rho(z)\|u\|^{2} \leq\left\|\overline{\mathcal{A}}_{z} u\right\|^{2}+\|u\|^{2}=\|u\|_{\mathcal{\mathcal { A }}_{z}}^{2}
$$

and

$$
\begin{aligned}
\|u\|_{\mathcal{\mathcal { A }}_{z}}^{2} & =\frac{1}{\rho(z)}\left(\rho(z)\left\|\overline{\mathcal{A}}_{z} u\right\|^{2}+\rho(z)\|u\|^{2}\right) \\
& \leq \frac{1}{\rho(z)}\left(\left\|\overline{\mathcal{A}}_{z} u\right\|^{2}+\rho(z)\|u\|^{2}\right)=\frac{1}{\rho(z)} g_{z}(u, u) .
\end{aligned}
$$

Hence in this case $g_{z}$ is positive definite and moreover its induced norm is equivalent to the graph norm of $\overline{\mathcal{A}}_{z}$. Consequently, the topology induced by $g_{z}$ and the topology on $\mathfrak{D}(\overline{\mathcal{A}})_{z}$ coincide.

Now we assume that $z \notin V$. Then $\rho(z)=0$ but we know that $\overline{\mathcal{A}}_{z}$ has a bounded inverse and accordingly there exists a constant $c_{z}$ such that $\left\|\overline{\mathcal{A}}_{z}^{-1} u\right\| \leq c_{z}\|u\|$ for all $u \in H$. We obtain $\|u\| \leq c_{z}\left\|\overline{\mathcal{A}}_{z} u\right\|$ for all $u \in \mathcal{D}(\overline{\mathcal{A}})_{z}$ and hence 


$$
\begin{aligned}
\|u\|_{\overline{\mathcal{A}}_{z}}^{2} & =\langle u, u\rangle+\left\langle\overline{\mathcal{A}}_{z} u, \overline{\mathcal{A}}_{z} u\right\rangle \\
& \leq\left(1+c_{z}^{2}\right)\left\langle\overline{\mathcal{A}}_{z} u, \overline{\mathcal{A}}_{z} u\right\rangle=\left(1+c_{z}^{2}\right) g_{z}(u, u) .
\end{aligned}
$$

Since we trivially have $g_{z}(u, u) \leq\|u\|_{\mathcal{A}_{z}}^{2}$ in this case, we infer again that $g_{z}$ is positive definite and induces the given topology on $\mathfrak{D}(\overline{\mathcal{A}})_{z}$.

In the following we denote by $B(r) \subset \mathfrak{D}(\overline{\mathcal{A}})$ the disc bundle of radius $r>0$ with respect to the metric defined in lemma 11.3 .10 and by $B_{z, r}(0) \subset \mathcal{D}(\overline{\mathcal{A}})_{z}$ the corresponding fibre over $z \in X \times \mathbb{R}$. Remember that, by lemma 11.3.7, we can assign to any compact subset $K$ of $X \times \mathbb{R}$, which has empty intersection with the singular set of $\overline{\mathcal{A}}$, a constant $\tilde{c}(K)$ such that the norms of the inverses of $\overline{\mathcal{A}}$ in $\mathcal{L}(H, \tilde{H})$ are uniformly bounded by this constant. In the following we denote throughout

$$
\tilde{c}:=\tilde{c}(\partial V)>0 .
$$

Now we choose $n \in \mathbb{N}$ such that $V \subset X \times[-n, n]$ and we expect from now on the following assumption:

(A) There exists $0<r<\min \left\{\frac{r_{0}}{\tilde{c}}, \frac{1}{M \tilde{c}^{2}}\right\}$ such that $0 \notin \bar{f}_{z}\left(\partial B_{z, r}(0)\right)$ for all $z \in X \times[-n, n]$,

where $r_{0}$ and $M$ are positive real numbers according to assumption i) from our theorem. The main part of the following argument is to obtain a contradiction to the assumptions of our theorem 11.2.6 and so to infer that (A) can not hold. In the final part we will explain why the negation of (A) implies the existence of a bifurcation point for the family $f$. We now fix once and for all an $r$ as in (A).

We consider the restriction of the disc bundle $B(r) \subset \mathfrak{D}(\overline{\mathcal{A}})$ to $X \times[-n, n]$. Since $X \times[-n, n]$ is a compact $\mathrm{CW}$-complex we can choose a global trivialisation

$$
\psi:\left.\mathfrak{D}(\overline{\mathcal{A}})\right|_{X \times[-n, n]} \rightarrow X \times[-n, n] \times H^{\prime}
$$

which we can assume to be fibrewise a unitary operator by lemma 11.3.6. Hence

$$
\psi(B(r))=X \times[-n, n] \times B,
$$

where $B \subset H^{\prime}$ denotes the closed unit ball in $H^{\prime}$. Moreover, $\bar{C} \circ\left\llcorner\circ \psi^{-1}: X \times[-n, n] \times H^{\prime} \rightarrow H\right.$ is compact by lemma 11.2 .4 and so

$$
f^{\prime}=\overline{\mathcal{A}} \circ \psi^{-1}+\bar{C} \circ \iota \circ \psi^{-1}: X \times[-n, n] \times H^{\prime} \rightarrow H
$$


is the sum of a continuous family of Fredholm operators of index 0 and a compact map such that $0 \notin f^{\prime}(X \times[-n, n] \times \partial B)$. Hence, according to theorem 11.3.3. for any sufficiently small $\varepsilon>0$ there exists a compact map

$$
\bar{C}^{\prime}: X \times[-n, n] \times B \rightarrow H
$$

such that $\left\|\bar{C}\left(\left(\iota \circ \psi^{-1}\right)(x, s, u)\right)-\bar{C}^{\prime}(x, s, u)\right\|<\varepsilon$ for all $(x, s, u) \in X \times[-n, n] \times B, 0 \notin$ $\left(\overline{\mathcal{A}} \circ \psi^{-1}+\bar{C}^{\prime}\right)(X \times[-n, n] \times \partial B)$ and $\bar{C}^{\prime}(X \times[-n, n] \times B)$ is contained in a finite dimensional subspace $W$ of $H$.

Accordingly we can find for any such $\varepsilon>0$ a continuous map

$$
\check{C}:\left.B(r)\right|_{X \times[-n, n]} \rightarrow X \times[-n, n] \times H
$$

and a finite dimensional subspace $W$ of $H$ such that $\check{C}\left(\left.B(r)\right|_{X \times[-n, n]}\right) \subset W$ and

$$
\left\|\bar{C}_{z}(u)-\check{C}_{z}(u)\right\|<\varepsilon,\left.\quad(z, u) \in B(r)\right|_{X \times[-n, n]} .
$$

Moreover, if we set

$$
\tilde{f}:\left.B(r)\right|_{\bar{V}} \rightarrow \bar{V} \times H, \quad \tilde{f}_{z}(u)=\overline{\mathcal{A}}_{z} u+\check{C}_{z}(u),
$$

then we obtain $\tilde{f}_{z}\left(\partial B_{z, r}(0)\right) \neq 0$ for all $z \in \bar{V}$.

In connection with our assumption (A) we fixed a constant $0<r<\min \left\{\frac{r_{0}}{\tilde{c}}, \frac{1}{M \tilde{c}^{2}}\right\}$. We now choose a sufficiently small $\varepsilon_{0}>0$ and a corresponding map $\breve{C}$ as above such that

$$
r \in\left[\frac{1}{2 M \tilde{c}^{2}}-\sqrt{\frac{1}{4 M^{2} \tilde{c}^{4}}-\frac{\varepsilon_{0}}{M \tilde{c}^{2}}}, \frac{1}{2 M \tilde{c}^{2}}+\sqrt{\frac{1}{4 M^{2} \tilde{c}^{4}}-\frac{\varepsilon_{0}}{M \tilde{c}^{2}}}\right]
$$

which just means that $r$ is in the interval 11.11) if and only if $\varepsilon_{0}+M \tilde{c}^{2} r^{2}<r$.

\section{Step 3: Sharpening the Sword of Topology}

We consider the image of s-ind $(\mathcal{A})$ under the isomorphism $\varphi: K(X \backslash Y \times \mathbb{R}) \rightarrow \tilde{K}\left((X \backslash Y \times \mathbb{R})^{+}\right)$ as defined in lemma B.2.1 which can be obtained as follows:

We can assume that the subspace $W \subset H$ in which the image of $\check{C}$ is contained is transversal to $\operatorname{im} \overline{\mathcal{A}}$ over $\bar{V}$. We denote by $E(\overline{\mathcal{A}}, \Theta(W))$ as before the total space of the bundle appearing in the definition of the index bundle 5.1.10. Now we take the relatively compact open neighbourhood $V$ of the singular set $\Sigma(\overline{\mathcal{A}}) \subset X \backslash Y \times \mathbb{R}$ and use $\overline{\mathcal{A}}$ in order to perform the clutching construction 
of $E(\overline{\mathcal{A}}, \Theta(W))$ restricted to $\bar{V}$ and $\left((X \backslash Y \times \mathbb{R})^{+} \backslash V\right) \times W$ along $\partial V$. In the following we denote the obtained bundle over $(X \backslash Y \times \mathbb{R})^{+}$throughout by $E$. So

$$
\varphi(\operatorname{s-ind}(\mathcal{A}))=[E]-[\Theta(W)] \in \tilde{K}\left((X \backslash Y \times \mathbb{R})^{+}\right) .
$$

Our next aim is to equip the bundle $E$ with a metric such that we can work with the associated sphere bundle below.

\subsubsection{Lemma. The map}

$$
g_{E}: E \rightarrow \mathbb{R}, \quad g_{z}(u, v)=\left\{\begin{array}{l}
\langle u, v\rangle_{H}, \quad z \in(X \backslash Y \times \mathbb{R})^{+} \backslash V, u, v \in W \\
\left\langle\overline{\mathcal{A}}_{z} u, \overline{\mathcal{A}}_{z} v\right\rangle_{H}+\rho(z)\langle u, v\rangle_{H}, z \in \bar{V}, u, v \in E(\overline{\mathcal{A}}, \Theta(W))_{z}
\end{array}\right.
$$

defines a bundle metric on $E$, where $\rho$ is the function defined by lemma 11.3.9.

Proof. Note at first that $g_{z}$ is indeed a scalar product in each fibre because on $V$ it is just the restriction of the metric $g$ we considered already in lemma 11.3.10

Moreover, on $\partial V$ both definitions coincide according to the identifications made in the clutching construction and hence $g$ is well defined and continuous by the universal property of the quotient topology.

\section{Step 4: (A) implies $J([E])=0$}

By the results of the third step, $\varphi(\operatorname{sind}(\mathcal{A}))$ is given by $[E]-[\Theta(W)] \in \tilde{K}\left((X \backslash Y \times \mathbb{R})^{+}\right)$and the bundle $E$ admits a bundle metric defined in lemma 11.3.11. Therefore we can define the associated sphere bundle $S(E ; r)$ of radius $r$ over $(X \backslash Y \times \mathbb{R})^{+}$. According to the definitions, the fibre of this bundle over a point $z \in \bar{V}$ is given by the intersection of $\partial B_{z, r}(0)$ and the corresponding fibre $E(\overline{\mathcal{A}}, W)_{z}$ of the index bundle.

Now the map $\tilde{f}$, which we constructed in the second step, yields a well defined continuous map from the restriction of $S(E ; r)$ to $\bar{V}$ into the finite dimensional space $W$ that is nowhere zero.

The next aim is to extend this map to a map $F: S(E ; r) \rightarrow W \backslash\{0\}$ on the whole bundle over $(X \backslash Y \times \mathbb{R})^{+}$. If $z \in \partial V, u \in \partial B_{z, r}(0)$ and $v \in W$ such that $[(z, u)]=[(z, v)] \in S(E ; r)$, then $\overline{\mathcal{A}}_{z} u=v$ and we have to define

$$
F(z, u)=\overline{\mathcal{A}}_{z} u+\tilde{C}_{z}(u)=v+\tilde{C}_{z}\left(\overline{\mathcal{A}}_{z}^{-1} v\right)=F(z, v) .
$$

Now we consider $z \in \partial V$ and $v \in W,\|v\|_{H}=r$. Then $g_{z}\left(\overline{\mathcal{A}}_{z}^{-1} v, \overline{\mathcal{A}}_{z}^{-1} v\right)=\|v\|_{H}^{2}=r^{2}$ and hence $\overline{\mathcal{A}}_{z}^{-1} v$ is an element of the fibre over $z$ of the disc bundle $B(r)$. We obtain from 11.10

$$
\left\|\check{C}_{z}\left(\overline{\mathcal{A}}_{z}^{-1} v\right)\right\|_{H}<\varepsilon_{0}+\left\|\bar{C}_{z}\left(\overline{\mathcal{A}}_{z}^{-1} v\right)\right\|_{H}
$$


Moreover, since $z \in \partial V$ we have by 11.9 .

$$
\left\|\overline{\mathcal{A}}_{z}^{-1} v\right\|_{\tilde{H}} \leq\left\|\overline{\mathcal{A}}_{z}^{-1}\right\|_{\mathcal{L}(H, \tilde{H})}\|v\|_{H} \leq \tilde{c} r
$$

and so we infer $\left\|\overline{\mathcal{A}}_{z}^{-1} v\right\|_{\tilde{H}} \leq r_{0}$ since we assumed $0<r<\min \left\{\frac{r_{0}}{\tilde{c}}, \frac{1}{M \tilde{c}^{2}}\right\}$ in the assumption (A). Hence we can use the assumption i) from our theorem and get

$$
\varepsilon_{0}+\left\|\bar{C}_{z}\left(\overline{\mathcal{A}}_{z}^{-1} v\right)\right\|_{H} \leq \varepsilon_{0}+M\left\|\overline{\mathcal{A}}_{z}^{-1} v\right\|_{\tilde{H}}^{2} .
$$

Finally, using 11.12 once again and the choice of $r$ in 11.11 we obtain

$$
\varepsilon_{0}+M\left\|\overline{\mathcal{A}}_{z}^{-1} v\right\|_{\tilde{H}}^{2} \leq \varepsilon_{0}+M \tilde{c}^{2} r^{2}<r=\|v\|_{H} .
$$

Summing up, we have found that

$$
\left\|\check{C}_{z}\left(\overline{\mathcal{A}}_{z}^{-1} v\right)\right\|_{H}<\|v\|_{H} \quad \text { for all } z \in \partial V, v \in W,\|v\|_{H}=r
$$

By assumption iii) of the theorem there exists a homeomorphism

$$
\phi:(X \backslash Y \times \mathbb{R})^{+} \backslash V \rightarrow B^{l},
$$

where $B^{l}$ denotes a closed Euclidean unit ball around 0 in $\mathbb{R}^{l}$. Let $\sigma:[0,1] \rightarrow[0,1]$ be any continuous function such that $\sigma(1)=1$ and $\sigma\left(\left[0, \frac{1}{2}\right]\right)=0$ and, moreover, $r: B \backslash\{0\} \rightarrow \partial B$ the radial retraction. We define

$$
F(z, v)=v+\sigma\left(\|\phi(z)\|_{\mathbb{R}^{l}}\right) \check{C}_{\phi^{-1}(r(\phi(z)))}\left(\overline{\mathcal{A}}_{\phi^{-1}(r(\phi(z)))}^{-1} v\right), \quad z \in(X \backslash Y \times \mathbb{R})^{+} \backslash V, v \in W,\|v\|=r .
$$

This is a continuous extension of $\left.F\right|_{\bar{V}}$ to all of $(X \backslash Y \times \mathbb{R})^{+}$which is the identity over an open subset of $(X \backslash Y \times \mathbb{R})^{+}$. Moreover, from 11.13 and $\sigma([0,1]) \subset[0,1]$ we obtain that $F(S(E ; r)) \subset W \backslash\{0\}$.

Finally, we define a map

$$
\tilde{F}: S(E ; r) \rightarrow S(\Theta(W) ; r), \quad \tilde{F}(z, u)=\left(z, r \frac{F(z, u)}{\|F(z, u)\|}\right)
$$

whose restriction to some fibre over $(X \backslash Y \times \mathbb{R})^{+} \backslash V$ is the identity and therefore in particular a homotopy equivalence. Now we want to cite the following fundamental theorem due to Albrecht Dold [Do80] ]

\footnotetext{
${ }^{1}$ Dold's theorem is also the main ingredient in the proof of theorem 11.3 .1 which we have omitted above.
} 
11.3.12 Theorem. Let $\left\{B_{1}, p_{1}, X, Y_{1}, G_{1}\right\}$ and $\left\{B_{2}, p_{2}, X, Y_{2}, G_{2}\right\}$ be two fibre bundles over the connected $C W$-complex $X$, where the typical fibres $Y_{1}, Y_{2}$ are assumed to be locally compact. If there exists a bundle map $h: B_{1} \rightarrow B_{2}$ such that $h_{x}: B_{1, x} \rightarrow B_{2, x}$ is a homotopy equivalence for some $x \in X$, then $h$ is a fibrewise homotopy equivalence.

A bundle map $h: B_{1} \rightarrow B_{2}$ is by definition a fibrewise homotopy equivalence if there exists a bundle map $g: B_{2} \rightarrow B_{1}$ such that $h \circ g$ and $g \circ h$ are homotopic to the respective identities through fibre preserving maps.

Now Dold's theorem 11.3 .12 implies that the map $\tilde{F}: S(E ; r) \rightarrow S(\Theta(W) ; r)$ is a fibrewise homotopy equivalence. We obtain $J([E])=0 \in J\left((X \backslash Y \times \mathbb{R})^{+}\right)$.

\section{Step 5: $\neg($ A) yields bifurcation}

In our theorem it is required that $J([E]) \neq 0$. Thus, by the results of the fourth step, assumption (A) can not hold in this case. Therefore we obtain:

For every $0<r<\min \left\{\frac{r_{0}}{\tilde{c}}, \frac{1}{M \tilde{c}^{2}}\right\}$ there exists $z=(x, s) \in X \times[-n, n]$ and an element $u \in \partial B_{z, r}(0)$ such that $\bar{f}_{z}(u)=0$.

Thus we can find a sequence $\left\{\left(z_{n}, u_{n}\right)\right\}_{n \in \mathbb{N}} \subset X \times[-n, n] \times H$ such that $u_{n} \in \partial B_{z_{n}, \frac{1}{n}}(0)$ and $\bar{f}_{z_{n}}\left(u_{n}\right)=0$ for all $n \in \mathbb{N}$. Since $X \times[-n, n]$ is sequentially compact, we can find a subsequence of $z_{n}=\left(x_{n}, s_{n}\right)$ which converges to an element $\left(x^{*}, s^{*}\right) \in X \times[-n, n]$. For the sake of simplicity we do not change the notation and denote the subsequence by $\left\{\left(x_{n}, s_{n}\right)\right\}$ as well.

Now we use once again the trivialisation $\psi:\left.\mathfrak{D}(\overline{\mathcal{A}})\right|_{X \times[-n, n]} \rightarrow X \times[-n, n] \times H^{\prime}$ which is unitary in each fibre. From $u_{n} \in \partial B_{z_{n}, \frac{1}{n}}(0), n \in \mathbb{N}$, we infer $\psi_{z_{n}}\left(u_{n}\right) \rightarrow 0$ in $H^{\prime}$ and since $\iota \circ \psi^{-1}: X \times[-n, n] \times H^{\prime} \rightarrow \tilde{H}$ is continuous by condition (C), we obtain that $u_{n} \rightarrow 0$ in $\tilde{H}$. Hence $\left(x^{*}, s^{*}\right)$ is a bifurcation point for the family $\bar{f}$ restricted to $X \times[-n, n]$ which we defined in 11.8 .

We will now finish the proof by showing that any bifurcation point of $\bar{f}$ is already a bifurcation point of $f$ under the assumptions of our theorem. The first part of our argument shows that any bifurcation point of $\bar{f}$ belongs to the singular set of $\mathcal{A}$ and hence in particular proves a further assertion from our theorem.

Assume that $z^{*}=\left(x^{*}, s^{*}\right)$ is such that $\overline{\mathcal{A}}_{z^{*}}$ belongs to $G \mathcal{C}(H)$. Then, since $G \mathcal{C}(H)$ is open in $\mathcal{C}(H)$ according to corollary 2.3.5 and $X$ is compact, there exists a compact neighbourhood $K \subset X \times[-n, n]$ of $z^{*}$ such that $\overline{\mathcal{A}}_{z} \in G \mathcal{C}(H)$ for all $z \in K$. By the assumed convergence of $\left\{z_{n}\right\}_{n \in \mathbb{N}}$ to $z^{*}$ we can find an $n_{0} \in \mathbb{N}$ such that $z_{n} \in K$ for all $n \geq n_{0}$. Moreover, we use lemma 11.3 .7 once again in order to choose a constant $c_{K}>0$ such that $\left\|\overline{\mathcal{A}}_{z}^{-1}\right\|_{\mathcal{L}(H, \tilde{H})} \leq c_{K}$ for all $z \in K$. Finally, since $u_{n} \rightarrow 0$ in $\tilde{H}$ we can assume $n_{0}$ to be sufficiently large such that $\left\|u_{n}\right\|_{\tilde{H}}<r_{0}$ for all $n \geq n_{0}$.

Now from $\bar{f}_{z_{n}}\left(u_{n}\right)=\overline{\mathcal{A}}_{z_{n}} u_{n}+\bar{C}_{z_{n}}\left(u_{n}\right)=0, n \in \mathbb{N}$ and assumption i) of our theorem, we obtain for all $n \geq n_{0}$ 


$$
\left\|u_{n}\right\|_{\tilde{H}}=\left\|\overline{\mathcal{A}}_{z_{n}}^{-1} \bar{C}_{z_{n}}\left(u_{n}\right)\right\|_{\tilde{H}} \leq c_{K}\left\|\bar{C}_{z_{n}}\left(u_{n}\right)\right\|_{H} \leq c_{K} M\left\|u_{n}\right\|_{\tilde{H}}^{2}
$$

in contradiction to $u_{n} \rightarrow 0$ in $\tilde{H}$.

We infer that $\overline{\mathcal{A}}_{z^{*}} \notin G \mathcal{C}(H)$ and hence, because of the selfadjointness of $\mathcal{A}$, that $s^{*}=0$ and $x^{*}$ belongs to the singular set of $\mathcal{A}$.

So far the found sequence fulfils $\mathcal{A}_{x_{n}}+C_{x_{n}}\left(u_{n}\right)=-i s_{n} u_{n}$ for all $n \in \mathbb{N}$ and to finish the proof we just have to ensure that $s_{n}=0$ for infinitely many of the $z_{n}=\left(x_{n}, s_{n}\right)$. But we know that $u_{n} \neq 0$ for all $n \in \mathbb{N}$ and $\left\|u_{n}\right\|_{\tilde{H}}, s_{n} \rightarrow 0, n \rightarrow \infty$. Thus there exists $n_{2} \in \mathbb{N}$ such that $s_{n} \in(-\varepsilon, \varepsilon)$ and $u_{n} \in \tilde{B}_{r_{1}}(0) \cap \mathcal{D}\left(\mathcal{A}_{z_{n}}\right)$ for all $n \geq n_{2}$, where $\varepsilon$ and $r_{1}$ are from assumption ii) of our theorem. Accordingly, $s_{n}=0$ for all $n \geq n_{2}$ and therefore $x^{*} \in X$ is indeed a bifurcation point of the family $f$. 


\section{Chapter 12}

\section{Examples of the Bifurcation Theorems}

In this chapter we consider two examples of our bifurcation theorems from the foregoing chapter. The first one treats a family of boundary value problems of nonlinear first order ordinary differential operators which are parametrised by maps in a Grassmannian (cf. [Pe88, §4]). We compute the index bundle of the corresponding linear part and obtain the existence of bifurcation points by using theorem 11.2.5. In the second example we consider nonlinear perturbations of a Floer family of elliptic boundary value problems and use our index theorem 8.1.1 and the bifurcation theorem 11.2.6 in order to conclude the existence of bifurcation points.

\subsection{Example I: A First Order Family Parametrised by a Grassmannian}

Let $X$ be a compact connected CW-complex and $b: X \rightarrow G_{n}\left(\mathbb{C}^{2 n}\right)$ a continuous map in the complex Grassmannian for some $n \in \mathbb{N}$. We consider the family of boundary value problems

$$
\left\{\begin{array}{l}
u^{\prime}+\varphi_{x}(u)=0 \\
(u(0), u(1)) \in b(x),
\end{array}\right.
$$

where $\varphi: X \times \mathbb{C}^{n} \rightarrow \mathbb{C}^{n}$ is a continuous function such that $\varphi_{x}(0)=0, \varphi_{x}: \mathbb{C}^{n} \rightarrow \mathbb{C}^{n}$ is real continuously differentiable, $x \in X$, and there exists some $M>0$ such that $\left\|\varphi_{x}^{\prime}(y)\right\|<M$ for all $(x, y) \in X \times \mathbb{C}^{n}$. We now restate the family of differential equations by defining

$$
\mathcal{A}_{x}: \mathcal{D}\left(\mathcal{A}_{x}\right)=\left\{u \in H^{1}\left(I, \mathbb{C}^{n}\right):(u(0), u(1)) \in b(x)\right\} \subset L^{2}\left(I, \mathbb{C}^{n}\right) \rightarrow L^{2}\left(I, \mathbb{C}^{n}\right), \quad \mathcal{A}_{x} u=u^{\prime}
$$


and

$$
C: X \times H^{1}\left(I, \mathbb{C}^{n}\right) \rightarrow L^{2}\left(I, \mathbb{C}^{n}\right), \quad C(x, u)=\varphi_{x}(u) .
$$

Then solutions of the boundary value problems correspond to zeroes of the nonlinear maps

$$
f_{x}=\mathcal{A}_{x}+C_{x}
$$

and the aim of this section is to prove the following proposition.

12.1.1 Proposition. Let $\bar{c}\left(\gamma^{n}\right)$ denote the total Chern class of the tautological bundle $\gamma^{n}$ over $G_{n}\left(\mathbb{C}^{2 n}\right)$. If $\kappa\left(b^{*} \bar{c}\left(\gamma^{n}\right)\right) \neq 0 \in H^{*}\left(X ; \mathbb{Z}_{2}\right)$ and there exists some $x_{0} \in X$ such that $b_{x_{0}}=\{0\} \times \mathbb{C}^{n}$, then there exists a bifurcation point of 12.1.

In order to prove the proposition we have to check the assumptions of corollary 11.2.8. We consider at first the nonlinearity $C: X \times H^{1}\left(I, \mathbb{C}^{n}\right) \rightarrow L^{2}\left(I, \mathbb{C}^{n}\right)$ and begin by proving its continuity. Note that by our assumptions on the function $\varphi$, there exists a constant $M>0$ such that

$$
\begin{aligned}
\left\|\varphi_{x}(y)-\varphi_{x_{0}}\left(y_{0}\right)\right\| & \leq\left\|\varphi_{x}(y)-\varphi_{x}\left(y_{0}\right)\right\|+\left\|\varphi_{x}\left(y_{0}\right)-\varphi_{x_{0}}\left(y_{0}\right)\right\| \\
& \leq M\left\|y-y_{0}\right\|+\left\|\varphi_{x}\left(y_{0}\right)-\varphi_{x_{0}}\left(y_{0}\right)\right\|
\end{aligned}
$$

for all $(x, y),\left(x_{0}, y_{0}\right) \in X \times \mathbb{C}^{n}$. Hence, if we fix some $\left(x_{0}, u_{0}\right) \in X \times H^{1}\left(I, \mathbb{C}^{n}\right)$ we obtain

$$
\begin{aligned}
\left\|C(x, u)-C\left(x_{0}, u_{0}\right)\right\|_{L^{2}\left(I, \mathbb{C}^{n}\right)}^{2} & =\int_{0}^{1}\left\|\varphi_{x}(u(t))-\varphi_{x_{0}}\left(u_{0}(t)\right)\right\|^{2} d t \\
& \leq 2 M^{2} \int_{0}^{1}\left\|u(t)-u_{0}(t)\right\|^{2} d t+2 \int_{0}^{1}\left\|\varphi_{x}\left(u_{0}(t)\right)-\varphi_{x_{0}}\left(u_{0}(t)\right)\right\|^{2} d t \\
& =2 M^{2}\left\|u-u_{0}\right\|_{L^{2}\left(I, \mathbb{C}^{n}\right)}^{2}+2 \int_{0}^{1}\left\|\varphi_{x}\left(u_{0}(t)\right)-\varphi_{x_{0}}\left(u_{0}(t)\right)\right\|^{2} d t \\
& \leq 2 M^{2}\left\|u-u_{0}\right\|_{H^{1}\left(I, \mathbb{C}^{n}\right)}^{2}+\mathcal{I}(x),
\end{aligned}
$$

where $\mathcal{I}: X \rightarrow \mathbb{R}$ is a continuous function vanishing at $x_{0}$. This shows that $C$ is continuous at $\left(x_{0}, u_{0}\right)$.

Next we note that $\varphi_{x}(u) \in H^{1}\left(I, \mathbb{C}^{n}\right)$ for any $u \in H^{1}\left(I, \mathbb{C}^{n}\right)$ and $\left(\varphi_{x}(u)\right)^{\prime}=\varphi_{x}^{\prime}(u) u^{\prime}$ by [Dob06, 5.19], $x \in X$. So $C\left(X \times H^{1}\left(I, \mathbb{C}^{n}\right)\right) \subset H^{1}\left(I, \mathbb{C}^{n}\right)$ and since 


$$
\begin{aligned}
\|C(x, u)\|_{H^{1}\left(I, \mathbb{C}^{n}\right)}^{2} & =\int_{0}^{1}\left\|\varphi_{x}(u(t))\right\|^{2} d t+\int_{0}^{1}\left\|\frac{d}{d t} \varphi_{x}(u(t))\right\|^{2} d t \\
& =\int_{0}^{1}\left\|\varphi_{x}(u(t))\right\|^{2} d t+\int_{0}^{1}\left\|\varphi_{x}^{\prime}(u(t)) u^{\prime}(t)\right\|^{2} d t \\
& \leq M^{2} \int_{0}^{1}\|u(t)\|^{2} d t+\int_{0}^{1}\left\|\varphi_{x}^{\prime}(u(t))\right\|^{2}\left\|u^{\prime}(t)\right\|^{2} d t \\
& \leq M^{2} \int_{0}^{1}\|u(t)\|^{2} d t+M^{2} \int_{0}^{1}\left\|u^{\prime}(t)\right\|^{2} d t \\
& =M^{2}\|u\|_{H^{1}\left(I, \mathbb{C}^{n}\right)}^{2}
\end{aligned}
$$

we infer that $C(X \times B) \subset H^{1}\left(I, \mathbb{C}^{n}\right)$ is bounded for every bounded subset $B \subset H^{1}\left(I, \mathbb{C}^{n}\right)$. Hence $C: X \times H^{1}\left(I, \mathbb{C}^{n}\right) \rightarrow L^{2}\left(I, \mathbb{C}^{n}\right)$ is compact because of the compactness of the inclusion $H^{1}\left(I, \mathbb{C}^{n}\right) \hookrightarrow L^{2}\left(I, \mathbb{C}^{n}\right)$.

Finally we show that each $C_{x}$ is real continuously differentiable at 0 . To do so we decompose $C_{x}$ as

$$
H^{1}\left(I, \mathbb{C}^{n}\right) \hookrightarrow C\left(I, \mathbb{C}^{n}\right) \stackrel{C_{x}}{\longrightarrow} C\left(I, \mathbb{C}^{n}\right) \hookrightarrow L^{2}\left(I, \mathbb{C}^{n}\right)
$$

and since the canonical inclusions are continuous, it is enough to show that $C_{x}$ is real continuously differentiable when regarded as a self-map of $C\left(I, \mathbb{C}^{n}\right)$. We find for $u, v \in C\left(I, \mathbb{C}^{n}\right)$

$$
\begin{aligned}
\left\|\varphi_{x}(u+v)-\varphi_{x}(u)-\varphi_{x}^{\prime}(u) v\right\|_{\infty} & =\sup _{t \in I}\left\|\varphi_{x}(u(t)+v(t))-\varphi_{x}(u(t))-\varphi_{x}^{\prime}(u(t)) v(t)\right\| \\
& =\sup _{t \in I}\left\|\int_{0}^{1} \frac{d}{d \lambda}\left(\varphi_{x}(u(t)+\lambda v(t))\right)-\varphi_{x}^{\prime}(u(t)) v(t) d \lambda\right\| \\
& =\sup _{t \in I}\left\|\int_{0}^{1} \varphi_{x}^{\prime}(u(t)+\lambda v(t)) v(t)-\varphi_{x}^{\prime}(u(t)) v(t) d \lambda\right\| \\
& \left.\leq \sup _{t \in I}\|v(t)\| \int_{0}^{1}\left\|\varphi_{x}^{\prime}(u(t)+\lambda v(t))-\varphi_{x}^{\prime}(u(t))\right\| d \lambda\right) \\
& \leq\|v\|_{\infty} \sup _{t \in I} \int_{0}^{1}\left\|\varphi_{x}^{\prime}(u(t)+\lambda v(t))-\varphi_{x}^{\prime}(u(t))\right\| d \lambda .
\end{aligned}
$$

Since the latter integral term tends to zero if $v$ tends to zero uniformly, we obtain that $C_{x}: C\left(I, \mathbb{C}^{n}\right) \rightarrow C\left(I, \mathbb{C}^{n}\right)$ is real differentiable on $C\left(I, \mathbb{C}^{n}\right)$ and its derivative is given by the continuous map

$$
D C_{x}: C\left(I, \mathbb{C}^{n}\right) \rightarrow \mathcal{L}\left(C\left(I, \mathbb{C}^{n}\right)^{\mathbb{R}}\right), \quad\left(D_{u} C_{x}\right) v=\varphi_{x}^{\prime}(u) v .
$$

Hence we have shown all assumptions on the map $C: H^{1}\left(I, \mathbb{C}^{n}\right) \rightarrow L^{2}\left(I, \mathbb{C}^{n}\right)$ that we need in order to obtain proposition 12.1 .1 . 
We now consider the family of linear operators $\mathcal{A}$ and note at first that it can be shown similarly as in the proof of theorem 8.1.1 that $\mathcal{A}$ is a gap continuous family of Fredholm operators of index 0. Moreover, we infer from 8.1.1 that $\mathfrak{D}(\mathcal{A})$ is a subbundle of $X \times H^{1}\left(I, \mathbb{C}^{n}\right)$ and so in particular condition $(\mathrm{C})$ from our bifurcation theorem is satisfied. Since the arguments are indeed very close to each other, we do not want to repeat them here.

As next step we compute the index bundle of $\mathcal{A}$. Again we refer to the proof of theorem 8.1.1 in order to note that the subspace of constant functions in $L^{2}\left(I, \mathbb{C}^{n}\right)$ is transversal to the image of $\mathcal{A}$ over $X$. Then we see that the total space of the index bundle of $\mathcal{A}$ is given by

$$
\left\{(x, u) \in X \times L^{2}\left(I, \mathbb{C}^{n}\right): u(t)=(1-t) v+t w, t \in I,(v, w) \in b(x)\right\}
$$

and so

$$
\text { ind } \mathcal{A}=b^{*}\left(\gamma^{n}-\Theta\left(\mathbb{C}^{n}\right)\right) \in K(X),
$$

where $\gamma^{n}$ denotes the tautological bundle over $G_{n}\left(\mathbb{C}^{2 n}\right)$. Hence the total Chern class of the index bundle is given by

$$
\bar{c}(\text { ind } \mathcal{A})=\bar{c}\left(b^{*}\left(\gamma^{n}-\Theta\left(\mathbb{C}^{n}\right)\right)\right)=b^{*} \bar{c}\left(\gamma^{n}\right) \in H^{*}(X ; \mathbb{Z}) .
$$

Finally, we note that

$$
\mathcal{A}_{x_{0}} u+\left(\left(D_{0} C_{x_{0}}\right) \circ \iota_{x_{0}}\right) u=u^{\prime}+\varphi_{x_{0}}^{\prime}(0) u
$$

maps $\mathcal{D}\left(\mathcal{A}_{x_{0}}\right)^{\mathbb{R}}=\left\{u \in H^{1}\left(I, \mathbb{C}^{n}\right)^{\mathbb{R}}: u(0)=0\right\}$ bijectively to $L^{2}\left(I, \mathbb{C}^{n}\right)^{\mathbb{R}}$ by well known existence and uniqueness results for initial value problems of linear first order systems. Hence $\mathcal{A}_{x_{0}}+\left(D_{0} C_{x_{0}}\right) \circ \iota_{x_{0}} \in G L\left(\mathfrak{D}(\mathcal{A})_{x_{0}}^{\mathbb{R}}, L^{2}\left(I, \mathbb{C}^{n}\right)^{\mathbb{R}}\right)$ by the open mapping theorem A.2.5 and now proposition 12.1.1 follows from corollary 11.2 .8 .

We conclude this section with a concrete example in the case $n=1$. We set $X=S^{2}=\mathbb{C} P^{1}$ and assume that $b: \mathbb{C} P^{1} \rightarrow G_{1}\left(\mathbb{C}^{2}\right)=\mathbb{C} P^{1}$ is the identity. Then $b^{*} \gamma^{1}=\gamma^{1}$ is the tautological line bundle over $\mathbb{C} P^{1}$ and, moreover, $c_{1}\left(\gamma^{1}\right)$ is a generator of the infinite cyclic group $H^{2}\left(\mathbb{C} P^{1} ; \mathbb{Z}\right)$ by [MS74, 14.4]. Now we consider the coefficient homomorphism $\kappa: H^{2}\left(\mathbb{C} P^{1} ; \mathbb{Z}\right) \rightarrow H^{2}\left(\mathbb{C} P^{1} ; \mathbb{Z}_{2}\right)$ which is part of the Bockstein sequence associated to

$$
0 \rightarrow \mathbb{Z} \stackrel{2 \cdot i d}{\longrightarrow} \mathbb{Z} \rightarrow \mathbb{Z}_{2} \rightarrow 0
$$

and which reads as 
$0=H^{1}\left(\mathbb{C} P^{1} ; \mathbb{Z}_{2}\right) \rightarrow H^{2}\left(\mathbb{C} P^{1} ; \mathbb{Z}\right) \stackrel{2 \cdot i d_{H^{2}\left(\mathbb{C} P^{1} ; \mathbb{Z}\right)}}{\longrightarrow} H^{2}\left(\mathbb{C} P^{1} ; \mathbb{Z}\right) \stackrel{\kappa}{\longrightarrow} H^{2}\left(\mathbb{C} P^{1} ; \mathbb{Z}_{2}\right) \rightarrow H^{3}\left(\mathbb{C} P^{1} ; \mathbb{Z}\right)=0$

Accordingly $\kappa: H^{2}\left(\mathbb{C} P^{1} ; \mathbb{Z}\right) \cong \mathbb{Z} \rightarrow H^{2}\left(\mathbb{C} P^{1} ; \mathbb{Z}_{2}\right) \cong \mathbb{Z}_{2}$ is the reduction modulo 2 and so in particular not trivial. Since $c_{1}\left(\gamma^{1}\right)$ is a generator of $H^{2}\left(\mathbb{C} P^{1} ; \mathbb{Z}\right)$, we infer that $\kappa\left(c_{1}\left(\gamma^{1}\right)\right) \neq 0 \in$ $H^{2}\left(\mathbb{C} P^{1} ; \mathbb{Z}_{2}\right)$. Hence all assumptions of proposition 12.1 .1 hold and we deduce the existence of a bifurcation point of the family of equations 12.1) for all functions $\varphi: S^{2} \times \mathbb{C} \rightarrow \mathbb{C}$ such that $\varphi_{x}(0)=0, \varphi_{x} \in C^{1}\left(\mathbb{R}^{2}, \mathbb{R}^{2}\right)$ and $\left\|\varphi_{x}^{\prime}(y)\right\|<M,(x, y) \in S^{2} \times \mathbb{C}$ for some $M>0$.

Finally, we want to mention that the foregoing example can easily be generalised to maps $b$ : $\mathbb{C} P^{k} \rightarrow G_{n}\left(\mathbb{C}^{2 n}\right)$ such that $b^{*} \gamma^{n}$ is isomorphic to the sum of the tautological line bundle over $\mathbb{C} P^{k}$ and $\Theta\left(\mathbb{C}^{n-1}\right)$. By the same argument as above one obtains that $\kappa\left(c_{1}\left(b^{*} \gamma^{n}\right)\right) \in H^{2}\left(\mathbb{C P}^{k} ; \mathbb{Z}_{2}\right)$ is non trivial. Moreover, such maps always exist by [Hu94, III.5.5] and one can see from their construction that they can be chosen such that $b\left(x_{0}\right)=\{0\} \times \mathbb{C}^{n} \in G_{n}\left(\mathbb{C}^{2 n}\right)$ for some $x_{0} \in \mathbb{C} P^{k}$.

\subsection{Example II: A Nonlinear Floer Family of Elliptic Bound- ary Value Problems}

Let $X$ be a compact topological space and $R: X \rightarrow S p(2, \mathbb{R})$ be a continuous family of symplectic matrices. We consider as in [Ni07] the Floer family defined by

$$
\mathcal{A}_{x}: \mathcal{D}\left(\mathcal{A}_{x}\right) \subset L^{2}\left(I, \mathbb{R}^{2}\right) \rightarrow L^{2}\left(I, \mathbb{R}^{2}\right), \quad \mathcal{A}_{x}=\sigma \frac{d u}{d t}+a(x, \cdot) u,
$$

where

$$
\sigma=\left(\begin{array}{cc}
0 & -1 \\
1 & 0
\end{array}\right),
$$

the domains are given by

$$
\mathcal{D}\left(\mathcal{A}_{x}\right)=\left\{u \in H^{1}\left(I, \mathbb{R}^{2}\right): u(0) \in\{0\} \times \mathbb{R}, u(1) \in R(x)(\{0\} \times \mathbb{R})\right\}
$$

and

$$
a: X \times I \rightarrow \mathbb{R}
$$

is a continuous function.

12.2.1 Lemma. The operators $\mathcal{A}_{x}, x \in X$, are selfadjoint on $L^{2}\left(I, \mathbb{R}^{2}\right)$. 
Proof. Since the multiplication by the continuous function $a_{x}: I \rightarrow \mathbb{R}$ is a bounded selfadjoint operator on $L^{2}\left(I, \mathbb{R}^{2}\right)$, we can use the Rellich perturbation theorem (cf. Ka76, Th. V.4.3]) in order to conclude that it suffices to show the selfadjointness of $u \mapsto \sigma u^{\prime}$ on $\mathcal{D}\left(\mathcal{A}_{x}\right)$. But this is a well known result which can be proved for example as in [We05, Bsp. VII.2 e)].

Our first aim is to compute s-ind $\left(\mathcal{A}^{\mathbb{C}}\right) \in K^{-1}(X, Y)$ explicitly if $X$ is a compact space and $Y \subset X$ a closed subspace having trivial intersection with the singular set of $\mathcal{A}$. Here we want to make use of the results we already obtained in theorem 8.1.1.

At first we note that

$$
\overline{\mathcal{A}}_{z}: \mathcal{D}\left(\overline{\mathcal{A}}_{z}\right) \rightarrow L^{2}\left(I, \mathbb{C}^{2}\right)
$$

is given by

$$
\overline{\mathcal{A}}_{z} u=\sigma \frac{d u}{d t}+a(x, \cdot) u+i s u
$$

where

$$
\mathcal{D}\left(\overline{\mathcal{A}}_{z}\right)=\left\{u \in H^{1}\left(I, \mathbb{C}^{2}\right): u(0) \in\{0\} \times \mathbb{C}, u(1) \in R(x)(\{0\} \times \mathbb{C})\right\} .
$$

According to theorem 8.1.1, this family is a bounded perturbation of a gap continuous family and hence itself gap continuous by lemma 6.1.10 Moreover, we obtain from 8.1.1 and lemma 6.1 .10 that $\mathfrak{D}\left(\overline{\mathcal{A}^{\mathbb{C}}}\right)$ is a subbundle of $X \times \mathbb{R} \times H^{1}\left(I, \mathbb{C}^{2}\right)$.

Now we consider the continuous family of invertible operators on $L^{2}\left(I, \mathbb{C}^{2}\right)$

$$
M: X \times \mathbb{R} \rightarrow G L\left(L^{2}\left(I, \mathbb{C}^{2}\right)\right), \quad\left(M_{z} u\right)(t)=e^{\sigma\left(\int_{0}^{t} a(x, \kappa) d \kappa+i s t\right)} u(t)
$$

and define

$$
N_{z}=M_{z}^{-1} \overline{\mathcal{A}}_{z} M_{z}, \quad \mathcal{D}\left(N_{z}\right)=M_{z}^{-1} \mathcal{D}\left(\overline{\mathcal{A}}_{z}\right), \quad z \in X \times \mathbb{R}
$$

We obtain

$$
\mathcal{D}\left(N_{z}\right)=\left\{u \in H^{1}\left(I, \mathbb{C}^{2}\right): u(0) \in\{0\} \times \mathbb{C}, u(1) \in e^{-\sigma\left(\int_{0}^{1} a(x, \kappa) d \kappa+i s\right)} R(x)(\{0\} \times \mathbb{C})\right\}
$$

and 


$$
\begin{aligned}
\left(N_{z} u\right)(t) & =M_{z}^{-1}\left(\sigma \frac{d}{d t}\left(e^{\sigma\left(\int_{0}^{t} a(x, \kappa) d \kappa+i s t\right)} u(t)\right)+(a(x, t)+i s) e^{\sigma\left(\int_{0}^{t} a(x, \kappa) d \kappa+i s t\right)} u(t)\right) \\
& =M_{z}^{-1}\left(\sigma^{2}(a(x, t)+i s) e^{\sigma\left(\int_{0}^{t} a(x, \kappa) d \kappa+i s t\right)} u(t)+\sigma e^{\sigma\left(\int_{0}^{t} a(x, \kappa) d \kappa+i s t\right)} \frac{d u}{d t}\right. \\
& \left.+(a(x, t)+i s) e^{\sigma\left(\int_{0}^{t} a(x, \kappa) d \kappa+i s t\right)} u(t)\right) \\
& =\sigma \frac{d u}{d t}
\end{aligned}
$$

Again we infer by theorem 8.1 .1 that $N$ is gap continuous and that $\mathfrak{D}(N)$ is a subbundle of $X \times \mathbb{R} \times H^{1}\left(I, \mathbb{C}^{2}\right)$.

Hence we can regard $M$ as a bundle isomorphism between $\mathfrak{D}(N)$ and $\mathfrak{D}\left(\overline{\mathcal{A}^{\mathbb{C}}}\right)$ and, moreover, $M^{-1}$ as a bundle automorphism of $X \times \mathbb{R} \times L^{2}\left(I, \mathbb{C}^{2}\right)$. Now, from the properties of the index bundle for morphisms between Banach bundles 5.2.1, 5.2.7 and 5.2.8, we obtain

$$
\begin{aligned}
\operatorname{s-ind}\left(\mathcal{A}^{\mathbb{C}}\right) & =\operatorname{ind}\left(\overline{\mathcal{A}^{\mathbb{C}}}\right)=\operatorname{ind}\left(M^{-1}\right)+\operatorname{ind}\left(\overline{\mathcal{A}^{\mathbb{C}}}\right)+\operatorname{ind}(M) \\
& =\operatorname{ind}\left(M^{-1} \overline{\mathcal{A}^{\mathbb{C}}} M\right)=\operatorname{ind}(N)
\end{aligned}
$$

and, moreover, theorem 8.1 .1 teaches us how to obtain ind $(N)$ explicitly. Accordingly, we have to compute the upper right entry of the matrix

$$
e^{-\sigma\left(\int_{0}^{1} a(x, \kappa) d \kappa+i s\right)} R(x) .
$$

12.2.2 Lemma. For any complex number $a \in \mathbb{C}$ we have

$$
e^{a \sigma}=\cos (a)+\sigma \sin (a)
$$

Proof. From $\sigma^{2}=-I$ we obtain

$$
\sigma^{k}=\left\{\begin{array}{l}
I, k=4 l, l \in \mathbb{N} \cup\{0\} \\
-I, k \text { even, } k \neq 4 l, l \in \mathbb{N} \cup\{0\} \\
-\sigma, k=4 l-1, l \in \mathbb{N} \\
\sigma, k \text { odd }, k \neq 4 l-1, l \in \mathbb{N}
\end{array}\right.
$$

Hence

$$
\begin{aligned}
e^{a \sigma} & =\sum_{k=0}^{\infty} \frac{a^{k} \sigma^{k}}{k !}=\sum_{k=0}^{\infty}(-1)^{k} \frac{a^{2 k}}{(2 k) !}+\sigma \sum_{k=0}^{\infty}(-1)^{k} \frac{a^{2 k+1}}{(2 k+1) !} \\
& =\cos (a)+\sigma \sin (a) .
\end{aligned}
$$


We now have for any $(0, b) \in\{0\} \times \mathbb{C}$

$$
\begin{aligned}
& \left(e^{-\sigma i s} e^{-\sigma \int_{0}^{1} a(x, \kappa) d \kappa} R(x)\right)(0, b) \\
& =(\cos (i s)-\sigma \sin (i s))\left(\cos \left(\int_{0}^{1} a(x, \kappa) d \kappa\right)-\sigma \sin \left(\int_{0}^{1} a(x, \kappa) d \kappa\right)\right)\left(\begin{array}{l}
R_{12}(x) b \\
R_{22}(x) b
\end{array}\right) \\
& =(\cosh (s)-i \sigma \sinh (s))\left(\cos \left(\int_{0}^{1} a(x, \kappa) d \kappa\right)-\sigma \sin \left(\int_{0}^{1} a(x, \kappa) d \kappa\right)\right)\left(\begin{array}{l}
R_{12}(x) b \\
R_{22}(x) b
\end{array}\right) \\
& =\left(\left(\cosh (s) \cos \left(\int_{0}^{1} a(x, \kappa) d \kappa\right)-i \sinh (s) \sin \left(\int_{0}^{1} a(x, \kappa) d \kappa\right)\right)\right. \\
& \left.-\sigma\left(\cosh (s) \sin \left(\int_{0}^{1} a(x, \kappa) d \kappa\right)+i \sinh (s) \cos \left(\int_{0}^{1} a(x, \kappa) d \kappa\right)\right)\right)\left(\begin{array}{l}
R_{12}(x) b \\
R_{22}(x) b
\end{array}\right) .
\end{aligned}
$$

Now the first column of this vector is given by

$$
\begin{aligned}
& \left(\cosh (s) \sin \left(\int_{0}^{1} a(x, \kappa) d \kappa\right)+i \sinh (s) \cos \left(\int_{0}^{1} a(x, \kappa) d \kappa\right)\right) R_{22}(x) b \\
& +\left(\cosh (s) \cos \left(\int_{0}^{1} a(x, \kappa) d \kappa\right)-i \sinh (s) \sin \left(\int_{0}^{1} a(x, \kappa) d \kappa\right)\right) R_{12}(x) b \\
& =\left(\sin \left(\int_{0}^{1} a(x, \kappa) d \kappa+i s\right) R_{22}(x)+\cos \left(\int_{0}^{1} a(x, \kappa) d \kappa+i s\right) R_{12}(x) b,\right.
\end{aligned}
$$

where we use that for any $a, b \in \mathbb{R}$

$$
\begin{gathered}
\sin (a+i b)=\sin (a) \cosh (b)+i \cos (a) \sinh (b) \\
\cos (a+i b)=\cos (a) \cosh (b)-i \sin (a) \sinh (b) .
\end{gathered}
$$

Finally, we obtain from theorem 8.1 .1

$$
\operatorname{s-ind}\left(\mathcal{A}^{\mathbb{C}}\right)=[\Theta(\mathbb{C}), \Theta(\mathbb{C}), G] \in K^{-1}(X, Y)
$$

where $G: X \times \mathbb{R} \rightarrow \mathbb{C}$ is defined by

$$
G(x, s)=\sin \left(\int_{0}^{1} a(x, \kappa) d \kappa+i s\right) R_{22}(x)+\cos \left(\int_{0}^{1} a(x, \kappa) d \kappa+i s\right) R_{12}(x) .
$$

We now restrict to the special case that $\int_{0}^{1} a(x, \kappa) d \kappa=0$ for all $x \in X$. Note that this assumption is not very unnatural because $\left\{g \in C(I, \mathbb{R}): \int_{0}^{1} g(t) d t=0\right\}$ is a 1-codimensional closed subspace of $C(I, \mathbb{R})$ with respect to the usual norm on this space. Then we have 


$$
G(x, s)=\sin (i s) R_{22}(x)+\cos (i s) R_{12}(x)=\cosh (s) R_{12}(x)+i \sinh (s) R_{22}(x)
$$

so that in particular s-ind $\left(\mathcal{A}^{\mathbb{C}}\right)$ does no longer depend on $a$.

After having computed the selfadjoint index of $\mathcal{A}^{\mathbb{C}}$, we now want to study bifurcation phenomena. Let

$$
f: H^{1}\left(I, \mathbb{R}^{2}\right) \rightarrow \mathbb{R}
$$

be a nonlinear bounded ${ }^{1}$ and continuous functional, where we assume that there exist $M>0$ and $r>0$ such that

$$
|f(u)| \leq M\|u\|_{H^{1}\left(I, \mathbb{R}^{2}\right)}, \quad u \in H^{1}\left(I, \mathbb{R}^{2}\right),\|u\|_{H^{1}\left(I, \mathbb{R}^{2}\right)} \leq r
$$

We consider the family of semilinear boundary value problems

$$
\left\{\begin{array}{l}
\sigma \frac{d u}{d t}+a(x, t) u(t)+f(u) u(t)=0, \quad t \in I \\
u(0) \in\{0\} \times \mathbb{R}, u(1) \in R(x)(\{0\} \times \mathbb{R})
\end{array}\right.
$$

parametrised by $X$ and define $B \subset X$ as the set of all $x^{*} \in X$ such that there exists a sequence $\left\{\left(x_{n}, u_{n}\right)\right\}_{n \in \mathbb{N}} \subset X \times H^{1}\left(I, \mathbb{R}^{2}\right)$, converging to $\left(x^{*}, 0\right)$ in $X \times H^{1}\left(I, \mathbb{R}^{2}\right)$ where $u_{n} \neq 0 \in H^{1}\left(I, \mathbb{R}^{n}\right)$, $n \in \mathbb{N}$, and each $u_{n}$ is a solution of 12.2 .

12.2.3 Proposition. Let $X$ be a simply connected compact topological space and $Y \subset X$ a closed subspace such that $R_{12}(x) \neq 0$ for all $x \in Y$.

If there exists a path $\gamma:(I, \partial I) \rightarrow(X, Y)$ and $t_{0} \in I$ such that $R_{12} \circ \gamma, R_{22} \circ \gamma \in C^{1}(I, \mathbb{R})$, $R_{12}(\gamma(t))=0$ if and only if $t=t_{0}$ and

$$
\left.\frac{d}{d t}\right|_{t=t_{0}} R_{12}(\gamma(t)) \neq 0
$$

then $X \backslash B$ is not path connected. If, moreover, $X$ is a manifold of dimension $n \geq 2$, then $B$ is not contractible and its dimension is at least $n-1$.

We now want to prove proposition 12.2 .3 by using proposition 11.2 .12 , where $H=L^{2}\left(I, \mathbb{C}^{2}\right)$, $\tilde{H}=H^{1}\left(I, \mathbb{C}^{2}\right)$ and our family of selfadjoint Fredholm operators is given by $\mathcal{A}^{\mathbb{C}}$ as already studied above. Moreover, we define

\footnotetext{
${ }^{1}$ By definition, $f$ is bounded if $f(B) \subset \mathbb{R}$ is bounded for any bounded subset $B \subset H^{1}\left(I, \mathbb{R}^{2}\right)$.
} 


$$
C: H^{1}\left(I, \mathbb{C}^{2}\right) \rightarrow L^{2}\left(I, \mathbb{C}^{2}\right), \quad u \mapsto f(\operatorname{Re}(u)) u,
$$

where $R e: H^{1}\left(I, \mathbb{C}^{2}\right) \rightarrow H^{1}\left(I, \mathbb{R}^{2}\right)$ is the continuous map defined by $\operatorname{Re}(u)=\frac{1}{2}(u+\bar{u})$. Note that once we have shown the existence of a sequence $\left\{\left(x_{n}, u_{n}\right)\right\}_{n \in \mathbb{N}} \subset X \times H^{1}\left(I, \mathbb{C}^{2}\right)$ as in the definition of a bifurcation point of $\mathcal{A}^{\mathbb{C}}+C$, then $\left\{\left(x_{n}, \operatorname{Re}\left(u_{n}\right)\right)\right\} \subset X \times H^{1}\left(I, \mathbb{R}^{2}\right)$ converges to a bifurcation point of 12.2 .

First of all, as already mentioned above, $\mathfrak{D}\left(\mathcal{A}^{\mathbb{C}}\right)$ is a subbundle of $X \times H^{1}\left(I, \mathbb{C}^{2}\right)$ and hence condition $(\mathrm{C})$ holds.

Next we want to show that $C: H^{1}\left(I, \mathbb{C}^{2}\right) \rightarrow L^{2}\left(I, \mathbb{C}^{2}\right)$ is compact. Note at first that $C$ is continuous since

$$
\begin{aligned}
\|C(u)-C(v)\|_{L^{2}\left(I, \mathbb{C}^{2}\right)} & =\|f(\operatorname{Re}(u)) u-f(\operatorname{Re}(v)) v\|_{L^{2}\left(I, \mathbb{C}^{2}\right)} \\
& \leq\|f(\operatorname{Re}(u)) u-f(\operatorname{Re}(u)) v\|_{L^{2}\left(I, \mathbb{C}^{2}\right)}+\|f(\operatorname{Re}(u)) v-f(\operatorname{Re}(v)) v\|_{L^{2}\left(I, \mathbb{C}^{2}\right)} \\
& \leq|f(\operatorname{Re}(u))|\|u-v\|_{H^{1}\left(I, \mathbb{C}^{2}\right)}+\mid f(\operatorname{Re}(u))-f(\operatorname{Re}(v))\|v\|_{H^{1}\left(I, \mathbb{C}^{2}\right)}
\end{aligned}
$$

for all $u, v \in H^{1}\left(I, \mathbb{C}^{2}\right)$. Moreover, since $f$ is assumed to be bounded, it is clear that $C$ maps bounded subsets of $H^{1}\left(I, \mathbb{C}^{2}\right)$ to bounded subsets in $H^{1}\left(I, \mathbb{C}^{2}\right)$. Hence $C: H^{1}\left(I, \mathbb{C}^{2}\right) \rightarrow L^{2}\left(I, \mathbb{C}^{2}\right)$ is compact because of the compactness of the inclusion $H^{1}\left(I, \mathbb{C}^{2}\right) \hookrightarrow L^{2}\left(I, \mathbb{C}^{2}\right)$.

Note that for all $u \in H^{1}\left(I, \mathbb{C}^{2}\right)$ such that $\|u\|_{H^{1}\left(I, \mathbb{C}^{2}\right)} \leq r$ we have

$$
\|C(u)\|_{L^{2}\left(I, \mathbb{C}^{2}\right)}=|f(\operatorname{Re}(u))|\|u\|_{L^{2}\left(I, \mathbb{C}^{2}\right)} \leq M\|u\|_{H^{1}\left(I, \mathbb{C}^{2}\right)}^{2}
$$

and hence the assumption i) in theorem 11.2 .6 is satisfied.

Next we show that there exists no non-trivial solution of

$$
\mathcal{A}_{x} u+f(\operatorname{Re}(u)) u=-i s u
$$

if $s \neq 0$.

Assume on the contrary that we have an $u \in \mathcal{D}(\mathcal{A})$ such that $(12.3)$ holds. Consider the operator

$$
\mathcal{A}_{x}+f(\operatorname{Re}(u)) \cdot I: \mathcal{D}\left(\mathcal{A}_{x}\right) \subset L^{2}\left(I, \mathbb{C}^{2}\right) \rightarrow L^{2}\left(I, \mathbb{C}^{2}\right)
$$

Since $f(\operatorname{Re}(u)) \in \mathbb{R}$, this operator is selfadjoint and hence its spectrum is entirely contained in $\mathbb{R}$. Accordingly, if $s \neq 0$, there exists no non-trivial solution of the linear equation

$$
\mathcal{A}_{x} v+f(\operatorname{Re}(u)) v=-i s v, \quad v \in \mathcal{D}\left(\mathcal{A}_{x}\right),
$$


and we infer that $u=0 \in \mathcal{D}\left(\mathcal{A}_{x}\right)$. This shows the assumption ii) from theorem 11.2.6.

Hence proposition 12.2 .3 follows from proposition 11.2 .12 once we can prove that under the remaining assumptions

$$
\operatorname{sf}\left(\mathcal{A}^{\mathbb{C}} \circ \gamma\right) \bmod 2 \neq 0
$$

According to the computations from above, we have

$$
\operatorname{s-ind}\left(\gamma^{*} \mathcal{A}^{\mathbb{C}}\right)=\left[\Theta(\mathbb{C}), \Theta(\mathbb{C}), \bar{\gamma}^{*} G\right] \in K^{-1}(I, \partial I),
$$

where

$$
\left(\bar{\gamma}^{*} G\right)(t, s)=\cosh (s) R_{12}(\gamma(t))+i \sinh (s) R_{22}(\gamma(t)), \quad(t, s) \in I \times \mathbb{R} .
$$

If now $\eta: S^{1} \rightarrow I \times \mathbb{R}$ is any simple curve surrounding $(0,1) \times\{0\} \subset I \times \mathbb{R}$, then

$$
\operatorname{sf}\left(\mathcal{A}^{\mathbb{C}} \circ \gamma\right)=c_{1}\left(\left[\Theta(\mathbb{C}), \Theta(\mathbb{C}), \bar{\gamma}^{*} G\right]\right)=w\left(\left(\bar{\gamma}^{*} G\right) \circ \eta, 0\right)
$$

according to proposition 7.3.1 and hence we want to compute the latter winding number.

Since the matrices $R(x)$ are in particular invertible, we infer $\left(R_{12}(x)\right)^{2}+\left(R_{22}(x)\right)^{2} \neq 0$ for all $x \in X$ and hence $\left(\bar{\gamma}^{*} G\right)(t, s)=0$ if and only if $s=0$ and $t=t_{0}$. Now the Jacobian of $\bar{\gamma}^{*} G$ at $\left(t_{0}, 0\right)$ is given by

$$
\begin{aligned}
J\left(t_{0}, 0\right) & =\left|\begin{array}{ll}
\left.\cosh (s) \frac{d}{d t} R_{12}(\gamma(t))\right|_{\left(t_{0}, 0\right)} & \left.\sinh (s) R_{12}(\gamma(t))\right|_{\left(t_{0}, 0\right)} \\
\left.\sinh (s) \frac{d}{d t} R_{22}(\gamma(t))\right|_{\left(t_{0}, 0\right)} & \left.\cosh (s) R_{22}(\gamma(t))\right|_{\left(t_{0}, 0\right)}
\end{array}\right| \\
& =\left.R_{22}\left(\gamma\left(t_{0}\right)\right) \frac{d}{d t}\right|_{t=t_{0}} R_{12}(\gamma(t)) \neq 0
\end{aligned}
$$

and according to [Dei85, §6.6] this shows

$$
w\left(\left(\bar{\gamma}^{*} G\right) \circ \eta, 0\right)=\operatorname{sgn} J\left(t_{0}, 0\right)= \pm 1 \neq 0 \bmod 2 .
$$

Hence proposition 12.2 .3 is proved.

We now consider examples of bounded and continuous functionals $f: H^{1}\left(I, \mathbb{R}^{2}\right) \rightarrow \mathbb{R}$ such that the growth condition

$$
|f(u)| \leq M\|u\|_{H^{1}\left(I, \mathbb{R}^{2}\right)}
$$


holds for some $M>0$ and for all $u \in H^{1}\left(I, \mathbb{R}^{2}\right)$ such that $\|u\|_{H^{1}\left(I, \mathbb{R}^{2}\right)}<r$ for a certain $r>0$. The main observation for obtaining such functionals is to require $f: H^{1}\left(I, \mathbb{R}^{2}\right) \rightarrow \mathbb{R}$ to be Gateaux-differentiable on some open ball $B_{r}(0)$ around 0 in $H^{1}\left(I, \mathbb{R}^{2}\right)$ and such that $\left\|D_{u} f\right\|<M$ for all $u \in B_{r}(0)$. If we moreover assume $f$ to vanish at 0 , then we obtain from the mean value theorem (cf. WWe5, III.5.4])

$$
|f(u)| \leq M\|u\|_{H^{1}\left(I, \mathbb{R}^{2}\right)}, \quad u \in B_{r}(0) .
$$

Typical examples of such functionals are of the form

$$
f: H^{1}\left(I, \mathbb{R}^{2}\right) \rightarrow \mathbb{R}, \quad f(u)=\int_{0}^{1} F(t, u(t)) d t
$$

for sufficiently regular functions $F: I \times \mathbb{R}^{2} \rightarrow \mathbb{R}^{2}$ (cf. AmM07, §1.3]).

A more elementary example is $f(u)=\|u\|_{H^{1}\left(I, \mathbb{R}^{2}\right)}^{p}$ for some $p \geq 1$. In this case we directly see from the definition that

$$
|f(u)| \leq\|u\|_{H^{1}\left(I, \mathbb{R}^{2}\right)}, \quad\|u\|_{H^{1}\left(I, \mathbb{R}^{2}\right)} \leq 1 .
$$

Moreover, by a similar argument we see that also the functionals

$$
\begin{aligned}
& f_{1}(u)=\|u\|_{L^{2}\left(I, \mathbb{R}^{2}\right)}^{p} \\
& f_{2}(u)=\left\|u^{\prime}\right\|_{L^{2}\left(I, \mathbb{R}^{2}\right)}^{p} \\
& f_{3}(u)=\|u\|_{\infty}^{p}
\end{aligned}
$$

satisfy the growth condition on their corresponding unit balls in $H^{1}\left(I, \mathbb{R}^{2}\right)$.

We now conclude this section by a concrete example. We set $X=S^{n}$ for some $n \geq 2$ and define $Y$ to consists of the north pole $(0, \ldots, 0,1)$ and south pole $(0, \ldots, 0,-1)$ of $S^{n}$. We consider the semi-circle

$$
S=\left\{\left(x_{1}, \ldots, x_{n+1}\right) \in S^{n}: x_{1}=\ldots=x_{n-1}=0, x_{n} \geq 0\right\}
$$

and assume to have a function $\varphi: S^{n} \rightarrow \mathbb{R}$ whose restriction to $S$ is given by

$$
\left.\varphi\right|_{S}: S \rightarrow \mathbb{R}, \quad \varphi(x)=\left\{\begin{array}{l}
\arctan \frac{x_{n+1}}{x_{n}}, x_{n} \neq 0 \\
\frac{\pi}{2}, \quad x_{n}=0, x_{n+1}=1 \\
-\frac{\pi}{2}, \quad x_{n}=0, x_{n+1}=-1
\end{array}\right.
$$


Note that such functions exist according to the Tietze extension theorem.

Now we define

$$
R(x)=\left(\begin{array}{cc}
\cos \varphi(x) & -\sin \varphi(x) \\
\sin \varphi(x) & \cos \varphi(x)
\end{array}\right)
$$

and consider the corresponding family of semilinear differential operators 12.2 for some bounded continuous functional $f: H^{1}\left(I, \mathbb{R}^{2}\right) \rightarrow \mathbb{R}$ satisfying the growth condition. We define a path

$$
\gamma:\left[-\frac{\pi}{2}, \frac{\pi}{2}\right] \rightarrow X, \quad \gamma(t)=(0, \ldots, 0, \cos t,-\sin t)
$$

which connects the north and the south pole in $S^{n}$ and note that $R_{12}(\gamma(t))=\sin t$ and $R_{22}(\gamma(t))=\cos t$. Hence $R_{12}(\gamma(t))=0$ if and only if $t=0$ and, moreover,

$$
\left.\frac{d}{d t}\right|_{t=0} R_{12}(\gamma(t))=1 \neq 0 .
$$

Now we obtain from proposition 12.2 .3 that the set of bifurcation points $B \subset S^{n}$ of the equation 12.2 is not contractible and has at least dimension $n-1$. 
Part V

Appendix 



\section{Appendix A}

\section{A Few Basic Definitions and Theorems of Functional Analysis}

The aim of this chapter is to recall some basics of generally unbounded linear operators. Proofs are usually omitted but individual references to the literature are given. We assume familiarity with the notions of Banach- and Hilbert spaces and bounded linear operators.

\section{A.1 Projections and Subspaces}

We consider two normed linear spaces $\left(U,\|\cdot\|_{U}\right)$ and $\left(V,\|\cdot\|_{V}\right)$. On the product $U \times V$ we can define norms by

$$
\|(u, v)\|_{p}=\left(\|u\|_{U}^{p}+\|v\|_{V}^{p}\right)^{\frac{1}{p}}, \quad 1 \leq p<\infty
$$

and

$$
\|(u, v)\|_{\infty}=\max \left\{\|u\|_{U},\|v\|_{V}\right\} .
$$

A.1.1 Lemma. The norms $\|\cdot\|_{p}, 1 \leq p<\infty$, and $\|\cdot\|_{\infty}$ are pairwise equivalent. Moreover, if $U$ and $V$ are Banach spaces, then $U \times V$ is a Banach space with respect to any of these norms.

Proof. [We05, I.3.3]

Now we assume that $U, V$ are subspaces of a Banach space $E$ such that $E=U \oplus V$ algebraically. In this case we have a unique projection $P: E \rightarrow E$ onto $U$ with respect to this decomposition and can now ask about the boundedness of $P$.

A.1.2 Lemma. $P: E \rightarrow E$ is bounded if and only if $U$ and $V$ are closed. 
Proof. ApVä05, Satz 4.10]

A strictly related problem concerns the following definition.

A.1.3 Definition. A closed subspace $U$ of a Banach space $E$ is called complemented if there exists a closed subspace $V$ of $E$ such that $E=U \oplus V$.

A well known example of a non complemented subspace of a Banach space (cf. eg. We05, Satz IV.6.5]) is given by

$$
\left\{\left\{x_{n}\right\}_{n \in \mathbb{N}} \subset \mathbb{C}: \lim x_{n}=0\right\}
$$

which is a closed subspace of

$$
l^{\infty}=\left\{\left\{x_{n}\right\}_{n \in \mathbb{N}} \subset \mathbb{C}: \sup _{n \in \mathbb{N}}\left|x_{n}\right|<\infty\right\}, \quad\left\|\left\{x_{n}\right\}_{n \in \mathbb{N}}\right\|=\sup _{n \in \mathbb{N}}\left|x_{n}\right| .
$$

However, we have the following two positive results.

A.1.4 Lemma. Any subspace $U$ of $E$ of finite dimension is complemented.

Proof. We05, IV.6.2]

A.1.5 Theorem. Any closed subspace $U$ of a Hilbert space $H$ is complemented.

Proof. [We05, V.3.4]

Note that the latter result is just the well known theorem on the orthogonal projection which gives rise to the decomposition $H=U \oplus U^{\perp}$ for any closed subspace $U \subset H$.

Lemma A.1.4 suggests the question if the same result holds if we require the codimension of $U$ instead of the dimension to be finite. That is, we assume to have a decomposition $E=U \oplus V$ where $V$ is of finite dimension. Since finite dimensional spaces are closed we obtain immediately that any closed subspace of $E$ of finite codimension is complemented. However, by the following result we can not omit the closedness assumption.

A.1.6 Lemma. In any Banach space $E$ of infinite dimension there exists a one codimensional subspace $U$ which is not closed.

Proof. ApVä05, Bsp. 7.14]

However, the following remarkable result states that $U$ is closed if it is the image of a bounded linear operator.

A.1.7 Lemma. If $E$ and $F$ are Banach spaces, $A \in \mathcal{L}(E, F)$ a bounded linear operator and $V \subset F$ a closed subspace such that $F=\operatorname{im} A \oplus V$, then $\operatorname{im} A$ is closed. 
Proof. ApVä05, Satz 7.6]

We conclude this section by mentioning the following result which connects complemented subspaces and the existence of one-sided inverses.

A.1.8 Lemma. Let $E, F$ be Banach spaces and $L: E \rightarrow F$ a bounded linear operator.

i) Let $L$ be surjective. Then $L$ has a bounded right inverse if and only if $\operatorname{ker} L$ is complemented in $E$.

ii) Let $L$ be injective. Then $L$ has a bounded left inverse if and only if $\operatorname{im} L$ is closed and complemented.

Proof. [GGK90, Theorem XI.6.1]

\section{A.2 Closed Operators}

A linear operator $T$ acting between the Banach spaces $E$ and $F$ is a linear map $T: \mathcal{D}(T) \rightarrow F$, where $\mathcal{D}(T)$ is a linear subspace of $E$. We usually write

$$
T: \mathcal{D}(T) \subset E \rightarrow F
$$

in order to emphasize the space $E$. We call $T$ densely defined if $\mathcal{D}(T)$ is dense in $E$ and bounded if there exists a constant $c>0$ such that

$$
\|T u\| \leq c\|u\|, \quad u \in \mathcal{D}(T) .
$$

In contrast to parts of the literature we call an operator $T$ invertible if it maps its domain $\mathcal{D}(T)$ bijectively onto its target space $Y$. If $A \subset \mathcal{C}(E, F)$ is a set of operators, we denote by $G A$ the invertible elements of $A$.

We let

$$
\operatorname{graph}(T)=\{(u, T u) \in E \times F: u \in \mathcal{D}(T)\} \subset E \times F
$$

be the graph of $T$ which is a linear subspace of the Banach space $E \times F$. Finally, we call $T$ closed if $\operatorname{graph}(T)$ is a closed subspace of $E \times F$. We denote the set of all closed operators acting between $E$ and $F$ by $\mathcal{C}(E, F)$.

We want to point out that in contrast to $\mathcal{L}(E, F)$, the space of all bounded operators having the whole space $E$ as domain, $\mathcal{C}(E, F)$ is not a linear space because the sum of two closed operators is in general not closed. 
A.2.1 Lemma. A bounded operator $T$ is closed if and only if $\mathcal{D}(T)$ is closed in $E$.

As a consequence of this lemma, $\mathcal{L}(E, F) \subset \mathcal{C}(E, F)$. On the other hand, we see that the product $0 \cdot T$ is in general not a closed operator.

A.2.2 Lemma. Let $T \in \mathcal{C}(E, F)$.

i) $\alpha T$ is closed for any complex number $\alpha \neq 0$.

ii) If $B$ is bounded and $\mathcal{D}(T) \subset \mathcal{D}(B)$, then $T+B$ is closed.

iii) If $T$ is invertible, then $T^{-1}$ is closed as well.

Proof. The first assertion is clear, because the map $E \times F \rightarrow E \times F,(u, v) \mapsto(u, \alpha \cdot v)$ is a homeomorphism mapping $\operatorname{graph}(T)$ to $\operatorname{graph}(\alpha \cdot T)$. The remaining assertions can be found in Ka76, Sect. III.5.2].

A.2.3 Remark. The second assertion of the previous lemma actually holds in the more general case that $B$ is relatively bounded with respect to $T$ and has T-bound less than 1 (cf. [Ka76, Theorem IV.1.1]).

The following theorem is usually known as the closed graph theorem.

A.2.4 Theorem. If $T \in \mathcal{C}(E, F)$ and $\mathcal{D}(T)=E$, then $T \in \mathcal{L}(E, F)$.

Proof. [Ka76, III.5.20]

Note as an immediate consequence that $\mathcal{D}(T) \neq E$ if $T \in \mathcal{C}(E, F)$ is unbounded. Moreover, if $T \in \mathcal{C}(E, F)$ is invertible, then $T^{-1} \in \mathcal{L}(F, E)$ and so a closed operator is invertible if and only if it possesses a bounded inverse.

Next we state two simple consequences of theorem A.2.4.

A.2.5 Corollary. If $T \in \mathcal{C}(E, F)$ is invertible, then $T^{-1} \in \mathcal{L}(F, E)$.

The foregoing corollary shows in particular that $G \mathcal{L}(E, F)=G L(E, F)$, where the right hand side consists as usual of all bijective bounded operators having a bounded inverse.

A.2.6 Corollary. If $E, F$ and $G$ are Banach spaces and $B \in \mathcal{L}(E, F), T \in \mathcal{C}(F, G)$ such that $\operatorname{im}(B) \subset \mathcal{D}(T)$, then $T B \in \mathcal{L}(E, G)$.

If $T$ is any operator acting between the Banach spaces $E$ and $F$, we can define for any $1 \leq p<\infty$ a norm on the domain $\mathcal{D}(T)$ by

$$
\|u\|_{T}=\left(\|u\|_{E}^{p}+\|T u\|_{F}^{p}\right)^{\frac{1}{p}}, \quad u \in \mathcal{D}(T)
$$

which is called the graph norm of $T$ for $p=2$. It is easy to show that all these norms are pairwise equivalent and in proofs we sometimes use the case $p=1$ which can help to avoid some 
technicalities.

Note that with this definition of the norm, $\mathcal{D}(T)$ is isometric to the subspace graph $(T)$ of $E \times F$ if we consider on the latter space the norm $\|\cdot\|_{2}$. Hence we obtain immediately the following result.

A.2.7 Lemma. If $T \in \mathcal{C}(E, F)$, then $\mathcal{D}(T)$ is a Banach space with respect to the graph norm.

The following lemma shows that these Banach space structures are already essentially determined by the underlying domain.

A.2.8 Lemma. If $T_{1}, T_{2} \in \mathcal{C}(E, F)$ have the same domain $D=\mathcal{D}\left(T_{1}\right)=\mathcal{D}\left(T_{2}\right)$, then the graph norms of $T_{1}$ and $T_{2}$ on $D$ are equivalent.

Proof. We denote by $D_{T_{1}}$ and $D_{T_{2}}$ the space $D$ with the graph norm of $T_{1}$ and $T_{2}$, respectively. Consider $T_{1}: D_{T_{2}} \rightarrow F$ and let $\left\{\left(u_{n}, T_{1} u_{n}\right)\right\}_{n \in \mathbb{N}} \subset \operatorname{graph}\left(T_{1}: D_{T_{2}} \rightarrow F\right)$ be any sequence which converges in $D_{T_{2}} \times F$ to an element $(u, v) \in D_{T_{2}} \times F$. Since $u_{n} \rightarrow u$ in $D_{T_{2}}$ implies that $u_{n}$ converges also to $u$ with respect to the norm of $E$, we obtain from the closedness of $T_{1}$ that $\left(u_{n}, T_{1} u_{n}\right) \rightarrow\left(u, T_{1} u\right)$ in $E \times F$. Hence $\left(u_{n}, T_{1} u_{n}\right) \rightarrow\left(u, T_{1} u\right)$ in $D_{T_{2}} \times F$ which implies that $T_{1}: D_{T_{2}} \rightarrow F$ is closed. Since $D_{T_{2}}$ is complete by lemma A.2.7, we obtain from theorem A.2.4 that $T_{1}: D_{T_{2}} \rightarrow F$ is bounded. Accordingly, there exists a constant $c>0$ such that

$$
\left\|T_{1} u\right\|_{F} \leq c\left(\|u\|_{E}+\left\|T_{2} u\right\|_{F}\right)
$$

and thus

$$
\|u\|_{E}+\left\|T_{1} u\right\|_{F} \leq(c+1)\left(\|u\|_{E}+\left\|T_{2} u\right\|_{F}\right) .
$$

By using elementary inequalities we finally obtain

$$
\left(\|u\|_{E}^{2}+\left\|T_{1} u\right\|_{F}^{2}\right)^{\frac{1}{2}} \leq \sqrt{2}(c+1)\left(\|u\|_{E}^{2}+\left\|T_{2} u\right\|_{F}^{2}\right)^{\frac{1}{2}}
$$

The assertion follows by interchanging $T_{1}$ and $T_{2}$.

We now consider special classes of closed Operators.

A.2.9 Definition. An operator $T \in \mathcal{C}(E, F)$ acting between Banach spaces $E$ and $F$ is called Fredholm if $\operatorname{ker} T$ is of finite dimension and $\operatorname{im} T$ of finite codimension. The Fredholm index of a Fredholm operator is defined as

ind $T=\operatorname{dim} \operatorname{ker} T-\operatorname{codimim} T$. 
The following result is often required as an additional assumption in the definition of a Fredholm operator.

A.2.10 Lemma. If $T \in \mathcal{C}(E, F)$ is Fredholm, then $\operatorname{im} T \subset F$ is closed.

Proof. If we consider $T$ on its domain $\mathcal{D}(T)$ with respect to the graph norm, then $T$ is bounded. Since a change of the norm in the domain does not affect im $T$, the assertion follows from lemma A.1.7.

We denote by $\mathcal{C F}_{k}(E, F)$ the set of all Fredholm operators of index $k \in \mathbb{Z}$ acting between the Banach spaces $E$ and $F$ and by $\mathcal{C F}(E, F)$ the union of all these spaces. Moreover, we set $\mathcal{B F}(E, F)=\mathcal{C F}(E, F) \cap \mathcal{L}(E, F)$.

We note an interesting result for bounded Fredholm operators that we use several times in the thesis.

A.2.11 Lemma. Suppose $A_{0}: V \rightarrow F$ is a restriction of $A \in \mathcal{L}(E, F)$ to a closed subspace $V$ of $X$ with $\operatorname{codim} V=n<\infty$. The $A$ is Fredholm if and only if $A_{0}$ is Fredholm, in which case ind $A=$ ind $A_{0}+n$.

Proof. [GGK90, XI.3.1]

One of the main theorems in the theory of Fredholm operators reads as follows.

A.2.12 Theorem. If $E, F, G$ are Banach spaces, $T \in \mathcal{C}(F, G)$ is a densely defined Fredholm operator and $S \in \mathcal{C}(E, F)$ is any Fredholm operator, then $T S$ is a Fredholm operator and

$$
\operatorname{ind}(T S)=\operatorname{ind}(T)+\operatorname{ind}(S)
$$

where as usual

$$
\mathcal{D}(T S)=\{u \in \mathcal{D}(S): S u \in \mathcal{D}(T)\}
$$

Proof. [GGK90, XVII.3.1]

Let $S$ and $T$ be two linear operators acting between the Banach spaces $E$ and $F$. We say that $S$ is $T$-compact if $\mathcal{D}(T) \subset \mathcal{D}(S)$ and $S: \mathcal{D}(T) \rightarrow F$ is compact with respect to the graph norm of $T$.

A.2.13 Theorem. If $T \in \mathcal{C}(E, F)$ is a Fredholm operator and $S$ is a $T$-compact operator acting between the Banach spaces $E$ and $F$, then $T+S$ is a Fredholm operator as well and

$$
\operatorname{ind}(T+S)=\operatorname{ind}(T) .
$$


Proof. [GGK90, XVII.4.3]

Finally we want to define the adjoint of an operator and consider symmetric and selfadjoint operators. In order to do so, we assume that $T$ is any (in particular not necessarily closed) densely defined operator acting on a Hilbert space $H$. We define

$$
\mathcal{D}\left(T^{*}\right)=\left\{v \in H: u \mapsto\langle T u, v\rangle_{H} \text { is bounded on } \mathcal{D}(T)\right\}
$$

and note that, since $\mathcal{D}(T)$ is assumed to be dense in $H$, each functional $u \mapsto\langle T u, v\rangle_{H}$, $v \in \mathcal{D}\left(T^{*}\right)$. has a continuous extension on all of $H$. Hence, by the Riesz representation theorem, we can associate to any $v \in \mathcal{D}\left(T^{*}\right)$ an element $T^{*} v \in H$ such that

$$
\langle T u, v\rangle_{H}=\left\langle u, T^{*} v\right\rangle_{H}, \quad u \in \mathcal{D}(T), v \in \mathcal{D}\left(T^{*}\right) .
$$

The resulting operator $T^{*}$ on $H$ with domain $\mathcal{D}\left(T^{*}\right)$ is linear and called the adjoint of $T$.

A.2.14 Lemma. If $T$ is densely defined, then $T^{*} \in \mathcal{C}(H)$.

Proof. [We05, Satz VII.2.4]

We want to point out that it can happen that $\mathcal{D}\left(T^{*}\right)=\{0\}$ for a densely defined operator $T$ (cf. We05, p. 338]). However, since we are solely interested in closed operators, such singular phenomena are excluded.

A.2.15 Lemma. If $T \in \mathcal{C}(H)$ is densely defined, then $T^{*}$ is densely defined as well and $T^{* *}=T$. Proof. Wei80, Theorem 5.3]

It is a common notation to write $S \subset T$ for two operators if $\mathcal{D}(S) \subset \mathcal{D}(T)$ and $S u=T u$ for all $u \in \mathcal{D}(S)$. Using this definition, a densely defined operator $T$ acting on a Hilbert space $H$ is called symmetric if $T \subset T^{*}$ and selfadjoint if $T=T^{*}$. Note that a selfadjoint operator is automatically closed by lemma A.2.14.

A.2.16 Lemma. Let $T$ be a densely defined operator acting on $H$.

i) $T$ is symmetric if and only if $T \subset T^{* *} \subset T^{*}=T^{* * *}$. In this case $T^{* *}$ is symmetric as well.

ii) $T \in \mathcal{C}(H)$ is symmetric if and only if $T=T^{* *} \subset T^{*}$.

iii) $T$ is selfadjoint if and only if $T=T^{* *}=T^{*}$.

Proof. [We05, VII.2.5]

If $A$ is a set of operators acting on a Hilbert space, then we denote by $A^{s a}$ the selfadjoint elements of $A$. 


\section{A.3 A Little Spectral Theory}

Let for the moment $T$ be any operator acting on a Banach space $E$. We call $\lambda \in \mathbb{C}$ an eigenvalue of $T$ if there exists an $u \in \mathcal{D}(T), u \neq 0$, such that $T u=\lambda u$. If on the other hand $\lambda$ is not an eigenvalue, then $\lambda-T$ is injective and hence the resolvent operator

$$
R(\lambda, T)=(\lambda-T)^{-1}, \quad \mathcal{D}(R(\lambda, T))=\operatorname{im} T \subset E
$$

is well defined. We define the resolvent set of $T$ by

$$
\rho(T)=\{\lambda \in \mathbb{C}: \lambda-T \text { bijective, } R(\lambda, T) \in \mathcal{L}(E)\} .
$$

A first important observation reads as follows:

A.3.1 Lemma. If $T \notin \mathcal{C}(E)$, then $\rho(T)=\emptyset$.

Proof. If $\lambda \in \rho(T) \neq \emptyset$, then $R(\lambda, T)=(\lambda-T)^{-1}$ is bounded and hence closed by lemma A.2.1. Then $\lambda-T$ is closed by lemma A.2.2 and so $T$ is closed as well.

Accordingly we assume in the following throughout that $T \in \mathcal{C}(E)$. Note that in this case we obtain from the closed graph theorem A.2.4 that

$$
\rho(T)=\{\lambda \in \mathbb{C}: \lambda-T \text { is bijective }\} .
$$

We define the spectrum $\sigma(T)$ of $T$ to be $\mathbb{C} \backslash \rho(T)$ and the point spectrum $\sigma_{p}(T) \subset \sigma(T)$ as the set of all eigenvalues of $T$.

A.3.2 Lemma. $\rho(T) \subset \mathbb{C}$ is open and, accordingly, $\sigma(T) \subset \mathbb{C}$ is closed.

Proof. Wei80, 5.14]

We want to point out that in contrast to operators in $\mathcal{L}(E)$, the spectrum can be unbounded and even empty.

There are various definitions of subsets of $\sigma(T)$ besides $\sigma_{p}(T)$. Here we just want to introduce the essential spectrum $\sigma_{\text {ess }}(T)$ which consists of all $\lambda \in \mathbb{C}$ such that $\lambda-T$ is not a Fredholm operator. In contrast to the case of bounded operators, it can happen that $\sigma_{\text {ess }}(T)=\emptyset$. Note that in general we neither have $\sigma(T)=\sigma_{p}(T) \cup \sigma_{\text {ess }}(T)$ nor $\sigma_{p}(T) \cap \sigma_{e s s}(T)=\emptyset$.

As next step we introduce the spectral projections. At first, we want to mention that, given an operator $T$ acting on a linear space $E$, a subspace $M \subset E$ is called $T$-invariant if $T(M \cap \mathcal{D}(T)) \subset$ $M$. In this case $\left.T\right|_{M}$ denotes the operator $T$ with domain $M \cap \mathcal{D}(T)$ and range in $M$. 
A.3.3 Theorem. Let $T \in \mathcal{C}(E)$ with spectrum $\sigma(T)=\sigma \cup \tau$, where $\sigma$ is contained in a bounded Cauchy domain $1^{1} \triangle$ such that $\bar{\triangle} \cap \tau=\emptyset$. Let $\Gamma$ be the oriented boundary of $\triangle$. Then

i) $P_{\sigma}:=\frac{1}{2 \pi i} \int_{\Gamma}(\lambda-T)^{-1} d \lambda$ is a projection,

ii) the subspaces $M=\operatorname{im} P_{\sigma}$ and $N=\operatorname{ker} P_{\sigma}$ are $T$-invariant,

iii) the subspace $M$ is contained in $\mathcal{D}(T)$ and $\left.T\right|_{M}$ is bounded,

(iv) $\sigma\left(\left.T\right|_{M}\right)=\sigma$ and $\sigma\left(\left.T\right|_{N}\right)=\tau$.

Proof. GGK90, Theorem XV.2.1]

We call a point $\lambda \in \sigma(T)$ an eigenvalue of finite type if $\lambda$ is isolated in $\sigma(T)$ and the

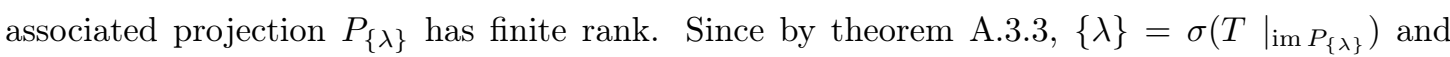
$\operatorname{im} P_{\lambda}$ is finite dimensional, it follows that $\lambda \in \sigma_{p}(T)$. A quite often appearing situation is described by the following theorem.

A.3.4 Theorem. Let $T \in \mathcal{C}(E)$ be such that $R\left(\lambda_{0}, T\right)=\left(\lambda_{0}-T\right)^{-1}$ is compact for some $\lambda_{0} \in \rho(T)$. Then $R(\lambda, T)$ is compact for any $\lambda \in \rho(T), \sigma(T)$ does not have a limit point in $\mathbb{C}$ and every point in $\sigma(T)$ is an eigenvalue of finite type. Moreover, for any $\lambda \in \mathbb{C}$ we have

$$
\operatorname{dim} \operatorname{ker}(\lambda-T)=\operatorname{codimim}(\lambda-T)<\infty
$$

so that each operator $\lambda-T$ is Fredholm of index 0 .

Proof. [GGK90, Theorem XV.2.3]

We call $T \in \mathcal{C}(E)$ an operator with compact resolvent if there exists $\lambda_{0} \in \mathbb{C}$ such that $R\left(\lambda_{0}, T\right)$ is compact. Note that $\sigma_{e s s}(T)=\emptyset$ in this case.

A.3.5 Lemma. If $T \in \mathcal{C}(E)$ has a compact resolvent and $B \in \mathcal{L}(E)$ is bounded, then $T+B$ is a Fredholm operator of index 0 .

Proof. We fix $\lambda \in \rho(T)$ and obtain a compact operator $(\lambda-T)^{-1}: E \rightarrow E$. Then $B(\lambda-T)^{-1}$ : $E \rightarrow E$ is compact as well since the composition of a bounded and a compact operator is still compact. We infer that

$$
B=\left(B(\lambda-T)^{-1}\right)(\lambda-T): \mathcal{D}(T) \rightarrow E
$$

is compact with respect to the graph norm of $T$ on $\mathcal{D}(T)$. Now the assertion follows from theorem A.2.13 and theorem A.3.4

\footnotetext{
${ }^{1}$ The definition of Cauchy domain can be found in GGK90, Sec. I.1]. For example, any open and connected subset of $\mathbb{C}$ whose boundary is a closed rectifiable Jordan curve is a Cauchy domain.
} 
Finally, we assume that $E=H$ is a Hilbert space and study spectra of symmetric and selfadjoint operators. We begin with the following remarkable result.

A.3.6 Lemma. Let $T \in \mathcal{C}(H)$ be densely defined and symmetric. Then precisely one of the following assertions hold:

i) $\sigma(T)=\mathbb{C}$;

ii) $\sigma(T)=\{\lambda \in \mathbb{C}: \operatorname{im} \lambda \geq 0\}$;

iii) $\sigma(T)=\{\lambda \in \mathbb{C}: \operatorname{im} \lambda \leq 0\}$;

iv) $\sigma(T) \subset \mathbb{R}$.

Moreover, $\sigma(T) \subset \mathbb{R}$ if and only if $T$ is selfadjoint.

Proof. [Ka76, Sec. V.3.4]

Note that in particular a closed symmetric operator is selfadjoint if $\rho(T) \cap \mathbb{R} \neq \emptyset$.

It is well known (cf. eg. Ka76, Sec. V.3.1] that

$$
\operatorname{ker}\left(T^{*}\right)=\operatorname{im}(T)^{\perp}
$$

for any densely defined operator $T \in \mathcal{C}(H)$. As a consequence we note

A.3.7 Lemma. If $T \in \mathcal{C}(H)$ is densely defined and selfadjoint, then

$$
\sigma(T)=\sigma_{p}(T) \cup \sigma_{e s s}(T) .
$$

Proof. Since $\sigma(T) \subset \mathbb{R}$, any $\lambda-T, \lambda \in \sigma(T)$, is selfadjoint as well. Hence it suffices to consider the case $\lambda=0$ and, accordingly, to assume that $0 \in \sigma(T)$.

Since $\operatorname{ker} T=\operatorname{im} T^{\perp}$ by A.1, we infer that either $T$ is not injective or has a dense image. If $T$ is not injective, then $0 \in \sigma_{p}(T)$. If on the other hand $T$ is injective, then $T$ has a dense image but $\operatorname{im}(T) \neq H$ because otherwise $0 \in \rho(T)$. Hence $\operatorname{im}(T)$ is not closed and so $T$ is not a Fredholm operator which implies $0 \in \sigma_{\text {ess }}(T)$.

Finally, we want to introduce the Cayley transform which is defined by

$$
\kappa(T)=(T-i)(T+i)^{-1}
$$

where $T$ is a selfadjoint operator. 
A.3.8 Lemma. $\kappa(T)$ is a unitary operator on $H$ for any selfadjoint operator $T \in \mathcal{C}(H)$. Moreover, $U$ is the Cayley transform of a selfadjoint operator $T \in \mathcal{C}(H)$ if and only if $1 \notin \sigma_{p}(U)$ and in this case $T$ can be recovered from $U$ by

$$
T v=i(I+U)(I-U)^{-1} v, \quad v \in \mathcal{D}(T) .
$$

Proof. [Co90, Cor. X.3.5] 


\section{Appendix B}

\section{$K$-Theory}

In this final chapter we give a brief survey of topological $K$-theory for pairs of locally compact spaces. Our main references are [At89], [LM89], [Fe91] and in particular [Se68. In the first section we define the $K$-theory groups and discuss their main properties. Since we do not use the most common definition we show in the second section that our definition is equivalent to the ordinary one. In the final third section we construct an explicit isomorphism $K^{-1}(I, \partial I) \rightarrow \mathbb{Z}$ which is induced by the first Chern number and turns out to be computable by means of the winding number.

\section{B.1 Basic Definitions and Properties}

Let $X$ be a locally compact topological space. We consider triples $\left\{E_{0}, E_{1}, a\right\}$, where $E_{0}$ and $E_{1}$ are vector bundles over $X$ and $a: E_{0} \rightarrow E_{1}$ is a bundle morphism. The support supp $E$ of such a triple $E=\left\{E_{0}, E_{1}, a\right\}$ is defined to be the subset of $X$ consisting of those points $x \in X$ for which $a_{x}: E_{0, x} \rightarrow E_{1, x}$ is not an isomorphism. By using the continuity of the determinant, it is clear that the support is a closed subset of $X$. $E$ is said to be trivial if its support is all of $X$. We call two triples $E^{0}=\left\{E_{0}^{0}, E_{1}^{0}, a_{0}\right\}$ and $E^{1}=\left\{E_{0}^{1}, E_{1}^{1}, a_{1}\right\}$ isomorphic, if there exist bundle isomorphisms $\varphi_{0}: E_{0}^{0} \rightarrow E_{0}^{1}$ and $\varphi_{1}: E_{1}^{0} \rightarrow E_{1}^{1}$ such that the diagram

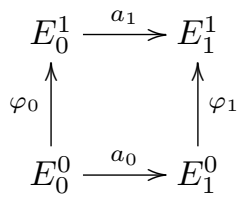

commutes.

If $A \subset X$ is a closed subspace, we denote by $L(X, A)$ the set of isomorphism classes of triples $E=\left\{E_{0}, E_{1}, a\right\}$ on $X$ such that $\operatorname{supp} E$ is a compact subset of $X \backslash A$. Note that $L(X, A)$ is a semigroup under the operation of direct sum and 


$$
\operatorname{supp} E^{0} \oplus E^{1}=\operatorname{supp} E^{0} \cup \operatorname{supp} E^{1}, \quad E^{0}, E^{1} \in L(X, A) .
$$

We call two elements $E^{0}=\left\{E_{0}^{0}, E_{1}^{0}, a_{0}\right\}$ and $E^{1}=\left\{E_{0}^{1}, E_{1}^{1}, a_{1}\right\}$ homotopic,$E^{0} \simeq E^{1}$, if there is an element in $L(X \times[0,1], A \times[0,1])$ such that its restriction to $X \times\{0\}$ and $X \times\{1\}$ are isomorphic to $E^{0}$ and $E^{1}$, respectively.

Finally we introduce an equivalence relation $\sim$ on $L(X, A)$ by $E^{0} \sim E^{1}$ if there are trivial elements $F^{0}, F^{1} \in L(X, A)$ such that

$$
E^{0} \oplus F^{0} \simeq E^{1} \oplus F^{1} .
$$

We define the K-theory $K(X, A)$ of the pair $(X, A)$ as the set of equivalence classes of $L(X, A)$ with respect to this equivalence relation. We denote elements in $K(X, A)$ by $\xi=$ $\left[E_{0}, E_{1}, a\right]$ and define a sum on $K(X, A)$ by

$$
\left[E_{0}^{0}, E_{1}^{0}, a_{0}\right]+\left[E_{0}^{1}, E_{1}^{1}, a_{1}\right]=\left[E_{0}^{0} \oplus E_{0}^{1}, E_{1}^{0} \oplus E_{1}^{1}, a_{0} \oplus a_{1}\right] \in K(X, A) .
$$

We omit the proof that the sum is well defined which is easy but a little bit tedious due to many appearing indices. Moreover, it is clear that $K(X, Y)$ is a monoid with this sum operation, where the neutral element is given as the equivalence class of any trivial element in $L(X, A)$.

In the following we treat the main properties of $K(X, Y)$.

We begin by discussing the functoriality of our construction and consider maps $f:(X, A) \rightarrow$ $(Y, B)$ of topological pairs which we assume throughout to be proper. We define

$$
f^{*}: K(Y, B) \rightarrow K(X, A), \quad f^{*}\left[E_{0}, E_{1}, a\right]=\left[f^{*} E_{0}, f^{*} E_{1}, f^{*} a\right]
$$

and note that $\left[f^{*} E_{0}, f^{*} E_{1}, f^{*} a\right]$ is indeed compactly supported in $X \backslash A$ because $f(A) \subset B$ and $f$ is proper.

B.1.1 Lemma. Let $g:(X, A) \rightarrow(Y, B)$ and $f:(Y, B) \rightarrow(Z, C)$ be two proper maps. Then

$$
(f \circ g)^{*}=g^{*} \circ f^{*}: K(Z, C) \rightarrow K(X, A) .
$$

Proof. This is an immediate consequence of the functoriality of the pullback construction for bundles. Indeed, we have for any $\left[E_{0}, E_{1}, a\right] \in K(Z, C)$

$$
\begin{aligned}
(f \circ g)^{*}\left[E_{0}, E_{1}, a\right] & =\left[(f \circ g)^{*} E_{0},(f \circ g)^{*} E_{1},(f \circ g)^{*} a\right]=\left[g^{*} f^{*} E_{0}, g^{*} f^{*} E_{1}, g^{*}\left(f^{*} a\right)\right] \\
& =g^{*}\left[f^{*} E_{0}, f^{*} E_{1}, f^{*} a\right]=g^{*}\left(f^{*}\left[E_{0}, E_{1}, a\right]\right) .
\end{aligned}
$$


Next we prove two different versions of homotopy invariance.

B.1.2 Lemma. If $f \simeq g:(X, A) \rightarrow(Y, B)$ are homotopic, then

$$
f^{*}=g^{*}: K(Y, B) \rightarrow K(X, A)
$$

Proof. Let $H: I \times(X, A)=(X \times I, A \times I) \rightarrow(Y, B)$ be a homotopy between $f$ and $g$. $H$ induces a map

$$
H^{*}: K(Y, B) \rightarrow K(X \times I, A \times I)
$$

and from the definition of $\simeq$ in $L(X, A)$ we infer

$$
\begin{aligned}
f^{*}\left[E_{0}, E_{1}, a\right] & =\left[f^{*} E_{0}, f^{*} E_{1}, f^{*} a\right]=\left[H_{0}^{*} E_{0}, H_{0}^{*} E_{1}, H_{0}^{*} a\right]=\left[H_{1}^{*} E_{0}, H_{1}^{*} E_{1}, H_{1}^{*} a\right] \\
& =\left[g^{*} E_{0}, g^{*} E_{1}, g^{*} a\right]=g^{*}\left[E_{0}, E_{1}, a\right]
\end{aligned}
$$

for all $\left[E_{0}, E_{1}, a\right] \in K(Y, B)$.

B.1.3 Lemma. Let $E_{0}$ and $E_{1}$ be vector bundles over $X$ and $a: I \rightarrow \operatorname{hom}\left(E_{0}, E_{1}\right)$, a path of bundle morphisms such that $\operatorname{supp}\left\{E_{0}, E_{1}, a_{t}\right\} \subset K \subset X \backslash A, t \in I$, for some compact set $K$. Then

$$
\left[E_{0}, E_{1}, a_{0}\right]=\left[E_{0}, E_{1}, a_{1}\right] \in K(X, A)
$$

Proof. If $p: X \times I \rightarrow X$ denotes the projection onto the first component, then $a$ induces a bundle morphism $a: p^{*} E_{0} \rightarrow p^{*} E_{1}$ and we can consider

$$
\left[p^{*} E_{0}, p^{*} E_{1}, a\right] \in K(X \times I, A \times I) .
$$

Now the assertion follows from the definition of $\simeq$ in $L(X, A)$.

We continue with a first consequence of the homotopy invariance.

B.1.4 Lemma. If $\left\{E_{0}, E_{1}, a_{0}\right\},\left\{E_{0}, E_{1}, a_{1}\right\} \in L(X, A)$ are such that there exists a compact neighbourhood $K \subset X \backslash A$ containing their supports and $\left.a_{0}\right|_{X \backslash K}=\left.a_{1}\right|_{X \backslash K}$, then

$$
\left[E_{0}, E_{1}, a_{0}\right]=\left[E_{0}, E_{1}, a_{1}\right] \in K(X, A) .
$$


Proof. Since $X \backslash A$ is locally compact, we can use Urysohn's lemma for locally compact spaces in order to find a relatively compact open neighbourhood $U$ of $K$ in $X \backslash A$ and a continuous function $\varphi: X \backslash A \rightarrow[0,1]$ such that $\left.\varphi\right|_{K} \equiv 1$ and $\left.\varphi\right|_{(X \backslash A) \backslash U} \equiv 0$. By extending constantly we can assume that $\varphi$ is defined on all of $X$ and $\left.\varphi\right|_{X \backslash U} \equiv 1$.

Now we consider the paths of bundle morphisms

$$
a_{i}(t)=((1-t)+(1-\varphi) t) a_{i}, t \in I, i=0,1,
$$

and note that $\operatorname{supp}\left\{E_{0}, E_{1}, a_{i}(t)\right\} \subset \bar{U} \subset X \backslash A$ for all $t \in I$ and $i=0,1$. Since $a_{0}(1) \equiv a_{1}(1)$ on all of $X$, the assertion follows by lemma B.1.3

Next we prove the so called logarithmic property of $K$ which is a further consequence of the homotopy invariance B.1.3.

B.1.5 Lemma. If $\xi_{0}=\left[E_{0}, E_{1}, a_{0}\right], \xi_{1}=\left[E_{1}, E_{2}, a_{1}\right] \in K(X, A)$, then their sum is given by

$$
\xi_{0}+\xi_{1}=\left[E_{0}, E_{2}, a_{0} \circ a_{1}\right] \in K(X, A)
$$

Proof. We note at first that we can decompose $a_{0} \oplus a_{1}$ as $\left(I \oplus a_{1}\right)\left(a_{0} \oplus I\right)$ where

$$
I \oplus a_{1}: E_{1} \oplus E_{1} \rightarrow E_{1} \oplus E_{2} \quad a_{0} \oplus I: E_{0} \oplus E_{1} \rightarrow E_{1} \oplus E_{1} .
$$

Now we define a path of bundle isomorphisms by

$$
I \rightarrow \operatorname{hom}\left(E_{1} \oplus E_{1}, E_{1} \oplus E_{1}\right), \quad t \mapsto\left(\begin{array}{cc}
\cos \left(\frac{\pi}{2} t\right) & -\sin \left(\frac{\pi}{2} t\right) \\
\sin \left(\frac{\pi}{2} t\right) & \cos \left(\frac{\pi}{2} t\right)
\end{array}\right)
$$

and obtain by composition a path $\rho: I \rightarrow \operatorname{hom}\left(E_{0} \oplus E_{1}, E_{1} \oplus E_{2}\right)$ given by

$$
\rho(t)=\left(\begin{array}{cc}
I & 0 \\
0 & a_{1}
\end{array}\right)\left(\begin{array}{cc}
\cos \left(\frac{\pi}{2} t\right) & -\sin \left(\frac{\pi}{2} t\right) \\
\sin \left(\frac{\pi}{2} t\right) & \cos \left(\frac{\pi}{2} t\right)
\end{array}\right)\left(\begin{array}{cc}
a_{0} & 0 \\
0 & I
\end{array}\right) .
$$

Note that the support of this path is constant and hence we can apply lemma B.1.3 to infer that

$$
\xi_{1}+\xi_{2}=\left[E_{0} \oplus E_{1}, E_{1} \oplus E_{2}, \rho(1)\right],
$$

where 


$$
\rho(1)=\left(\begin{array}{cc}
0 & -I \\
a_{1} a_{0} & 0
\end{array}\right)
$$

We obtain

$$
\begin{aligned}
\xi_{0}+\xi_{1}= & {\left[E_{0} \oplus E_{1}, E_{2} \oplus E_{1},\left(a_{1} \circ a_{0}\right) \oplus(-I)\right]=\left[E_{0}, E_{2}, a_{1} \circ a_{0}\right]+\left[E_{1}, E_{1},-I\right] } \\
& =\left[E_{0}, E_{2}, a_{1} \circ a_{0}\right] \in K(X, A) .
\end{aligned}
$$

Combining the last two results we now infer that $K(X, A)$ is actually a group. Indeed, if $\left[E_{0}, E_{1}, a\right] \in K(X, A)$ we use as in the proof of lemma B.1.4 Urysohn's lemma for locally compact spaces in order to choose a function $\varphi: X \rightarrow[0,1]$ such that $\varphi \equiv 1$ on a neighbourhood of the support of $\left\{E_{0}, E_{1}, a\right\}$ and $\varphi \equiv 0$ outside some larger compact subset of $X \backslash A$. Then the inverse of $\left[E_{0}, E_{1}, a\right]$ is given by

$$
-\left[E_{0}, E_{1}, a\right]=\left[E_{1}, E_{0},(1-\varphi) a^{-1}\right] \in K(X, A)
$$

which is an immediate consequence of the logarithmic property B.1.5 and lemma B.1.4 In particular, $K$ is a contravariant functor from the category of pairs of locally compact spaces and closed subspaces to the category of abelian groups.

We conclude this section by defining for any $n \in \mathbb{N}$ the higher $K$-theory groups as

$$
K^{-n}(X, A)=K\left(X \times \mathbb{R}^{n}, A \times \mathbb{R}^{n}\right) .
$$

\section{B.2 Relation to other Definitions of $K$-Theory}

In this section we want to compare the definition of $K(X, A)$ from the last section with the usual definition of $K$-theory.

At first we consider the case that $X$ is compact and $A=\emptyset$. Then $K(X)$ is usually defined as the Grothendiek group $\mathcal{G}(V e c t(X), \oplus)$ of the semigroup of isomorphism classes of vector bundles with respect to the direct sum $\oplus$ (cf. eg. At89, Ha09]). Accordingly, elements in $\mathcal{G}(\operatorname{Vect}(X), \oplus)$ are difference classes $\left[E_{0}\right]-\left[E_{1}\right]$ of vector bundles over $X$ and

$$
\left[E_{0}^{0}\right]-\left[E_{1}^{0}\right]=\left[E_{0}^{1}\right]-\left[E_{1}^{1}\right] \Longleftrightarrow E_{0}^{0} \oplus E_{1}^{1} \oplus \Theta\left(\mathbb{C}^{n}\right) \cong E_{0}^{1} \oplus E_{1}^{0} \oplus \Theta\left(\mathbb{C}^{n}\right)
$$

for some $n \in \mathbb{N}$.

We now define maps 


$$
\varphi_{X}: K(X) \rightarrow \mathcal{G}(V e c t(X), \oplus), \quad\left[E_{0}, E_{1}, a\right] \mapsto\left[E_{0}\right]-\left[E_{1}\right]
$$

and

$$
\varphi_{X}^{-1}: \mathcal{G}(\operatorname{Vect}(X), \oplus) \rightarrow K(X), \quad\left[E_{0}\right]-\left[E_{1}\right] \mapsto\left[E_{0}, E_{1}, 0\right] .
$$

Note that $\left[E_{0}, E_{1}, 0\right]$ is indeed in $K(X)$ due to the compactness of $X$ and, moreover, that $\left[E_{0}, E_{1}, a\right]=\left[E_{0}, E_{1}, 0\right]$ for any bundles $E_{0}, E_{1}$ over $X$ and any bundle morphism $a: E_{0} \rightarrow E_{1}$ which follows from lemma B.1.3. Hence we see that $\varphi_{X}$ and $\varphi_{X}^{-1}$ are indeed inverses of each other if they turn out to be well defined.

We begin by checking the well definedness of $\varphi_{X}$. Let $\left[E_{0}^{0}, E_{1}^{0}, a_{0}\right]=\left[E_{0}^{1}, E_{1}^{1}, a_{1}\right] \in K(X)$ and hence $\left[E_{0}^{0}, E_{1}^{0}, 0\right]=\left[E_{0}^{1}, E_{1}^{1}, 0\right]$. From the definition of equivalence in $K(X)$ we know that there exist bundles and isomorphisms $b_{0}: F_{0}^{0} \stackrel{\cong}{\longrightarrow} F_{1}^{0}$ and $b_{1}: F_{0}^{1} \cong F_{1}^{1}$ and an element $G \in L(X \times I)$ such that

$$
\begin{aligned}
\left.G\right|_{X \times\{0\}} & =\left\{E_{0}^{0} \oplus F_{0}^{0}, E_{1}^{0} \oplus F_{1}^{0}, 0 \oplus b_{0}\right\} \\
\left.G\right|_{X \times\{1\}} & =\left\{E_{0}^{1} \oplus F_{0}^{1}, E_{1}^{1} \oplus F_{1}^{1}, 0 \oplus b_{1}\right\} .
\end{aligned}
$$

Since homotopic bundles over paracompact spaces are isomorphic (cf. [Ha09, Prop. 1.7]), we infer that

$$
E_{0}^{0} \oplus F_{0}^{0} \cong E_{0}^{1} \oplus F_{0}^{1}, \quad E_{1}^{0} \oplus F_{1}^{0} \cong E_{1}^{1} \oplus F_{1}^{1}
$$

and so

$$
E_{0}^{0} \oplus E_{1}^{1} \oplus F_{0}^{0} \oplus F_{1}^{1} \cong E_{1}^{0} \oplus E_{0}^{1} \oplus F_{1}^{0} \oplus F_{0}^{1} .
$$

Now by [Ha09, Prop. 1.3] we can find a bundle $F$ over $X$ such that $F_{0}^{0} \oplus F_{1}^{1} \oplus F \cong F_{1}^{0} \oplus F_{0}^{1} \oplus F \cong$ $\Theta\left(\mathbb{C}^{n}\right)$ for some $n \in \mathbb{N}$ and we finally obtain

$$
E_{0}^{0} \oplus E_{1}^{1} \oplus \Theta\left(\mathbb{C}^{n}\right) \cong E_{0}^{1} \oplus E_{1}^{0} \oplus \Theta\left(\mathbb{C}^{n}\right) .
$$

Hence

$$
\left[E_{0}^{0}\right]-\left[E_{1}^{0}\right]=\left[E_{0}^{1}\right]-\left[E_{1}^{1}\right]
$$


and $\varphi_{X}$ is well defined.

In order to show that $\varphi_{X}^{-1}$ is well defined, assume that $\left[E_{0}^{0}\right]-\left[E_{1}^{0}\right]=\left[E_{0}^{1}\right]-\left[E_{1}^{1}\right] \in \mathcal{G}(\operatorname{Vect}(X), \oplus)$. Accordingly, there exists a bundle isomorphism

$$
E_{0}^{0} \oplus E_{1}^{1} \oplus \Theta\left(\mathbb{C}^{n}\right) \cong E_{1}^{0} \oplus E_{0}^{1} \oplus \Theta\left(\mathbb{C}^{n}\right)
$$

for some $n \in \mathbb{N}$ and hence

$$
\left[E_{0}^{0} \oplus E_{1}^{1} \oplus \Theta\left(\mathbb{C}^{n}\right), E_{1}^{0} \oplus E_{0}^{1} \oplus \Theta\left(\mathbb{C}^{n}\right), 0\right]=0 .
$$

Since $\left[\Theta\left(\mathbb{C}^{n}\right), \Theta\left(\mathbb{C}^{n}\right), 0\right]=0$ we infer

$$
\begin{aligned}
0 & =\left[E_{0}^{0} \oplus E_{1}^{1} \oplus \Theta\left(\mathbb{C}^{n}\right), E_{1}^{0} \oplus E_{0}^{1} \oplus \Theta\left(\mathbb{C}^{n}\right), 0\right]=\left[E_{0}^{0}, E_{1}^{0}, 0\right]+\left[E_{1}^{1}, E_{0}^{1}, 0\right]+\left[\Theta\left(\mathbb{C}^{n}\right), \Theta\left(\mathbb{C}^{n}\right), 0\right] \\
& =\left[E_{0}^{0}, E_{1}^{0}, 0\right]+\left[E_{1}^{1}, E_{0}^{1}, 0\right]
\end{aligned}
$$

and (B.1) yields

$$
\left[E_{0}^{0}, E_{1}^{0}, 0\right]=-\left[E_{1}^{1}, E_{0}^{1}, 0\right]=\left[E_{0}^{1}, E_{1}^{1}, 0\right] .
$$

Hence $\varphi_{X}^{-1}$ is well defined as well and we have shown that $K(X)$ and $\mathcal{G}(\operatorname{Vect}(X), \oplus)$ are bijective.

Finally, it is clear that $\varphi_{X}$ actually is a group isomorphism which is natural in the sense that for any two compact topological spaces $X$ and $Y$ and any map $f: Y \rightarrow X$ we have a commutative diagram

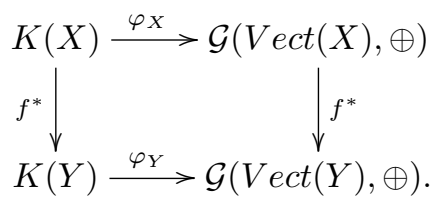

For a locally compact space $X$ the $K$-theory with compact supports is usually defined by $K_{c}(X):=\tilde{K}\left(X^{+}\right)$, where $\tilde{K}$ denotes the reduced $K$-theory group and $X^{+}$the one point compactification of $X$.

Now let $\left[E_{0}, E_{1}, a\right] \in K(X)$ be given and let $V \subset X$ be a relatively compact open neighbourhood of its support. Since $\bar{V}$ is compact, we can find a bundle $F$ over $\bar{V}$ such that $\left.E_{1}\right|_{\bar{V}} \oplus F \cong \Theta\left(\mathbb{C}^{n}\right)$. Setting $E^{\prime}=\left.E_{0}\right|_{\bar{V}} \oplus F$ and $a^{\prime}=a \oplus I$ we obtain a triple $\left\{E^{\prime}, \Theta\left(\mathbb{C}^{n}\right), a^{\prime}\right\} \in L(\bar{V})$ such that $\left.a^{\prime}\right|_{\partial V}:\left.E^{\prime}\right|_{\partial V} \rightarrow \partial V \times \mathbb{C}^{n}$ is an isomorphism. Now we can finally use this isomorphism in order 
to perform the well known clutching construction of $E^{\prime}$ and the trivial bundle $\Theta\left(\mathbb{C}^{n}\right)$ over $X^{+} \backslash V$ along $\partial V$. We denote the obtained bundle over $X^{+}$by $E^{\prime \prime}$ and define a map

$$
\varphi_{X}: K(X) \rightarrow K_{c}(X), \quad\left[E_{0}, E_{1}, a\right] \mapsto\left[E^{\prime \prime}\right]-\left[\Theta\left(\mathbb{C}^{n}\right)\right] .
$$

Conversely, if $[E]-\left[\Theta\left(\mathbb{C}^{n}\right)\right] \in \tilde{K}\left(X^{+}\right)=K_{c}(X)$, we take any trivialisation $\psi: \pi^{-1}(U) \rightarrow$ $U \times \mathbb{C}^{n}$ of $E$ on a closed neighbourhood of $\infty \in X^{+}$. Now we let $a$ be any extension of $\left.\psi\right|_{U \backslash\{\infty\}}$ to all of $X$ which exists according to the Tietze extension theorem and define a map

$$
\varphi_{X}^{-1}: K_{c}(X) \rightarrow K(X), \quad[E]-\left[\Theta\left(\mathbb{C}^{n}\right)\right] \mapsto\left[\left.E\right|_{X}, \Theta\left(\mathbb{C}^{n}\right), a\right] .
$$

We omit the proof that these maps are well defined and inverse to each other (cf. Pe12a, §3.1], [Se68, Prop. 3.1] or [LM89, §I.9]) but summarise these assertions in the following lemma.

B.2.1 Lemma. For any locally compact space $X$ there is an isomorphism

$$
\varphi_{X}: K(X) \rightarrow K_{c}(X)
$$

such that for any proper map $f: X \rightarrow Y$ we have a commutative diagram

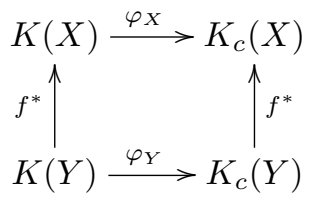

In particular, we obtain that for a compact topological space $X$

$$
K^{-n}(X)=K\left(X \times \mathbb{R}^{n}\right) \cong \tilde{K}\left(\left(X \times \mathbb{R}^{n}\right)^{+}\right)=\tilde{K}\left(\Sigma^{n}\left(X^{+}\right)\right)
$$

which is the usual definition of $K^{-n}$ (cf. [LM89, Def. 9.15]).

\section{B.3 An Example: $K^{-1}(I, \partial I) \cong \mathbb{Z}$}

In this section we construct an explicit isomorphism $c_{1}: K^{-1}(I, \partial I) \rightarrow \mathbb{Z}$.

In order to define $c_{1}$ let $\left[E_{0}, E_{1}, a\right] \in K^{-1}(I, \partial I)=K(I \times \mathbb{R}, \partial I \times \mathbb{R})$ be given. Since $I \times \mathbb{R}$ is contractible, we can find global trivialisations $\psi: E_{0} \rightarrow \Theta\left(\mathbb{C}^{n}\right)$ and $\varphi: E_{1} \rightarrow \Theta\left(\mathbb{C}^{n}\right)$. Then we define

$$
c_{1}\left(\left[E_{0}, E_{1}, a\right]\right)=w\left(\operatorname{det}\left(\varphi \circ a \circ \psi^{-1}\right) \circ \gamma, 0\right) \in \mathbb{Z},
$$


where $\gamma$ is any simple positively oriented curve surrounding the support of $\left\{E_{0}, E_{1}, a\right\}$ and $w(\cdot, 0)$ denotes the winding number for curves in $\mathbb{C}$ with respect to 0.

Before we study $c_{1}$ further, we want to recall the definition and some properties of the winding number $w(\gamma, 0)$ for curves $\gamma: S^{1} \rightarrow \mathbb{C} \backslash\{0\}$. One can give a simple definition of $w(\gamma, 0)$ by using algebraic topology. Indeed, by a well known result (cf. StZi94, 2.2.4]), any curve $\gamma: S^{1} \rightarrow \mathbb{C} \backslash\{0\}$ is homotopic to a curve $\gamma_{k}: S^{1} \rightarrow S^{1} \subset \mathbb{C} \backslash\{0\}, \gamma_{k}(z)=z^{k}$ for some $k \in \mathbb{Z}$ and, moreover, this $k$ is uniquely determined by $\gamma$ since $\gamma_{k} \simeq \gamma_{l}$ if and only if $k=l$. Now the winding number is defined by $w(\gamma, 0)=k$. From the definition we immediately obtain the following properties:

- $\gamma_{1} \simeq \gamma_{2}: S^{1} \rightarrow \mathbb{C} \backslash\{0\}$ if and only if $w\left(\gamma_{1}, 0\right)=w\left(\gamma_{2}, 0\right)$.

- If $\gamma_{1}, \gamma_{2}: S^{1} \rightarrow \mathbb{C} \backslash\{0\}$, then $w\left(\gamma_{1} \cdot \gamma_{2}, 0\right)=w\left(\gamma_{1}, 0\right)+w\left(\gamma_{2}, 0\right)$, where $\gamma_{1} \cdot \gamma_{2}$ denotes the pointwise multiplication in $\mathbb{C}$.

Since $\gamma: S^{1} \rightarrow \mathbb{C} \backslash\{0\}$ can be extended to $D^{2}$ if and only if $\gamma$ is homotopic to a constant map (cf. [StZi94, 2.3.3]), we obtain moreover

- $\gamma: S^{1} \rightarrow \mathbb{C} \backslash\{0\}$ can be extended to $D^{2}$ if and only if $w(\gamma, 0)=0$.

The aim of this section is to prove that $c_{1}$ is an isomorphism. We begin by showing that it is a homomorphism and in particular well defined.

B.3.1 Lemma. $c_{1}$ is a homomorphism.

Proof. First of all we note that $w\left(\operatorname{det}\left(\varphi \circ a \circ \psi^{-1}\right) \circ \gamma, 0\right)$ does not depend on the particular choice of the curve $\gamma$, because any two closed simple curves that surround the support of $\left\{E_{0}, E_{1}, a\right\}$ are homotopic.

We split the rest of the proof into three consecutive steps.

\section{Step 1: $c_{1}: L(I \times \mathbb{R}, \partial I \times \mathbb{R}) \rightarrow \mathbb{Z}$ is well defined}

At first we show the independence of $w\left(\operatorname{det}\left(\varphi \circ a \circ \psi^{-1}\right) \circ \gamma, 0\right)$ on the trivialisations. Let $\left\{E_{0}, E_{1}, a\right\} \in L(I \times \mathbb{R}, \partial I \times \mathbb{R})$ and

$$
\psi_{1}, \psi_{2}: E_{0} \rightarrow \Theta\left(\mathbb{C}^{n}\right), \quad \varphi_{1}, \varphi_{2}: E_{1} \rightarrow \Theta\left(\mathbb{C}^{n}\right)
$$

be two pairs of trivialisations. We obtain

$$
\begin{aligned}
& w\left(\operatorname{det}\left(\varphi_{1} \circ a \circ \psi_{1}^{-1}\right) \circ \gamma, 0\right)=w\left(\operatorname{det}\left(\varphi_{1} \circ \varphi_{2}^{-1} \circ \varphi_{2} \circ a \circ \psi_{2}^{-1} \circ \psi_{2} \circ \psi_{1}^{-1}\right) \circ \gamma, 0\right) \\
& =w\left(\operatorname{det}\left(\varphi_{1} \circ \varphi_{2}^{-1}\right) \circ \gamma, 0\right)+w\left(\operatorname{det}\left(\varphi_{2} \circ a \circ \psi_{2}^{-1}\right) \circ \gamma, 0\right)+w\left(\operatorname{det}\left(\psi_{2} \circ \psi_{1}^{-1}\right) \circ \gamma, 0\right) \\
& =w\left(\operatorname{det}\left(\varphi_{2} \circ a \circ \psi_{2}^{-1}\right) \circ \gamma, 0\right),
\end{aligned}
$$


where the last equality follows from the third property of the winding number stated above. Next, let

$$
\left\{E_{0}^{0}, E_{1}^{0}, a_{0}\right\}=\left\{E_{0}^{1}, E_{1}^{1}, a_{1}\right\} \in L(I \times \mathbb{R}, \partial I \times \mathbb{R})
$$

be isomorphic in the sense that there are bundle isomorphisms $\Phi_{1}: E_{0}^{0} \rightarrow E_{0}^{1}$ and $\Phi_{2}: E_{1}^{0} \rightarrow$ $E_{1}^{1}$ such that the diagram

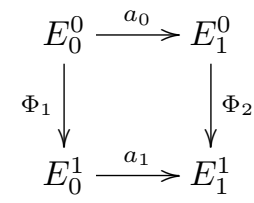

commutes. Furthermore, let

$$
\psi_{1}: E_{0}^{0} \rightarrow \Theta\left(\mathbb{C}^{n}\right), \psi_{2}: E_{0}^{1} \rightarrow \Theta\left(\mathbb{C}^{n}\right), \varphi_{1}: E_{1}^{0} \rightarrow \Theta\left(\mathbb{C}^{n}\right), \varphi_{2}: E_{1}^{1} \rightarrow \Theta\left(\mathbb{C}^{n}\right)
$$

be trivialisations. Then, using the independence already proved, we obtain

$$
\begin{aligned}
w\left(\operatorname{det}\left(\varphi_{1} \circ a_{0} \circ \psi_{1}^{-1}\right) \circ \gamma, 0\right) & =w\left(\operatorname{det}\left(\varphi_{1} \circ \Phi_{2}^{-1} \circ a_{1} \circ \Phi_{1} \circ \psi_{1}^{-1}\right) \circ \gamma, 0\right) \\
& =w\left(\operatorname{det}\left(\varphi_{2} \circ a_{1} \circ \psi_{2}^{-1}\right) \circ \gamma, 0\right) .
\end{aligned}
$$

Hence $c_{1}$ is well defined on $L(I \times \mathbb{R}, \partial I \times \mathbb{R})$.

Step 2: $c_{1}: L(I \times \mathbb{R}, \partial I \times \mathbb{R}) \rightarrow \mathbb{Z}$ is a homomorphism

Let $\left\{E_{0}^{0}, E_{1}^{0}, a_{0}\right\}$ and $\left\{E_{0}^{1}, E_{1}^{1}, a_{1}\right\}$ be two elements in $L(I \times \mathbb{R}, \partial I \times \mathbb{R})$. Using the same notation as in B.2. once again, $c_{1}$ is given on the sum

$$
\left\{E_{0}^{0}, E_{1}^{0}, a_{0}\right\}+\left\{E_{0}^{1}, E_{1}^{1}, a_{1}\right\}=\left\{E_{0}^{0} \oplus E_{0}^{1}, E_{0}^{1} \oplus E_{1}^{1}, a_{0} \oplus a_{1}\right\}
$$

by

$$
\begin{aligned}
& w\left(\operatorname{det}\left(\left(\varphi_{0} \oplus \varphi_{1}\right) \circ\left(a_{0} \oplus a_{1}\right) \circ\left(\psi_{0}^{-1} \oplus \psi_{1}^{-1}\right) \circ \gamma, 0\right)\right. \\
& =w\left(\operatorname{det}\left(\left(\varphi_{0} \circ a_{0} \circ \psi_{0}^{-1}\right) \oplus\left(\varphi_{1} \circ a_{1} \circ \psi_{1}^{-1}\right) \circ \gamma, 0\right)\right. \\
& =w\left(\operatorname{det}\left(\varphi_{0} \circ a_{0} \circ \psi_{0}^{-1}\right) \operatorname{det}\left(\varphi_{1} \circ a_{1} \circ \psi_{1}^{-1}\right) \circ \gamma, 0\right) \\
& =w\left(\operatorname{det}\left(\varphi_{0} \circ a_{0} \circ \psi_{0}^{-1}\right) \circ \gamma, 0\right)+w\left(\operatorname{det}\left(\varphi_{1} \circ a_{1} \circ \psi_{1}^{-1}\right) \circ \gamma, 0\right)
\end{aligned}
$$

Hence $c_{1}$ is a homomorphism. 
Step 3: $c_{1}: K(I \times \mathbb{R}, \partial I \times \mathbb{R}) \rightarrow \mathbb{Z}$ is well defined

First of all, $\left[E_{0}^{0}, E_{1}^{0}, a_{0}\right]=\left[E_{0}^{1}, E_{1}^{1}, a_{2}\right]$ if there exist trivial elements $\eta_{1}, \eta_{2} \in L(I \times \mathbb{R}, \partial I \times \mathbb{R})$ such that

$$
\left\{E_{0}^{0}, E_{1}^{0}, a_{0}\right\} \oplus \eta_{1} \simeq\left\{E_{0}^{1}, E_{1}^{1}, a_{2}\right\} \oplus \eta_{2} .
$$

From the second step and $c_{1}\left(\eta_{1}\right)=c_{1}\left(\eta_{2}\right)=0$ we infer that it suffices to show that homotopic elements in $L(I \times \mathbb{R}, \partial I \times \mathbb{R})$ are mapped to the same integer.

Accordingly, let $\left[G_{0}, G_{1}, a\right] \in L(I \times(I \times \mathbb{R}), I \times(\partial I \times \mathbb{R}))$. Since $I \times I \times \mathbb{R}$ is contractible we can find as above global trivialisations

$$
\varphi: G_{0} \rightarrow \Theta\left(\mathbb{C}^{n}\right), \quad \psi: G_{1} \rightarrow \Theta\left(\mathbb{C}^{n}\right)
$$

Consider the map

$$
\lambda \mapsto \psi_{\lambda} \circ a_{\lambda} \circ \varphi_{\lambda}^{-1}, \quad \lambda \in[0,1],
$$

which is a bundle endomorphism of the trivial bundle $\Theta\left(\mathbb{C}^{n}\right)$ over $I \times \mathbb{R}$ for each $\lambda \in[0,1]$. Now we take a simple positively oriented curve $\gamma$ surrounding the union of all supports of the $a_{\lambda}, \lambda \in I$, in $I \times \mathbb{R}$ which indeed exists because of the compactness of the support of $a$. Then we obtain by the homotopy invariance of the winding number

$$
w\left(\operatorname{det}\left(\psi_{0} \circ a_{0} \circ \varphi_{0}^{-1}\right) \circ \gamma, 0\right)=w\left(\operatorname{det}\left(\psi_{1} \circ a_{1} \circ \varphi_{1}^{-1}\right) \circ \gamma, 0\right) .
$$

Using the homotopy invariance of the degree, it turns out that the computation of $c_{1}\left(\left[E_{0}, E_{1}, a\right]\right)$ amounts to the well known computation of degrees of maps $S^{1} \rightarrow G L(n)$.

B.3.2 Lemma. For every $\left[E_{0}, E_{1}, a\right] \in K^{-1}(I, \partial I)$, there exists a unique $k \in \mathbb{Z}$ such that

$$
\left[E_{0}, E_{1}, a\right]=\left[\Theta(\mathbb{C}), \Theta(\mathbb{C}), a_{k}\right] \in K^{-1}(I, \partial I),
$$

where $a_{k}(z): \mathbb{C} \rightarrow \mathbb{C}, z \in I \times \mathbb{R}$, is given by multiplication by $z^{k}$. In particular, $c_{1}$ : $K^{-1}(I, \partial I) \rightarrow \mathbb{Z}$ is an isomorphism.

Proof. We can assume without loss of generality that $\left[E_{0}, E_{1}, a\right]=\left[\Theta\left(\mathbb{C}^{n}\right), \Theta\left(\mathbb{C}^{n}\right), a\right]$. Moreover, since the support of $\left\{E_{0}, E_{1}, a\right\}$ is a compact subset of $(I \backslash \partial I) \times \mathbb{R}$, we can choose a rectangle $R=I \times[-c, c], c>0$, such that the support of $\left\{E_{0}, E_{1}, a\right\}$ is contained in its interior. 
We note that any map $a: \partial R \rightarrow G L(n)$ is homotopic to a path in $U(n)$ since $U(n)$ is a deformation retract of $G L(n)$. Moreover, from the exact sequences of the fibre bundles

$$
U(N-1) \hookrightarrow U(N) \rightarrow S^{2 N-1}, \quad N \geq 2,
$$

we infer that the inclusions induce isomorphisms

$$
\iota_{*}: \pi_{1}(U(N-1)) \stackrel{\cong}{\longrightarrow} \pi_{1}(U(N)), \quad N \geq 2 .
$$

Since $U(1)=S^{1}$, we finally obtain that $a: \partial R \rightarrow G L(n)$ is homotopic to one and only one of the maps

$$
\tilde{a}_{k}: \partial R \rightarrow G L(n), \quad \tilde{a}_{k}(z)=\left(\begin{array}{cccc}
z^{k} & 0 & \cdots & 0 \\
0 & 1 & \cdots & 0 \\
\vdots & & \ddots & \vdots \\
0 & \cdots & \cdots & 1
\end{array}\right), \quad k \in \mathbb{Z} .
$$

Now, let $\left[\Theta\left(\mathbb{C}^{n}\right), \Theta\left(\mathbb{C}^{n}\right), a\right] \in K^{-1}(I, \partial I)$ be given. Since $\partial R$ is a deformation retract of

$$
A:=\partial R \cup(I \times[c, \infty)) \cup(I \times(-\infty,-c]),
$$

it is readily seen that there exist a uniquely determined $k \in \mathbb{Z}$ and a homotopy $H: I \times(I \times$ $\mathbb{R}) \rightarrow M(n, \mathbb{C})$ such that $H(I \times A) \subset G L(n), H(0, \cdot)=a$ and $H(1, z)=\tilde{a}_{k}(z), z \in A$, where now $\tilde{a}_{k}$ is defined on $I \times \mathbb{R}$ by the same formula as its restriction to $\partial R$ above. Now we infer by lemma B.1.3 and lemma B.1.4

$$
\left[\Theta\left(\mathbb{C}^{n}\right), \Theta\left(\mathbb{C}^{n}\right), a\right]=\left[\Theta\left(\mathbb{C}^{n}\right), \Theta\left(\mathbb{C}^{n}\right), \tilde{a}_{k}\right]
$$

and therefore

$$
\left[\Theta\left(\mathbb{C}^{n}\right), \Theta\left(\mathbb{C}^{n}\right), a\right]=\left[\Theta\left(\mathbb{C}^{n}\right), \Theta\left(\mathbb{C}^{n}\right), \tilde{a}_{k}\right]=\left[\Theta(\mathbb{C}), \Theta(\mathbb{C}), a_{k}\right]
$$

where $a_{k}(z): \mathbb{C} \rightarrow \mathbb{C}, z \in I \times \mathbb{R}$, is given by multiplication by $z^{k}$.

Finally, $c_{1}$ is an epimorphism, since any $k \in \mathbb{Z}$ has $\left[\Theta(\mathbb{C}), \Theta(\mathbb{C}), a_{k}\right]$ as a counterimage. Moreover, if $c_{1}\left(\left[E_{0}^{0}, E_{1}^{0}, b_{0}\right]\right)=c_{1}\left(\left[E_{0}^{1}, E_{1}^{1}, b_{1}\right]\right)=k$, we obtain that $\left[E_{0}^{0}, E_{1}^{0}, b_{0}\right]=\left[E_{0}^{1}, E_{1}^{1}, b_{0}\right]=$ $\left[\Theta(\mathbb{C}), \Theta(\mathbb{C}), a_{k}\right]$ by the uniqueness of $k$.

B.3.3 Remark. By using Chern-Weil theory, it is not difficult to show that $c_{1}: K^{-1}(I, \partial I) \rightarrow \mathbb{Z}$ is indeed the isomorphism induced by the first Chern number (cf. [Wa10, §3.1]). 


\section{Bibliography}

[AM09] A. Abbondandolo, P. Majer, Infinite Dimensional Grassmannians, J. Operator Theory 61, 2009, 19-62

[AmM07] A. Ambrosetti, A. Malchiodi, Nonlinear Analysis and Semilinear Elliptic Problems, Cambridge Studies in Advanced Mathematics 104, Cambridge University Press, 2007

[ApVä05] J. Appell, M. Väth, Elemente der Funktionalanalysis, Friedr. Vieweg \& Sohn Verlag, Wiesbaden, 2005

[Ar67] V.I. Arnold, A Characteristic Class Entering in Quantization Conditions, Func. Ana. Appl. 1, 1967, 1-14

[At89] M.F. Atiyah, K-Theory, Addison-Wesley, 1989

[AS69] M.F. Atiyah, I.M. Singer, Index Theory for Skew-Adjoint Fredholm Operators, Inst. Hautes Etudes Sci. Publ. Math. 37, 1969, 5-26

[APS76] M.F. Atiyah, V.K. Patodi, I.M. Singer, Spectral Asymmetry and Riemannian Geometry III, Proc. Cambridge Philos. Soc., 1979, 71-99

[BEE96] J.K. Beem, P.E. Ehrlich, K.L. Easley, Global Lorentzian Geometry, Marcel Dekker, 1996

[BJ82] T. Bröcker, K. Jänich, Introduction to Differential Topology, Cambridge University Press, 1982

[BJS03] U. Bunke, M. Joachim, S. Stolz, Classifying spaces and spectra representing the K-theory of a graded $C^{*}$-algebra, High-dimensional manifold topology, World Sci. Publishing, 2003, 80-114

[BLP05] B. Booß-Bavnbek, M. Lesch, J. Phillips, Unbounded Fredholm Operators and Spectral Flow, Canad. J. Math. 57, 2005, 225-250

[BoFu98] B. Booß-Bavnbek, K. Furutani, The Maslov Index: a Functional Analytic Definition and the Spectral Flow Formula, Tokyo J. Math. 21, 1998, 1-35 
[BoBl85] B. Booss, D. Bleecker, Topology and Analysis: The Atiyah-Singer Index Formula and Gauge-Theoretic Physics, Universitext, Springer, New York, 1985

[BW85] B. Booß, K. Wojciechowski, Desuspension of Splitting Elliptic Symbols I, Ann. Glob. Analysis and Geometry 3, 1985, 337-383

[Br93] G.E. Bredon, Topology and Geometry, Graduate Texts in Mathematics 139, Springer, 1993

[BS83] Y. Benyamini, Y. Sternfeld, Spheres in Infinite Dimensional Normed Linear Spaces are Lipschitz Contractible, Proc. Amer. Math. Soc. 88, 1983, 439-445

[CFP00] E. Ciriza, P.M. Fitzpatrick, J. Pejsachowicz, Uniqueness of Spectral Flow, Math. Comp. Mod. 32, 2000, 1495-1501

[CLM94] S.E. Cappel, R. Lee, E. Miller, On the Maslov Index, Comm. Pure Appl. Math. 47, 1994, 121-186

[Co90] J.B. Conway, A Course in Functional Analysis, Graduate Texts of Mathematics 96, second edition, Springer-Verlag, 1990

[CEPPT02] J.C. Correa Eidam, A.L. Pereira, P. Piccione, D.V. Tausk, On the Equality between the Maslov Index and the Spectral Index for the Semi-Riemannian Jacobi Operator, J. Math. Anal. Appl. 268, 2002, 564-589

[Dei85] K. Deimling, Nonlinear Functional Analysis, Springer-Verlag, 1985

[Do55] A. Dold, Über fasernweise Homotopieäquivalenz von Faserräumen, Mathematische Zeitschrift 62, 1955, 111-136

[Do80] A. Dold, Lectures on Algebraic Topology, Springer-Verlag, 2nd edition, 1980

[Dob06] M. Dobrowolski, Angewandte Funktionalanalysis, Springer, 2006

[Dr99] A. N. Dranishnikov, Cohomological dimension theory of compact metric spaces, Topology Atlas, 1999, arxiv:math/0501523v1

[Fed90] V.V. Fedorchuk, The Fundamentals of Dimension Theory, Encyclopaedia of Mathematical Sciences 17, General Topology I, 1990, 91-202

[Fe91] B.V. Fedosov, Index Theorems, Encyclopaedia of Mathematical Sciences 65, Partial Differential Equations VIII, 1991, 155-251

[FO91] K. Furutani, N. Otsuki, Spectral Flow and Maslov Index Arising from Lagrangian Intersections, Tokyo J. Math. 14, 1991, 135-150 
[FP88] P.M. Fitzpatrick, J. Pejsachowicz, The Fundamental Group of the Space of Linear Fredholm Operators and the Global Analysis of Semilinear Equations, Contemporary Mathematics 72, 1988, 47-87

[FP91] P.M. Fitzpatrick, J. Pejsachowicz, Nonorientability of the Index Bundle and Several-Parameter Bifurcation, Journal of Functional Analysis 98, 1991, 42-58

[FPR94] P.M. Fitzpatrick, J. Pejsachowicz, P.J. Rabier, Orientability of Fredholm Families and Topological Degree for Nonlinear Fredholm Mappings, Journal of Functional Analysis 124, 1994, 1-39

[FPR99] P.M. Fitzpatrick, J. Pejsachowicz, L. Recht, Spectral Flow and Bifurcation of Critical Points of Strongly-Indefinite Functionals-Part I: General Theory, Journal of Functional Analysis 162, 1999, 52-95

[FT94] P.M. Fitzpatrick, M. Testa, The Parity of Paths of Closed Fredholm Operators of Index Zero, Differential and Integral Equations 7, 1994, 823-846

[GGK90] I. Gohberg, S. Goldberg, M.A. Kaashoek, Classes of Linear Operators Vol. I, Operator Theory: Advances and Applications Vol. 49, Birkhäuser, 1990

[GMPT01] F. Giannoni, A. Masiello, P. Piccione, D.V. Tausk, A Generalized Index Theorem for Morse-Sturm Systems and Applications to Semi-Riemannian Geometry, Asian J. Math. 5, 2001, 441-472

[Gr73] M.J. Greenberg, Lectures on Algebraic Topology, Mathematics Lecture Note Series, W.A. Benjamin, Inc., 1973

[Ha02] A. Hatcher, Algebraic Topology, Cambridge University Press, 2002

[Ha09] A. Hatcher, Vector Bundles and K-Theory, work in progress

[Hel94] A.D. Helfer, Conjugate Points on Spacelike Geodesics or Pseudo-Selfadjoint Morse-Sturm-Liouville Systems, Pacific J. Math. 164, 1994, 321-340

[He92] H. Heuser, Funktionalanalysis, 3. edition, B.G. Teubner Stuttgart, 1992

[He95] H.Heuser, Gewöhnliche Differentialgleichungen, 3. edition, B.G. Teubner Stuttgart, 1995

[Hu94] D. Husemoller, Fibre Bundles, Graduate Texts in Mathematics 20, 3. edition, Springer, 1994

[HW48] W. Hurewicz, H. Wallmann, Dimension Theory, Princeton Mathematical Series 4, Princeton University Press, 1948 
[I165] L. Illusie, Contractibilité du groups linéaire des espaces de Hilbert de dimension infinie, Seminaire Bourbaki 17, 1965

[Jo03] M. Joachim, Unbounded Fredholm Operators and K-Theory, Highdimensional Manifold Topology,World Sci. Publishing, 2003, 177-199

[Ka76] T. Kato, Perturbation Theory of Linear Operators, Grundlehren der mathematischen Wissenschaften 132, 2nd edition, Springer, 1976

[KL04] P. Kirk, M. Lesch, The Eta-Invariant, Maslov Index, and Spectral Flow for Dirac Type Operators on Manifolds with Boundary, Forum Math. 16, 2004, $553-629$

[Kli82] W. Klingenberg, Riemannian Geometry, de Gruyter, 1982

[Kui65] N.H. Kuiper, The Homotopy Type of the Unitary Group of Hilbert Space, Topology 3, 1965, 19-30

[La95] S. Lang, Differential and Riemannian Manifolds, Graduate Texts in Mathematics 160, Springer, 1995

[LM89] H.B. Lawson, M-L Michelsohn, Spin Geometry, Princeton University Press, 1989

[Le97] J.M. Lee, Riemannian Manifolds, Graduate Texts in Mathematics 176, Springer, 1997

[Le05] M. Lesch, The Uniqueness of the Spectral Flow on Spaces of Unbounded Self-adjoint Fredholm Operators, Cont. Math. Amer. Math. Soc. 366, 2005, 193224

[Ma99] J.P. May, A Concise Course in Algebraic Topology, Chicago University Press, 2nd edition, 1999

[MDS95] D. McDuff, D. Salamon, Introduction to Symplectic Topology, Oxford Science, 1995

[MPT02] F. Mercuri, P. Piccione, D. Tausk, Stability of the Focal and the Geometric Index in Semi-Riemannian Geometry via the Maslov-Index, Pacific J. Math. 206, 2002, 375-400

[Mi69] J.W. Milnor, Morse Theory, Princeton University Press, 1969

[Mi70] B.S. Mityagin, The Homotopy Structure of the Linear Group of a Banach Space, Russ. Math. Surv. 25, 1970, 59-103

[Mi88] P.W. Michor, Gauge Theory for Diffeomorphism Groups, Differential Geometrical Methods in Theoretical Physics, 345-371 
[Mo34] M. Morse, The Calculus of Variations in the Large, Colloquium Publications Amer. Math. Soc. 18, 1934

[MS74] J.W. Milnor, J.D. Stasheff, Characteristic Classes, Princeton University Press, 1974

[MPP05] M. Musso, J. Pejsachowicz, A. Portaluri, A Morse Index Theorem for Perturbed Geodesics on Semi-Riemannian Manifolds, Topological Methods in Nonlinear Analysis 25, 2005, 69-99

[MPP07] M. Musso, J. Pejsachowicz, A. Portaluri, Morse Index and Bifurcation for pGeodesics on Semi-Riemannian Manifolds, ESAIM Control Optim. Calc. Var 13, 2007, 598-621

[Ne68] G. Neubauer, Homotopy Properties of Semi-Fredholm Operators in Banach Spaces, Math. Annalen 176, 1968, 273-301

[Ni93] L. Nicolaescu, The Maslov Index, the Spectral Flow and Splittings of Manifolds, C.R. Acad. Sci. Paris 317, 1993, 1515-1519

[Ni97] L. Nicolaescu, Generalized Symplectic Geometries and the Index of Families of Elliptic Problems, Memoirs AMS 128, 1997

[Ni07] L. Nicolaescu, On the Space of Fredholm Operators, An. Sti. Univ. Iasi. 53, 2007, 209-227

[Pa63] R.S. Palais, Morse Theory on Hilbert Manifolds, Topology 2, 1963, 299-340

[PT88] R.S. Palais, C-l Terng, Critical Point Theory and Submanifold Geometry, Springer-Verlag, 1988

[Pe88] J. Pejsachowicz, K-theoretic Methods in Bifurcation Theory, Cont. Math. 72, $1988,47-87$

[Pe01] J. Pejsachowicz, Index Bundle, Leray-Schauder Reduction and Bifurcation of Solutions of Nonlinear Elliptic Boundary Value Problems, Topological Methods in Nonlinear Analysis 18, 2001, 243-269

[Pe07] J. Pejsachowicz, Bifurcation of Homoclinics of Hamiltonian Systems, Proc. AMS 136, 2008, 2055-2065

[Pe08] J. Pejsachowicz, Topological Invariants of Bifurcation, $C^{*}$-Algebras and Elliptic Theory II, 239-250

[Pe12a] J. Pejsachowicz, Bifurcation of Fredholm Maps I; The Index Bundle and Bifurcation, Topological Methods in Nonlinear Analysis, to appear 
[Pe12b] J. Pejsachowicz, Bifurcation of Fredholm Maps II; The Dimension of the Set of Bifurcation Points, Topological Methods in Nonlinear Analysis, to appear

[Ph96] J. Phillips, Self-adjoint Fredholm Operators and Spectral Flow, Canad. Math. Bull. 39, 1996, 460-467

[PR82] H. Porta, L. Recht, Morse Theory for Bundles, Adv. Math., 45, 144-188

[PR05] Y. Pinchover, J. Rubinstein, An Introduction to Partial Differential Equations, Cambridge University Press, 2005

[PT00] P. Piccione, D.V. Tausk, The Maslov Index and a Generalized Morse Index Theorem for Non-Positive Definite Metrics, C.R. Acad. Sci. Paris, 331, Serie 1, 2000, 385-389

[PT02] P. Piccione, D.V. Tausk, The Morse Index Theorem in Semi-Riemannian Geometry, Topology 41, 2002, 1123-1159

[RS95] J. Robbin, D. Salamon, The Spectral Flow and the Maslov Index, Bull. London Math. Soc 27, 1995, 1-33

[Se68] G. Segal, Equivariant K-Theory, Publ. Math. Inst. Hautes Etudes Sci., 34, 1968 , $129-151$

[Sp81] E.H. Spanier, Algebraic Topology, Springer-Verlag, Corr. 3rd. printing, 1981

[St51] N. Steenrod, The Topology of Fibre Bundles, Princeton University Press, 1951

[StZi94] R. Stöcker, H. Zieschang, Algebraische Topologie, B.G. Teubner Stuttgart, 2. Aufl., 1994

[Sz08] D. Szeghi, On the Conjugate Locus of pseudo-Riemannian Manifolds, Indag. Mathem., N.S. 19, 2008, 465-480

[Te09] G. Teschl, Mathematical Methods in Quantum Mechanics, Graduate Studies in Mathematics 99, AMS, 2009

[Wa65] F. W. Warner, The Conjugate Locus of a Riemannian Manifold, American Journal of Mathematics 87, 1965, 575-604

[Wa07] N. Waterstraat, Der Spektralindex perturbierter semi-Riemannscher Geodäten als Windungszahl, diploma thesis, University of Göttingen, 2007

[Wa10] N. Waterstraat, A K-theoretic Proof of the Morse Index Theorem in semiRiemannian Geometry, arXiv:1007.1190

[Wa12] N. Waterstraat, A K-theoretic Proof of the Morse Index Theorem in semiRiemannian Geometry, Proc. Amer. Math. Soc., to appear 
[W08] C. Wahl, A New Topology on the Space of Unbounded Selfadjoint Operators, K-Theory and Spectral Flow, $C^{*}$-Algebras and Elliptic Theory II, Birkhäuser, Trends in Mathematics, 2008, 297-309

[Wei80] J. Weidmann, Linear Operators in Hilbert Spaces, Graduate Texts in Mathematics 68, Springer-Verlag, 1980

[We05] D. Werner, Funktionalanalysis, 5. Auflage, Springer, 2005

[Yo95] K. Yosida, Functional Analysis, Classics in Mathematics, Springer-Verlag, 6th edition, 1995

[ZKKP75] M.G. Zaidenberg, S.G. Krein, P.A. Kuchment, A.A. Pankov, Banach Bundles and Linear Operators, Russian Math. Surveys 30:5, 1975, 115-175 


\section{Curriculum Vitae}

\section{Personal}

$\begin{array}{ll}\text { Name } & \text { Nils Waterstraat } \\ \text { Address } & \text { Lärchenweg 20 } \\ & 37079 \text { Göttingen } \\ \text { Date of birth } & 24.05 .1983 \\ \text { Place of birth } & \text { Wolfsburg } \\ \text { Nationality } & \text { German }\end{array}$

\section{Academic}

Research assistant at department of mathematics

at University of Göttingen

Fellow of research training group 1493

"Mathematical Structures in Modern Quantum Physics"

funded by German Research Foundation (DFG)

University of Göttingen

$10 / 2004-12 / 2007$

Study of mathematics, minor theoretical physics

Certificate: Diploma (1,0; with distinction)

Technical University of Clausthal

10/2002-09/2004

Study of mathematics, minor physics

Certificate: intermediate diploma $(1,0)$

Secondary school "Theodor-Heuss-Gymnasium" in Wolfsburg

1995-2002

since $04 / 2011$

04/2008-03/2011

Certificate: Abitur $(1,4)$ 